\title{
THE IMPACT OF MUSIC ON HUMAN DEVELOPMENT AND WELL-BEING
}

EDITED BY: Michele Biasutti, Graham F. Welch, Jennifer MacRitchie,

Gary E. McPherson and Evangelos Himonides PUBLISHED IN: Frontiers in Psychology

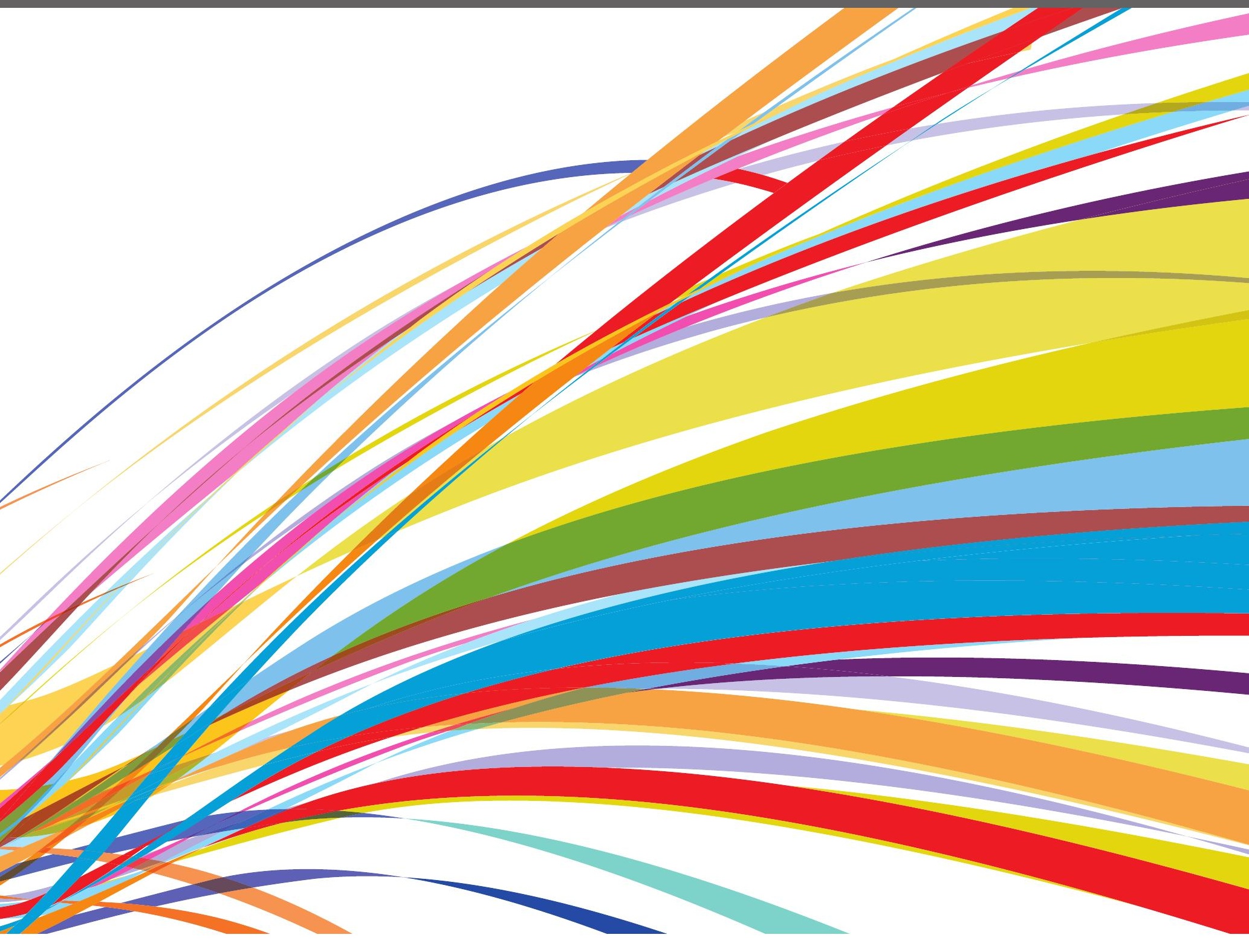

frontiers Research Topics 


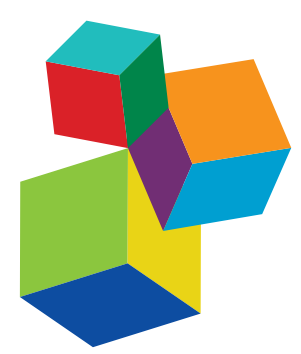

Frontiers eBook Copyright Statement

The copyright in the text of individual articles in this eBook is the property of their respective authors or their respective institutions or funders. The copyright in graphics and images within each article may be subject to copyright of other parties. In both cases this is subject to a license granted to Frontiers.

The compilation of articles constituting this eBook is the property of Frontiers.

Each article within this eBook, and the eBook itself, are published under the most recent version of the Creative Commons CC-BY licence. The version current at the date of publication of this eBook is CC-BY 4.0. If the CC-BY licence is updated, the licence granted by Frontiers is automatically updated to the new version.

When exercising any right under the CC-BY licence, Frontiers must be attributed as the original publisher of the article or eBook, as applicable.

Authors have the responsibility of ensuring that any graphics or other materials which are the property of others may be included in the

CC-BY licence, but this should be checked before relying on the

CC-BY licence to reproduce those materials. Any copyright notices relating to those materials must be complied with.

Copyright and source acknowledgement notices may not be removed and must be displayed in any copy, derivative work or partial copy which includes the elements in question.

All copyright, and all rights therein, are protected by national and international copyright laws. The above represents a summary only.

For further information please read Frontiers' Conditions for Website

Use and Copyright Statement, and the applicable CC-BY licence.

ISSN 1664-8714

ISBN 978-2-88963-683-9

DOI 10.3389/978-2-88963-683-9

\section{About Frontiers}

Frontiers is more than just an open-access publisher of scholarly articles: it is a pioneering approach to the world of academia, radically improving the way scholarly research is managed. The grand vision of Frontiers is a world where all people have an equal opportunity to seek, share and generate knowledge. Frontiers provides immediate and permanent online open access to all its publications, but this alone is not enough to realize our grand goals.

\section{Frontiers Journal Series}

The Frontiers Journal Series is a multi-tier and interdisciplinary set of open-access, online journals, promising a paradigm shift from the current review, selection and dissemination processes in academic publishing. All Frontiers journals are driven by researchers for researchers; therefore, they constitute a service to the scholarly community. At the same time, the Frontiers Journal Series operates on a revolutionary invention, the tiered publishing system, initially addressing specific communities of scholars, and gradually climbing up to broader public understanding, thus serving the interests of the lay society, too.

\section{Dedication to Quality}

Each Frontiers article is a landmark of the highest quality, thanks to genuinely collaborative interactions between authors and review editors, who include some of the world's best academicians. Research must be certified by peers before entering a stream of knowledge that may eventually reach the public - and shape society; therefore, Frontiers only applies the most rigorous and unbiased reviews.

Frontiers revolutionizes research publishing by freely delivering the most outstanding research, evaluated with no bias from both the academic and social point of view. By applying the most advanced information technologies, Frontiers is catapulting scholarly publishing into a new generation.

\section{What are Frontiers Research Topics?}

Frontiers Research Topics are very popular trademarks of the Frontiers Journals Series: they are collections of at least ten articles, all centered on a particular subject. With their unique mix of varied contributions from Original Research to Review Articles, Frontiers Research Topics unify the most influential researchers, the latest key findings and historical advances in a hot research area! Find out more on how to host your own Frontiers Research Topic or contribute to one as an author by contacting the Frontiers Editorial Office: researchtopics@frontiersin.org 


\section{THE IMPACT OF MUSIC ON HUMAN DEVELOPMENT AND WELL-BEING}

Topic Editors:

Michele Biasutti, University of Padova, Italy

Graham F. Welch, University College London, England

Jennifer MacRitchie, Western Sydney University, Australia

Gary E. McPherson, University of Melbourne, Australia

Evangelos Himonides, University College London, England

Music is one of the most universal ways of expression and communication in human life and is present in the everyday lives of people of all ages and from all cultures around the world. Music represents an enjoyable activity in and of itself, but its influence goes beyond simple amusement. Listening to music, singing, playing, composing and improvising, individually and collectively, are common activities for many people: these activities not only allow the expression of personal inner states and feelings, but also can bring many positive effects to those who engage in them. There is an increasing wealth of literature concerning the wider benefits of musical activity, and research in the sciences associated with music suggests that there are many dimensions of human life (physical, social, psychological-including cognitive and emotional) which can be affected positively by music. The impact that musical activity has on human life can be found in different processes, including a transfer of learning from the musical to another cognitive domain. Abilities that have been developed through music education and training may also be effectively applied in other cognitive tasks. Engagement in successful music activity may also have a positive impact on social skills and social inclusion, thus supporting the participation of the individual in collective and collaborative musical events. The promotion of social participation through music can foster many kinds of inclusion, including intercultural, intergenerational, and support for those who are differently abled. The aim of this Research Topic is to present a diverse range of original articles that investigate and discuss, in different ways, the crucial role that musical activity can play in human development and well-being.

Citation: Biasutti, M., Welch, G. F., MacRitchie, J., McPherson, G. E., Himonides, E., eds. (2020). The Impact of Music on Human Development and Well-Being.

Lausanne: Frontiers Media SA. doi: 10.3389/978-2-88963-683-9 


\section{Table of Contents}

05 Editorial: The Impact of Music on Human Development and Well-Being Graham F. Welch, Michele Biasutti, Jennifer MacRitchie, Gary E. McPherson and Evangelos Himonides

09 Understanding Wellbeing Among College Music Students and Amateur Musicians in Western Switzerland

Roberta Antonini Philippe, Céline Kosirnik, Noémi Vuichoud,

Aaron Williamon and Fabienne Crettaz von Roten

20 Beliefs and Values About Music in Early Childhood Education and Care: Perspectives From Practitioners

Margaret S. Barrett, Libby M. Flynn, Joanne E. Brown and Graham F. Welch

38 A 'Music, Mind and Movement' Program for People With Dementia: Initial Evidence of Improved Cognition

Olivia Brancatisano, Amee Baird and William Forde Thompson

58 Dancing to Metallica and Dora: Case Study of a 19-Month-Old

Laura K. Cirelli and Sandra E. Trehub

66 Using Music Technology Creatively to Enrich Later-Life: A Literature Review

Andrea Creech

80 "Telling me not to worry..." Hyperscanning and Neural Dynamics of Emotion Processing During Guided Imagery and Music

Jörg C. Fachner, Clemens Maidhof, Denise Grocke, Inge Nygaard Pedersen, Gro Trondalen, Gerhard Tucek and Lars O. Bonde

103 Short-Term Orchestral Music Training Modulates Hyperactivity and Inhibitory Control in School-Age Children: A Longitudinal Behavioural Study

Maria C. Fasano, Cristina Semeraro, Rosalinda Cassibba, Morten L. Kringelbach, Lucia Monacis, Valeria de Palo, Peter Vuust and Elvira Brattico

114 Flow and Satisfaction With Life in Elite Musicians and Top Athletes Katarina Habe, Michele Biasutti and Tanja Kajtna

125 Using Self-Determination Theory to Examine Musical Participation and Well-Being

Amanda E. Krause, Adrian C. North and Jane W. Davidson

137 Musical Instrument Practice Predicts White Matter Microstructure and Cognitive Abilities in Childhood

Psyche Loui, Lauren B. Raine, Laura Chaddock-Heyman, Arthur F. Kramer and Charles H. Hillman

147 Orff-Based Music Training Enhances Children's Manual Dexterity and Bimanual Coordination

Marta Martins, Leonor Neves, Paula Rodrigues, Olga Vasconcelos and São Luís Castro

159 Music, Rhythm and Trauma: A Critical Interpretive Synthesis of Research Literature

Katrina Skewes McFerran, Hsin I. Cindy Lai, Wei-Han Chang, Daniela Acquaro, Tan Chyuan Chin, Helen Stokes and Alexander Hew Dale Crooke 
171 Using Beatboxing for Creative Rehabilitation After

Laryngectomy: Experiences From a Public Engagement Project

Thomas Moors, Sanjeev Silva, Donatella Maraschin, David Young,

John M. Quinn, John de Carpentier, Johan Allouche and

Evangelos Himonides

184 The Heat is on: Effects of Synchronous Music on Psychophysiological Parameters and Running Performance in Hot and Humid Conditions

Luke Nikol, Garry Kuan, Marilyn Ong, Yu-Kai Chang and Peter C. Terry

193 Born to Speak and Sing: Musical Predictors of Language Development in Pre-schoolers

Nina Politimou, Simone Dalla Bella, Nicolas Farrugia and Fabia Franco

211 Neural Encoding of Pitch Direction is Enhanced in Musically Trained Children and is Related to Reading Skills

Vesa Putkinen, Minna Huotilainen and Mari Tervaniemi

218 Music Listening for Supporting Adolescents' Sense of Agency in Daily Life Suvi Helinä Saarikallio, William M. Randall and Margarida Baltazar

229 Music Use for Mood Regulation: Self-Awareness and Conscious Listening Choices in Young People With Tendencies to Depression

Joanna Stewart, Sandra Garrido, Cherry Hense and Katrina McFerran

241 Bridging the Clinic to Community: Music Performance as Social Transformation for Military Service Members

Rebecca Vaudreuil, Hannah Bronson and Joke Bradt

247 Exploring Wellbeing and Creativity Through Collaborative Composition as Part of Hull 2017 City of Culture

Caroline Waddington-Jones, Andrew King and Pamela Burnard

257 The Social Impact of Musical Engagement for Young Adults With Learning Difficulties: A Qualitative Study

Graeme B. Wilson and Raymond A. R. MacDonald 


\title{
Editorial: The Impact of Music on Human Development and Well-Being
}

\author{
Graham F. Welch ${ }^{1 *}$, Michele Biasutti ${ }^{2 *}$, Jennifer MacRitchie ${ }^{3}$, Gary E. McPherson ${ }^{4}$ and \\ Evangelos Himonides ${ }^{1}$
}

${ }^{1}$ Department of Culture, Communication and Media, University College London, London, United Kingdom, ${ }^{2}$ Department of Philosophy, Sociology, Education and Applied Psychology, University of Padua, Padua, Italy, ${ }^{3}$ School of Humanities and Communication Arts, Western Sydney University, Penrith, NSW, Australia, ${ }^{4}$ Melbourne Conservatorium of Music, University of Melbourne, Melbourne, VIC, Australia

Keywords: music, wider benefits, lifespan, health, well-being

\section{Editorial on the Research Topic}

\section{The Impact of Music on Human Development and Well-Being}

Music is one of the most universal ways of expression and communication for humankind and is present in the everyday lives of people of all ages and from all cultures around the world (Mehr et al., 2019). Hence, it seems more appropriate to talk about musics (plural) rather than in the singular (Goble, 2015). Furthermore, research by anthropologists as well as ethnomusicologists suggests that music has been a characteristic of the human condition for millennia (cf. Blacking, 1976; Brown, 1999; Mithen, 2005; Dissanayake, 2012; Higham et al., 2012; Cross, 2016). Nevertheless, whilst the potential for musical behavior is a characteristic of all human beings, its realization is shaped by the environment and the experiences of individuals, often within groups (North and Hargreaves, 2008; Welch and McPherson, 2018). Listening to music, singing, playing (informally, formally), creating (exploring, composing, improvising), whether individually and collectively, are common activities for the vast majority of people. Music represents an enjoyable activity in and of itself, but its influence goes beyond simple amusement.

These activities not only allow the expression of personal inner states and feelings, but also can bring about many positive effects in those who engage in them. There is an increasing body of empirical and experimental studies concerning the wider benefits of musical activity, and research in the sciences associated with music suggests that there are many dimensions of human lifeincluding physical, social, educational, psychological (cognitive and emotional) - which can be affected positively by successful engagement in music (Biasutti and Concina, 2013). Learning in and through music is something that can happen formally (such as part of structured lessons in school), as well as in other-than-formal situations, such as in the home with family and friends, often non-sequentially and not necessarily intentional, and where participation in music learning is voluntary, rather than mandated, such as in a community setting (cf. Green, 2002; Folkestad, 2006; Saether, 2016; Welch and McPherson, 2018).

Such benefits are evidenced across the lifespan, including early childhood (Gerry et al., 2012; Williams et al., 2015; Linnavalli et al., 2018), adolescence (McFerran et al., 2018), and older adulthood (Lindblad and de Boise, 2020). Within these lifespan perspectives, research into music's contribution to health and well-being provides evidence of physical and psychological impacts (MacDonald et al., 2013; Fancourt and Finn, 2019; van den Elzen et al., 2019). Benefits are also reported in terms of young people's educational outcomes (Guhn et al., 2019), and successful musical activity can enhance an individual's sense of social inclusion (Welch et al., 2014) and social cohesion (Elvers et al., 2017).

This special issue provides a collection of 21, new research articles that deepen and develop our understanding of the ways and means that music can impact positively on human development and well-being. The collection draws on the work of 88 researchers from 17 different countries 
across the world, with each article offering an illustration of how music can relate to other important aspects of human functioning. In addition, the articles collectively illustrate a wide range of contemporary research approaches. These provide evidence of how different research aims concerning the wider benefits of music require sensitive and appropriate methodologies.

In terms of childhood and adolescence, for example, Putkinen et al. demonstrate how musical training is likely to foster enhanced sound encoding in 9 to 15-year-olds and thus be related to reading skills. A separate Finnish study by Saarikallio et al. provides evidence of how musical listening influences adolescents' perceived sense of agency and emotional well-being, whilst demonstrating how this impact is particularly nuanced by context and individuality. Aspects of mental health are the focus for an Australian study by Stewart et al. of young people with tendencies to depression. The article explores how, despite existing literature on the positive use of music for mood regulation, music listening can be double-edged and could actually sustain or intensify a negative mood.

A Portuguese study by Martins et al. shifts the center of attention from mental to physical benefits in their study of how learning music can support children's coordination. They provide empirical data on how a sustained, 24-week programme of Orff-based music education, which included the playing of simple tuned percussion instruments, significantly enhanced the manual dexterity and bimanual coordination in participant 8year-olds compared to their active control (sports) and passive control peers. A related study by Loui et al. in the USA offers insights into the neurological impact of sustained musical instrument practice. Eight-year-old children who play one or more musical instruments for at least $0.5 \mathrm{~h}$ per week had higher scores on verbal ability and intellectual ability, and these correlated with greater measurable connections between particular regions of the brain related to both auditory-motor and bi-hemispheric connectivity.

Younger, pre-school children can also benefit from musical activities, with associations being reported between informal musical experiences in the home and specific aspects of language development. A UK-led study by Politimou et al. found that rhythm perception and production were the best predictors of young children's phonological awareness, whilst melody perception was the best predictor of grammar acquisition, a novel association not previously observed in developmental research. In another pre-school study, Barrett et al. explored the beliefs and values held by Australian early childhood and care practitioners concerning the value of music in young children's learning. Despite having limited formal qualifications and experience of personal music learning, practitioners tended overall to have positive attitudes to music, although this was biased toward music as a recreational and fun activity, with limited support for the notion of how music might be used to support wider aspects of children's learning and development.

Engaging in music to support a positive sense of personal agency is an integral feature of several articles in the collection. In addition to the Saarikallio team's research mentioned above, Moors et al. provide a novel example of how engaging in collective beatboxing can be life-enhancing for throat cancer patients in the UK who have undergone laryngectomy, both in terms of supporting their voice rehabilitation and alaryngeal phonation, as well as patients' sense of social inclusion and emotional well-being.

One potential reason for these positive findings is examined in an Australian study by Krause et al.. They apply the lens of self-determination theory to examine musical participation and well-being in a large group of 17 to 85-year-olds. Respondents to an online questionnaire signaled the importance of active music making in their lives in meeting three basic psychological needs embracing a sense of competency, relatedness and autonomy.

The use of public performance in music therapy is the subject of a US study by Vaudreuil et al. concerning the social transformation and reintegration of US military service members. Two example case studies are reported of service members who received music therapy as part of their treatment for post-traumatic stress disorder, traumatic brain injury, and other psychological health concerns. The participants wrote, learned, and refined songs over multiple music therapy sessions and created song introductions to share with audiences. Subsequent interviews provide positive evidence of the beneficial psychological effects of this programme of audience-focused musical activity.

Relatedly, McFerran et al. in Australia examined the ways in which music and trauma have been reported in selected music therapy literature from the past 10 years. The team's critical interpretive synthesis of 36 related articles led them to identify four different ways in which music has been used beneficially to support those who have experienced trauma. These approaches embrace the use of music for stabilizing (the modulation of physiological processes) and entrainment (the synchronization of music and movement), as well as for expressive and performative purposes-the fostering of emotional and social well-being.

The therapeutic potential of music is also explored in a detailed case study by Fachner et al.. Their research focuses on the nature of critical moments in a guided imagery and music session between a music therapist and a client, and evidences how these moments relate to underlying neurological function in the mechanics of music therapy.

At the other end of the age span, and also related to therapy, an Australian study by Brancatisano et al. reports on a new Music, Mind, and Movement programme for people in their eighties with mild to moderate dementia. Participants involved in the programme tended to show an improvement in aspects of cognition, particularly verbal fluency and attention. Similarly, Wilson and MacDonald report on a 10-week group music programme for young Scottish adults with learning difficulties. The research data suggest that participants enjoyed the programme and tended to sustain participation, with benefits 
evidenced in increased social engagement, interaction and communication.

The role of technology in facilitating access to music and supporting a sense of agency in older people is the focus for a major literature review by Creech, now based in Canada. Although this is a relatively under-researched field, the available evidence suggests that that older people, even those with complex needs, are capable of engaging with and using technology in a variety of ways that support their musical perception, learning and participation and wider quality of life.

Related to the particular needs of the young, children's general behavior can also improve through music, as exampled in an innovative, school-based, intensive 3month orchestral programme in Italy with 8 to 10 -year-olds. Fasano et al. report that the programme was particularly beneficial in reducing hyperactivity, inattention and impulsivity, whilst enhancing inhibitory control. These benefits are in line with research findings concerning successful music education with specific cases of young people with ADHD whose behavior is characterized by these same disruptive symptoms (hyperactivity, inattention, and impulsivity).

Extra-musical benefits are also reported in a study of college students (Bachelors and Masters) and amateur musicians in a joint Swiss-UK study. Antonini Philippe et al. suggest that, whilst music making can offer some health protective effects, there is a need for greater health awareness and promotion among advanced music students. Compared to the amateur musicians, the college music students evaluated their overall quality of life and general and physical health more negatively, as did females in terms of their psychological health. Somewhat paradoxically, the college students who had taken part in judged performances reported higher psychological health ratings. This may have been because this sub-group were slightly older and more experienced musicians.

Music appears to be a common accompaniment to exercise, whether in the gym, park or street. Nikol et al. in South East Asia explore the potential physical benefits of synchronous exercise to music, especially in hot and humid conditions. Their randomized cross-over study (2019) reports that "time-to-exhaustion" under the synchronous music condition was $2 / 3$ longer compared to the no-music condition for the same participants. In addition, perceived exertion was significantly lower, by an average of $22 \%$ during the synchronous condition.

Comparisons between music and sport are often evidenced in the body of existing Frontiers research literature related to performance and group behaviors. Our new collection contains a contribution to this literature in a study by Habe et al.. The authors investigated elite musicians and top athletes in Slovenia in terms of their perceptions of flow in performance and satisfaction with life. The questionnaire data analyses suggest that the experience of flow appears to influence satisfaction with life in these highfunctioning individuals, albeit with some variations related to discipline, participant sex and whether considering team or individual performance.
A more formal link between music and movement is the focus of an exploratory case study by Cirelli and Trehub. They investigated a 19-month-old infant's dance-like, motoricallycomplex responses to familiar and unfamiliar songs, presented at different speeds. Movements were faster for the more familiar items at their original tempo. The child had been observed previously as moving to music at the age of 6 months.

Finally, a novel UK-based study by Waddington-Jones et al. evaluated the impact of two professional composers who were tasked, individually, to lead a 4-month programme of group composing in two separate and diverse community settings-one with a choral group and the other in a residential home, both funded as part of a music programme for the Hull City of Culture in 2017. In addition to the two composers, the participants were older adults, with the residential group being joined by schoolchildren from a local Primary school to collaborate in a final performance. Qualitative data analyses provide evidence of multi-dimensional psychological benefits arising from the successful, group-focused music-making activities.

In summary, these studies demonstrate that engaging in musical activity can have a positive impact on health and well-being in a variety of ways and in a diverse range of contexts across the lifespan. Musical activities, whether focused on listening, being creative or re-creative, individual or collective, are infused with the potential to be therapeutic, developmental, enriching, and educational, with the caveat provided that such musical experiences are perceived to be engaging, meaningful and successful by those who participate.

Collectively, these studies also celebrate the multiplicity of ways in which music can be experienced. Reading across the articles might raise a question as to whether or not any particular type of musical experience is seen to be more beneficial compared with another. The answer, at least in part, is that the empirical evidence suggests that musical engagement comes in myriad forms along a continuum of more or less overt activity, embracing learning, performing, composing and improvising, as well as listening and appreciating. Furthermore, given the multidimensional neurological processing of musical experience, it seems reasonable to hypothesize that it is perhaps the level of emotional engagement in the activity that drives its degree of health and well-being efficacy as much as the activity's overt musical features. And therein are opportunities for further research!

\section{AUTHOR CONTRIBUTIONS}

The editorial was drafted by GW and approved by the topic Co-editors. All authors listed have made a substantial, direct and intellectual contribution to the Edited Collection, and have approved this editorial for publication.

\section{ACKNOWLEDGMENTS}

We are very grateful to all the contributing authors and their participants for their positive engagement with this Frontiers Research Topic, and also for the Frontiers staff for their commitment and support in bringing this topic to press. 


\section{REFERENCES}

Biasutti, M., and Concina, E. (2013). "Music education and transfer of learning," in Music: Social Impacts, Health Benefits and Perspectives, eds P. Simon and T. Szabo (New York, NY: Nova Science Publishers, Inc Series: Fine Arts, Music and Literature), 149-166.

Blacking, J. (1976). How Musical Is Man? London: Faber \& Faber.

Brown, S. (1999). “The 'musilanguage' model of music evolution," in The Origins of Music, eds N. L. Wallin, B. Merker, and S. Brown (Cambridge: The MIT Press), 271-301. doi: 10.7551/mitpress/5190.003.0022

Cross, I. (2016). "The nature of music and its evolution," in Oxford Handbook of Music Psychology, eds S. Hallam, I. Cross, and M. Thaut (New York, NY: Oxford University Press), 3-18. doi: 10.1093/oxfordhb/9780198722946.013.5

Dissanayake, E. (2012). The earliest narratives were musical. Res. Stud. Music Educ. 34, 3-14. doi: 10.1177/1321103X12448148

Elvers, P., Fischinger, T., and Steffens, J. (2017). Music listening as selfenhancement: effects of empowering music on momentary explicit and implicit self-esteem. Psychol. Music 46, 307-325. doi: 10.1177/0305735617707354

Fancourt, D., and Finn, S. (2019). What Is the Evidence on the Role of the Arts in Improving Health and Well-Being? A Scoping Review. Copenhagen: World Health Organisation.

Folkestad, G. (2006). Formal and informal learning situations or practices vs formal and informal ways of learning. Br. J. Music Educ. 23, 135-145. doi: $10.1017 /$ S0265051706006887

Gerry, D., Unrau, A., and Trainor, L. J. (2012). Active music classes in infancy enhance musical, communicative and social development. Dev. Sci. 15, 398-407. doi: 10.1111/j.1467-7687.2012.01142.x

Goble, J. S. (2015). Music or musics?: an important matter at hand. Act. Crit. Theor. Music Educ. 14, 27-42. Available online at: http://act.maydaygroup.org/articles/ Goble14_3.pdf

Green, L. (2002). How Popular Musicians Learn. Aldershot: Ashgate Press.

Guhn, M., Emerson, S. D., and Gouzouasis, P. (2019). A population-level analysis of associations between school music participation and academic achievement. J. Educ. Psychol. 112, 308-328. doi: 10.1037/edu0000376

Higham, T., Basell, L., Jacobi, R., Wood, R., Ramsey, C. B., and Conard, N.J. (2012). Testing models for the beginnings of the Aurignacian and the advent of figurative art and music: the radiocarbon chronology of GeißenklÃsterle. J. Hum. Evol. 62, 664-676. doi: 10.1016/j.jhevol.2012.03.003

Lindblad, K., and de Boise, S. (2020). Musical engagement and subjective wellbeing amongst men in the third age. Nordic J. Music Therapy 29, 20-38. doi: 10.1080/08098131.2019.1646791

Linnavalli, T., Putkinen, V., Lipsanen, J., Huotilainen, M., and Tervaniemi, M. (2018). Music playschool enhances children's linguistic skills. Sci. Rep. 8:8767. doi: $10.1038 / s 41598-018-27126-5$
MacDonald, R., Kreutz, G., and Mitchell, L. (eds.) (2013). Music, Health and Wellbeing. New York, NY: Oxford University Press. doi: 10.1093/acprof:oso/9780199586974.001.0001

McFerran, K. S., Hense, C., Koike, A., and Rickwood, D. (2018). Intentional music use to reduce psychological distress in adolescents accessing primary mental health care. Clin. Child Psychol. Psychiatry 23, 567-581. doi: $10.1177 / 1359104518767231$

Mehr, A., Singh, M., Knox, D., Ketter, D. M., Pickens-Jones, D., Atwood, S., et al. (2019). Universality and diversity in human song. Science 366:eaax0868. doi: $10.1126 /$ science.aax0868

Mithen, S. (ed.). (2005). Creativity in Human Evolution and Prehistory. London: Routledge. doi: 10.4324/9780203978627

North, A. C., and Hargreaves, D. J. (2008). The Social and Applied Psychology of Music. New York, NY: Oxford University Press. doi: 10.1093/acprof:oso/9780198567424.001.0001

Saether, M. (2016). Music in informal and formal learning situations in ECEC. Nordic Early Childhood Educ. Res. J. 13, 1-13. doi: 10.7577/nbf.1656

van den Elzen, N., Daman, V., Duijkers, M., Otte, K., Wijnhoven, E., Timmerman, H., et al. (2019). The power of music: enhancing muscle strength in older people. Healthcare 7:82. doi: 10.3390/healthcare7030082

Welch, G.F., and McPherson, G. E. (eds.). (2018). "Commentary: Music education and the role of music in people's lives," in Music and Music Education in People's Lives: An Oxford Handbook of Music Education (New York, NY: Oxford University Press), 3-18. doi: 10.1093/oxfordhb/9780199730810.0 13.0002

Welch, G. F., Himonides, E., Saunders, J., Papageorgi, I., and Sarazin, M. (2014). Singing and social inclusion. Front. Psychol. 5:803. doi: 10.3389/fpsyg.2014.00803

Williams, K. E., Barrett, M. S., Welch, G. F., Abad, V., and Broughton, M. (2015). Associations between early shared music activities in the home and later child outcomes: findings from the longitudinal study of Australian Children. Early Childhood Res. Q. 31, 113-124. doi: 10.1016/j.ecresq.2015.01.004

Conflict of Interest: The authors declare that the research was conducted in the absence of any commercial or financial relationships that could be construed as a potential conflict of interest.

Copyright (c) 2020 Welch, Biasutti, MacRitchie, McPherson and Himonides. This is an open-access article distributed under the terms of the Creative Commons Attribution License (CC BY). The use, distribution or reproduction in other forums is permitted, provided the original author(s) and the copyright owner(s) are credited and that the original publication in this journal is cited, in accordance with accepted academic practice. No use, distribution or reproduction is permitted which does not comply with these terms. 


\title{
Understanding Wellbeing Among College Music Students and Amateur Musicians in Western Switzerland
}

\author{
Roberta Antonini Philippe ${ }^{1 *}$, Céline Kosirnik ${ }^{1}$, Noémi Vuichoud ${ }^{1}$, Aaron Williamon ${ }^{2,3}$ and \\ Fabienne Crettaz von Roten ${ }^{4}$
}

1 Laboratoire PHASE, Faculté des Sciences Sociales et Politiques, Institut des Sciences du Sport, Université de Lausanne, Lausanne, Switzerland, ${ }^{2}$ Centre for Performance Science, Royal College of Music, London, United Kingdom, ${ }^{3}$ Faculty of Medicine, Imperial College London, London, United Kingdom, ${ }^{4}$ Faculté des Sciences Sociales et Politiques, Institut des

Sciences du Sport, Université de Lausanne, Lausanne, Switzerland

\section{OPEN ACCESS}

Edited by:

Michele Biasutti,

University of Padua, Italy

Reviewed by:

Brenda Hanna-Pladdy,

University of Maryland, Baltimore,

United States

Andrea Schiavio,

University of Graz, Austria

*Correspondence:

Roberta Antonini Philippe roberta.antoniniphilippe@unil.ch

Specialty section:

This article was submitted to

Performance Science,

a section of the journal

Frontiers in Psychology

Received: 26 December 2018

Accepted: 27 March 2019

Published: 03 May 2019

Citation:

Antonini Philippe R, Kosirnik C,

Vuichoud N, Williamon A and

Crettaz von Roten F (2019)

Understanding Wellbeing Among College Music Students and Amateur

Musicians in Western Switzerland.

Front. Psychol. 10:820.

doi: 10.3389/fpsyg.2019.00820
Musical performance requires the ability to master a complex integration of highly specialized motor, cognitive, and perceptual skills developed over years of practice. It often means also being able to deal with considerable pressure within dynamic environments. Consequently, many musicians suffer from health-related problems and report a large number of physical and psychological complaints. Our research aimed to evaluate and analyze the wellbeing of two distinct groups of musicians, college music students and amateur performers in the French-speaking part of Switzerland. A total sample of 126 musicians was recruited for the study (mean age $\pm S D=22.4 \pm 4.5$ years, 71 male). Wellbeing was assessed through the World Health Organization Quality of Life-BREF questionnaire evaluating two general measures, quality of life (QoL) and general health, and four specific dimensions: physical health, psychological health, social relationships, and environment. For both groups, respondents' QoL was high on each measure: median scores were higher than 4 for the two general measures and higher than 70 for the four specific dimensions. Among the dimensions, respondents had the highest mean score for environment (75.0), then social relationships and physical health (74.0 and 73.8, respectively), and finally, psychological health (70.3). Differences between groups of musicians emerged in terms of overall QoL and general health, as well as the physical health dimension, where college music students scored lower than the amateur musicians; conversely, college music students scored higher than the amateurs on social relationships. Our overview of musicians' wellbeing in Western Switzerland demonstrates that, while music making can offer some health protective effects, there is a need for greater health awareness and promotion among advanced music students. This research offers insight into musicians' wellbeing and points to the importance of involving different actors (teachers, administrators, support staff) in facilitating healthy music making.

Keywords: wellbeing, quality of life, health, college music students, amateur musicians, Switzerland

\section{INTRODUCTION}

Wellbeing is a major preoccupation for the World Health Organization (WHO), as outlined in the policy program Health 2020 (Lindert et al., 2015). European member states have agreed a unique measure to assess subjective wellbeing, life satisfaction. Although a universal definition or measure of subjective wellbeing does not exist, "In general, subjective wellbeing refers to a 
cognitive process of contentment, satisfaction or happiness derived from optimal functioning. Optimal functioning is a relative rather than an absolute concept as the benchmark for judging lives in an individual's perception of his or her own aspirations" (Lindert et al., 2015, p. 732).

Wellbeing is a multidimensional phenomenon and refers to emotional and cognitive dimensions of subjective experiences resulting from the individual evaluation of several facets of life. Research on wellbeing has revealed two fundamental perspectives (Disabato et al., 2016): hedonia (Diener, 1984) and eudaimonia (Ryff, 1989). The hedonic perspective emphasizes the attainment of pleasure and pain avoidance, focusing mainly on happiness. The eudaimonic perspective encompasses a person's optimal degree of functioning, focusing on meaning and selfrealization (Ryan and Deci, 2001) and dimensions of selfacceptance, autonomy, personal growth, positive relationships, environmental mastery, and goals in life (Ryff, 2014). According to Ryan and Deci (2002), if the fundamental needs of autonomy, relatedness and competence are fulfilled, individuals can experience personal growth and wellbeing.

In the field of music, wellbeing has been investigated in two different ways, with music as a facilitator but also as a disruptor of wellbeing. In terms of facilitation, much research has pointed out its strong and positive impact on people's lives (Pothoulaki et al., 2012; Västfjäll et al., 2012; Perkins and Williamon, 2014). Boyce-Tillman (2000), for instance, showed that music facilitates creativity and, in this way, promotes wellbeing. Also, singing in groups has been associated with positive wellbeing (Davidson, 2008; Boyce-Tillman, 2014). Evans (2015) demonstrated that music can fulfill the three fundamental needs identified by self-determination theory as necessary to wellbeing. Similarly, Dickinson (2018) has listed mechanisms by which music can influence wellbeing: it can motivate, the rhythm can diminish anxiety, music helps alleviate the effects of some disorders (e.g., obsessive-compulsive disorder and depression), it helps to find a balance between personal life and work, and it builds links and promotes exercise as well as release tension. Croom (2012) demonstrated that music can influence positively the five components of wellbeing outlined in Seligman's PERMA model: positive emotion, engagement, relationships, meaning, and accomplishment (Seligman, 1998). Using the same model, Ascenso et al. (2018) tested professional musicians on the five PERMA dimensions and found that musicians scored high on all five, suggesting that, even among professionals, music is linked with positive wellbeing.

However, research has also shown that making music can be a disruptor of wellbeing as it is linked with the many challenges that musicians face in their practice. Physical pain is one of the consequences in making music at a high level. Only 26.7\% reported that they had never experienced performance-related pain (Kenny and Ackermann, 2015). Furthermore, the large majority of professional musicians have experienced at least one medical problem (86\%), with $76 \%$ suffering at least once from a severe medical problem (Fishbein et al., 1988). They often experience pain (86\%) (Leaver et al., 2011), with some experiencing pain that profoundly impairs their performance (Croom, 2012). Prevalence of musculoskeletal disorders among music students has been reported to be anywhere between 35 to $80 \%$ (Zaza, 1998; Cruder et al., 2018), with musicians reporting more pain than other university students (e.g., medical students) (Kok et al., 2013). In a study concerning musicians' musculoskeletal problems, Chong et al. (1989) highlighted differences between student and amateur musicians who were seen in a clinic for such problems: $36 \%$ were students and $18 \%$ amateurs. Anxiety and distress during performance have also been studied (Kenny et al., 2014; Osborne et al., 2014; Antonini Philippe and Güsewell, 2016). These factors all pose risks to musicians' wellbeing (Williamon and Thompson, 2006).

Beyond the fact that music can have an influence, positive and negative, on wellbeing, it has been argued that wellbeing can have an impact on facilitating good performance (Williamon, 2004; Kenny, 2011), and yet, the literature shows that musicians engage poorly in health promoting behaviors. Kenny et al. (2014) conducted a survey of professional musicians in Australia, and the results reveal sub-optimal mental health and poor health behaviors. However, efforts are being made in some countries (e.g., United Kingdom, South Africa, and Australia) to reconfigure music training programs to incorporate insight from health professionals and active health education and literacy (Perkins et al., 2017).

The paradoxical bidirectional relationships between music and wellbeing as outlined above are puzzling and warrant further investigation. No doubt, there is large variation in the instruments and methods used when studying wellbeing, as well as cultural differences between countries and geographical regions. Indeed, many of the existing scales are based on morbidity, mortality, and the impact of disorders or disease on daily activities and behavior on perceived health, containing measures of disabilities (WHO, 1996). They are, as such, problematic as they do not capture QoL and are often culturally influenced depending on where they are developed.

In order to avoid these problems, the World Health Organization Quality of Life (WHOQOL) Group developed a multidimensional scale of QoL linked to their health policy program centered on a humanistic approach (WHOQOL Group, 1991, 1998). The resulting WHOQOL questionnaire focuses on functional and positive aspects and considers the social context as well as the environment in which people live (WHO, 1996). QoL is defined as "an individual's perception of their position in life, in the context of the culture and value systems in which they live and in relation to their goals, expectations, standards, and concerns. It is a broad ranging concept, affected in a complex way by the person's physical health, psychological state, level of independence, social relationships and their relationship to salient features of their environment" (WHOQOL Group, 1995, p. 1404). While the concepts of QoL and subjective wellbeing are similar (Diener et al., 1999), measurement of QoL is more widespread than that of subjective wellbeing, with QoL being more specific and commonly found in the methodological and scale development domain (Hawthorne et al., 2006). The Satisfaction with Life Scale (Diener et al., 1985; Pavot and Diener, 1993 ) is a notable exception and measures subjective wellbeing.

The WHO has developed many alternative measurement tools from the original WHOQOL questionnaire, which was composed 
of 100 items. For example, the WHOQOL-BREF examines life satisfaction and general health, as well as four component scores of physical health, psychological health, social relationships and environment. This 26-item questionnaire has been used in many studies, some in the field of music (Clift et al., 2007, 2010; Johnson et al., 2013; Garrido et al., 2016; Chang et al., 2018). These studies have focused on the four component scores of the questionnaire, but other studies have used only some dimensions or just the total WHOQOL-BREF score. For example, Dritsakis et al. (2017) investigated the positive effects of music on the wellbeing of patients with cochlear implants in three domains: physical health, psychological health, and social relationships. Mitchell et al. (2007) investigated the effects of music listening on the wellbeing of chronic pain sufferers, and their results revealed a higher total score for patients who listen frequently to music and who perceived it as personally important.

As mentioned before, culture can influence the ways in which people define and characterize wellbeing, and cultural differences also affect the impact of health promotion interventions within a given country or geographical region. If we refer to Switzerland, there seems to be a growing interest in understanding and promoting musicians' health. In 1997, the Swiss society for music medicine was founded in order to help musicians cope with performance-related physical problems. Recently, they initiated interdisciplinary consultations composed of musicians, doctors, psychologists, and diverse therapists in order to support musicians in their daily work (Berchtold-Neumann, 2018). Such consultations exist in the German- and Italian-speaking parts of Switzerland but not yet in the French-speaking region. Only a few Swiss studies have investigated the impact of music on the general population. One study by Thoma et al. (2012), conducted in Zurich, focused on the impact of music listening on emotion regulation and stress reactivity, as well as physiological and psychological functioning. Among other measures, they used the WHOQOL-5, a five-item version of the WHOQOL to test musicians' life satisfaction, but the psychometric properties of the questionnaire have not yet been validated. Another Swiss study was conducted on the influence of attending cultural and arts events on wellbeing. This longitudinal study with Swiss population aged 14 years and older (engaged actively or passively in cultural activities such as playing an instrument or singing, painting, sculpting) provided little evidence of a causal influence (Weziak-Białowolska, 2016). In fact, results showed that long-term health and wellbeing did not improve significantly as a result of any specific activity in the cultural arena.

Only a few studies have been devoted to the understanding of health and QoL among musicians during their formative years in conservatoire training (Williamon and Thompson, 2006; Kreutz et al., 2009). Therefore, our research aimed to evaluate and analyze the wellbeing of college music students compared with amateur musicians in the French-speaking part of Switzerland. This permits direct comparisons between these two groups, as well as the exploration of differences between those who take part in judged performances (e.g., competitions) and between men and women.

\section{MATERIALS AND METHODS}

\section{Respondents}

One hundred and thirty instrumental and vocal musicians and singers took part in the study, recruited via different music schools and music colleges by contacting directors, teachers and, also, via personal contacts. Four musicians were excluded from analyses because they performed computerassisted music but did not play an instrument such as those found in a symphony orchestra, leaving a final sample of 126 respondents (mean age $\pm S D=22.4 \pm 4.5$ years, 71 male) of two groups: HEM participants ("HEM" being, in French, "Haute Ecole de Musique") and non-HEM participants (i.e., amateur musicians) serving as the comparison group, as detailed in Table 1. A sample size of 126 is above the number required to calculate independent samples $t$-tests with a medium effect size [Cohen (1992), ES = 0.5], a first error alpha of 0.05 and a power of 0.8 , corresponding to 104 according to Gpower (version 3.1).

\section{Procedure and Methods}

All musicians provided socio-demographic and music-related information before completing the WHOQOL-BREF questionnaire. Sociodemographic variables included sex and age. Age was recoded into three groups: $\leq 20$ years, $21-24$ years, and $\geq 25$ years, using the higher group as the reference category. Data on performance specialism and music education was also collected. For specialism, musicians reported their instrument, and five categories were created: wind, strings, keyboard, percussion, and voice. Participants then indicated their music educational status: college music students seeking Bachelor or Master qualifications (i.e., HEM) or amateur musicians (i.e., non-HEM), and whether or not they participate in judged performances or competitions (Yes or No).

TABLE 1 | Characteristics of music college students (HEM) and amateur musicians (non-HEM) in the sample, including $n(\%)$ for categorical variables, mean (SD) for continuous variable, and Chi-square tests of independence.

\begin{tabular}{lcccc}
\hline & Total & HEM & Non-HEM & $\begin{array}{c}\text { Chi-square } \\
\text { test }\end{array}$ \\
\hline Characteristic & $n=126$ & $n=46$ & $n=80$ & \\
Sex (male) & $71(56.3 \%)$ & $25(54.3 \%)$ & $46(57.5 \%)$ & 0.118 \\
Age & $22.4(4.5)$ & $23(3.0)$ & $22(5.2)$ & $25.30^{*}$ \\
$\leq 20$ & $54(42.9 \%)$ & $7(15.2 \%)$ & $47(58.8 \%)$ & \\
$21-24$ & $40(31.7 \%)$ & $25(54.3 \%)$ & $15(18.8 \%)$ & \\
$\geq 25$ & $32(25.4 \%)$ & $14(30.4 \%)$ & $18(22.5 \%)$ & \\
Musical instrument & & & & $30.62 *$ \\
Wind & $46(36.5 \%)$ & $30(65.2 \%)$ & $16(20.0 \%)$ & \\
Strings & $18(14.4 \%)$ & $3(6.5 \%)$ & $15(18.8 \%)$ & \\
Keyboard & $30(23.8 \%)$ & $6(13.0 \%)$ & $24(30.0 \%)$ & \\
Percussion & $18(14.3 \%)$ & $1(2.2 \%)$ & $17(21.3 \%)$ & \\
Voice & $14(11.1 \%)$ & $6(13.0 \%)$ & $8(10.0 \%)$ & \\
Judged performance (Yes) & $96(76.2 \%)$ & $45(97.8 \%)$ & $51(64.6 \%)$ & $18.06 *$ \\
\hline * $<$ 0.05. & & & & \\
\hline & & &
\end{tabular}


The majority of respondents completed the paper version ( $n=117)$, but some completed the questionnaire online, with access provided by email. Information about the study was given to all participants and highlighted the confidentiality and anonymity of their participation. Musicians participated voluntarily and could stop answering the questionnaire at any time. Written informed consent was obtained from all participants.

The WHOQOL-BREF questionnaire, which consists of 26 items, was used to measure musicians' QoL. This version of the original 100-item WHO (1991) instrument has been adapted in various languages, including French, as used in this study. The WHOQOL-BREF is a self-administrated instrument that assesses general wellbeing. It consists, firstly, of two general items: overall QoL (rated from 1 Very poor to 5 Very good) and general health (from 1 Very dissatisfied to 5 Very satisfied). It then progresses to a series of 24 items on four QoL dimensions: physical health (7 items), psychological health (6 items), social relationships (3 items), and environment (8 items). Each item is scored on a 5-point intensity scale (1 Not at all to 5 Extremely), a 5-point evaluation scale (1 Very dissatisfied to 5 Very satisfied), a 5-point capacity scale (1 Very poor to 5 Very good), and a 5-point frequency scale (1 Never to 5 Always).

The physical health dimension includes questions on daily activities; dependence on medicinal substances and medical aids; energy and fatigue; mobility; pain and discomfort; sleep and rest; and work capacity. The psychological health dimension assesses knowledge of bodily image and appearance; negative feelings; positive feelings; self-esteem; spirituality/religion/personal beliefs; and thinking, learning, memory, and concentration. Social relationships are characterized through personal relationships; social support; and sexual activity. Finally, environment is captured through questions on financial resources; freedom, physical safety, and security; health and social care; accessibility and quality; home environment; opportunities for acquiring new information and skills; participation in and opportunities for recreation/leisure activities; physical environment (pollution/noise/traffic/climate); and transport.

The dimension's mean scores were calculated according to the procedure described by the WHO (1996) with a transformation to a $0-100$ scale. Scores are scaled in a positive direction, with a higher score corresponding to higher QoL. The reliability coefficients of the four dimensions were, respectively, Cronbach alpha $=0.68,0.75,0.64$, and 0.74 , which indicate minimally acceptable reliability for dimensions one and three, and respectable reliability for dimensions two and four (De Vellis, 2003).

This study was led by the sport psychology laboratory at the Institute of Sport Sciences, University of Lausanne, and ethical approval was granted by the Commission cantonale d'éthique de la recherche sur l'être humain (CER-VD). Authorization to use the WHOQOL-BREF was granted by the WHO through a standard user-agreement form.

\section{Data Analyses}

Descriptive statistics were calculated for each variable. Chisquare independence tests were performed to assess differences among HEM and non-HEM respondents in terms of sex, age group, musical instrument, and participation in judged performances. In comparisons of groups (e.g., sex, age groups) among the four QoL dimensions, MANOVAs were used. For comparing HEM vs. non-HEM, we used sex and age group as covariates whereas for comparing participants vs. non-participants in judged performances, we used sex, age group and education as covariates. Homogeneity of the matrix of variance-covariance was assessed with Box's $M$-test and normality with the Shapiro-Wilk test. When the MANOVA was significant, we performed univariate $F$-tests with a significance correction. We also calculated effect sizes (Cohen, 1992). Finally, a multivariate logistic regression was performed to compare the HEM and non-HEM groups on the four QoL dimensions after controlling for sex and age; the model meets Vittinghoff and McCulloch (2007) rule of 5 - 9 outcome events per predictor variable. The level of statistical significance was set at 0.05 . All analyses were performed using SPSS (version 25).

\section{RESULTS}

\section{Descriptive Characteristics of the Study Sample}

Within the sample, $56 \%$ of respondents were men and $43 \%$ women (Table 1). In musical terms, most played wind instruments (37\%), followed next by keyboard (24\%), and 76\% had participated in judged performances or competitions. A third of respondents followed HEM education.

Table 1 displays the descriptive statistics for HEM and nonHEM respondents. The distribution of age, musical instrument, and participation in judged performances varied significantly between HEM and non-HEM respondents: HEM musicians were more frequently in the higher age groups, they played more frequently wind instruments, and participated more frequently in adjudicated performances.

\section{Wellbeing}

The QoL of participants was high on each measure: median scores were higher than 4 for the first two measures and higher than 70 for the four QoL dimensions (Table 2). Among the dimensions, respondents had the highest mean score for environment (75.0), then on social relationships and physical health (74.0 and 73.8, respectively) and, finally, on psychological health (70.3).

TABLE 2 | Descriptive statistics for wellbeing, including overall QoL, general health, and each of the four dimensions of the WHOQOL-BREF (WHO, 1991).

\begin{tabular}{lcccr}
\hline & M (SD) & Median & Min & Max \\
\hline Overall QoL & $4.35(0.65)$ & 4 & 2 & 5 \\
General health & $4.09(0.85)$ & 4 & 2 & 5 \\
WHOQOL-BREF & & & & \\
Physical health & $73.81(13.14)$ & 75.0 & 32 & 100 \\
Psychological health & $70.34(14.35)$ & 71.0 & 29 & 100 \\
Social relationships & $73.99(17.37)$ & 75.0 & 8 & 100 \\
Environment & $75.00(13.33)$ & 75.0 & 31 & 100
\end{tabular}


Quality of Life varied little among sociodemographic groups. There was no significant relationship between each of the two general measures and sex (respectively, $\chi_{2}^{2}=2.96, p=0.227$, and $\left.\chi_{2}^{2}=0.201, p=0.904\right)$, nor for age groups $\left(\chi_{4}^{2}=5.19, p=0.269\right.$, and $\chi_{4}^{2}=1.79, p=0.775$ ).

The four QoL dimensions did not differ according to sex (Pillai $\left.=1.265, p=0.288, E t a^{2}=0.040\right)$. Nevertheless, the Psychological health dimension differed significantly as a function of $\operatorname{sex}\left(F_{1,124}=4.05, p=0.046, E t a^{2}=0.032\right)$, where female musicians had lower scores, $M(S D)=67.4$ (13.5), than male musicians, $M(S D)=72.6$ (14.69). The four QoL dimensions did not differ according to age group (Pillai $=0.714$, $\left.p=0.679, E t a^{2}=0.023\right)$.

\section{Relationships Between Wellbeing Factors and Education, Judged Performances, and Sex}

The two general measures of QoL were each significantly related to education. For overall QoL, a lower percentage of HEM respondents answered "very good" compared with non-HEM respondents $(26 \%$ vs. $54 \%), \chi_{2}^{2}=9.11, p=0.011$, and for general health, fewer HEM respondents answered "very satisfied" ( $22 \%$ vs. $41 \%), \chi_{2}^{2}=10.27, p=0.006$.

The four QoL dimensions were also related to education, with sex and age group as covariates (Pillai $=4.785, p=0.001$, $E t a^{2}=0.140$, with the two covariates not reaching significance). The QoL dimensions showed few significant differences (Table 3). The physical health dimension varied significantly by education $\left(p=0.000\right.$ and $\left.E t a^{2}=0.098\right)$ : HEM respondents had a lower QoL physical score than non-HEM respondents $(M=69.46$ vs. 76.31$)$.

The two general measures of QoL were not related with participation in judged performances: overall QoL $\left(\chi_{2}^{2}=4.49\right.$, $p=0.109)$ and general health $\left(\chi_{2}^{2}=1.20, p=0.549\right)$. The four QoL dimensions were related to taking part in judged performances, with sex, age group and education as covariates (Pillai $=3.47, p=0.010, E t a^{2}=0.107$, with education the only covariate reaching significance $p=0.000)$. The QoL dimensions showed few significant differences. Psychological health varied significantly according to judged performance as did Environment (see Table 4 respectively, $p=0.015$, $\left.E t a^{2}=0.049 ; p=0.035, E t a^{2}=0.037\right)$ where respondents

TABLE 3 | Comparisons of the four WHOQOL-BREF dimensions between music students (HEM) and amateur musicians (non-HEM).

\begin{tabular}{|c|c|c|c|c|c|c|c|}
\hline & \multicolumn{2}{|c|}{$\begin{array}{c}\text { HEM } \\
(n=46)\end{array}$} & \multicolumn{2}{|c|}{$\begin{array}{c}\text { Non-HEM } \\
(n=80)\end{array}$} & \multirow[b]{2}{*}{$F_{1,121}$} & \multirow[b]{2}{*}{$p$} & \multirow{2}{*}{$\begin{array}{c}\text { Effect } \\
\text { size }\end{array}$} \\
\hline & $M$ & $S D$ & $M$ & $S D$ & & & \\
\hline Physical health & 69.46 & 12.79 & 76.31 & 12.75 & 13.159 & $0.000^{*}$ & 0.098 \\
\hline Psychological health & 69.09 & 12.54 & 71.06 & 15.32 & 1.713 & 0.193 & 0.014 \\
\hline Social relationships & 77.02 & 17.19 & 72.25 & 17.35 & 0.932 & 0.336 & 0.008 \\
\hline Environment & 72.83 & 15.64 & 76.25 & 11.73 & 3.131 & 0.079 & 0.025 \\
\hline
\end{tabular}

TABLE 4 | Comparisons of the four WHOQOL-BREF dimensions between musicians who take part in judged performances and those who do not.

\begin{tabular}{|c|c|c|c|c|c|c|c|}
\hline & \multicolumn{2}{|c|}{$\begin{array}{l}\text { Not judged } \\
\quad(n=29)\end{array}$} & \multicolumn{2}{|c|}{$\begin{array}{l}\text { Judged } \\
(n=96)\end{array}$} & \multirow[b]{2}{*}{$F_{1,119}$} & \multirow[b]{2}{*}{$p$} & \multirow{2}{*}{$\begin{array}{c}\begin{array}{c}\text { Effect } \\
\text { size }\end{array} \\
E t a^{2}\end{array}$} \\
\hline & $M$ & $S D$ & $M$ & $S D$ & & & \\
\hline Physical health & 72.55 & 14.39 & 74.22 & 12.86 & 1.955 & 0.165 & 0.016 \\
\hline Psychological health & 64.62 & 15.70 & 71.84 & 13.45 & 6.123 & $0.015^{*}$ & 0.049 \\
\hline Social relationships & 73.45 & 14.94 & 74.22 & 18.18 & 0.730 & 0.394 & 0.006 \\
\hline Environment & 71.69 & 13.15 & 75.91 & 13.34 & 4.559 & $0.035^{*}$ & 0.037 \\
\hline
\end{tabular}

* significant at $p<0.05$.

TABLE 5 | Multivariate logistic regression on the four WHOQOL-BREF dimensions controlling for sex and age.

\begin{tabular}{lccccc}
\hline & Estimate & Std. error & Exp (B) & Wald stat. & $\boldsymbol{p}$ \\
\hline MODEL 1 & & & & & \\
Sex & -0.13 & 0.47 & 0.87 & 0.08 & 0.773 \\
Age group & & & & 21.85 & $0.000^{*}$ \\
=20 & -1.67 & 0.54 & 0.19 & 9.53 & $0.002^{*}$ \\
21-24 & 0.76 & 0.48 & 2.14 & 2.46 & 0.116 \\
MoDEL 2 & & & & & \\
Sex & 0.13 & 0.47 & 1.14 & 0.08 & 0.773 \\
Age group & & & & 22.48 & $0.000^{*}$ \\
S20 & -1.97 & 0.63 & 0.14 & 9.87 & $0.002^{*}$ \\
21-24 & 0.93 & 0.54 & 2.53 & 2.95 & 0.086 \\
Physical health & -0.08 & 0.03 & 0.92 & 9.82 & $0.002^{*}$ \\
Psychological & 0.001 & 0.02 & 1.00 & 0.004 & 0.947 \\
Social relationships & 0.04 & 0.02 & 1.04 & 5.31 & $0.021^{*}$ \\
Environment & 0.01 & 0.02 & 1.01 & 0.08 & 0.771 \\
\hline
\end{tabular}

*significant at $p<0.05$.

doing more judged performances had higher scores than those who did not.

We also performed a multivariate logistic regression comparing HEM and non-HEM respondents on the four QoL dimensions after controlling for sex and age. Model 2 explained the data well, with a Nagelkerke $R^{2}$ coefficient (i.e., a measure of the strength of the relationship) of 0.42 and $77 \%$ of the respondents correctly classified. Overall, three factors were significant: age group $\left(z_{2}=22.48, p=0.000\right)$, physical health $\left(z_{1}=9.83, p=0.002\right)$, and social relationships $\left.z_{1}=5.31, p=0.021\right)$ (Table 5). What distinguishes HEM and non-HEM musicians? Adding 1 to the physical health dimension score decreased the odds of being in the HEM group (odd ratios from 1 to 0.919 ), while conversely, adding 1 to social relationships dimension score increased the odds of being in the HEM group (odds ratio from 1 to 1.038 ).

\section{DISCUSSION}

The QoL of music college students (i.e., Bachelor and Master) and amateur performers who took part in this study was high, mirroring the positive wellbeing profiles reported by Ascenso et al. (2018) in their study of professional musicians. Median scores for overall QoL and general health were both higher than 4, 
and on the four QoL dimensions, they were higher than 70 . Nonetheless, the study highlights some intriguing differences between college music students and amateur musicians, for instance with amateur musicians scoring significantly higher than music students on overall QoL and general health.

\section{Overall Quality of Life}

When comparing music college students with amateur musicians, our results showed that music college students evaluated their overall QoL more negatively than amateur musicians, but this was not related to participation in judged performances and competitions.

As defined by the WHO (1996), QoL includes many domains of functioning. This subjective perception is affected by personal physical health, psychological states, social relationships, and environmental features (Saxena and Orley, 1997).

Our findings underline the results presented in Kreutz et al.'s (2009) study suggesting that music performance students tend to neglect health promoting behaviors (e.g., stress management, physical activity). Our results do not consider health promoting behaviors but highlight the fact that QoL among music college students seems to be influenced negatively by physical and psychological factors (Kreutz et al., 2009). These results can partially be explained by the unique educational context of music colleges, which generates both physical and psychological challenges to health. For example, Kenny (2004) showed that musicians at different stages of their careers, report different sources of stress. The top four sources among professional musicians were separation from family, irregular working hours, monotony of rehearsals, and traveling. By contrast, the top four stressors for student musicians were uncertainty about future employment, professional auditions, backstabbing, and irregular working hours (Steptoe, 1989). Therefore, our results are not surprising, as music college students face many difficulties that pose consequences for their overall QoL.

However, the unique educational context of music colleges can also present opportunities (Perkins et al., 2017). Indeed, support sources for health and wellbeing within conservatoires, including improved access to health professionals and welfare staff and specific health promotion initiatives, are now being developed (Perkins et al., 2017). In the French-speaking part of Switzerland, different initiatives are implemented to help music students in their daily work. For example, courses and training to manage stress during judged performances are offered to students, but these courses are often optional. And, as mentioned earlier, this region of Switzerland is less developed in this respect than the German- and Italian-speaking parts, which offer interdisciplinary consultations composed of musicians, doctors, psychologists, and other therapists (Berchtold-Neumann, 2018).

\section{General Health}

Our results also show that music students evaluated their general health more negatively than amateur musicians, unrelated to participation in judged performances, and competitions.

This matches findings among music students reported by Araújo et al. (2017) emphasizing that injury and ill-health among musicians are frequent and well documented in the literature. These issues mainly concern physical problems and suffering (Zander et al., 2010; Bonde et al., 2018). Also, as found in different studies (Ginsborg et al., 2009; Kreutz et al., 2009), PanebiancoWarrens et al. (2014) highlighted the fact that musicians have poor health habits especially concerning physical activity, stress management and nutrition. However, this concept of health is linked to physical aspects as well as to psychological states, for example coping with stress, dealing with negative feelings, and emotions. Williamon (2004) has underlined how physical activity can optimize musicians' skills by enhancing their physiological and psychological responses to performances (p. 163). However, a study comparing music performance students with non-music performance students revealed that musicians do not seem to engage in such activity and tend to have a less healthy lifestyle overall (Ginsborg et al., 2009).

Our results suggest that, as music college students' lives are centered on music and performance, these musicians' main focus may inhibit the importance they give to promoting their general health through physical activity, nutrition education or stress management training.

\section{Physical Health}

Concerning the physical health dimension, our results show that amateur musicians report better physical health than college music students. This is not surprising, as it is well known that music students frequently report pain or discomfort linked to bad posture, excessive practice on their instrument, and performance anxiety (Williamon and Thompson, 2006). The physical health score could be influenced by the pain and discomfort subscale of the WHOQOL-BREF. Pain and discomfort can be caused by performance-related injuries: muscle and tendon injuries, joint issues, nerve compression disorders, and central nervous system disorders. Moreover, the risk of injury increases with increased hours of practice (Kenny and Ackermann, 2009).

Sleep and rest, energy, and fatigue are also evaluated through different items. As some studies have shown that musicians report high levels of exhaustion, stomachaches, headaches, sleep disturbances (Kenny, 2004) and irregular sleep schedules (Araújo et al., 2017; Pecen et al., 2018), these sub-themes could impact on the physical health mean score.

Finally, several studies have highlighted that musicians tend to use drugs or substances: drinking alcohol (Kenny et al., 2014), using beta-blockers (Fishbein et al., 1988) and other prescribed medication (e.g., antidepressants and tranquilizers) or even illicit drugs (e.g., amphetamines, cannabis, cocaine, ecstasy, hallucinogens, and opiate) (West, 2004). Dependence on medicinal substances and medical aids' subscale could be a strong item influencing musicians' physical health.

Psychological factors may be involved in the genesis or maintenance of physical problems (Spahn et al., 2001). One study of musicians reported that psychosomatic aspects play a decisive role in musicians' somatic problems and that these should be addressed in treatment to avoid unwarranted medical interventions (Kenny and Ackermann, 2009).

Our results do not specify which aspect (e.g., fatigue, injuries, and use of substances) of physical health influences musicians 
the most. However, it clearly highlights the need for action to empower music college students in taking care of their physical health.

\section{Psychological Health}

Our results indicate higher psychological wellbeing among musicians who take part in judged performances and competitions and lower levels for female musicians when psychological health was examined as a function of sex.

Numerous studies have highlighted the undue psychological pressures of working in music (Kenny and Osborne, 2006; Seinfeld et al., 2013; Pecen et al., 2016). Therefore, our results seem counterintuitive. The finding that musicians who are confronted with judged performance situations have higher psychological wellbeing could be influenced by the thinking, learning, memory, and concentration subscale analyzed through the questionnaire. Musicians often seek perfection (Pecen et al., 2016; Araújo et al., 2017) and, to reach the highest levels of practice, they have to develop working strategies to enhance their performances. Also, musicians have a tendency to feel anxiety and stress when performing (Kenny, 2004; Nielsen et al., 2017; Wijsman and Ackermann, 2018), but the WHOQOL-BREF does not evaluate this aspect. However, as Biasutti and Concina (2014) showed, anxiety is also negatively correlated with experience, practice hours, and coping strategies. As college music students often practice more than amateurs, we could assume that they would be more prepared to play during judged performances. Therefore, musicians confronted with judged performances may develop strategies to face specific difficulties (Kaspersen and Goetestam, 2002) and seek help to be prepared (Williamon, 2004). These arguments could partially explain the present findings.

According to our results, female musicians present lower psychological health scores. This result is in line with previous large-scale normative studies using the WHOQOL-BREF, which report significantly lower means for women compared with men (Skevington et al., 2004). This psychological dimension score may be influenced by the self-esteem, body image, and negative and positive feelings sub-themes.

During the past two decades, a large number of studies have examined sex differences in self-esteem (Twenge and Campbell, 2001; Orth et al., 2010, 2012; Shaw et al., 2010). Researchers report that, at every age, men tend to have a higher level of self-esteem than women worldwide (Bleidorn et al., 2016). But, how can we explain this tendency? The concept of self-esteem has been investigated through the influence of sex-specific body satisfaction (Lerner et al., 1973, 1976). Body image is frequently linked to self-esteem as the evaluation of physical appearance is subjective and can either be positive or negative (Forrest and Stuhldreher, 2007).

Body image or physical appearance has been established as an important aspect of wellbeing. Physical appearance selfevaluation (i.e., body-esteem) is a specific domain of selfesteem, especially studied in female populations, showing that body esteem is a construct contained within the hierarchical framework of global self-esteem (Seo and Son, 2014). This construct emphasizes the person's affective evaluation of the body and feelings associated with personal body image. In the elite sporting context, different researchers have reported greater body dissatisfaction among women due to idealized shape or distorted and dissatisfied subjective body images (Smolak et al., 2000; Ferrand et al., 2005; de Bruin et al., 2007).

Finally, findings from different studies have repeatedly shown a higher prevalence of anxiety and depression diagnoses in women compared with men (Rae and McCambridge, 2004; Ryan, 2004; Yondem, 2007). A study conducted with singers showed that female musicians reported higher work demands and higher stress symptoms than their male colleagues (Holst et al., 2012). Female performers are a higher-risk group and more likely to need specific help. These results have been linked to the differences on how men and women respond to stress and the use of coping strategies (Barlow, 2001; Craske, 2003; Hammen, 2005). It is also crucial to take this aspect into account as positive and negative feelings are often observed to play an important role in health promoting behaviors (Bandura, 1997; Kreutz et al., 2009).

\section{Social Relationships and Support}

Finally, our results highlight that social relationships and social support increase the chance of reaching a high level in the field of music.

In a recent study conducted by Ascenso et al. (2017), musicians highlighted the importance of family, social and work-related connections to ensure positive functioning. Also, practicing music in groups seems to enhance positive social relationship (Clift et al., 2007, 2010; Ascenso et al., 2018). Some musicians even consider chamber and orchestral groups as part of their families, generating a group identity and positive feelings (Ascenso et al., 2017). However, Cooper and Wills (1989) have highlighted tense relationships between colleagues within music institutions, causing stress.

In contrast to musical groups, solo-oriented musicians often face isolation and loneliness. Therefore, they have to find other ways of establishing and maintaining social relationships (Ascenso et al., 2017), for example creating new social circles outside the music community through different activities. In the field of sport, it is well established that good social support generates higher levels of performance and wellbeing, especially among young athletes. Coach-, parent- and peer-support play an important role in enhancing athletes' motivation (Sheridan et al., 2014). Reis and Gable (2003) also highlighted the importance of strong relationships among the general population. It seems that this social dimension is central to musicians' wellbeing (Ascenso et al., 2017) and, as our results suggest, should be encouraged.

\section{Future Research}

Several additional directions should be taken into account in future research. First, our sample was not representative of Switzerland's musician population, nor wholly representative of the French-speaking part of Switzerland. The sample did not include students from all conservatories and music schools from the region. Regional culture is assumed to be similar across the French-speaking part of Switzerland; nevertheless, we could not determine the influence of musicians' personal cultural backgrounds on their health and wellbeing evaluations (Steptoe and Wardle, 2001; Wardle et al., 2004; Jylhä, 2009). Second, some external parameters that could have influenced 
musicians' answers and evaluations of their wellbeing require greater control. We did not know, for instance, the full extent to which each participating musician was individually exposed to health education initiatives, in their training or their personal lives. Also, participants answered the questionnaire at one specific moment, and we did not control for whether it was a particularly busy period (e.g., examinations, auditions) or a calm period. Third, our results are based on self-reports and could have been flavored by social desirability, stressful or difficult periods during which they filled in the questionnaire (e.g., injuries, personal issues), or career aspirations that could possibly have influenced their answers. Fourth, replication of the study with a larger sample would provide strong support for these findings and is therefore a task for future research. Finally, subsequent studies should investigate differences in wellbeing and health habits between classical, jazz, pop and rock musicians. Indeed, these musical styles convey different philosophies that could impact musicians' health attitudes, perceptions and behaviors.

\section{Conclusions and Practical Implications}

This research offers important insight into musicians' health and has implications for the future about the extent of health education programs in music education settings. Our overview of musicians' wellbeing in the French-speaking part of Switzerland underlines the importance of helping musicians to be aware of their health in order to take care of themselves. As Ascenso et al. (2017) said, "a clear sense of self appears as an overarching sustainer of wellbeing” (p. 65). Therefore, it is crucial to empower aspiring young musicians and accompany them in the process of health and wellbeing promotion. However, different specialists should be involved in ensuring musicians' health and wellbeing, not only their teachers and peers (Williamon, 2004; Williamon and Thompson, 2006; Williamon et al., 2017). Physical care has to be administered by professionals, specialized with musicians, ensuring their postural quality and overall musculoskeletal health. With regard to psychological health, musicians should have access to psychologists and counselors in case of clinical problems and performance coaches and psychologists to enhance their performance. Also, they could benefit from the help of, for example, relaxation therapists or hypnotherapists. Concerning social relationships and support, musicians should have the possibility to plan mediation with people concerned when facing interpersonal difficulties. However, it also falls on institutional structures (through administrators, teachers, and so on) to ensure possibilities for good social relations within places of work and study. Finally, regarding the environment, music institutions should provide suitable working conditions and easy access to training rooms.

\section{REFERENCES}

Antonini Philippe, R., and Güsewell, A. (2016). La simulation de concours d'orchestre: analyse qualitative et située de l'activité des musiciens. Cah. Soc. Québécoise Rech. Musique 17, 71-82. doi: 10.7202/1044 $671 \mathrm{ar}$

Araújo, L. S., Wasley, D., Perkins, R., Atkins, L., Redding, E., Ginsborg, J., et al. (2017). Fit to perform: an investigation of higher education music students'
Worldwide, efforts are being made to propose health education programs for college music students. Some authors, such as Braden et al. (2015), have already highlighted the positive impact of health and psychological skills enhancement programs within music school curricula (Matei et al., 2018). However, in Switzerland, more efforts could be invested in this regard.

The present study is only a beginning. As Ascenso et al. (2017) point out, a better understanding of the processes underpinning musicians' wellbeing and QoL are needed, both at a physical and a psychological level. This includes the influence of sleep, the use of substances and fatigue on the health of musicians, as well as the influence of self-esteem, body image, concentration, learning, and memory. Music making is great for health and wellbeing, but for those who commit to music professionally, more action is needed to support their health, both by musicians themselves as well as their teachers, administrators, and support staff.

\section{NOMENCLATURE}

\section{Resource Identification Initiative}

HEM: Haute École de Musique.

WHO: World Health Organization.

WHOQOL: World Health Organization Quality of Life.

QoL: Quality of Life.

\section{AUTHOR CONTRIBUTIONS}

RAP and CK contributed to the conception and design of the study. RAP, CK, NV, and FCvR organized the database. FCvR performed the statistical analysis. RAP, CK, NV, AW, and FCvR co-wrote the manuscript. All authors contributed to the manuscript revision, read, and approved the submitted version.

\section{FUNDING}

This research was funded by Fondation Chuard Schmid supporting original research, directed by teachers, at the University of Lausanne, Lausanne, Switzerland.

\section{ACKNOWLEDGMENTS}

We would like to thank the participating musicians and school directors and gratefully acknowledge Patricia Mendes De Carvalho and Guillaume Steiner for their help with data collection and data management.

perceptions, attitudes, and behaviors toward health. Front. Psychol. 8:1558. doi: 10.3389/fpsyg.2017.01558

Ascenso, S., Perkins, R., and Williamon, A. (2018). Resounding Meaning: a PERMA wellbeing profile of classical musicians. Front. Psychol. 9:1895. doi: 10.3389/fpsyg.2018.01895

Ascenso, S., Williamon, A., and Perkins, R. (2017). Understanding the wellbeing of professional musicians through the lens of Positive Psychology. Psychol. Music 45, 65-81. doi: 10.1177/0305735616646864 
Bandura, A. (1997). Editorial. Am. J. Health Promot. 12, 8-10. doi: 10.4278/08901171- 12.1 .8

Barlow, D. H. (2001). Anxiety and its Disorders: The Nature and Treatment of Anxiety and Panic, 2nd Edn. New York, NY: The Guilford Press.

Berchtold-Neumann, M. (2018). La médecine des musiciens: discipline insolite ou nécessité? Bull. Méd. Suisses 99, 1359-1360. doi: 10.4414/bms.2018.17184

Biasutti, M., and Concina, E. (2014). The role of coping strategy and experience in predicting music performance anxiety. Music. Sci. 18, 189-202. doi: 10.1177/ 1029864914523282

Bleidorn, W., Arslan, R. C., Denissen, J. J., Rentfrow, P. J., Gebauer, J. E., Potter, J., et al. (2016). Age and gender differences in self-esteem-A crosscultural window. J. Pers. Soc. Psychol. 111, 396-410. doi: 10.1037/pspp0000078

Bonde, L. O., Juel, K., and Ekholm, O. (2018). Associations between music and health-related outcomes in adult non-musicians, amateur musicians and professional musicians-Results from a nationwide Danish study. Nord. J. Music Ther. 27, 262-282. doi: 10.1080/08098131.2018.1439086

Boyce-Tillman, J. (2000). Promoting wellbeing through music education. Philos. Music Educ. Rev. 8, 89-98.

Boyce-Tillman, J. (2014). Music and wellbeing. J. Transdiscipl. Res. S. Afr. 10, 12-33. Braden, A. M., Osborne, M. S., and Wilson, S. J. (2015). Psychological intervention reduces self-reported performance anxiety in high school music students. Front. Psychol. 6:195. doi: 10.3389/fpsyg.2015.00195

Chang, B. H., Chen, B. W., Beckstead, J. W., and Yang, C. Y. (2018). Effects of a music-creation programme on the anxiety, self-esteem, and quality of life of people with severe mental illness: a quasi-experimental design. Int. J. Ment. Health Nurs. 27, 1066-1076. doi: 10.1111/inm.12414

Chong, J., Lynden, M., Harvey, D., and Peebles, M. (1989). Occupational health problems of musicians. Can. Fam. Physician 35, 2341-2348.

Clift, S., Hancox, G., Morrison, I., Hess, B., Kreutz, G., and Stewart, D. (2007). "Choral singing and psychological wellbeing: findings from English choirs in a cross-national survey using the WHOQOL-BREF," in Proceedings of the International Symposium on Performance Science (Utrecht: European Association of Conservatoires), 201-207.

Clift, S., Hancox, G., Morrison, I., Hess, B., Kreutz, G., and Stewart, D. (2010). Choral singing and psychological wellbeing: quantitative and qualitative findings from English choirs in a cross-national survey. J. Appl. Arts Health 1, 19-34. doi: 10.1386/jaah.1.1.19/1

Cohen, J. (1992). A power primer. Psychol. Bull. 112, 155-159. doi: 10.1386/jaah.1. $1.19 / 1$

Cooper, C. L., and Wills, G. I. (1989). Popular musicians under pressure. Psychol. Music 17, 22-36. doi: 10.1037/0033-2909.112.1.155

Craske, M. G. (2003). Origins of Phobias and Anxiety Disorders: Why more Women than Men? Amsterdam: Elsevier.

Croom, A. M. (2012). Music, neuroscience, and the psychology of wellbeing: a précis. Front. Psychol. 2:393. doi: 10.3389/fpsyg.2011.00393

Cruder, C., Falla, D., Mangili, F., Azzimonti, L., Araújo, L., Williamon, A., et al. (2018). Profiling the location and extent of musicians' pain using digital pain drawings. Pain Pract. 18, 53-66. doi: 10.1111/papr.12581

Davidson, J. W. (2008). Singing for self-healing, health and wellbeing. MCA Music Forum 29-33.

de Bruin, A. K., Oudejans, R. R., and Bakker, F. C. (2007). Dieting and body image in aesthetic sports: a comparison of Dutch female gymnasts and non-aesthetic sport participants. Psychol. Sport Exerc. 8, 507-520. doi: 10.1016/j.psychsport. 2006.10.002

De Vellis, R. (2003). Scale Development: Theory and Applications, 2nd Edn. Thousand Oaks, CA: Sage Publications.

Dickinson, S. C. (2018). 7 ways music can enhance wellbeing. Occupa. Health Wellbeing 70, 20-21.

Diener, E. (1984). Subjective wellbeing. Psychol. Bull. 95, 542-575. doi: 10.1037/ 0033-2909.95.3.542

Diener, E., Suh, E. M., Lucas, R. E., and Smith, H. L. (1999). Subjective wellbeing: three decades of progress. Psychol. Bull. 125, 276-302. doi: 10.1037/0033-2909. 125.2.276

Diener, E. D., Emmons, R. A., Larsen, R. J., and Griffin, S. (1985). The satisfaction with life scale. J. Pers. Assess. 49, 71-75. doi: 10.1207/s15327752jpa4901_13

Disabato, D. J., Goodman, F. R., Kashdan, T. B., Short, J. L., and Jarden, A. (2016). Different types of wellbeing? A cross-cultural examination of hedonic and eudaimonic wellbeing. Psychol. Assess. 28, 471-482. doi: 10.1037/pas0000209
Dritsakis, G., van Besouw, R. M., and O’Meara, A. (2017). Impact of music on the quality of life of cochlear implant users: a focus group study. Cochlear Implants Int. 18, 207-215. doi: 10.1080/14670100.2017.1303892

Evans, P. (2015). Self-determination theory: an approach to motivation in music education. Music. Sci. 19, 65-83. doi: 10.1177/1029864914568044

Ferrand, C., Magnan, C., and Antonini Philippe, R. (2005). Body-esteem, body mass index and risk for eating disorders among young French synchronised swimming athletes. Percept. Mot. Skills 101, 877-884. doi: 10.2466/PMS.101.7. 877-884

Fishbein, M., Middlestadt, S. E., Ottati, V., Straus, S., and Ellis, A. (1988). Medical problems among ICSOM musicians: overview of a national survey. Med. Probl. Perform. Art. 3, 1-8.

Forrest, K. Y. Z., and Stuhldreher, W. L. (2007). Patterns and correlates of body image dissatisfaction and distortion among college students. Am. J. Health Stud. $22,18-25$.

Garrido, G., Camps, L., Herrera, I., Guillamat, R., Vallés, V., Sanz, M., et al. (2016). Music and wellbeing. Int. J. Integr. Care 16, 1-8. doi: 10.5334/ijic.2734

Ginsborg, J., Kreutz, G., Thomas, M., and Williamon, A. (2009). Healthy behaviours in music and non-music performance students. Health Educ. 109, 242-258. doi: 10.1108/09654280910955575

Hammen, C. (2005). Stress and depression. Annu. Rev. Clin. Psychol. 1, 293-319. doi: 10.1146/annurev.clinpsy.1.102803.143938

Hawthorne, G., Herrman, H., and Murphy, B. (2006). Interpreting the WHOQOLBREF: preliminary population norms and effect sizes. Soc. Indic. Res. 77, 37-59. doi: 10.1007/s11205-005-5552-1

Holst, G. J., Paarup, H. M., and Baelum, J. (2012). A cross-sectional study of psychosocial work environment and stress in the Danish symphony orchestras. Int. Arch. Occup. Environ. Health 85, 639-649. doi: 10.1007/s00420-011-0710-z

Johnson, J. K., Louhivuori, J., Stewart, A. L., Tolvanen, A., Ross, L., and Era, P. (2013). Quality of life (QOL) of older adult community choral singers in Finland. Int. Psychogeriatr. 25, 1055-1064. doi: 10.1017/S1041610213000422

Jylhä, M. (2009). What is self-rated health and why does it predict mortality? Towards a unified conceptual model. Soc. Sci. Med. 69, 307-316. doi: 10.1016/j. socscimed.2009.05.013

Kaspersen, M., and Goetestam, K. (2002). A survey of music performance anxiety among Norwegian music students. Eur. J. Psychiatry 16, 69-80.

Kenny, D. T. (2004). Music performance anxiety: is it the music, the performance or the anxiety. Music Forum 10, 38-43.

Kenny, D. T. (2011). The Psychology of Music Performance Anxiety. Oxford: Oxford University Press. doi: 10.1093/acprof:oso/9780199586141.001.0001

Kenny, D. T., and Ackermann, B. (2009). "Optimizing physical and psychological health in performing musicians," in The Oxford Handbook of Music Psychology, eds S. Hallam, I. Cross, and M. Thaut (Oxford: Oxford University Press), 390-400. doi: 10.1093/oxfordhb/9780199298457.013.0036

Kenny, D. T., and Ackermann, B. (2015). Performance-related musculoskeletal pain, depression and music performance anxiety in professional orchestral musicians: a population study. Psychol. Music 43, 43-60. doi: 10.1177/ 0305735613493953

Kenny, D. T., Driscoll, T., and Ackermann, B. (2014). Psychological wellbeing in professional orchestral musicians in Australia: a descriptive population study. Psychol. Music 42, 210-232. doi: 10.1177/03057356124 63950

Kenny, D. T., and Osborne, M. S. (2006). Music performance anxiety: new insights from young musicians. Adv. Cogn. Psychol. 2, 103-112.

Kok, L. M., Vlieland, T. P. V., Fiocco, M., and Nelissen, R. G. (2013). A comparative study on the prevalence of musculoskeletal complaints among musicians and non-musicians. BMC Musculoskelet. Disord. 14:9. doi: 10.1186/1471-2474-14-9

Kreutz, G., Ginsborg, J., and Williamon, A. (2009). Health-promoting behaviours in conservatoire students. Psychol. Music 37, 47-60. doi: 10.1177/0305735607086047

Leaver, R., Harris, E. C., and Palmer, K. T. (2011). Musculoskeletal pain in elite professional musicians from British symphony orchestras. Occup. Med. 61, 549-555. doi: 10.1093/occmed/kqr129

Lerner, R. M., Karabenick, S. A., and Stuart, J. L. (1973). Relations among physical attractiveness, body attitudes, and self-concept in male and female college students. J. Psychol. 85, 119-129. doi: 10.1080/00223980.1973.9923870

Lerner, R. M., Orlos, J. B., and Knapp, J. R. (1976). Physical attractiveness, physical effectiveness, and self-concept in late adolescents. Adolescence 11, 313-326. 
Lindert, J., Bain, P. A., Kubzansky, L. D., and Stein, C. (2015). Wellbeing measurement and the WHO health policy Health 2010: systematic review of measurement scales. Eur. J. Public Health 25, 731-740. doi: 10.1093/eurpub/ cku193

Matei, R., Broad, S., Goldbart, J., and Ginsborg, J. (2018). Health education for musicians. Front. Psychol. 9:1137. doi: 10.3389/fpsyg.2018.01137

Mitchell, L. A., MacDonald, R. A., Knussen, C., and Serpell, M. G. (2007). A survey investigation of the effects of music listening on chronic pain. Psychol. Music 35, 37-57. doi: 10.1177/0305735607068887

Nielsen, C., Studer, R. K., Hildebrandt, H., Nater, U. M., Wild, P., Danuser, B., et al. (2017). The relationship between music performance anxiety, subjective performance quality and post-event rumination among music students. Psychol. Music 46, 136-152. doi: 10.1177/0305735617706539 doi: $10.1007 / \mathrm{s} 12160-008-9030-2$

Orth, U., Robins, R. W., and Widaman, K. F. (2012). Life-span development of self-esteem and its effects on important life outcomes. J. Pers. Soc. Psychol. 102, 1271-1288. doi: 10.1037/a0025558

Orth, U., Trzesniewski, K. H., and Robins, R. W. (2010). Self-esteem development from young adulthood to old age: a cohort-sequential longitudinal study. J. Pers. Soc. Psychol. 98, 645-658. doi: 10.1037/a0018769

Osborne, M. S., Greene, D. J., and Immel, D. T. (2014). Managing performance anxiety and improving mental skills in conservatoire students through performance psychology training: a pilot study. Psychol. Well Being 4, 1-17. doi: 10.1186/s13612-014-0018-3

Panebianco-Warrens, C. R., Fletcher, L., and Kreutz, G. (2014). Health-promoting behaviors in South African music students: a replication study. Psychol. Music 43, 779-792. doi: 10.1177/0305735614535829

Pavot, W., and Diener, E. (1993). The affective and cognitive context of selfreported measures of subjective well-being. Soc. Indic. Res. 28, 1-20. doi: 10. 1007/BF01086714

Pecen, E., Collins, D., and MacNamara, Á. (2016). Music of the night: performance practitioner considerations for enhancement work in music. Sport Exerc. Perform. Psychol. 5, 377-395. doi: 10.1037/spy0000067

Pecen, E., Collins, D. J., and MacNamara, Á. (2018). "It's your problem. deal with it." Performers' experiences of psychological challenges in music. Front. Psychol. 8:2374. doi: 10.3389/fpsyg.2017.02374

Perkins, R., Reid, H., Araújo, L. S., Clark, T., and Williamon, A. (2017). Perceived enablers and barriers to optimal health among music students: a qualitative study in the music conservatoire setting. Front. Psychol. 8:968. doi: 10.3389/ fpsyg.2017.00968

Perkins, R., and Williamon, A. (2014). Learning to make music in older adulthood: a mixed-methods exploration of impacts on well-being. Psychol. Music 42, 550-567. doi: 10.1177/0305735613483668

Pothoulaki, M., MacDonald, R., and Flowers, P. (2012). "The use of music in chronic illness: evidence and arguments," in Music, Health, and Well-Being, eds R. A. R. MacDonald, G. Kreutz, and L. Mitchell (Oxford: Oxford University Press), 239-256. doi: 10.1093/acprof:oso/9780199586974.003.0018

Rae, G., and McCambridge, K. (2004). Correlates of performance anxiety in practical music exams. Psychol. Music 32, 432-439. doi: $10.1177 / 0305735604046100$

Reis, H. T., and Gable, S. L. (2003). “Toward a positive psychology of relationships," in Flourishing: The Positive Person and the Good Life, eds C. L. Keyes and J. Haidt (Washington, DC: American Psychological Association), 129-159. doi: 10.1037/10594-006

Ryan, C. (2004). Gender differences in children's experience of musical performance anxiety. Psychol. Music 32, 89-103. doi: $10.1177 / 0305735604039284$

Ryan, R. M., and Deci, E. L. (2001). On happiness and human potentials: a review of research on hedonic and eudaimonic well-being. Annu. Rev. Psychol. 52, 141-166. doi: 10.1146/annurev.psych.52.1.141

Ryan, E. L., and Deci, R. M. (2002). "Overview of self-determination theory: an organismic-dialectical perspective," in Handbook of Self-Determination Research, eds E. L. Deci and R. M. Ryan (Rochester, NY: University of Rochester Press), 3-33.

Ryff, C. D. (1989). Happiness is everything, or is it? Explorations on the meaning of psychological well-being. J. Pers. Soc. Psychol. 57, 1069-1081. doi: 10.1037/ 0022-3514.57.6.1069
Ryff, C. D. (2014). Psychological well-being revisited: advances in the science and practice of eudaimonia. Psychother. Psychosom. 83, 10-28. doi: 10.1159/ 000353263

Saxena, S., and Orley, J. (1997). Quality of life assessment: the World Health Organization perspective. Eur. Psychiatry 12, 263-266. doi: 10.1016/S09249338(97)89095-5

Seinfeld, S., Figueroa, H., Ortiz-Gil, J., and Sanchez-Vives, M. V. (2013). Effects of music learning and piano practice on cognitive function, mood and quality of life in older adults. Front. Psychol. 4:810. doi: 10.3389/fpsyg.2013.00810

Seligman, M. E. (1998). What is the good life. APA Monit. 29, 1-4.

Seo, Y. S., and Son, Y. L. (2014). A study on body image, self-esteem, and family strengths of female university students. J. Korean Clin. Health Sci. 2, 90-97. doi: 10.15205/kschs.2018.03.31.1416

Shaw, B. A., Liang, J., and Krause, N. (2010). Age and race differences in the trajectories of self-esteem. Psychol. Aging 25, 84-94. doi: 10.1037/a0018242

Sheridan, D., Coffee, P., and Lavallee, D. (2014). A systematic review of social support in youth sport. Int. Rev. Sport Exerc. Psychol. 7, 198-228. doi: 10.1080/ 1750984X.2014.931999

Skevington, S. M., Lotfy, M., and O'Connell, K. A. (2004). The World Health Organization's WHOQOL-BREF quality of life assessment: psychometric properties and results of the international field trial. A report from the WHOQOL group. Qual. Life Res. 13, 299-310. doi: 10.1023/B:QURE. 0000018486.91360 .00

Smolak, L., Murnen, S. K., and Ruble, A. E. (2000). Female athletes and eating problems: a meta-analysis. Int. J. Eat. Disord. 27, 371-380. doi: 10.1002/(SICI)1098-108X(200005)27:4<371: AID-EAT1>3.0.CO;2-Y

Spahn, C., Ell, N., and Seidenglanz, K. (2001). Psychosomatic findings in musician patients at a department of hand surgery. Med. Probl. Perform. Art. 16, 144-151.

Steptoe, A. (1989). Stress, coping and stage fright in professional musicians. Psychol. Music 17, 3-11. doi: 10.1177/0305735689171001

Steptoe, A., and Wardle, J. (2001). Health behaviour, risk awareness and emotional well-being in students from Eastern Europe and Western Europe. Soc. Sci. Med. 53, 1621-1630. doi: 10.1016/S0277-9536(00) 00446-9

Thoma, M. V., Scholz, U., Ehlert, U., and Nater, U. M. (2012). Listening to music and physiological and psychological functioning: the mediating role of emotion regulation and stress reactivity. Psychol. Health 27, 227-241. doi: 10.1080/ 08870446.2011.575225

Twenge, J. M., and Campbell, W. K. (2001). Age and birth cohort differences in self-esteem: a cross-temporal meta-analysis. Pers. Soc. Psychol. Rev. 5, 321-344. doi: 10.1207/S15327957PSPR0504_3

Västfjäll, D., Juslin, P. N., and Hartig, T. (2012). "Music, subjective well-being, and health: the role of everyday emotions," in Music, Health, and Well-Being, eds R. A. R. MacDonald, G. Kreutz, and L. Mitchell (Oxford: Oxford University Press), 405-423. doi: 10.1093/acprof:oso/9780199586974.003.0027

Vittinghoff, E., and McCulloch, C. (2007). Relaxing the rule of ten events per variable in logistic and Cox regression. Am. J. Epidemiol. 165, 710-718. doi: 10.1093/aje/kwk052

Wardle, J., Steptoe, A., Guliš, G., Sartory, G., Sêk, H., Todorova, I., et al. (2004). Depression, perceived control, and life satisfaction in university students from Central-Eastern and Western Europe. Int. J. Behav. Med. 11, 27-36. doi: 10. 1207/s15327558ijbm1101_4

West, R. (2004). "Drugs and musical performance," in Musical Excellence: Strategies and Techniques to Enhance Performance, ed. A. Williamon (Oxford: Oxford University Press), 271-290. doi: 10.1093/acprof:oso/9780198525356. 003.0014

Weziak-Białowolska, D. (2016). Attendance of cultural events and involvement with the arts-impact evaluation on health and well-being from a Swiss household panel survey. Public Health 139, 161-169. doi: 10.1016/j.puhe.2016. 06.028

WHOQOL Group (1991). Assessment of Quality of Life in Health Care. Geneva: WHO.

WHOQOL Group (1995). The World Health Organization quality of life assessment (WHOQOL): position paper from the World Health Organization. Soc. Sci. Med. 41, 1403-1409. doi: 10.1016/0277-9536(95) 00112-K 
WHOQOL Group (1998). Development of the World Health Organization WHOQOL-BREF quality of life assessment. Psychol. Med. 28, 551-558. doi: $10.1017 /$ S0033291798006667

Wijsman, S., and Ackermann, B. J. (2018). Educating Australian musicians: are we playing it safe? Health Promot. Int. day030. doi: 10.1093/heapro/ day 030

Williamon, A. (ed.). (2004). Musical Excellence: Strategies and Techniques to Enhance Performance. Oxford: Oxford University Press. doi: 10.1093/acprof: oso/9780198525356.001.0001

Williamon, A., Clark, T., and Küssner, M. (2017). "Learning in the spotlight: approaches to self-regulating and profiling performance," in Musicians in the Making: Pathways to Creative Performance, eds J. Rink, H. Gaunt, and A. Williamon (Oxford: Oxford University Press), 206-221.

Williamon, A., and Thompson, S. (2006). Awareness and incidence of health problems among conservatoire students. Psychol. Music 34, 411-430. doi: 10. $1177 / 0305735606067150$

World Health Organization (1991). World Health Statistics. Geneva: World Health Organization.

World Health Organization (1996). WHOQOL-BREF: Introduction, Administration, Scoring, and Generic Version of the Assessment. Program on Mental Health. Geneva: World Health Organization.
Yondem, Z. D. (2007). Performance anxiety, dysfunctional attitudes and gender in university music students. Soc. Behav. Pers. Int. J. 35, 1415-1426. doi: 10.2224/sbp.2007.35.10.1415

Zander, M. R., Voltmer, E., and Spahn, C. (2010). Health promotion and prevention in higher music education. Med. Probl. Perform. Art. 25, $54-65$.

Zaza, C. (1998). Playing-related musculoskeletal disorders in musicians: a systematic review of incidence and prevalence. Can. Med. Assoc. J. 158, 1019-1025.

Conflict of Interest Statement: The authors declare that the research was conducted in the absence of any commercial or financial relationships that could be construed as a potential conflict of interest.

Copyright (c) 2019 Antonini Philippe, Kosirnik, Vuichoud, Williamon and Crettaz von Roten. This is an open-access article distributed under the terms of the Creative Commons Attribution License (CC BY). The use, distribution or reproduction in other forums is permitted, provided the original author(s) and the copyright owner(s) are credited and that the original publication in this journal is cited, in accordance with accepted academic practice. No use, distribution or reproduction is permitted which does not comply with these terms. 


\section{OPEN ACCESS}

Edited by:

Rafael Ramirez,

Universidad Pompeu Fabra, Spain

Reviewed by:

Ioulia Papageorgi,

University of Nicosia, Cyprus Eckart Altenmüller,

Hanover University of Music Drama and Media, Germany

*Correspondence:

Margaret S. Barrett

m.barrett@uq.edu.au

Specialty section:

This article was submitted to Performance Science, a section of the journal

Frontiers in Psychology

Received: 23 November 2018

Accepted: 15 March 2019

Published: 24 April 2019

Citation:

Barrett MS, Flynn LM, Brown JE and Welch GF (2019) Beliefs and Values

About Music in Early Childhood

Education and Care: Perspectives

From Practitioners.

Front. Psychol. 10:724.

doi: 10.3389/fpsyg.2019.00724

\section{Beliefs and Values About Music in Early Childhood Education and Care: Perspectives From Practitioners}

\author{
Margaret S. Barrett ${ }^{1 *}$, Libby M. Flynn ${ }^{1}$, Joanne E. Brown ${ }^{2}$ and Graham F. Welch ${ }^{3}$ \\ ${ }^{1}$ School of Music, The University of Queensland, Brisbane, QLD, Australia, ${ }^{2}$ School of Psychology, The University of \\ Queensland, Brisbane, QLD, Australia, ${ }^{3}$ Department of Culture, Communication and Media, Institute of Education, University \\ College London, London, United Kingdom
}

This paper reports the findings of a study that aimed to identify the music beliefs and values of educators in early childhood education and care settings in Australia. The aims of the study were 2-fold: to adapt and pilot a survey of music beliefs and values which might be implemented subsequently nationally in childcare settings; and, secondly, to identify the music beliefs and values held by early childhood and care educators concerning music in children's learning. The research questions that guided this component of the study were: What is the profile of early childhood and care educators? What beliefs and values for music engagement are held by early childhood and care educators? What shapes early childhood and care educators' music beliefs and values? Findings indicated that educators' beliefs and values on all items are above the mid-point indicating overall positive attitudes toward music despite the majority having no formal qualifications in music or a history of instrumental performance and/or singing. Given the overall positive attitudes toward music we suggest there is enormous potential within this population for further professional learning and development targeted at music and its potential wider benefits in young children's learning and lives.

Keywords: early childhood education and care, music education, early childhood educators, music beliefs and value, music practices in early childhood education and care

\section{INTRODUCTION}

In recent years there has been a documented steady decline in the provision of music education in the pre-service training of early childhood educators in Australia (Letts, 2015). Teacher education programs in early childhood have seen a reduction from some $72 \mathrm{~h}$ of music education over a 4-year Bachelor degree in 1988 (University of Tasmania, as one example) to current figures ranging from 0 to $17 \mathrm{~h}$ in total (Letts, 2015). Within the early education and care sector, professional qualifications such as certificates and diplomas have little consideration of music as either a content area or a teaching and learning strategy (Letts, 2015). Paradoxically, there is a substantial and growing body of literature that evidences the contributions of music learning and engagement to young children's development across a range of factors (for example, Moreno et al., 2009; Brown et al., 2010; Brown and Sax, 2013; Williams et al., 2015; Bugos and DeMarie, 2017).

Concurrent with this documented decline in educator preparation to teach and use music in early childhood education and care, there has been increasing recognition of the role early education and care plays in producing positive long-term development and learning outcomes (cf. Siraj-Blatchford et al., 2011). Governments internationally (Schober and Stahl, 2014; Department for Education, 2015) have focused on measuring and promoting access to quality 
early education and care; a development largely driven by the OECD. Australia is no exception to this with the Council of Australian Governments agreeing in 2009 to a National Early Childhood Development Strategy entitled Investing in the early years (Council of Australian Governments, 2009). Developments over the subsequent years include a bi-partisan initiative from successive federal governments to implement an Early Years Learning Framework (Department of Education Employment and Workplace Relations, 2009), an Educators' guide to use this framework (Department of Education Employment Workplace Relations, 2010), and a national monitoring and reporting process that assesses all childcare providers in the country against set criteria and quality standards (Australian Children's Education and Care Quality Authority (ACECQA), 2016). In short, there is significant recognition of the importance of early years learning and engagement and a strong commitment from a range of agencies in Australia to pursue a national agenda for improvement.

Despite the aforementioned body of evidence attesting to the importance of music in early learning and life, there is little recognition in official early years policy on the developmental role of music in early learning and engagement. Furthermore, there remains a concomitant lack of education and training for contemporary educators in the early childhood sector on how they might use music effectively as an educational tool. As an initial step to address this challenge, this paper aims to identify the values and beliefs of early childhood educators concerning their use of music. This will allow for a clearer and deeper understanding of the factors that facilitate or constrain the use of music in early childhood education and care programming.

This paper arises from a national investigation of young Australian children's experiences and engagement with music in the home, in Music Early Learning Programs (MELPs), and childcare (Barrett and Welch, 2013-2016). The overall investigation seeks to identify the ways in which Australian children and their families engage with music in these diverse settings, whilst noting the outcomes of such experience and engagement and the role of music-making in family life and parenting. The current paper reports findings from one strand of this project. Specifically, the analysis of music provision in long day-care settings in metropolitan and regional Queensland. The study focused on the experience, qualifications, and music beliefs and values of educators in these settings.

\section{THEORETICAL BACKGROUND}

Internationally, UNESCO (2016) has highlighted four main profile areas by which early childhood development can be assessed. These are executive function, social and emotional development, motor development, and early literacy and numeracy. There is a growing database of research literature that demonstrates how each of these profile areas can be nurtured through sustained engagement in musical activity. This is evidenced in studies on children's executive function (Moreno et al., 2011; Zuk et al., 2014; Bowmer et al., 2018), social and emotional development (Hallam, 2010; Barrett, 2011, 2016, 2017;
Welch et al., 2014), motor development (Derri et al., 2001) and early literacy and numeracy (Anvari et al., 2002; Moritz et al., 2013; Williams et al., 2015; Cohrdes et al., 2016).

In addition, it has been reported that early music experiences can have a beneficial impact on a wide range of developmental features embracing cognitive, emotional, physical, and social domains. Example studies include those by Bengtsson et al. (2005), Chen et al. (2012), Creech et al. (2016), Dingle et al. (2012), Eerola and Eerola (2013), Forgeard et al. (2008), Fujioka et al. (2006), Gaser and Schlaug (2003), Gordon et al. (2015), Habib et al. (2016), Halwani et al. (2011), Hetland (2000), Ho et al. (2003), Hyde et al. (2009), Knight et al. (2016), Masataka and Perlovsky (2012), Moreno and Besson (2006), Moreno et al. (2009), Nutley et al. (2014), Osborne et al. (2016), Pantev et al. (2001), Paulson et al. (2013), Rickard et al. (2010), Roden et al. (2012), Saunders et al. (2014), Schlaug et al. (2005), Seinfeld et al. (2013), Tierney et al. (2013), Trappe (2012), Welch et al. (2014), Welch et al. (2015), Wetter et al. (2009), and Williams et al. (2015). For overviews of such impacts, see Hallam (2015), Schlaug (2015), Silvia et al. (2016) and-for a more discursive narrative-see Henriksson-Macauley (2014).

Furthermore, such benefits have been documented in studies that have controlled for socio-economic status (SES) and ethnicity. Key features in such studies are that beneficial music making is characterized by being sustained, active (singing and playing instruments), involves generative opportunities (composing and improvising) whether undertaken in groups or individually, and is a "fun" positive experience for children (cf. Barrett, 2012; Hallam, 2015). Additionally, recent research indicates that individual and shared music making in family settings contributes to positive parenting practices (Barrett, 2009), and early identity development in young children (Barrett, 2011, 2016, 2017). For example, a large-scale Australian study suggested that children who participate in shared music making at age three are better prepared for school-related experiences at age five (Williams et al., 2015). Overall, the body of research indicates that music is commonly a key component in young children's learning and development and can be a vital tool in the learning and care practices of early childhood educators. Collectively, these studies (and others) are building an evidence base of music's potential and actual impacts on different aspects of aural perception related to sound and language, verbal memory, spatial reasoning, self-regulation, pro-social skills, and aspects of general school-related attainment.

Paradoxically, other literature (cf. Letts, 2015) indicates that music education is barely addressed in the pre-service preparation of early childhood educators in University and Technical and Further Education (TAFE) settings, both in Australia and (often) internationally. Research suggests that teachers of young children in community and school settings often have limited experience of music education, other than being able to draw on their own personal experience. Such experience, however, has its limitations, often leading to a reported "lack of confidence" in generalist music educators who are working in early childhood education and care (ECEC) settings and primary schools (Mills, 1989; Hennessy, 2000; McCullough, 2006; Seddon and Biasutti, 2008; Stakelum, 2008; 
Hallam et al., 2009; Stunell, 2010; Welch and Henley, 2014). Furthermore, this reported "lack of confidence" in the teaching of music tends not to be addressed sufficiently in Primary teachers' initial teacher education (pre-service) courses (e.g., Ballantyne and Packer, 2004; Ballantyne, 2006).

Teachers' beliefs concerning the nature of child education, development, and teaching and learning, are powerful shaping forces in their classroom practices. Early childhood pre-service teachers' beliefs about music as an active developmental tool rather than mere enrichment are shaped by their experience and knowledge of music (Austin and Reinhardt, 1999; Kim and Kemple, 2011). Investigation into 21 early childhood educators' self-efficacy beliefs for teaching across different subject areas indicated significantly lower scores for the arts in comparison to their perceived confidence for teaching mathematics and English (Garvis and Pendergast, 2011). Overall however, little is known of the music beliefs of early childhood education and care (ECEC) practitioners, particularly those working with children prior to entry into formal schooling. This investigation seeks to address this gap.

\section{AIMS}

The research aims for this study were 2-fold: first, to adapt and pilot a survey of music beliefs and values which might be implemented subsequently nationally in childcare settings; and, secondly, to identify the music beliefs and values held by early childhood and care educators concerning music in children's learning. The research questions that guided this component of the study were:

1. What is the profile of early childhood and care educators?

2. What beliefs and values for music engagement are held by early childhood and care educators?

3. What shapes early childhood and care educators' music beliefs and values?

\section{METHOD}

\section{Participants}

Data collection took place at seven, long (i.e., extended) daycare centers that were located across metropolitan and regional Queensland. A total of 88 participants (87 females and one male) each completed two questionnaires with the assistance of a project researcher. Purposive stratified sampling was conducted via deliberate selection of educators from rural and urban childcare center locations in proportion to the number of rural and urban childcare centers operating in Queensland. This was implemented with the intention of capturing a diverse cross-section of socioeconomic status, early childhood music education philosophies and business models (including not-forprofit, commercial, and community models). Center Directors ${ }^{1}$ $(n=10)$, lead educators $(n=32)$, and general educators $(n=46)$ constituted the final study participants.

\footnotetext{
${ }^{1}$ Ten Centre Directors participated from the 7 day care centers, reflecting personnel changes during data collection.
}

\section{Measures}

Two questionnaires were completed. The first was a demographic survey, which asked for information about practitioner educational qualifications, current (if any) education being undertaken, employment status, years of experience in the early childhood education sector, and past and present music engagement. Following this, participants completed the Music Beliefs Questionnaire (MBQ). The MBQ is a 37 -item survey, which gauges beliefs and values concerning the role of music within early childhood. Modified from the Austin and Reinhardt (1999) scale, the 37 items were designed to measure three key construct areas. These pertained to the beneficial outcomes of music for children in (1) creative and cultural development, (2) quality of life, and (3) social and emotional development. Responses to each statement were indicated on a 7-point Likerttype scale $(1=$ very untrue of what $I$ believe; $7=$ very true of what I believe; see Appendix A).

The MBQ piloted in the present study was an adaptation of Austin and Reinhardt's (1999) scale, originally designed to measure the music philosophy beliefs of pre-service teachers. A number of items in the Austin and Reinhardt scale were modified to suit the current study population and context, with an additional question included concerning music and special education. Items sought to gauge participants' beliefs about music by investigating concepts regarding: the outcomes of music education, music education significance for childhood development, music education relevance for child psychology, and music education importance in the creation and promotion of pro-social skills. Adaptations made for the revised scale also aimed to capture changes inherent in early childhood education theory, practice and policy that have occurred over time and across different countries of implementation. For example, Question 21 "Music education supports the development of a child's identity" reflects recent research that focuses on the formative role of music in identity (Hargreaves et al., 2017) and specifically in young children's identity (Barrett, 2011, 2016, 2017). Similarly, Question 36 "Music education offers a way to include children with special learning needs" acknowledges current policy and practice concerning inclusiveness in classrooms (Devarakonda, 2012; Jellison, 2018).

\section{Analytic Approach}

This study focused on illuminating educators' (a) demographic profile, (b) music engagement beliefs and values held, and (c) factors shaping music beliefs and values. Analyses were conducted in three initial stages. The first provided descriptive statistics on demographic details that outlined the profile of early childhood and care educators from the study (see Table 1). Exploration was also performed of the basic descriptive statistics from individual MBQ items in order to identify educators' highest and lowest endorsed beliefs regarding music (see Table 2). This provided general insight into the perceived outcomes and values attached to music education by these Australian early childhood and care educators.

In the second stage, a confirmatory factor analysis (CFA) was performed to validate the intended three-factor structure for the 37 MBQ survey items (see Figure 1 and Table 4). This 
TABLE 1 | Demographic profile of early childhood and care educators: frequency (n) and percentage (\%) of key categorical characteristics in the analytic sample.

\begin{tabular}{|c|c|c|}
\hline Characteristic & $n$ & $\%$ \\
\hline \multicolumn{3}{|l|}{ Identified Gender } \\
\hline Female & 87 & 98.86 \\
\hline Male & 1 & 1.14 \\
\hline \multicolumn{3}{|l|}{ Age of Participant } \\
\hline $18-24$ & 15 & 17.05 \\
\hline $25-34$ & 25 & 28.41 \\
\hline $35-44$ & 19 & 21.59 \\
\hline $45-54$ & 16 & 18.18 \\
\hline $55-64$ & 10 & 11.36 \\
\hline $65+$ & 2 & 2.27 \\
\hline Did Not State & 1 & 1.14 \\
\hline \multicolumn{3}{|l|}{ Educator Role } \\
\hline Director & 10 & 11.36 \\
\hline Lead Educator & 32 & 36.36 \\
\hline General Educator & 46 & 52.27 \\
\hline \multicolumn{3}{|l|}{ Educator Years of Experience } \\
\hline $0-4$ years & 24 & 27.27 \\
\hline $5-9$ years & 26 & 29.55 \\
\hline $10-14$ years & 20 & 22.73 \\
\hline $15-19$ years & 9 & 10.23 \\
\hline $20+$ years & 9 & 10.23 \\
\hline \multicolumn{3}{|l|}{ Employment Status } \\
\hline Full-Time & 56 & 63.64 \\
\hline Part-Time & 24 & 27.27 \\
\hline Casual & 8 & 9.09 \\
\hline \multicolumn{3}{|l|}{ Child Care Site Location } \\
\hline Urban & 65 & 73.86 \\
\hline Rural & 23 & 26.14 \\
\hline \multicolumn{3}{|c|}{ Highest Qualification Level Achieved } \\
\hline Certificate/Diploma & 69 & 78.41 \\
\hline Bachelors Degree & 14 & 15.91 \\
\hline Postgraduate Degree & 4 & 4.55 \\
\hline Missing & 1 & 1.14 \\
\hline \multicolumn{3}{|c|}{ Educator Qualification Field of Study } \\
\hline Early Childhood Education & 36 & 40.91 \\
\hline Child Care & 27 & 30.68 \\
\hline Primary/Secondary Education & 7 & 7.95 \\
\hline Nursing & 1 & 1.14 \\
\hline Other & 9 & 10.23 \\
\hline None/not complete & 8 & 9.09 \\
\hline \multicolumn{3}{|l|}{ Educator Current Study Status } \\
\hline Currently Studying & 18 & 20.45 \\
\hline Not Currently Studying & 70 & 79.55 \\
\hline \multicolumn{3}{|l|}{ Educator Current Field of Study } \\
\hline Early Childhood Education & 11 & 12.50 \\
\hline Child Care & 4 & 4.55 \\
\hline
\end{tabular}

TABLE 1 | Continued

\begin{tabular}{lcc}
\hline Characteristic & $\boldsymbol{n}$ & $\%$ \\
\hline Primary/secondary Education & 1 & 1.14 \\
Other & 2 & 2.27 \\
$\quad$ Not Currently Studying & 70 & 79.55 \\
Formal Music Qualifications & & \\
$\quad$ Yes & 2 & 2.27 \\
No & 86 & 97.73 \\
Ever Learnt Instrument/Sung & & \\
Yes & 42 & 47.73 \\
No & 46 & 52.27 \\
Currently Play Musical Instrument/Sing & & 15.91 \\
Yes & 14 & 31.82 \\
No & 28 & 52.27 \\
Never Played/Sung & 46 & \\
\hline$N=88$. Percentages may not sum to 100.00\% across categories within a demographic \\
variable due to rounding.
\end{tabular}

model was compared and contrasted against an alternative singlefactor structure. The single-factor model was found to provide an equally plausible explanation for the observed data, with both models displaying adequate overall model fit. However, the threefactor model demonstrated very high intercorrelations amongst its proposed latent constructs, almost to the point of singularity. Therefore, the more parsimonious single-factor structure was adopted. Given that some of the fit indices from both models were less than desirable, an exploratory factor analysis (EFA) using principal axis factoring extraction and direct oblimin (oblique) rotation was also conducted. This allowed for a data-driven approach to determine the underlying factor structure. Results provided by the scree plot further corroborated the use of a single-factor model to best represent the data. Thus, a unified MBQ scale score was utilized in further analyses.

The third stage of analysis made use of this overall MBQ score as the key outcome variable in a series of linear regressions, to explore which factors predict the general music beliefs and values held by early childhood and care educators regarding the benefits of music for young children (see Table 6). Specifically, the MBQ scale score was regressed onto educator age groupings (18-24, 25-34, 35-44, 45-54, 55-64, 65+ years), educator role (higher level educator [directors and lead educators], general educator), educator years of experience $(0-4,5-9$, $10-14,15-19,20+$ years), whether the educator had ever learnt a musical instrument or sung in choir (no, yes), highest level post-school qualification achieved by the educator (certificate/diploma, bachelor's degree, postgraduate degree), and the regional site from which the educator worked (rural, urban). These analyses were performed both as separate bivariate regressions with the individual predictors and as a standard multiple regression with all predictors entered simultaneously. This exploratory analytic approach allowed us to investigate not only the individual contributions from each factor, but also to identify any redundancy among predictors. As such, it permitted 
TABLE 2 | Descriptive statistics for highest- and lowest-rated items by early childhood and care educators on the music beliefs questionnaire (MBQ).

\begin{tabular}{|c|c|c|c|}
\hline Scale item & Mean (SD) & Minimum & Maximum \\
\hline \multicolumn{4}{|l|}{ HIGHEST-RATED MUSIC BELIEFS } \\
\hline Music education allows children to have fun (Q27) & $6.74(0.47)$ & 5.00 & 7.00 \\
\hline Music education enables children to develop their musical ability (Q14) & $6.50(0.66)$ & 4.00 & 7.00 \\
\hline Music education offers a way to include children from diverse cultures (Q3) & $6.49(0.68)$ & 4.00 & 7.00 \\
\hline Music education provides children with opportunities to improve their self-esteem (Q2) & $6.48(0.66)$ & 4.00 & 7.00 \\
\hline Music education encourages children to be creative (Q16) & $6.47(0.68)$ & 4.00 & 7.00 \\
\hline Music education helps to develop children's self-confidence (Q10) & $6.45(0.71)$ & 4.00 & 7.00 \\
\hline Music education provides children with a means of self-expression (Q15) & $6.45(0.71)$ & 4.00 & 7.00 \\
\hline Music education is an important part of a holistic approach to education (Q28) & $6.39(0.67)$ & 4.00 & 7.00 \\
\hline Music education encourages children to use their imagination (Q31) & $6.39(0.70)$ & 4.00 & 7.00 \\
\hline $\begin{array}{l}\text { Music education offers a way to include children who sometimes have trouble playing in a } \\
\text { group with other children (Q37) }\end{array}$ & $6.38(0.76)$ & 4.00 & 7.00 \\
\hline \multicolumn{4}{|l|}{ LOWEST-RATED MUSIC BELIEFS } \\
\hline Music education enables children to make meaning of their experiences of the world (Q7) & $5.72(1.01)$ & 3.00 & 7.00 \\
\hline Music education supports children to learn to control their behavior (Q4) & $5.72(1.04)$ & 4.00 & 7.00 \\
\hline Music education helps children to persist with challenging tasks (Q30) & $5.76(1.01)$ & 4.00 & 7.00 \\
\hline Music education helps children develop problem-solving skills (Q29) & $5.78(1.03)$ & 3.00 & 7.00 \\
\hline Music education enables children to improve the quality of their lives (Q19) & $5.82(1.03)$ & 4.00 & 7.00 \\
\hline Music education encourages children's understanding of different symbol systems (Q5) & $5.90(0.97)$ & 4.00 & 7.00 \\
\hline $\begin{array}{l}\text { Music education enables children to understand more sophisticated and complex } \\
\text { music (Q33) }\end{array}$ & $5.95(0.93)$ & 3.00 & 7.00 \\
\hline Music education supports children's skills in managing their own emotions (Q35) & $5.99(0.94)$ & 4.00 & 7.00 \\
\hline Music education is valuable in itself and needs no other justification (Q18) & $6.00(1.08)$ & 1.00 & 7.00 \\
\hline Music education supports the development of a child's identity (Q21) & $6.01(0.82)$ & 4.00 & 7.00 \\
\hline
\end{tabular}

$N=88$. All items on the $M B Q$ were scaled from 1 (very untrue of what I believe) to 7 (very true of what I believe).

illumination of the key facilitating factors promoting music beliefs and values of these early childhood and care educators and, thereby, provided an important contribution to our current understanding of the area. In the next section, we present the study results and discussion, linking findings back to the core research questions.

\section{RESULTS}

\section{What Is the Demographic Profile of Early Childhood and Care Educators?}

Descriptive statistics for the demographic profile of the analytic sample are presented in Table 1. The overwhelming majority of educator participants were female, which reflects the current over-representation of females globally in the early childhood education and care sector (Peeters et al., 2015). While slightly more participants were aged between 25 and 34 years, the range did vary. Indeed, the four youngest categories spanning 18-54 years best captured participants' age profile. Educators' years of experience also varied, but were best represented by the three lowest options spanning 0-14 years' experience. An additional basic descriptive analysis on the continuous variable of length of current employment revealed a more nuanced view on this. It showed that the average time educators had worked at their current care center was 6.25 years $(S D=4.81)$.

The majority of early childhood and care educators were employed on a full-time basis, with far fewer employed parttime or casually. Employment for educators in urban child care centers was far more common than in rural sites, which likely reflected service demand. Educators most frequently achieved a certificate or diploma as their highest qualification, with relatively few achieving a bachelor's or postgraduate university degree. Of those who had completed study beyond high school, the vast majority had chosen fields relevant to their current career, such as early childhood education, child care and Primary/Secondary education. A minority of educators were presently studying while concurrently working, either obtaining their ECEC qualifications or completing higher degrees usually with a clear focus on the relevant study fields of early childhood education, child care, and Primary/Secondary education.

In relation to music knowledge, few educators reported having attained any formal qualifications in music. However, approximately half of all participants stated some personal experience with music education, either having learnt to play an instrument and/or having sung in a choir themselves. Further basic descriptive analyses revealed that the average length of time that any had learnt a musical instrument or sung in choir was 3.42 years $(S D=2.66)$. Of this group, only one-third reported that they currently played and/or sang. This suggested that while 


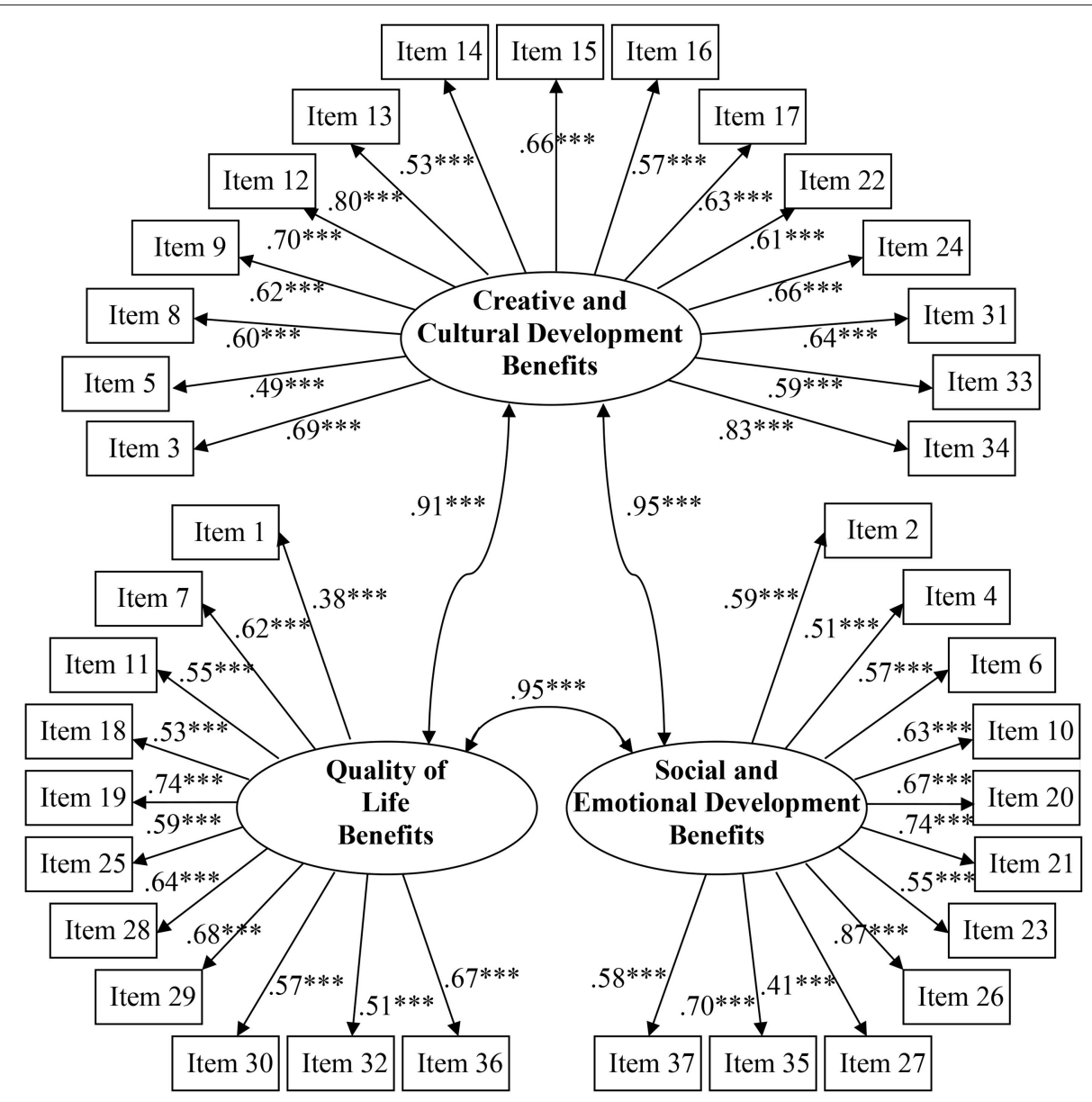

FIGURE 1 | Confirmatory factor analysis of the 37 Music Belief Questionnaire items: The hypothesized three-factor solution. All given regression weights are standardized parameter estimates. ${ }^{* *} p<0.001$.

half of participants had past experience with music, two-thirds of these had not continued active musical engagement. With this demographic profile in mind, we next sought to understand the beliefs and values these educators held about music education in the lives of young children in an effort to comprehend their teaching motivations and ethos better.

\section{What Beliefs and Values Regarding Music Engagement Are Held by Early Childhood and Care Educators?}

To evaluate the beliefs and values held by these educators pertaining to the role of music education in young children's early learning and development, a 37-item Music Beliefs Questionnaire (MBQ) was administered to study participants. It was observed that on the 1-7 response scale, the majority of items (78.38\%) displayed mean scores of 6.00 (true of what I believe) or higher, with the lowest mean of 5.72 being shown for two items (i.e., "Music education supports children to learn to control their behavior" and "Music education enables children to make meaning of their experiences of the world"). These consistently high mean item values, coupled with low standard deviations, suggested that the educators in this analytic sample expressed extremely positive, shared, global beliefs, and values regarding music and its place in the education of young Australian children. To help shed light on the most and least firmly held beliefs within these generally very high values, Table 2 presents the 10 highest and 10 lowest endorsed item statements from the MBQ.

As seen from these results, most strongly endorsed beliefs by early childhood and care educators included the concept of music as: (a) an essential creative outlet for young children; (b) a useful tool for the social inclusion of children; as well as (c) a method by which to bolster children's emotional development (i.e., selfesteem and self-confidence) in a fun and non-threatening space. Slightly less strongly endorsed were the utilitarian and adaptable skill aspects that music has to offer. Specifically, participants assigned relatively lower overall mean ratings to statements concerning ideas that children could use the skills gained from music education to understand the world, exert self-discipline (i.e., emotional and behavioral control), and bolster resilience, identity development, and quality of life. Furthermore, lower support was shown for concepts that key skills gained from music would transfer to aid general problem-solving, deciphering 
different symbol systems, or understanding more complex music (although it should be noted that these ratings still sat above the mid-point of the seven-point scale). Indeed, music education appeared not to be valued highly in and of itself, but rather viewed more as a fun and playful way to develop children's social and creative aspects to round out-rather than enhance transferable skills that can help develop and promote facets of-their traditional academic education.

This viewpoint appears to conflict somewhat with research findings that have reported the value of sustained music education in the nurturing of early literacy and numeracy (cf. Anvari et al., 2002; Moritz et al., 2013; Williams et al., 2015; Cohrdes et al., 2016), social and emotional development (Hallam, 2010; Welch et al., 2014), promotion of children's executive functioning (Moreno et al., 2011; Zuk et al., 2014; Bowmer et al., 2018), and motor skill development (Derri et al., 2001).

\section{Creation of the Music Beliefs Questionnaire (MBQ) Scale Score}

In line with the notable ceiling effects observed above, preliminary assessment of the score distributions for each MBQ item revealed that a large number displayed issues regarding skewness, and kurtosis. Specifically, the item $z$ scores for skewness and kurtosis were tested following the recommendations of Tabachnick and Fidell (2013), where an absolute value exceeding 3.29 was deemed significant (i.e., representative of the $p<0.001$ criterion). This revealed that 19 of the 37 MBQ scale items exhibited significant skew, whilst seven showed significant kurtosis (see Table 3). Given this violation of the assumption of criterion normality, a bootstrap approach was adopted to the ensuing confirmatory factor analysis (CFA) using 5,000 re-samples, employing the Bollen-Stine method, and robust bootstrap-adjusted fit indices recommended for use with non-normal data (Walker and Smith, 2017).

In an attempt to confirm the intended three-factor structure of the MBQ, the questionnaire items were submitted to a CFA. The proposed model was evaluated for fit regarding the 37 items measuring the three latent constructs they were designed to assess; namely, participant educators' beliefs and values in relation to the benefits engendered by music on children's (1) creative and cultural development, (2) quality of life, and (3) social and emotional development. These three factors aligned closely with-and were adapted from-those of Austin and Reinhardt (1999), who proposed the three factors of pre-service teachers' music philosophical beliefs to be (1) aesthetic benefits, (2) quality-of-life benefits, and (3) social-emotional benefits, respectively. Therefore, the previous administration of the Austin and Reinhardt scale from which our new measure was adapted formed the a priori theoretical basis that guided our choice of (a) number of factors, and (b) which items loaded onto each factor. As such, 15 items were specified to load on the creative and cultural development benefits factor ( $\alpha=0.91$, for items and content see Table 4), 11 items were confined to load on the quality of life benefits factor $(\alpha=0.85)$, and the remaining 11 items were constrained to the social and emotional development benefits factor $(\alpha=0.87)$. Each scale item was specified as loading on only one factor and, given that all latent constructs formed underlying facets regarding beliefs about music, the three factors were permitted to correlate. Intercorrelations amongst all the scale items are presented in Table 3, where these were performed as Spearman rho associations due to the non-normal nature of many item response distributions.

The latent structure underlying the music belief and value items was evaluated using a three-factor CFA model. This hypothesized structure provided good fit, whereby the observed data did not differ significantly from the proposed model, $\chi^{2}(626$, $N=88)=1,274.80, p=0.169$. However, the remaining fit indices did not meet the recommended 0.95 threshold for acceptable fit (Hu and Bentler, 1999): $\mathrm{CFI}_{\mathrm{adj}}=0.707, \mathrm{IFI}_{\mathrm{adj}}=0.712, \mathrm{TLI}_{\mathrm{adj}}=$ 0.688. Furthermore, the residual index for the proposed model was not below the recommended 0.06 cut-off ( $\mathrm{Hu}$ and Bentler, 1999): RMSEA $_{\mathrm{adj}}=0.109$.

Examination of the standardized parameter estimates showed that all of the items loaded significantly onto their respective hypothesized factors yet ranged somewhat in magnitude from 0.38 to 0.87 (see Figure 1). More specifically, only six items displayed strong loadings on the intended factor (i.e., $>0.70$ ), with three items demonstrating a weak loading (i.e., $>0.30$ ) and the remaining 28 showing moderate loadings on the prescribed factor (i.e., $>0.50$ ). In addition, while the three constructs were predicted to be related, these latent factors were too highly correlated with one another, almost to the point of singularity. These combined results suggested that, rather than the proposed three-factor solution, the MBQ items may be better captured by a single-factor scale, where the three factors are collapsed into one unifying latent construct assessing general educator beliefs and values held in relation to music. As such, this was evaluated as an alternative factor structure and compared to the original three-factor model.

To evaluate if a single general music beliefs factor better accounted for the current data, a one-factor solution was tested. For this, all $37 \mathrm{MBQ}$ items were specified to load upon the one factor $(\alpha=0.95)$. Similar to the three-factor structure, despite the overall model fitting the data well, $\chi^{2}(629, N=88)=1,277.87$, $p=0.155$, the other indices did not meet the recommended thresholds for good fit: $\mathrm{CFI}_{\mathrm{adj}}=0.707, \mathrm{IFI}_{\mathrm{adj}}=0.712, \mathrm{TLI}_{\mathrm{adj}}=$ 0.690, RMSEA $_{\text {adj }}=0.109$. As can be seen in Table 3, like the three-factor model, standardized item loadings for the singlefactor model were all significant, but ranged in magnitude from 0.38 to 0.86 . In this, four items presented with strong loadings, 28 items demonstrated moderate loadings, and the final five items exhibited weak loadings. Based upon the content of the items loading upon this single factor, the underlying construct perceived as captured was general favorable educator beliefs and values regarding the role of music in early childhood. Therefore, this factor was assigned the label "general favorable music beliefs."

The change in chi-square between the three-factor and onefactor models revealed no significant difference, $\Delta \chi^{2}(3)=3.06$, $p=0.382$. These findings showed there was no significant improvement in model fit by the more constrained and complex hypothesized three-factor model over the one-factor model, as both provided an equally plausible theoretical account for the 
TABLE 3 | Descriptive statistics, item loadings for single-factor model, and Spearman's Rho Intercorrelations for the MBQ scale items $(N=88)$.

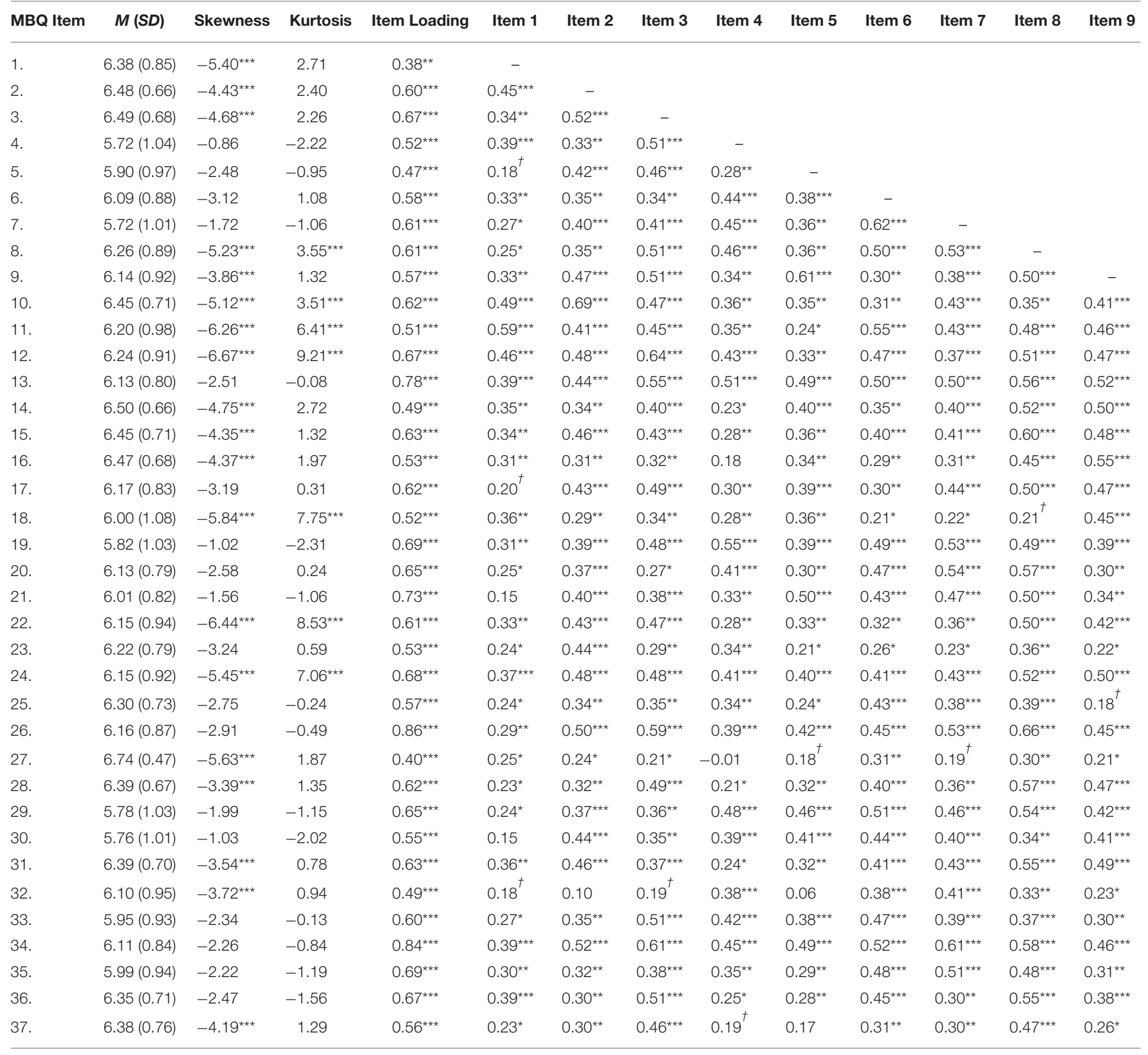

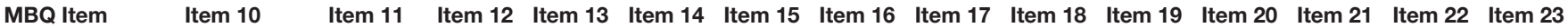


TABLE 3 | Continued

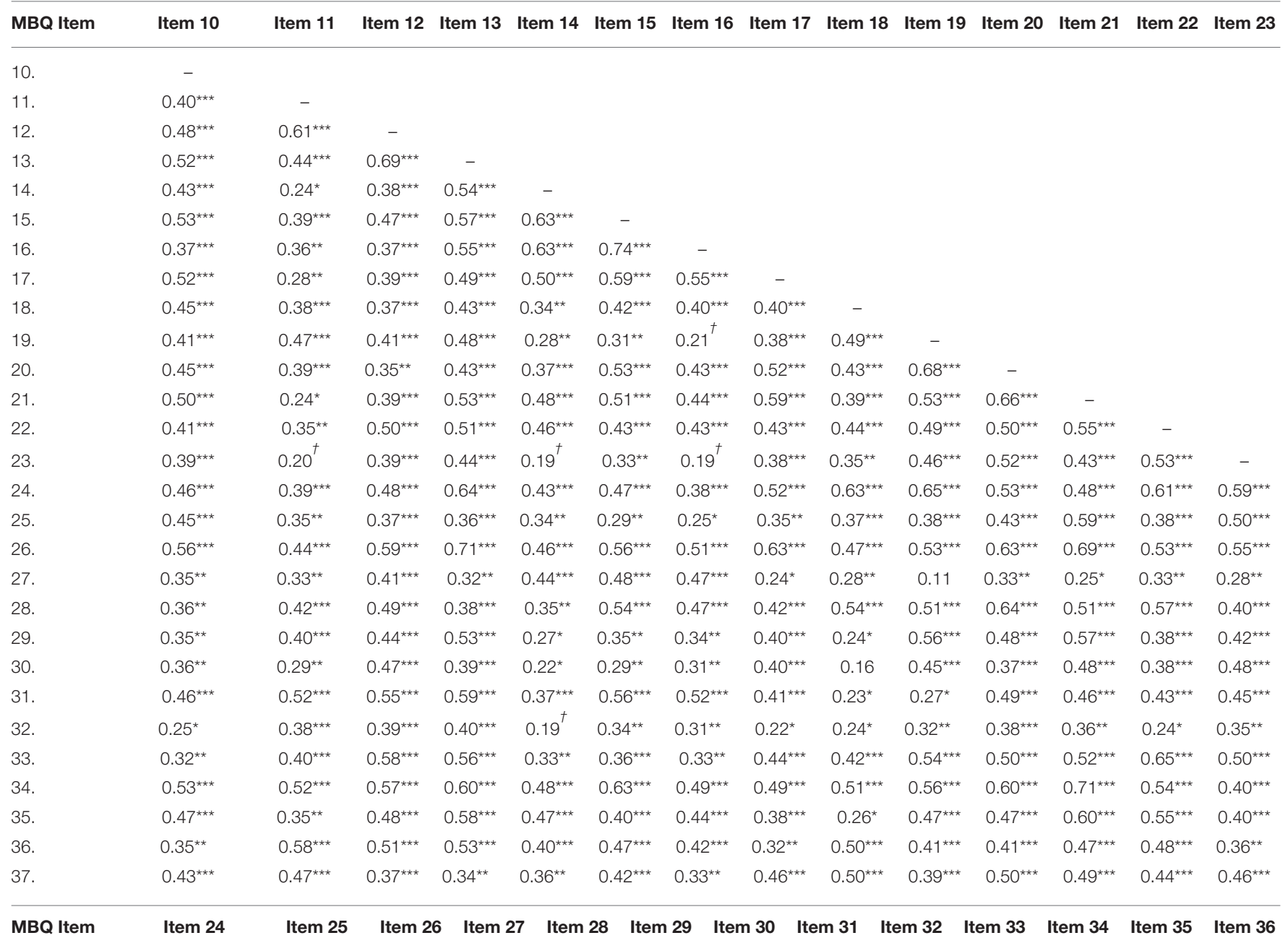


TABLE 3 | Continued

\begin{tabular}{|c|c|c|c|c|c|c|c|c|c|c|c|c|c|}
\hline MBQ Item & Item 24 & Item 25 & Item 26 & Item 27 & Item 28 & Item 29 & Item 30 & Item 31 & Item 32 & Item 33 & Item 34 & Item 35 & Item 36 \\
\hline \multicolumn{14}{|l|}{21.} \\
\hline \multicolumn{14}{|l|}{22.} \\
\hline \multicolumn{14}{|l|}{23.} \\
\hline 24. & - & & & & & & & & & & & & \\
\hline 25. & $0.45^{\star \star \star}$ & - & & & & & & & & & & & \\
\hline 26. & $0.62^{\star \star \star}$ & $0.62^{\star \star \star}$ & - & & & & & & & & & & \\
\hline 27. & $0.22^{\star}$ & $0.34^{\star \star}$ & $0.44^{\star \star \star}$ & - & & & & & & & & & \\
\hline 28. & $0.53^{\star \star \star}$ & $0.40^{\star \star \star}$ & $0.56^{\star \star \star}$ & $0.39^{\star \star \star}$ & - & & & & & & & & \\
\hline 29. & $0.39^{\star \star \star}$ & $0.43^{\star \star \star}$ & $0.51^{\star \star \star}$ & 0.13 & $0.41^{\star \star \star}$ & - & & & & & & & \\
\hline 30. & $0.38^{\star \star \star}$ & $0.34^{\star \star}$ & $0.36^{\star \star}$ & 0.11 & $0.33^{\star \star}$ & $0.70^{\star \star \star}$ & - & & & & & & \\
\hline 31. & $0.42^{\star \star \star}$ & $0.40^{\star \star \star}$ & $0.68^{\star \star \star}$ & $0.53^{\star \star \star}$ & $0.38^{\star \star \star}$ & $0.42^{\star \star \star}$ & $0.36^{\star \star}$ & - & & & & & \\
\hline 32. & $0.20^{\dagger}$ & $0.36^{\star \star}$ & $0.46^{\star \star \star}$ & $0.37^{\star \star \star}$ & $0.36^{\star \star \star}$ & $0.37^{\star \star \star}$ & $0.30^{\star \star}$ & $0.48^{\star \star \star}$ & - & & & & \\
\hline 33. & $0.55^{\star \star \star}$ & $0.44^{\star \star \star}$ & $0.57^{\star \star \star}$ & $0.23^{\star}$ & $0.51^{\star \star \star}$ & $0.55^{\star \star \star}$ & $0.53^{\star \star \star}$ & $0.39^{\star \star \star}$ & $0.40^{\star \star \star}$ & - & & & \\
\hline 34. & $0.55^{\star \star \star}$ & $0.59^{\star \star \star}$ & $0.78^{\star \star \star}$ & $0.37^{\star \star \star}$ & $0.58^{\star \star \star}$ & $0.53^{\star \star \star}$ & $0.42^{\star \star \star}$ & $0.55^{\star \star \star}$ & $0.46^{\star \star \star}$ & $0.54^{\star \star \star}$ & - & & \\
\hline 35. & $0.45^{\star \star \star}$ & $0.48^{\star \star \star}$ & $0.65^{\star \star \star}$ & $0.31^{* *}$ & $0.37^{\star \star \star}$ & $0.49^{\star \star \star}$ & $0.45^{\star \star \star}$ & $0.54^{\star \star \star}$ & $0.55^{\star \star \star}$ & $0.52^{\star \star \star}$ & $0.59^{\star \star \star}$ & - & \\
\hline 36. & $0.48^{\star \star \star}$ & $0.46^{\star \star \star}$ & $0.60^{\star \star \star}$ & $0.43^{\star \star \star}$ & $0.66^{\star \star \star}$ & $0.41^{\star \star \star}$ & $0.25^{\star}$ & $0.43^{\star \star \star}$ & $0.41^{\star \star \star}$ & $0.40^{\star \star \star}$ & $0.65^{\star \star \star}$ & $0.47^{\star \star \star}$ & - \\
\hline 37. & $0.40^{\star \star \star}$ & $0.54^{\star \star \star}$ & $0.65^{\star \star \star}$ & $0.42^{\star \star \star}$ & $0.64^{\star \star \star}$ & $0.28^{\star \star}$ & $0.21^{\dagger}$ & $0.36^{\star \star}$ & $0.41^{\star \star \star}$ & $0.38^{\star \star \star}$ & $0.59^{\star \star \star}$ & $0.48^{\star \star \star}$ & $0.75^{\star \star \star}$ \\
\hline
\end{tabular}

${ }^{\dagger} p<0.10^{\star} p<0.05^{\star \star} p<0.01^{\star \star \star} p<0.001$.

data. Consequently, use of the three latent constructs of creative and cultural development benefits, quality of life benefits, and social and emotional development benefits, did not capture and explain the present data set better than a single unified general set of beliefs about the role of music in childhood development. Taken together, these results suggested that the more parsimonious single-factor structure was preferred over the three-factor model and, as such, scores on the MBQ were calculated by averaging across all the items to obtain an overall single general measure of favorable beliefs and values held regarding the role of music in early childhood ${ }^{2}$.

It should be noted that neither of the tested models provided an adequate fit in terms of the absolute, incremental and residual fit indices. Therefore, neither provided a truly comprehensive account of the current data. This problem may be reflective of two core issues. Firstly, the factors may not be clearly defined by each set of items, as indicated by the predominantly moderate item loadings. This could suggest that the items did not measure the

\footnotetext{
${ }^{2}$ In light of the inadequate absolute, incremental, and residual fit indices displayed by the three- and one-factor models tested, we decided it prudent also to perform an exploratory factor analysis (EFA). This data-driven approach allowed the information scores gathered from participants to guide the best factor structure, which may illuminate more suitable models previously unconsidered. The EFA was conducted on the $37 \mathrm{MBQ}$ items using principle axis factoring (PAF) extraction and oblique direct oblimin rotation methods, employing a delta value of 0 . These choices acknowledged the measurement error inherent in self-report surveys and - since all underlying constructs were conceptualized to form facets regarding beliefs about music - permitted these extracted factors to be correlated to the extent shown in the data. Findings from the scree plot also suggested a singlefactor solution based upon the principle of discontinuity. This indicated a clear steep descent in the slope of the line (and thus discontinuous 'break') from Factor 1 to Factor 2, with a corresponding dramatic decrease in Eigenvalues from 14.52 to 2.30, respectively. Thus a one-factor structure for the MBQ items was also supported by the EFA results.
}

underlying construct as well as intended. This would have caused a discrepancy between the proposed model and the observed data, resulting in the lower fit indices, and higher residual indices than the recommended threshold cut-offs that was seen.

Secondly, the general lack of score variability-as seen from the restriction of range on many scale items-may have interfered with the appropriate clustering of scores that serve to differentiate the various factors more cleanly. This may have obstructed identification of the true magnitude of correlations among the various scale items and, similarly, identification of the groups of intercorrelations that form the basis of factors. This could explain why the three-factor model failed to emerge as the superior explanation for the data, as the data itself may not have contained the necessary spread of scores to better determine and discriminate among more than one factor. This is especially likely given that all scores gathered were at the high end of the 1-7 response scale and thus would have produced high intercorrelations with one another, assuming each participant provided the same or very similar scores to all items in the MBQ scale, representative of their overall general favorable music beliefs. This in turn, could also explain the very high correlations observed between the three latent constructs within the proposed three-factor model.

It also should be acknowledged, however, that these formulations are merely conjecture based upon the presence of restricted range issues within the present data set. In cases where score variability is lacking, it is more prudent and thus we would argue best practice to adopt an overall scale measure that has been shown to capture the current data, rather than make use of separate proposed scale factors (i.e., subscales) that are only theorized to bear out if greater score variability had have been achieved, as the latter may not form valid subscales. Reference 
TABLE 4 | Proposed three factors and associated MBQ items submitted to confirmatory factor analysis (CFA).

\section{FACTOR 1: CREATIVE AND CULTURAL DEVELOPMENT BENEFITS}

Music education offers a way to include children from diverse cultures (Q3)

Music education encourages children's understanding of different symbol systems (Q5)

Music education supports children's use of alternative forms of communication (Q8)

Music education enhances children's awareness and understanding of the arts (Q9)

Music education increases children's awareness of other cultures (Q12)

Music education provides children with new ideas and skills that can be used in their play (Q13)

Music education enables children to develop their musical ability (Q14)

Music education provides children with a means of self-expression (Q15)

Music education encourages children to be creative (Q16)

Music education encourages children to participate in home and community music making (Q17)

Music education helps children to appreciate and understand the role of music in their culture (Q22)

Music education increases the satisfaction that children are able to derive from music (Q24)

Music education encourages children to use their imagination (Q31)

Music education enables children to understand more sophisticated and complex music (Q33)

Music education provides children with access to a different form of intelligence or way of knowing (Q34)

\section{FACTOR 2: QUALITY OF LIFE BENEFITS}

Music education helps children develop and improve their motor-coordination skills (Q1)

Music education enables children to make meaning of their experiences of the world (Q7)

Music education helps children learn in other content areas (e.g., early literacy, numeracy) (Q11)

Music education is valuable in itself and needs no other justification (Q18)

Music education enables children to improve the quality of their lives (Q19)

Music education helps develop children's ability to focus their attention (Q25)

Music education is an important part of a holistic approach to education (Q28)

Music education helps children develop problem-solving skills (Q29)

Music education helps children to persist with challenging tasks (Q30)

Music education enhances the physical well-being of children (Q32)

Music education offers a way to include children with special learning needs (Q36)

FACTOR 3: SOCIAL AND EMOTIONAL DEVELOPMENT BENEFITS

Music education provides children with opportunities to improve their self-esteem (Q2)

Music education supports children to learn to control their behavior (Q4)

Music education helps children to learn about and understand emotions (Q6)

Music education helps to develop children's self-confidence (Q10)

Music education helps children develop relationships with others (Q20)

Music education supports the development of a child's identity (Q21)

Music education teaches children how to work together as a team (Q23)

Music education helps children to develop social skills (Q26)

Music education allows children to have fun (Q27)

Music education supports children's skills in managing their own emotions (Q35)

Music education offers a way to include children who sometimes have trouble playing in a group with other children (Q37)

to other similar scales for guidance in this matter-although wise-may not prove useful, as there exists some constructs for which people have a general tendency to express highly positive attitudes. Therefore, scores reflecting such variables will always be restricted in range and negatively skewed in its natural form. As such, use of scales measuring these types of constructs will be accompanied by extreme difficulty when trying to achieve the full range of response score options from participants and, consequently, will impede the ability to tease apart and identify the underlying theoretical constructs-should more than one exist to explain the data. As stated above, the most conservative and safest method in such instances is use of a single general measure to represent participants' views. Therefore, this approach was adopted as we sought to explore the early childhood and care educator characteristics that informed their beliefs and values about music.

\section{What Shapes Early Childhood and Care Educators' Music Beliefs and Values?}

To investigate which factors may influence educators' general beliefs and values about music, a series of six bivariate linear regressions were performed. Specifically, participants' average 
TABLE 5 | Intercorrelations between general MBQ scores and the six key educator characteristics.

\begin{tabular}{|c|c|c|c|c|c|c|}
\hline Variable & 2. & 3. & 4. & 5. & 6. & 7. \\
\hline 1. General MBQ scores & $0.20^{\dagger}$ & 0.15 & $0.26^{\star}$ & 0.08 & $-0.20^{\dagger}$ & 0.17 \\
\hline 2. Educator age & - & -0.05 & $0.60^{\star \star \star}$ & $0.18^{\dagger}$ & 0.09 & $0.34^{\star \star}$ \\
\hline 3. Educator role & & - & $0.22^{*}$ & 0.07 & 0.04 & -0.08 \\
\hline $\begin{array}{l}\text { 4. Years of educator } \\
\text { experience }\end{array}$ & & & - & 0.13 & 0.09 & 0.17 \\
\hline $\begin{array}{l}\text { 5. Ever learnt } \\
\text { instrument/sung }\end{array}$ & & & & - & 0.13 & 0.13 \\
\hline $\begin{array}{l}\text { 6. Highest post-school } \\
\text { qualification }\end{array}$ & & & & & - & 0.12 \\
\hline 7. Childcare region & & & & & & - \\
\hline
\end{tabular}

$N=86$ due to list wise deletion of cases. ${ }^{\dagger} p<0.10{ }^{*} p<0.05^{* *} p<0.01{ }^{* \star} p<0.001$

MBQ scores were regressed upon each of the predictors of (a) educator age, (b) educator role, (c) years of educator experience, (d) whether the educator had ever learnt a musical instrument or sung, (e) highest level of post-school qualification achieved by the educator, and (f) childcare regional site. These analyses were conducted individually to identify firstly any important contributing factors to the shaping of overall music beliefs.

Preliminary zero-order correlations among overall MBQ scores and the six key educator characteristics are presented in Table 5. It should be noted that a significant positive and moderate correlation was seen between educator age and years of educator experience, indicating a $36 \%$ overlap between these predictors. Likewise, educator age also shared a significant yet weak relationship with the predictor childcare regional site, where rural educators were more likely to be younger in age, with $12 \%$ overlap among these predictors. These overlaps indicated a considerable degree of shared variance and thus redundancy for these predictor variables.

As seen from Table 6, the only significant predictors of general beliefs about music held by early childhood and care educators were age and years of experience. In this, increased educator age and years of experience both were associated with more positive average ratings regarding general beliefs and values as to the beneficial role of music in the lives of young children. Also of note were marginally significant results in relation to the predictors of childcare regional site and highest educator qualification achieved. These findings suggested a trend for educators employed at rural (vs. urban) childcare sites and more qualified participants to express less favorable perspectives and philosophies concerning childhood music education. However, these results should be interpreted with caution as they were demonstrated to be trends only and not statistically reliable relationships.

A subsequent standard multiple regression was performed with all six predictors entered simultaneously. This additional analysis was conducted to identify whether these factors held as key individual predictors in their own right, or if redundancy existed among them. Findings revealed that once the other variables had been controlled, more qualified educators reported significantly less favorable general beliefs toward the role of music in early childhood education. Given the comparatively smaller number of participants who had obtained bachelor and postgraduate degrees to certificates/diplomas, this result suggested that those with university degrees must have exhibited not only lower overall MBQ ratings, but that these less favorable viewpoints concerning music were consistent and shared for these educators rather than driven by a few outliers (as evidenced by the reduced variability within the group that facilitated the significant finding). It should be noted, however, that overall music beliefs still remained favorable for these groups i.e., all average MBQ scores sat above the neutral mid-point on the 1-7 scale for educators with bachelor and postgraduate degrees.

Not surprisingly, educator age and years of experience were no longer significant as individual predictors of overall MBQ scores, suggesting a redundancy between these variables ${ }^{3}$. This indicated that while only one of these two educator characteristics was required to capture adequately an increase in general music beliefs, the most logical and best choice was years of experience, as educator age displayed too much overlap with other factors in the model (which inflated its shared variance and diminished any unique contribution it may offer). Collectively,

${ }^{3}$ Calculation of variance inflation factors (VIFs) revealed that although educator age and years of experience produced considerably higher values than the other model predictors (confirming a high degree of shared variance and thus redundancy for these variables), no multicollinearity was present in the SMR model, $V I F_{\text {age }}=1.82, V I F_{\text {experience }}=1.73, V I F_{\text {site }}=1.16, V I F_{\text {role }}=1.12$, $V I F_{\text {qualification }}=1.03, V I F_{\text {musichistory }}=1.06$ (where values of 1.00 indicate no shared variance between a given predictor and other model predictors, and values above 5.00 indicate multicollinearity). Further testing through SMR models run without each of educator age and years of experience, in turn, supported the notion of redundancy. Specifically, when educator age was excluded from the overall model, years of experience became a significant positive individual predictor of overall MBQ scores, whereby increased years of educator experience predicted more positive general music beliefs after the other variables had been controlled, $\beta=0.22, p=0.042$. This was in addition to the previously identified significant negative effect of highest educator qualification achieved, where more qualified participants expressed less favorable viewpoints concerning childhood music education, $\beta=-0.25, p=0.017$. The remaining predictors of educator role, childcare regional site and history of ever learning a musical instrument/singing remained non-significant, $\beta s<0.17, p s>0.127$. However, when years of educator experience was excluded from the SMR model, age did not become a significant individual predictor of participants' MBQ scores once the other predictors had been controlled, $\beta=0.17, p=0.125$. Yet, again, the previously recognized significant negative influence of highest educator qualification achieved held, $\beta=-0.25, p=0.022$, and the remaining predictors were non-significant as unique contributors to the model, $\beta s<0.18$, $p s>0.097$. It should be noted that, as stated earlier, educator age also displayed a considerable correlation and thus degree of overlap with childcare regional site as well as with years of experience. This likely explained the inability of age to achieve significance as a unique individual predictor in the SMR, as the vast majority of variance it contributed to the explanation of MBQ ratings was shared with other variables in the model. Therefore, the combined results here supported the idea that the high level of shared variance-and thus redundancy-between predictors within the model prevented educator years of experience and age emerging as significant individual predictors in the original SMR model, rather than the respective bivariate regression results with these predictors being indicative of Type I errors. This being said, it should be acknowledged that these findings speak more strongly to the redundancy of educator age over years of experience, due to the considerable overlap the former also displayed with childcare regional site, which resulted in it not demonstrating significance as an individual predictor within this additional SMR analysis. 
TABLE 6 | Regression analyses predicting general MBQ scores based on six key educator characteristics.

\begin{tabular}{|c|c|c|c|c|c|c|}
\hline Educator characteristic predictor(s) & B & $B_{S E}$ & $\beta$ & $R^{2}$ & $d f$ & $\boldsymbol{F}$ \\
\hline \multicolumn{7}{|l|}{ INDIVIDUAL BIVARIATE REGRESSION ANALYSES } \\
\hline Educator age $\quad(18-24,25-34,35-44,45-54,55-64,65+$ years $)$ & 0.09 & 0.04 & 0.22 & 0.05 & 1,85 & $4.47^{\star}$ \\
\hline Educator role $(1=$ general educator, 2 = higher level educator [directors and lead educators] $)$ & 0.17 & 0.11 & 0.17 & 0.03 & 1,86 & 2.42 \\
\hline Educator years of experience $\quad(0-4,5-9,10-14,15-19,20+$ years $)$ & 0.11 & 0.04 & 0.28 & 0.08 & 1,86 & $7.18^{\star \star}$ \\
\hline Ever learnt instrument/sung $\quad(0=$ no, $1=$ yes $)$ & 0.11 & 0.11 & 0.10 & 0.01 & 1,86 & 0.92 \\
\hline Highest post-school qualification $\quad(1=$ certificate/diploma, 2 = bachelors degree, $3=$ postgraduate degree $)$ & -0.19 & 0.10 & -0.19 & 0.04 & 1,85 & $3.25^{\dagger}$ \\
\hline Childcare Region $\quad(1=$ rural, $2=$ urban) & 0.24 & 0.13 & 0.20 & 0.04 & 1,86 & $3.51^{\dagger}$ \\
\hline STANDARD MULTIPLE REGRESSION & & & & 0.16 & 6,79 & $2.49^{\star}$ \\
\hline Educator age & 0.02 & 0.05 & 0.06 & & & \\
\hline Educator role & 0.13 & 0.11 & 0.13 & & & \\
\hline Educator years of experience & 0.08 & 0.06 & 0.19 & & & \\
\hline Ever learnt instrument/sung & 0.06 & 0.11 & 0.05 & & & \\
\hline Highest post-school qualification & -0.25 & 0.10 & $-0.25^{\star}$ & & & \\
\hline Childcare region & 0.18 & 0.13 & 0.15 & & & \\
\hline
\end{tabular}

${ }^{\dagger} p<0.10^{*} p<0.05^{* *} p<0.01$.

these findings revealed that while in isolation age, years of experience and qualification level shaped early childhood and care educator beliefs about the benefits of music in young children's education, only the characteristics of qualification and years of experience were required to negatively and positively predict these beliefs, respectively, due to redundancy of age with other key educator characteristics.

\section{DISCUSSION}

The first aim of this study was to adapt and pilot a survey of music beliefs and values for wider implementation in Australian early childhood and care settings. An existing survey, the Music Beliefs Questionnaire (MBQ; Austin and Reinhardt, 1999), was adapted for the participant group.

\section{The Music Beliefs Questionnaire (MBQ): Scale Factor Structure}

The original Austin and Reinhardt (1999) survey had three key construct areas related to (1) aesthetic benefits, (2) quality-oflife benefits, and (3) social-emotional benefits, respectively. The proposed three-factor structure of the MBQ, modeled on this earlier work, was not better at capturing the expressed music beliefs of educators than a universal single-factor model for the current study sample. While both provided equivalently plausible explanations for the data, the MBQ was best gauged through a single average, generalized scale score, as it offered a more parsimonious account as to the underlying latent constructs.

A closer review of the original Austin and Reinhardt (1999) scale development identifies potential reasons why we did not discover the same factor structure. These researchers espoused their three-factor model following comparison and interpretation of three-, four-, and five-factor structures from their data, as suggested by a scree plot. This, along with adoption of very liberal item weights of 0.35 and above as evidence of salient factor loadings, would indicate a degree of ambiguity in identification of the underlying latent constructs. Further, they also reported a substantial reduction in Eigenvalues between their model Factor 1 and Factor 2 (i.e., a drop from 10.22 to 2.25). Based on the discontinuity principle, this could also have suggested a single-factor solution. Yet this option was overlooked in favor of a more complex multi-scale latent structure. This issue was compounded by the fact that performance of the exploratory factor analysis (EFA) to establish their initial factor structure was not followed up by a confirmatory factor analysis (CFA) with a new sample. Hence confirmation and generalizability of their chosen factor structure for the scale was not actually established. Therefore, it is difficult to have confidence that the original Austin and Reinhardt (1999) scale was indeed best represented as three distinct factors. Thus, the inability of the current study to confirm the intended three-factor model for our 37 Music Beliefs Questionnaire items, based on the previous work of Austin and Reinhardt, is unlikely to be a cause for concern.

It should be noted that four key differences were identified between the sample employed by Austin and Reinhardt (1999) that were used to claim a three-factor structure to educators' music beliefs and the sample employed in the current study that helped discover a single-factor explanation for these same beliefs. Specifically, sample characteristics differed in terms of (1) participant type, (2) country, (3) sample size, and (4) gender breakdown. While our study made use of actual educators within the early childhood education and care sector, participants within the previous study were pre-service music teachers and therefore, undergraduate university students studying music education majors. The present study was carried out in Queensland Australia, while the previous study was presumably performed in the United States of America (though this was never stated explicitly by the authors and can only be inferred). The sample size for the present research was smaller $(N=88)$ than that 
for the Austin and Reinhardt study $(N=137)$, where the latter exhibited a much more equal gender distribution than was shown in the current study (i.e., 77 females: 60 males vs. 87 females: 1 male). However, none of these differences in sample characteristics offers an explanation as to the difference in factor structure claimed. This may be attributed to Austin's and Reinhardt's use of unsuitably low item weights as factor loadings for the three-factor solution and disregard of the notable evidence for a single-factor model within their data (despite working with a larger, more powerful and more equal gender-distributed sample). We suggest the findings from their research offer more support to our proposed singlefactor solution. We therefore conclude that a single latent factor capturing generalized beliefs about the role of music in young children's lives may offer the simplest and most suitable explanation.

\section{Music Education Beliefs and Values Held by Early Childhood and Care Educators}

The second aim of the study was to identify the music beliefs and values held by Australian early childhood and care educators concerning music in children's learning and development.

Overall, favorable global viewpoints were expressed by all educators in the present analytic sample in relation to their beliefs about music and its value in the education of young Australian children. However, within this, degrees of favorability were shown for some specific beliefs over others. Beliefs that received the strongest support by these educators were those concerned with music offering a fun and creative vehicle for children's self-expression, enhancing their self-image, and facilitating the social inclusion of other children. However, music education was relatively less strongly endorsed for its contribution to children's education outright, or concerning the transferable skills that might help establish and cultivate other academic and self-development areas of a child's life. Therefore, it would appear that music education was most strongly and pervasively perceived as primarily an aesthetic activity for children, with less recognition, or perhaps understanding, of its utilitarian value to the broader academic and learning spheres. This finding is not surprising, with Yazejian and Peisner-Feinberg (2009) highlighting how arts activities are often placed as secondary to activities that are considered to help children in areas of cognitive development such as numeracy and literacy.

It should be acknowledged, however, that the ceiling effects observed for a large number of the Music Beliefs Questionnaire (MBQ) scale items did not allow for further discrimination between the extent of educator endorsement toward potential consequences of music education in young children's lives. This may have prevented us from further teasing apart educators' philosophical views as to the perceived outcomes of music education, including its potential significance in childhood cognitive and motor development, child psychology, and the formation and advancement of children's interpersonal skills.

\section{Demographic Profile of Participant Early Childhood and Care Educators, and Factors That Shape Their Beliefs and Values About Music In Education}

The prototypical composition of the early childhood and care educator workforce found in the present study were young females, with a little over 6 years of experience, and who tended to work full-time in urban childcare centers. The proportion of female to male participants $(87: 1)$ may be viewed as a limitation of the study. As noted earlier, females are disproportionately represented in the early childhood education and care professions globally (Peeters et al., 2015) and the composition of this study sample reflects this global pattern. Most achieved their highest education through means of a certificate or diploma in a field relevant to the care and education of children. Very few possessed formal music qualifications, though roughly half had some personal music experience through having sung in a choir or played a musical instrument previously. However, only approximately one-sixth of the overall educator group had demonstrated continued active musical engagement, while the other one-third with musical experience had withdrawn their involvement over the years.

The standard educator profile, combined with the individual music belief item endorsements and the key educator characteristic of experience as a predictor of music beliefs, is highly informative. Specifically, it is possible to speculate that less experienced educators-who also make up the bulk of the current workforce-may hold less favorable beliefs about music because they themselves have not been educated systematically on the evidence concerning likely benefits and how best to utilize music as a tool in their teaching arsenal. Within the current early childhood education and care training programs that have been implemented in recent years in Australia, there is limited time allocated to teaching educators about the potential benefits of effective musical experiences within the classroom (0-17 $\mathrm{h}$ according to Letts, 2015), with little if any focus on how to use it to enhance other core academic areas for children. This may explain higher beliefs within the current educator cohort that music is primarily an aesthetic and creative device in the classroom, and relatively lower beliefs that skills gained from music can be translated to promote and accomplish academic outcomes.

This "knowledge deficit" is receiving increasing attention within the music education literature. Repeated studies reiterate the importance of improving teacher training by (re)integrating music in the teaching curriculum in order to attempt to close the gap and provide educators with a strong foundational knowledge base that draws on an increasingly rich evidence base regarding the use and role of music within early childhood education and development (Kim and Kemple, 2011; Reynolds and Burton, 2017). Corroborating this very point, Kim and Choy (2008) sought to examine whether pre-service teacher's knowledge, skills and attitudes toward music and music education improved following the completion of a specific music education course. Statistically significant improvements were shown for pre-service teachers' knowledge of musical concepts 
and confidence for teaching musical concepts, suggesting a correlation between knowledge and confidence-a finding echoed in other literature concerning generalist Primary teachers and music (cf. Seddon and Biasutti, 2008). Similarly, a new study of music teacher identity in Singapore found that the development of music teacher identity is highly contextualized in terms of personal biography in music, professional teacher identity and opportunities to experience successful music education practice in situ (Chua, 2018). Findings such as these highlight the "missing component" (see below) that exists within pre-service teacher education and warrants urgent attention.

In order to maximize the potential strengths, frameworks of practice, and learning in and through music for early childhood service providers, a shared language between the different contributory disciplines needs to exist. This would allow for a shared understanding and identification of essential characteristics of practice that support and encourage optimal child health, education and well-being. For this type of systemic change to take place, it has been argued that appropriate preservice education is the "missing component" (Grant et al., 2018). For example, Kim and Kemple (2011) report that there is a clear positive correlation between a practitioner's musical knowledge and their beliefs concerning music's value. In their study of preservice teachers, four domains were identified as influencing participants' beliefs: personal music experiences, experience of music in the field (as teaching), teacher training coursework, and their self-efficacy in implementing music activities in the classroom. Formative experiences as teachers can cast a long shadow on teachers' willingness to engage with and use music in the classroom.

Interestingly, aside from years of educator experience, the only other characteristic shown to shape the general music beliefs held by these childhood and care educators was qualification level. Specifically, more qualified educators tended to hold less favorable beliefs regarding the role of music education in early childhood. Although this finding was unexpected, we speculate it may reflect a rise in part from current emphases in teacher education. That is, more qualified educators are likely to have spent more of their professional preparation engaged with more formal childhood and childcare education knowledge and techniques. As a consequence, they are more likely to put greater trust in more traditional and familiar academic methods of teaching that, customarily, have not provided experience in music education as a strategy to promote child learning and engagement.

The wider implications of this can be evidenced in RussellBowie (2009) cross-cultural study which reported that, across five countries, Australian students gave a significantly lower rating than peers from other countries in relation to how much priority schools should place on music; suggesting an overall lower belief in music education within Australian schools. In that study, the most highly rated barriers to implementing music in the primary school classroom included factors such as "lack of priority for music" and "lack of personal musical experiences." The authors concluded that in order for music to be a central tenet of education, teachers need to feel confident and competent in the act of teaching, learning, and making music themselves. Only then will we see change.

The picture presented above is cause for concern, as there is an implied neglect of a curriculum area (music) in our children's education and development due to teacher's not feeling confident (or convinced) about music's place and value. There is evident need to ensure that pre-service teacher education builds teachers' confidence and capacity to provide the full range of curriculum areas, and draw on the benefits of music for children's learning and development.

Knowledge of the overall early childhood and care educator profile can be used to our advantage to inform potential interventions and likelihood of intervention success. Although the stereotypical educator worked in an urban area, the rural areas cannot be neglected. This is especially true in light of the trend for rural educators to express relatively less favorable beliefs concerning the role of music in young children's education. This may stem from a lack of appropriate allocated resources and training provided to rural centers and staff for music programs, especially those that highlight and support the positive outcomes that music can create in terms of academic, developmental, and psychological advancement. It may also stem from having a greater priority on an academic "core." Therefore, construction and availability of online resources regarding the value of music in the nurturing of young individuals' overall academic achievement and well-being may be beneficial in the uptake of music education to promote more favorable beliefs. These resources should include not only the most appropriate techniques for implementation, but also supply an explanation as to the research findings that underpin arguments for the implementation of music in children's learning and development. Such information is likely to engender greater support and uptake from potential educators, as users are more inclined to employ techniques they feel they understand, which have been communicated clearly, and for which the underlying mechanisms have been made transparent. Furthermore, provision of such repositories may help to further address and bridge the divide between educators' personal music experiences and knowledge and their professional understanding of music's use in early childhood education and development. Echoing this sentiment, Reynolds and Burton (2017) provided a list of recommendations to policy makers suggesting a stronger emphasis on the provision of better training and resources to early childhood teachers in relation to how and why we should be considering musically inclusive classrooms, in an effort to close the current gap that exists between early childhood education provision and music.

\section{Concluding Remarks}

The aims of this study were 2-fold: first to adapt and pilot a survey of music beliefs and values for wider implementation in childcare settings; and second, to identify those musical beliefs and values held by early childhood and care educators concerning music in children's learning. As indicated above, the survey was successful in capturing these beliefs. However, the analysis suggested that a single latent generalized factor may offer the best explanation of educators' beliefs and values over the previously-conceived three-factor structure account. 
Findings indicated that educators' beliefs and values on all items were above the mid-point, indicating overall positive attitudes toward music. It is noteworthy that this was despite the majority not having formal qualifications in music (98\%) less than half having previously learnt a musical instrument and/or sung in a choir (48\%) and only $16 \%$ currently playing a musical instrument or singing on a regular basis. It should be noted that greater years of teaching experience correlated positively with more positive attitudes, perhaps reflecting a greater emphasis on music education in these educators initial training. As noted in the literature above, for those educators who completed their training in recent years there has been limited opportunity to develop music knowledge and skills.

Approximately $20 \%$ of the sample reported graduate qualifications (Bachelor degree and above). This group reported relatively less favorable beliefs and values toward music. This finding may well-reflect the recent structuring to graduate qualifications of workforce training in the early childhood and care sector in which music education has played a lessor role. As noted above, the gender profile of the study participants might be viewed as a limitation. Further research might investigate the perspectives of male early childhood and care educators.

Cumulatively, the current study findings indicate that there is enormous potential within this population for further professional learning and development targeted at music and its conceivable wider benefits in young children's learning and lives. Given the growing evidence pool concerning the importance of music in children's lives in the home setting (Williams et al., 2015), better training programs, government and curriculum policies, and advocacy are warranted in order to challenge

\section{REFERENCES}

Anvari, S. H., Trainor, L. J., Woodside, J., and Levy, B. A. (2002). Relations among musical skills, phonological processing, and early reading ability in preschool children. J. Exp. Child Psychol. 83, 111-130. doi: 10.1016/S0022-0965(02)00124-8

Austin, J. R., and Reinhardt, D. (1999). Philosophy and advocacy: an examination of preservice music teachers' beliefs. J. Res. Music Educ. 47, 18-30.

Australian Children's Education and Care Quality Authority (ACECQA) (2016). NQF Snapshot Q2 2016. Sydney, NSW: ACECQA.

Ballantyne, J. (2006). Reconceptualising preservice teacher education courses for music teachers: the importance of pedagogical content knowledge and skills and professional knowledge and skills. Res. Stud. Music Educ. 26, 37-50. doi: 10.1177/1321103X060260010101

Ballantyne, J., and Packer, J. (2004). Effectiveness of preservice music teacher education programs: perceptions of early-career music teachers. Music Educ. Res. 6, 299-312. doi: 10.1080/1461380042000281749

Barrett, M. S. (2009). Sounding lives in and through music: a narrative inquiry of the 'everyday' musical engagement of a young child. J. Early Childhood Res. 7, 115-134. doi: 10.1177/1476718X09102645

Barrett, M. S. (2011). Musical narratives: a study of a young child's identity work in and through music-making. Psychol. Music 39, 403-423. doi: $10.1177 / 0305735610373054$

Barrett, M. S. (2012). "Music learning and education in early childhood: an overview," in Oxford Handbook of Music Education, eds G. E. McPherson and G. F. Welch (Oxford: Oxford University Press), 227-228. "old" assumptions and integrate "new" music education and development knowledge.

\section{ETHICS STATEMENT}

This project was approved by the Institutional Human Research Ethics approval committee (approval notice 2013001040) on July 20, 2015. Written informed consent was obtained from all participants in the study.

\section{AUTHOR CONTRIBUTIONS}

MB designed the study, oversaw data generation, led the article draft, and revision. LF generated data in the field, undertook literature review and contributed to the article draft, and revision. JB undertook the statistical analysis and contributed to the article draft and revision. GW co-designed the study and contributed to the article draft and revision.

\section{ACKNOWLEDGMENTS}

This research was funded by the Australian Research Council grant DP130102488 (MB and GW, Being and becoming musical: Toward a cultural ecological model of early musical development).

\section{SUPPLEMENTARY MATERIAL}

The Supplementary Material for this article can be found online at: https://www.frontiersin.org/articles/10.3389/fpsyg. 2019.00724/full\#supplementary-material
Barrett, M. S. (2016). Attending to "culture in the small": a narrative analysis of the role of play, thought, and music in young children's world-making. Res. Stud. Music Educ. 38, 41-54. doi: 10.1177/1321103X15603557

Barrett, M. S. (2017). "From small stories: laying the foundations for narrative identities in and through music," in Handbook of Musical Identities eds R. MacDonald, D. Hargreaves, and D. Miell (Oxford: Oxford University Press), 63-78.

Barrett, M. S., and Welch, G. F. (2013-2016). Being and Becoming Musical: Toward a Cultural Ecological Model of Early Musical Development. Australian Research Council Discovery Grant DP130102488.

Bengtsson, S. L., Nagy, Z., Skare, S., Forsman, L., Forssberg, H., and Ullen, F. (2005). Extensive piano practicing has regionally specific effects on white matter development. Nat. Neurosci. 8, 1148-1150. doi: 10.1038/ nn1516

Bowmer, A., Mason, K., Knight, J., and Welch, G. (2018). Investigating the impact of a musical intervention on preschool children's executive function. Front. Psychol. 9:2389. doi: 10.3389/fpsyg.2018.02389

Brown, E. D., Benedett, B., and Armistead, M. E. (2010). Arts enrichment and school readiness for children at risk. Early Childhood Res. Q. 25, 112-124. doi: 10.1016/j.ecresq.2009.07.008

Brown, E. D., and Sax, K. L. (2013). Arts enrichment and preschool emotions for low-income children at risk. Early Childhood Res. Q. 28, 337-346. doi: 10.1016/j.ecresq.2012.08.002

Bugos, J. A., and DeMarie, D. (2017). The effects of a short-term music program on preschool children's executive functions. Psychol. Music 45, 855-867. doi: 10.1177/0305735617692666 
Chen, J. L., Rae, C., and Watkins, K. E. (2012). Learning to play a melody: an fMRI study examining the formation of auditory-motor associations. Neuroimage 59, 1200-1208. doi: 10.1016/j.neuroimage.2011.08.012

Chua, S. L. (2018). Growing Music Teacher Identity and Agency: Influencers and Inhibitors. Unpublished PhD Thesis, UCL Institute of Education.

Cohrdes, C., Grolig, L., and Schroeder, S. (2016). Relating language and music skills in young children: a first approach to systemize and compare distinct competencies on different levels. Front. Psychol. 7:1616. doi: 10.3389/fpsyg.2016.01616

Council of Australian Governments. (2009). Investing in the Early Years. Retrieved from: https://www.startingblocks.gov.au/media/1104/national_ecd_ strategy.pdf (accessed April 2, 2019).

Creech, A., Gonzalez-Moreno, P., Lorenzino, L., and Waitman, G. (2016). El Sistema and Sistema-Inspired Programmes, 2nd Edn. San Diego, CA: Sistema Global.

Department for Education. (2015). 2010 to 2015 Government Policy: Childcare and Early Education. Retrieved from https:/www.gov.uk/government/ publications/2010-to-2015-government-policy-childcare-and-earlyeducation (accessed April 2, 2019).

Department of Education Employment and Workplace Relations. (2009). Belonging, Being and Becoming: The Early Years Learning Framework for Australia. Canberra, ACT: The Australian Government, Commonwealth of Australia.

Department of Education Employment and Workplace Relations. (2010). Educators, Belonging, Being and Becoming: The Educators' Guide to the Early Years Learning Framework for Australia. Canberra, ACT: The Australian Government, Commonwealth of Australia.

Derri, V., Tsapikidou, A., Zachopoulou, E., and Kioumourtzoglou, E. (2001). Effect of a music and movement programme on development of locomotor skills by children 4 to 6 years of age. Eur. J. Phys. Educ. 6, 16-25. doi: 10.1080/1740898010060103

Devarakonda, C. (2012). Diversity and Inclusion in Early Childhood. London: Sage Publications Ltd.

Dingle, G. A., Brander, C., Ballantyne, J., and Baker, F. A. (2012). 'To be heard': the social and mental health benefits of choir singing for disadvantaged adults. Psychol. Music 41, 405-421. doi: 10.1177/0305735611430081

Eerola, P.-S., and Eerola, T. (2013). Extended music education enhances the quality of school life. Music Educ. Res. 16, 88-104. doi: 10.1080/14613808.2013.829428

Forgeard, M., Winner, E., Norton, A., and Schlaug, G. (2008). Practicing a musical instrument in childhood is associated with enhanced verbal ability and nonverbal reasoning. PLOS ONE 3:e3566. doi: 10.1371/journal.pone.0003566

Fujioka, T., Ross, B., Kakigi, R., Pantev, C., and Trainor, L. J. (2006). One year of musical training affects development of auditory cortical-evoked fields in young children. Brain 129, 2593-2608. doi: 10.1093/brain/awl247

Garvis, S., and Pendergast, D. (2011). An investigation of early childhood teacher self-efficacy beliefs in the teaching of arts education. Int. J. Educ. Arts 12, 1-15. Available online at: http://www.ijea.org/v12n9/v12n9.pdf

Gaser, C., and Schlaug, G. (2003). Brain structures differ between musicians and non-musicians. J. Neurosci. 23, 9240-9245. doi: 10.1523/JNEUROSCI.23-27-09240.2003

Gordon, R. L., Fehd, H. M., and McCandliss, B. D. (2015). Does music training enhance literacy skills? A meta-analysis. Front. Psychol. 6:1777. doi: 10.3389/fpsyg.2015.01777

Grant, J., Gregoric, C., Jovanovic, J., Parry, Y., and Walsh, K. (2018). Educating professionals who will work with children in the early years: an evidence-informed interdisciplinary framework. Early Years. doi: 10.1080/09575146.2018.1488819

Habib, M., Lardy, C., Desiles, T., Commeiras, C., Chobert, J., and Besson, M. (2016). Music and dyslexia: a new musical training method to improve reading and related disorders. Front. Psychol. 7:26. doi: 10.3389/fpsyg.2016.00026

Hallam, S. (2010). The power of music: its impact on the intellectual, social and personal development of children and young people. Int. J. Music Educ. 28, 269-289. doi: 10.1177/0255761410370658

Hallam, S. (2015). The Power of Music. London: International Music Education Research Centre, University College London.

Hallam, S., Burnard, P., Robertson, A., Saleh, C., Davies, V., Rogers, L., et al. (2009). Trainee primary-school teachers' perceptions of their effectiveness in teaching music. Music Educ. Res. 11, 221-240. doi: 10.1080/14613800902924508
Halwani, G. F., Loui, P., Ruber, T., and Schlaug, G. (2011). Effects of practice and experience on the arcuate fasciculus: comparing singers, instrumentalists, and non-musicians. Front. Psychol. 2:156. doi: 10.3389/fpsyg.2011.00156

Hargreaves, D. J., MacDonald, R., and Miell, D. (2017). Oxford Handbook of Musical Identities. New York, NY: Oxford University Press.

Hennessy, S. (2000). Overcoming the red-feeling: the development of confidence to teach music in primary school amongst student teachers. Br. J. Music Educ. 17, 183-196. doi: 10.1017/S02650517000 00243

Henriksson-Macauley, L. (2014). The Music Miracle: The Scientific Secret to Unlocking Your Child's Full Potential. London: Earnest House Publishing.

Hetland, L. (2000). Learning to make music enhances spatial reasoning. J. Aesthetic Educ. 34, 179-238. doi: 10.2307/33 33643

Ho, Y. C., Cheung, M. C., and Chan, A. S. (2003). Music training improves verbal but not visual memory: cross-sectional and longitudinal explorations in children. Neuropsychology 17, 439-450. doi: 10.1037/0894-4105.17.3.439

$\mathrm{Hu}, \mathrm{L}$., and Bentler, P. M. (1999). Cutoff criteria for fit indices in covariance structure analysis: conventional criteria versus new alternatives. Struct. Equ. Model. 6, 1-55. doi: 10.1080/10705519909540118

Hyde, K. L., Lerch, J., Norton, A., Forgeard, M., Winner, E., Evans, A. C., et al. (2009). Musical training shapes structural brain development. J. Neurosci. 29, 3019-3025. doi: 10.1523/JNEUROSCI.5118-08.2009

Jellison, J. (2018). "Inclusive music classrooms and programs," in Special Needs, Community Music and Adult Learning: An Oxford Handbook of Music Education eds G. McPherson and G. F. Welch (New York, NY: Oxford University Press) 63-79.

Kim, H. K., and Kemple, K. M. (2011). Is music an active developmental tool or simply a supplement? Early childhood preservice teachers' beliefs about Music. J. Early Childhood Teach. Educ. 32, 135-147. doi: 10.1080/10901027.2011.572228

Kim, J., and Choy, D. (2008). Learning to toot your own horn: preservice teachers integrating music into a childhood classroom. J. Res. Childhood Educ. 22, 405-423. doi: 10.1080/02568540809594636

Knight, J., Bowmer, A., and Welch, G. F. (2016). 'Music for Change' 2015-2018: Collaborating With Speech and Language Therapists: A Multi-Perspective Report. London: Creative Futures.

Letts, R. (2015). Tertiary pre-service courses for primary school specialist music teachers. Music Trust of Australia. Retrieved from http://musicinaustralia. org.au/index.php?title=Tertiary_Preservice_Courses_for_Primary_School_ Specialist_Music_Teachers\#Author (accessed April 2, 2019).

Masataka, N., and Perlovsky, L. (2012). The efficacy of musical emotions provoked by Mozart's music for the reconciliation of cognitive dissonance. Sci. Rep. 2:694. doi: $10.1038 /$ srep00694

McCullough, L. (2006). "I don't Know Anything About Music": An Exploration of Primary Teachers' Knowledge About Music in Education. Unpublished PhD Thesis, Northumbria University, UK.

Mills, J. (1989). The generalist primary teacher of music: a problem of confidence. Br. J. Music Educ. 6, 125-138. doi: 10.1017/S0265051700007002

Moreno, S., and Besson, M. (2006). Musical training and language-related brain electrical activity in children. Psychophysiology 43, 287-291. doi: 10.1111/j.1469-8986.2006.00401.x

Moreno, S., Bialystok, E., Barac, R., Schellenberg, E. G., Cepeda, N. J., and Chau, T. (2011). Short-term music training enhances verbal intelligence and executive function. Psychol. Sci. 22, 1425-1433. doi: 10.1177/0956797611416999

Moreno, S., Marques, C., Santos, A., Santos, M., Castro, S. L., and Besson, M. (2009). Musical training influences linguistic abilities in 8-year-old children: more evidence for brain plasticity. Cereb. Cortex 19, 712-723. doi: 10.1093/cercor/bhn120

Moritz, C., Yampolsky, S., Papadelis, G., Thomson, J., and Wolf, M. (2013). Links between early rhythm skills, musical training, and phonological awareness. Read. Writing 26, 739-769. doi: 10.1007/s11145-0129389-0

Nutley, S. B., Darki, F., and Klingberg, T. (2014). Music practice is associated with development of working memory during childhood and adolescence. Front. Hum. Neurosci. 7:926. doi: 10.3389/fnhum.2013.00926

Osborne, M., McPherson, G. E., Faulkner, R., Davidson, J., and Barrett, M. (2016). Exploring the academic and psychosocial impact of El Sistema-inspired 
music programs within two low socio-economic schools. Music Educ. Res. 18, 156-175. doi: 10.1080/14613808.2015.1056130

Pantev, C., Engelien, A., Candia, V., and Elbert, T. (2001). Representational cortex in musicians. Plastic alterations in response to musical practice. Ann. N. Y. Acad. Sci. 930, 300-314. doi: 10.1111/j.1749-6632.2001.tb05740.x

Paulson, S., Barucha, J., Iyer, V., Limb, C., and Tomaino, C. (2013). Music and the mind: the magical power of sound. Ann. N. Y. Acad. Sci. 1303, 63-79. doi: $10.1111 /$ nyas. 12183

Peeters, J., Rohrmann, T., and Emilsen, K. (2015). Gender balance in ECEC: why is there so little progress? Eur. Early Childhood Educ. Res. J. 23, 302-314. doi: 10.1080/1350293X.2015.1043805

Reynolds, A. M., and Burton, S. L. (2017). Serve and return: communication foundations for early childhood music policy stakeholders. Arts Educ. Policy Rev. 118, 140-153. doi: 10.1080/10632913.2016.1244779

Rickard, N., Vasquez, J., Murphy, F., Gill, A., and Toukhsati, S. (2010). Benefits of a classroom based instrumental music program on verbal memory of primary school children: a longitudinal study. Austr. J. Music Educ. 2010, 36-47. Available online at: https://files.eric.ed.gov/fulltext/EJ912414.pdf

Roden, I., Kreutz, G., and Bongard, S. (2012). Effects of a schoolbased instrumental music program on verbal and visual imagery in primary school children: a longitudinal study. Front. Psychol. 3:572. doi: $10.3389 /$ fpsyg.2012.00572

Russell-Bowie, D. (2009). What me? Teach music to my primary class? Challenges to teaching music in primary schools in five countries. Music Educ. Res. 11, 23-36. doi: 10.1080/14613800802699549

Saunders, J., Knight, J., Hobsbaum, A., Himonides, E., and Welch, G. F. (2014). “'Literacy through music' - a multidisciplinary and multi-layered creative collaboration," in Collaborative Creative Thought and Practice in Music, ed M. Barrett (Farnham: Ashgate Press), 253-268.

Schlaug, G. (2015). Musicians and music making as a model for the study of brain plasticity. Progr. Brain Res. 217, 37-55. doi: 10.1016/bs.pbr.2014.11.020

Schlaug, G., Norton, A., Overy, K., and Winner, E. (2005). Effects of music training on brain and cognitive development. Ann. N. Y. Acad. Sci. 1060, 219-230. doi: 10.1196/annals.1360.015

Schober, P. S., and Stahl, J. F. (2014). Childcare trends in Germany: Increasing socio-economic disparities in East and West. DIW Econ. Bull. 11, 51-58. Available online at: https://www.diw.de/sixcms/detail.php?id=diw_ 01.c.491982.de

Seddon, F., and Biasutti, M. (2008). Non-music specialist trainee primary school teachers' confidence in teaching music in the classroom. Music Educ. Res. 10, 403-421. doi: 10.1080/14613800802280159

Seinfeld, S., Figueroa, H., Ortiz-Gil, J., and Sanchez-Vives, M. V. (2013). Effects of music learning and piano practice on cognitive function, mood and quality of life in older adults. Front. Psychol. 4:810. doi: 10.3389/fpsyg.2013.00810

Silvia, P. J., Thomas, K. S., Nusbaum, E. C., Beaty, R. E., and Hodges, D. A. (2016). How does music training predict cognitive abilities? A bifactor approach to musical expertise and intelligence. Psychol. Aesthet. Creat. Arts 10, 184-190. doi: $10.1037 /$ aca0000058

Siraj-Blatchford, I., Mayo, A., Melhuish, E., Taggart, B., Sammons, S., and Sylva, K. (2011). Performing Against the Odds: Developmental Trajectories of Children in the EPPSE 3-16 Study. London: Department for Education.

Stakelum, M. (2008). Creating a musical world in the classroom: application of a Bourdieuan approach towards understanding teacher practice. Br. J. Music Educ. 25, 91-102. doi: 10.1017/S0265051707007747

Stunell, G. (2010). Not musical? Identity perceptions of generalist primary school teachers in relation to classroom music teaching in England. Action Critic
Theory Music Educ. 9, 79-107. Available online at: http://act.maydaygroup.org/ articles/Stunell9_2.pdf

Tabachnick, B. G., and Fidell, L. S. (2013). Using Multivariate Statistics, 6th Edn, New International Edition. Harlow: Pearson Education Limited.

Tierney, A., Krizman, J., Skoe, E., Johnston, K., and Kraus, N. (2013). High school music classes enhance the neural processing of speech. Front. Psychol. 4:855. doi: 10.3389/fpsyg.2013. 00855

Trappe, H. J. (2012). The effect of music on human physiology and pathophysiology. Music Med. 4, 100-105. doi: 10.1177/1943862112438106

UNESCO (2016). Global Education Monitoring Report. Education for People and Plant: Creating Sustainable Futures for All, 2nd Edn. Paris: UNESCO.

Walker, D. A., and Smith, T. J. (2017). Computing robust, bootstrap-adjusted fit indices for use with nonnormal data. Measure. Evalu. Counsell. Dev. 50, 131-137. doi: 10.1080/07481756.2017.1326748

Welch, G. F., and Henley, J. (2014). Addressing the challenges of teaching music by generalist school teachers. Revista ABEM. 22, 12-38. Available online at: http://www.abemeducacaomusical.com.br/revistas/revistaabem/index.php/ revistaabem/article/view/459

Welch, G. F., Himonides, E., Saunders, J., Papageorgi, I., and Sarazin, M. (2014). Singing and social inclusion. Front. Psychol. 5:803. doi: 10.3389/fpsyg.2014.00803

Welch, G. F., Saunders, J., Edwards, S., Palmer, Z., Himonides, E., Knight, J., et al. (2015). Using singing to nurture children's hearing? A pilot study. Cochlear Implants Int. 16, S63-70. doi: 10.1179/1467010015Z.000000000276

Wetter, O. E., Koerner, F., and Schwaninger, A. (2009). Does musical training improve school performance? Instruct. Sci. 37, 365-374. doi: 10.1007/s11251-008-9052-y

Williams, K. E., Barrett, M. S., Welch, G. F., Abad, V., and Broughton, M. (2015). Associations between early shared music activities in the home and later child outcomes: findings from the Longitudinal Study of Australian Children. Early Childhood Res. Q. 31, 113-124. doi: 10.1016/j.ecresq.2015. 01.004

Yazejian, N., and Peisner-Feinberg, E. A. (2009). Effects of a preschool music and movement curriculum on children's language skills. Natl. Head Start Assoc. Dialog 12, 327-341. doi: 10.1080/15240750903075255

Zuk, J., Benjamin, C., Kenyon, A., and Gaab, N. (2014). Behavioural and neural correlates of executive functioning in musicians and non-musicians. PLoS ONE 9:e99868. doi: 10.1371/journal.pone.0099868

Conflict of Interest Statement: There are no perceived conflicts of interest for any of the research team in relation to this article submission and the research which it reports. It should be noted that GW is an editor of the Research Topic "The impact of music on human development and well-being," to which this article belongs.

The remaining authors declare that the research was conducted in the absence of any commercial or financial relationships that could be construed as a potential conflict of interest.

Copyright (ㄷ) 2019 Barrett, Flynn, Brown and Welch. This is an open-access article distributed under the terms of the Creative Commons Attribution License (CC BY). The use, distribution or reproduction in other forums is permitted, provided the original author(s) and the copyright owner(s) are credited and that the original publication in this journal is cited, in accordance with accepted academic practice. No use, distribution or reproduction is permitted which does not comply with these terms. 
OPEN ACCESS

Edited by: Michele Biasutti,

University of Padova, Italy

Reviewed by:

Johannes Tröger,

German Research Center for Artificial

Intelligence, Germany

Maria Paula Foss,

University of São Paulo, Brazil

*Correspondence: William Forde Thompson

bill.thompson@mq.edu.au

Specialty section

This article was submitted to Performance Science, a section of the journal

Frontiers in Psychology

Received: 27 December 2018 Accepted: 04 June 2019

Published: 16 July 2019

Citation:

Brancatisano O, Baird A and Thompson WF (2019) A 'Music, Mind and Movement' Program for People With Dementia: Initial Evidence of

Improved Cognition.

Front. Psychol. 10:1435.

doi: 10.3389/fpsyg.2019.01435

\section{A 'Music, Mind and Movement' Program for People With Dementia: Initial Evidence of Improved Cognition}

\author{
Olivia Brancatisano ${ }^{1,2}$, Amee Baird ${ }^{1,2}$ and William Forde Thompson ${ }^{1,2 *}$ \\ ${ }^{1}$ Department of Psychology, Faculty of Human Sciences, Macquarie University, Sydney, NSW, Australia, ${ }^{2}$ Centre for \\ Scaffolding the Ageing Mind, Faculty of Human Sciences, Macquarie University, Sydney, NSW, Australia
}

Background: Music is being increasingly used as a therapeutic tool for people with dementia. Research has uncovered several qualities of music that are responsible for its beneficial effects. Based on the identification of seven therapeutic capacities of music, we devised the Music, Mind, and Movement (MMM) program and evaluated whether it had therapeutic benefit for people with dementia (various types) in the areas of cognition, mood, identity, and motor fluency.

Methods: The MMM program involved seven 45-min weekly group sessions, and individual 15-min "booster" sessions. Twenty people with mild to moderate dementia participated. Group $1(n=10)$ completed the MMM program first and Group $2(n=10)$ acted as a wait list control for 7 weeks, receiving standard care and completing the MMM program after the first group. Assessments of global cognition (Addenbrooke's Cognitive Examination, ACE-III), mood (Geriatric Depression Scale short form), identity ('I am' task), and fine motor skills (9-Hole peg task) were conducted at baseline (T1), time 2 (T2, post treatment), and time 3 (T3, 1 month post MMM program).

Results: Within group comparisons were conducted with 12 participants from the MMM program and 10 participants receiving standard care. Global cognition (total ACEIII score) improved in 8/12 participants after the MMM program, whilst it decreased in 8/10 participants after the period of standard care. MMM participants showed increases in ACE-III subdomain scores of attention $(p=0.007)$ and verbal fluency $(p=0.056)$.

Conclusion: Our preliminary findings suggest that the MMM program may improve cognition, particularly verbal fluency and attention, in people with dementia.

Keywords: music, movement, dementia, cognition, fluency, attention, therapeutic

\section{INTRODUCTION}

Dementia is an umbrella term used to describe a group of neurodegenerative disorders that cause a decline in cognitive function, impacting on everyday skills. Currently, dementia affects approximately 50 million people worldwide (Alzheimer's Disease International). The most common form of dementia is the Alzheimer's type, accounting for approximately $70 \%$ of cases. 
The hallmark symptom of Alzheimer's dementia (AD) is impaired memory. There is no cure for dementia, although certain pharmacological treatments can improve some symptoms (e.g., Howard et al., 2012). Nevertheless, many of these pharmacological treatments have side effects and are ineffective for some individuals. Therefore, there is a demand for non-pharmacological treatments, especially if such alternative therapies confer behavioral and psychological benefits that are equal to those observed for pharmacological therapies, without any of the adverse events (Dyer et al., 2018).

Music has been used as a therapeutic intervention for people with numerous neurological disorders, particularly in dementia care (for review, see Altenmüller and Schlaug, 2013; Thompson and Schlaug, 2015). It has been proposed that the therapeutic value of music may be attributed to seven distinct capacities of music. Namely, that music is persuasive, engaging, emotional, personal, physical, and social, and it affords synchronization (Thompson and Schlaug, 2015). Together, these capacities comprise a robust blend of affordances that can be used in a therapeutic setting to address many of the symptoms of dementia, such as memory decline, decreased language fluency, and an altered sense of self (Brancatisano and Thompson, in press). These capacities form the basis of the Therapeutic Music Capacities Model (TMCM, Figure 1), which outlines the capacities and the therapeutic outcomes that arise as a result of their therapeutic potential. We evaluated the efficacy of a newly developed music-based program, the Music, Mind, and Movement (MMM) program, which is based on this model.

There are three fundamental advantages of using music for therapy with people with dementia. Firstly, music is easy to access and deliver as therapy. Particularly with recent advances in technology, music is more ubiquitous than ever before. We have access to thousands of songs, spanning culture and time, in a range of settings, from individual music listening with iPods to group settings. This makes music suitable to the dementia population since individuals are able to partake in the experience (whether through listening, moving or music making) irrespective of their level of functioning. Furthermore, the negative side effects of such interventions are rare. Negative experiences of music can arise if the music is intrusive or otherwise unappealing (Chang et al., 2010; Nair et al., 2011), or if the music reinforces depressive tendencies (Garrido et al., 2017, 2018), but such negative effects tend to be transient and easily managed by removing the individual from the musical source.

Secondly, musical functions, including some forms of musical memory, are often spared in the face of $\mathrm{AD}$, even during the most severe stage. Some of these preserved musical abilities include the detection of wrong notes in familiar songs (Cuddy and Duffin, 2005), learning new songs in both musicians with dementia (Cowles et al., 2003) and non-musicians with dementia (Prickett and Moore, 1991; Samson et al., 2009; Baird et al., 2017), the ability to detect emotional meaning in music (Drapeau et al., 2009) and show emotional responses to music such as joy (Norberg et al., 2003; Baird and Thompson, 2018). These observations of spared music abilities in the face of dementia opened the door to the possibility of using it as a means of therapy in dementia care.
Lastly, music can prime or scaffold other (non-musical) functions. For example, music can stimulate autobiographical memory (e.g., Irish et al., 2006; Baird et al., 2018). It also engages the individual in new learning, exercise and cognitive training, and thus can reinforce the processes of 'neural scaffolding.' This is a process, originally defined by Park and Reuter-Lorenz (2009) in their 'Scaffolding Theory of Aging and Cognition' (STAC) model, which explains how life-course factors can enhance or deplete neural resources, influencing the developmental course of cognition and brain function. The STAC model proposes that as individuals age, certain enriching factors may enable 'compensatory scaffolding' to occur, which may protect against cognitive decline. In the face of dementia, neuropathology may undermine the brain's ability to provide effective compensation. Music and its broad network of capacities can engage brain regions that are involved in neural scaffolding, such as frontal areas that are relatively preserved in people with the most common type of dementia, AD.

In the last decade music-based activities have been implemented as a way of alleviating negative symptomology associated with dementia (primarily for AD), such as agitation (Raglio et al., 2010), anxiety (Sung et al., 2010, 2012) and depression (Ray and Mittelman, 2017). Further, cognitive function has been shown to improve during or immediately after music-based treatments (Van de Winckel et al., 2004; Chu et al., 2014; Särkämö et al., 2014). There have been two Cochrane reviews that have examined the effectiveness of music interventions for behavioral, emotional and cognitive outcomes in people with dementia (Vink et al., 2003; Van der Steen et al., 2017). Vink et al. (2003) included ten randomized control trials (RCTs) and the second, more recently, by Van der Steen et al. (2017) included 17 RCTs. Vink et al. (2003) stated that no conclusions could be drawn as the quality of methods was poor overall. The later 2017 study was able to conclude that the evidence was 'moderately strong' to support the use of music to improve symptoms of depression, but not agitation or aggression. Evidence that music can improve mood, cognition, anxiety and social interaction was low, with issues such as risk of bias and small sample sizes. Thus, whilst there is support for music as a treatment for certain symptoms of dementia, ambiguity exists surrounding its effectiveness for various other symptoms of dementia.

Part of the reason for this ambiguity may be the multitude of different ways music is used therapeutically. Music interventions range from traditional music therapy, an evidence-based practice involving a trained music therapist, to music-based programs led by a musician or facilitator with no music training. These music-based programs can involve listening to music (receptive) or music making (active), such as singing or using an instrument. The music used in these interventions can be researcher or participant chosen depending on the outcome desired, such as using personal music to promote reminiscence. In addition, these activities can be experienced either individually or in a group. Each of these modes of therapies has a variety of beneficial effects. Active music therapy in a group setting has been shown to improve general cognition, measured using the Mini-Mental State Examination (MMSE, Bruer et al., 2007; 


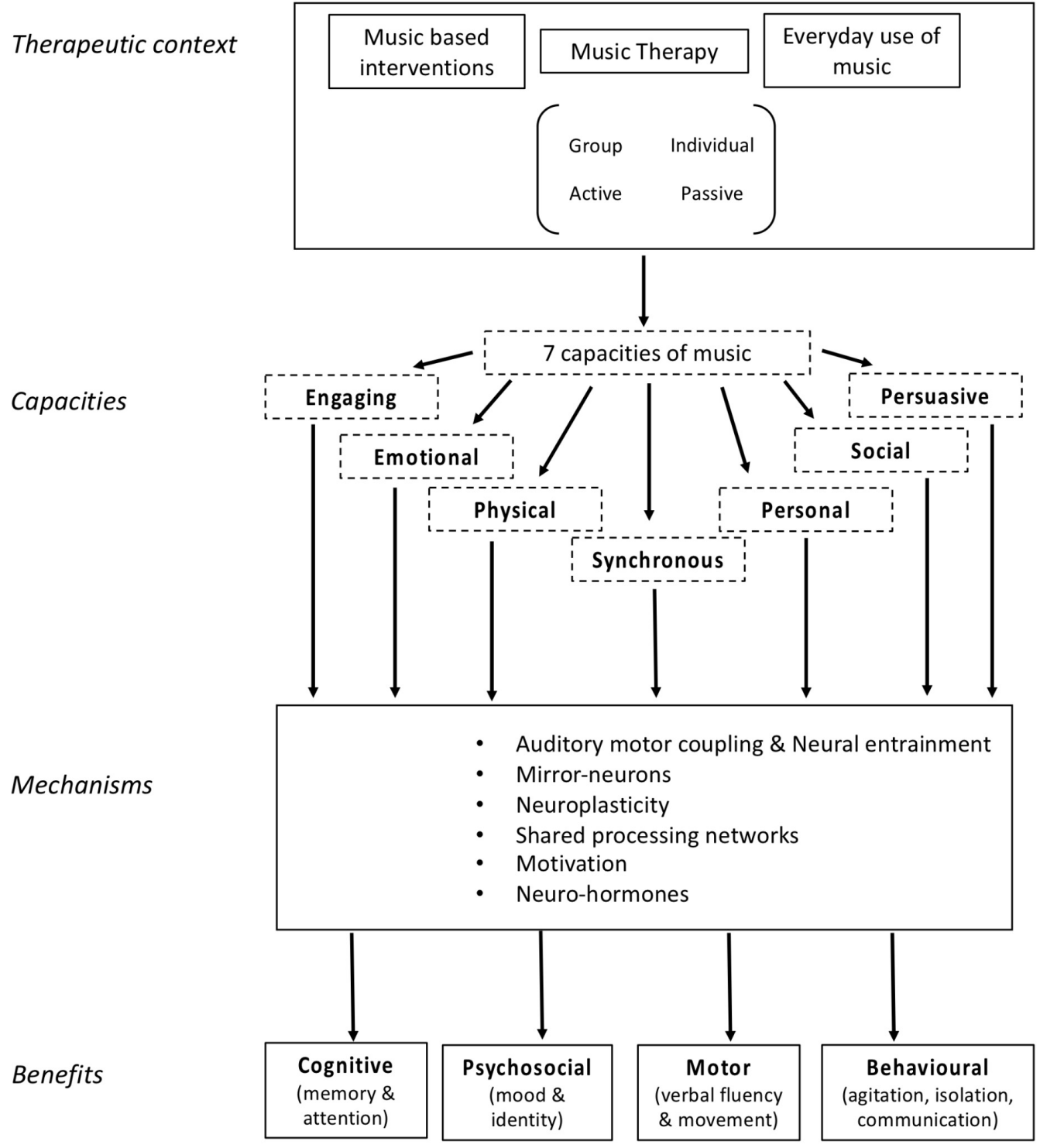

FIGURE 1 | The Therapeutic Music Capacities Model (TMCM).

Chu et al., 2014), and specific cognition functions, such as verbal fluency (Brotons and Koger, 2000; Lyu et al., 2018). Group music therapy has also demonstrated benefits by reducing associated symptoms of dementia such as depression (Chu et al., 2014) and agitation (Raglio et al., 2010; Lin et al., 2011; Vink et al., 2013; Tsoi et al., 2018). Group music-based treatments (as distinct from music therapy), such as music listening and making or moving to music, have also been shown to improve overall cognition (Van de Winckel et al., 2004; Särkämö et al., 2014; Cheung et al., 2018; Tang et al., 2018), in addition to specific cognitive functions, such as attention and various executive functions (Särkämö et al., 2014) verbal fluency and memory (Cheung et al., 2018). Interestingly, group music- based activities designed specifically for cognitive stimulation in older adults with and without cognitive decline have been shown to offer greater benefits for cognition (measured using the MMSE) and executive function (such as verbal fluency and attention tasks) than physical activity alone (Biasutti and Mangiacotti, 2018). As with music therapy, group music-based treatments can also reduce symptoms of apathy (Tang et al., 2018), agitation (Choi et al., 2009; Ho et al., 2018) and depression (Ashida, 2000). Additionally, individualized music treatments, such as the use of personalized playlists, have been used to encourage cessation of antipsychotic medication (Thomas et al., 2017). Overall, it is clear that music has a multitude of ways in which it interacts as a therapeutic context for people with dementia.

Many interventions for people with dementia contain some of the same therapeutic qualities as music. For example, cooking and art therapy are engaging and social, and can invite personal reflection. In some instances, these therapies have offered similar 
benefits to music-based therapies. In one study, a cooking intervention and music intervention (involving singing and instrument playing) resulted in similar short-term reduction of behavioral disorders in people with AD (Narme et al., 2014), but it was only the music intervention that continued to have this effect long term. Other forms of therapy do not include some of music's additional qualities that may lead to its extra therapeutic benefits. For example, music allows us to synchronize our actions, which promotes social bonding. Music can also induce spontaneous movement, which may confer cognitive benefits (Verghese et al., 2003). In addition, music has the innate ability to move us emotionally in the same manner as other stimuli that affect the hedonic centers in the brain, such as food and drugs (Blood and Zatorre, 2001). It is this combination of a number of capacities that makes music an 'all in one' therapeutic approach. Whilst this is one of music's strengths, it also makes it a complex treatment tool to understand experimentally.

Experimental and review studies are contributing significant knowledge to devising successful music programs to improve dementia related symptoms. The majority of this research, however, has not identified the various capacities by which music can confer beneficial therapeutic effects. Moreover, there is no overarching theoretical model of the therapeutic value of music. To address this issue, Thompson and Schlaug (2015) proposed seven capacities of music that explain why it may be an ideal treatment tool for neurological disorders such as dementia. These seven capacities extend the framework outlined by MacDonald et al. (2012) who described ten qualities of music that may account for the links between music, health and wellbeing. As described in detail below, the seven capacities are that music is engaging, emotional, physical, personal, social, persuasive and permits synchronization. Each capacity represents a class of active ingredients of music-based treatments, and can be further analyzed into more concrete ingredients of effective interventions. Understanding these capacities, and associated active ingredients, should lead to more effective music interventions for people with dementia.

\section{The Seven Capacities of Music}

Music is engaging. Music activates multiple systems in the brain simultaneously, including frontal, parietal, temporal, and cerebellar regions to deeper subcortical structures (e.g., Blood and Zatorre, 2001; Zatorre and Salimpoor, 2013). By engaging multiple processes, it places the brain in an 'enriched' and challenging setting, triggering neuroplasticity. In addition, by casting a 'wide net' of engagement, this offers multiple opportunities for addressing deficits. In particular, music can facilitate the encoding of verbal material by enhancing neural coherence during new learning (Peterson and Thaut, 2007). Whilst healthy individuals may not need to rely on musicenhanced encoding because they have intact cortical structures for memory, the mnemonic benefits provided by music may be necessary for individuals with AD (Kilgour et al., 2000). In effect, music provides a comprehensive, neurological scaffold for memory. Music also captures our attention such that we are likely to pursue the therapy in an undistracted manner, thereby reaping maximum benefits.
Music is emotional. One of the most significant purposes of music is to convey emotional meaning. Some brain regions involved in emotion processing, namely the medial frontal areas are relatively spared from degeneration in $\mathrm{AD}$ (Jacobsen et al., 2015). The ability of music to heighten emotions can be utilized to reduce apathy (loss of interest and a lack of or blunted emotional responses) in people with moderate to severe $\mathrm{AD}$ (Massaia et al., 2018; Tang et al., 2018). Receptive music-based treatments have been shown to significantly improve apathy (Massaia et al., 2018; Tang et al., 2018) and increase smiling behaviors compared to a control intervention of standard care (Raglio et al., 2008). Music also plays an important role in re-gaining access to emotions and memories, particularly in people with AD (e.g., El Haj et al., 2012; Baird et al., 2018). Interestingly, episodic memories evoked by music in people with $\mathrm{AD}$ tend to contain more emotional content and are more positively valanced, than episodic memories evoked in silence (El Haj et al., 2012; Cuddy et al., 2017), implying that the effects of music can benefit people with AD not only by eliciting memories, but also by inducing a positive state of mind.

Music is inherently a very physical stimulus. It is hard to separate the experiences of music and movement, and when we hear certain types of music we get a strong urge to move our body to the music. Engaging in physical exercise has been known to delay the onset of dementia (for review, see Laurin et al., 2001; Larson et al., 2006; Carvalho et al., 2014). Furthermore, in a longitudinal review over 5 years, engagement in leisure activities, such as dancing, reduced the risk of dementia (Verghese et al., 2003). Interventions that have paired music and exercise in people with dementia have reported a decrease in depression, as well as improvements in specific cognitive functions such as verbal fluency and memory (e.g., Cheung et al., 2018). Exercise and its associated benefits for memory may be accompanied by an increase in the production of brain derived neurotrophic factor which mediates neurogenesis (Erickson et al., 2011). Pairing music and movement therefore encourages exercise and subsequently benefits cognition, mood, and behavior.

Music affords synchronization. We have an instinctive ability to synchronize our body's movements, and speech, to music. Simply moving in time with one another to music has many positive therapeutic benefits. For example, in synchronous drumming there is a release of endorphins and neurochemicals that are responsible for feelings of social bonding, empathy, and trust (Tarr et al., 2014). The tendency to move in time to music may assist in learning new movement sequences in people with AD (Moussard et al., 2014). Music treatments that have emphasized synchronizing the playing of musical instruments have resulted in improvement in cognitive functions such as verbal fluency, supported by neuroimaging results which demonstrate an increase in the level of cerebral blood flow to the prefrontal cortex (Shimizu et al., 2017).

Music is personal through its ability to reinforce our sense of self as it is commonly linked with our identity. Music that is heard repeatedly during significant or pivotal times in our personal development eventually seems to signify that time of life. There is increasing interest in using personalized playlists as a therapeutic tool for people with dementia (Garrido et al., 2017). 
Levels of agitation have been reported to decrease after listening to personally preferred music compared to relaxing classical music (Gerdner, 2000). Preferred music listening interventions can also reduce anxiety levels in people with dementia compared to standard care with no music (Sung and Chang, 2005; Sung et al., 2010). Familiar music can also be used to help people with dementia become more oriented within a new environment or maximize their sense of familiarity in a current one (Son et al., 2002).

Music is social. Isolation is one of the most significant challenges associated with dementia, owing to the decline in behavioral and cognitive functions. Music acts as a catalyst for bringing people together and also enhances group experiences. The social nature of music may be beneficial in boosting the healing process via cohesion, collective enjoyment and a sense of support for one another. Improvements have been demonstrated in cognitive functions (e.g., attention), behavior, mood, and wellbeing after participating in group singing and music activities (Sakamoto et al., 2013; Narme et al., 2014; Särkämö et al., 2014). Importantly, many people with dementia also indicate that group singing helped them to accept and cope with their condition (Osman et al., 2016).

Music is persuasive, and belief in a treatment is crucial for participation, motivation, and recovery. The positive belief in a treatment may make participating in therapy more likely (Rosenstock, 1974). In other words, merely believing that a treatment will lead to positive outcomes can amplify the therapeutic benefits. Music has the capacity to persuade or influence us and has been used historically as a tool to reinforce, change or inspire beliefs. For example, messages in advertisements or political movements are highlighted and enriched by music. It is persuasive also in the sense that the sheer enjoyment it stimulates leads to an optimistic outlook, which is beneficial in a therapeutic setting.

We have taken these seven capacities of music and developed the TMCM (Figure 1) (Brancatisano and Thompson, in press). The model begins by identifying contexts in which music can be experienced in a therapeutic way, previously identified by MacDonald et al. (2012). These contexts are broken down into the seven capacities that form the core of the model. A number of biological and psychological processes are then listed that may underlie the link between the seven individual capacities of music and their beneficial outcomes. Finally, arising from the seven capacities through the underlying mechanisms are multiple potential benefits, including cognitive, psychosocial, motor, and behavioral benefits. As it stands, most musicbased treatments or music therapy practices incorporate one or more of these attributes, but not all. These capacities have not yet been combined to form an intervention in a systematic way, which may in turn maximize the effect of a musicbased intervention.

We devised the MMM program based on the TMCM. The MMM program was then evaluated in people with dementia to (a) determine the potential benefits that including all seven capacities of music would have on the participants' cognition, mood, identity and motor function, (b) examine the impact that certain individual or a combination of capacities would have on the participants' cognition, mood, identity and motor function, and (c) explore the relationship between factors which may affect session attendance and cognitive performance.

\section{MATERIALS AND METHODS}

\section{Ethical Approval}

Ethical approval was granted by the Macquarie University Human Sciences Ethics committee and the residential aged care facility. Verbal consent to approach the participants was first sought from their significant other (family member or partner). Written and informed consent was obtained from all participants, who underwent an initial visit to explain the study and gauge their interest. If participants were interested, a second visit was organized for verbal and written consent from the participant and their significant other. Continuing consent was monitored each week with each participant being re-informed of the nature of the program and asked if they were happy to participate. If the participant declined, they were invited again to take part the following week. If they declined 2 weeks in a row, they were excluded from the study.

\section{Participants}

We recruited participants from a local residential aged care facility who had either a clinical diagnosis of any type of dementia (mild to moderate) or indication of cognitive impairment (e.g., mild cognitive impairment, 'memory impairment') as specified in their medical records. Evidence of probable dementia was confirmed in all participants by cognitive assessment results using Addenbrooke's Cognitive Examination III (ACE-III total score at or below 82). Only one participant scored above 82 (a score of 84) which was indicative of possible dementia. Inclusion criteria were fluent English language skills, no severe psychiatric disorder (e.g., schizophrenia) and no hearing or language impairment that would prevent communication or ability to hear music. We approached the residential aged care facility manager who provided a list of 44 names of individuals who matched these criteria. The potential participants' family members were then contacted, fourteen of whom declined to participate. Thirty participants were then visited individually at the residential facility to determine their suitability and interest in the program. Ten participants refused to participate leaving a final sample of 20 . These 20 participants then completed baseline assessments.

The 20 participants were divided into two groups for logistical reasons. Group 1 completed the whole MMM program first and Group 2 completed the program second (immediately after Group 1), with Group 2 acting as a 'waitlist control.' The demographic and clinical characteristics of the participants are presented in Table 1. The clinical diagnoses were obtained from the medical records at the residential facility. In 10 out of 20 participant no formal diagnosis of dementia noted in the medical records, but the ACE-III scores revealed that all participants met the cut off for probable dementia. The two groups were well matched in that they included various dementia types and one participant in each group had a diagnosis of Parkinson's disease and associated dementia (see Table $\mathbf{1}$ ). 
TABLE 1 | Demographic and clinical characteristics of the two groups of participants $(n=20)$.

\begin{tabular}{lcc}
\hline & Group 1 ( $\boldsymbol{n}=\mathbf{1 0})$ & Group 2 ( $\boldsymbol{n}=\mathbf{1 0})$ \\
\hline Age (years) & $84.4(7.1)$ & $82.20(8.0)$ \\
Gender (M/F) & $3 / 7$ & $1 / 9$ \\
Education (years) & $16.0(0.9)$ & $15.11(1.0)$ \\
Time at residential facility (months) & $27.2(20.2)$ & $18.20(18.5)$ \\
Musical background (yes/no) & $4 / 6$ & $4 / 6$ \\
Clinical diagnoses (number of participants) & & 2 \\
Alzheimer's dementia & 3 & 1 \\
Vascular dementia & 2 & 2 \\
Mild cognitive impairment & 0 & 2 \\
Memory disturbance & 4 & 3 \\
Other & 1 & \\
\hline
\end{tabular}

Data presented as mean (standard deviation) unless otherwise stated. Memory disturbance specified as 'amnesia,' 'memory changes/issues,' 'short term memory loss' on medical records. Other specified as mood disorders $(n=1)$, Parkinson's disease $(n=2)$, depression $(n=1)$.

\section{Conditions}

\section{The MMM Program}

The MMM program incorporates music-based activities that highlight all seven capacities of music. The capacities represent distinct qualities of music and the outcomes are the benefits that arise as a consequence of tapping into these capacities to target relevant areas of decline, such as cognitive, behavioral, motor, and identity, as outlined in the TMCM (see Figure 1). The MMM program encompasses activities that possess a specific set of 'active ingredients' to enrich the environment and maximize the activation of the seven capacities outlined in the TMCM. We suggest that there are five active ingredients that apply to various activities in the MMM program:

(a) Familiarity of the music (novel or familiar)

(b) Complexity of music activity (number of functions engaged in an activity)

(c) Intensity of music activity (quantity of each task)

(d) Difficulty of music activity (easy or hard)

(e) Empathic focus (degree to which an activity engages empathy)

Through the activities, participants are encouraged to play simple instruments (such as bucket drums and egg shakers), sing, move and interact with one another.

\section{Sessions}

We devised seven weekly sessions broken into four blocks and each block contained activities that focussed on two or three related capacities of music (a summary of the activities can be seen in Table 2). The four blocks were designed to determine the relative contribution of each individual or combination of comparable capacities on specific outcomes, for example, the contribution of the personal and emotional capacities of music (sessions 3 and 4) related to the outcome of 'mood' and 'identity' (Table 2).

Block one (sessions 1 and 2), focussed on the engaging and persuasive attributes which may benefit attention and memory functions. The active ingredients in these sessions include familiarity, novelty, complexity, simplicity, and empathic focus. For example, in the first session the activity is relatively simple, as the participants attempt to complete verbal tasks to novel musical structures (e.g., how melody and rhythm can be used to help to remember verbal information, such as names or short phrases).

TABLE 2 | MMM sessions and their targeted capacities, active ingredients, and outcomes.

\begin{tabular}{|c|c|c|c|c|}
\hline & Sessions 1 and 2 & Sessions 3 and 4 & Sessions 5 and 6 & Session 7 \\
\hline Capacities & Engaging and persuasive & Emotional and personal & $\begin{array}{l}\text { Social, physical and } \\
\text { synchronization }\end{array}$ & All attributes \\
\hline Active ingredients & $\begin{array}{l}\text { Familiarity, Novelty, Complexity, } \\
\text { Simplicity, Empathic focus }\end{array}$ & Familiarity, Empathic focus & $\begin{array}{l}\text { Familiarity, Complexity, Intensity, } \\
\text { Novelty }\end{array}$ & All ingredients \\
\hline Example activities & $\begin{array}{l}\text { Novelty, Simplicity and Complexity } \\
\text { (i) Introducing each musical instrument } \\
\text { with songs (egg shakers, drums) } \\
\text { (ii) Learning names and personal } \\
\text { description to melodic and rhythmic } \\
\text { framework } \\
\text { Familiarity } \\
\text { (i) Game: Guess that advertisement's } \\
\text { song (and singing along) } \\
\text { Empathic focus } \\
\text { (i) Game: Which advert jingle is more } \\
\text { convincing? (discussing how music } \\
\text { makes adverts convincing) } \\
\text { (ii) Game: Where does this song } \\
\text { belong? (guessing the context of the } \\
\text { song and discussing why it fits there) }\end{array}$ & $\begin{array}{l}\text { Familiarity } \\
\text { (i) Game: Who sang that } \\
\text { song? (guessing the name of } \\
\text { artist and song) } \\
\text { (ii) Finish the next line of the } \\
\text { song lyrics (singing along to } \\
\text { favorite songs and guessing } \\
\text { the missing lyrics) } \\
\text { Familiarity and Empathic focus } \\
\text { (i) Reminiscence with music } \\
\text { (play each individual's favorite } \\
\text { sons and discuss memories } \\
\text { evoked) } \\
\text { (ii) Game: Guess which movie } \\
\text { the soundtrack belongs to? } \\
\text { (iii) Music and our emotions } \\
\text { (playing to express emotions } \\
\text { and how you are feeling) }\end{array}$ & $\begin{array}{l}\text { Familiarity and simplicity } \\
\text { (i) Warm up movement } \\
\text { exercises; start with slow } \\
\text { songs (e.g., "My Bonnie") and } \\
\text { progress to faster (e.g., "In } \\
\text { the Mood") } \\
\text { Familiarity, Complexity, Intensity } \\
\text { Musical instrument playing } \\
\text { (moving and making music in } \\
\text { synchrony, turning to face } \\
\text { partners and exchanging } \\
\text { instruments and making eye } \\
\text { contact). } \\
\text { Novelty } \\
\text { Game: Match the dance song } \\
\text { to the photo (discuss why the } \\
\text { music fits with each song) }\end{array}$ & $\begin{array}{l}\text { All ingredients } \\
\text { Combine activities from other } \\
\text { sessions (e.g., reminiscence, } \\
\text { singing, playing along and } \\
\text { engaging socially). }\end{array}$ \\
\hline Outcomes & Cognitive (memory and attention) & $\begin{array}{l}\text { Psychosocial (mood and } \\
\text { identity) }\end{array}$ & $\begin{array}{l}\text { Motor (verbal and movement } \\
\text { fluency) }\end{array}$ & All \\
\hline
\end{tabular}


Participants are invited to listen to how messages in familiar advertisements are highlighted and enriched by music, making them extremely memorable.

Block two (sessions 3 and 4) focussed on the emotional and personal attributes. The active ingredients in these sessions are familiarity and empathetic focus, which are designed to facilitate mood and identity. Familiarity is emphasized by including the participants' personally selected favorite songs as well as songs from their reminiscence bump period. The aim of this block is to encourage reminiscence and discussion about personal memories and to stimulate emotions through playing and listening to music, embodying empathy.

Block three (sessions 5 and 6) emphasized the social, physical and synchronous attributes. The active ingredients in this session are familiarity, simplicity, complexity, and intensity to stimulate positive change in both motor and verbal fluency. For these sessions, we used familiar music that had a strong beat and paired it with body movements to promote physical activity. Participants were encouraged to play their instruments in synchrony with each other and to engage socially whilst doing so (such as turning to the person beside them). Rhythmical phrases played in time to the music started off simply, to allow participants to gain a sense of mastery, and became more complex as the session progressed.

Block four (session 7) drew upon all the capacities and involved live music, encompassing all active ingredients. As it was the last session, an emphasis was placed on intensity and familiarity. The active ingredients in this session were 'intensive' and 'familiar', as it is the last session. Musicians from the local Conservatorium of Music played the residents favorite songs. Live music can be especially engaging for participants and playing preferred and familiar songs may help to bring back personal memories and provide a rich emotional experience. During this session, participants were encouraged to respond to the music with physical movements and singing along, allowing them to synchronize their movements and voice with the music and with other participants, ensuring that they would have a powerfully social experience.

\section{Music choice}

Before starting the program, the researchers asked the participants for at least one or two favorite songs, artists or genres of music. This was primarily for the reminiscence sessions in Block 2. All other music used in the sessions was from the participants reminiscence bump period (aged 10-30 years). This would ensure the participants would be highly familiar with the songs and the personal nature of the program would be maximized. A list of songs used in the program can be found in Appendix.

\section{Musical instruments}

Each participant had a bucket drum, two drum sticks and an egg shaker set up in front of them at the start of each session. The researchers ensured they were easily accessible (for example, if the participants had trouble reaching the bucket drum two or more buckets were stacked on top of each other).

\section{Program set-up}

The duration of each session was approximately $45 \mathrm{~min}$. This allowed approximately $10 \mathrm{~min}$ for each activity (see Table 2 ). The sessions took place in a medium sized room at the residential aged care facility. Power-point slides were devised to both prompt the researchers and show pictures and videos to the residents (mainly for sessions 1-4). Participant chairs were set up in a semi-circle to face the projector screen where the powerpoint slides were displayed. The intervention was conducted by two facilitators. Facilitator one (F1, JC) had experience with prior group music programs in schools and elderly care, and facilitated all sessions. The second facilitator (F2, OB), assisted by encouraging discussion and ensuring all participants had the relevant musical instruments. A third facilitator (F3, AB) was present for Group 1 and had a similar role to F2. One facilitator would typically sit at the front by the projector screen and the other approximately half way around the circle to facilitate a social environment.

Approximately half of the participants required wheelchairs for transportation to and from the venue and stayed in their wheelchair during the sessions. These participants demonstrated a minimal to medium range of motion and were able to move upper and lower limbs voluntarily.

\section{Booster sessions}

On a separate day after each group session, F1 visited each participant individually for 10-15 min as part of the 'Booster Sessions.' The booster sessions were designed as a way to increase dosage and allow the participants to reflect on their experience of the previous session. F1 would first ask the participants a series of open-ended questions pertaining to whether they remembered the previous MMM session, and if so, did they enjoy it and specifically what they enjoyed about it. F1 then engaged the participant in completing two activities that were covered in the previous session as a "refresher" for the participant.

\section{Standard Care}

Group 2 served as a waitlist group for 7 weeks and continued their standard care and activities until their follow-up assessments. Activities that were available to them by the residential aged care facility on a weekly basis were group discussions, miniature bowling, physical exercise, 'name that tune' and craft. Nine out of the 10 participants answered a series of yes/no questions to determine their level of activity during this period of time; 2/9 participants said they participated in physical exercise; 5/9 participants said they engaged in social activities; $3 / 9$ participates said they sang regularly; 6/9 participants said they listened to music regularly and 0/9 participants said they played a musical instrument currently.

\section{Design}

The study design is illustrated in Figure 2. Two groups of participants completed the MMM program (Group 1 and Group 2). Group 1 completed the MMM program first, whilst recruitment of Group 2 took place. Group 2 then served as a waitlist control, with a period of no intervention during which they received standard care only (henceforth referred to as 
the SC group). Group 2 then completed the MMM program immediately after Group 1. All participants who completed the MMM program are henceforth referred to as the 'MMM group.' Assessments of global cognition, mood, identity, verbal and motor fluency were taken at baseline (1 week prior to MMM program/SC start, T1) and at a follow-up immediately after the program/SC (T2). For the MMM group only, assessments were also taken at an extended follow-up 1 month after participation in the program (T3).

A subset of the measures (brief assessments for a total duration of $5 \mathrm{~min}$ ) were taken at the end of the last session of the three MMM blocks (in week 2, 4, and 6). This was designed to assess the distinct effect that each of the seven attributes might have on specific domains of functioning. Baseline assessments of Group 1 were performed by authors 1 and 3 (F2 and F3; $\mathrm{OB}$ and $\mathrm{AB}$, respectively). Baseline assessments of Group 2 were performed by an independent researcher, blind to participant group membership, who also conducted all other follow-up assessments for both groups (excluding Group 2 follow-up SC assessment, due to logistic restrictions which were done by F2 who was blinded to the original baseline assessment scores).

\section{Measures \\ Cognitive Function: Addenbrooke's Cognitive Examination (ACE-III) Australian Version III}

The ACE-III is a cognitive measure used in screening for dementia and developed as a "theoretically motivated extension of the MMSE" (Mathuranath et al., 2000; Mioshi et al., 2006). Cut-offs at $88 / 100$ (sensitivity $=94 \%$, specificity $=89 \%$ ) and $82 / 100$ (sensitivity $=84 \%$, specificity $=100 \%$ ) for the suspicion of dementia have been defined. The ACE-III has very good reliability (alpha coefficient $=0.8$ ).

The ACE-III tests five subdomains of cognitive skills: attention (/18), memory (/26), verbal fluency (/14), language (/26), and visuospatial skills $(/ 16)$ with a total score out of 100 . The subdomain of attention tests the participants' ability to: recall the date, the current season and location, repeat back immediately three simple words and serial subtraction. The memory items first test the participants' ability to: recall the three simple words, then asks them to verbalize them, memorize and recall a fictional name and address, and remember several well-known historical facts. Fluency tests the ability to: list as many animals they can in $1 \mathrm{~min}$, followed by as many words they can beginning with the letter ' $\mathrm{P}$ ' in $1 \mathrm{~min}$. The language subdomain requires the participant to: complete a series of physical tasks that are verbally directed by the researcher using a pencil and piece of paper (e.g., "pick up the pencil but not the paper"), write two complete sentences, repeat four complex words and two short proverbs, name 12 simple drawings of objects and animals, answer four semantic questions relating to the drawings, and read aloud five words that are easily mispronounced. Lastly, the visuospatial abilities subdomain tests participants' ability to: copy two figures, draw a clock face and the hands set at a particular time, count multiple dots in a square and recognize four partially obscured letters. Questions in the attention, memory and verbal fluency domains were also taken at the end of week 2, and verbal fluency domain at the end of week 6 .

\section{Mood: Geriatric Depression Scale Short-Form (GDS-SF)}

The Geriatric Depression Scale Short-Form (GDS-SF) has been used to screen for depression in the elderly (Sheikh and Yesavage, 1986). It was initially developed as a 30 -item tool but the short form (15-item) was established for use in time constraints. The GDS-SF has been reported to be sensitive to depression in people with dementia. Scores greater than 5 points suggest depression and scores greater than 10 are almost always depression. It has good sensitivity (92\%), specificity (89\%), and high correlation $(r=0.84, p<0.001)$.

\section{Self-Identity: ‘I Am/I Was’ Task}

In the original form of this task (Rathbone et al., 2008), participants are asked to list 10 'I am' statements that strongly define their identity (e.g., "I am a grandfather," "I am shy"). They are then asked to select the three most relevant statements and recall a personal memory that is linked to each one. For this study, the task was modified to involve the participant listing as many ' $I$ am' and 'I was' statements as they could in 1 min per category (e.g., "I was a dancer," "I was a nurse") in order to measure the participant's change in identity over time. We also omitted the task of recalling memories associated with the statements and adapted the task for people with dementia by asking them to list the statements verbally rather than in written form, similar to that of Gridley et al. (2016). This task was also repeated at the end of week 4 .

\section{Autobiographical Memory Fluency: Autobiographical Fluency Task (AFL)}

In the original version of this task (Dritschel et al., 1992), participants are asked to recall list personal events and names of friends/acquaintances from different lifetime periods: ages 5-11 years, 11-17 years, 5 years post high school and currently. They are given $90 \mathrm{~s}$ for each stage of life and each category (events and names). For this study, we omitted the events category and asked participants to list names only. This task was also repeated at the end of week 4 .

\section{Motor Fluency: Nine-Hole Pegboard Task}

This task was initially introduced by Kellor et al. (1971) and is used to measure finger dexterity in neurological disorders, particularly stroke and Parkinson's disease. Participants were timed on how long it takes to insert all nine pegs one by one into the holes and remove them one by one. Measurements were taken for both the dominant (self-reported preferred writing hand) and non-dominant hands. This task was also repeated at the end of week 6 .

\section{Data Analysis}

Data analysis was completed using SPSS. Due to the cross-over structure of the study, the small sample size and several variables violating the assumptions of normality, we analyzed data using the Wilcoxon Signed Rank test within the SC and MMM groups. We compared the outcome measures (cognition, mood, selfidentity, autobiographical memory fluency and motor fluency) 


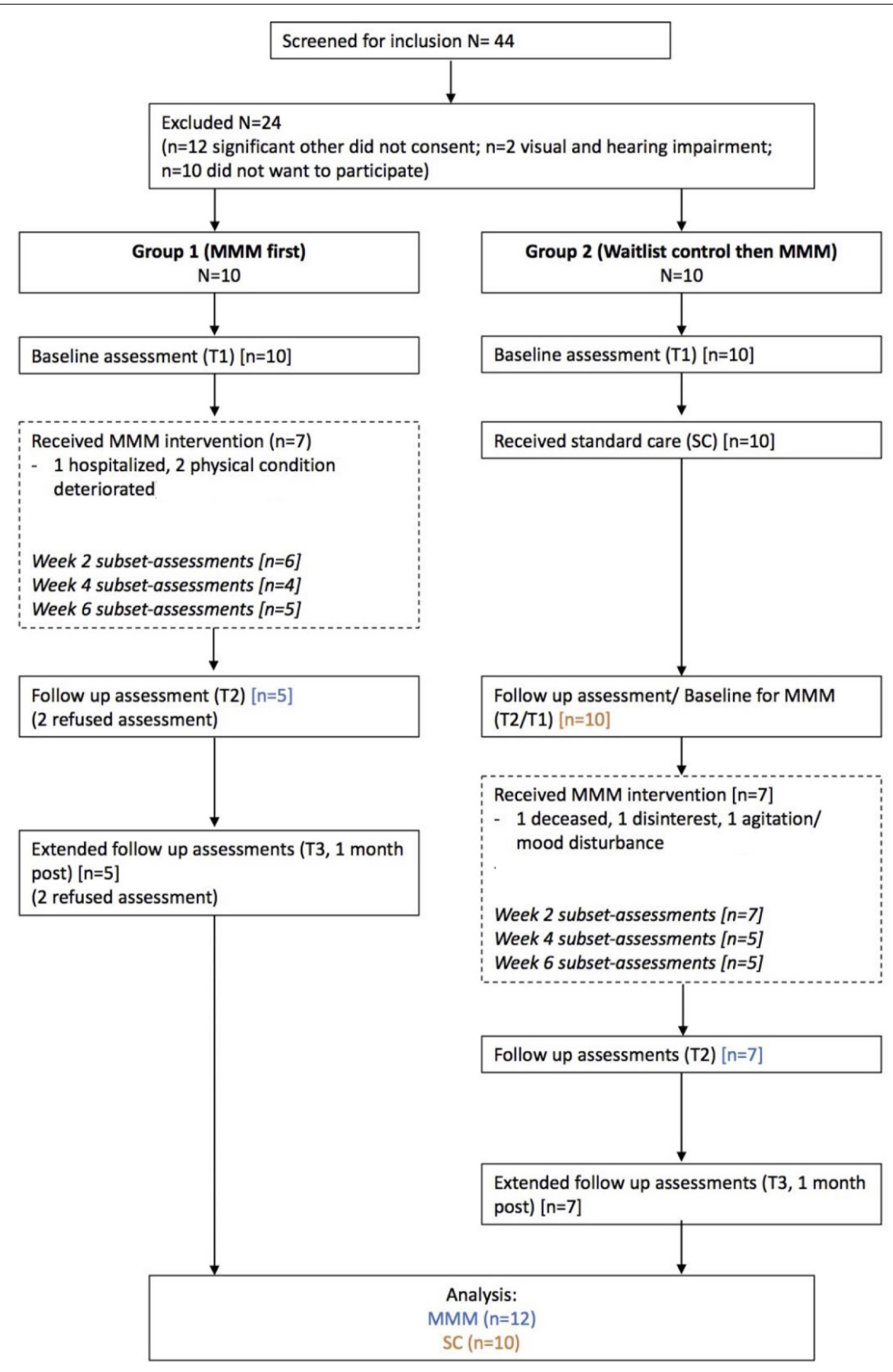

FIGURE 2 | Flow chart of the study design.

within the MMM and $\mathrm{SC}$ groups at $\mathrm{T} 1$ versus $\mathrm{T} 2$, and $\mathrm{T} 2$ versus T3 (MMM group only) to determine the effects of the MMM program overall.

We also sought to determine the distinct contribution that each of the seven capacities had on a subset of outcome measures. To do this, we conducted pairwise comparisons of assessments taken at baseline and 'test' phase (either week, 2, 4, or 6 of the MMM program). At the end of week 2 (following sessions focussing on engaging and persuasive capacities) assessments consisted of subsets of cognition (attention, memory and verbal fluency). At the end of week 4 (following sessions on the personal and emotional capacities), assessments consisted of tasks assessing autobiographical fluency (AFL) and identity ('I $\mathrm{am} / \mathrm{I}$ was' task). Finally, at the end of week 6 (following sessions on the social, physical, and synchronous capacities) 
assessments consisted of motor fluency (peg hole task) and verbal fluency tasks.

The answers provided by the participants to the open-ended questions during the booster sessions were categorized into each of the seven capacities of music. This was completed by F2, according to the key words the participant used to describe what they enjoyed. For example, if participants said that they "enjoyed moving to the music" this was categorized into the physical capacity. If participants stated that they "enjoyed talking with one another" or "being a part of something" this was categorized into the social capacity. The categories of the seven capacities of music were not mutually exclusive, as some responses included many key words describing several capacities. For example, some participants stated that they "enjoyed talking about old memories, with everyone and hearing their stories" this was classified as personal and social.

\section{RESULTS}

\section{Group Characteristics}

There were no significant differences between the Group 1 and Group 2 in age, gender, level of education, or musical background (whether they had spent time learning an instrument or singing, see Table 1).

\section{Drop-Out Rates}

Figure 2 shows the drop-out rates per week for the MMM group and the associated reasons. In Group 1, 7/10 participants completed the MMM program (three drop-outs due to hospitalization and deterioration of physical condition) and in Group 2, 7/10 participants completed the MMM program (three drop-outs due to death, disinterest and agitation/mood disturbance). Regarding post program assessment, in Group 1, five participants were included in the analysis (two refused final assessment). In Group 2, all 10 participants completed assessments pre/post the waitlist period of standard care, and all seven participants who finished the MMM program completed post assessments. Thus, analyses of cognition, mood, identity and fine motor skills at T1, T2, and T3 were conducted on 12 participants in the MMM group (five from Group 1 and seven from Group 2) and 10 participants in the SC group.

We compared the participants who dropped out of the MMM program $(n=6)$ with those who completed the intervention $(n=12)$ on several potential influencing factors such as age, duration of residing at facility and total ACE-III score (pre MMM intervention). There were no significant differences between those that dropped out and those who completed the intervention on any of these measures.

\section{Cognitive Function}

Table 3 shows results for total scores and subdomain scores of ACE-III for participants in the SC and MMM groups. We examined both the total ACE-III scores and the ACE-III subdomain scores for the period of standard care $(n=10)$ and for the MMM program $(n=12)$, comparing the scores at T1 versus T2, and T1 versus T3 (for MMM group only).

On average, there was a decrease in the mean total ACE-III score for participants in the SC group from T1 (59.40) to T2 (55.20). The majority of participants $(8 / 10)$ in the SC group showed a decline in total ACE-III score, eliciting a marginally significant median decrease, $z=-1.89, p=0.059$. For participants in the MMM group, the mean total ACE-III scores improved between T1 (57.00) and T2 (60.58). Overall, 8/12 participants had an increase in total ACE-III scores after participating in the MMM intervention, and the remaining four participants had a decrease. Statistical analysis showed a marginally significant median increase in total ACE-III scores, $z=-1.93$, $p=0.054$ (Figure 3).

The MMM group showed an increase in mean total ACEIII score from T1 (57.00) to T3 (60.50), 1 month after the MMM program. Again, 8/12 participants in the MMM group had an increase in total ACE-III scores 1 month after the MMM program, and the remaining four participants had a decrease, but this median difference did not reach statistical significance.

Statistical analysis on the ACE-III subdomain scores revealed there was no significant change in attention, memory, fluency, language or visuospatial ability scores from T1 to T2 in the SC group. In contrast, the MMM group showed an increase in mean 'attention' scores from T1 (10.67) to T2 (12.92). Nine participants showed an improvement in attention scores, three showed stable scores and no participant showed decreased attention scores, producing a statistically significant median increase in the subset of attention, $z=-2.68, p=0.007$. There was also a slight increase in mean 'verbal fluency' scores from T1 (4.33) to T2 (5.58). Seven participants showed an improvement in verbal fluency scores, three showed stable scores and two participants showed decreased scores, producing a marginally significant median increase in the subset of verbal fluency, $z=-1.91$, $p=0.056$ (Figure 4).

Comparisons of the ACE-III subdomain scores between baseline (T1) and the extended follow-up (T3) for participants in the MMM program revealed there was no significant differences in the subdomain ACE-III scores of attention, verbal fluency, memory, visuospatial skills or language between the time intervals (T1 and T3). When observing individual total ACE-III scores from T1 to T3 in the MMM group, we found that the total ACE-III score increased for eight out of the 12 participants after the extended follow up, whereas four participants exhibited a decrease in these scores.

Whilst the design of our study did not permit direct between- group statistical comparisons, Figure 4 illustrates the changes in the five ACE-III subdomains over the 8-week period for participants in the SC and MMM groups. We can see that whilst participants in the SC group decreased slightly in attention, memory and verbal fluency scores, participants in the MMM group showed slight increases in these three subdomain scores. Both groups had a negligible decrease in language and visuospatial subdomains scores over the 8 weeks, with the SC group showing a slightly greater decline. 


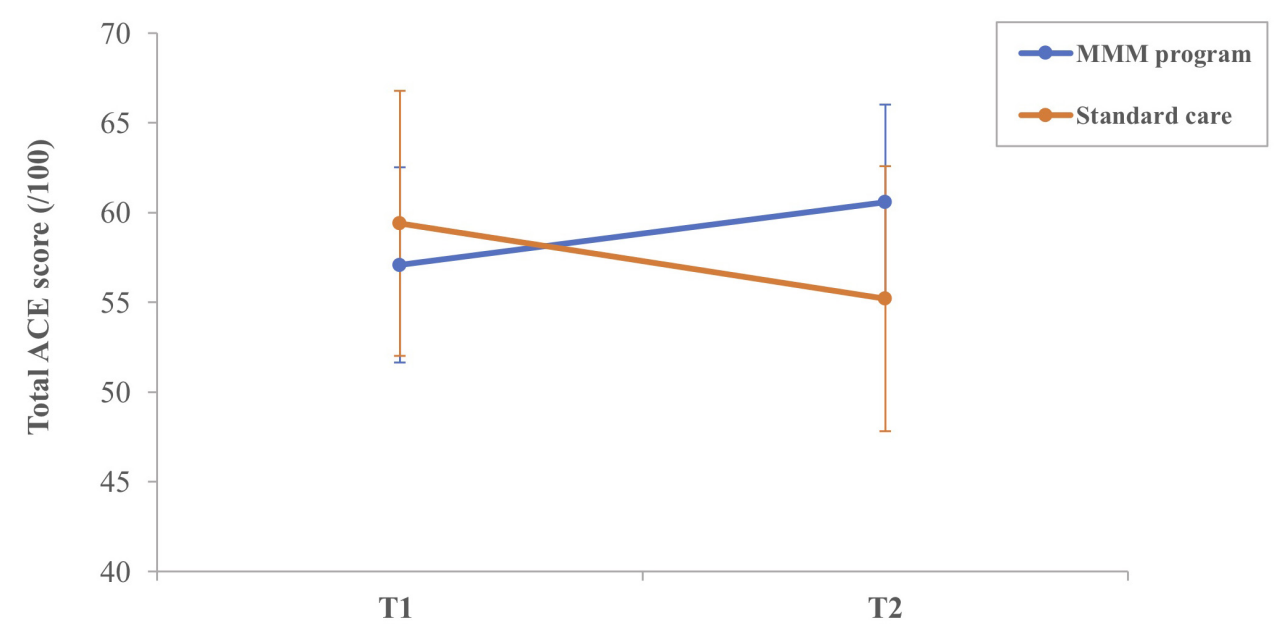

FIGURE 3 | Mean total ACE-III score for participants in the MMM $(n=12)$ and standard care $(n=10)$ groups at T1 (time 1, baseline) and T2 (time 2 , follow-up) (error bars $=$ mean \pm standard error of the mean).

\section{Mood}

The mean GDS-SF scores of participants in both the SC and MMM groups remained stable between T1 and T2 and, T1 and T3 for the MMM group. In other words, there was no difference in reported depression symptoms before and after the period of intervention or standard care (see Table 3).

\section{Self-Identity}

All 20 participants were able to generate 'I am/I was' statements at T1. However, one participant, who had minimal expressive language at T1, was unable to generate any statements at T2 after the period of standard care. This participant later withdrew from the MMM program at week 2. The mean total number of 'I am' and 'I was' statements were analyzed separately. There were no significant differences in the mean number of 'I am/I was' statements between T1 and T2 in the SC or MMM groups, and $\mathrm{T} 2$ and $\mathrm{T} 3$ in the MMM group (see Table 3 ).

\section{Autobiographical Memory Fluency}

For analysis purposes, the total number of names listed in each lifetime period was collapsed to create a total AFL score. There was no difference in the total number of names produced by participants in the SC and MMM groups at T1 compared with T2, and T2 compared with T3 in the MMM group (see Table 3 ).

\section{Motor Fluency}

Within the SC and MMM groups we compared the time taken (in seconds) for participants to complete the pegboard task with their

TABLE 3 | Outcome measures, mean (SD), for standard care and MMM groups at T1 (baseline), T2 (follow-up), and T3 (extended follow-up, MMM only).

\begin{tabular}{|c|c|c|c|c|c|c|}
\hline \multirow[t]{2}{*}{ Standard care $(n=10)$} & & \multicolumn{5}{|c|}{ MMM $(n=12)$} \\
\hline & T1 & $\mathrm{T} 2$ & $\mathbf{T 1}$ & T2 & T3 & \\
\hline \multicolumn{2}{|l|}{ Total ACE-III score (/100) } & $59.40(24.87)$ & $55.20(22.56)^{*}$ & $57.00(16.64)$ & $60.58(18.85)$ & $60.50(18.54)$ \\
\hline Attention (/18) & $12.00(4.83)$ & $10.70(4.42)$ & $10.67(3.20)$ & $12.92(3.20)^{* *}$ & $11.42(3.89)$ & \\
\hline Memory (/26) & $14.20(6.23)$ & $13.40(8.45)$ & $12.08(6.23)$ & $12.75(5.99)$ & $14.08(5.42)$ & \\
\hline Fluency (/14) & $5.80(3.82)$ & $3.90(3.14)$ & $4.33(2.74)$ & $5.58(2.39)^{*}$ & $5.0(2.92)$ & \\
\hline Language (/26) & $18.70(8.37)$ & $19.40(7.04)$ & $20.58(4.83)$ & $20.42(5.93)$ & $20.5(5.93)$ & \\
\hline Visuospatial (/16) & $8.70(4.44)$ & $7.80(3.88)$ & $9.42(4.38)$ & $8.92(4.29)$ & $9.5(3.87)$ & \\
\hline \multicolumn{2}{|l|}{ GDS-SF (/11) } & $5.33(2.91)$ & $5.89(3.10)$ & $3.83(2.29)$ & $4.17(2.59)$ & $3.33(2.10)$ \\
\hline \multicolumn{7}{|l|}{ Identity statements } \\
\hline \multicolumn{2}{|l|}{ 'I was' } & $7.10(3.48)$ & $5.40(3.06)$ & $6.33(2.81)$ & $6.67(3.82)$ & $5.92(3.34)$ \\
\hline \multicolumn{2}{|l|}{ 'I am’ } & $5.20(1.75)$ & $3.70(2.83)$ & $3.67(2.71)$ & $4.75(1.71)$ & $4.58(3.31)$ \\
\hline \multicolumn{2}{|c|}{ Autobiographical fluency score (total) } & $12.00(10.99)$ & $10.50(10.28)$ & $9.92(9.95)$ & $10.75(9.84)$ & $10.58(12.97)$ \\
\hline \multicolumn{7}{|l|}{ Peg task (seconds) } \\
\hline Dominant hand & $53.58(10.67)$ & $69.89(17.48)$ & $58.3(15.91)$ & $56.84(23.12)$ & $49.93(5.78)$ & \\
\hline Non-dominant hand & $55.34(16.03)$ & $62.64(12.43)$ & $61.31(10.09)$ & $61.67(21.61)$ & $54.66(10.98)$ & \\
\hline
\end{tabular}

Data presented as mean (standard deviation) unless otherwise stated. * $p<0.056$ (T2 vs. T1), ** $p<0.007$ (T2 vs. T1). 


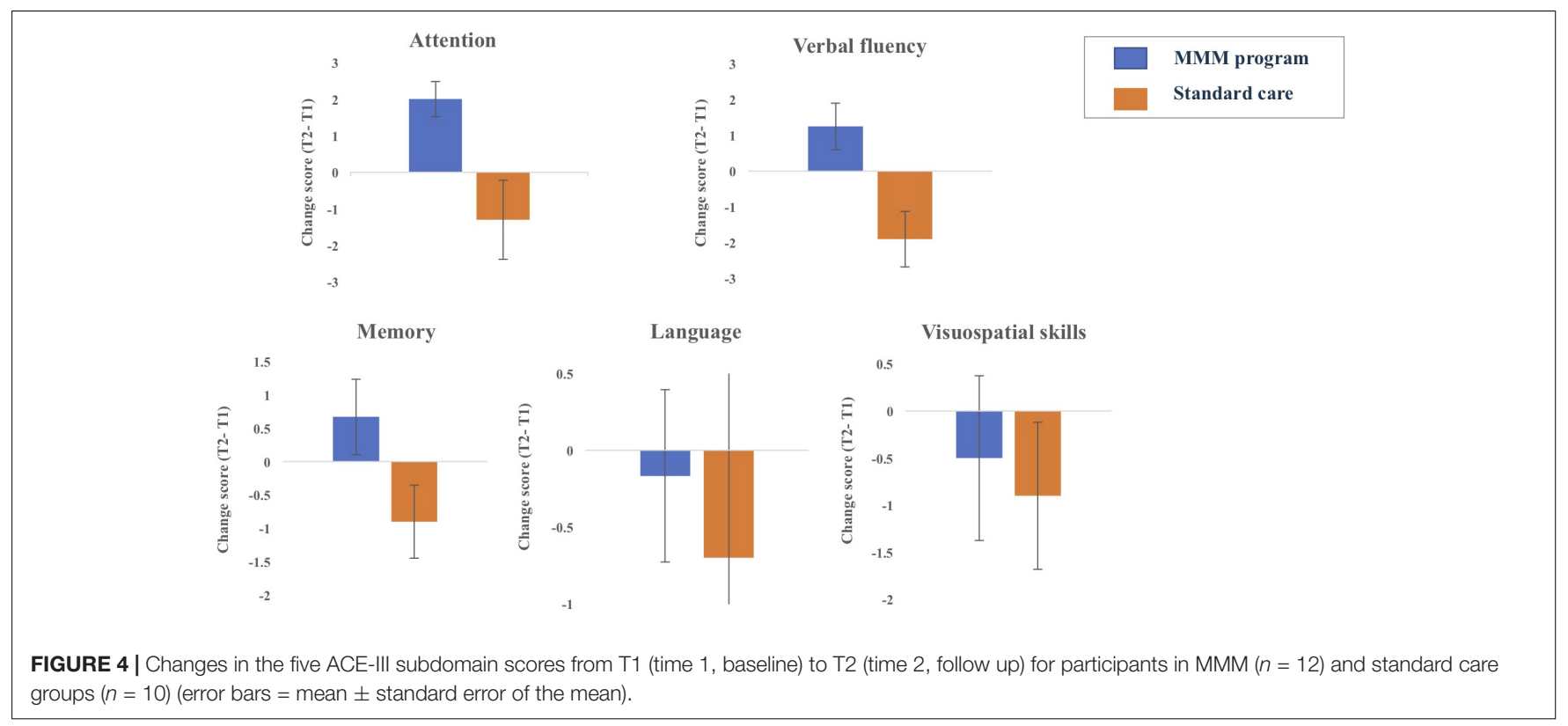

dominant and non-dominant hand at T1 versus T2. In the SC group, there was a trend for the time taken to complete the motor task to increase for both the dominant and non-dominant hands (from $53.58 \mathrm{~s}$ to $69.89 \mathrm{~s}$, and from $55.34 \mathrm{~s}$ to $62.64 \mathrm{~s}$, respectively), but these differences did not reach statistical significance. In the MMM group, the time taken for participants to complete the task with the dominant and non-dominant hands remained stable from T1 to T2. For participants in the MMM group, there was a trend for the time taken to complete the task with the dominant hand to decrease, from $56.84 \mathrm{~s}$ (T2) to $49.93 \mathrm{~s}$ (T3), but this was not statistically significant (Table 3 ).

\section{Assessment of Individual Seven Capacities}

To determine the distinct contribution that each of the seven capacities had on the subset measures we conducted pairwise comparisons between assessments taken at baseline and test phase (either week, 2, 4, or 6). Table 4 displays the assessments conducted at week 2, 4, and 6. Results showed that there were no significant differences between baseline and test phase on any of the subset of measures at week 2, 4, or 6 .

\section{Exploratory Analyses}

\section{Prediction of Change in Cognition}

We wanted to determine whether certain variables (age, years of education, time in residency and, number of sessions attended for MMM group only) predicted the change in cognition from $\mathrm{T} 1$ to T2, as measured by the total ACE-III score. To do this, we calculated a 'change score' by subtracting the total ACEIII score at T1 from the total ACE-III score at T2. We then conducted a multiple regression analysis to determine if there was a relationship between these variables and the total ACE-III change score for participants in both MMM and SC groups.
In the MMM group, there was a statistically significant regression equation, $F(3,8)=4.78, p=0.034$, adj. $R^{2}=0.508$. The only variable to predict change in total ACE-III score was the participants' time in residency, $p=0.040$ (Figure 5). In other words, the longer the participant had been a resident at the aged care facility the less improvement in overall cognition after the MMM program. Age, years of education, and number of sessions attended were not significant predictors. For participants in the SC group, the model was non-significant, demonstrating no relationship between any of the demographic variables (age, years of education, and time in residency) and the total ACEIII change score.

We then further examined the significant negative relationship between participants' time in residency and the total ACE-III change score the MMM group. We removed the participant with the longest duration of residency (52 months), their being an outlier by more than 2 standard deviations (see Figure 5). With this outlier removed, the data did not violate the assumption of Normality determined by the Shapiro-Wilk statistic. We then reanalyzed the comparison of total ACEIII scores pre and post the MMM program. We found a statistically significant increase in total ACE-III scores from T1 $(M=56.27, S D=17.25)$ to $\mathrm{T} 2(M=60.45, S D=19.76)$, $t(10)=-2.4, p=0.037$, and from $\mathrm{T} 1(56.27)$ to $\mathrm{T} 3(M=61.18$, $S D=19.29), t(10)=-3.03, p=0.013$ for participants in the MMM group.

\section{Prediction of Attendance of MMM Sessions}

To examine predictors of session attendance in the MMM program we conducted a multiple regression analysis with the variables of age, time in residency and GDS-SF score. The multiple regression model statistically significantly predicted the number of MMM program sessions attended $F(2,17)=4.79$, $p=0.022$, adj. $R^{2}=0.285$. The GDS-SF score was the only variable to significantly predict MMM session attendance, $p=0.017$. In 
TABLE 4 | Subset measures, mean (SD), for participants who completed measures at the relevant sessions (baseline and test phase) during the MMM program.

\begin{tabular}{|c|c|c|c|c|c|c|}
\hline Measures & Baseline & & & Test phase & & \\
\hline $\begin{array}{l}\text { Engaging and } \\
\text { persuasive }\end{array}$ & Attention (/8) & Memory- word list (/3) & $\begin{array}{l}\text { Verbal fluency- } \\
\text { letter (/7) }\end{array}$ & Attention (/8) & $\begin{array}{c}\text { Memory-word list } \\
\text { (/3) }\end{array}$ & $\begin{array}{l}\text { Verbal fluency- } \\
\text { letter (/7) }\end{array}$ \\
\hline Week $2(n=12)$ & $3.58(1.83)$ & $0.92(1.16)$ & $2.83(0.39)$ & $3.50(1.38)$ & $1.17(1.11)$ & $2.67(1.50)$ \\
\hline $\begin{array}{l}\text { Emotional and } \\
\text { personal }\end{array}$ & AFL total & 'I was' & 'I am’ & AFL total & 'I was' & 'I am’ \\
\hline Week $4(n=11)$ & $6.0(5.06)$ & $5.27(2.57)$ & $3.73(2.24)$ & $5.54(4.76)$ & $5.18(3.06)$ & $4.0(2.0)$ \\
\hline $\begin{array}{l}\text { Physical, } \\
\text { synchronous and } \\
\text { social }\end{array}$ & Peg task (dominant) & Peg task (non-dominant) & $\begin{array}{l}\text { Verbal fluency-total } \\
\qquad(/ 14)\end{array}$ & Peg task (dominant) & $\begin{array}{c}\text { Peg task } \\
\text { (non-dominant) }\end{array}$ & $\begin{array}{l}\text { Verbal fluency-total } \\
\text { (/14) }\end{array}$ \\
\hline Week $6(n=10)$ & $55.30(23.45)$ & $56.64(15.07)$ & $3.0(1.43)$ & 47.25 (10.20) & 43.25 (12.51) & $4.0(1.45)$ \\
\hline
\end{tabular}

AFL, autobiographical fluency task; dominant, dominant hand; non-dominant, non-dominant hand.

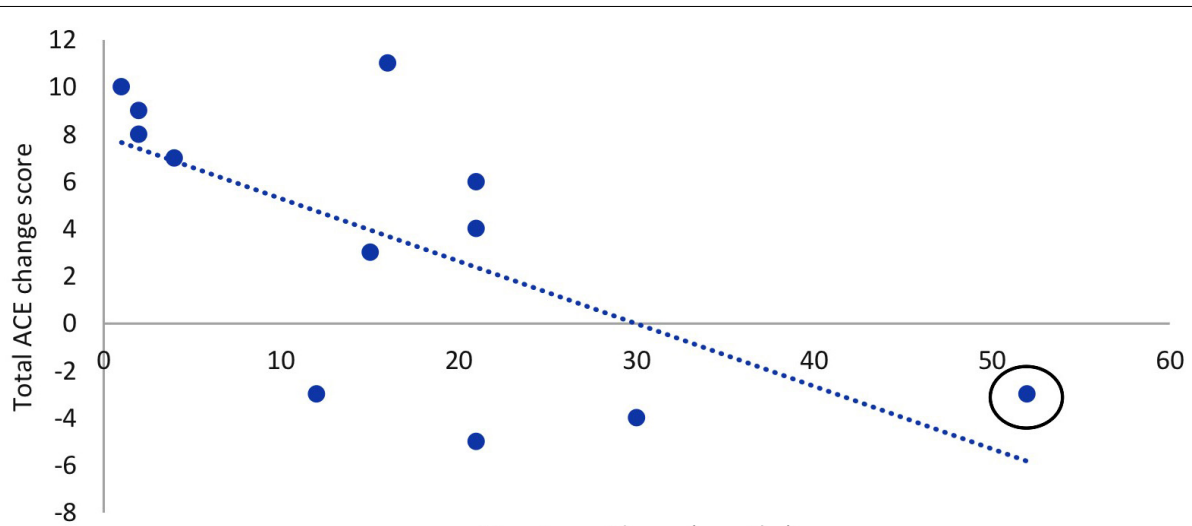

Time in residency (months)

FIGURE $\mathbf{5}$ | Relationship between time in residency and total ACE-III change score (T2-T1) after the MMM intervention ( $n=12)$. Outlier circled.

other words, the higher the GDS-SF score (indicative of possible depression), the less MMM sessions attended (Figure 6).

We then compared the GDS-SF scores of the participants who dropped out of the MMM program with those who completed the program. Those who dropped out of the MMM program had a higher GDS-SF score prior to the MMM program (7.33), suggestive of mild depression, compared to those who remained in the program, who had no indication of depression prior to the MMM program (4.21), $t(18)=2.64, p=0.017$.

\section{Subjective Responses From Booster Sessions}

The weekly booster session visits were made by F1 to participants who attended the corresponding MMM session. The availability of participants varied on this day and was influenced by factors such as health, visitors and their activity schedule. Thus, $67.3 \%$ of participants on average each week were seen for booster sessions. Figure 7 depicts the distribution of the participants' responses to the open-ended questions determining what aspects they enjoyed in the MMM program. The social capacity of music in the MMM program was the most valued by the participants, accounting for $36 \%$ of the responses. These responses included statements about enjoying the company of others in the group, making new friends, being in a group scenario, participating in discussions or 'getting out of their room.' The engaging and personal capacities were the second most common theme of responses each accounting for $17 \%$ of the responses. These included statements about enjoying the experience of learning new things and the sounds of the musical instruments. Responses pertaining to the personal capacity included enjoying reminiscing and listening to their old songs. References to the emotional capacity of music were present in $13 \%$ of responses and included statements of feeling "lifted," "happy" and "ready to party" after the sessions. Finally, 4\% of responses included reference to the synchronous capacity of music and included statements such as doing two actions at one time. Other types of reflections accounted for $13 \%$ of all responses and included statements pertaining specifically to the MMM program, for example that it was "entertaining," "something different' and that the program was "delivered well."

\section{DISCUSSION}

We conducted a pilot MMM program for people with mild to moderate dementia (of various types) and examined its effects on 


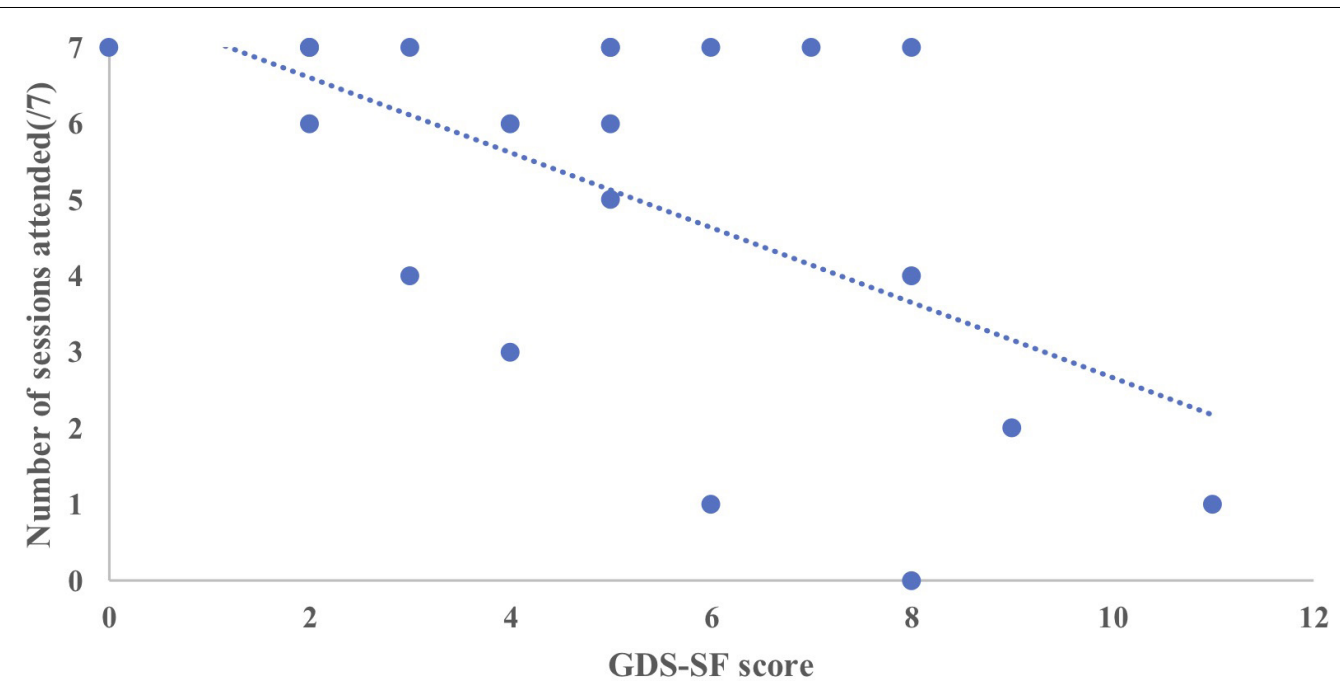

FIGURE 6 | Relationship between the number of MMM sessions attended (maximum 7) and GDS-SF score prior to starting the intervention ( $n=20$ ).

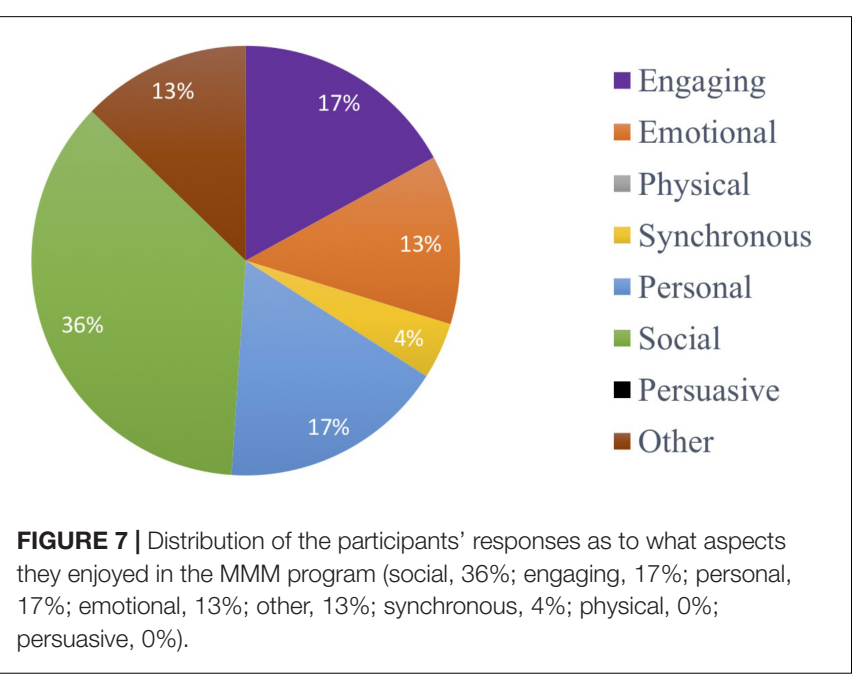

cognition, mood, identity and fine motor function. The MMM program is group-based, comprising of seven weekly sessions broken into four blocks which draw upon the seven capacities of music in the TMCM (Figure 1). The main finding was that compared with people receiving standard care who showed a slight decline in global cognition (ACE III total score) over time, those who participated in the MMM program showed a slight improvement in global cognition. In particular, significant improvements occurred in the subdomain scores of attention and verbal fluency. In contrast, we did not find any change in mood, sense of identity or motor function after MMM participation. To our knowledge, the MMM program is the first music program based on a theoretical model underpinning the beneficial capacities of music for neurological impairment.

Our primary finding was a marginal improvement of global cognition after the 7-week MMM program. This result is in keeping with previous studies which have shown that both musicbased interventions and music therapy can improve or stabilize global cognition in people with dementia (Suzuki et al., 2004; Van de Winckel et al., 2004; Bruer et al., 2007; Chu et al., 2014; Särkämö et al., 2014; Cheung et al., 2018; Tang et al., 2018). Our findings of improved performance in the specific cognitive subdomains of verbal fluency and attention are also consistent with previous research. Specifically, improvements in verbal fluency have been reported by Brotons and Koger (2000), Van de Winckel et al. (2004), Cheung et al. (2018), and Lyu et al. (2018). Särkämö et al. (2014) also found an improvement in general cognition and attention (as measured by the MMSE and Frontal Assessment Battery) after 10 weeks of either group singing or group music listening interventions. Taken together, these results suggest that a music program that involves actively applying seven crucial capacities of music is cognitively enhancing, particularly for verbal fluency and attention.

Importantly, our results also showed that the time in aged care residency predicted the degree of cognitive improvement that occurred after the MMM program. In other words, the longer a participant had spent in residency, the less global cognitive improvement they showed after MMM participation. Interestingly, post hoc analysis showed that by removing one participant who was an outlier with the longest time in residency, the comparison of participants' total ACE-III scores from T1 to T2 in the MMM group became statistically significant. This suggests that early engagement with the MMM program is most beneficial. Implementation of the MMM program in people with early stages of dementia may help to optimize cognitive function and delay admission to a residential aged care facility.

One mechanism underlying the beneficial cognitive effects we observed could be neural scaffolding, facilitated by the 'enriched environment' that the MMM program provided. Including all seven capacities of music may have preserved the natural scaffolding process which was under threat by neurological impairment. The MMM program also incorporated activities 
which emphasized several 'active' ingredients, for example activities that were 'novel' or 'challenging', which may lead to 'scaffolding enhancement' (Park and Reuter-Lorenz, 2009). These active ingredients may facilitate neural scaffolding and improve cognition by placing the individual in a state of deep engagement. A similar phenomenon has been observed in other enriching group-based activities with healthy elderly individuals that involve learning novel and challenging skills, such as quilting or digital photography (Reuter-Lorenz and Park, 2014). Thus, the MMM program, through combining the multitude of therapeutic capacities of music, may provide the enriched environment needed for compensatory scaffolding.

We propose that the cognitive benefits of the MMM program can be attributed to the relative contribution of each of the seven capacities of music, activating neural networks and providing this compensatory scaffolding. Firstly, we utilized multiple music activities (singing, playing, moving) which may maximize widespread engagement of frontal, parietal, temporal, limbic/paralimbic, and cerebellar regions. In particular, the cognitive effects may have been mediated through enhanced arousal, which facilitates attention and verbal fluency. Secondly, listening to personal music evokes emotions and corresponds with neural activity in subcortical and medial frontal regions (Koelsch, 2010). Simultaneously, the limbic and paralimbic regions, associated with the processing of emotions, are activated (Blood and Zatorre, 2001). Frontal regions relatively spared from degeneration in $\mathrm{AD}$ and may be a hub for the intersection between music, memories, and emotions (Jacobsen et al., 2015). The physical movement involved in the program may have increased temporal arousal, stimulating cognitive activity. Indeed, improvement in cognition in people with dementia has been found in similar studies looking at music and movement (Van de Winckel et al., 2004; Satoh et al., 2014; Shimizu et al., 2017; Cheung et al., 2018). Adding to this effect, playing rhythmical musical instruments in a social setting allowed individuals to synchronize with the music and also with each other, which may have stimulated neural activation in the prefrontal cortex and improved cognition (Shimizu et al., 2017). Lastly, the potential therapeutic benefits of the program were enhanced by the persuasive nature of music. Participants retained the motivation to keep attending the MMM program as they experienced it as being an enjoyable activity, demonstrated by their qualitative statements of the sessions being 'social' and 'engaging.' In this therapeutic setting, their enjoyment lead to increased motivation for participation. Thus, by involving all seven capacities of music and placing individuals in an enriched setting, the MMM program may maximize the potential of stimulating compensatory neural scaffolds.

Our finding of a decline in global cognition in the standard care group is consistent with other research findings. For example, Tang et al. (2018) reported cognitive decline in people with dementia following standard care in a nursing home facility over 12 weeks and attributed this decline to apathy. The high occurrence of social withdrawal and isolation in people with dementia may be related to higher levels of apathy, which is associated with cognitive impairment (e.g., Benoit et al., 2012). Whilst the individuals in the standard care group had the option of attending various activities (provided by the residential aged care facility), these activities did not involve all seven capacities of the MMM program. Furthermore, many individuals did not attend the activities available. Therefore, a lack of stimulation may account for the decline in their cognition. A lack of engagement in stimulating activities later in life may hasten the decline in cognition that typically arises in aging as a result of age associated disuse (Mahncke et al., 2006).

In addition to cognition, we also assessed identity, mood and motor function. Whilst music is commonly linked with identity in dementia (McDermott et al., 2014; Baird and Thompson, 2018), this is the first study, to the best of our knowledge, to undertake an empirical investigation of this issue to examine the effect of a music-based program on identity in people with dementia. We found no significant change in our identity measure (using the 'I am/I was' task) after participation in the MMM program. This identity measure may not have been sensitive enough to capture changes that may have been more subtle or qualitative in nature. These constructs may have been better explored via interviews, as shown in previous research in group singing for people with dementia (e.g., Camic et al., 2013; Osman et al., 2016). Furthermore, the use of observational measures might have been more sensitive in detecting symptoms of depression and altered self identity. These measures could be more reliable than the self-assessment instruments use as they do not rely on the participant to provide a direct response and thus, may be suitable for people who have communication and other cognitive difficulties associated with dementia.

To assess mood, we used a standard measure of depression specifically designed for elderly populations (GDS-SF). Unlike other similar studies that have reported improvement on this measure after participation in music activities (e.g., Guétin et al., 2009; Cooke et al., 2010), we did not find significant differences between standard care and MMM groups. We would have expected that the assessments of mood may have reflected the subjective feedback about feeling 'happy' and 'elated' after the MMM sessions. We did, however, observe a significant correlation between number of sessions attended and level of depression (total GDS score). Thus, our finding of no change in mood scores could be a dosage related finding, in that the participants who reported lowest mood at the start of the program attended the least amount of MMM sessions, meaning they did not get the full benefit of the entire program. These results also highlight how comorbidities that exist prior to the start of a therapeutic program, such as depression, may reduce session attendance; stunting the potential for rehabilitation.

Participants responded well to the program and were able to participate in all activities. Participants' subjective responses from the booster sessions revealed that the most enjoyed and valued aspects of the program were its social, engaging, personal and emotional nature. Through the semi-formal interviews and observations during the sessions, we found that discussing personal memories, emotional reactions to songs and also moving in time with one another enabled the creation of social connections. This reinforces the value of conducting musicbased interventions in a group scenario. Similarly, participants enjoyed listening to their personally chosen songs and those 
chosen by the other members and reminiscing. Furthermore, we deemed the visit in session 7 from the student musicians from the local conservatorium of Music an important aspect of the study that could be implemented more often in programs. This intergenerational aspect to the program could be a way to reduce stigma associated with dementia whilst also decreasing social isolation, as previously demonstrated by Harris and Caporella (2014).

\section{Limitations and Future Directions}

We evaluated our program as a prospective cohort study, but a number of factors should be considered when interpreting the results. First, as participation was voluntary and recruitment was from a single residential care facility, our sample is only representative of individuals in aged care with an interest in music, and not necessarily representative of all individuals with dementia. Future research should examine the benefits of the MMM program to a broader sample of participants.

Second, our cohort of participants with probable dementia was a heterogeneous group that included those with a clinical diagnosis of dementia, MCI and memory disturbance. Pooling responses from a heterogenous group of individuals makes it difficult to determine how music programs can be designed to optimally benefit people with different types of dementia. On the other hand, the heterogeneous nature of our sample means that the benefits observed may be generally applicable the wider dementia population. Indeed, the majority of participants had no musical background, suggesting that anyone can participate in and receive benefits from the MMM program, even those with no prior music training.

Third, our sample size was small, primarily owing to attrition rates from illness, death, visitors, and timing of sessions after lunch when many participants were tired and often slept. That we observed a small improvement in global cognitive function following a pilot MMM program justifies future research involving a larger sample size, and a RCT design.

Fourth, it is important to consider the impact of practice effects for the measures that were used at week 2, 4, and 6 in the MMM group (but not throughout the period of standard care). The repeated measures design may have familiarized participants with test measures, thereby confounding the effects of the MMM intervention (Goldberg et al., 2015). However, the categorical verbal fluency test, which was used most frequently in the study, has been shown not to be susceptible to practice effects in people with dementia over short time intervals, such as weeks (Cooper et al., 2004). Thus, it is unlikely that practice effects can account for these specific results.

Fifth, as an initial examination of the MMM program, the research provides little insight into the optimal dosage of the intervention. Future research should evaluate the duration and dosage of such a program to potentially maximize the cognitive benefits of the MMM program. Although visiting each participant on a weekly basis may not be feasible by residential aged care facility staff, the improvement in cognition in our results indicated that potentially one full group MMM session plus an individual booster MMM session per week is valuable. Similarly, it would be worthwhile exploring whether the observed benefits of the MMM program for verbal fluency and attention might generalize to an improvement in communication, social skills or activities of daily living.

Sixth, although the MMM program is based on a model of the active ingredients (capacities) of music-based interventions, the current investigation does not provide a full understanding of the specific benefits of each capacity and its therapeutic outcome, as seen in the TMCM. Future research could include comparisons of the full MMM program with programs that focus on more specific sets of capacities, such as music and reminiscence (emotional and personal) or music and movement (physical and synchronous). Further research could also compare the MMM with other arts-based programs to determine whether music is unique in its effects, or whether any social activity can lead to benefits (as seen in Narme et al., 2014 who found no short-term behavioral differences between music and cooking).

Finally, the MMM program could be trialed with people with other neurological disorders such as Parkinson's disease or stroke. In our cohort of participants, we had two individuals with Parkinson's disease and one with post stroke aphasia. These individuals were capable of participating in all activities in the MMM program to the same extent as the participants with dementia.

\section{CONCLUSION}

We have devised and evaluated a music-based treatment that is grounded in a model of the therapeutic application of music, the TMCM. A primary advantage of the MMM program is that activities are linked with plausible mechanisms of intervention. This investigation not only furthers our understanding of music-based treatments but, unlike other protocols, addresses individual goals. Therapists can emphasize specific activities that target specific problems faced by individuals with neurological disorders. Our results demonstrate that the MMM program may benefit cognition, particularly attention and verbal fluency, in people with various types of dementia. By including all seven capacities of music in one program, individuals were placed in a particularly enriched environment likely to promote neural scaffolding. Our findings highlight the importance of engaging people in such programs soon after admission to a residential aged care facility (or even earlier) to gain optimal cognitive benefits. The small and heterogenous sample limits the conclusions that can be drawn, and underscores the need for additional research to be conducted on these effects. Nevertheless, our findings encourage the development and refinement of music-based interventions that build on evidence and theory, and that may be personalized to suit the various needs of people with dementia.

\section{ETHICS STATEMENT}

The application was considered by the Macquarie University Human Research Ethics Committee [HREC (Medical Sciences)]. Reference No: 5201700523. Initial contact was made to the 
participant by aged care facility staff that they are familiar with. If the participant was willing, the aged care facility staff then communicated the suitability of participants to the investigators. Consent for participation and review of medical records was obtained from the significant other of potential participants and participants themselves. Given the mobile fragility of many of the participants they were asked to only move within their limits. Any emotional distress was dealt with by having contact with the staff at the care facility who knew the participants mental state well. Before the beginning of each session participants were assessed as to whether they were well enough to participate.

\section{AUTHOR CONTRIBUTIONS}

$\mathrm{OB}$ and $\mathrm{AB}$ coordinated testing and data collection. $\mathrm{OB}$ was responsible for data analysis and all authors contributed to data interpretation. OB wrote the first draft of the manuscript and all authors contributed to further revisions. All authors approved the final version of the manuscript and contributed to the design and development of the study.

\section{REFERENCES}

Altenmüller, E., and Schlaug, G. (2013). Neurologic music therapy: the beneficial effects of music making on neurorehabilitation. Acoust. Sci. Technol. 34, 5-12. doi: $10.1250 /$ ast. 34.5

Ashida, S. (2000). The effect of reminiscence music therapy sessions on changes in depressive symptoms in elderly persons with dementia. J. Music Ther. 37, 170-182. doi: 10.1093/jmt/37.3.170

Baird, A., Brancatisano, O., Gelding, R., and Thompson, W. F. (2018). Characterization of music and photograph evoked autobiographical memories in people with Alzheimer's disease. J. Alzheimers Dis. 66, 693-706. doi: 10.3233/ JAD- 180627

Baird, A., and Thompson, W. F. (2018). The Impact of music on the self in dementia. J. Alzheimers Dis. 61, 827-841. doi: 10.3233/JAD-170737

Baird, A., Umbach, H., and Thompson, W. F. (2017). A non-musician with severe Alzheimer's dementia learns a new song. Neurocase 23, 36-40. doi: 10.1080/ 13554794.2017.1287278

Benoit, M., Berrut, G., Doussaint, J., Bakchine, S., Bonin-Guillaume, S., Frémont, P., et al. (2012). Apathy and depression in mild Alzheimer's disease: a crosssectional study using diagnostic criteria. J. Alzheimers Dis. 31, 325-334. doi: 10.3233/JAD-2012-112003

Biasutti, M., and Mangiacotti, A. (2018). Assessing a cognitive music training for older participants: a randomised controlled trial. Int. J. Geriatr. Psychiatry 33, 271-278. doi: 10.1002/gps.4721

Blood, A. J., and Zatorre, R. J. (2001). Intensely pleasurable responses to music correlate with activity in brain regions implicated in reward and emotion. Proc. Natl. Acad. Sci. U.S.A. 98, 11818-11823. doi: 10.1073/pnas.191355898

Brancatisano, O., and Thompson, W. F. (in press). "Seven capacities of music that underpin its therapeutic value in dementia care," in Music and Dementia: From Cognition to Therapy, eds A. Baird, S. Garrido, and J. Tamplin (Oxford: Oxford University Press).

Brotons, M., and Koger, S. M. (2000). The impact of music therapy on language functioning in dementia. J. Music Ther. 37, 183-195. doi: 10.1093/jmt/37.3.183

Bruer, R. A., Spitznagel, E., and Cloninger, C. R. (2007). The temporal limits of cognitive change from music therapy in elderly persons with dementia or dementia-like cognitive impairment: a randomized controlled trial. J. Music Ther. 44, 308-328. doi: 10.1093/jmt/44.4.308

Camic, P. M., Williams, C. M., and Meeten, F. (2013). Does a 'Singing Together Group' improve the quality of life of people with a dementia and their carers? a pilot evaluation study. Dementia 12, 157-176. doi: 10.1177/1471301211422761

\section{FUNDING}

We would like to acknowledge the generous support of the Hunter New England and Central Coast Primary Health Network for the funding necessary to establish this pilot program (Collaborative Health Innovation Project Funding), and Scott White for facilitating this funding opportunity. This work was also supported by the National Health and Medical Research Council and the Australian Research Council [NHMRC-ARC Dementia Research Development Fellowship to AB (Grant No. 1104833) and ARC funding grants to WFT].

\section{ACKNOWLEDGMENTS}

We would like to thank the participants, caregivers, and staff at BaptistCare Warabrook Centre, for their participation and facilitation during the pilot studies. We especially thank Emma Chesterfield for her support in enabling the study to go ahead, Jo Cook for facilitating the MMM program and booster sessions, and Tamara Paulin for conducting all the participant assessments.

Carvalho, A., Rea, I. M., Parimon, T., and Cusack, B. J. (2014). Physical activity and cognitive function in individuals over 60 years of age: a systematic review. Clin. Intervent. Aging 9, 661-682. doi: 10.2147/CIA.S55520

Chang, F. Y., Huang, H. C., Lin, K. C., and Lin, L. C. (2010). The effect of a music programme during lunchtime on the problem behaviour of the older residents with dementia at an institution in Taiwan. J. Clin. Nurs. 19, 939-948. doi: 10.1111/j.1365-2702.2009.02801.x

Cheung, D. S. K., Lai, C. K. Y., Wong, F. K. Y., and Leung, M. C. P. (2018). The effects of the music-with-movement intervention on the cognitive functions of people with moderate dementia: a randomized controlled trial. Aging Ment. Health 22, 306-315. doi: 10.1080/13607863.2016.1251571

Choi, A. N., Lee, M. S., Cheong, K. J., and Lee, J. S. (2009). Effects of group music intervention on behavioral and psychological symptoms in patients with dementia: a pilot-controlled trial. Int. J. Neurosci. 119, 471-481. doi: 10.1080/ 00207450802328136

Chu, H., Yang, C. Y., Lin, Y., Ou, K. L., Lee, T. Y., O’Brien, A. P., et al. (2014). The impact of group music therapy on depression and cognition in elderly persons with dementia: a randomized controlled study. Biol. Res. Nurs. 16, 209-217. doi: 10.1177/1099800413485410

Cooke, M., Moyle, W., Shum, D., Harrison, S., and Murfield, J. (2010). A randomized controlled trial exploring the effect of music on quality of life and depression in older people with dementia. J. Health Psychol. 15, 765-776. doi: $10.1177 / 1359105310368188$

Cooper, D. B., Lacritz, L. H., Weiner, M. F., Rosenberg, R. N., and Cullum, C. M. (2004). Category fluency in mild cognitive impairment: reduced effect of practice in test-retest conditions. Alzheimer Dis. Assoc. Disord. 18, 120-122. doi: 10.1097/01.wad.0000127442.15689.92

Cowles, A., Beatty, W. W., Nixon, S. J., Lutz, L. J., Paulk, J., Paulk, K., et al. (2003). Musical skill in dementia: a violinist presumed to have alzheimer's disease learns to play a new song. Neurocase 9, 493-503. doi: 10.1076/neur.9.6.493. 29378

Cuddy, L. L., and Duffin, J. (2005). Music, memory, and Alzheimer's disease: is music recognition spared in dementia, and how can it be assessed? Med. Hypotheses 64, 229-235. doi: 10.1016/j.mehy.2004.09.005

Cuddy, L. L., Sikka, R., Silveira, K., Bai, S., and Vanstone, A. (2017). Music evoked autobiographical memories in Alzheimer's Disease: evidence for a positivity effect. Cogent Psychol. 4:1277578. doi: 10.1080/23311908.2016.1277578

Drapeau, J., Gosselin, N., Gagnon, L., Peretz, I., and Lorrain, D. (2009). Emotional recognition from face, voice, and music in dementia of the alzheimer type. Ann. N. Y. Acad. Sci. 1169, 342-345. doi: 10.1111/j.1749-6632.2009.04768.x 
Dritschel, B. H., Williams, J. M. G., Baddeley, A. D., and Nimmo-Smith, I. (1992). Autobiographical fluency: a method for the study of personal memory. Mem. Cogn. 20:133. doi: 10.3758/BF03197162

Dyer, S., Harrison, S., Laver, K., Whitehead, C., and Crotty, M. (2018). An overview of systematic reviews of pharmacological and non-pharmacological interventions for the treatment of behavioral and psychological symptoms of dementia. Int. Psychogeriatr. 30, 295-309. doi: 10.1017/S1041610217002344

El Haj, M., Fasotti, L., and Allain, P. (2012). The involuntary nature of musicevoked autobiographical memories in Alzheimer's disease. Conscious Cogn. 21, 238-246. doi: 10.1016/j.concog.2011.12.005

Erickson, K. I., Voss, M. W., Prakash, R. S., Basak, C., Szabo, A., Chaddock, L., et al. (2011). Exercise training increases size of hippocampus and improves memory. Proc. Natl. Acad. Sci. U.S.A. 108, 3017-3022. doi: 10.1073/pnas.1015950108

Garrido, S., Dunne, L., Chang, E., Perz, J., Stevens, C. J., and Haertsch, M. (2017). The use of music playlists for people with dementia: a critical synthesis. J. Alzheimer's Dis. 60, 1129-1142. doi: 10.3233/JAD- 170612

Garrido, S., Stevens, C. J., Chang, E., Dunne, L., and Perz, J. (2018). Music and dementia: individual differences in response to personalized playlists. $J$ Alzheimers Dis. 64, 933-941. doi: 10.3233/JAD- 180084

Gerdner, L. A. (2000). Effects of individualized versus classical "relaxation" music on the frequency of agitation in elderly persons with Alzheimer's disease and related disorders. Int. Psychogeriatr. 12, 49-65. doi: 10.1017/ s1041610200006190

Goldberg, T. E., Harvey, P. D., Wesnes, K. A., Snyder, P. J., and Schneider, L. S. (2015). Practice effects due to serial cognitive assessment: implications for preclinical Alzheimer's disease randomized controlled trials. Alzheimer's Dementia 1, 103-111. doi: 10.1016/j.dadm.2014.11.003

Gridley, K., Brooks, J., Birks, Y., Baxter, K., and Parker, G. (2016). Improving Care for People with Dementia: Development and Initial Feasibility Study for Evaluation of Life Story Work in Dementia Care. Southampton: NIHR Journals Library;Health Services and Delivery Research.

Guétin, S., Portet, F., Picot, M. C., Pommié, C., Messaoudi, M., Djabelkir, L., et al. (2009). Effect of music therapy on anxiety and depression in patients with Alzheimer's type dementia: randomised, controlled study. Dement. Geriatr. Cogn. Disord. 28, 36-46. doi: 10.1159/000229024

Harris, P. B., and Caporella, C. A. (2014). An intergenerational choir formed to lessen alzheimer's disease stigma in college students and decrease the social isolation of people with alzheimer's disease and their family members: a pilot study. Am. J. Alzheimers Dis. Other Demen. 29, 270-281. doi: 10.1177/ 1533317513517044

Ho, R. T., Fong, T. C., Sing, C. Y., Lee, P. H., Leung, A. B., Chung, K. S., et al. (2018). Managing behavioral and psychological symptoms in Chinese elderly with dementia via group-based music intervention: a cluster randomized controlled trial. Dementia doi: 10.1177/1471301218760023 [Epub ahead of print].

Howard, R., McShane, R., Lindesay, J., Ritchie, C., Baldwin, A., Barber, R., et al. (2012). Donepezil and memantine for moderate-to-severe alzheimer's disease. N. Engl. J. Med. 366, 893-903. doi: 10.1056/NEJMoa1 106668

Irish, M., Cunningham, C. J., Walsh, J. B., Coakley, D., Lawlor, B. A., Robertson, I. H., et al. (2006). Investigating the enhancing effect of music on autobiographical memory in mild Alzheimer's disease. Dement Geriatr. Cogn. Disord. 22, 108-120. doi: 10.1159/000093487

Jacobsen, J. H., Stelzer, J., Fritz, T. H., Chételat, G., La Joie, R., and Turner, R. (2015). Why musical memory can be preserved in advanced Alzheimer's disease. Brain 138, 2438-2450. doi: 10.1093/brain/awv135

Kellor, M., Frost, J., Silberberg, N., Iversen, I., and Cummings, R. (1971). Hand strength and dexterity. Am. J. Occup. Ther. 25, 77-83.

Kilgour, A. R., Jakobson, L. S., and Cuddy, L. L. (2000). Music training and rate of presentation as mediators of text and song recall. Mem. Cogn. 28, 700-710. doi: $10.3758 / \mathrm{bf} 03198404$

Koelsch, S. (2010). Towards a neural basis of music-evoked emotions. Trends Cogn. Sci. 14, 131-137. doi: 10.1016/j.tics.2010.01.002

Larson, E. B., Wang, L., Bowen, J. D., McCormick, W. C., Teri, L., Crane, P., et al. (2006). Exercise is associated with reduced risk for incident dementia among persons 65 years of age and older. Ann. Intern. Med. 144, 73-81.

Laurin, D., Verreault, R., Lindsay, J., MacPherson, K., and Rockwood, K. (2001). Physical activity and risk of cognitive impairment and dementia in elderly persons. Arch. Neurol. 58, 498-504.
Lin, Y., Chu, H., Yang, C. Y., Chen, C. H., Chen, S. G., Chang, H. J., et al. (2011). Effectiveness of group music intervention against agitated behavior in elderly persons with dementia. Int. J. Geriatr. Psychiatry 26, 670-678. doi: 10.1002/gps. 2580

Lyu, J., Zhang, J., Mu, H., Li, W., Champ, M., Xiong, Q., et al. (2018). The effects of music therapy on cognition. psychiatric symptoms, and activities of daily living in patients with Alzheimer's Disease. J. Alzheimers. Dis. 64, 1347-1358. doi: 10.3233/JAD- 180183

MacDonald, R. A. R., Kreutz, G., and Mitchell, L. A. (2012). "What is music health and wellbeing and why is it important," in Music, Health and Wellbeing MacDonald, eds R. A. R. Kreutz and G. Mitchell (Oxford: Oxford University Press).

Mahncke, H. W., Bronstone, A., and Merzenich, M. M. (2006). Brain plasticity and functional losses in the aged: scientific bases for a novel intervention. Prog. Brain Res. 157, 81-109. doi: 10.1016/s0079-6123(06)57006-2

Massaia, M., Reano, A., Luppi, C., Santagata, F., Marchetti, M., and Isaia, G. C. (2018). Receptive music interventions improve apathy and depression in elderly patients with dementia. Geriatr. Care 4, 8-15. doi: 10.4081/gc.2018.7248

Mathuranath, P. S., Nestor, P. J., Berrios, G. E., Rakowicz, W., and Hodges, J. R. (2000). A brief cognitive test battery to differentiate Alzheimer's disease and frontotemporal dementia. Neurology 55, 1613-1620. doi: 10.1212/wnl.55.11. 1613

McDermott, O., Orrell, M., and Ridder, H. M. (2014). The importance of music for people with dementia: the perspectives of people with dementia, family carers, staff and music therapists. Aging Mental Health 18, 706-716. doi: 10. 1080/13607863.2013.875124

Mioshi, E., Dawson, K., Mitchell, J., Arnold, R., and Hodges, J. R. (2006). The Addenbrooke's cognitive examination revised (ACE-R): a brief cognitive test battery for dementia screening. Int. J. Geriatr. Psychiatry 21, 1078-1085. doi: $10.1002 /$ gps. 1610

Moussard, A., Bigand, E., Belleville, S., and Peretz, I. (2014). Music as a mnemonic to learn gesture sequences in normal aging and alzheimer's disease. Front. Hum. Neurosci. 8:294. doi: 10.3389/fnhum.2014.00294

Nair, B. K., Heim, C., Krishnan, C., D’Este, C., Marley, J., and Attia, J. (2011). The effect of baroque music on behavioural disturbances in patients with dementia. Australas. J. Ageing 30, 11-15. doi: 10.1111/j.1741-6612.2010. 00439.x

Narme, P., Clément, S., Ehrlé, N., Schiaratura, L., Vachez, S., Courtaigne, B., et al. (2014). Efficacy of musical interventions in dementia: evidence from a randomized controlled trial. J. Alzheimers Dis. 38, 359-369. doi: 10.3233/JAD130893

Norberg, A., Melin, E., and Asplund, K. (2003). Reactions to music, touch and object presentation in the final stage of dementia: an exploratory study. Int. J. Nurs. Stud. 40, 473-479. doi: 10.1016/S0020-7489(03)00062-2

Osman, S. E., Tischler, V., and Schneider, J. (2016). 'Singing for the Brain': a qualitative study exploring the health and well-being benefits of singing for people with dementia and their carers. Dementia 15, 1326-1339. doi: 10.1177/ 1471301214556291

Park, D. C., and Reuter-Lorenz, P. (2009). The adaptive brain: aging and neurocognitive scaffolding. Annu. Rev. Psychol. 60, 173-196. doi: 10.1146/ annurev.psych.59.103006.093656

Peterson, D. A., and Thaut, M. H. (2007). Music increases frontal EEG coherence during verbal learning. Neurosci. Lett. 412, 217-221. doi: 10.1016/j.neulet.2006. 10.057

Prickett, C. A., and Moore, R. S. (1991). The use of music to aid memory of Alzheimer's patients. J. Music Ther. 28, 101-110. doi: 10.1093/jmt/28.2.101

Raglio, A., Bellelli, G., Traficante, D., Gianotti, M., Ubezio, M. C., Gentile, S., et al. (2010). Efficacy of music therapy treatment based on cycles of sessions: a randomised controlled trial. Aging Ment. Health 14, 900-904. doi: 10.1080/ 13607861003713158

Raglio, A., Bellelli, G., Traficante, D., Gianotti, M., Ubezio, M. C., Villani, D., et al. (2008). Efficacy of music therapy in the treatment of behavioral and psychiatric symptoms of dementia. Alzheimer Dis. Assoc. Disord. 22, 158-162. doi: 10.1097/WAD.0b013e3181630b6f

Rathbone, C. J., Moulin, C. J., and Conway, M. A. (2008). Self-centered memories: the reminiscence bump and the self. Mem. Cognit. 36, 1403-1414. doi: 10.3758/ MC.36.8.1403 
Ray, K. D., and Mittelman, M. S. (2017). Music therapy: a nonpharmacological approach to the care of agitation and depressive symptoms for nursing home residents with dementia. Dementia 16, 689-710. doi: 10.1177/ 1471301215613779

Reuter-Lorenz, P. A., and Park, D. C. (2014). How does it STAC up? Revisiting the scaffolding theory of aging and cognition. Neuropsychol. Rev. 24, 355-370. doi: 10.1007/s11065-014-9270-9

Rosenstock, I. M. (1974). Historical origins of the health belief model. Health Educ. Monogr. 2, 328-335. doi: 10.1177/109019817400200403

Sakamoto, M., Ando, H., and Tsutou, A. (2013). Comparing the effects of different individualized music interventions for elderly individuals with severe dementia. Int. Psychogeriatr. 25, 775-784. doi: 10.1017/S1041610212002256

Samson, S., Dellacherie, D., and Platel, H. (2009). Emotional power of music in patients with memory disorders. Ann. N. Y. Acad. Sci. 1169, 245-255. doi: 10.1111/j.1749-6632.2009.04555.x

Särkämö, T., Tervaniemi, M., Laitinen, S., Numminen, A., Kurki, M., Johnson, J. K., et al. (2014). Cognitive, emotional, and social benefits of regular musical activities in early dementia: randomized controlled study. Gerontologist 54, 634-650. doi: 10.1093/geront/gnt100

Satoh, M., Ogawa, J., Tokita, T., Nakaguchi, N., Nakao, K., Kida, H., et al. (2014). The effects of physical exercise with music on cognitive function of elderly people: mihama-kiho project. PLoS One 9:e95230. doi: 10.1371/journal.pone. 0095230

Sheikh, J. I., and Yesavage, J. A. (1986). Geriatric depression scale (GDS): recent evidence and development of a shorter version. Clin. Gerontol. 5, 165-173. doi: 10.1300/J018v05n01_09

Shimizu, N., Umemura, T., Matsunaga, M., and Hirai, T. (2017). Effects of movement music therapy with a percussion instrument on physical and frontal lobe function in older adults with mild cognitive impairment: a randomized controlled trial. Aging Ment. Health 22, 1-13. doi: 10.1080/13607863.2017. 1379048

Son, G. R., Therrien, B., and Whall, A. (2002). Implicit memory and familiarity among elders with dementia. J. Nurs. Scholarsh. 34, 263-267. doi: 10.1111/j. 1547-5069.2002.00263.x

Sung, H. C., and Chang, A. M. (2005). Use of preferred music to decrease agitated behaviours in older people with dementia: a review of the literature. J. Clin. Nurs. 14, 1133-1140. doi: 10.1111/j.1365-2702.2005.01218.x

Sung, H. C., Chang, A. M., and Lee, W. L. (2010). A preferred music listening intervention to reduce anxiety in older adults with dementia in nursing homes. J. Clin. Nurs. 19, 1056-1064. doi: 10.1111/j.1365-2702.2009.03016.x

Sung, H. C., Lee, W. L., Li, T. L., and Watson, R. (2012). A group music intervention using percussion instruments with familiar music to reduce anxiety and agitation of institutionalized older adults with dementia. Int. J. Geriatr. Psychiatry 27, 621-627. doi: 10.1002/gps.2761

Suzuki, M., Kanamori, M., Watanabe, M., Nagasawa, S., Kojima, E., Ooshiro, H., et al. (2004). Behavioral and endocrinological evaluation of music therapy for elderly patients with dementia. Nurs. Health Sci. 6, 11-18. doi: 10.1111/j.14422018.2003.00168.x
Tang, Q., Zhou, Y., Yang, S., Thomas, W. K. S., Smith, G. D., Yang, Z., et al. (2018). Effect of music intervention on apathy in nursing home residents with dementia. Geriatr. Nursing 39, 471-476. doi: 10.1016/j.gerinurse.2018.02.003

Tarr, B., Launay, J., and Dunbar, R. I. M. (2014). Music and social bonding: "selfother” merging and neurohormonal mechanisms. Front. Psychol. 5:1096. doi: 10.3389/fpsyg.2014.01096

Thomas, K. S., Baier, R., Kosar, C., Ogarek, J., Trepman, A., and Mor, V. (2017). Individualized music program is associated with improved outcomes for U.S. nursing home residents with dementia. Am. J. Geriatr. Psychiatry 25, 931-938. doi: 10.1016/j.jagp.2017.04.008

Thompson, W. F., and Schlaug, G. (2015). The healing power of music. Sci. Am. Mind 26, 32-41. doi: 10.1038/scientificamericanmind0315-32

Tsoi, K. K. F., Chan, J. Y. C., Ng, Y. M., Lee, M. M. Y., Kwok, T. C. Y., and Wong, S. Y. S. (2018). Receptive music therapy is more effective than interactive music therapy to relieve behavioral and psychological symptoms of dementia: a systematic review and meta-analysis. J. Am. Med. Dir. Assoc. 19, 568.e-576.e. doi: 10.1016/j.jamda.2017.12.009

Van de Winckel, A., Feys, H., De Weerdt, W., and Dom, R. (2004). Cognitive and behavioural effects of music-based exercises in patients with dementia. Clin. Rehabil. 18, 253-260. doi: 10.1191/0269215504cr750oa

Van der Steen, J. T., Van Soest-Poortvliet, M. C., Van der Wouden, J. C., Bruinsma, M. S., Scholten, R. J., and Vink, A. C. (2017). Music-based therapeutic interventions for people with dementia. Cochr. Database Syst. Rev. 5, CD003477. doi: 10.1002/14651858.CD003477.pub3

Verghese, J., Lipton, R. B., Katz, M. J., Hall, C. B., Derby, C. A., Kuslansky, G., et al. (2003). Leisure activities and the risk of dementia in the elderly. N. Engl. J. Med. 348, 2508-2516. doi: 10.1056/NEJMoa022252

Vink, A. C., Bruinsma, M. S., and Scholten, R. J. P. M. (2003). Music therapy for people with dementia. Cochr. Database. Syst. Rev. 4, CD003477. doi: 10.1002/ 14651858.CD003477.pub2

Vink, A. C., Zuidersma, M., Boersma, F., De Jonge, P., Zuidema, S. U., and Slaets, J. P. (2013). The effect of music therapy compared with general recreational activities in reducing agitation in people with dementia: a randomised controlled trial. Int. J. Geriatr. Psychiatry 28, 1031-1038. doi: 10.1002/gps. 3924

Zatorre, R. J., and Salimpoor, V. N. (2013). From perception to pleasure: Music and its neural substrates. PNAS 110, 10430-10437. doi: 10.1073/pnas.1301228110

Conflict of Interest Statement: The authors declare that the research was conducted in the absence of any commercial or financial relationships that could be construed as a potential conflict of interest.

Copyright (c) 2019 Brancatisano, Baird and Thompson. This is an open-access article distributed under the terms of the Creative Commons Attribution License (CC BY). The use, distribution or reproduction in other forums is permitted, provided the original author(s) and the copyright owner(s) are credited and that the original publication in this journal is cited, in accordance with accepted academic practice. No use, distribution or reproduction is permitted which does not comply with these terms. 


\section{APPENDIX A:}

\section{List of Songs}

1. Glen Miller - In the Mood

2. Glen Miller - Chattanooga Choo Choo

3. Judy Garland - Somewhere Over the Rainbow

4. Gene Kelly - Singing in the Rain

5. Nat King Cole - Too Young

6. Doris Day - Que Sera Sera

7. Doris Day - Black Hills

8. Dinah Shore - Buttons and Bows

9. Bing Crosby and The Andrews Sisters - Don't Fence Me In

10. Bing Crosby - Swing on a star

11. Bing Crosby - White Christmas

12. Perry Como - Catch a falling star

13. Frank Sinatra - Come fly with me

14. Little Eva - The Locomotion

15. Buddy Holly - Peggie Sue

16. Sam Cooke - Cupid

17. Ella Fitzgerald - A-Tisket, A-Tasket

18. Bill Haley and his Comets - Joey's song

19. Elvis - It's now or never

20. The Beatles - Hey Jude

21. Some Enchanted Evening

22. My Bonnie lies over the ocean

23. Oklahoma 'Oh what a beautiful morning' 


\title{
Dancing to Metallica and Dora: Case Study of a 19-Month-Old
}

\author{
Laura K. Cirelli ${ }^{1,2 \star}$ and Sandra E. Trehub ${ }^{2}$ \\ ${ }^{1}$ Department of Psychology, University of Toronto Scarborough, Toronto, ON, Canada, ${ }^{2}$ Department of Psychology, \\ University of Toronto Mississauga, Mississauga, ON, Canada
}

Rhythmic movement to music, whether deliberate (e.g., dancing) or inadvertent (e.g., foot-tapping), is ubiquitous. Although parents commonly report that infants move rhythmically to music, especially to familiar music in familiar environments, there has been little systematic study of this behavior. As a preliminary exploration of infants' movement to music in their home environment, we studied $\mathrm{V}$, an infant who began moving rhythmically to music at 6 months of age. Our primary goal was to generate testable hypotheses about movement to music in infancy. Across nine sessions, beginning when $\checkmark$ was almost 19 months of age and ending 8 weeks later, she was video-recorded by her mother during the presentation of 60-s excerpts from two familiar and two unfamiliar songs presented at three tempos - the original song tempo as well as faster and slower versions. $V$ exhibited a number of repeated dance movements such as head-bobbing, arm-pumping, torso twists, and bouncing. She danced most to Metallica's Now that

OPEN ACCESS

Edited by:

Jennifer MacRitchie,

Western Sydney University, Australia

Reviewed by:

Clemens Wöllner,

Universität Hamburg, Germany

Bettina E. Bläsing,

Technical University

Dortmund, Germany

*Correspondence:

Laura K. Cirelli

laura.cirelli@utoronto.ca

Specialty section:

This article was submitted to

Performance Science,

a section of the journal

Frontiers in Psychology

Received: 30 January 2019 Accepted: 24 April 2019

Published: 15 May 2019

Citation:

Cirelli LK and Trehub SE (2019) Dancing to Metallica and Dora: Case

Study of a 19-Month-Old.

Front. Psychol. 10:1073.

doi: 10.3389/fpsyg.2019.01073
We're Dead, a recording that her father played daily in V's presence, often dancing with her while it played. Its high pulse clarity, in conjunction with familiarity, may have increased V's propensity to dance, as reflected in lesser dancing to familiar music with low pulse clarity and to unfamiliar music with high pulse clarity. $\vee$ moved faster to faster music but only for unfamiliar music, perhaps because arousal drove her movement to familiar music. Her movement to music was positively correlated with smiling, highlighting the pleasurable nature of the experience. Rhythmic movement to music may have enhanced her pleasure, and the joy of listening may have promoted her movement. On the basis of behavior observed in this case study, we propose a scaled-up study to obtain definitive evidence about the effects of song familiarity and specific musical features on infant rhythmic movement, the developmental trajectory of dance skills, and the typical range of variation in such skills.

Keywords: music, infancy, rhythm, movement, dance, development

\section{INTRODUCTION}

Active musical engagement, whether by attentive listening, singing, or rhythmic movement, is pervasive across the lifespan and across cultures (Savage et al., 2015; Trehub et al., 2019). These activities play a critical role in mood regulation (Laukka, 2007), social bonding (Cirelli et al., 2014; Tarr et al., 2014), personal identity (North and Hargreaves, 1999), and other aspects of well-being (Groarke and Hogan, 2016). The seeds of such musical engagement are planted in infancy when the primary caregiver serves as musical partner and mentor (Cirelli and Trehub, 2018; Trehub and Gudmundsdottir, 2019). Caregivers around the world sing to infants in a warm and distinctive manner 
(Trehub and Trainor, 1998; Ilari, 2005) that is often accompanied by rhythmic movement (Longhi, 2009). Infants are highly engaged by the infant-directed singing style (Trainor, 1996; Costa-Giomi, 2014) and by the songs themselves. Unfamiliar individuals who sing one of the caregiver's songs elicit more social interest (Mehr et al., 2016) and assistance (Cirelli and Trehub, 2018) from infants than those who sing an unfamiliar song. Although infants exhibit more rhythmic movement to music than to speech (Zentner and Eerola, 2010), the factors that underlie early movement to music remain poorly understood. The present case study was motivated by an interest in generating testable hypotheses about the development of movement to music in infancy.

Moving to the beat of music requires precise temporal processing and auditory-motor integration, including predictive timing and planning at various levels of the motor system (Patel and Iversen, 2014). Music listening, even without movement, activates motor areas in the adult brain (Grahn and Brett, 2007; Fujioka et al., 2009). The newborn's brain tracks sound onsets, offsets, and musical tempo (Winkler et al., 2009; Háden et al., 2015). By 2 months, infants differentiate rhythmic patterns (Demany et al., 1977) and tempo differences (Baruch and Drake, 1997) and, by 12 months, culture-specific exposure influences rhythm perception (Hannon and Trehub, 2005).

Infants exhibit spontaneous movement to metrically regular sounds by 5 months of age, but their movements are not aligned with the beat and change little between 5 and 24 months of age (Zentner and Eerola, 2010). Although infants' pace of movement is reportedly faster for faster music than for slower music (Zentner and Eerola, 2010), that does not seem to be the case for preschoolers (Eerola et al., 2006). In general, mature synchronization to music is typically achieved by 10 years of age (Drake et al., 2000; McAuley et al., 2006). Children's preferred tempo is faster at younger than at older ages, and the tempo range over which they can coordinate their attention and movement increases progressively with age (Drake et al., 2000; McAuley et al., 2006). Little is known, however, about infants' preferred tempo.

Groove is often used to describe musical patterns that evoke a desire to move (Madison, 2006). Music rated high in groove activates motor areas in the brain (Stupacher et al., 2013), prompting spontaneous bursts of coordinated movement (Janata et al., 2012). High-groove music tends to have moderate rhythmic complexity (Witek et al., 2014), tempo corresponding to spontaneous tapping rates (Madison et al., 2011; Etani et al., 2018), high pulse clarity, and predominant energy in lowfrequency bands (Burger et al., 2013; Stupacher et al., 2016). It is unclear, however, whether those musical features promote rhythmic movement in infants and young children.

If musical tempo influences infants' movement tempo (Zentner and Eerola, 2010), those links may be mediated by arousal (i.e., excitement) and pleasure. Music listening activates adult brain networks linked to pleasure and reward (Blood and Zatorre, 2001). Moreover, dancing to music increases pleasure relative to music listening alone (Dunbar et al., 2012; Bernardi et al., 2017). Musical engagement clearly evokes pleasure in infants. By 5 months, infants smile considerably more to interactive singing than to speech (Mehr et al., 2016). Infants also smile more when moving to music, especially during periods of greater coordination to the music (Zentner and Eerola, 2010).

Gaps in knowledge about infants' movement responses to music, such as the role of music familiarity, testing context, arousal, and pleasure should be addressed systematically. For the moment, however, there is a disconnect between the available laboratory research, which reveals relatively limited movement to music and no apparent change between 5 and 24 months (Zentner and Eerola, 2010), and parent reports of frequent infant "dancing" to music along with dramatic change over the first 2 years. A similar disconnect was evident between young children's reported inability to match the pitch contours and pitch range of songs (e.g., Rutkowski and Miller, 2002) and toddlers' effective matching of those features when singing their favorite songs at home (Gudmundsdottir and Trehub, 2018). As a preliminary exploration of infants' movement to music in their home environment, we undertook a case study of $\mathrm{V}$, a Canadian girl who reportedly began moving rhythmically to music at 6 months of age and joined our study when she was almost 19 months of age. Across nine sessions, V's mother video-recorded her during the presentation of musical excerpts of familiar and unfamiliar songs at three tempos-the original song tempo as well as a faster and slower tempo. Our immediate goal focused on the influence of song familiarity, song type, and tempo on V's duration and tempo of movement as well as possible links between movement to music and visible pleasure. Our broader goal was to generate hypotheses for further research in home or laboratory environments.

\section{METHODS}

\section{Participant}

$\mathrm{V}$ is part of a bilingual (French/English) household in a small city in Northern Ontario, where she lives with her mother, father, and 7 -year-old brother. She was 18 months, 27 days of age on the first recording session and 20 months, 18 days on the final session. According to maternal report, she began moving regularly to music at 6 months of age. $\mathrm{V}$ had not participated in music programs in the community, but her parents and brother exposed her to music by singing and dancing with her and playing musical recordings. No member of her family had formal music training, but her father is a self-taught amateur guitarist. Written informed consent was obtained from the mother for the publication of this case report and the inclusion of identifiable information including images, age, and gender.

\section{Stimuli}

The stimuli featured four songs, two familiar and two unfamiliar. When asked to report V's "favorite songs", her parents identified Dora the Explorer Theme Song (119 beats per minute [bpm]) (Sitron and Straus, 2010), heard regularly from 6 months (once weekly at study onset, more frequently in earlier months), and Metallica's Now that We're Dead (128 bpm) (Hetfield and Ulrich, 2017), heard regularly from 12 months (once daily at study onset), resulting in greater cumulative exposure to Dora but more consistent recent exposure to Metallica. The 
Dora theme was typically played from YouTube, but V was visibly excited by the music and relatively disinterested in the visuals. Exposure to Now that We're Dead (Metallica), her father's favorite song, was from an audio-recording, but father and daughter often danced together to the song, with father bobbing his head to the music. An unfamiliar song chosen to match Dora was Hey It's Franklin, the theme song from Franklin the Turtle, another animated children's TV show (Cockburn, 2000). An unfamiliar song chosen to match Metallica was the Backstreet Boys' Everybody (Pop and Martin, 1997), a song for adults that has been used previously to investigate cross-species dancing (Patel et al., 2009). We used Audacity 2.1.0, to create versions of each song at $100 \mathrm{bpm}$ (designated slow) and $140 \mathrm{bpm}$ (designated fast), maintaining original pitch levels. We equated maximum amplitude across clips, which were comparable in subjective loudness. For intermediate tempo, familiar songs were maintained at original bpm, and unfamiliar songs were set to $120 \mathrm{bpm}$. The usual tempo range for dance music is $94-176 \mathrm{bpm}$, with $120-150$ being most common (van Noorden and Moelants, 1999). Pulse clarity (i.e., strength of rhythmic periodicities) and mean fundamental frequency (F0) were calculated at the intermediate tempo using MIRtoolbox 1.7 running on Matlab 2016a. Metallica and Backstreet had high pulse clarity $(0.79 ; 0.67)$ and low mean F0 $(120 \mathrm{~Hz} ; 203 \mathrm{~Hz})$, respectively. Dora and Franklin had low pulse clarity (0.49; $0.31)$ and high or low F0 $(304 \mathrm{~Hz} ; 225 \mathrm{~Hz})$, respectively. The 12 recordings (4 songs $\mathrm{X} 3$ tempos) were trimmed to $60 \mathrm{~s}$, with 1-s fade-out. The first minute of each recording was always used to optimize familiarity for Metallica and Dora. Except for Metallica, which was instrumental ${ }^{1}$, the other songs contained vocals and instrumentals. Excerpts of these clips at each tempo can be accessed in the supplementary section (see Supplementary Audios 1-4). Individual audio files for each test session had six song clips (trials) separated by 10 -s silence. The song clips were ordered across sessions so that each session included two clips at each of the three tempos, and each of the 4 songs as heard at least once but not more than twice. Across all 8 sessions, the 12 clips ( 4 songs at 3 tempos) were presented 4 times each ${ }^{2}$. A ninth session replaced trials lost because of V's fussiness (four trials) or camera malfunction (one trial).

\section{Procedure}

Audio files for each session were provided via Google Drive. The mother was instructed to play the audio files for $\mathrm{V}$ at her convenience and to capture V's responses with video-recordings. She was asked to refrain from moving to the music or singing during the recording sessions and to ensure that no other family members (brother or father, if present) did so. However, she was free to offer verbal encouragement (e.g., asking $\mathrm{V}$ to dance). In fact, she provided such encouragement only when $\mathrm{V}$ had not initiated dancing on a trial, doing so comparably on familiar (65\%) and unfamiliar (65\%) song trials. The music was presented (at $\sim 60 \mathrm{~dB}$ ) by means of a personal tablet, and sessions were recorded by cell phone. After each session, the mother reported

\footnotetext{
${ }^{1}$ The first minute of the original recording is instrumental.

${ }^{2}$ An error resulted in having 3 Dora Fast trials and 5 Franklin Fast trials.
}

time of day, those present, and any notable circumstances, transmitting all recordings and information via Google Drive.

\section{Data Analysis}

An assistant coded the videos with ELAN software (https://tla. mpi.nl/tools/tla-tools/elan/, Lausberg and Sloetjes, 2009). She was trained extensively by the lead author, and had years of experience working with young children. The assistant used audio only to locate the start of each trial, subsequently coding the target behaviors with the audio turned off. For each trial, she
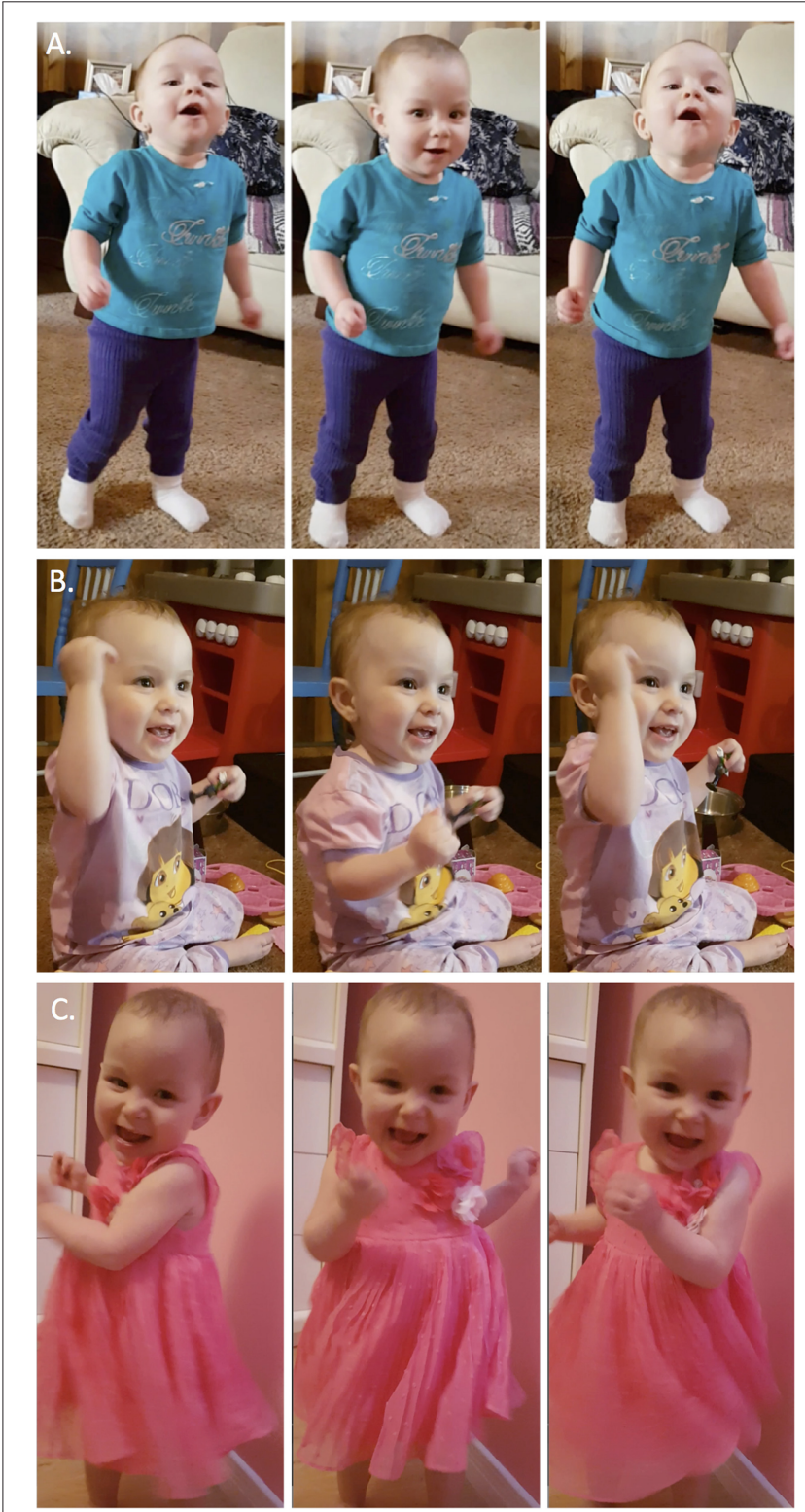

FIGURE 1 | Examples of V's common dance moves. (A) Head bobs, (B) Arm pumps, and (C) Torso twists. Video examples can be found in the Supplementary Materials. Written informed consent obtained from the parents of the child for the publication of these images. 
identified number of dance bursts, burst duration, and percentage of time smiling when V's face was visible. Dance bursts were defined as time windows with two or more rhythmic movements within 2,000 ms. Independent coding of $27 \%$ of the trials yielded high interrater reliability for the three measures $(r=0.97, r$ $=0.98, r=0.82$, for number of dance bursts, burst duration, and smiling, respectively). $\mathrm{V}$ exhibited various dance patterns including head bobbing, arm pumps, swaying, torso twists, and bouncing (examples in Figure 1). For each dance burst, the coder indicated the time of peak movement position (lowest head position for head-bobbing, fully extended arm for arm pump). V's requests for more music ("d'autre?") were coded during the silent interval between songs.

\section{RESULTS}

Dance bursts occurred in all recording sessions and in 32 of 48 trials. For trials with dance bursts, bursts per trial ranged from 1 to $5(M=2.22, S D=1.21)$. Although dance bursts occurred in $67 \%$ of the trials, overall dancing per trial was limited, from
$1.3 \mathrm{~s}$ to $22.1 \mathrm{~s}(M=8.1 \mathrm{~s}, S D=5.1 \mathrm{~s}$ for trials with at least 1 dance burst). Mean dancing per trial also varied across song selections. As can be seen in Figure 2A, V danced more to Metallica's Now That We're Dead, a familiar pop song (heavy metal genre) that had considerably greater pulse clarity than the other familiar song and lower mean F0 than the other songs.

Dance time as a function of tempo is shown in Figure 2B. V's propensity to dance most to Metallica was driven by the tempomodified (fast and slow) conditions. Otherwise, tempo exerted no clear effects on dance time.

Table 1 shows the incidence of each recurring dance pattern (see Figure 1 and Supplementary Videos 1-3 for examples) for each song. Arm pumps were common during three of the four songs, and distinctive head-bobbing was especially notable during Metallica trials.

A Pearson correlation between dance time and percentage of time smiling per trial, $r=0.60, p<0.001$ (Figure 3) highlighted the link between musical movement and positive affect.

For each dance burst, we calculated interonset intervals (ms) between successive movement peaks. For each trial, mean interonset interval was converted to movement rate
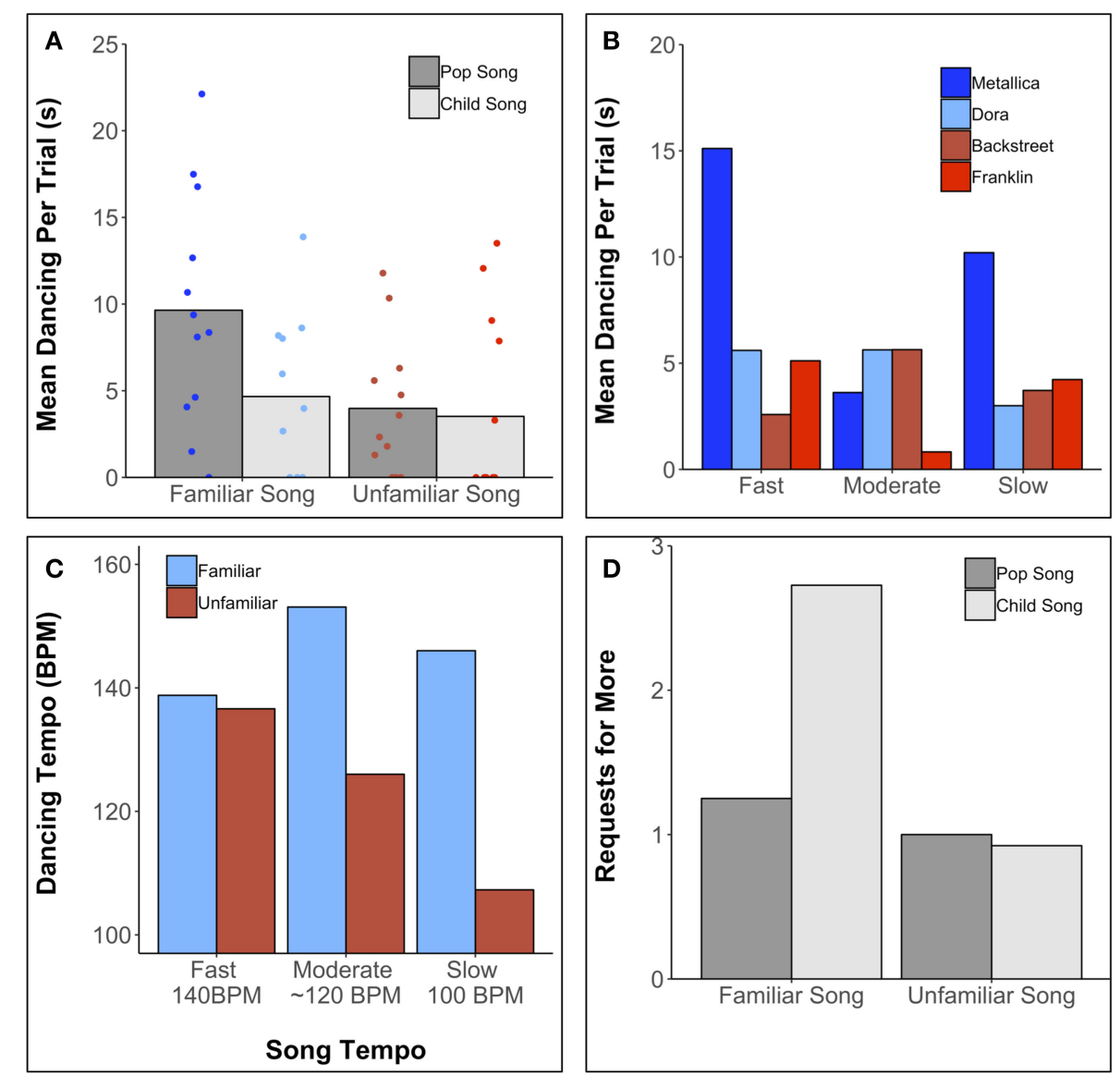

FIGURE 2 | (A) Mean time dancing during the 60-s trials for familiar (Metallica, Dora) and unfamiliar (Backstreet Boys, Franklin) songs. Individual trial data are displayed as jittered scatter points. (B) Mean time dancing per trial as a function of tempo and song. (C) Mean dancing tempo across Song Tempo and Song Familiarity conditions. (D) Mean number of requests for more (“d'autre?") after song trials featuring Metallica (familiar pop song), Dora (familiar children's song), Backstreet Boys (unfamiliar pop song), and Franklin (unfamiliar children's song). 
TABLE 1 | Number of trials in which V performed specific dance patterns.

\begin{tabular}{lccccc}
\hline Song & Head bobbing & Arm pumps & Swaying & Torso twists & Bouncing \\
\hline Dora & 2 & 5 & 0 & 2 & 1 \\
Metallica & 11 & 4 & 0 & 1 & 3 \\
Backstreet & 3 & 5 & 0 & 3 & 3 \\
Franklin & 1 & 1 & 2 & 2 & 0
\end{tabular}

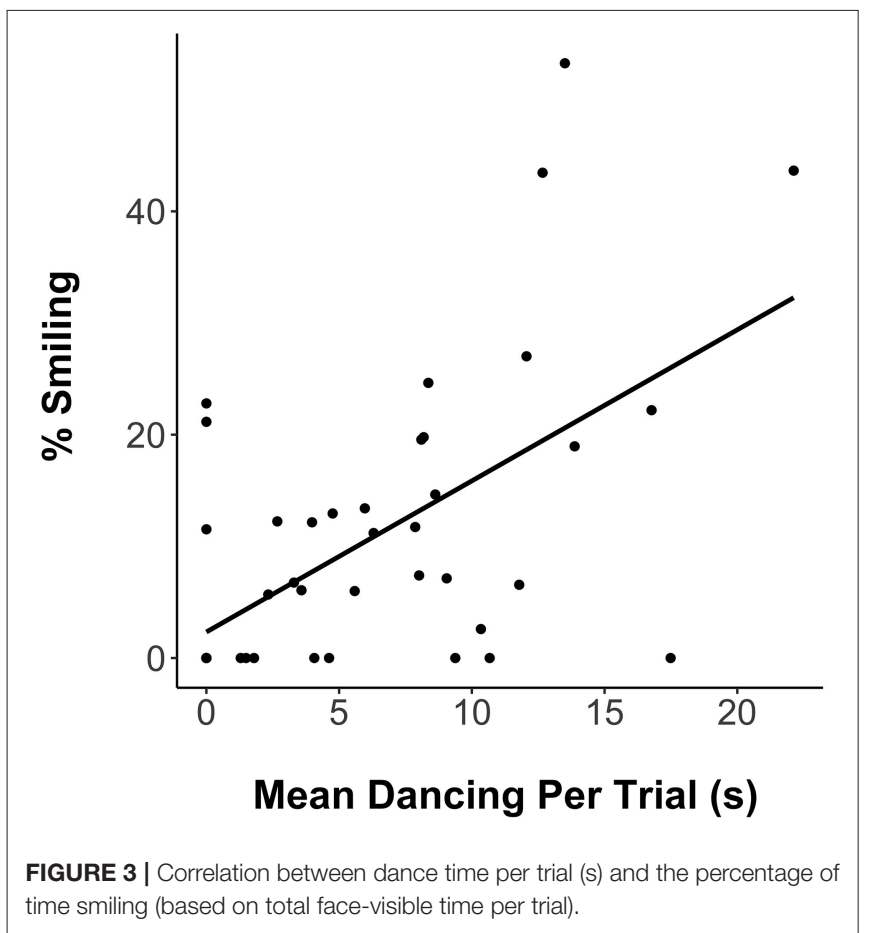

(bpm). Movement rate across tempo (Fast, Moderate, Slow) and song familiarity is displayed in Figure 2C. For familiar songs, movement tempo was rapid for all musical tempos. For unfamiliar songs, there is suggestive evidence of slower movement tempo for slower songs. Moreover, there is a suggestion of tempo convergence (coordination of movement with musical tempo) at the fastest song tempo regardless of familiarity.

During the 10-s silent intervals between songs, $\mathrm{V}$ asked for “more" (“d'autre?") after 27 of 48 trials (56\%), doing so more frequently (sometimes repeatedly) after hearing familiar songs (70\%) than unfamiliar songs (44\%) (see Figure 2D) and most frequently after hearing the Dora song.

\section{DISCUSSION}

The present case study explored the feasibility of studying infant dance or rhythmic movement to music in the home environment. We focused specifically on the influence of song familiarity and tempo on the incidence and tempo of movement as well as links between movement and pleasure. $\mathrm{V}$ danced in every recording session, supporting the home environment as a promising venue for eliciting movement to music. Overall, she danced $9 \%$ of the time, which is modest but somewhat higher than the $6 \%$ of rhythmic movement for 5- to 24-month-olds in a laboratory context (Zentner and Eerola, 2010). Note that the laboratory study excluded data from a third of infants who fussed or attended excessively to the caregiver. Moreover, infants sat on their parents' lap, reducing the likelihood of observing typical patterns of movement to music. As for 2- to 4-year-olds, $40 \%$ were unwilling to dance in the laboratory (Eerola et al., 2006).

V's head-bobbing, arm pumping, and body twists were more dance-specific and motorically complex than the rhythmic limb movements observed in previous studies with infants (Zentner and Eerola, 2010; Fujii et al., 2014) but less complex than the rhythmic body movements observed with 2- to 4-year-olds (Eerola et al., 2006). Age-related changes in the complexity of V's "dancing" can be seen in her limb movements at 8 months (Supplementary Video 4) before data collection began, body twists at 19 months (Supplementary Video 5), and hopping at 29 months (Supplementary Video 6) after data collection ended. V's most common dance move was head-bobbing, especially for Metallica. Such motion has powerful effects on the vestibular system and on rhythm perception (Phillips-Silver and Trainor, 2008). Adult head-bobbing is often evoked by music with a strong bass line (Burger et al., 2013), as in the Metallica song. The contribution of bass sounds to the nature and frequency of young children's movements can be explored in future investigations with a wider range of stimuli and a large sample of children across a broad age range. V's head-bobbing to Metallica probably stemmed from dancing with her father, who modeled such head movements to that song.

V's dance duration was greater for familiar music $(12 \%$ of the time) than for unfamiliar music (6\%), which was driven by her response to Metallica (16\%). For example, V danced no more to the familiar and well-liked Dora theme than to the unfamiliar excerpts. Familiarity may influence rhythmic movement in infants but only in conjunction with other factors such as pulse clarity or beat salience, which is correlated with groove (Madison et al., 2011; Witek et al., 2014; Stupacher et al., 2016). Now that We're Dead has high pulse clarity, in contrast to the low pulse clarity of Dora. On its own, high pulse clarity may be insufficient to enhance movement in infants, as evident in V's modest dance duration to Everybody (Backstreet Boys), which was high in pulse clarity but unfamiliar. V's experience with Now that We're Dead as a "dance song" may be relevant. The relative contributions of familiarity and pitch clarity to movement could be explored in future research that involves independent manipulation of these factors.

Unexpectedly, $\mathrm{V}$ danced most to Metallica on trials with altered tempo (21\%)-15.1 s with increased tempo, $10.2 \mathrm{~s}$ with decreased tempo, and $3.6 \mathrm{~s}$ with the original tempo. Presumably she had long-term memory for the original tempo, perhaps finding the altered versions amusing or exciting, as reflected in increased smiling during the altered versions. Just as younger infants perceive absurd versions of familiar events as humorous (Mireault et al., 2018), older infants may react with heightened excitement when their expectations of highly familiar music are violated. Musical tempo may be most memorable for music with 
a highly salient bass line or pulse and least memorable for music with a less salient bass line and more salient melody line.

With respect to tempo flexibility, V's tempo of movement was faster for the familiar songs than for the unfamiliar songs (see Figure 2C), perhaps because of heightened arousal. For the familiar songs, moreover, $\mathrm{V}$ danced fastest for the original tempo and slowest for the fastest tempo, precluding orderly relations between dance tempo and musical tempo. For the unfamiliar music, there was tentative evidence of tempo flexibility in the sense of faster dancing to faster music. Infants may accord greater attention to timing details in the context of unfamiliar songs that elicit moderate arousal levels. Although 5- to 24-month-olds move modestly faster to faster music than to slower music (Zentner and Eerola, 2010), preschool children show little evidence of doing so (Eerola et al., 2006). Further study of the effects of age, song familiarity, and social context (dancing alone or with parent) on tempo flexibility is warranted.

Developmental changes in coordinated movement to music have been linked to changes in spontaneous tempo (characteristic rate of rhythmic movement without sound) and preferred tempo (optimal tempo for perception and coordinated movement) (McAuley et al., 2006). V's movement timing was closest to the target tempo for the fastest songs $(140 \mathrm{bpm})$, both familiar and unfamiliar (Figure 2C). Spontaneous motor tempo changes from $\sim 200 \mathrm{bpm}$ at 4 years of age to $90 \mathrm{bpm}$ in elderly adults, and synchronization is most accurate when the target tempo matches the preferred tempo (McAuley et al., 2006). The developmental timetable may be influenced by age-related changes in internal timing mechanisms (Vanneste et al., 2001; McAuley et al., 2006) and biomechanical factors involving limb length and weight (Goodman et al., 2000). Future research with infants should encompass a wider range of tempos, including the preferred tempo of 4-yearolds (200 bpm), and spontaneous motor tempo should be documented.

V's dance duration per trial was positively related to smiling (Figure 3), highlighting the links between dance and pleasure. In previous research, smiling was related to ratings of movement coordination to music in 5- to 24-month-olds (Zentner and Eerola, 2010). Does movement to music generate pleasure, or do pleasurable responses to music motivate movement? Both may well be the case. Adults' motivation to move to music has been linked to pleasure and to perceived musical groove (Janata et al., 2012; Witek et al., 2014). Musical features such as syncopation have independent effects on pleasure and desire to move (Witek et al., 2014). Moreover, musical movement heightens pleasure through increased arousal (Tarr et al., 2015).

Although Metallica prompted V to dance more and to dance distinctively, Dora prompted the most requests for more music. Aside from its familiarity, the high pitch and prominent vocals may have contributed to its appeal. Infants' preference for high pitch in speech (Fernald, 1992) and song (Trainor and Zacharias, 1998) is well-documented.

Obviously, a case study of a normally developing child warrants cautious interpretation and cannot speak to issues of individual differences or to development in general. Nevertheless, V's responses reveal dance behavior that is possible but not necessarily typical for 19- or 20-month-olds. A study of supine 3 - and 4-month-olds revealed less movement in the context of music than in silence, but 2 of the 30 infants moved more to the music and also exhibited some coordination to the musical beat (Fujii et al., 2014). That study underlines the importance of considering individual behavior patterns even in group studies. With respect to the present study, a scaled-up version with large sample size could provide definitive evidence about the effects of song familiarity and various musical features on infant movement. When familiarity and pulse clarity are manipulated independently, we would expect familiar music to prompt more dancing than unfamiliar music and music with high pulse clarity to prompt more dancing than music with low pulse clarity. In light of the current findings, we would also expect young children to demonstrate greater tempo flexibility when moving to unfamiliar compared to familiar music. An expanded age range (e.g., 1-4 years of age) could provide information about the developmental trajectory of dance skills (e.g., characteristic movements, tempo flexibility, synchrony), the typical range of variation, and the effects of dance experience and musical exposure. Systematic testing in the home environment with specified, parent-administered protocols would provide multiple benefits such as a comfortable and familiar context for participating children, convenience for parents, access to non-local as well as local participants, and a wealth of information about the early development of dancing.

\section{ETHICS STATEMENT}

This study was carried out in accordance with the recommendations of the University of Toronto Research Ethics Board with written informed consent from the participant's legal guardian.

\section{AUTHOR CONTRIBUTIONS}

Both LC and ST contributed to study design, data analysis, and manuscript preparation.

\section{FUNDING}

This research was funded by a grant from the Natural Sciences and Engineering Research Council of Canada to ST and by a postdoctoral fellowship from the Social Sciences and Humanities Research Council of Canada to LC.

\section{ACKNOWLEDGMENTS}

We would like to acknowledge $\mathrm{V}$ and her family for their participation in this study. We would also like to thank Samantha Cottrell, Chella Velkannan, and Jovana Miladinovic for assistance with video coding. 


\section{SUPPLEMENTARY MATERIAL}

The Supplementary Material for this article can be found online at: https://www.frontiersin.org/articles/10.3389/fpsyg. 2019.01073/full\#supplementary-material

Supplementary Video 1 | The arm pump.

Supplementary Video 2 | The head bob.

Supplementary Video 3 | The torso twist.

Supplementary Video 4 | V at 8 months responding to Dora.

\section{REFERENCES}

Baruch, C., and Drake, C. (1997). Tempo discrimination in infants. Infant Behav. Dev. 20, 573-577. doi: 10.1016/S0163-6383(97)90049-7

Bernardi, N. F., Bellemare-Pepin, A., and Peretz, I. (2017). Enhancement of pleasure during spontaneous dance. Front. Human Neurosci. 11:572. doi: $10.3389 /$ fnhum.2017.00572

Blood, A. J., and Zatorre, R. J. (2001). Intensely pleasurable responses to music correlate with activity in brain regions implicated in reward and emotion. Proc. Natl. Acad. Sci. U.S.A. 98, 11818-11823. doi: 10.1073/pnas.191355898

Burger, B., Thompson, M. R., Luck, G., Saarikallio, S., and Toiviainen, P. (2013). Influences of rhythm- and timbre-related musical features on characteristics of music-induced movement. Front. Psychol. 4:183. doi: 10.3389/fpsyg.2013.00183

Cirelli, L. K., Einarson, K. M., and Trainor, L. J. (2014). Interpersonal synchrony increases prosocial behavior in infants. Dev. Sci. 17, 1003-1011. doi: $10.1111 /$ desc. 12193

Cirelli, L. K., and Trehub, S. E. (2018). Infants help singers of familiar songs. Music Sci. 1:205920431876162. doi: 10.1177/2059204318761622

Cockburn, B. (2000). Hey, it's Franklin. On Hey, It's Franklin [MP3 file]. Los Angeles, CA: Kid Rhino

Costa-Giomi, E. (2014). Mode of presentation affects infants preferential attention to singing and speech. Music Percep. 32, 160-169. doi: 10.1525/MP.2014.32.2.160

Demany, L., McKenzie, B., and Vurpillot, E. (1977). Rhythm perception in early infancy. Nature 266, 718-719. doi: 10.1038/266718a0

Drake, C., Jones, M. R., and Baruch, C. (2000). The development of rhythmic attending in auditory sequences: attunement, referent period, focal attending. Cognition 77, 251-288. doi: 10.1016/S0010-0277(00)00106-2

Dunbar, R. I. M., Kaskatis, K., MacDonald, I., and Barra, V. (2012). Performance of music elevates pain threshold and positive affect: implications for the evolutionary function of music. Evol. Psychol. 10, 688-702. doi: $10.1177 / 147470491201000403$

Eerola, T., Luck, G., and Toiviainen, P. (2006). “An investigation of preschoolers' corporeal synchronization with music," in Proceedings of the 9th International Conference on Music Perception and Cognition (Bologna), 472-476.

Etani, T., Marui, A., Kawase, S., and Keller, P. E. (2018). Optimal tempo for groove: its relation to directions of body movement and Japanese nori. Front. Psychol. 9:462. doi: $10.3389 /$ fpsyg.2018.00462

Fernald, A. (1992). "Meaningful melodies in mothers speech to infants. in Nonverbal Vocal Communication: Comparative and Developmental Approaches, Papoušek, H., Jürgens, B., and Papoušek, M. (Cambridge: Cambridge University Press), 262-282.

Fujii, S., Watanabe, H., Oohashi, H., Hirashima, M., Nozaki, D., and Taga, G. (2014). Precursors of dancing and singing to music in three- to four-monthsold infants. PLoS ONE 9:e97680. doi: 10.1371/journal.pone.0097680

Fujioka, T., Trainor, L. J., Large, E. W., and Ross, B. (2009). Beta and gamma rhythms in human auditory cortex during musical beat processing. Ann. N. Y. Acad. Sci. 1169, 89-92. doi: 10.1111/j.1749-6632.2009.04779.x

Goodman, L., Riley, M. A., Mitra, S., and Turvey, M. T. (2000). Advantages of rhythmic movements at resonance: minimal active degrees of freedom, minimal noise, and maximal predictability. J. Motor Behav. 32, 3-8. doi: $10.1080 / 00222890009601354$
Supplementary Video $\mathbf{5}$ | V at 19 months responding to Dora.

Supplementary Video 6 | V at 29 months responding to Dora.

Supplementary Audio 1 | 30-s clips of Dora at slow, moderate, and fast tempos.

Supplementary Audio 2 | 30-s clips of Metallica at slow, moderate, and fast tempos.

Supplementary Audio 3 | 30-s clips of Franklin at slow, moderate, and fast tempos.

Supplementary Audio 4 | 30-s clips of Everybody at slow, moderate, and fast tempos.

Grahn, J. A., and Brett, M. (2007). Rhythm and beat perception in motor areas of the brain. J. Cogn. Neurosci. 19, 893-906. doi: 10.1162/jocn.2007.19.5.893

Groarke, J. M., and Hogan, M. J. (2016). Enhancing wellbeing: an emerging model of the adaptive functions of music listening. Psychol. Music 44, 769-791. doi: $10.1177 / 0305735615591844$

Gudmundsdottir, H., and Trehub, S. E. (2018). Adults recognize toddlers' song renditions. Psychol. Music 46, 281-291. doi: 10.1177/0305735617711762

Háden, G. P., Honing, H., Török, M., and Winkler, I. (2015). Detecting the temporal structure of sound sequences in newborn infants. Int. J. Psychophysiol. 96, 23-28. doi: 10.1016/j.ijpsycho.2015.02.024

Hannon, E. E., and Trehub, S. E. (2005). Tuning in to musical rhythms: Infants learn more readily than adults. Proc. Natl. Acad. Sci. U.S.A. 102, 12639-12643. doi: $10.1073 /$ pnas.0504254102

Hetfield, J., and Ulrich, L. (2017). Now that we're dead [Recorded by Metallica]. On Hardwired... to Self-Destruct [MP3 file]. San Rafael, CA: Blackened.

Ilari, B. (2005). On musical parenting of young children: Musical beliefs and behaviors of mothers and infants. Early Child Dev. Care 175, 647-660. doi: 10.1080/0300443042000302573

Janata, P., Tomic, S. T., and Haberman, J. M. (2012). Sensorimotor coupling in music and the psychology of the groove. J. Exp. Psychol. 141, 54-75. doi: $10.1037 / \mathrm{a} 0024208$

Laukka, P. (2007). Uses of music and psychological well-being among the elderly. J. Happi. Studies 8, 215-241. doi: 10.1007/s10902-006-9024-3

Lausberg, H., and Sloetjes, H. (2009). Coding gestural behavior with the NEUROGES-ELAN system. Behav. Res. Methods 41, 841-849. doi: 10.3758/BRM.41.3.841

Longhi, E. (2009). 'Songese': Maternal structuring of musical interaction with infants. Psychol. Music 37, 195-213. doi: 10.1177/0305735608097042

Madison, G. (2006). Experiencing groove induced by music: consistency and phenomenology. Music Percept. 24, 201-208. doi: 10.1525.mp.2006.24.2.201

Madison, G., Gouyon, F., Ullén, F., and Hörnström, K. (2011). Modeling the tendency for music to induce movement in humans: first correlations with low-level audio descriptors across music genres. J. Exp. Psychol. 37, 1578-1594. doi: $10.1037 / \mathrm{a} 0024323$

McAuley, J. D., Jones, M. R., Holub, S., Johnston, H. M., and Miller, N. S. (2006). The time of our lives: life span development of timing and event tracking. J. Exp. Psychol. General 135, 348-367. doi: 10.1037/0096-3445.135. 3.348

Mehr, S. A., Song, L. A., and Spelke, E. S. (2016). For 5-month-old infants, melodies are social. Psychol. Sci. 27, 486-501. doi: 10.1177/0956797615626691

Mireault, G. C., Crockenberg, S. C., Heilman, K., Sparrow, J. E., Cousineau, K., and Rainville, B. (2018). Social, cognitive, and physiological aspects of humour perception from 4 to 8 months: two longitudinal studies. Br. J. Dev. Psychol. 36, 98-109. doi: 10.1111/bjdp.12216

North, A. C., and Hargreaves, D. J. (1999). Music and adolescent identity. Music Educ. Res. 1, 75-92. doi: 10.1080/1461380990010107

Patel, A. D., and Iversen, J. R. (2014). The evolutionary neuroscience of musical beat perception: the action simulation for auditory prediction (ASAP) hypothesis. Front. Syst. Neurosci. 8:57. doi: 10.3389/fnsys.2014.00057

Patel, A. D., Iversen, J. R., Bregman, M. R., and Schulz, I. (2009). Experimental evidence for synchronization to a musical beat in a nonhuman animal. Curr. Biol. 19, 827-830. doi: 10.1016/j.cub.2009.03.038 
Phillips-Silver, J., and Trainor, L. J. (2008). Vestibular influence on auditory metrical interpretation. Brain Cogn. 67, 94-102. doi: 10.1016/j.bandc.2007.11.007

Pop, D., and Martin, M. (1997). Everybody [Recorded by Backstreet Boys]. On Backstreet's Back [MP3 file]. New York, NY: Jive.

Rutkowski, J., and Miller, M. S. (2002). A longitudinal study of elementary children's acquisition of their singing voices. Update: Appli. Res. Music Educ. 22, 5-14. doi: 10.1177/87551233030220010102

Savage, P. E., Brown, S., Sakai, E., and Currie, T. E. (2015). Statistical universals reveal the structures and functions of human music. Proc. Natl. Acad. Sci. U.S.A. 112, 8987-8992. doi: 10.1073/pnas.1414495112

Sitron, J., and Straus, B. (2010). Dora the Explorer Theme Song. On We did it! Dora's Greatest Hits [MP3 file]. New York, NY: Legacy Records

Stupacher, J., Hove, M. J., and Janata, P. (2016). Audio features underlying perceived groove and sensorimotor synchronization in music. Music Percept. 33, 571-589. doi: $10.1525 / \mathrm{mp} .2016 .33 .5 .571$

Stupacher, J., Hove, M. J., Novembre, G., Schütz-Bosbach, S., and Keller, P. E. (2013). Musical groove modulates motor cortex excitability: a TMS investigation. Brain Cogn. 82, 127-136. doi: 10.1016/j.bandc.2013.03.003

Tarr, B., Launay, J., Cohen, E., and Dunbar, R. I. (2015). Synchrony and exertion during dance independently raise pain threshold and encourage social bonding, Biol. Lett. 11:20150767. doi: 10.1098/rsbl.2015.0767

Tarr, B., Launay, J., and Dunbar, R. I. M. (2014). Music and social bonding: "Self-other" merging and neurohormonal mechanisms. Front. Psychol. 5:1096. doi: 10.3389/fpsyg.2014.01096

Trainor, L. J. (1996). Infant preferences for infant-directed versus noninfantdirected playsongs and lullabies. Infant Behav. Dev. 19, 83-92. doi: 10.1016/S0163-6383(96)90046-6

Trainor, L. J., and Zacharias, C. A. (1998). Infants prefer higher-pitched singing. Infant Behav. Dev. 21, 799-805. doi: 10.1016/S0163-6383(98)90047-9

Trehub, S., and Trainor, L. (1998). Singing to infants: lullabies and play songs. $A d v$. Infancy Res. 12, 43-77.
Trehub, S. E., and Gudmundsdottir, H. R. (2019). "Mothers as singing mentors for infants," in The Oxford Handbook of Singing. eds Welch, G., Howard, D., and Nix, J (Oxford, UK: Oxford University Press), 455-469. doi: 10.1093/oxfordhb/9780199660773.013.25

Trehub, S. E., Weiss, M. W., and Cirelli, L. K. (2019). "Musicality across the lifespan," in Foundations of Music Psychology: Theory and Research. eds Levitin, D. J. and Rentfrow, J (Cambridge, MA: MIT Press), 265-303

van Noorden, L., and Moelants, D. (1999). Resonance in the perception of musical pulse. J. N. Music Res. 28, 43-66.

Vanneste, S., Pouthas, V., and Wearden, J. H. (2001). Temporal control of rhythmic performance: a comparison between young and old adults. Exp. Aging Res. 27, 83-102. doi: 10.1080/03610730125798

Winkler, I., Háden, G. P., Ladinig, O., Sziller, I., and Honing, H. (2009). Newborn infants detect the beat in music. Proc. Natl. Acad. Sci. U.S.A. 106, 2468-2471. doi: 10.1073/pnas.0809035106

Witek, M. A. G., Clarke, E. F., Wallentin, M., Kringelbach, M. L., and Vuust, P. (2014). Syncopation, body-movement and pleasure in groove music. PLoS ONE 9:e94446. doi: 10.1371/journal.pone.0094446

Zentner, M., and Eerola, T. (2010). Rhythmic engagement with music in infancy. Proc. Natl. Acad. Sci. U.S.A. 107, 5768-5773. doi: 10.1073/pnas. 1000121107

Conflict of Interest Statement: The authors declare that the research was conducted in the absence of any commercial or financial relationships that could be construed as a potential conflict of interest.

Copyright (ㅇ 2019 Cirelli and Trehub. This is an open-access article distributed under the terms of the Creative Commons Attribution License (CC BY). The use, distribution or reproduction in other forums is permitted, provided the original author(s) and the copyright owner(s) are credited and that the original publication in this journal is cited, in accordance with accepted academic practice. No use, distribution or reproduction is permitted which does not comply with these terms. 
OPEN ACCESS

Edited by:

Jennifer MacRitchie,

Western Sydney University, Australia

Reviewed by:

Jeanette Tamplin, Faculty of Fine Arts and Music,

University of Melbourne, Australia Alexander Refsum Jensenius,

University of Os/o, Norway

${ }^{*}$ Correspondence:

Andrea Creech

andrea.creech@mus.ulaval.ca

Specialty section This article was submitted to Performance Science, a section of the journal

Frontiers in Psychology

Received: 12 November 2018 Accepted: 14 January 2019 Published: 30 January 2019

Citation:

Creech A (2019) Using Music Technology Creatively to Enrich Later-Life: A Literature Review.

Front. Psychol. 10:117. doi: 10.3389/fpsyg.2019.00117

\section{Using Music Technology Creatively to Enrich Later-Life: A Literature Review}

\author{
Andrea Creech* \\ Faculté de Musique, Université Laval, Québec, QC, Canada
}

Background: A growing body of evidence has demonstrated significant social and emotional benefits of music-making amongst senior citizens. However, several as-yet unresolved age-related barriers to "musicking" have been identified. Positioned within the emergent field of gerontechnology, concerned with the interface between aging and technology research, this review of literature thus explores the potential for music technologies to function as a vehicle for creative musical opportunities in later-life.

Methods: ERIC, Psychlnfo, and Web of Science databases were searched, focusing on the intersection between music, technology, and aging. The criteria for inclusion were that the paper should: (1) be in English; (2) report empirical research involving the use of music technologies intended to support receptive (listening, interpreting, reflecting) or active (playing, creating, performing) engagement with music amongst older persons, defined as being aged 60 years or above (United Nations, 2017); (3) be published as a peer reviewed journal article.

Results: Of 144 papers screened, 18 papers were retained. 10 studies focused on using technology to support musicking in the form of listening, reflecting, and interpreting. Just five studies explored the utility of technology in promoting singing or playing instruments, while a further three were focused on music and movement.

Conclusions: Overall, the literature reviewed suggests that older people, even those with complex needs, are capable of, and interested in using music technologies to access and create personally meaningful music. The limited research that does exist points to multiple and significant benefits that may be derived from receptive or active musicking supported by a range of music technologies.

Keywords: music, technology, creative, aging, later-life

\section{INTRODUCTION}

Questions concerned with the potential for music technology to support creative and positive aging are framed within a context where we are witnessing an unprecedented longevity revolution. The world's population aged 60 and above is set to rise from 962 million in 2017 to over two billion in 2050 (United Nations, 2017). Particularly steep increases in absolute numbers of older people will occur in developing countries. All over the world, though, the proportion of the population aged 60 and over is increasing; by the year 2050 some of the most aged countries will be Japan, Spain, Italy, Germany, and Portugal, where those aged 60+ will account for between 38 and 43 per cent of their populations (United Nations, 2013). The year 2050 will see half of the global population 
living in countries where at least 20 per cent of people are aged 60 years or above (United Nations, 2017). The aging population internationally represents a triumph of public health policy yet poses significant challenges with regards to sustained quality of life, with particular risks of depression and loneliness, chronic disease, cognitive impairments, and sensory declines (ChangQuan et al., 2010; Sixsmith and Gutman, 2013; World Health Organization, 2015; Chambers et al., 2016)

In response to these quality of life challenges, a growing body of evidence has demonstrated significant social and emotional benefits of music-making amongst senior citizens (Creech et al., 2013a, 2014b; Fung and Lehmberg, 2016). However, several as-yet unresolved age-related barriers to "musicking," referring to listening, playing, creating, performing, interpreting, and reflecting (Elliott and Silverman, 2015), have been identified (Jansen, 2005; Withnall, 2010; Hallam et al., 2012). These barriers include ageist attitudes, psychosocial factors, as well as issues relating to the social or physical environment, accessibility of conventional tools (e.g., musical instruments), inclusion, and modes of communication. There is now a pressing need to consolidate and develop our knowledge concerning the potential for innovative music technologies to mitigate these barriers, thus maximizing the potential for access to the creative, psychological, and physiological benefits of musical engagement in later-life.

In this paper I therefore review the literature concerned with the utility and efficacy of music technology in later life contexts, addressing the following question: What is the potential for music technologies to function as a vehicle for promoting access to, and engagement in, musicking in later-life?

\section{BACKGROUND}

\section{The Power of Music in Later-Life}

Listening to music and playing musical instruments have been described by community-dwelling elders as "restorative activities" (Jansen and von Sadovszky, 2004): activities that "enable a person to feel refreshed, rested, at peace, clear-headed, and mentally able to take on new tasks and challenges" (Jansen, 2005, p. 37). By "restorative," Jansen (2005) refers to activities that enhance well-being through fostering a sense of "being away" ( $p$. 37), either physically in a new location or metaphorically allowing the mind to wander to another place. Restorative activities such as music listening or music-making offer the scope for a person to "feel in a whole other world," with their interest fully engaged and a high degree of "fit and harmony between the activity and the person's interests, wishes, and abilities" (p. 37).

From a more proactive perspective, it has been argued that creative spaces offer older people the opportunity to protest against a narrative of decline and instead perform creative acts, explore new ways of "becoming" and experience a humanized old age and continuing sense of citizenship. Writing through the lens of very old age, Erikson and Erikson (1998) reflect that the final life stage need not be solely concerned with withdrawal but, rather, may be experienced as a period of growth and creative expression that is accessed through "contact with one another and $[\ldots]$ a regaining of lost skills, including play, activity, joy, and song $[\ldots]$ ". (p. 227).
A body of research supports the view that receptive or active musical engagement may function both as a restorative and as a creative space. (Jenkins, 2011), for example, reported that participation in music was one significant predictor of positive changes in well-being amongst a sample drawn from the English Longitudinal Study of Aging $(n \approx 6,000)$. In a similar vein, Cohen (2006) found that creative musical opportunities were associated with improvements in general health and a reduction in health risk factors. It has been reported that older people who were actively engaged in musical activities felt that they had greater control over their lives, experienced more pleasure and felt more cared for than those participating in other leisure activities; these positive outcomes were found amongst older people who identified themselves as "beginners" in music as well as those who said they were more experienced (Creech et al., 2013b). Creech et al. (2014a) reported that older people formulated aspirations in relation to possible musical selves through playing and performing in musical groups. Reported cognitive advantages include improvements in attention and concentration (Bugos et al., 2007). Describing an ethnographic study in the context of residential care for older persons, Allison (2008) reported that a song writing group provided a space where older people could transcend the limitations of chronic ill health and institutional care, engaging in creative music-making. Thus, the accumulated evidence suggests that engagement with music, including listening as well as making music, offers a context where older people may continue to experience a positive and creative quality of life.

\section{The Digital Divide}

Within our current context, characterized by an aging population alongside the accelerated use of digital technology (Warschauer and Matuchniak, 2010; Poushter, 2016), the field of gerontechnology has emerged, concerned with the scientific study of the interface between aging and technology (Fozard et al., 2000). Increasingly, attention has been directed toward the possibilities offered by technology for enhancing the quality of later life, for example with technological innovations relating to connection with the outside world, ambient-assisted living, e-health, home-monitoring, robotics for independent living, and digital games (Kaplan et al., 2013; Helfert et al., 2015). According to the World Health Organization (2015, p. 36) "emerging technologies, particularly those used to foster communication and engagement," may make the goal of aging in place "more achievable."

Although anxieties relating to technology use have been noted amongst older people, Heinz et al. (2013) also found an openness to using technology, particularly innovations that offered potential enhancements to quality of life and independence. This suggests important opportunities exist to explore the potential benefits of integrating technology within the domain of practice of later-life musicking (Stige, 2012; Elliott and Silverman, 2015). Music technology with younger people is now well-established within special education (Farrimond et al., 2011), music education (Ruthmann and Hebert, 2012) and within music therapy contexts (Hahna et al., 2012; Magee, 2013; Stensaeth and Magee, 2016). However, there has been 
limited attention focused on the use of music technology as a medium to promote access to the holistic and social praxis of musicking (Elliott and Silverman, 2015) in later life, particularly in non-therapeutic contexts.

Notwithstanding these technological potentials and some evidence of increasing later-life engagement with technology, a persistent generational digital divide has been noted, whereby older people, in comparison with younger people, remain less likely to engage with technology (Charness and Boot, 2009; Allen, 2013; Poushter, 2016). This so-called digital divide may be related to attitudinal barriers, including those expressed by older people themselves about what it means to be an older person (Jansen, 2005). The generational digital divide has furthermore been linked to obstacles relating to "age-related changes in perceptual, cognitive, and motor systems" (Charness and Boot, p. 255) which may include lack of training; physical challenges when using peripheral equipment such as keyboards; accessibility issues related to tremors, arthritis or limited vision; or cognitive challenges such as difficulty in understanding menu structures.

Amongst music technology users, a digital divide has likewise been observed, contrasting "digital natives" who have grown up with technology with "digital immigrants," for whom technology represents a foreign and somewhat unwelcoming territory (Magee, 2013). Ambivalence toward music technology, amongst older people, may be related to "an industry-wide focus on youth which has led to a systematic disregard for the needs, preferences, and capabilities of older adults" (Damant and Knapp, 2015, p. 18). Accordingly, technological challenges and accessibility issues may be conceptualized as limits of the technology and its application, not deficits in the users. This approach is derived from the cognitive system engineering framework (Hollnagel and Woods, 2005) which takes the perspective that the human agent and the information system form a single unit (the joint cognitive system). Consequently, the purpose of the system-here, music technologies-is to work in conjunction with the human user in an efficient and integrated manner. In other words, one must adapt a system to fit the human capacities and limits. In this way, music technologies may be mobilized and adapted in creative ways that address quality of life issues in later-life contexts.

\section{Creative Music Technologies in Later Life}

According to Himonides and Purves (2010, p 123-124), music technology can "enhance our lives through experiencing music in new ways; facilitate the communication of our musics [...]; provide wider access to other people's musics [...]; and provide access to music for people with special needs and requirements." Four key terms, drawing upon these principles of inclusion and access to communicative musical experience, frame a design ethos that underpins the development of creative technologies that may be relevant for older people. Accessible or assistive devices differentiate for specific physical or cognitive constraints, while inclusive or universal devices are intended to enhance usability through recognition of a wide spectrum of capabilities (Samuels, 2014).

Broadly, creative music technologies comprise electrical or digital tools to select, listen to, create, manipulate, analyse, or record musical sounds. These technologies comprise listening devices, sound generators (e.g., synthesizers or samplers), musical instruments and interfaces (e.g., MIDI controller keyboards; distance sensors; assistive digital instruments) and visual realities (e.g., software that translates musical sound into visual representations and feedback (Farrimond et al., 2011). Sound generators provide a rich musical palette that can be easily accessed and manipulated to meet individual musical preferences. Digital musical interfaces offer the vehicle through which individuals may access and control the sound generators; a range of interfaces are available, and specific interfaces may be chosen for creative reasons or to meet the physical, sensory or cognitive abilities and needs of an individual, while visual realities may be used as motivational feedback tools, and to promote multisensory creative musical expression. Owing to the availability of inexpensive and powerful technology, there is everincreasing availability of creative music technologies that meet a wide range of diverse needs, including specific needs that may be related to aging.

Cappelen and Anderson (2014, p. 4) have coined the term "Musicking Tangibles" designed to function as an arena for "positive and empowering" musicking experiences within special needs contexts. Arguing that Midi-based assistive music technology, with its emphasis on control of the interface, can be aesthetically limiting and tiring, Cappelen and Anderson advocate a focus on "motivating social interaction, co-creation, and musicking." Four generations of Musicking Tangible design are described, including multi-sensory and interactive soft objects and spaces that can be "open to many interpretations, interaction forms and activity levels" (p. 5). To date, the reported applications of Musicking Tangibles have focused on families with children with severe disabilities, although the principles of co-creation and empowerment may have significant implications for the design and use of music technology across many life stages and contexts.

Weisberger (2013), who facilitated an elders' song writing group within a Hispanic community adult day center, has described the potential for widely available technologies to support collaborative musicking with older people. Her aim with the group, which included several participants who lived with dementia as well as physical constraints, was to use culturallyrelevant music as a unifying medium for the group, many of whose participants had experienced significant isolation related to their cognitive and physical challenges. Early sessions focused around acoustic instruments, which posed many barriers to participation. As Weisberger (2013, p. 284) explains, although the group members could identify the music that "moved" them, "we would lose the beat, get out of tune, forget the words, and get distracted." GarageBand was adopted as a tool for exploring potential solutions to the limitations experienced with the acoustic instruments, and the group proceeded to create an album of 20 original songs. GarageBand provided a framework within which the group could explore tempo, rhythm, timbre, harmony, and melody. Group members also became confident in using the microphone to experiment and record their singing, learnt new technological language, and contributed to editorial decisions. Weisberger notes that in addition to supporting the exploration of communication and expression through music, 
GarageBand served as a vehicle for helping to normalize the wider technological world. She further notes that a range of nondirective as well as directive facilitation strategies were necessary in order to support the participants effectively in reaching individual as well as group goals. For example, while some participants strove to enhance memory or language, others used the musical activities as a context for reducing anxiety, and these goals were situated within a group context that prioritized mutual support and reciprocal recognition of individual expressions of feelings or experience. Weisberger captured individual expressive ideas using multiple tracks, loops and layering, achieving a sense of the whole being aesthetically, greater than the sum of its parts. Finally, Weisberger observes that the option of listening back to recorded performance contributed to and sense of pride in the group identity, as well as boosting self-awareness and self-esteem.

The background literature thus suggests that there may be an important role for music technology to play in facilitating access to creative music opportunities for older people, including frail elders who may be living with significant cognitive or physical challenges. However, to date there has been limited empirical research in this area. The purpose of this review was therefore to synthesize the previous research concerned with music technology in later life, with a view to identifying key messages and themes, as well as areas for future exploration.

\section{METHODS}

Three databases were searched: ERIC, PsychInfo and Web of Science, with the Web of Science database refined to include items in the categories of music, education, gerontology, interdisciplinary social sciences, rehabilitation, and multidisciplinary psychology. Focusing on abstracts, search terms were: music, technology, aging (aging, elderly, seniors, older people, older adults). Searches were therefore carried out in the following manner: (1) music + technology + aging; (2) music + technology + aging; (3) music + technology + elderly; (4) music + technology + seniors; and so on. The criteria for inclusion were that the paper should: (1) be in English; (2) report empirical research involving the use of music technologies intended to support receptive (listening, interpreting, reflecting) or active (playing, creating, performing) engagement with music amongst older persons; (3) include research participants who may be classified as "older people," defined as being aged 60 years or above (United Nations, 2017); (4) be published as a peer reviewed journal article. After removing duplicates, a total of 144 records were found through database searches, and their titles and abstracts were screened against the inclusion criteria. One hundred and twenty-six records were excluded at that stage, while 18 records were retained. A further five records were identified and added, through the process of scanning the reference lists of these 18 retained records (Figure 1). Full texts

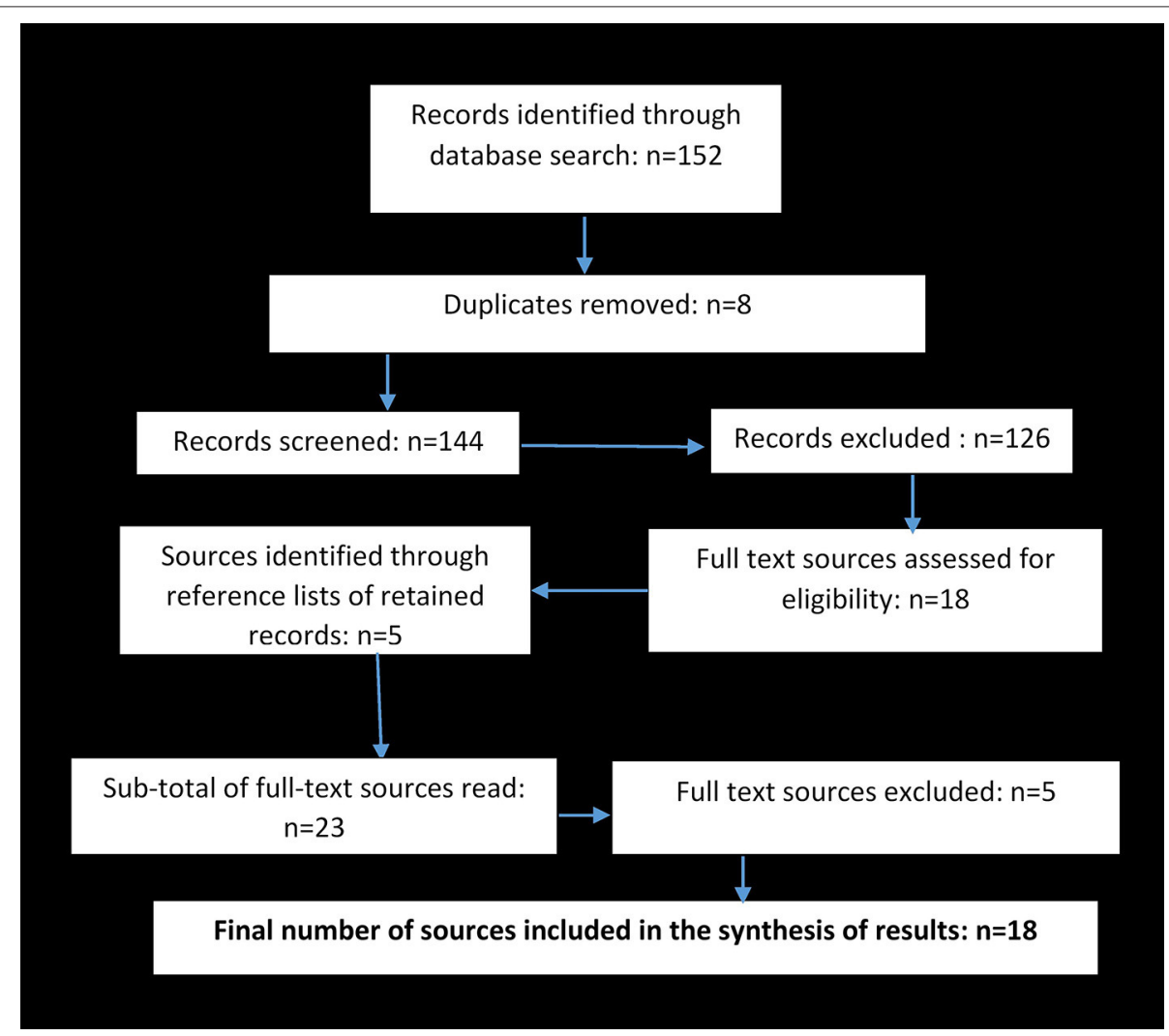

FIGURE 1 | Records searched and sources retained. 
for the sub-total of 23 retained records were located and read in full. Five papers were excluded for reasons that included: (1) the role of music was as a stimulus rather than as a medium for musicking, according to the categories proposed by Elliott and Silverman (2015); (2) the focus was not on musicking, but on media more broadly; (3) the paper was descriptive or theoretical rather than empirical; (4) the paper was not a peer reviewed article. Thus, a final total of 18 full-texts were retained for inclusion in the review.

\section{RESULTS}

The 18 papers included in this review are listed in Table 1. Information extracted included study design, purpose of the study, facets of musicking addressed in the study, number and ages of participants and whether the study focused on participants living with dementia, and key findings. Overall, the majority (10) of studies focused on using technology to support musicking in the form of listening, reflecting, and interpreting. Just five studies from the total of 18 explored the utility of technology in promoting singing or playing instruments, while a further three were focused on music and movement. Ten studies focused on persons living with dementia, and of those, six focused on using technology to support listening, while three included an element of promoting movement with music and just one included making music with a digital instrument.

\section{Using Technology for Accessing Preferred Music}

A growing body of evidence indicates that older people are capable of learning to use music technology to support access to listening and reflecting on their preferred music. For example, Lancioni et al. (2014) reported that older people living with mild to moderate Alzheimer's Disease were able to engage with a technology set-up comprising a laptop computer, amplifier, user interface (switch), and speaker intended to support the process of choosing and accessing their preferred music. Four participants aged 75-89 took part in an intervention (preceded by guided practice attempts) that comprised $10 \mathrm{~min}$ sessions where they were invited to use the technology to access their music choices. Prior to this experimental intervention, control baseline sessions took place where staff played recordings for the participants, without the aid of the technology set-up. Video recordings of the baseline control sessions and the experimental intervention sessions were evaluated using a social validation check that included indicators of happiness, self-determination, and social image. Results revealed that participants were able to use the technology, and the social validation scale differentiated between the control and experimental (technology) sessions, with more positive scores found in the experimental sessions where participants used the technology.

The idea of using simple technologies as an interface for enabling access to preferred music was also explored by Davison et al. (2016). In their within-subjects experimental study, 11 participants aged 76-95 and living with dementia completed a protocol that involved learning to use a Memory Box-a laptop computer configured in such a way as to provide easy access to personally meaningful music, photographs, movies, or messages. Over 8 weeks, each participant spent a total of two and a half hours learning to use the Memory Box with researcher support (the experimental intervention), and an equivalent amount of time in visits from a researcher who read the newspaper and discussed local events (the control condition). Measures for anxiety, agitation, and depression were completed pre and post intervention. Overall, participants showed a preference for music and photographs, and several (six out of 11) were able to use the system independently. Anxiety and depression were both found to decrease, over the period of the intervention. These findings added to an earlier study by Garland et al. (2007), who carried out a within-subjects experimental study with older people living with dementia. Thirty participants were exposed to a music listening condition, which was compared to a simulated visit (audio tape of a familiar person), a placebo (audio tape of a gardening book), and no intervention. Observations were made, using a time sampling approach before, during, and following each of the conditions. Listening to personally meaningful music was found to have moderate and variable positive effects for physical agitation. A key finding here was that a simple technology (in this study, a cassette player and headphones, with a playlist comprising personally significant music) could function as a tool to alleviate physically aggressive behavior among older persons with dementia.

In a similar vein, Sixsmith et al. (2007) demonstrated that older persons with a dementia diagnosis expressed a desire to use technology and were able to do so in order to access preferred music. Their observational study aimed to understand the factors that could best support older people with dementia in engaging with computer activities. Twenty-seven nursing home residents with an average age of 85 were offered the opportunity to take part in daily computer activities (including a range of choices that included music, games, video, email, and internet searching) over the course of 7 weeks. On average, participants voluntarily attended three sessions per week of approximately $25 \mathrm{~min}$ each. Participants engaged successfully with an average of two activities per session, requiring just $2 \mathrm{~min}$ (on average) of technical support during each session. The researchers noted that the most liked activity was listening to personally meaningful music that accompanied slideshows or videos.

With a similar focus on using technology to enable autonomous creative choices and access to preferred music, Sixsmith et al. (2007) describe a user-led research and development initiative in the UK, focusing on the creation of a simple music-playing device that would meet the user needs of older people. An ecological model of quality of life, taking account of personal attributes (e.g., cognitive and psychological characteristics, preferences), contextual factors (e.g. social support, physical characteristics of the immediate environment), and socio-cultural factors, framed an iterative process that led to the development of a device intended for use by individuals living with dementia. This ecological model "focused on the everyday activities of the person and highlighted opportunities for targeting technology and design 
TABLE 1 | Full text sources retained.

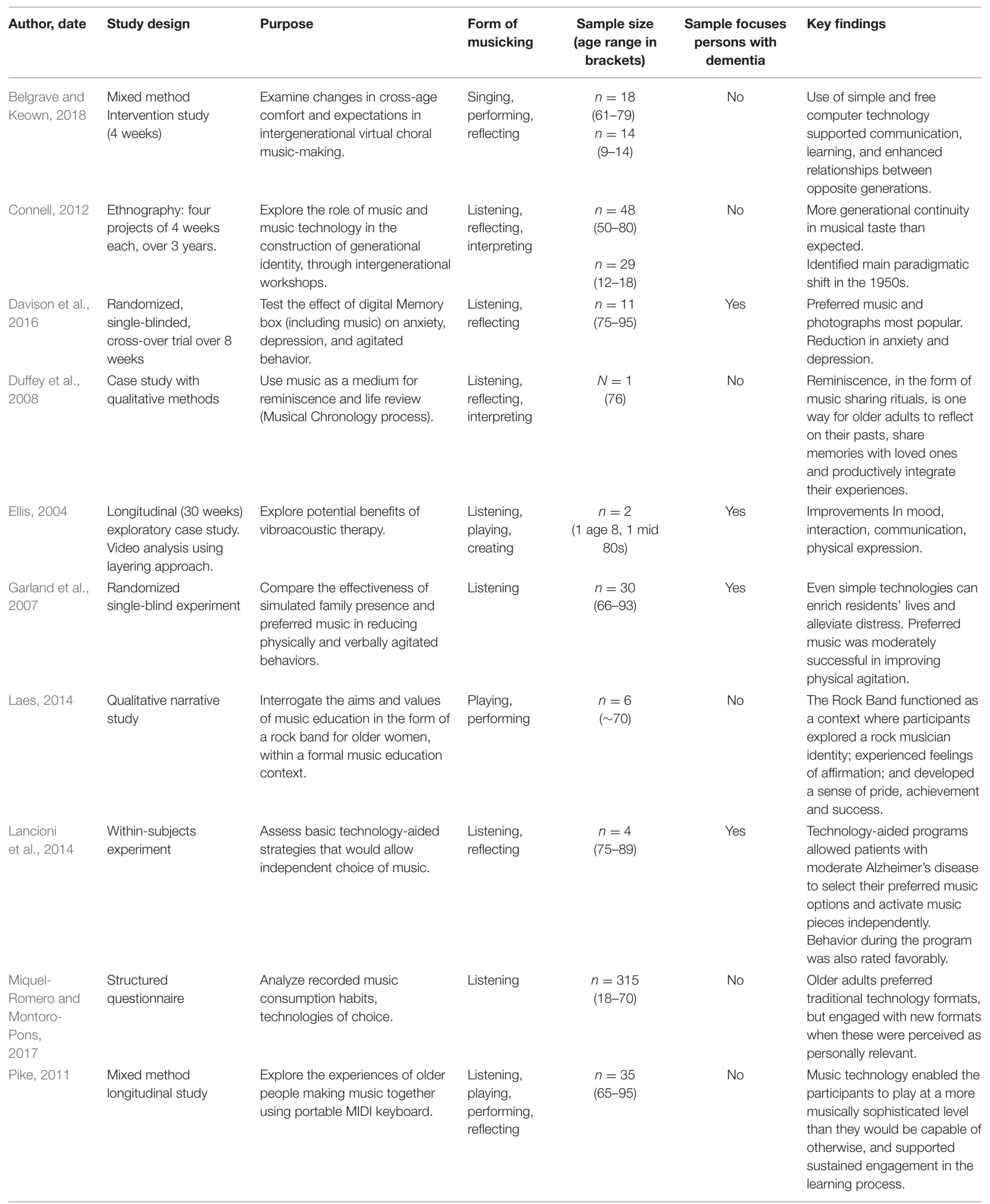


TABLE 1 | Continued

\begin{tabular}{|c|c|c|c|c|c|c|}
\hline Author, date & Study design & Purpose & $\begin{array}{l}\text { Form of } \\
\text { musicking }\end{array}$ & $\begin{array}{l}\text { Sample size } \\
\text { (age range in } \\
\text { brackets) }\end{array}$ & $\begin{array}{l}\text { Sample focuses } \\
\text { persons with } \\
\text { dementia }\end{array}$ & Key findings \\
\hline $\begin{array}{l}\text { Reid et al., } \\
2017\end{array}$ & $\begin{array}{l}\text { Between-groups } \\
\text { experimental design }\end{array}$ & $\begin{array}{l}\text { Test a technology-based method } \\
\text { for conducting longitudinal } \\
\text { studies of regular singing and } \\
\text { song learning in older adults. }\end{array}$ & $\begin{array}{l}\text { Listening, } \\
\text { singing }\end{array}$ & $\begin{array}{c}n=4 \\
(65-84)\end{array}$ & No & $\begin{array}{l}\text { Technology-based singing } \\
\text { and song learning protocol } \\
\text { was shown to be feasible for } \\
\text { use with older adults }\end{array}$ \\
\hline $\begin{array}{l}\text { Rosseland, } \\
2016\end{array}$ & $\begin{array}{l}\text { Qualitative research- } \\
\text { through-design study, } \\
\text { using observational } \\
\text { methods }\end{array}$ & $\begin{array}{l}\text { Explore interpersonal synchrony } \\
\text { and entrainment between paired } \\
\text { participants with Alzheimer's } \\
\text { Disease, aided by an interactive } \\
\text { system that synchronizes the } \\
\text { musical tempo and the tempo of } \\
\text { the users' movements. }\end{array}$ & $\begin{array}{l}\text { Listening, } \\
\text { movement to } \\
\text { music }\end{array}$ & $\begin{array}{c}n=9 \\
\text { ("elderly") }\end{array}$ & Yes & $\begin{array}{l}\text { Most participants were able to } \\
\text { use the device and } \\
\text { experienced entrainment and } \\
\text { synchronous movement, } \\
\text { when working in pairs. }\end{array}$ \\
\hline $\begin{array}{l}\text { Rosseland } \\
\text { and Culén, } \\
2016\end{array}$ & & $\begin{array}{l}\text { Describe and discuss a } \\
\text { Research \& Development (R\&D) } \\
\text { project focused on designing a } \\
\text { rhythmic interaction device for } \\
\text { older people. }\end{array}$ & $\begin{array}{l}\text { Listening, } \\
\text { movement to } \\
\text { music }\end{array}$ & $\begin{array}{c}n \approx 12 \\
\text { ("older people") }\end{array}$ & Yes & $\begin{array}{l}\text { Highlights how R\&D involving } \\
\text { users can generate new } \\
\text { knowledge. Repmoves } \\
\text { promoted freedom of } \\
\text { movement, and entrainment. }\end{array}$ \\
\hline $\begin{array}{l}\text { Scott Reis } \\
\text { et al., } 2012\end{array}$ & $\begin{array}{l}\text { Research \& } \\
\text { development project }\end{array}$ & $\begin{array}{l}\text { Develop a bespoke music } \\
\text { technology for use by the elderly } \\
\text { that allows musical } \\
\text { expressiveness through motion }\end{array}$ & $\begin{array}{l}\text { Listening, } \\
\text { movement to } \\
\text { music }\end{array}$ & $\begin{array}{c}n=8 \\
\text { (Mean age 83) }\end{array}$ & No & $\begin{array}{l}\text { Seven out of eight succeeded } \\
\text { in using the technology to } \\
\text { create expressive movement } \\
\text { and sound. }\end{array}$ \\
\hline $\begin{array}{l}\text { Sixsmith } \\
\text { et al., } 2007\end{array}$ & $\begin{array}{l}\text { User-led research and } \\
\text { development }\end{array}$ & $\begin{array}{l}\text { Design simple music player for } \\
\text { persons with dementia, with } \\
\text { user-led process. }\end{array}$ & $\begin{array}{l}\text { Listening, } \\
\text { reflecting }\end{array}$ & $\begin{array}{l}n=26 \\
(62-96)\end{array}$ & Yes & $\begin{array}{l}\text { Integrated theoretical, } \\
\text { methodological, and design } \\
\text { framework, leading to } \\
\text { development of prototype, }\end{array}$ \\
\hline $\begin{array}{l}\text { Smith et al., } \\
2017\end{array}$ & $\begin{array}{l}\text { Repeated measures } \\
\text { within participants } \\
\text { design }\end{array}$ & $\begin{array}{l}\text { Determine if a self-administered } \\
\text { computer-based rehabilitation } \\
\text { program, could improve music } \\
\text { appreciation and speech } \\
\text { understanding in adults who } \\
\text { have a cochlear implant. }\end{array}$ & $\begin{array}{l}\text { Listening, } \\
\text { reflecting, } \\
\text { interpreting }\end{array}$ & $\begin{array}{l}n=21 \\
(32-82)\end{array}$ & No & $\begin{array}{l}\text { Significant pre-post } \\
\text { improvements for low musical } \\
\text { ability participants, with } \\
\text { regards music enjoyment, } \\
\text { aural acuity, music and } \\
\text { speech perception. }\end{array}$ \\
\hline $\begin{array}{l}\text { Tak et al., } \\
2015\end{array}$ & $\begin{array}{l}\text { Observational } \\
\text { descriptive study }\end{array}$ & $\begin{array}{l}\text { Identify characteristics that are } \\
\text { crucial for the success of a } \\
\text { computer-assisted intervention } \\
\text { for persons with dementia. }\end{array}$ & $\begin{array}{l}\text { Listening, } \\
\text { reflecting }\end{array}$ & $\begin{array}{l}n=27 \\
(78-97)\end{array}$ & Yes & $\begin{array}{l}\text { On average, participants } \\
\text { engaged in } 3 \text { sessions per } \\
\text { week of } 25 \text { min each. They } \\
\text { required an average of } 2 \text { min } \\
\text { technical assistance } \\
\text { per session. Listening to } \\
\text { music was the most preferred } \\
\text { activity. }\end{array}$ \\
\hline $\begin{array}{l}\text { Vahia et al., } \\
2017\end{array}$ & Observational study & $\begin{array}{l}\text { To investigate the feasibility, } \\
\text { safety, and utility of tablet } \\
\text { devices in managing older } \\
\text { psychiatric inpatients with } \\
\text { agitation and dementia. }\end{array}$ & Listening & $\begin{array}{c}n=36 \\
\text { (Mean age 80) }\end{array}$ & Yes & $\begin{array}{l}\text { All participants, regardless of } \\
\text { dementia severity, were able } \\
\text { to use apps that included } \\
\text { music apps, and were rated } \\
\text { by staff to derive clinical } \\
\text { benefit. Music apps were } \\
\text { among the most frequently } \\
\text { used type of App. }\end{array}$ \\
\hline
\end{tabular}

solutions" (p. 87). Through initial interviews with 26 participants living with dementia and subsequent stakeholder focus groups, both music and creativity were identified as key themes related to quality of life. "Music was important ... Music provided a context for a variety of forms of interaction and participation and had beneficial emotional effects" (p. 89), and "promoting the enjoyment and use of music" (p. 90) emerged as a priority area on the technology wish list. A prototype was developed, involving input from a range of perspectives (e.g., engineers, designers, gerontologists, dementia researchers), including testing and feedback from end-users (persons living with dementia). Sixsmith et al. (p. 92) explain that "this ensured that technology development took place in a way that was based on the experiences and needs of people with dementia and their caregivers rather than being based on a technology-driven approach."

Focusing on accessibility and the potential wider benefits of technology, Vahia et al. (2017) investigated the use of tablets as a non-pharmacologic intervention intended to reduce anxiety amongst patients at a geriatric behavior health inpatient 
unit. Two iPads were each loaded with 70 applications (apps), including music and musical instruments as well as communication, games, video entertainment, news, and photography apps, rated for level of complexity. When patients became agitated or restless, they were given an iPad and directed toward apps that matched their individual preferences. Music playing apps, and particularly harp and piano, were amongst the five most commonly used. Time spent engaged with apps, the specific apps chosen, and a rating of restlessness after app use were recorded. The authors reported that all of their 39 participants tolerated the use of the tablets, although time spent using the app as well as the level of app complexity were negatively related to severity of dementia. Those diagnosed with mild dementia benefitted the most with regards to reduction in agitation.

The specific technologies of choice for accessing music, among community-dwelling adults, have also been explored. MiquelRomero and Montoro-Pons (2017) carried out a structured questionnaire study, using a quota sampling method to achieve a representative sample of 315 Spanish residents aged 18-70, $34 \%$ of whom were aged $46-70$. The questionnaire gathered information about music listening habits including devices used, opinions about music, and socioeconomic data. The researchers found that overall older people were not enthusiastic about accessing music using new digital formats, preferring to rely on hi-fi systems, DVDs, or the television, suggesting that the music industry must recognize the continuing need for supporting these formats. However, the authors also point out individual differences, and highlight that older adults are only likely to engage with new technological interfaces when these are perceived as being personally relevant and meaningful.

Overall, the research concerned with using technology to enable access to preferred music in later life contexts demonstrates that technologies can mitigate barriers to personally meaningful musicking in the form of listening and reflecting. The key messages are that the technology must be relevant to the needs of the user, and that "under caregiver supervision, even persons with severe impairment may use simple and intuitive apps, especially when they are matched to each individual's preferences and level of cognitive function (Vahia et al., 2017, p. 863).

\section{Using Music Technology for Reminiscence}

The significance of music technology for supporting reminiscence has been highlighted. For example, Lazar et al. (2014) carried out a systematic review of technology as an aid for reminiscence therapy and found that eight out of ten studies that described using multi-sensory reminiscence approaches had integrated recorded music alongside other stimuli. Reminiscence therapists used technology, in these instances, as a tool to harness musical responsiveness, described as a continuing ability that persists long after other abilities may have become compromised.

Music technology as a specific tool for reminiscence and lifereview was investigated by Duffey et al. (2008), who developed a Musical Chronology process, a structured approach whereby clients recollect and share popular songs that are accessed using technology and mapped against significant points in their life journeys. The Musical Chronology has been applied with older adults where there have been reports of "multiple areas of personal growth, including increased self-awareness and selfacceptance, the development of new relationships, increased comfort with being open and authentic in relationships, progress in dealing with grief, a renewed appreciation for life, and hope for the future" [Somody, 2010, p. v-vi]. Duffey et al. (2008) describe the adaptation of the Musical Chronology as a framework for collaborative life review between adult children and their parents, using one case study to demonstrate how music sharing rituals, supported by technology, offered the opportunity "for older adults to reflect on their pasts, share memories with loved ones, and productively integrate their experience" (p. 60), the latter point referring to a major later-life developmental task (Erikson and Erikson, 1998).

Intergenerational musical review was also investigated by Connell (2012), who explored the role of music and music technology in the construction of generational identity. Over 4 years, four community projects took place, involving a total of 48 older people aged $50+$ to $80+$, alongside a total of 29 young people aged between 12 and 18. The participants were trained in the use of DJ equipment, and given access to this equipment as well as a supply of vinyl records, over a series of four worksops. The workshops were found to provide a powerful context for enhancing intergenerational understandings. Overall, although the 1950s was identified as a period where there had been a paradigmatic shift in musical identities, the workshop activities revealed more generational accord than discord. As Connell reports, "one of the more striking findings so far is that actually, for older people aged 70-something or less, many of their tastes are not so far away from those of teenagers as initial stereotyping might suggest" (p. 275).

In these examples, it is clear that the technology becomes a means to an end, functioning as the tool with which users may explore their unique lifecourse narratives. Technology may also serve as a tool for using music as the focus of intergenerational dialogue, enhancing relationships and reciprocal understandings of generational values and identities.

\section{Music Technology to Support Singing}

Significant wider benefits of singing in later life have been demonstrated (Clift et al., 2008), but there has been limited research focused on ways in which technology can be harnessed to support older people in engaging with singing. Reid et al. (2017) adapted a commercially available iPad application (SingFit) and tested its efficacy as a technology-based method for conducting longitudinal studies of regular singing and song learning in older adults. Forty-eight musically inactive participants (i.e., they had not engaged actively with musicmaking for at least 40 years) aged 65-84 were allocated to singing, listening, or control groups. The singing group used the app as a "sing-along" aid to learning and re-learning to sing favorite songs. The listening group used the app as a listening device, also focused on their favorite songs, while the control group did not use the app. The researchers were interested in the feasibility of using the app to promote singing, and whether daily singing or 
listening over a period of 5 weeks could have a positive effect on cognitive function. Although no significant effect was found for cognitive function (possibly because the 5 week protocol was too short to reveal any such effects), a key finding of this study was that the app functioned as an accessible gateway to singing. Older adults used the app with minimal support, with the majority of participants finding it motivating and enjoyable some reporting that they intended to continue singing after the research protocol had ended.

\section{Technology to Support Music Perception and Appreciation}

It is known that hearing loss is widespread among older people, and that there are significant challenges associated with this (Lin et al., 2011). One such challenge may be the loss of music perception, and consequently diminished enjoyment of musicking, in any of its forms. Accordingly, some researchers have focused on the potential for technology to serve as a tool to support rehabilitation in relation to music perception, among adults with cochlear implants (Smith et al., 2017).

Smith et al. (2017) recruited 21 adults aged 32-82 and living with a cochlear implant, who participated in a study to determine if a self-administered computer-based rehabilitation program, HearTunes (Rehab), could improve their music appreciation and speech understanding. The software presents musical patterns structured in such a way as to incrementally improve listening and focus. A novel feature of this software was its holistic nature, encompassing all aspects of musical perception rather than focusing solely on isolated musical tasks. Participants, who were categorized as having high or low musical ability (according to data gathered regarding musical background), engaged with the software for an average of $3.5 \mathrm{~h}$ per week, over 4 weeks. Pre and post tests were carried out, measuring music enjoyment, music perception, and speech understanding; the tests were repeated again 6 months after the study had finished. Significant improvements in enjoyment of music were found after 4 weeks, for the low-ability music group. At the 6 month follow-up, improvements in enjoyment of music were also found for those designated as having high musical ability. With regards to the tests for aural acuity, perceptual ability, and speech recognition, the software seemed to be most effective for those in the low musical ability group, although the significant improvements from pre to post training were not always evident at the 6 month follow-up. The researchers suggest that a longer rehabilitation period, initiated closer to the activation of the cochlear implant (CI), may be necessary in order to achieve long-lasting results, and reflect that "more complex and challenging versions of the software may also be necessary to develop to elicit more significant improvements in those CI users with more experience and background in music" (p. e267). Overall, a key finding of the study is that technology can be mobilized for supporting older adults in regaining and sustaining the capacity to engage with music, notwithstanding hearing loss. However, once again this study demonstrates the need for technology to differentiate for specific user needs and characteristics.

\section{Collaborative Musicking Supported by Technology}

Very few studies have focused on the potential for laterlife active and collaborative musicking supported by music technology. However, technology as a vehicle for group music making was investigated by Pike (2011), who explored the experiences of older people making music together using portable MIDI keyboards connected through a mixer, with the cohesive group sound broadcast through portable speakers. Thirty-five older people aged between 65 and 95 participated in the group over the course of 8 years. Data were collected from the participants via questionnaires, interviews, and recorded observation. While none of the participants had previous experience of using MIDI keyboards, this did not pose a barrier to learning but rather enhanced the overall musical experience. Specifically, the music technology was perceived to have enabled the participants to play at a more musically sophisticated level than they would be capable of otherwise, and supported sustained engagement in the learning process. Participants highlighted that the MIDI technology enabled participants to hear their individual parts within the group, served as an important tool for supporting the group in developing an internalized sense of pulse, and that the option to gain immediate aural feedback promoted discussion focused around creative choices relating to timbre, orchestration, and articulation.

The implications for music education values and priorities were explored by Laes (2014), who carried out narrative interviews with older women who, as musical novices, participated in a rock band within a formal music education context. As Laes (p. 11) explains, “ music pedagogues of today are challenged to have a responsibility to engage third-age learners with music learning, for example by exploring the use of technology and the design of collaborative learning environments, and both these goals are easily realized in a rock band context for third-age learners." The key themes emerging from Laes' study were that the rock band was a context for learning and participation where the participants had the opportunity to explore their musical identities, and where they experienced a sense of social affirmation, pride and success, as successive challenges were overcome.

Intergenerational asynchronous collaboration using an online platform was the focus of a study reported by Belgrave and Keown (2018). In their research, an established music therapy choir comprising older adults aged 61-79 collaborated in a project with a youth choir, aged 9-14. The groups engaged in two "virtual exchanges," in the form of recordings of themselves shared via Dropbox. The first of these recorded exchanges consisted of an introduction to their choir, followed by a recorded performance of a piece of music that was deemed to be representative of the choir. The second exchange comprised a teaching demonstration, whereby each choir provided an audio recording of themselves teaching a favorite song to their counterparts. Following the virtual exchanges, the two choirs met in a face-to-face workshop and performance. The participants filled in pre and post measure of intergenerational attitudes and 
expectations of the intergenerational experience. A key finding of the project was that the use of a simple and free computer technology supported communication, learning, and enhanced relationships between the opposite generations. As the authors noted, "both generations reacted positively to the interactions and cited the [virtual] interactions as one of the top enjoyable factors of the project" (p. 7).

The reported research concerned with using technology to support collaborative playing and performing is scarce. However, there seems to be some indications that even simple technologies can indeed support musical learning amongst older people, that older adults happily engage with musical activities involving technology, and that there may be important potential wider benefits associated with the use of music technology in intergenerational contexts.

\section{Music and Movement With Creative Music Technologies}

One interactive and creative music technology that has perhaps been under-researched (although used widely in special needs contexts) is the Soundbeam, described as an "elastic keyboard in space that allows sound to be created without the need for physical contact with any equipment" [(Swingler, 1998), p. 2]. The Soundbeam works on the same principle as the Thereminvox, in that high frequency ultrasound beams, inaudible to the human ear, are emitted and movements within those beams are transformed into musical sounds. However, in contrast to the Thereminvox, the Soundbeam, which translates distance and movement data into a digital code that is interpreted by any electronic instrument or sound, offers infinite possibilities for the quality of soundscape that is created. A second key difference that distinguishes the Soundbeam from the Thereminvox is that the beams emitted by Soundbeam can be adjusted to lengths varying from a few centimeters to several meters, making it possible to control musical sound with movements ranging from very small to very big. In addition, the sounds created can be transposed, making it possible to integrate the Soundbeam within collaborative musicmaking involving acoustic instruments. Finally, the Soundbeam offers scope for adjusting the number and sequence of notes captured within each beam (e.g., a particular scale with a specific number of octaves), to define the articulation required to trigger sound, and to adjust specific qualities of the sound produced. Thus, individuals with varying degrees of mobility are enabled to participate in intentional and collaborative musical activities, with the scope for making aesthetic choices, expressing imaginative ideas, exercising choice, developing listening skills, enhancing confidence, developing spatial awareness, and refining motor skills (Russell, 1996; Swingler and Brockhouse, 2009).

The Soundbeam has been found to be "the single most frequently used electronic music technology" by UK music therapists (Magee and Burland, 2008, p. 125). A survey of 22 special educational needs schools in the UK revealed that $30 \%$ of music therapists within those contexts had used music technology, and of those $30,76 \%$ had used the Soundbeam in their practice (Farrimond et al., 2011). However, the overall use of assistive technology in music therapy is not yet widespread, and much of the published evidence in that domain is concerned with the use of electronic music technologies with children or adolescents (Magee, 2013). For example, Hahna et al. (2012) carried out a survey of technology use within music therapy contexts in Australia, Canada, the UK and the USA. Overall, $71 \%$ of survey respondents reported using music technology in their clinical practice, but of those just $16 \%$ used technology with clients over the age of 65 .

A notable exception is Ellis (2004), who reports a longitudinal case study example of using digital music technologies with an elderly resident (aged mid-80s) within a long-term care context, who had been languishing following a stroke. Aiming to improve communication, motor control, and well-being, Ellis had developed the Vibroacoustic Sound Therapy (VAST), comprising exploration with a microphone and sound processor (supporting enhanced interactive communication skills), musicmaking with the Soundbeam (supporting independent physical movement and control, extending listening range, awakening curiosity, enabling self-expression), and dedicated time relaxing in the "Soundchair," where recorded low-frequency calming music is intended to promote physical and mental well-being and to potentially trigger memories and reminiscence.

Ellis (2004) describes a layered approach to analysis of video recordings of several sessions over time, where VAST was applied. In the layered approach, significant moments from each session are extracted and layered one after another, creating a chronological representation of progression concerning specific behaviors or responses. Engaging with the Soundbeam, the participant "spontaneously started to use her left arm to control sound in most expressive ways," and after several weeks she was reported to be generally more calm, communicative, cheerful, and sociable. These findings are framed by a summary of field notes gathered over several years, documenting the effects of VAST for over 35 individuals in later-life therapeutic contexts., where Ellis reports having observed improved communication, mobility, opportunities for individual exploration and control, deep relaxation, pleasure, well-being, and self-esteem. The importance of facilitation strategies is highlighted by Ellis, who cautions that technology must be mobilized in such a way as to support intrinsic motivation and a locus of control in the user.

With a similar focus on using technology to promote interaction with sound, a more recent Research through Design project, whereby prototypes are developed through a reflective process involving user engagement in the field, addressed the question of whether a music player could inspire seniors to be more physically active (Rosseland, 2016; Rosseland and Culén, 2016). The researchers describe the development of RepMoves, articulating "a generic interaction design concept to motivate physical activity through rhythmic interaction with music" (Rosseland and Culén, 2016, p. 108). Using a motion sensor, the RepMoves prototypes adjusted the tempo of its music to the tempo of five specific movement patterns performed by the user. Essentially, the user's role is conceptualized rather like an orchestral conductor, with his or her body movements such as arm swings or body sways dictating the tempo of the "orchestra" (in this case, the music player RepMoves). RepMoves prototypes 
were tested with groups of older people diagnosed with early stage Alzheimers Disease. Through exploratory sessions using familiar songs, collaborative movement with RepMoves was found to be possible, stimulating entrainment, or moving in synchrony with one another, a phenomenon that has been associated with positive well-being (Gill, 2012). Visual feedback in the form of photographs and videos was also added, offering an experience close to a virtual reality. The researchers illustrate that, through the conceptual exploration with older people, they "were able to explore and understand how a range of contextual, personal and social factors could influence the future adoption of the RepMoves concept in specific contexts" (Rosseland and Culén, 2016, p. 116).

The idea of using motion sensor technology was likewise explored by Scott Reis et al. (2012). Their research and development project was framed by the underpinning principles of accessibility, inclusion and social participation. The aim was to develop a bespoke music technology for use by the elderly that would "allow musical expressiveness through motion, solely using the resources available in an ordinary home computer" (2012, p. 211). A prototype was developed that recognized motion (using a computer camera), and translated it to an audio signal that was then reproduced as audio, with low latency. The prototype was tested with a group of eight older people with an average age of 83 , none of whom had background experience with music-making or with using computers. The participants were curious, excited and happy to explore the new technology, and seven out of the eight participants succeeded in making expressive musical sounds within 10 min trials.

Overall, the limited research that has thus far addressed the question of how technology can encourage expressive movement with music points to strong potential within gerontechnology for the development of music technologies that support enhanced mobility, expressivity through music, with implications for positive well-being.

\section{DISCUSSION}

This review of literature has demonstrated that the design and use of creative music technologies intended to enrich later life remains an under-researched area. Much of the literature that does exist focuses on music technology as an assistive tool within therapeutic contexts, while there is very little research, to date, that explores the affordances or challenges relating to engagement with music technologies amongst older people living independently within the community. Furthermore, there is limited research concerned with what inclusive practices using music technologies in the community, with intergenerational or later-life groups, might entail. However, this review does synthesize a growing body of research, design and practice that has recognized the significance of music as a continuing ability that may be supported in personally meaningful, creative and differentiated ways with accessible music technologies (Lazar et al., 2014).

The literature reviewed in this paper suggests that older people, even those with complex needs, are capable of engaging with technology and using technology in a range of ways that support their musical perception, learning and participation (e.g., Pike, 2011; Laes, 2014; Smith et al., 2017). This evidence is in accordance with research concerned with later-life musical engagement more generally, where it has been demonstrated that older learners develop compensatory strategies to mitigate physical or cognitive constraints (Gembris, 2008). Technologies that support receptive musicking (e.g., listening devices) as well as active musicking (e.g., motion sensor devices, digital musical instruments, singing apps and music composition or improvisation technologies) have been found to support access to the multiple personal, social, cognitive, and physical benefits that have been associated with musicking (e.g., Ellis, 2004; Rosseland, 2016; Reid et al., 2017; Vahia et al., 2017). Furthermore, music technologies have been shown to function as creative tools that may have the capacity to provide "restorative spaces" (Jansen, 2005) that privilege reflection and reminiscence, personal healing and problem solving (Duffey et al., 2008; Somody, 2010; Connell, 2012; Lazar et al., 2014). These activities may be of particular importance at a stage of life where the potential for creative growth and expression (Erikson and Erikson, 1998; Weisberger, 2013) may be overshadowed by narratives of decline (Findsen, 2005). Thus, the creative use of music technologies, as evidenced in this literature review, offer strong potential to serve as a vehicle whereby older people may overcome barriers to musicking and, through music, make substantive connections in their lives (Duffey et al., 2008) and engage in purposeful and enriching activities.

What then are the underpinning theoretical principles that could be said to frame the design of creative music technologies that enrich the lives of older people? The literature reviewed here demonstrated that that effective and innovative design of music technologies for older adults requires cognizance of the diversity amongst the intended audience. Indeed, it has been argued that as people accumulate life experience they become more different than similar, and that attempts to characterize older people as a homogeneous group thus risks being /misguided and ageist" (Withnall, 2010, p. 119). The evidence that diverse groups of older people can and do engage with music technologies may thus encourage designers to reflect upon their own underlying assumptions about older people and to engage with research and development approaches that place the users at the heart of a design process with a strong element of user testing and feedback in the field (Sixsmith et al., 2007). Approaches to the design of later-life music technologies may be particularly salient. When they are: (1) inclusive in embracing an ecological model of quality of life (Sixsmith et al., 2007) that recognizes the prior experience and wider socio-economic and cultural characteristics of participants' lives; (2) accessible, in facilitating engagement and allowing older adults to recognize and implement compensatory user strategies; (3) are assistive, in enabling interdependent creative expression, learning and participation, and (4) universal, in the sense that the technologies function as a space for the collaborative and social practice of musicking.

Some limitations to this review relate to the rapid advances in technology itself. As the review was limited to published peerreviewed journal articles, some cutting-edge innovations in the 
use of music technology in later-life contexts, not yet the focus of published empirical user-studies, may have been omitted. Indeed, excluded records included, for example, conference proceedings that reported the design and development of piano-playing robots intended to foster positive affective states among older people (Park et al., 2008), computer software using motion technology to foster musical expressiveness (Reis et al., 2012), as well as mobile Apps intended to promote easy access to personally meaningful music (Nezerwa et al., 2014; Wang and Tan, 2015). Notwithstanding their non-inclusion in the systematic review reported here, these sources reinforce the key finding that older people can and do engage with technology, and that the development of technologies that facilitate (1) access to preferred music and (2) the use of music to reduce stress, particularly among persons living with dementia, has been of particular interest. A further limitation is language. This systematic review represents peer reviewed studies published in English; yet our aging population is a global issue of concern to researchers worldwide working in the interdisciplinary fields of gerontechnology and music, health, and well-being.

In conclusion, notwithstanding the limitations noted above, this review provides a strong rationale for exploring and developing the landscape comprising music technologies for older people in a range of diverse contexts. Arguments focused

\section{REFERENCES}

Allen, M. K. (2013). Consumption of Culture by Older Canadians on the Internet (75 006 X). Available online at: http://www.statcan.gc.ca/pub/75-006-x/2013001/ article/11768-eng.htm

Allison, T. A. (2008). "Songwriting and transcending institutional boundaries in the nursing home," in The Oxford Handbook of Medical Ethnomusicology, ed. B. D. Koen (NewYork, NY: Oxford University Press), 218-245.

Belgrave, M. J., and Keown, D. J. (2018). Examining cross-age experiences in a distance-based intergenerational music project: comfort and expectations in collaborating with opposite generation through "virtual" exchanges. Front. Med. 5:214. doi: 10.3389/fmed.2018.00214

Bugos, J. A., Perlstein, W. M., McCrae, C. S., Brophy, T. S., and Bedenbaugh, P. H. (2007). Individualized Piano Instruction enhances executive functioning and working memory in older adults. Aging Ment. Health 11, 464-471. doi: 10.1080/13607860601086504

Cappelen, B., and Anderson, A.-P. (2014). "Designing four generations of Musicking Tangibles," in Music, Health, Technology and Design, ed. K. Stansaeth (Oslo: NMH-publications), 1-19.

Chambers, L. W., Bancej, C., and McDowell, I. (2016). Prevalence and Monetary Costs of Dementia in Canada: Population Health Expert Panel. Toronto: Alzheimer Society Canada in collaboration with the Public Health Agency of Canada.

Chang-Quan, H., Xue-Mei, Z., Bi-Rong, D., Zhen-Chan, L., Ji-Rong, Y., and Qing-Xiu, L. (2010). Health status and risk for depression among the elderly: a meta-analysis of published literature. Age Ageing 39, 23-30. doi: 10.1093/ageing/afp187

Charness, N., and Boot, W. (2009). Aging and information technology use: potentials and barriers. Curr. Direc. Psychol. Sci. 18, 253-258. doi: 10.1111/j.1467-8721.2009.01647.x

Clift, S., Hancox, G., Staricoff, R., and Whitmore, C. (2008). Singing and Health: Summary of a Systematic Mapping;and Review of Non-Clinical Research. Canterbury.

Cohen, G. (2006). Research on creativity and aging: the positive impact of the arts on health and illness. Generations 30, 7-15. Available online around quality of life, cognitive function, social interaction, and supporting mobility all point to the need for research and development in this area. Perhaps the strongest argument of all though is the view that innovative music technologies may enable access to joyful, creative, and restorative experience, throughout our later lives.

\section{AUTHOR CONTRIBUTIONS}

The author confirms being the sole contributor of this work and has approved it for publication.

\section{FUNDING}

This review was supported by funding from the Social Sciences Research Council of Canada, grant number 890-2017-0021: Creative later-life in a digital age: mobilizing music and creative technologies for inclusive later-life musical learning and participation, creative expression, digital literacy, and quality of life.

\section{ACKNOWLEDGMENTS}

The author wishes to acknowledge the support of the Social Sciences and Humanities Research Council of Canada.

at: https:/www.agingkingcounty.org/wp-content/uploads/sites/185/2016/07/ RESEARCH-ON-CREATIVITY-AND-AGING.pdf

Connell, M. (2012). Talking about old records: generational musical identity among older people. Pop. Music 31, 261-278. doi: 10.1017/S0261143012000074

Creech, A., Hallam, S., McQueen, H., and Varvarigou, M. (2013a). The power of music in the lives of older adults. Res. Stud. Music Educ.35, 83-98. doi: 10.1177/1321103X13478862

Creech, A., Hallam, S., Varvarigou, M., Gaunt, H., McQueen, H., and Pincas, A. (2014a). The role of musical possible selves in supporting subjective well-being in later life. Music Educ. Res. 16, 32-49. doi: 10.1080/14613808.2013.788143

Creech, A., Hallam, S., Varvarigou, M., and McQueen, H. (2014b). Active Ageing With Music: Supporting Well Being in the Third and Fourth Ages. London: IOE Press.

Creech, A., Hallam, S., Varvarigou, M., McQueen, H., and Gaunt, H. (2013b). Active music making: a route to enhanced subjective well-being amongst older people. Perspect. Public Health 133, 36-43. doi: 10.1177/17579139124 66950

Damant, J., and Knapp, M. (2015). What are the Likely Changes in Society and Technology Which Will Impact Upon the Ability of Older Adults to Maintain Social (extra-familial) Networks of Support Now, in 2025 and in 2040? London: Foresight, Government Office for Science Available online at: www.gov.uk/goscience

Davison, T. E., Nayer, K., Coxon, S., de Bono, A., Eppingstall, B., Jeon, Y. H., et al. (2016). A personalized multimedia device to treat agitated behavior and improve mood in people with dementia: a pilot study. Geriatr. Nurs. 37, 25-29. doi: 10.1016/j.gerinurse.2015.08.013

Duffey, T., Somody, C., and Clifford, S. (2008). Conversations with my father: adapting a musical chronology and the emerging life song with older adults. J. Creativity Mental Health 2, 45-63. doi: 10.1300/J456v02n04_05

Elliott, D. J., and Silverman, M. (2015). Music Matters 2nd ed. New York, NY: Oxford University Press.

Ellis, P. (2004). Vibroacoustic sound therapy: case studies with children with profound and multiple learning difficulties and the elderly in long-term residential care. Stud. Health Technol. Inform. 103, 36-42. doi: 10.3233/978-1-60750-946-2-36 
Erikson, E. H., and Erikson, J. M. (ed.). (1998). The Life Cycle Completed: Extended Version with New Chapters on the Ninth Stage of Development. Kindle Edition. New York, NY: W.W. Norton \& Company.

Farrimond, B., Gillard, D., Bott, D., and Lonie, D. (2011). Engagement with Technology in Special Educational and Disabled Music Settings. Available online at: https://network.youthmusic.org.uk/file/5694/download?token=I-1K0qhQ

Findsen, B. (2005). Learning Later. Malabar, FL: Krieger Publishing Co.

Fozard, J. L., Rietsema, J., Bouma, H., and Graafmans, J. A. M. (2000). Gerontechnology: creating enabling environments for the challenges and opportunities of aging. Educ. Gerontol. 26, 331-344. doi: 10.1080/036012700407820

Fung, V., and Lehmberg, L. (2016). Music for Life: Music Participation and Quality of Life of Senior Citizens. New York, NY: Oxford University Press and Oxford Scholarship Online. doi: 10.1093/acprof:oso/9780199371686.001.0001

Garland, K., Beer, E., Eppingstall, B., and O'Connor, D. W. (2007). A comparison of two treatments of agitated behavior in nursing home residents with dementia: simulated family presence and preferred music. Am. J. Geriatr. Psychiatry 15, 514-521. doi: 10.1097/01.JGP.0000249388.37080.b4

Gembris, H. (2008) 10-12 September. "Musical activities in the third age: an empirical study with amateur musicians," in Proceedings from the Second European Conference on Developmental Psychology of Music, eds. A. Daubney, E. Longhi, A. Lamont, and D. J. Hargreaves (England: Roehampton University), 103-108

Gill, S. P. (2012). Rhythmic synchrony and mediated interaction: toward a framework of rhythm in embodied interaction. AI Society 27, 111-127. doi: 10.1007/s00146-011-0362-2

Hahna, N. D., Hadley, S., Miller, V. H., and Bonaventura, M. (2012). Music technology usage in music therapy: a survey of practice. Arts Psychother. 39, 456-464. doi: 10.1016/j.aip.2012.08.001

Hallam, S., Creech, A., Varvarigou, M., and McQueen, H. (2012). The characteristics of older people who engage in community music making, their reasons for participation and the barriers they face. J. Adult Contin. Educ. 18, 21-43. doi: 10.7227/JACE.18.2.3

Heinz, M., Martin, P., Margrett, J., Yearns, M., Franke, W., Yang, H., et al. (2013). Perceptions of technology among older adults. J. Gerontol. Nurs. 39, 42-51. doi: 10.3928/00989134-20121204-04

Helfert, M., Holzinger, A., Ziefle, M., Fred, A., O’Donoghue, J., and Röcker, C. (2015). "Information and Communication Technologies for Ageing Well and e-Health: Revised selected papers," in The First International Conference, May 20-22 (ICT4AgeingWell 2015, Lisbon).

Himonides, E., and Purves, R. (2010). "The role of technolog," in Music Education in the 21st Century in the United Kingdom, eds. S. Hallam and A. Creech (London: Institute of Education, University of London), 123-140

Hollnagel, E., and Woods, D. D. (2005). Joint Cognitive Systems: Foundations of Cognitive Systems Engineering. Boca Raton, FL: CRC Press. doi: 10.1201/9781420038194

Jansen, D. A. (2005). Perceived barriers to participation in mentally restorative activities by community-dwelling elders. Act. Adapt. Aging 29, 35-53. doi: 10.1300/J016v29n02_03

Jansen, D. A., and von Sadovszky, V. (2004). Restorative activities of communitydwelling elders. West. J. Nurs. Res. 26, 381-399. doi: $10.1177 / 0193945904263010$

Jenkins, A. (2011). Participation in learning and wellbeing among older adults. Int. J. Lifelong Educ. 30, 403-420. doi: 10.1080/02601370.2011.570876

Kaplan, M., Sánchez, M., Shelton, C., and Bradley, L. (2013). Using Technology to Connect Generations. Penn State University \& Washington D.C. Generations United. Available online at: http://extension.psu.edu/youth/intergenerational/ program-areas/technology

Laes, T. (2014). Empowering later adulthoodmusic education: a case study of a rock band for third-age learners. Int. J. Music Educ. 33, 51-65. doi: $10.1177 / 0255761413515815$

Lancioni, G. E., Singh, N. N., O’Reilly, M. F., Sigafoos, J., D’Amico, F., Sasnelli, G., et al. (2014). Persons with moderate Alzheimer's disease use simple technology aids to manage daily activities and leisure occupation. Res. Dev. Disabil. 35, 55-63. doi: 10.1016/j.ridd.2014.05.002

Lazar, A., Thompson, H., and Demiris, G. (2014). A systematic review of the use of technology for reminiscence therapy. Health Educ. Behav. 41(1 Suppl.), 51S-61S. doi: 10.1177/1090198114537067
Lin, F. R., Thorpe, R., Gordon-Salant, S., and Ferrucci, L. (2011). Hearing loss prevalence and risk factors among older adults in the United States. J. Gerontol. 66A, 582-590. doi: 10.1093/gerona/glr002

Magee, W. L. (Ed.). (2013). Music Technology in Therapeutic and Health Settings. London: Jessica Kingsley Publishers.

Magee, W. L., and Burland, K. (2008). An exploratory study of the use of electronic music technologies in clinical music therapy. Nord. J. Music Ther. 17, 124-141. doi: 10.1080/08098130809478204

Miquel-Romero, M. J., and Montoro-Pons, J. D. (2017). Consumption habits, perception and positioning of content-access devices in recorded music. Int. J. Arts Manag. 19, 4-18.

Nezerwa, M., Wright, R., Howansky, S., Terranova, J., Carlsson, X., Robb, J., et al. (2014). "Alive inside: developing mobile apps for the cognitively impaired," in IEEE Long Island Systems, Applications and Technology (LISAT) Conference 2014 (Farmingdale, NY).

Park, K.-H., Jeong, S.-H., Pelczar, C., and Bien, Z. Z. (2008). Beat gesture recognition and finger motion control of a piano playing robot for affective interaction of the elderly. Intell. Serv. Rob. 1, 185-193. doi: 10.1007/s11370-008-0018-3

Pike, P. D. (2011). Using technology to engage third-age (retired) leisure learners: a case study of a third-age MIDI piano ensemble. Int. J. Music Educ. 29, 116-123. doi: $10.1177 / 0255761410396965$

Poushter, J. (2016). Smartphone Ownership and Internet Usage Continues to Climb in Emerging Economies: But advanced economies Still Have Higher Rates of Technology Use (202.419.4372). Washington DC Available online at: http:// www.pewglobal.org/2016/02/22/smartphone- ownership-and-internet-usagecontinues-to-climb-in-emerging-economies/

Reid, A. G., Rakhilin, M., Patel, A. D., Urry, H. L., and Thomas, A. K. (2017). New technology for studying the impact of regular singing and song learning on cognitive function in older adults: a feasibility study. Psychomusicology 27 , 132-144. doi: 10.1037/pmu0000179

Reis, Lee, S.cott., Reis, G., Barroso, J., and Pereira, A., n. (2012). "AMIGA An interactive musical environment for Gerontechnology," in The Proceedings of the 4th International Conference on Software Development for Enhancing Accessibility and Fighting Info-Exclusion (DSAI 2012), (Douro Region, Portugal).

Rosseland, R. B. (2016). Design and evaluation of an interactive music system for exercise and physical activity with Alzheimer's patients. SoundEffects 6, 4-22. Available online at: https://www.soundeffects.dk/article/view/24910

Rosseland, R. B., and Culén, A. L. (2016). Repmoves: stories that a rhythmic interaction device for seniors can tell. Int. J. Comp. Sci. Inform. Syst. 11, 104-118. Available online at: http://www.iadisportal.org/ijcsis/papers/ 2016190208.pdf

Russell, K. (1996). Imagining the music, exploring the movement: soundbeam in the sunshine state. Qld. J. Music Educ. 4, 41-48.

Ruthmann, S. A., and Hebert, D. (2012). "Music learning and new media in virtual and online environments," in Oxford Handbook of Music Education, Vol. 2, eds. G. McPherson and G. Welch. (New York: Oxford University Press). 567-583

Samuels, K. (2014). "Enabling Creativity: inclusive music practices and interfaces," in The International Conference on Live Interfaces (ICLI), (Lisbon).

Scott Reis, L., Reis, G., Barroso, J., and Pereira, A. (2012). AMIGA - an interactive musical environment for gerontechnology. Proc. Comp. Sci. 14, 208-217. doi: 10.1016/j.procs.2012.10.024

Sixsmith, A., and Gutman, G. (2013). Technologies of Active Aging (Vol. 9). New York: Springer. doi: 10.1007/978-1-4419-8348-0

Sixsmith, A., Orpwood, R., and Torrington, J. (2007). Quality of life technologies for people with dementia. Top. Geriatr. Rehabil. 23, 85-93. doi: 10.1097/00013614-200701000-00011

Smith, L., Bartel, L., Joglekar, S., and Chen, J. (2017). Musical rehabilitation in adult cochlear implant recipients with a self-administered software. Otol. Neurotol. 38, E262-E267. doi: 10.1097/MAO.00000000000 01447

Somody, C. (2010). Meaning and Connections in Older Populations: A Phenomenological Study of Reminiscence Using a Musical Chronology and the Emerging Life Song. Doctor of Philosophy in Counselor Education and Supervision Dissertation, The University of Texas at San Antonio, San Antonio. Stensaeth, K., and Magee, W. (2016). "The future of technology in music therapy: towards collaborative models of practice," in Envisioning the Future 
of Music Therapy, ed. C. Dileo (Philadelphia, PA: Temple University's Arts and Quality of Life Research Center). Available online at: www.temple.edu/boyer/ researchcenter 148-157.

Stige, B. (2012). "Health Musicking: a perspective on music and health as action and performance," in Music, Health and Wellbeing eds. R. Macdonald, G. Kreutz, and L. Mitchell (Chapter 14) (Oxford: Oxford University Press; Oxford Scholarship Online). doi: 10.1093/acprof:oso/9780199586974.003.0014

Swingler, T. (1998). That Was Me $i$ : Applications of the "Soundbeam MIDI Controller as a Key to Creative Communication, Learning, Independence and Joy," in The California State University Northridge Conference on Technology and Persons with Disabilities, (Los Angeles, CA).

Swingler, T., and Brockhouse, J. (2009). Getting better all the time: using music technology for learners with special needs. Austral. J. Music Educ. 2, $49-57$.

Tak, S. H., Zhang, H. M., Patel, H., and Hong, S. H. (2015). Computer activities for persons with dementia. Gerontologist 55, S40-S49. doi: 10.1093/geront/gnv003

United Nations (2017). World Population Ageing (ST/ESA/SER.A/408). Available online at: http://www.un.org/en/development/desa/population/publications/ pdf/ageing/WPA2017_Report.pdf

United Nations, Department of Economic and Social Affairs, Population Division. (2013). World Population Prospects: The 2012 Revision, Highlights and Advance Tables. Working Paper No. ESA/P/WP.228. Available online at: https:// population.un.org/wpp/Publications/Files/WPP2012_HIGHLIGHTS.pdf

Vahia, I. V., Kamat, R., Vang, C., Posada, C., Ross, L., Oreck, S, et al. (2017). Use of tablet devices in the management of agitation among inpatients with dementia: an open-label study. Am. J. Geriatr. Psychiatry 25, 860-864. doi: 10.1016/j.jagp.2016.07.011
Wang, D., and Tan, A. H. (2015). "MyLife: An Online Personal Memory Album," in The 2015 Ieee/Wic/Acm International Conference on Web Intelligence and Intelligent Agent Technology (Singapore). doi: 10.1109/WI-IAT. 2015.148

Warschauer, M., and Matuchniak, T. (2010). New technology and digital worlds: analyzing evidence of equity in access, use, and outcomes. Rev. Res. Educ. 34, 179-225. doi: 10.3102/0091732X09349791

Weisberger, A. (2013). "Garageband as a digital co-facilitator: creating and capturing moments with adults and elderly people with chronic health conditions," in Music Technology in Therapeutic and Health Settings, ed W. L. Magee (London: Jessica Kingsley Publishers), 279-293.

Withnall, A. (2010). Improving Learning in Later Life. Abingdon, Oxon: Routledge. World Health Organization (2015). World report on Ageing and Health. Retrieved from Geneva: Available online at: http://apps.who.int/iris/ bitstream/handle/10665/186463/9789240694811_eng.pdf;jsessionid= 894E75AD6F26056D2FD4CA2366A89D19? sequence $=1$

Conflict of Interest Statement: The author declares that the research was conducted in the absence of any commercial or financial relationships that could be construed as a potential conflict of interest.

Copyright (c) 2019 Creech. This is an open-access article distributed under the terms of the Creative Commons Attribution License (CC BY). The use, distribution or reproduction in other forums is permitted, provided the original author(s) and the copyright owner(s) are credited and that the original publication in this journal is cited, in accordance with accepted academic practice. No use, distribution or reproduction is permitted which does not comply with these terms. 


\section{OPEN ACCESS}

Edited by: Michele Biasutti, University of Padova, Italy

Reviewed by: Mats B. Küssner, Humboldt University of

Berlin, Germany

Dan Zhang,

Tsinghua University, China

*Correspondence: Jörg C. Fachner jorg.fachner@anglia.ac.uk

Specialty section: This article was submitted to Performance Science, a section of the journal

Frontiers in Psychology

Received: 31 January 2019 Accepted: 20 June 2019 Published: 25 July 2019

Citation:

Fachner JC, Maidhof C, Grocke D, Nygaard Pedersen I, Trondalen G,

Tucek G and Bonde LO (2019) "Telling me not to worry..." Hyperscanning and Neural Dynamics of Emotion Processing During Guided Imagery and Music. Front. Psychol. 10:1561. doi: 10.3389/fpsyg.2019.01561

\section{"Telling me not to worry..." Hyperscanning and Neural Dynamics of Emotion Processing During Guided Imagery and Music}

\author{
Jörg C. Fachner ${ }^{1,2 *}$, Clemens Maidhof ${ }^{1,2}$, Denise Grocke ${ }^{3}$, Inge Nygaard Pedersen ${ }^{4}$, \\ Gro Trondalen ${ }^{5}$, Gerhard Tucek ${ }^{2}$ and Lars O. Bonde ${ }^{4,5}$ \\ ${ }^{1}$ Cambridge Institute for Music Therapy Research, Anglia Ruskin University, Cambridge, United Kingdom, ${ }^{2}$ Josef Ressel \\ Centre for Personalised Music Therapy, IMC University of Applied Sciences Krems, Krems an der Donau, Austria, \\ ${ }^{3}$ Melbourne Conservatorium of Music, University of Melbourne, Melbourne, VIC, Australia, ${ }^{4}$ Department of Communication \\ and Psychology, The Faculty of Humanities, Aalborg University, Aalborg, Denmark, ${ }^{5}$ Centre for Research in Music and \\ Health, Norwegian Academy of Music, Oslo, Norway
}

To analyze how emotions and imagery are shared, processed and recognized in Guided Imagery and Music, we measured the brain activity of an experienced therapist ("Guide") and client ("Traveler") with dual-EEG in a real therapy session about potential death of family members. Synchronously with the EEG, the session was video-taped and then micro-analyzed. Four raters identified therapeutically important moments of interest $(\mathrm{MOI})$ and no-interest $(\mathrm{MONI})$ which were transcribed and annotated. Several indices of emotion- and imagery-related processing were analyzed: frontal and parietal alpha asymmetry, frontal midline theta, and occipital alpha activity. Session ratings showed overlaps across all raters, confirming the importance of these MOls, which showed different cortical activity in visual areas compared to resting-state. $\mathrm{MOI} 1$ was a pivotal moment including an important imagery with a message of hope from a close family member, while in the second $\mathrm{MOI}$ the Traveler sent a message to an unborn baby. Generally, results seemed to indicate that the emotions of Traveler and Guide during important moments were not positive, pleasurably or relaxed when compared to resting-state, confirming both were dealing with negative emotions and anxiety that had to be contained in the interpersonal process. However, the temporal dynamics of emotion-related markers suggested shifts in emotional valence and intensity during these important, personally meaningful moments; for example, during receiving the message of hope, an increase of frontal alpha asymmetry was observed, reflecting increased positive emotional processing. EEG source localization during the message suggested a peak activation in left middle temporal gyrus. Interestingly, peaks in emotional markers in the Guide partly paralleled the Traveler's peaks; for example, during the Guide's strong feeling of mutuality in $\mathrm{MOI} 2$, the time series of frontal alpha asymmetries showed a significant cross-correlation, indicating similar emotional processing in Traveler and Guide. Investigating the moment-to-moment interaction in music therapy showed how 
asymmetry peaks align with the situated cognition of Traveler and Guide along the emotional contour of the music, representing the highs and lows during the therapy process. Combining dual-EEG with detailed audiovisual and qualitative data seems to be a promising approach for further research into music therapy.

Keywords: music therapy, moments of interest, dyadic interaction, imagery, emotion, EEG, alpha asymmetry, social neuroscience

\section{INTRODUCTION}

Musical imagery research investigates imagination of intervals, melodies and other musical elements in order to compare them to the listening process (Hubbard, 2010). This may include any imagery of sound and music where there is no physical source, e.g. when conductors study scores or composers compose without a piano, or when participants are asked to recall and imagine what they've heard before (Schaefer et al., 2011). However, in this explorative study, we were primarily interested in shared visual imagery and emotion related to music listening occurring in a therapeutic setting with a therapist and a client, while the client is in an altered state of consciousness (ASC). Listening to music can completely absorb people, cutting off other sensory input, but absorption skills seem to be linked to music preference, imagery, hypnotizable and intensity of emotions evoked (Snodgrass and Lynn, 1989; Kreutz et al., 2008; Schäfer et al., 2013). The goal of this case study was to test a series of subsequent propositions regarding emotion-, imagery-, and event-related activity in frontal and parietal regions of the brain.

\section{Spontaneous Imagery, Emotion and Gaining Insight}

In the Bonny Method of Guided Imagery and Music (BMGIM or here GIM), a client (in GIM often referred to as "the Traveler" doing an imaginary "journey," while the therapist is referred to as "the Guide") describes images, feelings, or thoughts that occur spontaneously while eyes-closed listening to special music programs in an induced ASC (Bonny, 2002). Imagery or image refers to:

"experiences of music during the listening phase of BMGIM, including images in all sensory modalities, kinesthetic images, body sensations, feelings, thoughts and noetic images (an intuitive sense of imaginal events that arise outside of other imagery modes)." (Goldberg, 2002, p. 360).

Commonly, certain passages during the imagery process will have pivotal meaning for the Traveler and become a focus in the therapy process (Grocke, 1999). A pivotal moment is a turning point in which a client makes a transition. Getting in touch with one's own emotions and gaining personal insight during such moments in the imagery process seems to be a

Abbreviations: AA, Alpha Asymmetries; ASC, Altered States of Consciousness; EEG, Electroencephalogram; FAA, Frontal Alpha Asymmetry; FMT, Frontal Midline Theta; GIM, Guided Imagery and Music; MOI, Moments of Interest; MONI, Moments of No-Interest; MTG, Middle Temporal Gyrus; PAA, Parietal Alpha Asymmetry; ROI, Region of Interest. driving force of change (Maack and Nolan, 1999). Specifically, "gaining new insight" or discovery of new perspectives is considered to be an important theme during such "transforming" moments (Lin et al., 2010), although the imagery itself is diverse and multimodal, including visual, auditory, somatic, direct memories, involuntary and unbidden imagery, images of significant people, places and events from the person's history (Bonde, 2019). For an overview of imagery categories in GIM see (Grocke, 1999, p. 15).

\section{Spontaneous Imagery and Interactive Processes}

A typical GIM session comprises an initial discussion of the Traveler's concerns, and defining a focus for the music and imagery experience. The Guide provides a relaxation induction for the Traveler who reclines with eyes closed, chooses a predetermined music program, or spontaneously chooses music to match the Traveler's imagery. As the music plays the Traveler describes any imagery, feeling, or thoughts, while the Guide facilitates occasionally with non-direct inquiries. Bruscia defined the Bonny method of GIM as

"an individual form of exploring consciousness, which involves spontaneous imaging in an expanded state of consciousness, to predesigned programs of classical music, while interacting with a guide who uses non-directive, non-analytical, music-based interventions within a client-centered orientation, ..." (Bruscia, 2002, p. 46; italics from author).

Of interest for this research is engaging in the client-therapist dialogue during the spontaneously occurring imagery during music listening in an ASC. The client is not just reporting on what he or she "sees" during the imagery process, it is surrendering to an intense process (Blom, 2011, 2014), it is a "lived experience during the imagery ... that occurs as the client interacts with the images" (Heinschel, 2002, p. 332) facilitated by the therapist as a "personalized response "(ibid. p. 326) during the therapy process.

In this research, we wanted to study the interaction between a client and a therapist during a real-world GIM session, in which a therapist accompanies and interacts to deepen the experience of the client's emerging images. We were interested in how spontaneously evoked and guided imagery is processed and shared with the therapist and investigated the temporal dynamics of emotional processing with EEG during imagery of important moments in GIM. Spontaneous imagery is essential for the therapeutic process (Trondalen, 2016), but how meaningful imagery emerges in the time-course of the therapy and how its temporal dynamics relate to emotional processing have not 
been investigated so far. Many randomized controlled trials showed that music therapy works, but how it works remains unclear (Maratos et al., 2011). As the therapeutic relationship is essential to music therapy action, investigating brain correlates of dyadic therapeutic processes is crucial to understand and explain how music therapy works (Fachner, 2014; Hunt, 2015). As dyadic recordings have not been done before in a music therapy context (and especially in GIM), this the first EEG hyperscanning study investigating shared emotional processing and temporal dynamics of its neural correlates.

\section{GIM and Temporal Neural Dynamics of Affective States}

Alan Lem, in a first but technically limited attempt to capture the continuous GIM process with EEG, music analysis and verbal reports, described frontal responses (slowing of the EEG and increase of amplitude) during distinct music passages, relaxation and occurrence of visual imagery indicating a "specific time of occurrence of a particular imagery response" (Lem, 1998, p. 15). A formal music analysis and profiling the music's affective expression divided the piece (G. Pierne's Concertstück for harp and orchestra) into 26 segments within 3 larger sections. EEG amplitude and frequency were synchronously plotted against the wave form of the music and correlations were explored. After an initial EEG slowing in the first section, fluctuations in the latter 2 sections, and an association between EEG bursts and reported occurrence of visual imagery at the end of the music's section 1 and 2 (see p. 9), several segment specific observations were described.

Of interest for us here is that the reported "correlations and associations" seem to convey a temporal dynamic between the identified affective expressions of the piece, the state changes as indicated in the frontal EEG and the corresponding imagery processes along the timeline of the GIM process of the 27 participants. The EEG changes are pointing to what Helen Bonny coined as the guiding "affective contour" (Bonny, 1978, p. 39), which is determined through the music and gives the therapist a more or less predictive tool to elicit particular emotions within an interactive narrative structure of the session.

Hunt (2011) went a step further in analyzing the imagery process, in a quasi-experimental condition, during a controlled Music \& Imagery session and analyzed responses to 6 probe modalities of the imagery experience (visual, kinesthetic, body, affect, memory and also interaction). Hunt provided a verbal probe for each of the categories and then analyzed the following $10 \mathrm{~s}$ of EEG. Additionally, she interviewed the participants about their experience based on a video of their session. In the interactive imagery condition (a probe of dialoguing with an imaginary person), stronger coherence was observed relating to localizations of the language centers (Broca and Wernicke) and a general observation was that "beta and gamma frequencies played a significant role in how participants ... made meaning out of the imagery" (Hunt, 2011, p. III). This may give a hint on how clients integrate new information and meaning of the imagery.

A neurometric GIM single case study of a GIM Traveler by Fachner et al. (2015) described distinct neurometric resting-state and ASC induction differences. ASC exhibited more Alpha zscore deviations than the resting-state when comparing the single case against a normative database. In addition, exploring source localization patterns of an important imagery segment of the therapy session returned highest cortical activation in cuneus and pre-cuneus. However, how the emotional involvement during the imagery can be studied and linked to the interaction of therapist and client is still an open question.

\section{Alpha Band, Emotion and Imagery}

Schaefer et al. (2011) investigated alpha band signatures induced by imagining and perceiving short natural, well-known musical phrases. Here imagery was interpreted as internally directed attention that would inhibit activity in visual areas bilaterally distributed in parieto-occipital areas of the brain. Indeed, participants exhibited individually shifting but increased parietooccipital alpha power topographies while imagining music. Moreover, Cooper et al. (2003) described frontal and occipital alpha responses while imagining pure tones and discussed increased alpha power not as idling but as an "index [of] active inhibition of non-task relevant cortical areas" (p. 73). In other words, if subjects turn their attention inwardly and start imagining, then alpha power indexes those parts in the brain topography that are actively inhibited (or idling) and thus show less cortical activity.

However, with regards to emotional responses evoked by music, particularly alpha power over frontal brain areas seems to indicate positive or negative emotions. Schmidt and Trainor (2001) showed that frontal alpha power distinguished the valence of musical excerpts. Participants exhibited greater relative left frontal EEG activity to "joy" and "happy" musical excerpts and greater relative right frontal EEG activity to fear and sad musical excerpts. When listening to music rated as representing positive valence, significant left frontal activity changes in DC-EEG were found (Altenmuller et al., 2002). Mikutta et al. (2012, p. 423) "aimed to identify the physiological correlates of continuous changes in subjective emotional states" while listening to a longer piece of music. They interpreted the observed rightfrontal suppression of alpha activity as being related to the emotional content independent of the dynamics expressed in loudness parameters. However, although subjective ratings were continuously recorded, temporal dynamics of the correlations and accordingly changes in the frontal processing were not investigated. Interestingly, Jäncke et al. (2015) presented an aria repeatedly to 16 participants and described "a remarkable variability in EEG oscillations, both within and between the repeated presentations of the aria" (p. 1) and an increase of eventrelated synchronization in all frequency bands. They concluded that the state of listening and attention on internal processes may best be characterized as mind wandering but "that the neurophysiological activations occurring during music listening are dynamic and not stationary" (ibid.).

\section{AIMS}

In this research we aim to explore spontaneous emerging imagery and how this is emotionally processed in a dyadic therapeutic 
relationship. We are interested how imagery and emotion processing are interacting in the therapeutic process and how the corresponding time series of neural correlates is related to the identified events in particular moments of therapeutic interest. As healing processes are known to deepen and intensify during particular moments in which meaningful events are situating cognitive processes, we aim to analyze authentic data of a music therapy process (Fachner, 2016, 2017).

In therapy, therapists and clients are immersed through the senses, interaction, embodiment and narrative experience in real-time contextually performed interpersonal action. Clinical experience and qualitative research suggests that spontaneously emerging imagery in the client is recognized by the therapist, but also from both as a mutual spontaneously occurring feeling (Blom, 2011, 2014). Such events may be accompanied with an unspecific emotion, recognizing a spontaneous occurring thought, making the therapist sense that "something" in the client has happened (Blom, 2014; Trondalen, 2016, 2019). Indices of such events may include a range of non-verbal indices and - as Rothschild (1962) and Watzlawick et al. (2014) would frame it biosemiotic interpunctions of the internal process, for example a change of prosodic features while talking, a different toning and intentionality of body posture, a change of facial expression and gesture and other context-related indicators of emotional processing. These indices may occur as serial and parallel biosemiotic epiphenomena of the internal imagery processes. Rothschild was interested in a system of semiotic symbols transmitted and received via bodily processes, while Watzlawick discussed that during interaction we can see a time series of interpunctions, i.e., sequences or moments in the communicative process that define the quality of the relationship. Therapists are trained to recognize such processes while they also become aware of sudden ideas which are not usually their own, and would interpret this as material that they recognize from the client:

\begin{abstract}
"The therapist was sensitive to small and subtle changes in the patient's gestures, pace, intensity and tone of voice, body language and facial expression. During the sessions, any changes in patients' language expressing imagery and their personal challenges or problems were observed. Gaining new insight, the patients attempted to change or control their negative emotions..." (Lin et al., 2010, p. 1146).
\end{abstract}

Recording a dual-EEG of a Traveler and a Guide in a GIM session may capture such emergence of spontaneous imagery with meaningful emotional content and allow for analysis of its time course.

\section{Spontaneous Imagery, Music, Shared Emotional States and Therapeutic Change}

"Unexpected change in musical features intensity and tempoand thereby enhanced tension and anticipation-is proposed to be one of the primary mechanisms by which music induces a strong emotional response in listeners" (Arjmand et al., 2017, p. 1). Other research on musical emotions supports this proposition (Huron, 2006; Juslin, 2013; Wärja and Bonde, 2014; Koelsch, 2015). However, Arjmand and colleagues investigated subjective and averaged, as well as temporal dynamics of EEG frontal asymmetry (FA) measures of experienced emotion of participants listening to self-selected music. They described a left pre-frontal response (indicating positive emotions) during pleasurable music but also peak FA responses "to co-occur with key musical events relating to change" (ibid).

However, a GIM session is not solely focusing on the music, but there is a "healing contract" (Fachner, 2017); that is, a therapeutic relationship is established between the Traveler and the Guide, who uses both the occurring imagery for psychotherapeutic purposes while verbally interacting to deepen the experience of the emerging images. According to the shared network hypothesis "observing or imaging another in a particular affective state activates a representation of the same state in the observer" (Acquadro et al., 2016, p. 7), while also activating similar emotion-specific networks with a "specificity in the temporal flow of the affect information from the sender to the perceiver" (ibid). In other words, there are moments in which we recognize and share meaningful emotional states in and with others and this seems to be mediated by shared neural representations and brain-to-brain coupling processes; the latter seem to be signified by increased theta- and alpha power crosscorrelations (for an overview, see Acquadro et al., 2016).

However, to find out about the meaning, the timing (synchronously or interpunctive) and the (sensory) mode of the emerging imagery (visual, kinesthetic, tactile, somatic gustatory, olfactory, auditory but in a therapeutic context also affect, memory, interaction; see Hunt, 2011), we need to know about its specific content and importance for the therapeutic process at a particular time-point during the therapy session.

\section{Moment of Interest (MOI) Identification}

Usually therapists select particular parts from one or a series of sessions to describe a narrative of therapeutic change processes in therapy (Bruscia, 1991; Hibben, 1999; Aldridge, 2004; Bonde, 2004). In a case study, there is usually one first-person perspective (from the therapist) on the therapy process and a selection of important and thus interesting episodes of a session or a series of sessions (Grocke and Moe, 2015; Hunt, 2016). These moments of interest may be indicative of a particular string of therapy progress, describe encounter between therapist and client, or represent pivotal change of behavior following the emergence of a particular important imagery and insight. In other words, therapists rate some segments of therapy to be of indicative importance for clients' development in therapy. Moments of interest (MOI) refer to the selected target sequences in a time series of clinically relevant events that are recognized and selected based on personal and/or professional preferences and interests (Fachner, 2017).

To analyze the particular parts of the process in more detail, microanalytic approaches to music therapy (Wosch and Wigran, 2007) utilize the selections and ratings of other therapists or non-therapists and try to achieve a degree of content validity of the chosen parts. Because asking only the Guide might lead to missing other aspects of the therapy (Spiro and Himberg, 2016), we asked both the Guide and the Traveler for their MOIs and additionally requested MOI selections from two other GIM 
therapy experts. If there would be important moments indicating change in the therapy progress, then these moments should be selected by all raters, resulting in an overlap of their selections, thus indicating a higher degree of content validity.

Utilizing an objectivist case study approach (Ridder and Fachner, 2016), we studied these MOIs and how they are contextualized in the overall progression of the therapy with a set of objective data, namely an EEG, which has an objective reality in itself, and allows inferences and propositions about the time series data of brain processing of both therapist and client.

\section{Brain Activity and Asymmetries}

Davidson (2004) attributed a leading role in processing emotion to the function of the frontal cortex regulating approach/withdrawal behavior. Thus, positive emotion and approach behavior would be processed in the left, while negative emotions and withdrawal would be linked to right frontal lobe activity (Harmon-Jones, 2003). According to Davidson, EEG activity reflects this activity on the cortical level as measured in alpha asymmetries at frontal leads, and for depression most prominently on the pre-frontal leads F3/4 (Gold et al., 2013), i.e., frontal alpha asymmetry (FAA). FAA is a well-established measure of emotional processing (Harmon-Jones et al., 2010; Smith et al., 2017) and offers a simple connectivity ratio (ln F4$\ln$ F3) between the homolog electrode parameters of the left and right frontal lobe. Positive values indicate dominant activity on the left frontal cortical side, while negative values vice versa. FAA and frontal measures indicated positive or negative valence of musical emotions (Field et al., 1998; Schmidt and Trainor, 2001; Altenmuller et al., 2002).

Heller and Nitscke (1997) introduced asymmetries of parietal alpha power indicating arousal and intensity of emotional processing, i.e., increased right parietal activity would indicate the arousal and therefore the intensity of emotions experienced (Schmidt and Trainor, 2001; Jakobi, 2009). Valence and arousal are discussed to represent the intensity of perceived and felt musical emotions (Eerola and Vuoskoski, 2013). However, parietal alpha asymmetries (PAA) are also discussed to indicate anxiety and withdrawal, but with inconclusive results (Stewart et al., 2011).

FAA and frontal biomarkers have been successfully applied in music therapy research correlating therapy effectiveness and brain activity changes in depression (Fachner et al., 2013), cancer and palliative care (Lee et al., 2012; Ramirez et al., 2018) and Disorders of Consciousness (O'Kelly et al., 2013). Comparing the effect of music therapy on depressed patients' resting-state EEG, frontal brain activity, as indicated in alpha power and asymmetries, was significantly different to those not receiving music therapy. Further, Frontal Midline Theta (FMT) correlated positively with the reduction of anxiety measures after music therapy (Fachner et al., 2013).

FMT has been utilized as an anxiety marker for anxiolytics (Mizuki et al., 1989; Mitchell et al., 2008). Increased FMT power is related to lower levels of anxiety, and has indicated states of internalized attention and positive emotional experience (Aftanas and Golocheikine, 2001). In music research comparing non- / preferred and non- / pleasurable music, an increase of FMT along the listening of pleasurable preferred music was observed (Sammler et al., 2007). Recently another study confirmed FMT increases over the time-course of listening to pleasurable music (Nemati et al., 2019).

Continuous measures of frontal activities seem also to be indicative of leading and following in social interaction (Konvalinka et al., 2014) and during duet guitar improvisation (Müller et al., 2013). Furthermore, mobile EEG applications allow authentic in situ recordings (Debener et al., 2012) and complex data integration (Maidhof et al., 2014). In short, frontal EEG measures are sufficient and promising tools to research therapy processes in real-world settings of music therapy (Fachner, 2016).

In this study, we were interested how the temporal FAA dynamics change according to the emotional impact of the emerging imagery and how this is related to the therapy process, whereby analysis of temporal FAA dynamics focuses on the asymmetry peaks in the time series (Allen and Cohen, 2010). In our study, we expect peaks to appear during important MOI segments and we will study the biosemiotic interpunctions of the peaks in those segments.

\section{Propositions Regarding Emotional Valence and Arousal During Selected MOls}

\section{Emotion-Related Activity}

Based on the studies discussed in above sections on "Spontaneous Imagery and Interactive Processes" and "Brain Activity and Asymmetries," we will explore the differences in several markers of emotional processing.

I. We expect differences between emotional processing during important moments and during the resting-state, and thus a difference in the alpha asymmetry (AA) measures.

- More specifically, compared to the resting-state, we expect during MOIs more left-shifted frontal AA (see Field et al., 1998), possibly indicating positive emotional valence, and more right-shifted parietal AA, possibly indicating emotional intensity/arousal (see Schmidt and Trainor, 2001).

II. We expect FMT power to increase during positive emotional processing (Aftanas and Golocheikine, 2001; Sammler et al., 2007).

\section{Imagery-Related Activity}

As discussed in section "Alpha Band, Emotion and Imagery," increased alpha power may index those brain areas that are less active during imagery and when attention is turned inwardly (see Cooper et al., 2003; Schaefer et al., 2011).

III. We expect the visual cortex to be more active (to exhibit less alpha power) during the imagery process than during the resting-state.

IV. Additionally, during GIM (compared to rest), we expect that temporo-parietal areas are less active (i.e. more alpha power) then occipital areas. 


\section{Event-Related Activity}

In the analysis of the temporal dynamics of emotional processing we will inspect the moment-to-moment interaction and the cooccurring event-related F/PAA during interesting moments.

V. We expect strong peaks to occur within imagery events of strong emotional valence (see Arjmand et al., 2017).

VI. We expect the peaks to indicate a temporal dynamic of dyadic biosemiotic interpunction of the therapy process, i.e., peaks indicating shared emotional processing (see Acquadro et al., 2016).

\section{MATERIALS AND METHODS}

\section{Participants}

Two right-handed, female healthy participants (age: 72 and 65 years old) took part in the EEG study. One of them was the Guide (therapist) and the other the Traveler (client), both with more than 20 years of experience in music therapy. Two experienced GIM therapists (age: 62 and 67 years) with about 20 years of GIM practice watched the video-recorded session and selected important moments of interest.

\section{Therapy Background}

The Traveler reported to the Guide that her son's pregnant girlfriend had recently been diagnosed with a life-threatening illness and that therefore the birth of the (grand) child was in danger. An operation had been put on hold to keep the child healthy. The Traveler's motivation for the therapy was to find out (1) how to cope with the helplessness and anxiety of losing the child and (2) how to keep hope. The intentions of the Guide were to (1) be present to the Traveler's situation and (2) provide a supportive music experience that would contain the Traveler while exploring strong emotions. Both Traveler and Guide are experienced therapists with more than 30 years of clinical practice in music therapy and the Bonny Method of GIM and are colleagues. Nevertheless, a therapy healing contract was established, as the Traveler was asking for help with an actual personal problem.

Participants gave informed written consent prior to the study (including written informed consent for the publication of their identifiable data), which was approved by the local ethics committee of Anglia Ruskin University and conducted in accordance with the Declaration of Helsinki.

\section{Materials and Apparatus \\ Procedure}

Before and after the therapy session, a resting-state EEG was recorded for ca. $4 \mathrm{~min}$. while participants had their eyes closed (for EEG details, see below). During the therapy, the Guide had her eyes open while the Traveler had her eyes closed.

The session followed the usual structure. It began with a pretalk (ca. $12 \mathrm{~min}$ ) in which the Traveler described a situation she wanted to explore, which became the focus for the session. This was followed by the induction of an ASC (ca. $12 \mathrm{~min}$ ), during which the Guide provided an autogenic relaxation induction. The chosen "Nurturing" music program (Bonny, 1980b) lasted around $34 \mathrm{~min}$ (see below for details) and was not modified. When the music program was finished, a return phase from the ASC followed, in which the Traveler and the Guide remained silent. After the session, the Guide and Traveler had a post-talk for ca. $6 \mathrm{~min}$.

\section{Music}

For this session, the Guide chose the original Nurturing program from Bonny created in 1980 (Grocke, 2002). In an interview with D. Grocke, Bonny explained the title and her intentions for this GIM listening program:

"...we wanted something that was indicative of nurturing of all sorts, not only childhood experiences of nurturing but a close warm feeling, which I feel we have. (...) The Nurturing tape was designed to do just that: to nurture. (...) A lot of it goes back to childhood, their lack of comfort and nurturing, or their perceived lack of it. They have to get beyond their anger and get down to positive feelings."

The pieces listened to during the journey are from Britten: Simple Symphony: Sentimental Sarabande; Vaughan-Williams: Prelude on Rhosymedre (4:10 min); Berlioz: L'Enfance du Christ (Flight into Egypt, Overture (6:55 min); Shepherd's Chorus (5:00 min); Puccini: Madame Butterfly (Humming Chorus) (2:47 min); Massenet: Scenes Alsaciennes (Sous les Tilleuls) (4:58 min); Canteloube: Songs of the Auvergne (Brezairola) (3:13 min).

A recent study by Dukic et al. (2019), testing the GIM process of the "Nurturing" program with 23 participants, confirmed that music of this type can have the psychological function of creating an emotional-scenic background.

\section{Data Recording and Analyses Content and Video Analysis}

A verbal transcript of the video-taped GIM session was annotated with ELAN (Version 5.3, 2018, Max Planck Institute for Psycholinguistics, Nijmegen, The Netherlands). ELAN (Wittenburg et al., 2006) was also used in the event-related analysis of the FAA and PAA (see section "Event-Related Emotional Processing" below). For this, the time series of the FAA values were imported into ELAN and events were located correspondingly.

About 4 weeks after the session, we instructed Traveler, Guide, and two independent raters (see section "Participants") via email to identify three moments of interest (MOI) based on the video recording of the session, i.e., parts that they think are of special interest and importance as well as a moment that was of noimportance (MONI) for the GIM therapy session. We did not ask for any specific emotional content to be identified but to describe, analyze and reflect upon these moments from a therapeutic perspective. See Supplementary Material for a short video excerpt relating to Table 3 and Figure 4.

Based on the identified individual MOIs from each rater, we identified two overlaps in MOIs across raters; that is, parts of the session all raters evaluated as being interesting (see Figure 1). Based on these overlaps, all further analyses focused on two time periods that ranged from the earliest beginning of an individual MOI included in each overlap to the latest end of an 


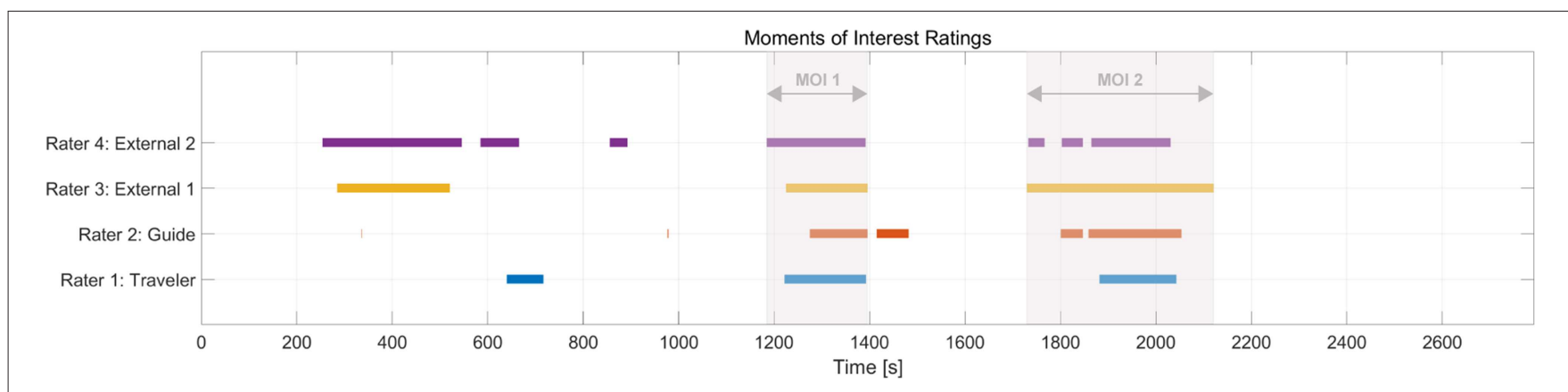

FIGURE 1 | Ratings and overlaps of the Guide-Traveler dyad and 2 independent raters. The red shaded areas show the segments that entered the subsequent analyses ("MOIs"). Note: Timeline displays amounts of seconds related to EEG recording; colors match with Table 1.

individual MOI included in the same overlap; in other words, ranges of overlaps of individual MOIs (see two red-shaded boxes in Figure 1; see Table 1 for details related to the content). This procedure allowed us to focus on parts of the session that all raters agreed upon while not having to analyze only parts of individual MOIs. For reasons of simplicity, henceforth, we refer to these ranges of overlaps as MOI 1 and MOI 2 (in contrast to individual MOIs, identified by the raters). The duration of MOI 1 and MOI 2 were $3.31 \mathrm{~min}$ and $6.31 \mathrm{~min}$, respectively. When necessary, parts of MOIs will be referred to as segments of MOIs. The duration of the MONI was $3.32 \mathrm{~min}$.

The analysis of these MOIs followed a micro-analytic approach (Wosch and Wigran, 2007): the therapy process was submitted to a moment-to-moment analysis, in combination to the parallel analysis of the EEG (see below).

\section{EEG}

The EEG was recorded with $500 \mathrm{~Hz}$ from $32 \mathrm{Ag} / \mathrm{AgCl}$ active electrodes (ActiCap, Brain Products GmbH, Germany) for each participant, placed according to the extended 10-20 system (FP1, FP2, F7, F8, F3, F4, Fz, FT9, FT10, FC5, FC6, FC1, FC2, T7, T8, C3, C4, TP9, TP10, CP5, CP6, CP1, CP2, Pz, P7, P8, P4, $\mathrm{P} 3, \mathrm{Oz}, \mathrm{O} 1, \mathrm{O} 2)$. The ground electrodes were placed on Fpz on each participant, and were recorded by using a ground distributor connected to an ActiCHamp amplifier (Brainproducts $\mathrm{GmbH}$, Germany). This amplifier implements a reference-free design, i.e., there is no dedicated hardware reference electrode and a reference electrode ( $\mathrm{Cz}$ of participant 1 ) was only chosen in the recording software (Brainvision Recorder, version 1.21.030, Brain Products $\mathrm{GmbH}$ ). As only a single reference electrode can be chosen there, the first offline pre-processing step involved rereferencing the EEG signals of participant 2 to $\mathrm{Cz}$ of participant 2 , thereby eliminating the influence of the online shared reference on the data.

Simultaneous video and audio was recorded with a Sony HVR Z1E camera, connected via Firewire to the EEG-recording PC. EEG and video signals were synchronized with the BrainVision Video Recorder (Brain Products GmbH, Germany).

Data were offline first analyzed with the BrainVision Analyzer software (version 2.1.2; Brain Products $\mathrm{GmbH}$ ). An Independent Component Analysis was performed (Infomax extended) and components reflecting horizontal and vertical eye movements and blinks were subtracted from the data. Data were then filtered with a 4th order Butterworth IIR filter (1-40 Hz; $50 \mathrm{~Hz}$ notch).

Due to a poor scalp-electrode contact during recording, four electrodes were found - based on visual inspection - to show large amounts of noise (mostly in the $50 \mathrm{~Hz}$ line noise range, but in lower frequency ranges as well as). Therefore, electrodes O2, P8 and TP10 of the Guide and FP1 of the Traveler were interpolated by spherical (4th order) splines.

After computing current source densities (CSD) from the scalp-recorded voltages, data were divided into $1 \mathrm{~s}$ epochs with $0.5 \mathrm{~s}$ overlap and artifactual epochs were automatically detected and manually checked (based on scalp-recorded voltage fluctuations). Automatic rejection criteria were: maximal $50 \mu \mathrm{V}$ voltage step, maximal allowed difference of $200 \mu \mathrm{V}$ in $200 \mathrm{~ms}$ intervals, absolute threshold of $\pm 100 \mu \mathrm{V}$, and lowest activity of $0.5 \mu \mathrm{V}$ in $100 \mathrm{~ms}$ intervals. A Fast Fourier Transformation (FFT) was performed (max. resolution $0.977 \mathrm{~Hz}$ ) on all artifact-free epochs, which were Hanning windowed (10\%).

Power values for all Fourier coefficients were exported and further analyzed in Matlab (version 9.2.0, Mathworks, Inc.). Alpha power for each segment was calculated as the band (8$13 \mathrm{~Hz}$ ) value sum and the alpha asymmetry scores were computed by taking the differences between the natural-log transformed power values of frontal electrodes $\mathrm{F} 4$ and $\mathrm{F} 3$, and between $\mathrm{P} 3$ and P4. In addition, power in the theta $(4-8 \mathrm{~Hz})$ frequency band was calculated for electrode Fz.

For investigating the time course of alpha asymmetries, missing values (due to artifactual epochs) were linearly interpolated and the time series were filtered (Savitzky-Golay, 3 rd order, 11 frames). In addition, mean alpha power across different conditions (rest, moments of interest, moment of no interest) was calculated for an occipital region of interest (ROI) with electrodes $\mathrm{O} 2, \mathrm{Oz}$, and $\mathrm{O} 1$. For EEG source localization, data were re-referenced to average reference and submitted to a Low Resolution Electromagnetic Tomography (Pascual-Marqui et al., 1994) analysis as implemented in BrainVision Analyzer.

Results of occipital alpha power and frontal alpha asymmetry during resting-state and moments of (no) interest were statistically analyzed with paired samples $t$-tests in SPSS using case-by-case exclusion for missing values (Version 24; IBM, 
TABLE 1 | Detailed MOI selections, descriptions and themes of the session.

\begin{tabular}{|c|c|c|c|c|}
\hline Person & Start & End & MOI description-Statements & MOI Overlaps - Extract \\
\hline Rater 4 & 13:53:09 & $13: 58$ & $\begin{array}{l}\text { stable (touching the wood) - new branches of my own tree - many roots - } \\
\text { pregnancy - birth - traumatic events- nobody there - needed "hand on my } \\
\text { head" }\end{array}$ & \\
\hline Rater 3 & 13.53 .40 & 13.57 .35 & $\begin{array}{l}\text { In this darker, minor section of the music the image of the TREE is } \\
\text { developed, and related to memories of childbirths and difficult } \\
\text { feelings/family traumas. }\end{array}$ & \\
\hline Rater 4 & 14:03:11 & 14:03:47 & $\begin{array}{l}\text { A dream of looking at her (unborn) son. A message from him "don't worry - I } \\
\text { went up - to the breast cave so I am safe" }\end{array}$ & \\
\hline Guide & $14: 05: 12$ & 14:05:12 & Empowerment of the women in the roots of her family & \\
\hline Rater 4 & 14:08:40 & 14:09:52 & $\begin{array}{l}\text { Not recognized by mother because being pregnant without being married } \\
\text { "Couldn't you have it removed?". }\end{array}$ & $\begin{array}{l}\text { MOI } 1 \text { - SEGMENT } 1 \\
\text { 1) Negative emotion } \\
\text { 2) Positive emotion emerging } \\
\text { image of the Grandmother } \\
\text { 3) Message from } \\
\text { Grandmother }\end{array}$ \\
\hline Traveler & 14.09.17 & 14.12 .07 & $\begin{array}{l}\text { Grandmother singing. Being told not to worry. Music coming into the body } \\
\text { and Heart. Experienced from inside as a pivotal moment }\end{array}$ & \\
\hline Rater 3 & 14.09 .20 & 14.12 .10 & $\begin{array}{l}\text { Grandmother sends a message. Music for the body and the heart (long } \\
\text { sequence without talking). }\end{array}$ & \\
\hline Rater 4 & 14:09:52 & $14: 12: 06$ & The music - really coming into her body: "Coming into my heart" & $\begin{array}{l}\text { MOI } 1 \text { - SEGMENT } 2 \\
\text { Positive emotion: Music fills } \\
\text { her heart }\end{array}$ \\
\hline Guide & $14: 10: 10$ & $14: 12: 10$ & Music coming into her heart & \\
\hline Guide & $14: 12: 30$ & 14:13:36 & Client sees the green light around her daughter in Law (strong visual image) & \\
\hline Rater 3 & 14.17 .45 & 14.22 .45 & "Music is nurturing" - Connecting to unborn baby and wishing all the best. & $\begin{array}{l}\text { MOI } 2 \text { - SEGMENT } 1 \\
\text { 1) Music is nurturing } \\
\text { 2) A message to the baby }\end{array}$ \\
\hline Rater 4 & 14:20:00 & 14:21:00 & the music is "Singing is for the baby" & \\
\hline Traveler & 14.20 .17 & 14.22 .57 & $\begin{array}{l}\text { The tree is singing. From inside the tree and the roots. Embracing the tree } \\
\text { with my Family hand in hand }\end{array}$ & \\
\hline Rater 4 & 14:21:00 & $14: 22: 45$ & Hand in hand around the tree. The tree is singing-from inside-roots & \\
\hline Rater 3 & 14.22 .45 & 14.24 .15 & $\begin{array}{l}\text { Therapist summarizes the session with focus on the TREE experience. Very } \\
\text { close contact. }\end{array}$ & \\
\hline
\end{tabular}

Colors indicate raters (corresponding with Figure 1) and overlap; time refers to the actual time of day of the recording, comments/statements are entered subsequently, according to their start time.

USA). $P$ values were Bonferroni corrected by multiplying the observed $p$-values with 9 (for FAA in the Traveler) or 6 (FAA for Guide, PAA, FMT, occipital alpha power). Cross-correlations were computed in R (R Development Core Team, 2008).

\section{RESULTS}

\section{General Comments on the GIM Session}

The external GIM therapists examined the overall content and range of core topics of the therapy session as:
External rater 1: "A very authentic and moving session; The travel is a mixture of memories-body experiencescore imagery-and even a message; guiding is sparse, mostly connecting imagery to body and emotions."

External rater 2: "The images in the journey are rich: colors, memories, feelings, body sensations, spiritual experiences (feeling a presence very close-her boy), dialogue and messages. Themes are trees, roots, family history, females and giving birth (reproduction), love. Enclosed are images/messages that stood out." 
The Guide stated: "I am aware that the analysis could focus on different types of experiences (visual, memories, embodied imagery, transpersonal, and transference to the music). I was also aware of moments when I felt very close to the Traveler's experiences, and for want of a better word I've called those moments "mutuality." I felt almost a leap in my heart when she mentions that her mother experienced the same problem, realizing the generational impact of it. I also felt very close to the Traveler's experience during the Berlioz Chorus-the 3rd verse of the chorus where there is a strong crescendo in the music. Another point was during the whole of the Brezairola[the Traveler] has a strong transference to the female voice, and I am silent so I don't interfere with it. But I felt very connected with her for the duration of that piece."

The Traveler was also asked for her experiences. She described her experience of parts of the session as "pivotal" and which will be reported in more detail below.

\section{Moments of Interest and Imagery}

As can be seen in Figure 1, all raters identified moments of interest in the second half of the session that clearly overlapped with each other. All subsequent analyses will focus around these overlaps (red-shaded area in Figure 1, henceforth just called "MOIs," see section "Content and Video Analysis") and smaller segments within these MOIs. Table 1 lists the corresponding detailed MOI selections, descriptions and themes of the session returned from Traveler and Guide, and from the independent raters.

In contrast to the above-mentioned moments, the moment of no-interest (MONI) was from the beginning of the session (13:49:15-13:52:47) and marks the exploration in the starting phase of the journey in which particular colors are imagined and the imagery has not yet developed a particular focus. All raters agreed that it was not easy to select MONIs.

\section{Differences in Imagery-Related Activity}

Figure 2 shows averaged occipital alpha power during the resting-state, the two overlapping segments of important moments, and during the non-important moment for the Guide and the Traveler. For both participants, alpha power was larger during the resting-state compared to therapeutically interesting moments and compared to the non-interesting moment. In the Traveler, alpha power was higher during the moment of no interest compared to both interesting moments, which in turn did not differ in terms of alpha activity. In the Guide, alpha power differed between the two important moments and between MOI 2 and MONI (for statistical results, see Table 2).

These findings suggest increased activity in the visual cortex during important moments compared to the resting-state for both participants, as well as increased activity in visual areas during important compared to non-important moments in the Traveler.

As shown in Figure 3, this difference in power is likely due to a shift of the topographical distribution of alpha power. Whereas during the resting-state, alpha power showed a predominantly occipital distribution, there appeared to be less occipital power and a change to a more temporo-parietal power distribution during MONI.

TABLE 2 | Summary of paired sample $t$-tests for occipital alpha power during rest, important and non-important moments for the Traveler and the Guide.

\begin{tabular}{lcccc}
\hline & & $\boldsymbol{t}$-value & Degrees of freedom & $\boldsymbol{p}$-value \\
\hline TRAVELER & & & & \\
Rest vs. & $\mathrm{MOI} 1$ & 7.69 & 381 & 0.0006 \\
Rest vs. & $\mathrm{MOI} 2$ & 7.92 & 452 & 0.0006 \\
Rest vs. & $\mathrm{MONI}$ & 6.88 & 395 & 0.0006 \\
MOI 1 vs. & $\mathrm{MOI} 2$ & -0.064 & 381 & 0.949 \\
MOI 1 vs. & $\mathrm{MONI}$ & -3.62 & 381 & 0.0006 \\
MOI 2 vs. & $\mathrm{MONI}$ & -3.68 & 395 & 0.0006 \\
GUIDE & & & & 0.0006 \\
Rest vs. & $\mathrm{MOI} 1$ & 9.71 & 400 & 0.0006 \\
Rest vs. & $\mathrm{MOI} 2$ & 12.19 & 443 & 0.0006 \\
Rest vs. & $\mathrm{MONI}$ & 9.37 & 388 & 0.0006 \\
MOI 1 vs. & $\mathrm{MOI} 2$ & 5.23 & 400 & 0.224 \\
MOI 1 vs. & $\mathrm{MONI}$ & 1.22 & 388 & $<0.0006$ \\
MOI 2 vs. & $\mathrm{MONI}$ & -4.6 & 388 &
\end{tabular}

MOI, Moment of interest; MONI, moment of no interest.
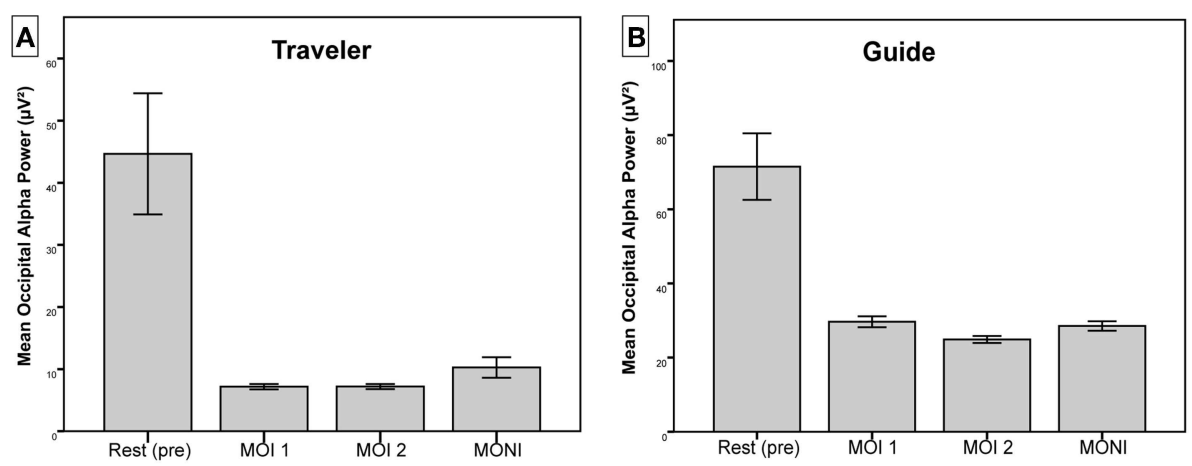

FIGURE 2 | Mean alpha power over occipital ROI for (A) Traveler and (B) Guide. Error bars represent \pm 2 standard error of the mean. 
Resting state

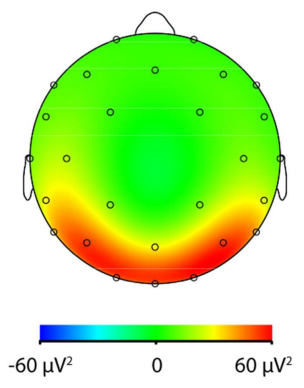

Moment of Interest 1

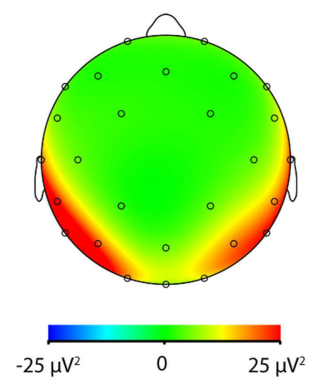

Moment of Interest 2

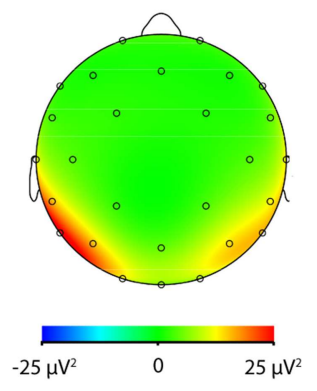

Moment of No-Interest

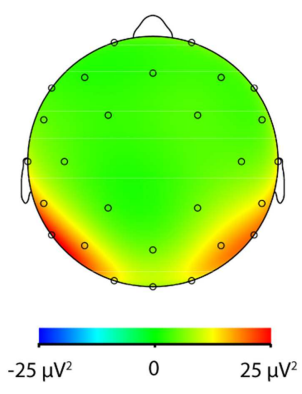

FIGURE 3 | Topographic distribution of alpha power of the Traveler during Rest, MOI 1, MOI 2, and MONI. Red colors represent more alpha power, indicating less cortical activity.

\section{Differences in Emotion-Related Activity Frontal Alpha Asymmetry}

The frontal alpha asymmetry of the Traveler was more positive (indicating positive emotional processing) during therapeutically important moments (MOI 1: $M=0.13, S D=0.7$; MOI 2: $M=$ $0.06, S D=0.74)$ compared to the resting-state (Rest1) prior to the session $(M=-0.17, S D=0.75$; Paired sample $t$ tests Rest 1 vs. MOI 1: $t(361)=-5.6, p<0.0009$; Rest1 vs. MOI $2: t_{(421)}=$ $-5.14, p<0.0009)$. Further, the FAA was more positive during the moment of no interest $(M=0.84, S D=0.73)$ compared to both moments of interest $\left(t_{(359)}=-13.12, p<0.0009 ; t_{(365)}=\right.$ $-14.21, p<0.0009)$. There were no differences in FAA between the two different MOIs $\left(t_{(354)}=1.5, p=0.14\right)$. There were no differences between the resting-state prior and after the session $\left(t_{(428)}=-0.49, p=0.62\right)$.

In contrast, the FAA of the Guide did not differ between the Rest1 $(M=0.05, S D=0.65)$ and the first important moment $(M=-0.02, S D=0.65)$; paired sample $t$ tests Rest1 vs. MOI 1: $t_{(393)}=1.56, p=0.119$. However, the FAA was more negative during the second important moment $(M=-0.3, S D=0.69)$ than during the resting-state (paired samples $t$-test Rest1 vs. MOI 2: $\left.t_{(389)}=7.35, p<0.0006\right)$. Further, the FAA was more positive during the moment of no interest $(M=0.16, S D=$ $0.57)$ compared to both moments of interest $\left(t_{(371)}=-4.58, p<\right.$ 0.0006 and $t_{(333)}=-9.24, p<0.0006$, respectively). Additionally, the second MOI showed a more negative FAA compared to the first MOI $\left(t_{(351)}=5.51, p<0.0006\right)$. There was no difference $\left(t_{(437)}=2.32, p=0.16\right)$ between pre and post resting-states.

\section{Parietal Alpha Asymmetry}

The parietal alpha asymmetry (PAA) of the Traveler was more positive during the Rest1 $(M=0.095, S D=0.58)$ compared to the first, but did not differ compared to the second MOI (MOI 1: $M=-0.031, S D=0.49$; MOI 2: $M=0.015, S D=0.52$ : paired sample $t$ test Rest1 vs. MOI 1: $t_{(361)}=3.18, p=0.012$; Rest1 vs. MOI 2: $\left.t_{(421)}=1.85, p=0.39\right)$. There were no differences in PAA between the two MOIs, nor between the interesting and noninteresting moments (all $p$ 's $>0.103$ ). The PAA was significantly more negative during Rest 2 compared to Rest $1\left(t_{(428)}=4.279\right.$, $p<0.0006)$.

The PAA of the Guide was more positive during the Rest1 ( $M$ $=0.229, S D=0.823$ ) compared to both MOIs (MOI $1: M=$ $-0.53, S D=0.69$; MOI 2: $M=-0.72, S D=0.64$ : paired sample $t$-test Rest1 vs. MOI 1: $t_{(393)}=13.86, p<0.0006$; Rest1 vs. MOI $\left.2: t_{(389)}=19.19, p<0.0006\right)$. Additionally, the PAA was more negative during the second MOI compared to the first one $t_{(351)}$ $=3.17, p=0.012$ ), as well as compared to the moment of nointerest $\left(t_{(333)}=-3.191, p=0.012\right)$. There was no difference in PAA during the first MOI compared to the moment of no-interest $\left(M=-0.57, S D=0.67 ; t_{(371)}=0.93, p=0.35\right)$, and there was no difference between the pre- and post-resting-state $(p=0.952)$.

\section{Frontal Midline Theta}

For the Traveler, frontal midline theta power (FMT) was increased during the resting-state $(M=43.01, S D=28.91)$ compared to both MOIs (MOI 1: $M=2.81, S D=2.345$; MOI $2: M=2.7, S D=1.56$; rest vs. MOI $1: t_{(361)}=26.13, p<0.0006$; rest vs. MOI 2: $\left.t_{(421)}=27.47, p<0.0006\right)$. Compared to MOI 1 and 2, FMT was increased during the moment of no-interest $\left(M=11.33, S D=6.71\right.$; MONI vs. MOI $1: t_{(359)}=-22.78, p$ $<0.0006$; MONI vs. MOI 2: $\left.t_{(365)}=-24.28, p<0.0006\right)$. FMT did not differ between both MOIs $(p=0.63)$. FMT was higher in the resting-state before the session compared to after the session $\left(t_{(428)}=14.05, p<0.0006\right)$.

Similarly, frontal midline theta power (FMT) of the Guide was also increased during the resting-state $(M=101.21, S D=54.45)$ compared to both MOIs (MOI 1: $M=4.63, S D=2.21$; MOI 2: $M$ $=5.27, S D=2.78$; rest vs. MOI 1: $t_{(393)}=35.19, p<0.0006$; rest vs. MOI 2: $\left.t_{(389)}=35.26, p<0.0006\right)$. The FMT during the moment of no-interest $(M=5.15, S D=2.56)$ was increased compared to MOI $1\left(t_{(371)}=-2.72, p=0.042\right)$, but did not differ compared to MOI $2\left(t_{(333)}=0.04, p=0.97\right)$. In addition, MOI 2 showed larger FMT values than MOI $1\left(t_{(351)}=-3.42, p=\right.$ 
0.006). Similar to the findings of the Traveler, FMT was higher in the resting-state before the session compared to after the session $\left(t_{(437)}=19.63, p<0.006\right)$.

\section{Event-Related Emotional Processing MOI 1: Pivotal Moment-Message From Grandma}

Here we are looking at two interrelated segments (see Tables 3, 4). The first describes a "message from the grandma" and the second is a description how the "music is filling up her heart." We asked the Traveler to expand on her experiences in more detail in order to contextualize the data accordingly. The Traveler described this MOI as a pivotal moment. Before this segment she spoke about negative emotions relating to her son's birth. Her mother was critical of her because she was pregnant without being married. "Couldn't you have it removed?" she had asked, and the Traveler felt very angry. But despite this her parents had been very loving and caring toward her son. After she told this, she listened to the music (2nd verse of Shepherd's Farewell to the Holy Family-Berlioz' L'enfance du Christ) and then reported after the imagery that she just had received a message from her grandma, who always had a positive influence on her. Seeing her grandmother telling her "not to worry," was a pivotal moment (see Table 3 below and Supplementary Material for a short video excerpt of this segment).

Written comment from Traveler:

"Like the music is becoming the spirit of my grandmother and the voices bring the message. I feel relieved and feels as if the music gets into my body and I feel some lightness in the body and at the same time feel spiritually connected to my grandmother. Suddenly

TABLE 3 | MOI 1 segment1, pivotal imagery, transcript of Traveler and Guide and event-related frontal alpha asymmetry (FAA) peak values.

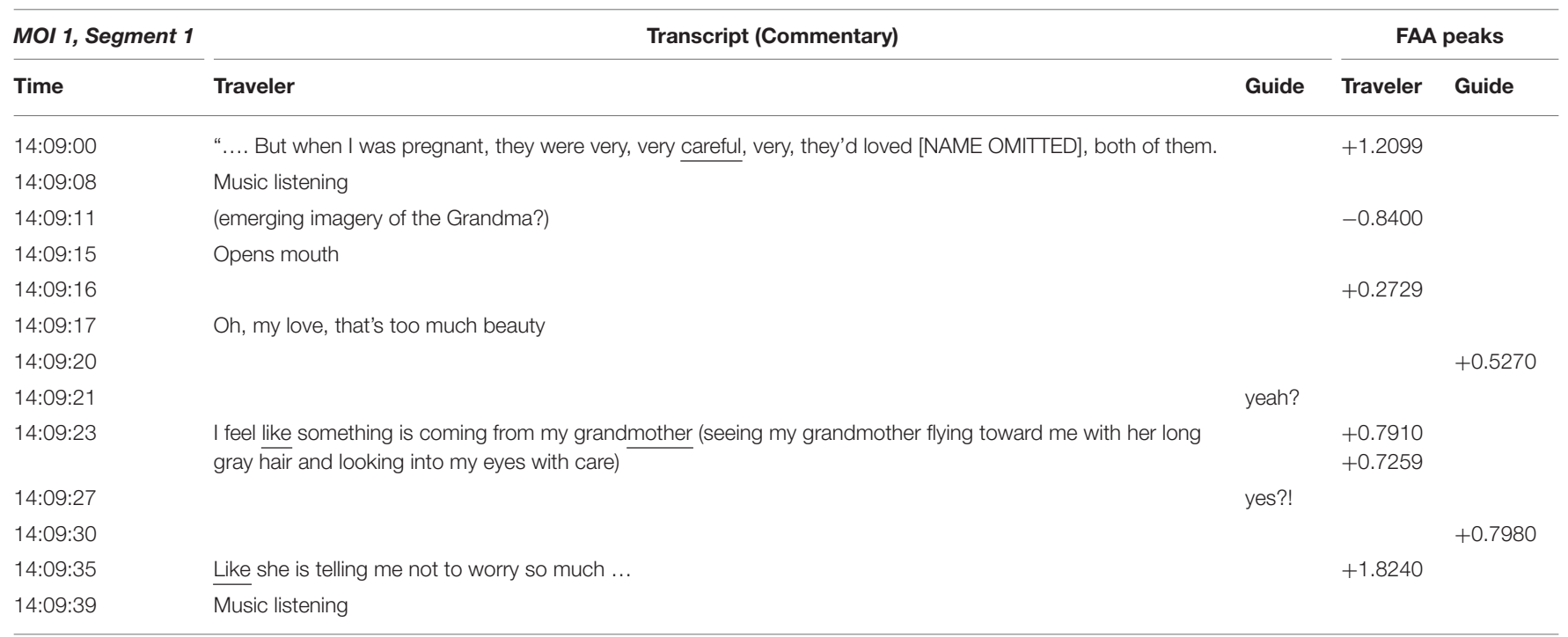

Underlined words correspond with peaks. FAA peaks are log-transformed (In) power differences of relative AA (here In F4 - In F3). For example, positive values (like +1.8240 ) indicate relative higher activity in the left-frontal cortex during the corresponding time segment (14:09:35).

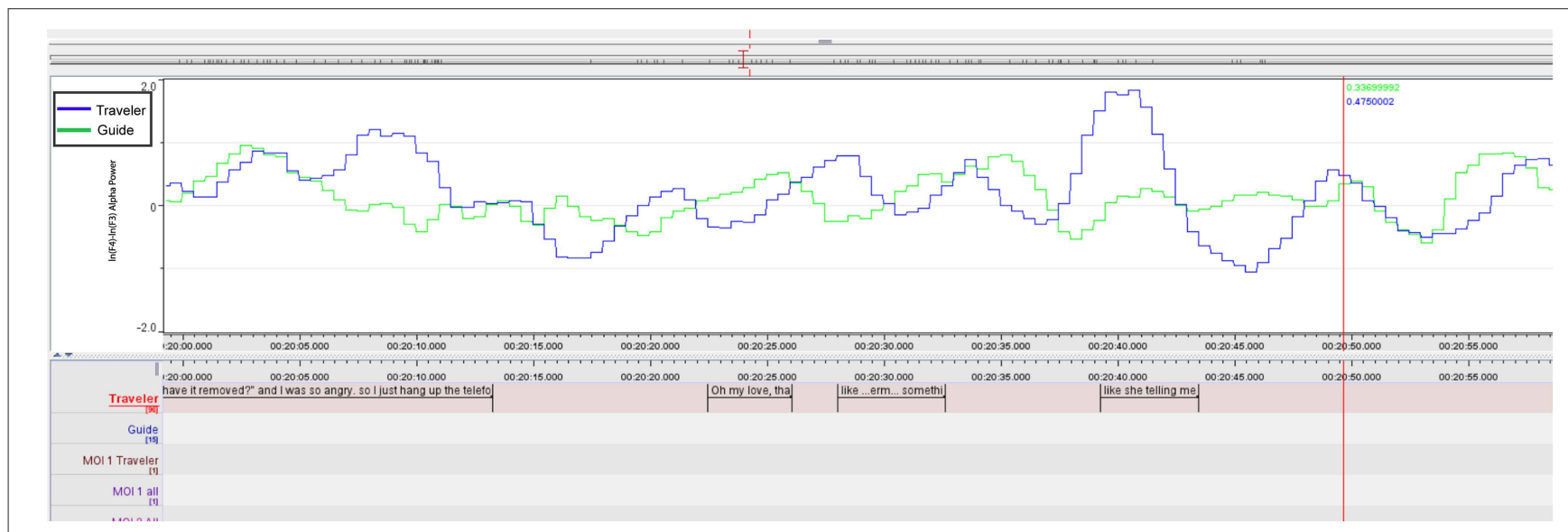

FIGURE 4 | MOI 1, Segment 1. Time course of frontal alpha asymmetry (F3/4, $0.5 \mathrm{~s}$ bins), indicating interplay of emotional processing of Guide (Green) and Traveler (Blue). For a description of event-related peaks, see Table 3. 
it feels like my heart is opening towards the music and the music and the spiritual sound is filling my heart - thus experiencing my heart much bigger and warm and thankful. I am touched and get into tears. It feels very relieving. I feel like being very peaceful."

We will now look how this moment unfolds along the conversation and the parallel peaks of the FAA representing the continuous emotional processing. When peaks coincided with spoken words they are underlined in the table. In the description of the two segments of MOI 1 we can study the interpunction of emotional processing in the Traveler and in the Guide.

\section{MOI 1 - Segment 1}

The first positive peak corresponds with talking about the Traveler's parents' loving care for her son (after telling that her mother was first suggesting to abort). This is followed by section in which the pivotal image of the grandmother probably emerges, interestingly accompanied with a negative peak. Then she tells about her emergent imagery and successive positive peaks culminate in the pivotal message sent from her grandma "not to worry."

Inspecting the emotional contour of both we can see how the peaks of the Guide (see Table 3 and Figure 4) increase in parallel, especially when she recognizes that there is an important imagery emerging, signified with the Traveler's words: "oh, my love ...," but culminate a second later than the Traveler's FAA peaks (See Supplementary Material for a short video excerpt).

\section{MOI 1 - Segment 2}

Both share this moment by knowing about the importance of this message and, while the negative peak of the Guide relates to adjusting loudness on the iPod, her next two peaks (14:10:32) mark the advice to the Traveler to "take in as much as you like." This leads into a longer part, in which the Traveler enjoys the deep relief until the end of this "woman's choir" (Berlioz: L'Enfance du Christ, 3rd verse of Shepherd's Farewell), as the Traveler describes it. Results of the FAA cross-correlation between Traveler and Guide during this segment (Figure 5 and Table 4) are reported below.
For completeness here are the lyrics of the $3 \mathrm{rd}$ verse sung by the choir:
Dearest infant, may God bless you; God bless your happy parents too. May injustice never darken the love that God bestows on you. Guardian angels guide, protect you. May they lead you safely through! ${ }^{1}$

All raters overlapped from 14:10:10-14:12:06 were agreeing that the part in which the music fills the heart and a woman's choir accompanying the relief constituted an important MOI.

\section{MOI 2: Mutuality}

\section{MOI 2-Segment 1}

The second MOI that all raters agreed upon and the range of ratings covered (see Figure $\mathbf{1}$ above) was described and annotated (see also Table 5 below) from the Traveler as follows:

\section{T: 'The singing is for the baby'}

COMMENT T: (feeling like being connected to the unborn baby through the music-like me singing to the baby. Feel the three (husband, son and daughter in law around me) -

T: '...we are all standing hand in hand around the tree...'

COMMENT T: A feeling like the tree is singing from inside - from inside the roots- like a very strong power - giving a deep feeling of grounding and really connected to the tree and the roots and my family. Like all of us are filled out and grounded and connected by this strong and spiritual voice. A feeling of power."

The following two segments of MOI 2 were annotated with the corresponding FAA and PAA peaks.

\section{MOI 2-Segment 2}

In the following segment the therapist asked the Traveler, after connecting to the unborn baby, (see Table 6) if the Traveler

\footnotetext{
${ }^{1}$ Translation from French as to http://www3.cpdl.org/wiki/index.php/L \%27adieu_des_bergers_(Hector_Berlioz)
}

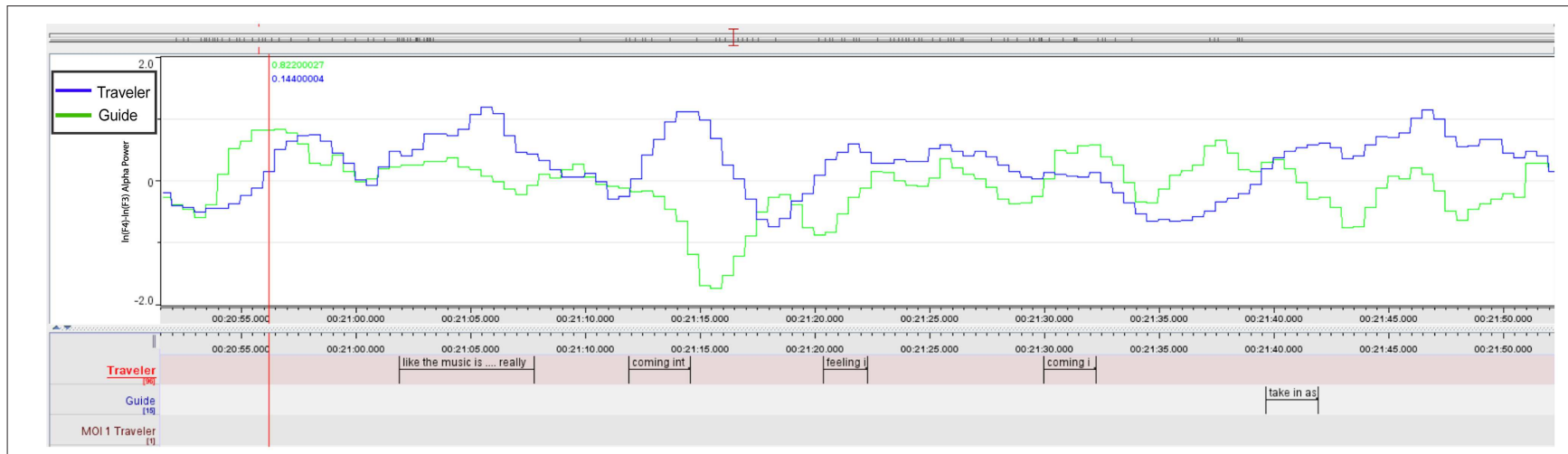

FIGURE 5 | MOI 1, Segment 2. Time course of frontal alpha asymmetry (F3/4, $0.5 \mathrm{~s}$ bins), indicating interplay of emotional processing of Guide (Green) and Traveler (Blue). For a description of event-related peaks, see Table 4. 
wants to send something "to the baby." With the second sending the voice of the female singer started into another verse of Canteloube's "Brezairola" (a lullaby) and here the Guide "felt very close" to the Traveler, signifying this part as a strong "moment of mutuality" (see above).

We can trace this mutuality graphically in the plot of the F3/4 FAA dynamics in Figure 6 and in the cross-correlation reported

TABLE 4 | MOI 1, segment 2; pivotal imagery, transcript of Traveler and Guide and event-related frontal alpha asymmetry (FAA) peak values.

\begin{tabular}{|c|c|c|c|c|}
\hline \multirow{2}{*}{$\begin{array}{l}\text { MOI 1, Segment } 2 \\
\text { Time }\end{array}$} & \multicolumn{2}{|c|}{ Transcript (Commentary) } & \multicolumn{2}{|c|}{ FAA peaks } \\
\hline & Traveler & Guide & Traveler & Guide \\
\hline 14:09:51 & & & & +0.8220 \\
\hline 14:09:53 & & & +0.7490 & \\
\hline 14:09:57 & Like the music is ... really & & +1.0839 & \\
\hline 14:10:07 & ...Coming into the body... & & +1.12 & \\
\hline $14: 10: 10$ & & $\begin{array}{l}\text { (adjusts } \\
\text { volume } \\
\text { on } \\
\text { iPod) }\end{array}$ & & -1.7379 \\
\hline $14: 10: 15$ & ... feeling it... & & +0.4610 & \\
\hline $14: 10: 25$ & $\begin{array}{l}\ldots \text { and, coming into the } \\
\text { heart }\end{array}$ & & & \\
\hline $14: 10: 27$ & & & & +0.5766 \\
\hline $14: 10: 29$ & Music listening & & -0.6609 & \\
\hline $14: 10: 32$ & & & & +0.6490 \\
\hline $14: 10: 35$ & & $\begin{array}{l}\text { Take in } \\
\text { as } \\
\text { much } \\
\text { as you } \\
\text { want }\end{array}$ & & \\
\hline $14: 10: 41$ & Music listening & & +1.1450 & \\
\hline
\end{tabular}

Underlined words correspond with peaks. FAA peaks are log-transformed (In) power differences of relative AA (here In F4 - In F3). For example, positive values (like +1.1450) indicate relative higher activity in the left-frontal cortex during the corresponding time segment (14:10:41). in the next paragraph. Both ELAN screenshots contextualize Traveler and Guide's verbalizations of guidance and imagery, and also the asymmetry data inspected along the time course of therapy.

\section{FAA Cross-Correlation During MOI 1 and 2}

Based on the comments of the Guide about her feeling very close to the experiences of the Traveler (see section "General comments on the GIM Session" above), we further explored a possible relationship between the emotional processing in the Traveler and Guide. For that, we calculated the crosscorrelation between the FAA time series during two segments of MOI 1 (Berlioz piece: $2.18 \mathrm{~min}, 14: 09: 52-14: 12: 10$ ) and MOI 2 (Brezairola piece, 3.14 min, 14:19:54-14:23:08) that were explicitly highlighted by the Guide (see Figure 8). Results showed a significant cross-correlation during the first MOI at lag $5(p=0.031$; cross-correlation coefficient $=$ $0.138)$, as well as significant cross-correlations for lag $0(p$ $=0.011)$ and $-1(p=0.001)$ during the second MOI (cross-correlation coefficients 0.138 and 0.178 for lag -1 and 0 , respectively).

\section{LORETA Analysis of Emerging Imagery}

The highest peak in FAA in the Traveler occurred in the first segment of MOI 1, when the Traveler expressed that her Grandma is telling her not to worry. Thus, it is likely that this part of the session is of pivotal importance. The imagery may have emerged from 14:09:08 to 14:09:17, prior to the verbalization of the imagery (see Table 4). To further explore brain activity during this particular segment, we submitted the time-domain EEG (14:09:12-17) to a source localization analysis with LORETA (see Figure 9). Results showed a best match at $3 \mathrm{~mm}$ in BA 39 within the posterior parts of the Middle Temporal Gyrus $(X=52, Y=-67, Z=$ 8). A comparison with a segment of the same length $(5 \mathrm{~s})$ during the moment of no-interest during which an image of a color was processed showed a peak activation at the same cortical areas. However, the strength of the activation during the MOI was nominally clearly higher than during

TABLE 5 | MOI 2 Segment 1. Transcript of Traveler and Guide and event-related alpha asymmetry peak values.

\begin{tabular}{|c|c|c|c|c|}
\hline \multirow{2}{*}{$\begin{array}{l}\text { MOI 2, Segment } 1 \\
\text { Time }\end{array}$} & \multicolumn{2}{|l|}{ Transcript (Commentary) } & \multicolumn{2}{|c|}{ FAA and PAA peaks } \\
\hline & Traveler & Guide & Traveler & Guide \\
\hline & .... Like the music is very nurturing... & & & \\
\hline $14: 17: 48$ & listening & & +0.6212 & \\
\hline 14:18:08 & Sadly it is also containing the sad thing about it, don't know... in a nurturing way & & +0.9589 & \\
\hline $14: 18: 30$ & Exhales deeply & & $\begin{array}{l}\text { P3-4: }+0.8598 \\
\text { F4-3: }-0.8358\end{array}$ & \\
\hline \multirow[t]{2}{*}{$14: 18: 53$} & I feel like almost being connected to the baby & & +0.1879 & \\
\hline & 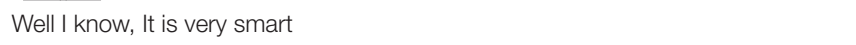 & & & \\
\hline 14:19:03 & listening & & +1.0179 & \\
\hline
\end{tabular}

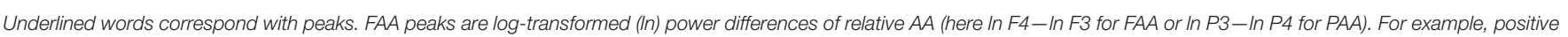

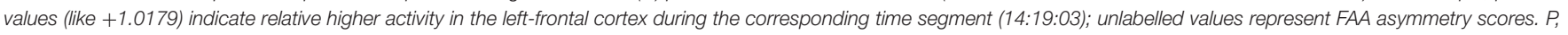
Parietal; F, Frontal Asymmetries. 
TABLE 6 | MOI 2 segment 2. Transcript of Traveler and Guide and event related alpha asymmetry peak values.

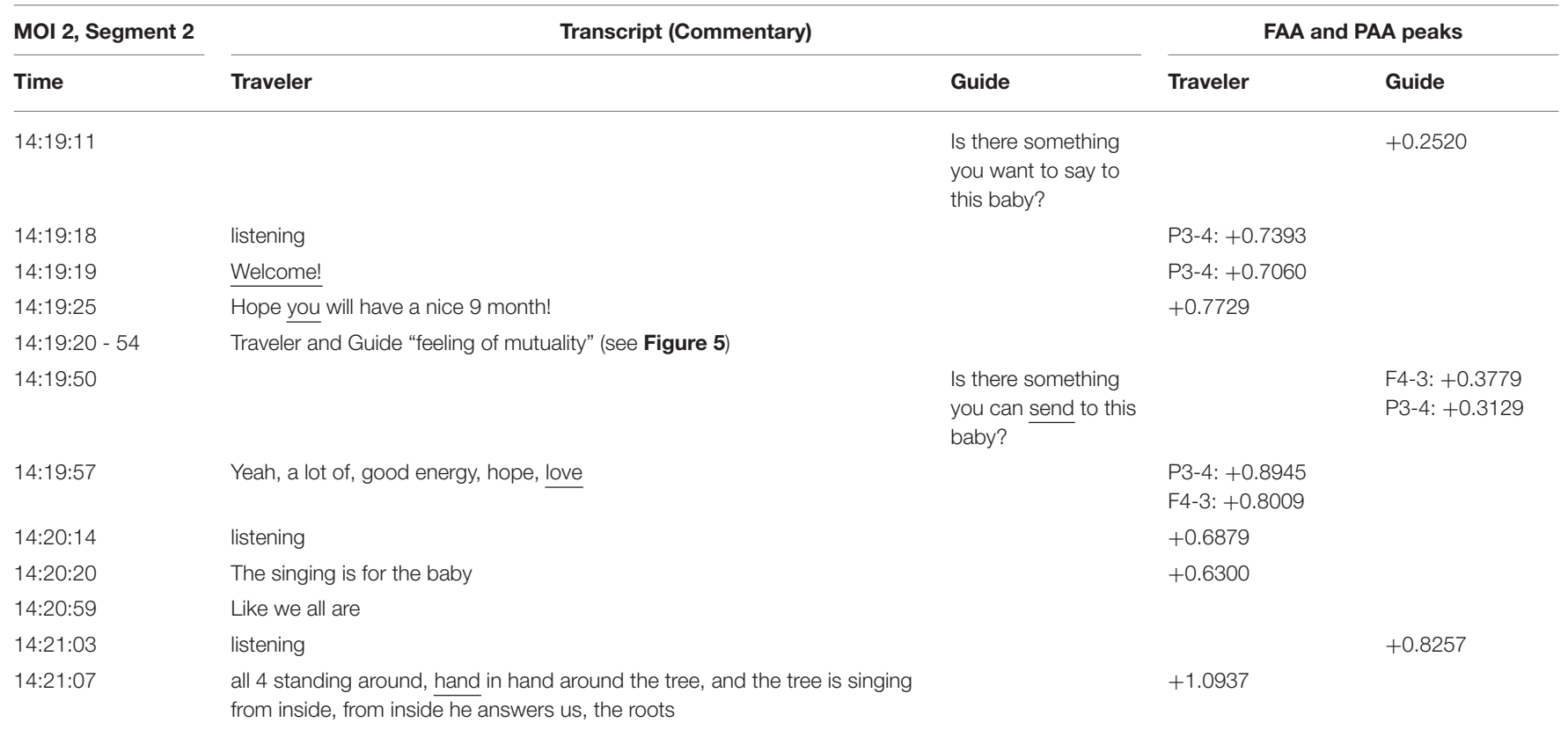

Underlined words correspond with peaks. FAA peaks are log-transformed power (In) differences of relative AA (here In F4-In F3 for FAA or In P3-In P4 for PAA). For example, positive values (like +1.0937) indicate relative higher activity in the left-frontal cortex during the corresponding time segment (14:21:07); unlabelled values represent FAA asymmetry scores. $P$, Parietal; F, Frontal Asymmetries.

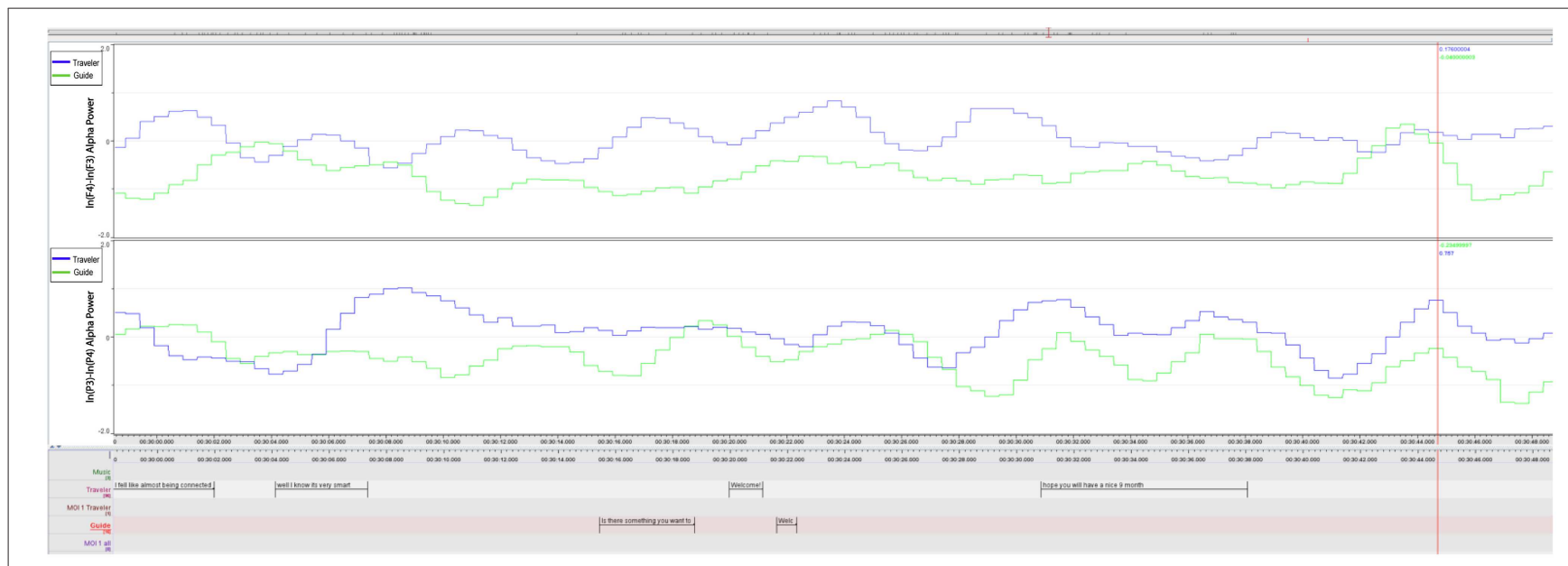

FIGURE 6 | MOI 2, Segment 1. Time course of frontal and parietal alpha asymmetry ( $F 3 / 4, P 3 / 4,0.5 \mathrm{~s}$ bins), indicating interplay of emotional processing of Guide (Green) and Traveler (Blue). For a description of event-related peaks, see Table $\mathbf{5}$.

the moment of no-interest $\left(0.00106 \mu \mathrm{A} \mathrm{mm} \mathrm{m}^{2}\right.$ vs. 0.000255 $\mu \mathrm{A} \mathrm{mm}^{2}$, respectively).

\section{FAA Difference Between Induction of Altered State and Rest}

The FAA in the Traveler was more negative during the induction of the altered state of consciousness $(M=-0.91, S D=0.77)$ compared to the resting-state $\left(M=-0.208, S D=0.71 ; t_{(372)}=\right.$ $-13.24, p<0.0009)$, as well as compared to both moments of interest (all p's < 0.0009).

\section{DISCUSSION}

In this first Hyperscanning recording of a music therapy session, we were investigating the natural GIM session flow and investigated the continuous time series of a dual-EEG of a Traveler (client) and a Guide (therapist). This provided a dataset with high ecological validity, including an authentic therapeutic relationship between a Traveler and a Guide.

The analysis of session ratings showed overlaps between all raters, confirming the importance of two particular shared moments of interest (MOIs) in the session and that these 
moments are of distinct content from moments with noimportance (MONI). The range and the particular overlaps (see Figure 1) offered a reliable base to select relevant material to further study the experienced imagery as it emerged in more detail.

For the exploration of imagery processing, we used occipital alpha power as an index of activity in cortical visual areas. For the exploration of emotional processing, several central markers of emotion were used, namely the frontal and parietal alpha asymmetry and frontal midline theta power. These markers were used (a) to investigate general differences between the restingstate and therapeutically important and non-important moments and (b) to explore the time course of emotional processing and a possible relationship between Traveler and Guide during the session. For this objectivist case study (see Ridder and Fachner, 2016) we formulated a few propositions (see end of "Aims" section) and will discuss the corresponding results.

\section{Moment of Interest 1: Message From Grandma}

Proposition V: We expect strong peaks to occur within imagery events of strong emotional valence.

In the first MOI, an important personal visual imagery and a corresponding dialogue with an imagined significant person constituted a pivotal moment. In this moment, the Traveler received a message from her grandma, which was accompanied by a negative peak in FAA (see Table 3) indicating a small sequence of negative emotion processing; however, verbalizing the message to the Guide, and here especially the meaning"telling me not to worry"-, showed the largest positive FAA peak in that sequence (see Figure 3) and this confirmed our proposition V. It is likely that the Traveler immediately recognized the positive impact of the message, but that the negative emotional impact of the previous narrative about being asked to abort (that was still present when the message occurred) transformed into a positive emotion when being told "not to worry" (see Table 3). This all happens during a passage in the music in which the choir sings blessings to parents and a child (see results section "MOI 1-Segment 1"). For both Traveler and Guide, being experienced GIM therapists themselves, this coincidence of the message and the music may have added to the emotional significance this moment has on several layers.

In previous electrophysiological and imaging studies, a right hemispheric MTG asymmetry has been associated with face recognition (Ojemann et al., 1992; Gur et al., 1993). In particular attractiveness of faces (Vartanian et al., 2013) seems to be processed in the right MTG. In a recent functional magnetic resonance imaging study on negative emotion in GIM, Lee et al. (2016) reported stronger peak intensity in the left MTG when comparing a GIM condition with a condition that included only a verbal instruction, whereas the comparison between GIM and only music listening showed no lateralization effects. Compared to that study, our source localization results (see Figure 9) showed a more posteriorly peak MTG activation. One might speculate that a verbal instruction as heard from the Traveler's Grandma ("not to worry") may also be processed in the left MTG.

However, recent investigations into Social Anxiety Disorder reported impaired activity in the left MTG (Yun et al., 2017). Given the significance of the message delivered from the Grandma in terms of the change that was brought forward in the therapy, the moment in which the image appeared may have been accompanied with the general fear and anxiousness of losing the child, which in turn fits with the observation of reduced overall FMT values and less positive PAA shifts during MOI 1.

For completeness it has to be reported that the FAA displayed a negative peak, and that the Traveler was swallowing during that moment ${ }^{2}$. Because swallowing rates have been shown to be modulated by affective states (Cuevas et al., 1995; Ritz and Thöns, 2006), this reaction might also point to the strong emotional impact of the emerging image that had to be "digested" here. Nevertheless, after the image of the Grandma appeared, a successive increase of positive FAA peaks appeared (Table 3) culminating in the verbal report of the Traveler that the Grandma told her "not to worry too much."

\section{Shared Temporal Dynamics of Emotion}

Proposition VI: We expect the peaks to indicate a temporal dynamic of dyadic biosemiotic interpunction of the therapy process, i.e., peaks indicating shared emotional processing.

Schall et al. (2015) pointed out that music therapy action can be traced in the time series of therapy events and reported corresponding decreases and increases of situational wellbeing and emotional expression during different phases of the intervention. In our study, in Tables 3-6, we can see how the emotional contour of the session unfolds and how the biosemiotic interpunction regarding emotional processing aligns between Guide and Traveler, thus confirming proposition VI.

Thus, by inspecting the AA peaks, we can trace the emotional intensity occurring during both, verbal and non-verbal events, for example when the Traveler reported the message but also when the Traveler was only listening/imagining without any verbal utterances (14:10:41). It also showed how the situated emotional processing builds up to culminate in a positive peak when the reassuring message of the grandma was verbalized. Here the culminating peaks may indicate a situated cognitive process in which the Traveler gains insight into her own family roots and history of childbirth and 'emotions signifying meaning are evoked through the interplay of music and the people' (Fachner, 2014, p 793); in this case with Berlioz's music and lyrics (see section "MOI 1-Segment 2" above) as a soundtrack to the Traveler's inner journey. Situated cognition refers to the inseparability of embodied perception and action and that meanings of the experiences are cognitively processed according

\footnotetext{
${ }^{2}$ Note that the part of the EEG segment containing the swallowing artifact has been rejected (duration: ca. $1.7 \mathrm{~s}$ ), which resulted in 3 missing data points in the FAA time series. These have been linearly interpolated. Because the peak, measured from zero-crossing before and after the peak, consisted of 11 data points, it appears likely that the observed peak, even though partly based on interpolated and filtered data, reflects a peak in FAA and not just an artifact due to the interpolation.
} 
to the affordances of the social and physical context in which we are situated (Iyer, 2002; Schwarz, 2002); in this case, the contextrelated insight that is based on the successive emotional peaks and meaning of the message shared with the Guide. Grandma's message is perceived and recognized as important by both subsequently and shared in an interpunction of increasing peaks.

The FAA peaks of the Guide and the Traveler appeared to be aligned during both MOIs (inspect Figures 5-7). Especially during the second segment of the second MOI, which has been described by a "strong feeling of mutuality" by the Guide (see section "General Comments on the GIM Session"), a close alignment of both FAA peaks can be observed (see Figures 6, 7; Table 6). This is also indexed by a significant crosscorrelation between the two FAA time series (see Figure 8), perhaps suggesting that shared temporal dynamics of emotional processing is represented in the FAA. Here the Guide described that the Traveler had a "strong transference to the female voice and I am silent so I don't interfere with it. But I felt very connected with her for the duration of that piece."

\section{Differences in Cortical Activity During Rest and Moments of (no) Interest in GIM}

During a real-world GIM session, a multitude of different processes are co-occurring. To ensure that our observations reflect imagery- and emotion-related activity, we tested if there are differences in cortical activity in areas known to be involved during imagery and emotion.

\section{Imagery: Occipital and Temporo-Parietal Differences}

Proposition III: We expect the visual cortex to be more active (to exhibit less alpha power) during the imagery process than during the resting-state.

Proposition IV: Additionally, during GIM (compared to rest), we expect that temporo-parietal areas are less active (i.e. more alpha power) then occipital areas.

Confirming proposition III and in line with other imagery research (Hunt, 2011; Schaefer et al., 2011) our results showed that cortical activity with regards to occipital alpha power differed between the resting-state and the therapy session (both conditions with no external visual input for the Traveler). Because previous studies have shown the involvement of visual areas in the occipital cortex during music-related imagery (visual and musical; Hunt, 2011; Schaefer et al., 2011), our results of increased alpha power during the resting-state (i.e., less activity) suggest that occipital areas are more activated during the GIM session and hence imagery processes might have been more pronounced.

Furthermore, inspection of the topographical distribution of alpha power suggests that temporo-parietal areas became more inhibited during the therapy, confirming proposition IV. Interestingly, alpha power increases over temporo-parietal regions has been associated with creative-related demands and creative ideation, and could reflect more internally oriented attention (Benedek et al., 2014; Fink and Benedek, 2014). Thus, one might speculate that increased temporo-parietal alpha activity during interesting moments (compared to rest and to non-interesting moments) reflects creative processes, which play an important role in music therapy.

However, there appears to be a difference in topographical distribution between the two moments of interest. Benedek et al. (2014) concluded that focused internal attention shows alpha power increases in the right parietal cortex. In the second MOI we observe a topography in which the right parietal alpha power seems to be decreased and the Traveler is focusing on sending messages to the unborn baby. Additionally, the Guide describes a strong feeling of mutuality with the Traveler during these passages (see section "General Comments on the GIM Session"). So, the inverse to what Benedek described may have been observed here: while focusing attention on internal mental imagery, the intentional direction of the imagery is turned outward and along with music and the feeling of mutuality while being in a therapeutic relationship alpha power in the right temporo-parietal cortex decreases. Further research is needed to investigate these ideas, including the role of creative ideation during therapy, in more detail.

\section{Emotion: Frontal and Parietal Differences}

\begin{abstract}
Proposition I: We expect differences between emotional processing during important moments and during the resting-state, and thus a difference in the alpha asymmetry (AA) measures.

More specifically, compared to the resting-state, we expect during MOIs more left-shifted frontal AA, possibly indicating positive emotional valence, and more right-shifted parietal $A A$, possibly indicating emotional intensity/arousal.

Proposition II: We expect FMT power to increase during positive emotional processing.
\end{abstract}

Because previous studies have shown changes in frontal brain activity during and after receiving music therapy (Lem, 1998; Lee et al., 2012; Fachner et al., 2013; O'Kelly et al., 2013), we investigated a few propositions regarding frontal and also parietal brain activity during MOIs, which was compared to rest and MONI. Previous studies reported a frontal asymmetry shift toward positive emotional processing after listening to preferred music (Field et al., 1998) or during musical emotions (Schmidt and Trainor, 2001; Altenmuller et al., 2002). Whereas the Field et al. study was embedded in a therapeutic framework of treating depressed mothers and FAA was extracted from pre/post-listening resting-state recordings, our subjects were doing a GIM session and imaging while listening to music, which again is different to listening only conditions, as used in the studies by Altenmuller et al. (2002) and Schmidt and Trainor (2001).

Although the Traveler's asymmetry scores were more left-shifted (FAA) during MOIs compared to rest (which confirmed the FAA part of proposition I; see section "EmotionRelated Activity"), MONI FAA returned more positive frontal asymmetries (indicating a more positive processing) then during the MOIs. There were no differences between the MOIs for the Traveler. Schmidt and Trainor (2001) used increased relative right PAA as a marker of arousal and intensity of emotional processing, but found no clear evidence for representing 


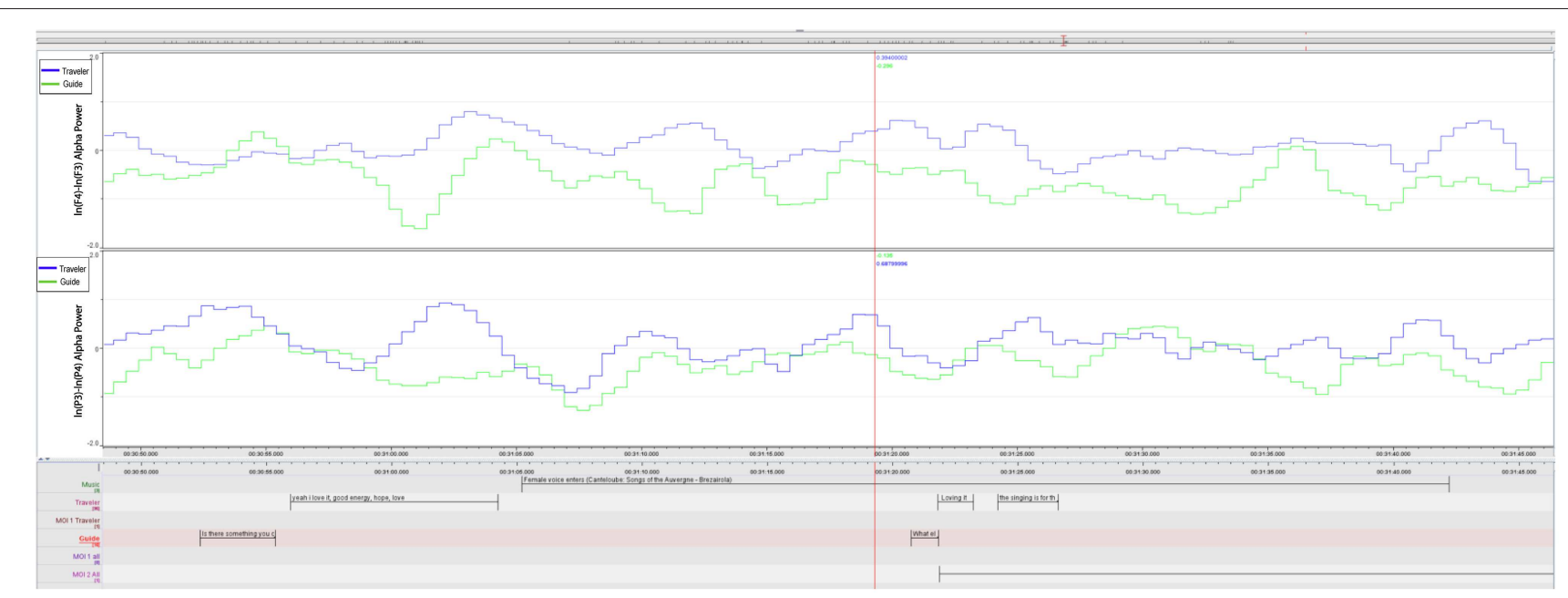

FIGURE 7 | MOI 2, Segment 2. Time course of frontal and parietal alpha asymmetry (F3/4, P3/4, 0.5 s bins), indicating interplay of emotional processing of Guide (Green) and Traveler (Blue). For a description of event-related peaks, see Table 6.

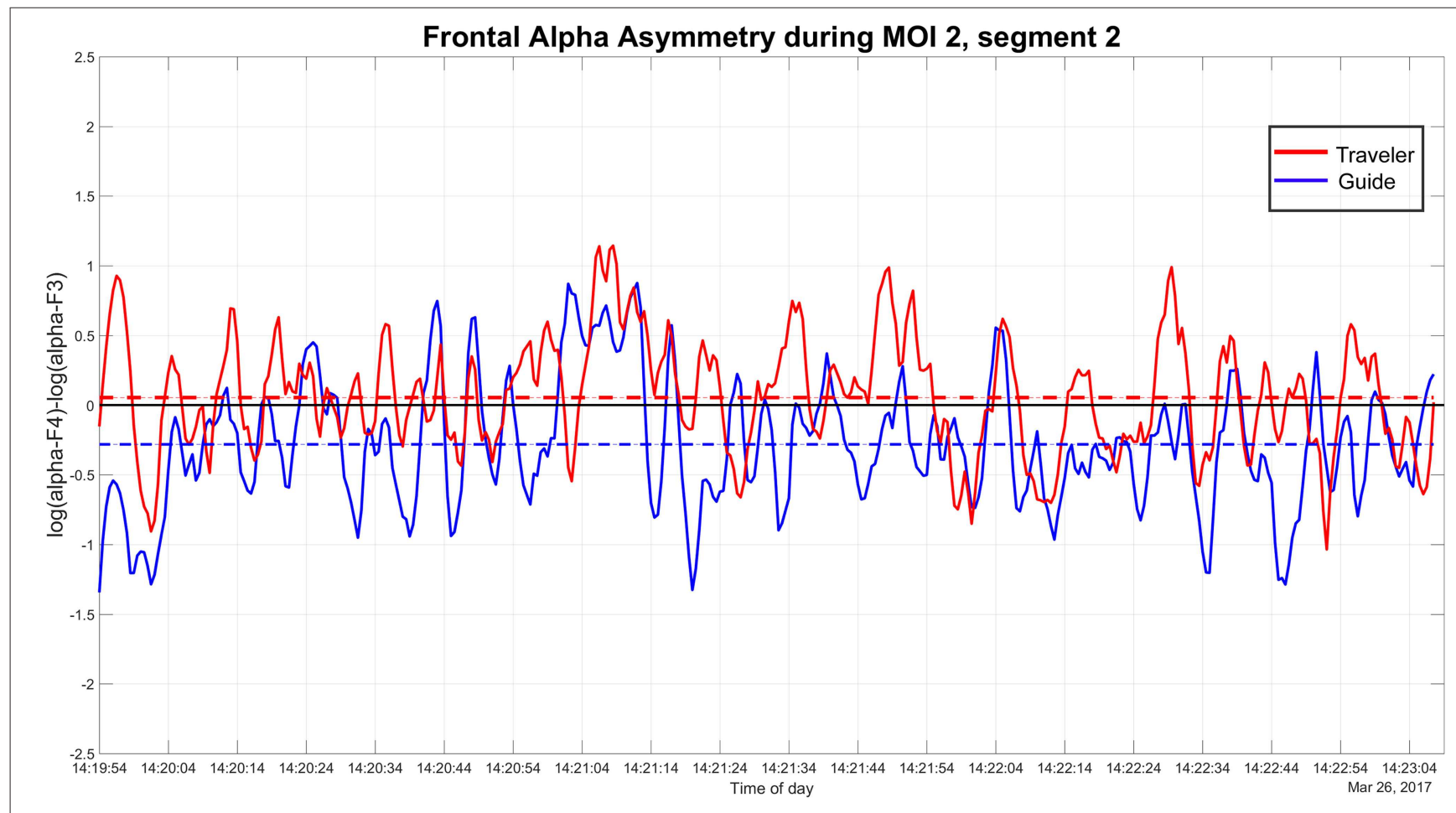

FIGURE 8 | MOI 2, Extended Segment 2. Time course of frontal alpha asymmetry at electrode F3/F4 of Guide and Traveler that entered cross-correlation analysis.

intensity. In our study, the Guide's MOI PAA was less positive compared to rest (not confirming the PAA of proposition I). Further the Guide's FAA differed between the two MOIs and rest and $\mathrm{P} / \mathrm{FAA}$ indicated more negative processing during the second MOI.

Interestingly emotion processing during MOIs was not that positive as proposed. This was also confirmed with FMT, which we expected to indicate increased positive emotional processing
(Proposition II; see Aftanas and Golocheikine, 2001; Sammler et al., 2007; Nemati et al., 2019). In general, rest and MONI had higher FMT power indicating relaxation, while less power was observed during the MOIs and during Rest2.

Thus, MOI AA and FMT results returned a more "negative" overall emotional processing. This may reflect the therapeutic work on a potential harmful expected life event of a close relative. Even when experiencing a message from grandma and being 


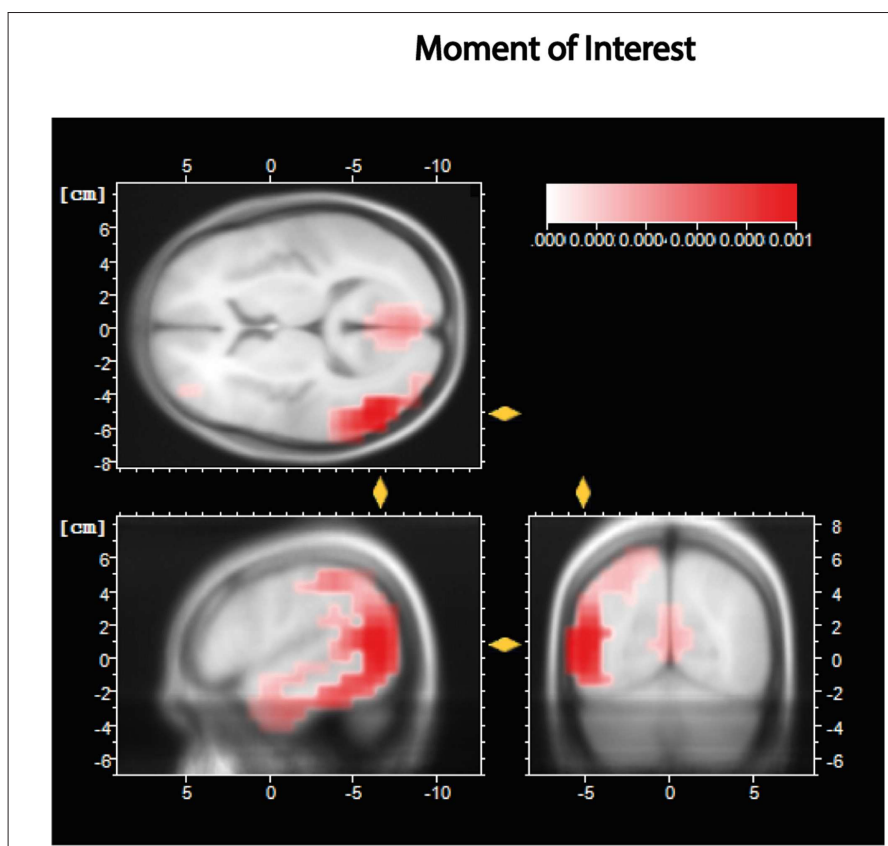

$X=-52, Y=-67, Z=9$

Best Match at $3 \mathrm{~mm}$

Brodmann area 39

Temporal Lobe

Middle Temporal Gyrus

\section{Moment of No-Interest}

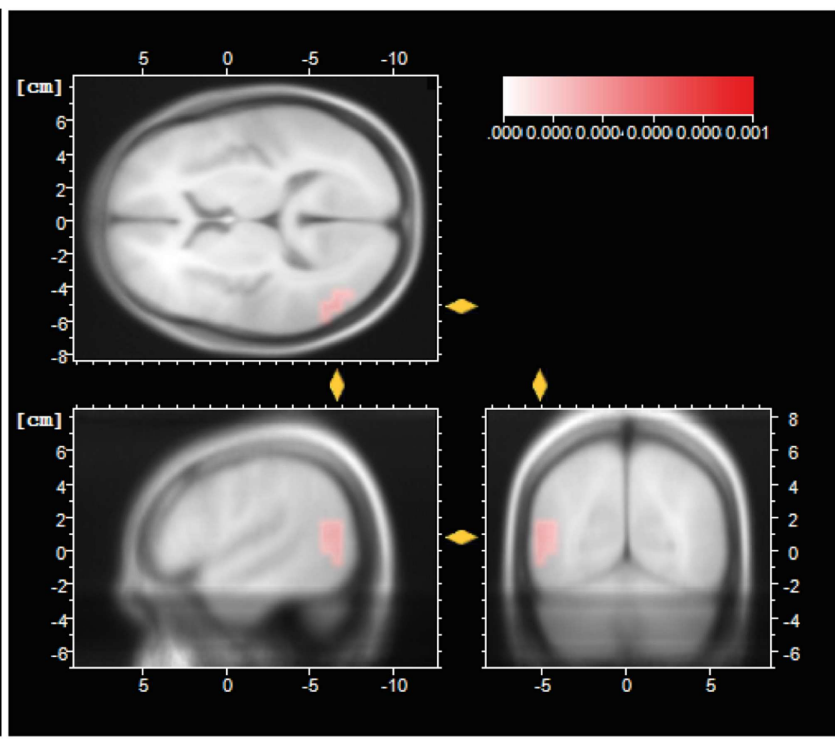

$X=-52, Y=-67, Z=8$

Best Match at $3 \mathrm{~mm}$

Brodmann area 39

Temporal Lobe

Middle Temporal Gyrus

FIGURE 9 | LORETA source localization results. The left panel shows localization estimates for a 5 s segment of MOI 1, While the right panel shows estimates for a 5 s segment of MONI. In both conditions, the peak activation was estimated to occur in the posterior part of the Middle Temporal Gyrus (BA 39), but was less pronounced during the MONI.

connected to the family tree (a more positive image), still the possibility existed that the grandchild will not survive nor will the daughter-in-law. Further, the emotional processing of the Guide may reflect the empathic therapeutic concern for the Traveler and realizing and partly feeling the impact on the Traveler. A recent study indicated that right-frontal processing is related to empathic concern (Tullett et al., 2012), and we may speculate that the more negative asymmetry values of the therapist are related to this.

In contrast to previous studies investigating the processing of preferred, liked or disliked music in neutral laboratory settings (Eerola and Vuoskoski, 2013), in the real-world therapy there were real threads to be processed and coping with fear and anxiety was the reason to do the journey. Indeed, this processing is likely reflected in the F/PAA and FMT MOI measures of Guide and Traveler and the experts did identify the parts of the session where meaningful spontaneous imagery occurred that offered hope and promising change.

\section{Role of the Altered State of Consciousness (ASC)}

In a former study, we reported a neurometric difference between the resting-state and the altered state induction as indicated by $z$-scores (compared to a normative EEG database) on the alpha range (Fachner et al., 2015). In this study, we found that during the induction of the ASC, the Traveler showed a more negative FAA compared to the resting-state. This suggests that the induction was effective and that the Traveler entered an altered state of consciousness, which in turn has to be kept in mind when considering imagery and emotion-related processing of the Traveler during the session.

GIM has evolved out of a pharmaco-supported psychotherapy setting in which the ASC has been evoked with strong psychedelic drugs (Bonny and Pahnke, 1972). However, Helen Bonny observed that inducing an ASC with deep relaxation methods and a subsequent journey in a reclined or supine position elicited sufficient imagery for the therapy session (Bonny, 1980a). Nevertheless, we know from recent research into MDMA or LSD that the vividness of imagery is stronger due to the breakdown of ego defense mechanisms (Carhart-Harris and Friston, 2010; Kaelen et al., 2015, 2016, 2018a,b) and the emergence of important imagery along with the music, for example in Amazonian Ayahuasca (De Rios, 1972; Shannon, 2011) yield strong imagery patterns with a culture specific taxonomy and noetic structure that may well yield a vast amount of important emerging imagery that can be used for therapy (Bonny, 1980a). Recent research investigating psychedelics have shown imagery enhancement under the influence (Kaelen et al., 2015, 2016, 2018a,b). However, the integration and conscious experience yielded with the relaxation induction methods can 
be controlled voluntarily while the imagery intensity under the influence may put the Traveler in a "floodlight state" that needs longer time to be processed and integrated (Grocke, 1999; Kaelen et al., 2018a,b). However, research into deep relaxation with music and imagery (Pfeifer et al., 2016) and reviewing common features of music and altered states stresses that setting, performance rites, suggestibility traits, and personal willingness to go into an altered state are of importance (Fachner, 2011). Thus, a proper investigation into the role of the ASC according to the induction method during these interventions is needed.

\section{Explorative vs. Naturalistic Design to Study Spontaneous Imagery}

A recent review stressed "the importance of the ecological characteristic of the experiments as [...] this is a key feature when investigating human interactions" (Acquadro et al., 2016, p. 9; see also Debener et al., 2012). In this objectivist case study, we addressed this demand for investigating ecological situations and explored the underlying neural correlates of a therapy process and tested a few propositions. Thus, the main intention was not to set up an experimental study on imagery and emotion in the brain, but to accompany a real-world music therapy session with EEG and video (which however lacks the experimental control typical for lab-based studies) (Fachner, 2014; 2016). This therapy took place to help a person cope with potential suffering, namely the life-threatening illness of a family member awaiting a baby, while we were interested in emerging meaningful imagery (see above) that is of pivotal importance for the patient in order to achieve change.

However, technological and methodological advances in hyperscanning and multi-modal data recording possibilities (Müller et al., 2013; Maidhof et al., 2014; Zamm et al., 2017) allowed for this exploratory dual-EEG study into shared imagery and emotion in the therapy process. Thus, a reflexive research process started, linking the first (subjective experience, imagery and emotions) and third (objective: physiological measures) person perspective (Fachner, 2014; Hunt, 2015; 2016; 2017).

Michel et al. (1993) and Lehmann et al. (1998) were studying spontaneous mentation and imagery by probing imagery and thought processes. Other more recent studies into the time-course of alpha power changes in creative ideation investigated continuous data in order to capture emerging thought elicited under experimental control (Schwab et al., 2014). Such experimental set-ups clearly yield in sum more defined spontaneous imagery or thought processes that can be repeated and therefore allow firmer inferences than can be drawn from a single trial during a real-world session.

Clearly, more repetitions of such case studies are needed that investigate focused questions about action mechanisms of artsbased interventions. However, in order to accompany an artsbased healing process, we have to adapt the tools that allow investigating brain processes in situ (Fachner, 2014; Hunt, 2015). Creative art process strives for unique results accounting all contingencies and situatedness and are not meant to be repeated, while scientific processes are unique in how they can and ought to be repeated. As we are researching healing processes, we have to accept the uniqueness of the therapeutic interaction (Fachner, 2017) while being able to observe these processes (for example, with EEG and video). After observing the processes in different ecological situations, we may then meta-analyze series of objectivist case studies in order to describe commonalities in procedures regarding emotion and imagery, turn-taking and co-activity, or other assorted phenomena of social interaction that are part of the therapeutic relationship. By doing so, we may also be able to describe a neural signature of what makes the therapeutic relationship in music therapy unique and at the same time comparable to the "sociophysiology" of other psychotherapeutic relationships (Schiepek, 2011, p. 351ff).

\section{Limitations}

This is an explorative single case study investigating a reallife music therapy session. Therefore, findings need to be interpreted with caution and cannot be generalized. In a strict sense, this study cannot be repeated and findings not be replicated. Nevertheless, our initial observations can be addressed in future studies more specifically. For this, and to derive at an understanding of the mechanisms underlying music therapy, we believe a dual strategy consisting of series of case studies (allowing meta-analyses to find commonalities) in parallel to controlled experiments with healthy participants (allowing testing of hypotheses derived from case studies) can be a fruitful approach.

Both participants were listening to the same music, and thus any similarity in emotional processing could be in principle due to the common factor of music-evoked emotions in both participants. However, we also found several differences in emotional processing as well as imagery-processes between the participants. For example, whereas the FAA of the Traveler was more positive during therapeutically important moments, it did not differ for the Guide. Given the different roles of the participants and the dynamic relationship between musical intensity, the Traveler's imagery and the sharing with the Guide, we believe that a simple explanation in terms of commonly evoked musical emotions in both participants seems rather unlikely. In contrast, this study may call for a special awareness toward emotional peaks of intensity and close connection between musical intensity (profile) and (peak, pivotal) experiences. For future work, it is even conceivable to combine the moment-to-moment interaction with neurofeedback approaches (e.g., bio-guided music therapy; see Miller, 2011) to apply patient-specific peak thresholds as targets for emotion regulation and awareness.

One could argue that wearing EEG caps, being connected to an EEG amplifier and filmed could have influenced the authenticity and thus quality of the session (possibly influencing imagery, emotional processes, etc.). However, the Traveler remarked interestingly in the post-session talk (i.e., without being specifically asked about it by the researchers, which might have introduced a bias) to the Guide that she completely forgot about the whole "lab-like" situation, including wearing an EEG cap and the video camera-it appeared she was only realizing this situation after coming out of the ASC. This confirms ideas about the feasibility of music therapy in-situ EEG-hyperscanning 
studies that do not affect the therapy process itself significantly (Fachner, 2016).

Another limitation might be that both independent raters as well as Guide and Traveler were experienced GIM therapists. Therefore, the therapy, the selection of interesting moments and their descriptions were based on expert knowledge, given that Guide and Traveler are (international) colleagues and have more than 30 years of experience in music therapy. Spiro and Himberg (2016) pointed out that studies based only on the perspective of therapists may miss out on "other aspects what may be going on" (p. 4) and therefore expert bias is potentially accumulated here (see section "Moment of Interest (MOI) Identification"). On the other hand, the addition of two external raters not involved in the therapy and the reports from the Travelerbeing experienced in interpreting and reflecting about therapy processes herself-seems to be, at least in this instance, an appropriate strategy to gain insights into what happened during the session and to achieve a higher content validity of the MOIs. However, this expert knowledge might have influenced the flow and emergence of the imagery and emotional processing, i.e., access to important imagery might be enhanced in experienced Travelers; nevertheless, it is likely that a personal pivotal meaning for the experienced Traveler was the same as it would have been for any other Traveler or client (Grocke, 1999). Still, this calls for further research into emerging imagery and the role of background knowledge and different relationship situations (Bonde and Beck, 2019; Dukic et al., 2019).

We used well-known indices of cortical emotional processing that have also been used in a clinical context in numerous studies (for overviews, see e.g., Allen et al., 2004; Mitchell et al., 2008; Allen and Reznik, 2015; Smith et al., 2017) and also in music and emotion studies (Schmidt and Trainor, 2001; Altenmuller et al., 2002; Sammler et al., 2007). However, future studies could employ more recently developed indices of brain-to-brain coupling. In the music domain, these measures have been mainly used to investigate interpersonal action coordination (Sänger et al., 2013; D'Ausilio et al., 2015; Zamm et al., 2017). Given the importance of emotional processes in social interactions including music performance (Acquadro et al., 2016), it will be interesting to develop other measures of brain-to-brain coupling related to emotion, which have the added benefit of a higher temporal resolution due to the time-frequency decomposition of the data.

Another limitation is that differences in FAA between restingstate (no interaction) and moments during the session might be only due to differences in the social interaction including verbalizations, and do not reflect differences in emotional processing. However, when comparing the FAA during MOIs and MONI, where, importantly, a comparable interaction (including verbal interactions) took place, the FAA differed, suggesting that not only physical differences have contributed to the change in alpha asymmetries between resting-state and MOIs. To clarify this issue future studies could compare similar social interactions but differing in their emotional (verbal) content.

Whether age has an influence on our FAA results cannot be estimated given the sparse research available with FAA and music and older people. One study focusing on neurofeedback training with depressed older people (Ramirez et al., 2015) reported a decrease of relative left alpha activity indicating an improvement of their depression condition. Future studies may investigate potential effect of age on markers of emotional processing.

\section{CONCLUSIONS}

In this explorative objectivist case study of Guided Imagery and Music, a Traveler was working with the assistance of a Guide on a threatening problem of losing family members. In this case, we observed that intense emotional processing of imagery in GIM has a temporal dynamic and was shared with the Guide. The EEG-derived biomarkers, indexing imagery- and emotion-related processes, suggested that during the important moments of therapy, emotions were not positive, pleasurable and relaxed (even though the Traveler was in an ASC) as opposed to our proposition. The significant differences between restingstate and therapy in the emotional valence and intensity, as measured with frontal and parietal alpha asymmetries as well as frontal midline theta power, confirm that both were dealing with negative emotions and anxiety that had to be contained in the interpersonal process. Nevertheless, the therapeutic setting offered a secure base to work on negative emotions, fear, and anxiety, and allowed the Traveler to develop hope and a perspective that change is still possible and that there may be a good end.

During a pivotal moment captured in this study, in which an imagined significant person delivered a message of hope ("not to worry too much"), we could study in detail how emotional processing changed from negative to positive valence and how Traveler and Guide shared the dynamics of this emotional processing, in particular how the Guide's emotional peak processing followed the one of the Traveler until the message was verbalized by the Traveler.

Even though this is an explorative case study and findings need to be interpreted with caution, we believe that the methodological approach of combining dual-EEG and detailed video-based and qualitative data is a promising approach for future research in this domain.

By analyzing the moment-to-moment interaction in music therapy, we were able to observe how frontal and parietal asymmetry peaks aligned with the situated cognition of Traveler and Guide along the emotional contour of the music, representing the highs and lows during the therapy process. This might have implications for a further understanding of the underlying mechanisms of music therapy and may contribute in the future to reveal what interventions (and when delivered) may be more effective.

\section{ETHICS STATEMENT}

This study was carried out in accordance with the recommendations of Code of practice for applying for ethical approval at Anglia Ruskin University with written informed consent from all subjects. All subjects gave written informed consent in accordance with the Declaration of Helsinki. The 
protocol was approved by the ARU Departments Research Ethics Panel.

\section{AUTHOR CONTRIBUTIONS}

JF and CM drafted the manuscript, developed methods, design of the study, and conducted measurements and analysis. DG and IN contributed to design, procedure and draft. GTr and LB contributed to design and draft. GTu contributed to the methods and LB initiated the EEGIM research.

\section{FUNDING}

This research has been enabled with a research grant from the Music Therapy Charity, London, UK and from the Christian Doppler Foundation, Vienna, Austria. Open Access funding was granted from Anglia Ruskin University.

\section{REFERENCES}

Acquadro, M. A. S., Congedo, M., and De Riddeer, D. (2016). Music performance as an experimental approach to hyperscanning studies. Front. Hum. Neurosci. 10:242. doi: 10.3389/fnhum.2016.00242

Aftanas, L. I., and Golocheikine, S. A. (2001). Human anterior and frontal midline theta and lower alpha reflect emotionally positive state and internalized attention: high-resolution EEG investigation of meditation. Neurosci. Lett. 310, 57-60. doi: 10.1016/S0304-3940(01)02094-8

Aldridge, D. (2004). Case Studies in Music Therapy Research. London. Jessica Kingsley Publishers.

Allen, J. J. B., Coan, J. A., and Nazarian, M. (2004). Issues and assumptions on the road from raw signals to metrics of frontal EEG asymmetry in emotion. Biol. Psychol. 67, 183-218. doi: 10.1016/j.biopsycho.2004.03.007

Allen, J. J. B., and Cohen, M. X. (2010). Deconstructing the 'resting' state: exploring the temporal dynamics of frontal alpha asymmetry as an endophenotype for depression. Front. Hum. Neurosci. 5:12. doi: 10.3389/fnhum.2010.00232

Allen, J. J. B., and Reznik, S. J. (2015). Frontal EEG asymmetry as a promising marker of depression vulnerability: summary and methodological considerations. Curr. Opin. Psychol. 4, 93-97. doi: 10.1016/j.copsyc.2014.12.017

Altenmuller, E., Schurmann, K., Lim, V. K., and Parlitz, D. (2002). Hits to the left, flops to the right: different emotions during listening to music are reflected in cortical lateralisation patterns. Neuropsychologia 40, 2242-2256. doi: 10.1016/S0028-3932(02)00107-0

Arjmand, H.-A., Hohagen, J., Paton, B., and Rickard, N. S. (2017). Emotional responses to music: shifts in frontal brain asymmetry mark periods of musical change. Front. Psychol. 8:2044. doi: 10.3389/fpsyg.2017.02044

Benedek, M., Schickel, R. J., Jauk, E., Fink, A., and Neubauer, A. C. (2014). Alpha power increases in right parietal cortex reflects focused internal attention. Neuropsychologia 56, 393-400. doi: 10.1016/j.neuropsychologia.2014.02.010

Blom, K. M. (2011). Transpersonal-spiritual BMGIM experiences and the process of surrender AU Nordic J. Music Ther. 20, 185-203. doi: 10.1080/08098131.2010.487645

Blom, K. M. (2014). Experiences of Transcendence and the Process of Surrender in Guided Imagery And Music (GIM). (PhD Monograph) Aalborg University, Denmark.

Bonde, L. O. (2004). "To draw from bits and pieces a more supportable narrative". An introduction to Paul Ricoeur's theory of metaphor and narrative and a discussion of its relevance for a hermeneutic understanding of music-assisted imagery in The Bonny Method of Guided Imagery and Music (BMGIM). Can. J. Music Ther. 11, 31-62.

Bonde, L. O. (2019). "Multi-modal imagery in the receptive music therapy model Guided Imagery and Music (GIM)," in Oxford Handbook of Sound \& Imagination, eds. M. Grimshaw, M. Walther-Hansen and M. Knakkergaard (Oxford: Oxford University), 427-444.

\section{ACKNOWLEDGMENTS}

Thanks to the study participants, to Jodie Bloska for proofreading and comments and to the EEGIM study group of the MT consortium.

\section{SUPPLEMENTARY MATERIAL}

The Supplementary Material for this article can be found online at: https://www.frontiersin.org/articles/10.3389/fpsyg. 2019.01561/full\#supplementary-material

Video 1 | Video excerpt of the identified Moment of Interest 1 - Segment 1 of the GIM session. The Guide is sitting on the left side, while the Traveler is laying down with eyes closed (right side). In this excerpt, the Traveler describes her imagery in which her grandmother told her "not to worry so much". The music during this segment is H. Berlioz: L'enface du Christ (Shepard's Farewell). See Figure 1 for the time course of the frontal alpha asymmetry and Table $\mathbf{3}$.

Bonde, L. O., and Beck, B. D. (2019). "Imagining nature during music listening. An exploration of the meaning, sharing and therapeutic potential of nature imagery in guided imagery and music," in Nature in Psychotherapy and Arts Therapies, Vol. 2, ed E. Pfeifer [under constant involvement of H. H. Decker-Voigt] (Gießen: Psychosozial-Verlag), 147-168.

Bonny, H. (1978). The Role of Taped Music Programs in the GIM process. Baltimore, MD: ICM.

Bonny, H. (1980a). GIM Therapy - Past, Present and Future Implications. Salina, KS: The Bonny Foundation.

Bonny, H. (1980b). Nurturing. A GIM Music Program. Baltimore MD: Institute for Consciousness and Music.

Bonny, H. (2002). Music and Consciousness: The Evolution of Guided Imagery and Music. Gilsum, NH: Barcelona Publishers.

Bonny, H. L., and Pahnke, W. N. (1972). The use of music in psychedelic (LSD) psychotherapy. J. Music Ther. 9, 64-87. doi: 10.1093/jmt/9.2.64

Bruscia, K. E. (1991). Case Studies in Music Therapy. Phoenixville, PA: Barcelona Publishers.

Bruscia, K. E. (2002). "The boundaries of Guided Imagery and Music and the Bonny method," in Guided Imagery and Music: The Bonny method and beyond, eds. K.E. Bruscia and D. Grocke. (Gilsum NH: Barcelona Publishers), 37-62.

Carhart-Harris, R. L., and Friston, K. J. (2010). The default-mode, ego-functions and free-energy: a neurobiological account of Freudian ideas. Brain 133(Pt 4), 1265-1283. doi: 10.1093/brain/awq010

Cooper, N. R., Croft, R. J., Dominey, S. J. J., Burgess, A. P., and Gruzelier, J. H. (2003). Paradox lost? Exploring the role of alpha oscillations during externally vs. internally directed attention and the implications for idling and inhibition hypotheses. Int. J. Psychophysiol. 47, 65-74. doi: 10.1016/S0167-8760(02)00107-1

Cuevas, J. L., Cook, E. W., Richter, J. E., McCutcheon, M., and Taub, E. (1995). Spontaneous swallowing rate and emotional state. Digest. Dis. Sci. 40, 282-286. doi: 10.1007/BF02065410

D'Ausilio, A., Novembre, G., Fadiga, L., and Keller, P. E. (2015). What can music tell us about social interaction? Trends Cogn. Sci. 19, 111-114. doi: 10.1016/j.tics.2015.01.005

Davidson, R. J. (2004). What does the prefrontal cortex "do" in affect: perspectives on frontal EEG asymmetry research. Biol. Psychol. 67, 219-233. doi: 10.1016/j.biopsycho.2004.03.008

De Rios, M. D. (1972). Visionary Vine: Hallucinogenic Healing in the Peruvian Amazon. San Francisco, CA: Chandler.

Debener, S., Minow, F., Emkes, R., Gandras, K., and de Vos, M. (2012). How about taking a low-cost, small, and wireless EEG for a walk? Psychophysiology 49, 1449-1453. doi: 10.1111/j.1469-8986.2012.01471.x

Dukic, H., Parncutt, R., and Bunt, L. (2019). Narrative archetype elicitation in the imagery of clients in guided imagery and music therapy sessions. Psychol. Music. doi: 10.1177/0305735619854122 
Eerola, T., and Vuoskoski, J. K. (2013). A review of music and emotion studies: approaches, emotion models, and stimuli. Music Percept. 30, 307-340. doi: $10.1525 / \mathrm{mp} .2012 .30 .3 .307$

Fachner, J. (2011). "Time is the key - Music and ASC," in Altering Consciousness: A Multidisciplinary Perspective, eds. E. Cardeña and M. Winkelmann. (Santa Barbara: Praeger), 355-376.

Fachner, J. (2014). Communicating change - meaningful moments, situated cognition and music therapy - a reply to North (2014). Psychol. Music 42, 791-799. doi: 10.1177/0305735614547665

Fachner, J. (2016). "The future of music therapy research in neuroscience," in Envisioning the Future of Music Therapy, ed. C. Dileo (Philadelphia: Temple University), 133-139.

Fachner, J. (2017). "Music, moments and healing processes: music therapy," in Routledge Companion to Music Cognition, eds. R. Ashley and R. Timmers. (London: Routledge), 89-100. doi: 10.4324/9781315194738-8

Fachner, J., Ala-Ruona, E., and Ole Bonde, L. (2015). "Guided Imagery in Music - a neurometric EEG/LORETA case study," in Ninth Triennial ESCOM Conference, eds. J. Ginsberg, A. Lamont and S. Bramley (Manchester, UK: European Society for the Cognitive Sciences of Music), 80.

Fachner, J., Gold, C., and Erkkil,ä, J. (2013). Music therapy modulates frontotemporal activity in the rest-EEG in depressed clients. Brain Topogr. 26, 338-354. doi: 10.1007/s10548-012-0254-x

Field, T., Martinez, A., Nawrocki, T., Pickens, J., Fox, N. A., and Schanberg, S. (1998). Music shifts frontal EEG in depressed adolescents. Adolescence 33, 109-116.

Fink, A., and Benedek, M. (2014). EEG alpha power and creative ideation. Neurosci. Biobehav. Rev. 44, 111-123. doi: 10.1016/j.neubiorev.2012.12.002

Gold, C., Fachner, J., and Erkkilä, J. (2013). Validity and reliability of electroencephalographic frontal alpha asymmetry and frontal midline theta as biomarkers for depression. Scand. J. Psychol. 54, 118-126. doi: $10.1111 /$ sjop. 12022

Goldberg, F. S. (2002). "A holographic field theory model of the bonny method of guided imagery and Music (BMGIM)," in Guided Imagery and Music. The Bonny Method and Beyond, eds. K. E. Brusica and D. E. Grocke. (Gilsum, NH: Barcelona Publishers), 359-378.

Grocke, D. (1999). A Phenomenological Study of Pivotal Moments in Guided Imagery and Music (GIM) Therapy. (PhD Monograph) Melbourne, VIC: University of Melbourne.

Grocke, D. (2002). "The Bonny music programs," in Guided Imagery and Music: The Bonny Method and Beyond, eds. K.E. Bruscia \& D. Grocke. (Gilsum, NH: Barcelona Publishers), 99-13.

Grocke, D., and Moe, T. (2015). Guided Imagery and Music (GIM) and Music Imagery Methods for Individual and Group Therapy. London and Philadelphia: Jessica Kingsley Publishers.

Gur, R. C., Jaggi, J. L., Ragland, J. D., Resnick, S. M., Shtasel, D., Muenz, L., et al. (1993). Effects of memory processing on regional brain activation: cerebral blood flow in normal subjects. Int. J. Neurosci. 72, 31-44. doi: 10.3109/00207459308991621

Harmon-Jones, E. (2003). Early career award. clarifying the emotive functions of asymmetrical frontal cortical activity. Psychophysiology 40, 838-848. doi: 10.1111/1469-8986.00121

Harmon-Jones, E., Gable, P. A., and Peterson, C. K. (2010). The role of asymmetric frontal cortical activity in emotion-related phenomena: a review and update. Biol. Psychol. 84, 451-462. doi: 10.1016/j.biopsycho.2009.08.010

Heinschel, J. A. (2002). A descriptive study of the interactive guided imagery experience. J. Holist. Nursing 20, 325-346. doi: 10.1177/089801002237591

Heller, W., and Nitscke, J. B. (1997). Regional brain activity in emotion: a framework for understanding cognition in depression. Cogn. Emot. 11, 637-661. doi: 10.1080/026999397379845a

Hibben, J. (1999). Inside Music Therapy: Client Experiences. Gilsum, NH: Barcelona Publishers.

Hubbard, T. L. (2010). Auditory imagery: empirical findings. Psychol. Bull. 136, 302-329. doi: 10.1037/a0018436

Hunt, A. (2015). Boundaries and potentials of traditional and alternative neuroscience research methods in music therapy research. Front. Hum. Neurosci. 9:342. doi: 10.3389/fnhum.2015.00342

Hunt, A. (2016). "First-Person Research," in Music Therapy Research, 3 edn ed. B. Wheeler. (Dallas: Barcelona Publishers), 443-467.
Hunt, A. M. (2011). A Neurophenomenological Description of the Guided Imagery and Music Experience. (PhD). Temple University.

Huron, D. B. (2006). Sweet Anticipation: Music and the Psychology of Expectation. Cambridge: MIT Press

Iyer, V. (2002). Embodied mind, situated cognition, and expressive microtiming in African-American music. Music Percept. 19, 387-414. doi: $10.1525 / \mathrm{mp} .2002 .19 .3 .387$

Jakobi, U. E. (2009). A Meta-Analysis About EEG Alpha Asymmetries and Depression in Adults: Proof of a Vulnerability Marker for Depression. Saarbrücken: VDM Verlag Dr. Müller.

Jäncke, L., Kühnis, J., Rogenmoser, L., and Elmer, S. (2015). Time course of EEG oscillations during repeated listening of a well-known aria. Front. Hum. Neurosci. 9:401. doi: 10.3389/fnhum.2015.00401

Juslin, P. N. (2013). From everyday emotions to aesthetic emotions: Towards a unified theory of musical emotions. Phys. Life Rev. 10, 235-266. doi: 10.1016/j.plrev.2013.05.008

Kaelen, M., Barrett, F. S., Roseman, L., Lorenz, R., Family, N., Bolstridge, M., et al. (2015). LSD enhances the emotional response to music. Psychopharmacology 232, 3607-3614. doi: 10.1007/s00213-015-4014-y

Kaelen, M., Giribaldi, B., Raine, J., Evans, L., Timmerman, C., Rodriguez, N., et al. (2018a). The hidden therapist: evidence for a central role of music in psychedelic therapy. Psychopharmacology 235, 505-519. doi: 10.1007/s00213-017-4820-5

Kaelen, M., Giribaldi, B., Raine, J., Evans, L., Timmermann, C., Rodriguez, N., et al. (2018b). Correction to: the hidden therapist: evidence for a central role of music in psychedelic therapy. Psychopharmacology 235:1623. doi: 10.1007/s00213-018-4886-8

Kaelen, M., Roseman, L., Kahan, J., Santos-Ribeiro, A., Orban, C., Lorenz, R., et al. (2016). LSD modulates music-induced imagery via changes in parahippocampal connectivity. Eur Neuropsychopharmacol. 26, 1099-1109. doi: 10.1016/j.euroneuro.2016.03.018

Koelsch, S. (2015). Music-evoked emotions: principles, brain correlates, and implications for therapy. Ann N Y Acad Sci. 1337, 193-201. doi: 10.1111/nyas. 12684

Konvalinka, I., Bauer, M., Stahlhut, C., Hansen, L. K., Roepstorff, A., and Frith, C. D. (2014). Frontal alpha oscillations distinguish leaders from followers: multivariate decoding of mutually interacting brains. Neuroimage 94, 79-88. doi: 10.1016/j.neuroimage.2014.03.003

Kreutz, G., Ott, U., Teichmann, D., Osawa, P., and Vaitl, D. (2008). Using music to induce emotions: Influences of musical preference and absorption. Psychol. Music 36, 101-126. doi: 10.1177/0305735607082623

Lee, E. J., Bhattacharya, J., Sohn, C., and Verres, R. (2012). Monochord sounds and progressive muscle relaxation reduce anxiety and improve relaxation during chemotherapy: a pilot EEG study. Compl. Ther. Med. 20, 409-416. doi: 10.1016/j.ctim.2012.07.002

Lee, S. E., Han, Y., and Park, H. (2016). Neural activations of guided imagery and music in negative emotional processing: a functional MRI study. J. Music Ther. 53, 257-278. doi: 10.1093/jmt/thw007

Lehmann, D., Strik, W. K., Henggeler, B., Koenig, T., and Koukkou, M. (1998). Brain electric microstates and momentary conscious mind states as building blocks of spontaneous thinking: I. Visual imagery and abstract thoughts. Int. J. Psychophysiol. 29, 1-11. doi: 10.1016/S0167-8760(97)00098-6

Lem, A. (1998). EEG reveals potential connections between selected categories of imagery and the psycho-acoustic profile of music. Austr. J. Music Ther. 9, 3-17.

Lin, M.-F., Hsu, M.-C., Chang, H.-J., Hsu, Y.-Y., Chou, M.-H., and Crawford, P. (2010). Pivotal moments and changes in the Bonny method of guided imagery and music for patients with depression. J. Clin. Nursing 19, 1139-1148. doi: 10.1111/j.1365-2702.2009.03140.x

Maack, C., and Nolan, P. (1999). The effects of guided imagery and music therapy on reported change in normal adults. J. Music Ther. 36, 39-55. doi: $10.1093 / \mathrm{jmt} / 36.1 .39$

Maidhof, C., Kästner, T., and Makkonen, T. (2014). Combining EEG, MIDI, and motion capture techniques for investigating musical performance. Behav. Res. Methods 46, 185-195. doi: 10.3758/s13428-0130363-9

Maratos, A., Crawford, M. J., and Procter, S. (2011). Music therapy for depression: it seems to work, but how? Br. J. Psychiatry 199, 92-93. doi: 10.1192/bjp.bp.110.087494 
Michel, C. M., Henggeler, B., Brandeis, D., and Lehmann, D. (1993). Localization of sources of brain alpha/theta/delta activity and the influence of the mode of spontaneous mentation. Physiol. Measure. 14:A21. doi: 10.1088/0967-3334/14/4A/004

Mikutta, C., Altorfer, A., Strik, W., and Koenig, T. (2012). Emotions, arousal, and frontal alpha rhythm asymmetry during Beethoven's 5th Symphony. Brain Topogr. 25, 423-430. doi: 10.1007/s10548-012-0227-0

Miller, E. B. (2011). Bio-Guided Music Therapy. London: Jessica Kingsley Publishers.

Mitchell, D. J., McNaughton, N., Flanagan, D., and Kirk, I. J. (2008). Frontalmidline theta from the perspective of hippocampal "theta". Prog. Neurobiol. 86, 156-185. doi: 10.1016/j.pneurobio.2008.09.005

Mizuki, Y., Suetsugi, M., Imai, T., Kai, S., Kajimura, N., and Yamada, M. (1989). A physiological marker for assessing anxiety level in humans: frontal midline theta activity. Psychiatry Clin. Neurosci. 43, 619-626. doi: 10.1111/j.1440-1819.1989.tb03096.x

Müller, V., Sänger, J., and Lindenberger, U. (2013). Intra- and inter-brain synchronization during musical improvisation on the guitar. PLoS ONE. 8:e73852. doi: 10.1371/journal.pone.0073852

Nemati, S., Akrami, H., Salehi, S., Esteky, H., and Moghimi, S. (2019). Lost in music: neural signature of pleasure and its role in modulating attentional resources. Brain Res. 1711, 7-15. doi: 10.1016/j.brainres.2019.01.011

Ojemann, J. G., Ojemann, G. A., and Lettich, E. (1992). Neuronal activity related to faces and matching in human right nondominant temporal cortex. Brain 115 (Pt 1), 1-13. doi: 10.1093/brain/115.1.1

O’Kelly, J., James, L., Palaniappan, R., Taborin, J., Fachner, J., and Magee, W. L. (2013). Neurophysiological and behavioral responses to music therapy in vegetative and minimally conscious states. Front. Hum. Neurosci. 7:884. doi: 10.3389/fnhum.2013.00884

Pascual-Marqui, R. D., Michel, C. M., and Lehmann, D. (1994). Low resolution electromagnetic tomography: a new method for localizing electrical activity in the brain. Int. J. Psychophysiol. 18, 49-65. doi: 10.1016/0167-8760(84)90014-X

Pfeifer, E., Sarikaya, A., and Wittmann, M. (2016). Changes in states of consciousness during a period of silence after a session of depth relaxation music therapy (DRMT). Music Med. 8, 180-186.

R Development Core Team (2008). R: A Language and Environment for Statistical Computing, Statistics. 360 edn. (Vienna, Austria: R Foundation for Statistical Computing). Avaialble online at: http://www.R-project.org (accessed April 1, 2019)

Ramirez, R., Palencia-Lefler, M., Giraldo, S., and Vamvakousis, Z. (2015). Musical neurofeedback for treating depression in elderly people. Front. Neurosci. 9:354. doi: 10.3389/fnins.2015.00354

Ramirez, R., Planas, J., Escude, N., Mercade, J., and Farriols, C. (2018). EEGbased analysis of the emotional effect of music therapy on palliative care cancer patients. Front. Psychol. 9:254. doi: 10.3389/fpsyg.2018.00254

Ridder, H. M., and Fachner, J. (2016). "Objectivist case study research. Singlesubject and small-n research," in Music Therapy Research, 3 edn, eds. B. Wheeler and K. Murphy (Dallas: Barcelona Publishers), 291-302.

Ritz, T., and Thöns, M. (2006). Affective modulation of swallowing rates: Unpleasantness or arousal? J. Psychosomat. Res. 61, 829-833. doi: 10.1016/j.jpsychores.2006.05.008

Rothschild, F. S. (1962). Laws of symbolic mediation in the dynamics of self and personality *. Ann. NY. Acad. Sci. 96, 774-784. doi: 10.1111/j.1749-6632.1962.tb50161.x

Sammler, D., Grigutsch, M., Fritz, T., and Koelsch, S. (2007). Music and emotion: electrophysiological correlates of the processing of pleasant and unpleasant music. Psychophysiology 44, 293-304. doi: 10.1111/j.1469-8986.2007.00497.x

Sänger, J., Müller, V., and Lindenberger, U. (2013). Directionality in hyperbrain networks discriminates between leaders and followers in guitar duets. Front. Hum. Neurosci. 7:234. doi: 10.3389/fnhum.2013.00234

Schaefer, R. S., Vlek, R. J., and Desain, P. (2011). Music perception and imagery in EEG: Alpha band effects of task and stimulus. Int. J. Psychophysiol. 82, 254-259. doi: 10.1016/j.ijpsycho.2011.09.007

Schäfer, T., Fachner, J., and Smukalla, M. (2013). Changes in the representation of space and time while listening to music. Front. Psychol. 4:508. doi: 10.3389/fpsyg.2013.00508

Schall, A., Haberstroh, J., and Pantel, J. (2015). Time series analysis of individual music therapy in dementia: effects on communication behavior and emotional well-being. GeroPsych. 28, 113-122. doi: 10.1024/1662-9647/a000123

Schiepek (2011). Neurobiologie der Psychotherapie. Stuttgart: Schattauer.
Schmidt, L. A., and Trainor, L. J. (2001). Frontal brain electrical activity (EEG) distinguishes valence and intensity of musical emotions. Cogn. Emot. 15, 487-500. doi: 10.1080/02699930126048

Schwab, D., Benedek, M., Papousek, I., Weiss, E. M., and Fink, A. (2014). The timecourse of EEG alpha power changes in creative ideation. Front. Hum. Neurosci. 8:310. doi: 10.3389/fnhum.2014.00310

Schwarz, N. (2002). "Situated cognition and the wisdom of feelings: Cognitive tuning," in The Wisdom in Feelings, eds. L. F. Barrett \& P. Salovey. (New York: Guildford Press), 144-166.

Shannon, B. (2011). "Ayahuasca and music," in Music and Consciousness, eds. E. Clarke and D. Clarke. (Oxford: Oxford University Press), 281-294.

Smith, E. E., Reznik, S. J., Stewart, J. L., and Allen, J. J. B. (2017). Assessing and conceptualizing frontal EEG asymmetry: an updated primer on recording, processing, analyzing, and interpreting frontal alpha asymmetry. Int. J. Psychophysiol. 111, 98-114. doi: 10.1016/j.ijpsycho.2016.11.005

Snodgrass, M., and Lynn, S. J. (1989). Music absorption and hypnotizability. Int. J. Clin. Exp. Hypn. 37, 41-54. doi: 10.1080/00207148908410532

Spiro, N., and Himberg, T. (2016). Analysing change in music therapy interactions of children with communication difficulties. Phil. Trans. R. Soc. B 371:20150374. doi: 10.1098/rstb.2015.0374

Stewart, J. L., Towers, D. N., Coan, J. A., and Allen, J. J. B. (2011). The oftneglected role of parietal EEG asymmetry and risk for major depressive disorder. Psychophysiology 48, 82-95. doi: 10.1111/j.1469-8986.2010.01035.x

Trondalen, G. (2016). Relational Music Therapy: An Intersubjective Perspective. Dallas, TX: Barcelona Publishers.

Trondalen, G. (2019). "GIM and Life Transitions: A relational perspective," in Guided Imagery and Music: The Bonny Method and Beyond, 2 edn, ed. D. Grocke. (Dallas, TX Barcelona Publishers), 97-114.

Tullett, A. M., Harmon-Jones, E., and Inzlicht, M. (2012). Right frontal cortical asymmetry predicts empathic reactions: support for a link between withdrawal motivation and empathy. Psychophysiology 49, 1145-1153. doi: 10.1111/j.1469-8986.2012.01395.x

Vartanian, O., Goel, V., Lam, E., Fisher, M., and Granic, J. (2013). Middle temporal gyrus encodes individual differences in perceived facial attractiveness. Psychol. Aesthet. Creat. Arts 7:38. doi: 10.1037/a0031591

Wärja, M., and Bonde, L. O. (2014). Music as co-therapist: towards a taxonomy of music in therapeutic music and imagery work. Music Med. 6, 16-27.

Watzlawick, P., Bavelas, J. B., and Jackson, D. D. (2014). Pragmatics of Human Communication: a Study of Interactional Patterns, Pathologies, and Paradoxes. NewYork, NY: W. W. Norton \& Company.

Wittenburg, P., Brugman, H., Russel, A., Klassmann, A., and Sloetjes, H. (2006) "ELAN: a professional framework for multimodality research", in Proceedings of LREC 2006, Fifth International Conference on Language Resources and Evaluation, (Genoa: LREC).

Wosch, T., and Wigran, T. (2007). "Microanalysis in music therapy: introduction and theoretical basis," in Microanalysis in Music Therapy: Methods, Techniques and Applications for Clinicians, Researchers, Educators and Students, eds. T. Wosch, T. Wigran and B. Wheeler. (London: JKP), 13-26.

Yun, J.-Y., Kim, J.-C., Ku, J., Shin, J.-E., Kim, J.-J., and Choi, S.-H. (2017). The left middle temporal gyrus in the middle of an impaired social-affective communication network in social anxiety disorder. J. Affect. Disord. 214, 53-59. doi: 10.1016/j.jad.2017.01.043

Zamm, A., Palmer, C., Bauer, A.-K. R., Bleichner, M. G., Demos, A. P., and Debener, S. (2017). Synchronizing MIDI and wireless EEG measurements during natural piano performance. Brain Res. 1716, 27-38. doi: 10.1016/j.brainres.2017.07.001

\section{Conflict of Interest Statement: DG, IN, GTr, and LB are GIM therapists.}

The authors declare that the research was conducted in the absence of any commercial or financial relationships that could be construed as a potential conflict of interest.

Copyright (C) 2019 Fachner, Maidhof, Grocke, Nygaard Pedersen, Trondalen, Tucek and Bonde. This is an open-access article distributed under the terms of the Creative Commons Attribution License (CC BY). The use, distribution or reproduction in other forums is permitted, provided the original author(s) and the copyright owner(s) are credited and that the original publication in this journal is cited, in accordance with accepted academic practice. No use, distribution or reproduction is permitted which does not comply with these terms. 


\section{OPEN ACCESS}

Edited by: Michele Biasutti,

University of Padova, Italy

Reviewed by:

Annet Bluschke,

Dresden University of Technology,

Germany

Alexander Refsum Jensenius, University of Oslo, Norway

*Correspondence:

Maria C. Fasano

mariacelestefasano@clin.au.dk

Specialty section:

This article was submitted to

Performance Science,

a section of the journal

Frontiers in Psychology

Received: 23 November 2018

Accepted: 18 March 2019

Published: 03 April 2019

Citation:

Fasano MC, Semeraro C

Cassibba R, Kringelbach ML,

Monacis L, de Palo V, Vuust $P$ and

Brattico E (2019) Short-Term

Orchestral Music Training Modulates

Hyperactivity and Inhibitory Control

in School-Age Children:

A Longitudinal Behavioural Study.

Front. Psychol. 10:750

doi: 10.3389/fpsyg.2019.00750

\section{Short-Term Orchestral Music} Training Modulates Hyperactivity and Inhibitory Control in School-Age Children: A Longitudinal Behavioural Study

\author{
Maria C. Fasano ${ }^{1 *}$, Cristina Semeraro ${ }^{2}$, Rosalinda Cassibba ${ }^{2}$, Morten L. Kringelbach ${ }^{1,3,4}$, \\ Lucia Monacis $^{5}$, Valeria de Palo ${ }^{5}$, Peter Vuust ${ }^{1}$ and Elvira Brattico ${ }^{1}$ \\ ${ }^{1}$ Center for Music in the Brain, Department of Clinical Medicine, Aarhus University - The Royal Academy of Music, Aarhus, \\ Denmark, ${ }^{2}$ Department of Psychology, Educational Sciences, Communication, University of Bari, Bari, Italy, ${ }^{3}$ Department \\ of Psychiatry, University of Oxford, Oxford, United Kingdom, ${ }^{4}$ Institut D'études Avancées de Paris, Paris, France, \\ ${ }^{5}$ Department of Humanities, University of Foggia, Foggia, Italy
}

Survey studies have shown that participating in music groups produces several benefits, such as discipline, cooperation and responsibility. Accordingly, recent longitudinal studies showed that orchestral music training has a positive impact on inhibitory control in school-age children. However, most of these studies examined long periods of training not always feasible for all families and institutions and focused on children's measures ignoring the viewpoint of the teachers. Considering the crucial role of inhibitory control on hyperactivity, inattention and impulsivity, we wanted to explore if short orchestral music training would promote a reduction of these impulsive behaviors in children. This study involved 113 Italian children from 8 to 10 years of age. 55 of them attended 3 months of orchestral music training. The training included a 2-hour lesson per week at school and a final concert. The 58 children in the control group did not have any orchestral music training. All children were administered tests and questionnaires measuring inhibitory control and hyperactivity near the beginning and end of the 3-month training period. We also collected information regarding the levels of hyperactivity of the children as perceived by the teachers at both time points. Children in the music group showed a significant improvement in inhibitory control. Moreover, in the second measurement the control group showed an increase in self-reported hyperactivity that was not found in the group undergoing the music training program. This change was not noticed by the teachers, implying a discrepancy between self-reported and observed behavior at school. Our results suggest that even an intense and brief period of orchestral music training is sufficient to facilitate the development of inhibitory control by modulating the levels of self-reported hyperactivity. This research has implications for music pedagogy and education especially in children with high hyperactivity. Future investigations will test whether the findings can be extended to children diagnosed with ADHD.

Keywords: collective music training, hyperactivity, inhibitory control, children, El Sistema, ADHD, impulsivity 


\section{INTRODUCTION}

Survey studies have shown benefits, such as discipline, cooperation and responsibility, from participating in music groups and needing to work together toward a common goal, especially in children (Hallam, 2010). In music education, several methods have acknowledged the benefits of playing in groups and incorporated this in the training. The diffusion of these innovative approaches has led to a rising interest in exploring the effects of collective music training in children (Moreno et al., 2011; Schellenberg et al., 2015; Jaschke et al., 2018). In particular, in the last years a growing number of studies have focused on the effects of orchestral training, such as the El Sistema approach (Habibi et al., 2014, 2016; Alemán et al., 2017; Holochwost et al., 2017; Sachs et al., 2017), which is a well-documented form of collective music education (Majno, 2012). This music program aims to promote the inclusion of at-risk children by providing them with high quality music training and instruments for free. Compared with other types of music training, playing in an orchestra requires fine motor, rhythmic and visual skills, as well as the discipline to sit patiently in silence for the entire execution of a piece waiting for your turn and, sometimes not playing for several minutes. Moreover, performing in an orchestra or a choir requires constant attention to the gestures of the conductor and, at the same time, the ability to synchronize to his rhythm and dynamics. Finally and most importantly, playing with others also necessitates listening to and synchronizing with the other players in order for the performers to blend their sounds (Biasutti, 2013). All of this represents a real and constant cognitive and emotional training, amplified by the experience of being part of a real orchestra performing in front of a large, live audience.

In support of this, some researchers recently showed that children involved in an orchestral program inspired by the El Sistema approach for one, two, or three years, exhibited better performance in inhibitory control and self-control tasks (Alemán et al., 2017; Holochwost et al., 2017). The careful planning and monitoring of performance (Palmer and Drake, 1997), the control over the focus of attention (Duke et al., 2011), and the integration of sensorimotor information (Münte et al., 2002) make ensemble and orchestral performance a proper vehicle for neuroplastic and neurocognitive changes. A recent functional magnetic resonance imaging (fMRI) study did indeed found that children who had participated in a similar music program for 2 years had greater involvement of brain regions known to be involved in cognitive control (supplementary motor cortex, anterior cingulate cortex, inferior frontal gyrus, and insula bilaterally) compared to children who did not participate in the music program in trials that require inhibitory control (Sachs et al., 2017).

Executive functions, such as attention and inhibitory control, are critical for children's development and mental health because they represent the basis for the self-regulation needed throughout life (Diamond and Lee, 2011). These cognitive skills are impaired in children with symptoms of Attention Deficit Hyperactivity Disorder (ADHD) (Barkley, 1997; Whelan et al., 2012), a diagnosis that, according to the most recent statistics, has a high incidence. For this reason, some researchers have started looking at the inhibitory control as the possible object of early treatment devoted to prevent or reduce the presence of hyperactivity, inattention, and impulsivity, better known as ADHD symptoms (Thorell et al., 2009; Halperin et al., 2013; Re et al., 2015). Re et al. (2015) conducted a study where they looked at the effect that a training program focused on executive functions had on inhibitory control, hyperactivity, inattention, and impulsivity in children. In this study, they involved both children with and without a diagnosis of ADHD who were or were not involved in this cognitive training program. Both children with ADHD symptoms and children with typical development attending the training program improved their performance in tasks measuring control of attention and impulsive behavior compared with the children that did not participate in the training program.

Considering the capability that long-lasting orchestral playing has in enhancing inhibitory control, we therefore wanted to investigate whether orchestral training could be used to promote a reduction of the levels of hyperactivity, inattention and impulsivity together with an improvement of inhibitory control in children.

Furthermore, until now the studies focusing on the effects of orchestral music training have considered music programs (provided outside regular school hours) lasting at least one year. However, a music program that spans the course of a year or more could be financially challenging for the institutions and can represent a significant drain on the parents' time, as they need to bring their children to the training in the afternoon, the course often being external. All this can make this valuable and very promising training difficult to replicate, especially in the schools. Optimizing the feasibility of this music training could be beneficial for many children who would not otherwise have access to it. Therefore, in our study we wanted to investigate the effectiveness of orchestral training when implemented over a shorter period of time. Indeed, Moreno et al. (Moreno et al., 2011) showed that children between 4 and 6 years old randomly assigned to a short-term collective music training program of just 4 weeks (20 sessions in total) had better performance on inhibitory control tasks compared to children who completed a comparable program of visual art training. Nevertheless, in their experiment they used a training program requiring daily commitment from the children. Moreover, this program was based primarily on listening activities without any instrumental training. Here, we wanted to investigate if a short-term orchestral program would also lead to an improvement in inhibitory control, as well as in impulsive and hyperactive behaviors. To do so, we focused on a short-term intense orchestral training program of 10 lessons provided in schools.

Since school is one of the most important settings in which it is possible to observe the children's behavior during activities that require attention and self-control (Re and Cornoldi, 2007), teachers could be potential valid informants for these scientific investigations. However, their point of view has not been adequately investigated in previous studies. Here, we decided to include questionnaires for our participants' teachers in order to test whether they would provide consistent and complementary 
measures of hyperactivity, inattention and impulsivity of the children as perceived by an observer's perspective.

Overall, with this longitudinal study we aimed to explore both near- and far-transfer effects of short orchestral music training, near being a transfer occurring between similar learning contexts and far a transfer involving skills that are very different from each other (Biasutti and Concina, 2013). For this purpose, we selected an inhibitory control task involving auditory processing to test whether a transfer effect on inhibitory control could be seen even after less than 3 months of orchestral music training, when assessing it using auditory stimulation. At the same time, we administered both to children and teachers questionnaires and tasks measuring hyperactivity, inattention and impulsivity to test whether short orchestral training is able to produce far transfer effects in these specific areas.

\section{MATERIALS AND METHODS}

\section{Participants}

We recruited 130 children 8-10 years of age, enrolled in public schools in the Apulia region in Southern Italy. Based on interviews with the teachers and on the outcomes of COM questionnaires (Cornoldi et al., 2004), we excluded from the sample children with a psychiatric diagnosis, traits of problematic behaviors, poor intellectual abilities or other relevant problems $(n=6)$. Furthermore, we did not include in the analysis four children who left the music training program and six children who were absent during one of the two assessments. The final number of participants included in our study was 113 third $(n=66)$ and fourth $(n=47)$ graders from four schools. The sample comprised 57 girls and 56 boys with a mean age of 8 years and 11 months at the baseline. Approximately, 36\% of the mothers and $11 \%$ of the fathers of our participants were unemployed. The majority of mothers and fathers had either a high school degree (around 40\%) or a secondary school degree (around 40\%). For more details on the demographic details, please refer to Table 1.

Our sample included two groups: a music group and a control group. Both groups were invited to this project from the schools by means of emails and direct communication with the teachers. Children in the music group $(n=55)$ attended 3 months of an innovative orchestral music training program that was implemented for free as extra-curricular course in the schools involved. The participation in the music training program (lasting around 3 months) was optional. Since the music program had a limited number of participants, the teachers selected a group of children without any music education/background to take part in the music program. Children in the control group $(n=58)$ did not participate in the music program and were recruited from the same schools following a similar procedure.

This study was part of the project "Armonie per la salute a scuola" approved and financed by the Apulia region for the years 2015/2016 and 2016/2017. The project provided a music training program implemented as part of the school curriculum and included the administration of tests and questionnaires for monitoring its effects ${ }^{1}$. Hence, this study conducted in an educational setting belongs to the special case described by both the national and the APA guidelines regarding the conduct of practicing psychologists ${ }^{2,3}$ for which ethical approval is not required. However, as part of the school activities, the parents were provided with detailed information concerning the music program and the administration of tests and questionnaires and gave their written informed consent for both. All data was anonymized upon completion of the study. In sum, this study was carried out in accordance with the ethical guidelines of the Declaration of Helsinki.

\section{Music Training Program}

In our study we focused on an innovative standardized orchestral music training program provided by the association "Music ' $n$ ' play." Music ' $n$ ' play (MusicaInGioco in Italian) is associated with the Italian Sistema delle Orchestre e dei Cori Giovanili e Infantili and has in the last decade involved more than 2,000 Italian children in the Apulia region through different initiatives, becoming deeply rooted in the Southern Italian context. Our study fell within one of the projects providing this specific training called "Armonie per la salute a scuola," approved and financed by the Apulia region for the years 2015/2016 and 2016/2017. Since 2014, this project has each year offered orchestral training in elementary schools as an after-school course of 13 lessons monitored by tests and questionnaires to evaluate its effects. All the children are given instruments free of charge, as well as the opportunity to practice at home every day. In particular, in our study the children were given violin, cello, percussions, flute and piano. Each lesson of this music training program lasts $2 \mathrm{~h}$ and $15 \mathrm{~min}$ : for $45 \mathrm{~min}$ the children play in small groups (instrument sections, each including four to five children with the same instrument), and for one and a half hours they play with the entire orchestra. The last lesson consists of a concert performed in high-profile events. This final concert represents a crucial part in this specific training program, due to the considerable emotional challenge that it implies. In our study the post-test was performed after the 10th lesson instead of the 13th for practical reasons: the beginning of the music training program was delayed and the last three lessons were provided after the end of the school year (more details can be found in the Limitations). For this reason, the Director supervising the music program organized two additional concerts in order for all of the children to perform during the 10th lesson. In particular, half of the children participating in the present study performed in a famous theatre in the Apulia region and the other half in an anti-mafia event aired on the main Italian TV channel Raiuno ${ }^{1,4}$

This orchestral music program, called Music ' $n$ ' play, is inspired by the El Sistema approach but, at the same time, it includes other pedagogical aspects from Freire, Sloboda, Dewey, Vygotskij, Orff, Dalcroze, Kodàli, Rolland and Gordon

\footnotetext{
${ }^{1}$ https://musicaingiocobari.wordpress.com/2016/08/02/armonie-per-la-salute-ascuola-ed-201516/

${ }^{2}$ https://www.aipass.org/node/11560

${ }^{3}$ http://www.apa.org/ethics/code/

${ }^{4}$ https://musicaingiocobari.wordpress.com/tag/armonie-per-la-salute-a-scuola/
} 
(Gargiulo and Altomare, 2017). The element shared with the ElSistema approach is the centrality of orchestral playing, seen as the starting point instead of the ending point of the musical experience. Children start playing together in an orchestra from their first lesson and, at the end of each year, they perform in one or more concerts in theatres with an orchestral ensemble that includes anywhere from 50 to 1000 instruments with a custom-made repertoire (for more details about this approach, see Gargiulo and Altomare, 2017). Music ' $n$ ' play works on three main areas of music learning, combining formal and informal learning procedures: imitation and repetition (call and response, body percussion, etc.), improvisation and creativity (including the use of multimedia contents), and formal learning (music reading, technique, intonation). Each of these three areas are developed mainly by means of body percussion, instrumental and vocal activity. One of the novel principles of this music program is the "reticularity," namely the ability of the teacher to adapt each lesson to the needs and the feedback of the children, with a primary goal of maintaining their engagement. The variety of exercises, including body percussion, call and response, improvisation, multimedia contents, repertoire, singing, sight reading, respiration, direction etc., offers the teachers the possibility to pique the attention and interest of the children by switching from one exercise to another depending on the children's feedback. Thanks to this learner-centered approach, the children receive a great deal of different information, mainly "by doing," and they have the possibility to process all this information individually and with peers. In this way interest and engagement in learning are continuously strengthened by activities that stimulate the initiative, finding original solutions to problems and interaction with peers. Moreover, the use of informal learning and the diversity of different music activities offer the performers the possibility to express their own music creativity through different channels. This strategy, combined with the reconceptualization of mistakes as learning opportunities instead of limits, has allowed this pedagogical approach to be used also with children diagnosed with ADHD and other neurodevelopmental disorders. Promising results have been observed, but not yet scientifically proven. A crucial aim of this music program is indeed to promote the integration of children who are experiencing difficulties for many reasons. Therefore, when this program is offered in schools, the headmasters and the teachers are always invited to include especially children with a psychiatric diagnosis or with social or behavioral problems. However, in our study we included only the healthy children involved in the program, in order to determine its general effects before moving to identifying its impact on specific pathologies.

\section{Procedures}

Data collection took place between February and March for the pre-test and early June for the post-test over two subsequent school years, 2015/2016 and 2016/2017. The pre-test was followed by 10 2-hours music lessons once a week, distributed over a 3-month period, for the music group. The last lesson consisted in a performed concert in theater or on live TV. One week after the concert, the teachers and the children from both groups underwent the post-test. The testing sessions took place in the schools, where the tests and questionnaires were administered individually by Italian licensed psychologists. The children were administered a stop-signal test (Walk-No Walk Test - Ranette) (Marzocchi et al., 2010), an impulsivity control test (Matching Figures MF-14) (Marzocchi et al., 2010) and a rating Scale assessing the levels of Inattention and Hyperactivity-impulsivity (SDAB) (Marzocchi et al., 2010). The teachers were also given the instructor version of this last Scale measuring the levels of Inattention and Hyperactivityimpulsivity (SDAI) (Cornoldi et al., 1996). In addition to these tests that provided the dependent variables for the current study on hyperactivity, impulsivity and inhibitory control, we administered to the children the Academic Self-Regulation Questionnaire (ASRQ) for another research question that will be reported separately. Moreover, qualitative data were also collected, but they will be discussed in a separate article. All measures were administered at both time points to children and teachers (Figure 1).

\section{Measures}

\section{Walk-No Walk Test (Ranette; Frogs)}

The Walk-No Walk Test (Marzocchi et al., 2010) is a paper-andpencil test that evaluates selective and sustained attention and the inhibition of an ongoing response. It is derived from the "stop signal task" of Logan and Cowan (1984). The test includes two A4 sheets of paper in which 20 sets of stairs (each including 12 steps) are drawn with a small frog on the first step. The task requires children to listen to a tape that will play two sounds: GO tone and NO-GO tone. The child is asked to fill in a step each time he or she hears the GO tone, while she/he has to stop every time she/he hears the NO-GO tone. The difficulty of the task lies in the fact that the first $208 \mathrm{~ms}$ of both tones are identical, but the NOGO tone is marked by a concluding vocal exclamation ("D'oh!"). Therefore, the task requires the children to listen to the entire sound before providing the response in order to understand if it is a $\mathrm{GO}$ or NO-GO signal. For each set of stairs, there are many GO signals and only one NO-GO signal. The number of correct trials defines the score.

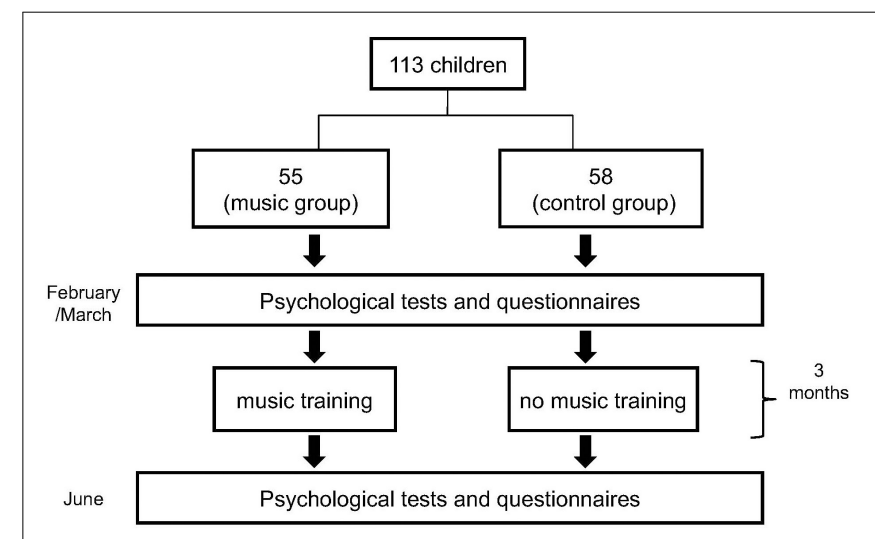

FIGURE 1 | Trial profile showing the different phases of the experiment. 


\section{SDAB and SDAI}

The SDAB and SDAI scales (Marzocchi et al., 2010) are used to measure the levels of hyperactivity-impulsivity and inattention. The SDAI scale is for teachers and includes 18 items and the SDAB is for children and includes 14 items. Half of the items relate to hyperactivity-impulsivity and the other half relate to inattention. High scores indicate a larger presence of hyperactivity-impulsivity/inattention.

\section{MF-14 Test}

The MF-test (Marzocchi et al., 2010) assesses several executive components, in particular sustained attention and impulsivity control, and is derived from the impulsivity control MFFT test (Kagan, 1966). We chose the short version of the test to reduce time spent on test administration and to comply with the school board's time requirements. This version, validated for children from early-primary school, consists of 14 items, including a target picture and six alternative pictures similar to the target. Among these pictures, only one is identical to the target, and the child has to identify it. The pictures represent objects from everyday life. Numbers of errors and response time are considered for the scoring of this test.

\section{COM/Teachers Scale}

The teachers were asked to fill out the COM scale (Cornoldi et al., 2004) used to detect the presence of possible traits of conduct disorder, oppositional defiant disorder, high-functioning autism, depression, anxiety, and Tourette syndrome in children. This questionnaire was added to ensure that the results were not skewed by possible pathologies in the participating children. The children with a score $>21$ were not included in the sample.

\section{Statistical Analysis}

Data analysis was conducted using the Statistical Package for the Social Sciences (SPSS version 25). Equality of variance at the premeasurement for age and all the tests and questionnaires was analyzed using Levene's test; a chi-square test was performed for the variables: sex, mother/father education and occupation. Mixed-design Analyses of Variance (ANOVAs) were performed examining scores as a function of one repeated measure (Time) and one between-subjects factor (Group). For each outcome measure (Walk-No Walk Test, SDAB, SDAI, MF-14) we wanted to investigate whether the difference between pre- and posttest varied as a function of Group (music or control). Thus, each participant had two scores (pre-test and post-test) for each outcome measure. Post hoc analyses were performed for each measure to indicate differences between pre-test and post-test in each group. We corrected the multiple testing with false discovery rate (FDR) method using a false discovery rate of 0.05 (Table 2).

\section{RESULTS}

Analyses of the groups' demographic variables at pre-test confirmed that the music and the control group did not differ at pre-test in terms of age, sex, COM questionnaire scores, mother's education, father's education, mother's occupation, and father's occupation (Table 1). Furthermore, a comparison of means indicated no significant differences at pre-test across groups for all the outcome measures used except for Walk-No Walk Test, $t(105)=4.31, p<0.01$ with children in the control group scoring higher than the music group.

Considering the performance on the Walk-No Walk Test, we found a significant main effect of Time, $F(1,105)=21.59$, $p<0.001, \eta 2 p=0.17$ and a significantly strong interaction between Group and Time, $F(1,105)=9.45, p=0.003, \eta 2 p=0.083$ (Figure 2). Moreover, a main effect of Group was found, $F(1,105)=12.33, p=0.001$ with children in the control group scoring higher than the music group. The post hoc test showed that the music group improved significantly $(p<0.001)$ between the two measurements, whereas the slight increase in performance of the control group was not significant $(p=0.27)$. In order to control for the difference between the groups at pre-test, we performed further analysis (ANCOVAs) for the other tests having the inhibitory control scores at pretest as a covariate.

Regarding our additional test MF-14, for the errors we found a main effect of Time, $F(1,111)=29.01, p<0.001, \eta 2 p=0.2$ indicating a reduction of errors in the post-test compared to the pre-test, but no significant interaction emerged between Group and Time, $F(1,111)=0.32, p>0.5, \eta 2 p=0.003$. We did not find a significant main effect of Group, $F(1,111)=1.41$, $p=0.24$. The covariate inhibitory control at pre-test was not significantly related to the MF-14 errors, $F(1,104)=1.42, p=0.24$. After controlling for the effect of inhibitory control, we did not obtain a main effect of Time, $F(1,104)=3.18$, $p=0.07$, Group, $F(1,104)=0.81, p>0.1$, nor an interaction between Group and Time, $F(1,104)=1.11, p>0.1$. Considering the MF-14 response time, we did not find any effect of Time, $F(1,111)=1.51$, $p>0.1$, Group, $F(1,111)=2.33, p=0.13$, or interaction between Group and Time, $(F<1)$. The covariate inhibitory control at pre-test was not significantly linked to the MF-14 response time, $F(1,104)=1.42, p=2.37$, and the results did not change after controlling for the covariate [Time, $F(1,104)=3.18$, $p=0.08$; Group, $F(1,104)=0.82, p=0.37$; Group $\times$ Time: $F(1,104)=1.11, p=0.29$.

For the hyperactivity-impulsivity dimension measured by the SDAB scale, we found no significant main effect of Time, $F(1,106)=2.14, p=0.15$ nor Group $(F<1)$, but we found a significant interaction between Group and Time, $F(1,106)=7.69, p=0.007, \eta 2 p=0.068$ (Figure 3 ). The post hoc test showed that children who did not follow any intense music program had a significant increase of hyperactivityimpulsivity ( $p=0.003$ ) from the pre- to the post-test, while the music group did not yield a significant difference between the two measurements $(p=0.37)$. However, a further post hoc test showed a significant difference between the two groups at post-measurement $t(108)=2.42, p=0.017$, reflecting less hyperactivity in the music group as compared with the control group (for the means, see Table 2). The covariate inhibitory control at pre-test was not significantly related to the hyperactivity-impulsivity, $F(1,99)=2.75, p=0.10$. Also in this case, a significant interaction between Group and Time was found, $F(1,99)=6.06, p=0.016, \eta 2 p=0.06$ and no 
TABLE 1 | Demographic details of the children and their parents at pre-test.

\begin{tabular}{|c|c|c|c|c|c|c|}
\hline & \multicolumn{2}{|c|}{ Music Group } & \multicolumn{2}{|c|}{ Control Group } & \multirow[b]{2}{*}{$t$} & \multirow[b]{2}{*}{$p$} \\
\hline & $M$ & $S D$ & $M$ & $S D$ & & \\
\hline Age (months) & 106.18 & 6.33 & 107.60 & 6.84 & 1.15 & 0.25 \\
\hline \multirow[t]{2}{*}{ COM questionnaire } & 4.00 & 5.06 & 4.48 & 5.50 & 0.49 & 0.63 \\
\hline & \multicolumn{2}{|l|}{$\%$} & \multicolumn{2}{|l|}{$\%$} & $x^{2}$ & $p$ \\
\hline Sex (female) & \multicolumn{2}{|l|}{$41,8 \%$} & \multicolumn{2}{|l|}{$58,6 \%$} & 3.19 & 0.07 \\
\hline Mother occupation & $\begin{array}{l}17 \% \text { em } \\
0 \% \text { entr } \\
3 \% \text { freel } \\
22 \% \text { un } \\
6 \% \text { othe }\end{array}$ & & $\begin{array}{l}22 \% \text { em } \\
3 \% \text { entr } \\
11 \% \text { fre } \\
16 \% \text { un } \\
5 \% \text { othe }\end{array}$ & & 8.54 & 0.07 \\
\hline Mother education & \multicolumn{2}{|c|}{$\begin{array}{l}4 \% \text { primary school } \\
23 \% \text { secondary } \\
\text { school } \\
16 \% \text { high school } \\
6 \% \text { bachelor/master } \\
1 \% \text { other }\end{array}$} & \multicolumn{2}{|c|}{$\begin{array}{l}0 \% \text { primary school } \\
21 \% \text { secondary } \\
\text { school } \\
24 \% \text { high school } \\
11 \% \text { bachelor/master } \\
0 \% \text { other }\end{array}$} & 7.85 & 0.1 \\
\hline Father occupation & $\begin{array}{l}23 \% \text { em } \\
2 \% \text { entr } \\
13 \% \text { fre } \\
7 \% \text { uner } \\
1 \% \text { othe }\end{array}$ & & $\begin{array}{l}31 \% \text { em } \\
3 \% \text { entr } \\
19 \% \text { fre } \\
3 \% \text { uner } \\
0 \% \text { othe }\end{array}$ & & 4.17 & 0.384 \\
\hline Father education & $\begin{array}{l}5 \% \text { prim } \\
21 \% \text { se } \\
\text { school } \\
16 \% \text { hig } \\
5 \% \text { bacl } \\
1 \% \text { othe }\end{array}$ & & $\begin{array}{l}0 \% \text { prim } \\
22 \% \text { sec } \\
\text { school } \\
27 \% \text { hig } \\
5 \% \text { bacl } \\
1 \% \text { othe }\end{array}$ & vline & 8.26 & 0.08 \\
\hline
\end{tabular}

significant effect of Time, $(F<1)$ and Group, $F(1,99)=0.1$, $p=0.76$ emerged after controlling for inhibitory control at pretest. To further explore the results in hyperactivity-impulsivity, we considered the items related to the hyperactivity and

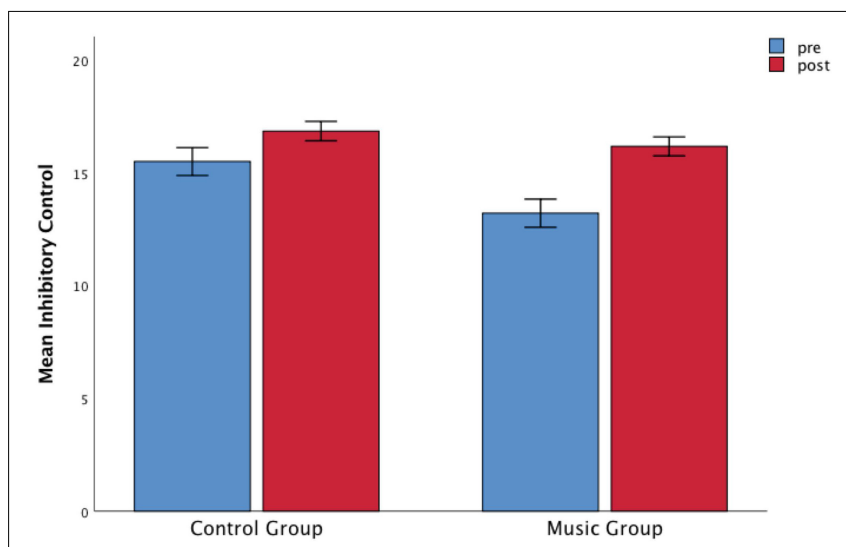

FIGURE 2 | The music group was associated with an increase in inhibitory control scores starting from a significantly lower score at pre-test compared to the control group. Children's mean scores as a function of Time (pre-test and post-test) and Group (music and control). Error bars are standard errors. the ones referring to the impulsivity separately, performing two separate ANOVAs (see Supplementary Material). The inattention measured with the SDAB scale did not yield any main effect of Time, $F(1,106)=3.21, p=0.076$, Group $(F<1)$, nor an interaction between Group and Time, $(F<1)$. Also in this case, the covariate inhibitory control was not related to the dependent variable $(F<1)$ and the results did not change after controlling for the covariate [Time, $F<1$; Group, $F<1$; Group $\times$ Time, $F<1$ ].

Finally, we analyzed the ratings given to the children on the SDAI rating scale by their teachers and found no significant main effect of Time for the hyperactivity-impulsivity subscale, $F(1,84)=1.36, p>0.1$, nor a main effect of Group $(F<1)$. Moreover, there was no significant interaction between Group and Time $(F<1)$. The covariate inhibitory control at pre-test was not related to the hyperactivity-impulsivity perceived by the teachers, $F(1,78)=2.13, p=0.15$. We did not find any main effect of Group $(F<1)$ and interaction between Group and Time $(F<1)$. However, we observed a main effect of Time, $F(1,78)=9.45, p=0.003, \eta 2 p=0.11$ after controlling for the inhibitory control at pre-test. For the inattention subscale of SDAI, we found a main effect of Time, $F(1,84)=7.26, p=0.009$, $\eta 2 p=0.08$ indicating a reduction of inattention levels in the post-test compared to the pre-test, but a non-significant main effect of Group, $F(1,84)=1.91, p=0.17$, and a non-significant 
TABLE 2 | Means (M) and standard deviation (SD) of the scores obtained by the two groups (music group and control group) at pre-test and post-test and ANOVA results.

\begin{tabular}{|c|c|c|c|c|c|c|c|c|c|c|}
\hline & \multicolumn{4}{|c|}{ Music Group } & \multicolumn{4}{|c|}{ Control Group } & & \\
\hline & \multicolumn{2}{|c|}{ pre } & \multicolumn{2}{|c|}{ post } & \multicolumn{2}{|c|}{ pre } & \multicolumn{2}{|c|}{ post } & \multicolumn{2}{|c|}{ pre-post } \\
\hline & $M$ & $S D$ & $M$ & $S D$ & $M$ & $S D$ & $M$ & $S D$ & p-value & FDR-corrected \\
\hline \multicolumn{11}{|l|}{ Children } \\
\hline Walk-No Walk & 13.68 & 4.04 & 16.23 & 3.16 & 16.57 & 2.8 & 17.09 & 2.88 & 0.003 & 0.02 \\
\hline MF errors & 8.33 & 4.59 & 6.09 & 4.18 & 7.21 & 5.25 & 5.40 & 3.93 & 0.57 & 0.1 \\
\hline MF time & 9.18 & 4.74 & 8.82 & 5.23 & 10.76 & 6.21 & 10.04 & 5.37 & 0.68 & 0.68 \\
\hline SDAB hyperactivity-impulsivity & 16.83 & 2.68 & 16.35 & 2.65 & 16.13 & 2.76 & 17.68 & 3.31 & 0.007 & 0.025 \\
\hline SDAB inattention & 19.44 & 2.85 & 18.52 & 2.99 & 19.13 & 2.94 & 18.62 & 3.59 & 0.6 & 0.84 \\
\hline \multicolumn{11}{|l|}{ Teachers } \\
\hline SDAI hyperactivity-impulsivity & 1.14 & 2.15 & 1.03 & 2.99 & 1.33 & 2.41 & 0.80 & 1.54 & 0.44 & 1.03 \\
\hline SDAI Inattention & 2.32 & 3.80 & 1.57 & 3.08 & 3.41 & 3.80 & 2.31 & 2.92 & 0.62 & 0.72 \\
\hline
\end{tabular}

interaction between Group and Time $(F<1)$ (Figure 4). Our ANCOVA showed that the inhibitory control at pre-test was not related to the levels of inattention indicated by the teachers, $F(1,78)=1.63, p=0.21$. There was no significant effect of Time, $F(1,78)=1.59, p=0.21$, Group $F(1,78)=1.72, p=0.19$, or Interaction Group by Time $(F<1)$ after controlling for the effects of inhibitory control.

\section{DISCUSSION}

This study was designed to examine if a short-term orchestral music training program implemented in schools is able to enhance inhibitory control and reduce hyperactivity, inattention and impulsivity in children. For this purpose, we selected a systematic and intense orchestral training program that enables children who have never played a musical instrument before, to play in an orchestra in front of a large audience after just 3 months of training. In order to explore the effects of this music program better, we included tasks measuring near- and fartransfer effects, and we administered questionnaires to both the children and their teachers.

Concerning the near-transfer effects, our results suggest that even short orchestral music training can facilitate the development of inhibitory control when mediated by auditory stimulation (Figure 2). However, we noticed lower scores in inhibitory control at baseline measurement in children from the music group compared to the control group. The reason for this could be related to the use of a community-based quasiexperimental design without randomization of participants: the inclusive philosophy focused on children with special needs inspiring the music training might have implicitly guided the selection of children for the experimental group. It could be argued that the significantly higher scores at the pre-test in the control group could be the reason why we did not find a significant increase of inhibitory control in this group compared to the music group which, on the contrary, may have had more room for improvement. Nevertheless, we found an equality of variance between the two groups (music and control) for all the

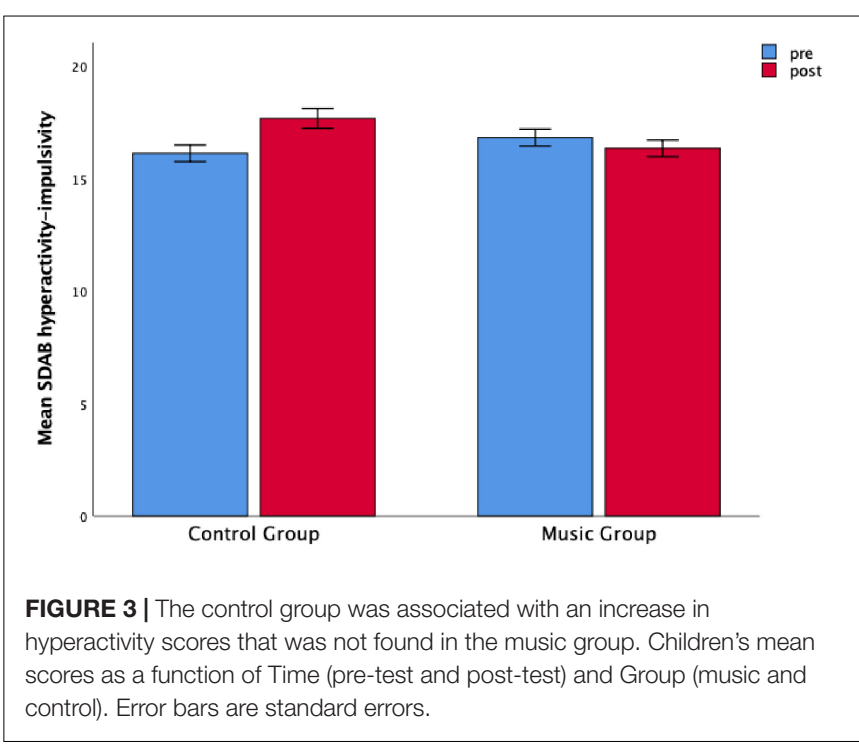

other variables considered in the study. In order to control for the difference in inhibitory control between the two groups at pre-test, we performed further analyses inserting this variable as covariate. The ANCOVAs did not alter the main result found for the SDAB questionnaire (for children) nor any other interaction including Group as factor. However, some effects of the covariate were obtained on the main effect of Time for the MF errors and the SDAI questionnaires (for teachers).

Although we found a significant difference at the baseline between the experimental and the control group, our post hoc analysis showed that the children who underwent music training had a clear-cut improvement $(p=0.004)$ in the Walk-No Walk test requiring selective attention, sustained attention and inhibition of an ongoing response. Considering that the orchestral music program selected for this study is directed especially to children with behavioral, social or psychological difficulties, these results are encouraging and very relevant for pedagogical implications. Moreover, this finding shows that a short orchestral training program of just 10 session over 3 months 


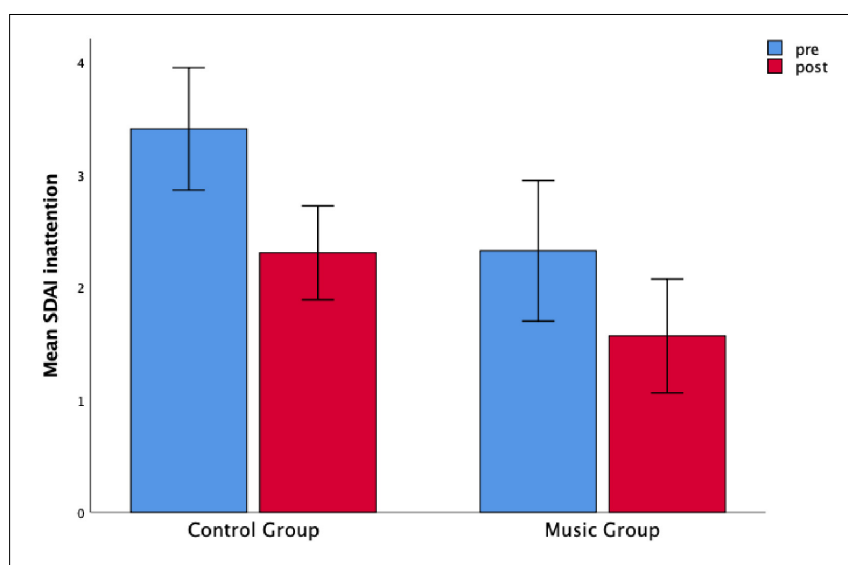

FIGURE 4 | Both the control group and music groups showed a decrease in inattention scores rated by the teachers. Teachers' mean scores as a function of Time (pre-test and post-test) and Group (music and control). Error bars are standard errors.

results in a transfer effect on inhibitory control in 8-10 yearold children. This can have important implications for music pedagogy and education. All the previous studies conducted with collective instrumental music training have always taken into consideration music programs lasting at least one year and, therefore, requiring an amount of financial resources not always affordable for institutions such as public schools. The possibility to implement short-term, and therefore less expensive but efficient orchestral music programs involving only few teachers for a limited period of time, could make music training more accessible for schools. Given that public schools are accessible to all, and provide the opportunity for early intervention (Diamond and Lee, 2011), this would represent a big step forward in education.

Although we did not find a significant effect of this music program on inattention and impulsivity as perceived by the children, a far-transfer effect of this innovative music program on hyperactivity-impulsivity was recorded by the SDAB scale (see Figure 3). Comparing the post- with the pre-test, the children belonging to the control group showed a strong increment of hyperactivity-impulsivity over the three months that was not found in the music group (Figure 3). This effect was clear especially for hyperactivity (see Supplementary Material). Considering the items used to measure the levels of hyperactivity ("Do you have difficulties in staying seated? Do you like to dangle your feet or have something in your hands to play with? Do you find difficult to not leave the seat in situations where remaining seated is expected? Do you have difficulties in playing or engaging in leisure activities quietly? Have you been told that you are not able to stay put?"), we can hypothesize that we found an increase in the control group at the post-test because the assessment had been conducted in the last days of the schools when the children are usually already in "holiday mode." On the contrary, the children who had followed the music program had experienced call and response improvisations that implied waiting for a response. They also had to sit still in the orchestra constantly paying attention to the multisensory inputs deriving from the director and the other players. They had to do this for the duration of the entire rehearsal, and carry out many other regulatory activities of the Music ' $n$ ' play program. They did not show an increase in hyperactive behaviours, and it does indeed appear likely that this was a consequence of the demands of the music training program.

The engagement produced in the children by the ability of the conductor to mediate and adapt the different needs and music competences, together with the incentive salience of music per se, may have played a crucial role in modulating these functional behaviors in the children. This result opens the door to a new way of approaching and treating hyperactive behaviors and, potentially, ADHD. The interventions that have been used up until now to train children in regulating their hyperactive behavior have been directed only to children already diagnosed with ADHD and have been focused on cognitivebehavioral strategies and, more recently, on training of executive functions (Re and Cornoldi, 2007; Salvaguardia et al., 2009; Bergman Nutley et al., 2011; Röthlisberger et al., 2012; Halperin et al., 2013; Re et al., 2015). Although some positive results are emerging from the interventions focusing on executive functions (Re et al., 2015), they do not offer the children the same degree of engagement that music is able to produce. Rewarding and pleasurable activities, such as music listening, playing and going to concerts, peak in early adolescence (North et al., 2000). Therefore, the use of music training as a tool to improve executive functions and the regulation of behaviors could lead to even better results, reinforced exponentially by the strong engagement that music implies, especially for children. Some researchers have tried to use music therapy to treat children with ADHD. However, the therapies usually consist of music treatments reserved exclusively for children with ADHD (Rickson, 2006) and with poor results. Oftentimes, the subscription to programs explicitly created for children with inattention and hyperactivity problems further amplifies their social marginalization, reinforcing their dysfunctional behaviors. Therefore, compared to treatments focused on the enhancement of specific skills that children diagnosed with ADHD lack, a music program involving both children with and without a specific diagnosis of ADHD and presented just as music training may represent a way to avoid the inevitable tendency to marginalization. We hypothesize that a teaching method similar to the one adopted in the music program of this study may lead to an improvement of inhibitory control and regulation of hyperactive behaviors especially in children with ADHD or other developmental diseases thanks to the inclusiveness of this approach and the role of the conductor of acknowledging the contributions and competences of each performer without labeling any creative contribution as a mistake.

However, the effects of the music training program were less tangible on hyperactivity and inattention as rated by the teachers. A global significant reduction of inattention was found from pre- to post-test. Similarly, the hyperactivity indicated by the teachers was significantly smaller in the post-test for both the groups after correcting for the inhibitory control at pre-test. Therefore, part of the improvements seemed to be due to the general activities in which the children were involved during 
this period and to their associated maturation. However, it is important to emphasize that the teachers played an active role in the selection of the music group. Therefore, it is possible that the teachers tended to be more receptive to the symptoms expressed by the children undergoing the music program, adopting a more critical view. This could have affected the evaluation of the reduction of the dysfunctional behaviors, such as inattention, in the music group. This obtained discrepancy between the teachers' attitudes toward the children and the actual school behavior of the students reveals some level of subjectivity that should be taken into consideration when evaluating the effects of an extra-curricular intervention on children and on the school context in general.

\section{Limitations}

One limitation in this study is the absence of randomization of the two groups of participants, as a result of this communitybased approach aiming at studying a music training program already successfully implemented in the area. For this reason, our results should be treated with caution and further investigations providing randomized controlled trials are needed. Nevertheless, these results have intrinsic value since they examine an existing phenomenon of a large music intervention involving thousands of children in Southern Italy, attracting media attention and funding from local agencies ${ }^{5}$.

Moreover, our results are limited by the absence of an active control group that would have allowed us to better identify effects linked specifically to learning to play a musical instrument in an orchestra. However, the improvement of the music group in the auditory inhibitory control task attests an interaction between the cognitive control system and the auditory domain that is consistent with previous studies (Amer et al., 2013; Bialystok and DePape, 2009). Music experience has been shown to enhance subcortical processing of auditory stimuli in noisy environments with a related improvement of cognitive control (Parbery-Clark et al., 2009; Strait et al., 2014). At the same time, the inclusion of a passive control group not engaging in intensive training allowed us to control the naturally occurring behavioral and psychological maturation in this age range, characterized by rapid brain development.

In this study, as an additional measure of impulsivity seen as the negative counterpart of inhibitory control, we used the short version of the MF-test, MF-14 (Marzocchi et al., 2010). We did not administer the full MF-20 test, due to the request from the school of having assessments as brief as possible. This test did not show any differences between groups after the music training program, hence mismatching what was found with the test on inhibitory control. However, the lack of findings here might be attributed to the shortened version of the test. The administration of the long version MF-20 (Marzocchi et al., 2010) may possibly have shown a different result on impulsivity measures.

Finally, the limited amount of music lessons between the two measurements may not have been enough to enhance far transfer

${ }^{5}$ http://www.musicaingioco.net effects on impulsivity. Since we wanted to perform our posttest at school to avoid absences and missing data, we needed to do it before the end of the school year, slightly before the music program was completed. After the post-test, the children received two more lessons and performed in an additional final concert (for a total of 13 lessons). Therefore, it may be possible that the effect of this short training program would have been stronger if measured 1 month later, after the entire program was completed.

Despite these limitations, this study, to our knowledge, provides the first experimental evidence on the effects of a short orchestral music training program on inhibitory control and hyperactivity.

\section{CONCLUSION}

Children with a tendency toward hyperactive or impulsive behaviors are usually left without any treatment or training until they are diagnosed with ADHD. Once the child is labeled with this diagnosis, his/her symptoms become more resistant and harder to treat due to the social implications that accompany a diagnosis. The results illustrated here suggest that even a short, but systematic and engaging orchestral training program can facilitate the development of inhibitory control modulating the levels of hyperactivity self-reported by the children. Therefore, short, systematic music interventions rationalizing financial and teachers' resources and well-grounded on pedagogical principles might represent a potential educational tool for school-age children. Moreover, it can prevent tendencies toward hyperactive behavior that could further develop into a diagnosis and eventually treat ADHD symptoms. Nevertheless, future investigations should be conducted to test whether the findings that we are presenting here with healthy children can be extended to children diagnosed with ADHD.

\section{AUTHOR CONTRIBUTIONS}

EB and MF contributed equally to the conception and design of the work. MF, CS, LM, and VdP executed data collection supervised by EB, RC, and MK. MF performed the analysis and created the figures. PV had an important role in the fund raising for the project. MF wrote the article. All authors critically revised and approved the final version of the manuscript.

\section{FUNDING}

This work was supported by Center for Music in the Brain (MIB), funded by the Danish National Research Foundation (DNRF 117).

\section{ACKNOWLEDGMENTS}

We wish to thank all the children participating in this study and their teachers. We also thank other members of our 
team who helped with data collection, data entry, and initial analysis, in particular Giada Della Rocca, Valentina Massaro, Alessia Destratis, Valeria Ruggieri, Eleonora Russo, Anna Chiara Cattedra, Adriana Giancaspro, Ida Siemens Lorenzen, Johanna Pardon, Leonardo Bonetti, and Marina Kliuchko. We gratefully acknowledge the association "MusicaInGioco" (Music ' $n$ ' play), in particular Director Andrea Gargiulo, for their commitment in organizing extra concerts with the children before our second measurement. We also thank the primary schools for their

\section{REFERENCES}

Alemán, X., Duryea, S., Guerra, N. G., McEwan, P. J., Muñoz, R., Stampini, M., et al. (2017). The effects of musical training on child development: a randomized trial of el sistema in venezuela. Prev. Sci. 18, 865-868. doi: 10.1007/s11121-0160727-3

Amer, T., Kalender, B., Hasher, L., Trehub, S. E., and Wong, Y. (2013). Do older professional musicians have cognitive advantages? PLoS One 8:e71630. doi: 10.1371/journal.pone.0071630

Barkley, R. A. (1997). Behavioral inhibition, sustained attention, and executive functions: constructing a unifying theory of ADHD. Psychol. Bull. 121, 65-94. doi: 10.1037/0033-2909.121.1.65

Bergman Nutley, S., Söderqvist, S., Bryde, S., Thorell, L. B., Humphreys, K., and Klingberg, T. (2011). Gains in fluid intelligence after training non-verbal reasoning in 4-year-old children: a controlled, randomized study. Dev. Sci. 14, 591-601. doi: 10.1111/j.1467-7687.2010. 01022.x

Bialystok, E., and DePape, A. M. (2009). Musical Expertise, Bilingualism, and Executive Functioning. J. Exp. Psychol. Hum. Percept. Perform. 35, 565-574. https://doi.org/10.1037/a0012735

Biasutti, M. (2013). Orchestra rehearsal strategies: conductor and performer views. Music. Sci. 17, 57-71. doi: 10.1177/10298649124 67634

Biasutti, M., and Concina, E. (2013). Music education and the transfer of learning. J. Commun. Res. 5, 397-413.

Cornoldi, C., Gardinale, M., and Masi, A. P. L. (1996). Impulsività e Autocontrollo. Trento: Erikson.

Cornoldi, C., Molin, A., and Marcon, V. (2004). Il Questionario COM: uno strumento di identificazione di problematiche associate al DDAI. Difficoltà di Apprendimento 9, 391-412.

Diamond, A., and Lee, K. (2011). Interventions shown to aid executive function development in children 4 to 12 years old. Science 333, 959-964. doi: 10.1126/ science. 1204529

Duke, R. A., Cash, C. D., and Allen, S. E. (2011). Focus of attention affects performance of motor skills in music. J. Res. Music Educ. 59, 44-55. doi: 10. $1177 / 0022429410396093$

Gargiulo, A., and Altomare, E. (2017). Musicabilia - Disabilità, "El Sistema Abreu" e Neuroscienze. Bari: Radici Future.

Habibi, A., Cahn, B. R., Damasio, A., and Damasio, H. (2016). Neural correlates of accelerated auditory processing in children engaged in music training. Dev. Cogn. Neurosci. 21, 1-14. doi: 10.1016/j.dcn.2016. 04.003

Habibi, A., Ilari, B., Crimi, K., Metke, M., Kaplan, J. T., Joshi, A. A., et al. (2014). An equal start: absence of group differences in cognitive, social, and neural measures prior to music or sports training in children. Front. Hum. Neurosci. 8:690. doi: 10.3389/fnhum.2014 .00690

Hallam, S. (2010). The power of music: its impact on the intellectual, social and personal development of children and young people. Int. J. Music Edu. 28, 269-289. doi: 10.1177/025576141037 0658

Halperin, J. M., Marks, D. J., Bedard, A. C. V., Chacko, A., Curchack, J. T., Yoon, C. A., et al. (2013). Training executive, attention, and motor skills: a proof-ofconcept study in preschool children with ADHD. J. Atten. Disord. 17, 711-721. doi: $10.1177 / 1087054711435681$ cooperation in the process of recruitment and Hella Kastbjerg and Olivia Foster Vander Elst for proofreading the article.

\section{SUPPLEMENTARY MATERIAL}

The Supplementary Material for this article can be found online at: https://www.frontiersin.org/articles/10.3389/fpsyg. 2019.00750/full\#supplementary-material

Holochwost, S. J., Propper, C. B., Wolf, D. P., Willoughby, M. T., Fisher, K. R., Kolacz, J., et al. (2017). Music education, academic achievement, and executive functions. Psychol. Aesthet. Creat. Arts 11, 147-166. doi: 10.1037/aca000 0112

Jaschke, A. C., Honing, H., and Scherder, E. J. A. (2018). Longitudinal analysis of music education on executive functions in primary school children. Front. Neurosci. 12:103. doi: 10.3389/fnins.2018. 00103

Kagan, J. (1966). Reflection-impulsivity: the generality and dynamics of conceptual tempo. J. Abnorm. Psychol. 71, 17-24. doi: 10.1037/ h0022886

Logan, G. D., and Cowan, W. B. (1984). On the ability to inhibit thought and action: a theory of an act of control. Psychol. Rev. 121, 66-95. doi: 10.1037/0033-295X. 91.3.295

Majno, M. (2012). From the model of El Sistema in Venezuela to current applications: learning and integration through collective music education. Ann. N. Y. Acad. Sci. 1252, 56-64. doi: 10.1111/j.1749-6632.2012. 06498.x

Marzocchi, G. M., Re, A. M., and Cornoldi, C. (2010). BIA - Batteria Italiana per l'ADHD. Trento: Erikson.

Moreno, S., Bialystok, E., Barac, R., Schellenberg, E. G., Cepeda, N. J., and Chau, T. (2011). Short-term music training enhances verbal intelligence and executive function. Psychol. Sci. 22, 1425-1433. doi: 10.1177/095679761141 6999

Münte, T. F., Altenmüller, E., and Jäncke, L. (2002). The musician's brain as a model of neuroplasticity. Nat. Rev. Neurosci. 3, 473-478. doi: 10.1038/nrn843

North, A. C., Hargreaves, D. J., and O'Neill, S. A. (2000). The importance of music to adolescents. Br. J. Educ. Psychol. 70(Pt 2), 255-272. doi: 10.1348/ 000709900158083

Palmer, C., and Drake, C. (1997). Monitoring and planning capacities in the acquisition of music performance skills. Can. J. Exp. Psychol. 51:369. doi: 10. 1037/1196-1961.51.4.369

Parbery-Clark, A., Skoe, E., and Kraus, N. (2009). Musical experience limits the degradative effects of background noise on the neural processing of sound. J. Neurosci. 29, 14100-14107. doi: 10.1523/JNEUROSCI.3256-09. 2009

Re, A. M., Capodieci, A., and Cornoldi, C. (2015). Effect of training focused on executive functions (attention, inhibition, and working memory) in preschoolers exhibiting ADHD symptoms. Front. Psychol. 6:1161. doi: 10.3389/ fpsyg.2015.01161

Re, A. M., and Cornoldi, C. (2007). ADHD at five: a diagnosis-intervention program. Adv. Learn. Behav. Disabil. 20, 223-240. doi: 10.1016/S0735-004X(07) 20009-6

Rickson, D. J. (2006). Instructional and improvisational models of music therapy with adolescents who have attention deficit hyperactivity disorder (ADHD): a comparison of the effects on motor impulsivity. J. Music Ther. 43, 39-62. doi: $10.1093 /$ jmt/43.1.39

Röthlisberger, M., Neuenschwander, R., Cimeli, P., Michel, E., and Roebers, C. M. (2012). Improving executive functions in 5-and 6-year-olds: evaluation of a small group intervention in prekindergarten and kindergarten children. Infant Child Dev. 21, 411-429. doi: 10.1002/icd.752

Sachs, M., Kaplan, J., Der Sarkissian, A., and Habibi, A. (2017). Increased engagement of the cognitive control network associated with music training in children during an fMRI Stroop task. PLoS One 12:e0187254. doi: 10.1371/ journal.pone.0187254 
Salvaguardia, F., Re, A. M., Caponi, B., and Cornoldi, C. (2009). Esperienza di un training sulla memoria di lavoro con bambini con tratti di disattenzione e iperatttività (experience with a working memory training with children with disattention/hyperactivity traits). Disturbi di Attenzione e Iperattività 4, 171-187.

Schellenberg, E. G., Corrigall, K. A., Dys, S. P., and Malti, T. (2015). Group music training and children's prosocial skills. PLoS One 10:e0141449. doi: 10.1371/ journal.pone.0141449

Strait, D. L., O'Connell, S., Parbery-Clark, A., and Kraus, N. (2014). Musicians' enhanced neural differentiation of speech sounds arises early in life: developmental evidence from ages 3 to 30. Cereb. Cortex 24, 2512-2521. doi: $10.1093 /$ cercor/bht103

Thorell, L. B., Lindqvist, S., Nutley, S. B., Bohlin, G., and Klingberg, T. (2009). Training and transfer effects of executive functions in preschool children. Dev. Sci. 12, 106-113. doi: 10.1111/j.1467-7687.2008. 00745.x
Whelan, R., Conrod, P. J., Poline, J. B., Lourdusamy, A., Banaschewski, T., Barker, G. J., et al. (2012). Adolescent impulsivity phenotypes characterized by distinct brain networks. Nat. Neurosci. 15, 920-925. doi: 10.1038/nn. 3092

Conflict of Interest Statement: The authors declare that the research was conducted in the absence of any commercial or financial relationships that could be construed as a potential conflict of interest.

Copyright (C) 2019 Fasano, Semeraro, Cassibba, Kringelbach, Monacis, de Palo, Vuust and Brattico. This is an open-access article distributed under the terms of the Creative Commons Attribution License (CC BY). The use, distribution or reproduction in other forums is permitted, provided the original author(s) and the copyright owner(s) are credited and that the original publication in this journal is cited, in accordance with accepted academic practice. No use, distribution or reproduction is permitted which does not comply with these terms. 


\title{
Flow and Satisfaction With Life in Elite Musicians and Top Athletes
}

\author{
Katarina Habe ${ }^{1 *}$, Michele Biasutti ${ }^{2}$ and Tanja Kajtna ${ }^{3}$ \\ ${ }^{1}$ Academy of Music, University of Ljubljana, Ljubljana, Slovenia, ${ }^{2}$ Department FISPPA, University of Padova, Padova, Italy, \\ ${ }^{3}$ Faculty of Sport, University of Ljubljana, Ljubljana, Slovenia
}

OPEN ACCESS

Edited by: Rosie Perkins,

Royal College of Music, United Kingdom

Reviewed by: Bruno Gingras,

University of Vienna, Austria Paula Thomson,

California State University, Northridge, United States

*Correspondence: Katarina Habe katarina.habe@ag.uni-lj.si

Specialty section: This article was submitted to Performance Science, a section of the journal Frontiers in Psychology

Received: 10 December 2018 Accepted: 13 March 2019 Published: 29 March 2019

Citation: Habe K, Biasutti $M$ and Kajtna $T$ (2019) Flow and Satisfaction With Life in Elite Musicians and Top Athletes. Front. Psychol. 10:698. doi: 10.3389/fpsyg.2019.00698
Although flow has been studied extensively in music and sport, there is a lack of research comparing these two domains. With the aim of filling this gap, elite musicians and top athletes in Slovenia were contrasted in the current study. Differences for flow and satisfaction with life between elite musicians and top athletes were explored. Individual versus group performance setting and gender differences were considered. 452 participants; 114 elite Slovenian musicians (mean age 23.46 years) and 338 top Slovenian athletes (mean age 22.40 years) answered questions about flow and satisfaction with life measures. The results show differences between elite musicians and top athletes in four flow dimensions: transformation of time and autotelic experience were higher in musicians while clear goals and unambiguous feedback were higher in athletes. However, differences in global flow were not confirmed. Elite musicians and top athletes experienced flow more often in group than in individual performance settings and surprisingly it was experienced more in male than in female top performers. Satisfaction with life has a positive correlation with all nine dimensions of flow, but only challenge-skill balance was a significant predictor for satisfaction with life.

Keywords: flow, satisfaction with life, expert performance, top athletes, elite musicians

\section{INTRODUCTION}

There is a growing interest in research into flow in music and sport. These two fields have several aspects in common, such as that both music and sport are performative activities in which the performers have to give their best. The concert is the typical situation in which musicians have to produce a peak performance, and as contests and matches are also where athletes have to produce performance excellence. There are several studies that have associated flow with peak performance (O’Neill, 1999; MacDonald et al., 2006; Baker and MacDonald, 2013; Marin and Bhattacharya, 2013). Flow was extensively studied in top athletes (Lindsay et al., 2005; Nicholls et al., 2005; Sugiyama and Inomata, 2005; Aherne et al., 2011; Pain et al., 2011; Pates et al., 2012; Briegel-Jones et al., 2013; Pates and Cowen, 2013; Hodge et al., 2014; Koehn et al., 2014; Norsworthy et al., 2017; for review, see Swann et al., 2012), but there is a scarcity of data exploring flow in elite musicians (Sinnamon et al., 2012). Further, flow in music and sport has typically been considered separately without comparing these two domains.

There may also be other relevant points of comparison for musicians and athletes, such as satisfaction with life. Satisfaction with life is connected to the stressful life that musicians and athletes lead, but few studies have investigated the relationship between flow and satisfaction with life in top/elite performers. Although previous studies have concentrated mainly on the measurement of affective well-being - considering flow as an emotional phenomenon - the 
measurements of life satisfaction could complement traditional affective measurements which has proven useful in predicting reactions to stressful events (Huebner and Dew, 1996; Bradley and Corwyn, 2004).

Trying to fill this gap, the current study contrasted elite musicians and top athletes for flow and satisfaction with life. Individual versus group performance settings and gender differences were considered. The literature review covers several issues, including defining performance excellence, similarities and differences between sport and music performance, flow and music, flow and sports, and flow and satisfaction with life.

\section{Defining Performance Excellence}

There are many synonyms for performance excellence, such as peak performance, expert performance, elite performance, top performance, optimal performance and performance expertise (Berkopec, 2016). Peak performance is defined as: "quantitatively and/or qualitatively absolutely outstanding action results achieved under regular conditions" (Nitsch and Hackfort, 2016, p. 15). In this concept the following three features are emphasized: "peak performance implies acting at one's own limits, it requires full commitment to the expert activity and often neglecting all other life areas and it brings publicity to the performer" (Nitsch and Hackfort, 2016, p. 15). There are several differences in music and sport when defining excellence. The criteria are often far more subjective in the music domain than in sport. In sport scores and timings are all that counts, and the main criteria of success involve quantity. Conversely, musical excellence is a result of a subtle unreachable interplay between musical piece, performer/s, performance setting and audience (Waddell et al., 2018). When talking about performance excellence in the case of music and sport, a performance should be referred to as work rather than as leisure (Juniu et al., 1996; Delle Fave et al., 2011c; Delle Fave and Zager Kocjan, 2016).

\section{Similarities and Differences Between Sport and Music Performance}

Sport and music performances share many similarities, such as requirements for prolonged training, endurance, perfectionism, high self-regulation, strategic decisions, emotional expressivity, and social skills. Music facilitates several elements of sport performance such as arousal regulation, synchronization, acquisition of motor skills (Pates et al., 2003).

With regard to the differences, music training often starts earlier than sports training involves fine-motor skills, and takes place in extremely competitive environments, that often cause anxiety even in top performers (Biasutti and Concina, 2014). Anxiety in top performers is not necessarily due to the competitive environment, but also because the evaluation of what makes a good musical performance is subjective, thus introducing a larger degree of uncertainty than in sports. However, also sport performances take place in particularly competitive settings which could be subjected to external factors and evaluations. In sports such as figure skating, dancing, artistic gymnastics the performance is assessed by judges and this could cause additional stress for the characteristics of the evaluation process. In addition, emotional states and expressivity are frequently part of success, because for athletes it is important to express their feelings as opposed to holding them down and thus increasing tension, which might disrupt performance (Kajtna and Jeromen, 2013).

Musical and sport performances are frequently linked to emotional states, such as flow (Martin, 2008; Altenmüller and Ioannou, 2016). One common aspect of music and sport performance is that both have a strong potential to elicit flow. Csikszentmihalyi $(1990,1993)$ found that artists and athletes seemed more likely to experience flow, especially during their work. Csikszentmihalyi (1975, p. 36) defined flow as "the holistic sensation that people feel when they act with total involvement." Flow refers to a state of mind which brings together cognitive, physiological and affective aspects and corresponds to an optimal psychophysical state (Biasutti, 2017). In music and sport total immersion with, absorption in the activity, high internal enjoyment and intrinsic motivation are frequently observed. Entering a flow state involves developing a balance between the perceived abilities of a person and their perceived opportunities (Nakamura and Csikszentmihalyi, 2002), and balancing challenges and skills is the golden rule of flow (Jackson and Csikszentmihalyi, 1999). The experience of flow is characterized by the following nine dimensions: a challengeskill balance, merging of action and awareness, clear goals, unambiguous feedback, total concentration, sense of control, loss of self-consciousness, transformation of time, and autotelic experience (Csikszentmihalyi, 1990).

\section{Flow and Music}

Since music is noted as one of the activities which induces flow most often (Lowis, 2002), it is not surprising that in recent years, many studies have been conducted on flow in the musical domain (O’Neill, 1999; Custodero, 2002, 2005; MacDonald et al., 2006; Smolej Fritz and Avsec, 2007; Biasutti and Frezza, 2009; de Manzano et al., 2010; Diaz, 2013; Fullagar et al., 2013; Wrigley and Emmerson, 2013; Biasutti, 2015; Chirico et al., 2015; Hart and Di Blasi, 2015). Music in its origins is a kind of flow of structured sounds in time and space with emotional and cognitive content. When we are immersed in music with full concentration - with the whole of our being - we have the opportunity to access the state of flow. According to Croom $(2012,2015)$ the analysis of flow in musical contexts is a rapidlydeveloping field. Chirico et al. (2015) reported that when studying flow in music, it is necessary to concentrate on different music activities, such as musical composition (Byrne et al., 2003; John, 2006; MacDonald et al., 2006; Hart and Di Blasi, 2015), musical listening (Diaz, 2013) and musical performance (O’Neill, 1999; Sawyer, 2006, 2007; Smolej Fritz and Avsec, 2007; Kirchner, 2011). Most studies of flow in the musical domain have focused on musical performance.

\section{Flow and Sports}

There are even more studies exploring flow in the sports domain than in the music domain (Csikszentmihalyi et al., 1977; Massimini and Carli, 1988; Jackson, 1996; Jackson and Marsh, 1996; Jackson et al., 1998; Jackson and Csikszentmihalyi, 1999; Bakker et al., 2011; Muzio et al., 2012; Swann et al., 2012). 
In sports, the majority of studies have been conducted with top athletes and only a few with recreational athletes (Jackson, 1996; Jackson and Marsh, 1996; Jackson et al., 1998; Bakker et al., 2011). Many of these studies used qualitative methodology as a source of in-depth data for confirming the nine dimensional constructs of flow, proposed by Jackson and Csikszentmihalyi (1999). Jackson (1992) in a qualitative study of figure skaters concluded that flow is defined by high levels of challenges and skills, attention and a clear, perfect focus. Similar conclusions were drawn with Japanese University athletes coming from different areas of sports such as swimming, athletics, and figure skating (Sugiyama and Inomata, 2005), and with elite swimmers (Bernier et al., 2009).

\section{Differences in Experiencing Flow With Regard to Gender and Individual/Group Performance Settings}

Considering gender differences in experiencing flow Csikszentmihalyi (1990, p. 4) describes, that "Flow is reported in essentially the same words by men and women." This descriptive finding suggests that flow is broadly reported beyond gender (Bonaiuto et al., 2016). Altought most of the studies show no differences in experiencing flow between men and women (Russell, 2001; Kee and Wang, 2008; Moreno et al., 2008; Bonaiuto et al., 2016), some studies suggest, that flow is experienced more frequently by female than by male (Gnezda, 2016; Habe and Berkopec, 2016; Habe and Tement, 2016). For example a higher sense of control (flow dimension) in boy athletes compared to girl athletes between 12 and 16 years old was reported by Moreno et al. (2008).

In the current study gender differences in flow are expected because women tend to experience positive emotional states in a more intensive and vivid way than men do (Fujita et al., 1991).

In addition, differences in experiencing flow according to performance setting (individual/group) are expected. Sawyer (2003) defined group flow as a collective state that occurs when a group is performing at the peak of its abilities. The conditions that encourage individual flow could also influence group flow (Sawyer, 2006). Duncan and West (2018) offer a simplified model of group flow, consisting of three dimensions: vision, ownership \& contribution, communication. When groups cooperate to agree on goals and patterns, social flow, commonly known as group cohesion, is more likely to occur than individual flow (Walker, 2010). Interestingly previous studies did not show significant differences in experiencing flow between individual and team sport performance (Fossmo, 2006; Elbe et al., 2010). Conversely, in music performance flow is experienced more frequently in group performance than in individual performance, because the level of performance anxiety is lower (Bloom and Skutnick-Henley, 2005; Berkopec, 2016).

\section{Flow and Satisfaction With Life}

Flow can be considered one of the indicators of well-being. Experiencing flow brings performers a sense of accomplishment and internal success. In top performers being subjected to flow conveys not only emotional fulfillment, but also a general feeling of satisfaction with life. Satisfaction with life could be considered a cognitive and global evaluation of the quality of one's life as a whole (Pavot and Diener, 1993).

Top/elite performers face stressful and challenging events on an almost everyday basis. Satisfaction with life, could be considered a cognitive indicator of subjective well-being. Flow is also frequently experienced during performance, which is a core component of a professional career. Flow can be viewed as a facilitator for perceiving internal success in performance, which can reflect satisfaction with life. Flow can also be perceived as positive stress (eustress), which is defined as a stress that results in positive outcomes (Selye, 1975). Eustress is usually the result of more manageable levels of stress (Le Fevre et al., 2003). The idea of eustress as "good stress" relates to the Yerkes-Dodson Law, which states that increasing arousal is beneficial to performance until some optimum level is reached (O'Sullivan, 2011). Perceived positive stress is positively related to life satisfaction (Abolghasemi and Taklavi Varaniyab, 2010). If we consider flow as a kind of positive stress, then it requires extensive activation at all levels of human functioning. To reach peak performance, a cognitive component of flow seems to play a crucial role in gaining personal well-being (Bloom and SkutnickHenley, 2005). Flow has received attention as being among the indicators of an individual's well-being (Csikszentmihalyi, 1975; Bassi and Delle Fave, 2012).

One of the basic functions of music is to promote well-being (Thorgaard et al., 2004; Hays and Minichiello, 2005), which also happens in recreational sports (Arent et al., 2000; Peluso and de Andrade, 2005; Ströhle, 2009; Häkkinen et al., 2010). However there is a difference regarding the expertise of performers. As opposed to amateur musicians or recreational athletes, many performing experts often report on the negative aspects of their elite performing career, such as anxiety, depression and stress (Kenny et al., 2003; Murko Feguš, 2016).

Well-being can be viewed from a hedonic or eudemonic perspective. The hedonic paradigm is represented by subjective well-being, divided into cognitive (satisfaction with life) and emotional (positive/negative emotionality) components (Diener et al., 1985). Conversely, the eudemonic paradigm is represented by psychological well-being which comprises self-acceptance, personal growth, purpose in life, environmental mastery, autonomy, and positive relations with others (Ryff, 1989).

To our knowledge there is little research that has explored well-being in professional athletes. Previous studies on flow and well-being reveal that experiencing more intense flow correlates positively with hedonic and eudemonic well-being (Smolej Fritz and Avsec, 2007; Delle Fave et al., 2011a; Moneta, 2012; Bassi et al., 2014; Baker et al., 2015).

Other studies about the relationship between flow and satisfaction with life in musicians show that satisfaction with life is weakly positively associated with flow, while having clear goals supports a sense of satisfaction with life (Chirico et al., 2015). Clear goals are important predictors of satisfaction with life (Smolej Fritz and Avsec, 2007), and two dimensions of flow, balance between challenge and skills and autotelic experience, correlated positively with satisfaction with life (Sedlár, 2014). Smolej Fritz and Avsec (2007) concluded that the experience of flow in musicians is more related to emotional than cognitive 
aspects of subjective well-being. In accordance with previous research findings on flow, we hypothesized that:

- H1: There would be differences in flow between elite musicians and top athletes.

- H2: There would be differences in flow regarding group versus individual performance settings.

- H3: There would be gender differences in flow with female top/elite performers experiencing flow more often than male.

- H4: Flow in top/elite performers would positively correlate with satisfaction with life.

\section{MATERIALS AND METHODS}

\section{Participants}

Four hundred and fifty two elite musicians and top athletes participated in the study. Elite musicians are expert active performers that regularly perform nationally and internationally as soloists or as members of small chamber groups (duo, trio, quartet, quintet) in eminent solo concerts and festivals and/or have gained renowned rewards (first, second, third prize) in national and/or international competitions. The inclusion criteria for elite musicians is to have a career as a musician and to regularly perform internationally or having gained a prize in national or international competitions. Top athletes are athletes that play individually or in teams at national level. The inclusion criteria for top athletes is registration in the Olympic committee of Slovenia - the Association of Sports Federations. Participant ages ranged between 14 and 58 years, with a mean age of 23.46 years $(S D=7.06$ years). There were 114 top Slovenian musicians with a mean age of 26.59 years $(S D=9.46$ years $)$ and 338 top Slovenian athletes with a mean age of 22.40 years $(S D=5.68$ years). Musicians included wind instrument players (27.2\%), pianists and accordion players (24.6\%), singers $(21.1 \%)$ and string instrument players (22.6\%). Athletes were $63.0 \%$ individual sports athletes (swimming, track and field athletes, tennis players, martial arts athletes) and $37.0 \%$ team sports athletes (handball, football, basketball and volleyball). The difference in age between musicians and athletes was significant $[t=-4.46 ; p(t)=0.00]$. 149 participated in team events (team sports or bands and choirs) and 303 in individual sports or mainly undertook solo performances. The differences in age between participants who participated in team events and those who perform individually were not significant $[p(t)=0.68] .224$ participants were male and 224 were female, the differences in age were significant $[t=2.43 ; p(t)=0.02]$ - the male mean age was 24.25 years $(S D=7.54$ years $)$ and the female was 22.65 years old on average $(S D=6.45)$.

This study was carried out in accordance with the recommendations of the Slovenian Psychological Society. In accordance with the Declaration of Helsinki consent obtained from all adult participants and from the parents of all 6 participants under the age of 16 was both written and informed. Research was approved by the Ethical committee of the Faculty of Arts at the University of Maribor.

\section{Instruments}

Dispositional Flow Scale - 2 (DFS-2; Jackson and Eklund, 2002)

DFS-2 is a self-descriptive instrument, based on the following nine dimensions of flow theory (Csikszentmihalyi, 1990): challenge-skill balance, merging of action and awareness, clear goals, unambiguous feedback, total concentration, sense of control, loss of self-consciousness, transformation of time, autotelic experience. It includes 36 items, 4 items per dimension. The items refer to athletic and physical dimensions, during which people experience intense positive emotions. Each item is evaluated on a five level Likert scale, where 1 means "never" and 5 "always." The respondents are asked to evaluate how often they experience the sensation described for each item. A global flow score was computed, considering the sum of all items. The reliability of the scale was high, with Cronbach alpha coefficients ranging between 0.78 and 0.86 (Phillips, 2005).

\section{Satisfaction With Life Scale (SWLS; Diener et al., 1985)}

SWLS is a scale composed of five items, which measure the overall self-estimation of quality of life. Participants evaluate their level of agreement with each item on a seven level Likert scale, where 1 means "completely disagree" and 7 "completely agree." The score was obtained by summing up the five items, 35 being the maximum score. The questionnaire has shown high internal consistency and stability over time, ranging up to 4 years (Pavot and Diener, 1993). The initial study had a Cronbach alpha of 0.87 and test-retest reliability of 0.82 (Diener et al., 1985).

\section{Procedure}

The participants were recruited through national sports associations and musical schools and orchestras. The measures

TABLE 1 | Comparison of athletes and musicians in flow dimensions and satisfaction with life.

\begin{tabular}{|c|c|c|c|c|c|c|}
\hline \multirow[t]{2}{*}{ Measures } & \multicolumn{2}{|c|}{ Athletes } & \multicolumn{2}{|c|}{ Musicians } & \multirow[b]{2}{*}{$\boldsymbol{F}$} & \multirow[b]{2}{*}{$p$} \\
\hline & $M$ & $S D$ & $M$ & $S D$ & & \\
\hline Challenge-skill balance & 15.74 & 2.25 & 15.64 & 2.12 & 0.41 & 0.68 \\
\hline $\begin{array}{l}\text { Merging of action and } \\
\text { awareness }\end{array}$ & 14.27 & 2.83 & 14.55 & 2.32 & -1.06 & 0.29 \\
\hline Clear goals & 17.24 & 2.24 & 16.61 & 2.39 & 2.56 & 0.01 \\
\hline Unambiguous feedback & 15.89 & 2.45 & 14.72 & 2.64 & 4.33 & 0.00 \\
\hline Total concentration & 15.86 & 2.38 & 15.63 & 2.32 & 0.87 & 0.38 \\
\hline Sense of control & 15.12 & 2.45 & 15.13 & 2.31 & -0.04 & 0.97 \\
\hline Loss of self-consciousness & 13.75 & 3.70 & 13.66 & 3.86 & 0.22 & 0.83 \\
\hline Transformation of time & 14.08 & 3.82 & 16.03 & 2.91 & -5.68 & 0.00 \\
\hline Autotelic experience & 16.20 & 2.64 & 16.84 & 2.20 & -2.56 & 0.01 \\
\hline Global flow score & 138.14 & 16.63 & 138.81 & 14.81 & -0.38 & 0.70 \\
\hline Satisfaction with life & 24.45 & 5.57 & 25.82 & 5.20 & -2.13 & 0.03 \\
\hline
\end{tabular}

$M$, mean; SD, standard deviation; $p$, statistical significance of parameter $F$. The measures highlighted in gray had significant differences between athletes and musicians. 
TABLE 2 | Comparison of team and individual performers in flow dimensions and satisfaction with life.

\begin{tabular}{|c|c|c|c|c|c|c|}
\hline \multirow[b]{2}{*}{ Measures } & \multicolumn{2}{|c|}{ Team } & \multicolumn{2}{|c|}{ Individual } & \multirow[b]{2}{*}{$t$} & \multirow[b]{2}{*}{$p$} \\
\hline & $M$ & $S D$ & $M$ & $S D$ & & \\
\hline Challenge-skill balance & 16.13 & 2.05 & 15.51 & 2.27 & 2.84 & 0.0 \\
\hline $\begin{array}{l}\text { Merging of action and } \\
\text { awareness }\end{array}$ & 14.91 & 2.38 & 14.06 & 2.82 & 3.32 & 0.0 \\
\hline Clear goals & 17.42 & 1.98 & 16.91 & 2.42 & 2.21 & 0.0 \\
\hline Unambiguous feedback & 15.91 & 2.50 & 15.44 & 2.56 & 1.86 & 0.0 \\
\hline Total concentration & 16.17 & 2.23 & 15.62 & 2.41 & 2.40 & 0.02 \\
\hline Sense of control & 15.46 & 2.26 & 14.96 & 2.48 & 2.06 & 0.0 \\
\hline Loss of self-consciousness & 13.73 & 3.53 & 13.72 & 3.84 & 0.03 & 0.97 \\
\hline Transformation of time & 15.28 & 3.34 & 14.22 & 3.83 & 2.86 & 0.00 \\
\hline Autotelic experience & 16.98 & 2.26 & 16.06 & 2.62 & 3.85 & 0.00 \\
\hline Global flow score & 141.98 & 14.65 & 136.50 & 16.61 & 3.42 & 0.00 \\
\hline Satisfaction with life & 25.42 & 4.75 & 24.82 & 5.68 & 0.82 & 0.41 \\
\hline
\end{tabular}

$M$, mean; $S D$, standard deviation; $p$, statistical significance of parameter $t$. The measures highlighted in gray had significant differences between teams and individuals.

were administered individually in face-to-face meetings in small groups, mainly after practice or rehearsal sessions.

\section{RESULTS AND DISCUSSION}

The data was processed with the statistical package IBM SPSS 21.0. Differences between athletes and musicians and male and female participants, were computed with Ancova as we controlled the effect of age. Participants, who mainly perform individually, and those who mainly perform in groups were compared using a $t$-test, and correlations between flow dimensions and satisfaction with life was calculated with Pearson's $r$. Linear regression was used to predict satisfaction with life on the basis of flow experience.
The first analysis was of reliability on all the measures for assessing the quality of the data collected. The results have shown high reliability: Cronbach's Alpha was 0.85 for SWLS and from 0.74 to 0.83 for the subscales of DFS-2, while global flow reliability was 0.83 .

Regarding the first hypothesis (There would be differences in some dimensions of flow between elite musicians and top athletes) the comparison of elite musicians and top athletes in flow dimensions and satisfaction with life shows that there are significant differences in four dimensions of flow (see Table 1).

These differences have different trends: when athletes experience flow they have a clear idea of what their goals are and they have clear feedback, which tells them that they are on the way to achieving them. Conversely, elite musicians experience more transformation of time, which can either go faster or slower and they have a more autotelic experience of their performance than the top athletes. These findings are consistent with the different characteristics of the two disciplines: it is probably easier to set goals in sport where you can easily control their achievement. For example when you are running 100 meters you can tell using a chronometer whether you have run below a specific time, and can develop feedback mechanisms for monitoring the achievement of goals. Conversely, music is an artistic discipline subject to individual interpretation, and is more difficult than in sports to set clear and shared goals and to control them. Elite musicians demonstrated a deeper level of transformation of time than top athletes, which is a characteristic connected to the nature of music. Music is developing over time and can induce trance and altered states of consciousness (this issue will be discussed in more depth later). Our results about the differences in transformation of time between elite musicians and top athletes are not in line with previous findings by Thomson and Jaque (2016), that showed no differences between talented dancers, singers and athletes. A possible reason could be due to the different population of participants included in the current and in the Thomson and Jaque's (2016) studies. The difference

TABLE 3 | Comparison of team and individual performers in musicians and athletes in flow dimensions and satisfaction with life.

\begin{tabular}{|c|c|c|c|c|c|c|c|c|c|c|}
\hline \multirow[t]{3}{*}{ Measures } & \multicolumn{5}{|c|}{ Athletes } & \multicolumn{5}{|c|}{ Musicians } \\
\hline & \multicolumn{2}{|c|}{ Team } & \multicolumn{2}{|c|}{ Individual } & \multirow[b]{2}{*}{$t$} & \multicolumn{2}{|c|}{ Team } & \multicolumn{2}{|c|}{ Individual } & \multirow[b]{2}{*}{$t$} \\
\hline & $M$ & $S D$ & $M$ & $S D$ & & $M$ & $S D$ & $M$ & $S D$ & \\
\hline Challenge-skill balance & 16.26 & 2.08 & 15.44 & 2.30 & $3.28^{* *}$ & 15.50 & 1.79 & 15.68 & 2.20 & -0.36 \\
\hline Merging of action and awareness & 14.92 & 2.38 & 13.89 & 3.01 & $3.49 * *$ & 14.83 & 2.44 & 14.48 & 2.29 & 0.67 \\
\hline Clear goals & 17.56 & 1.99 & 17.05 & 2.36 & $2.04^{*}$ & 16.67 & 1.76 & 16.59 & 2.54 & 0.17 \\
\hline Unambiguous feedback & 16.13 & 2.44 & 15.75 & 2.45 & 1.37 & 14.79 & 2.57 & 14.70 & 2.68 & 0.15 \\
\hline Total concentration & 16.26 & 2.22 & 15.62 & 2.44 & $2.45^{*}$ & 15.71 & 2.24 & 15.61 & 2.35 & 0.18 \\
\hline Sense of control & 15.47 & 2.30 & 14.92 & 2.52 & $2.02^{*}$ & 15.38 & 2.08 & 15.07 & 2.37 & 0.58 \\
\hline Loss of self-consciousness & 13.83 & 3.52 & 13.69 & 3.81 & 0.33 & 13.21 & 3.56 & 13.78 & 3.94 & -0.64 \\
\hline Transformation of time & 14.91 & 3.46 & 13.59 & 3.94 & $3.11^{* *}$ & 17.17 & 1.69 & 15.72 & 3.10 & $3.05^{* *}$ \\
\hline Autotelic experience & 16.90 & 2.31 & 15.79 & 2.73 & $4.00^{* *}$ & 17.38 & 2.04 & 16.70 & 2.23 & 1.34 \\
\hline Global flow score & 142.24 & 14.82 & 135.73 & 17.19 & $3.53^{* *}$ & 140.63 & 13.95 & 138.32 & 15.07 & 0.68 \\
\hline Satisfaction with life & 24.43 & 5.09 & 24.45 & 5.75 & -0.03 & 27.46 & 3.19 & 25.39 & 5.54 & $2.37^{*}$ \\
\hline
\end{tabular}

M, mean; SD, standard deviation; *t significant at 0.05 level; **t significant at 0.00 level. The differences that were significant are highlighted in gray. 
TABLE 4 | Comparison of male and female participants in flow dimensions and satisfaction with life.

\begin{tabular}{|c|c|c|c|c|c|c|}
\hline \multirow[t]{2}{*}{ Measures } & \multicolumn{2}{|c|}{ Male } & \multicolumn{2}{|c|}{ Female } & \multirow[b]{2}{*}{$\boldsymbol{F}$} & \multirow[b]{2}{*}{$p$} \\
\hline & $M$ & $S D$ & $M$ & $S D$ & & \\
\hline Challenge-skill balance & 15.96 & 2.11 & 15.46 & 2.30 & 2.39 & 0.02 \\
\hline $\begin{array}{l}\text { Merging of action and } \\
\text { awareness }\end{array}$ & 14.46 & 2.71 & 14.22 & 2.71 & 0.95 & 0.34 \\
\hline Clear goals & 17.28 & 2.10 & 16.87 & 2.46 & 1.91 & 0.06 \\
\hline Unambiguous feedback & 15.97 & 2.41 & 15.21 & 2.63 & 3.22 & 0.00 \\
\hline Total concentration & 16.08 & 2.24 & 15.51 & 2.46 & 2.60 & 0.01 \\
\hline Sense of control & 15.46 & 2.23 & 14.78 & 2.55 & 3.06 & 0.00 \\
\hline Loss of self-consciousness & 14.44 & 3.44 & 13.00 & 3.89 & 4.18 & 0.00 \\
\hline Transformation of time & 14.46 & 3.83 & 14.68 & 3.59 & -0.61 & 0.54 \\
\hline Autotelic experience & 16.58 & 2.37 & 16.14 & 2.69 & 1.86 & 0.06 \\
\hline Global flow score & 140.71 & 14.50 & 135.86 & 17.41 & 3.21 & 0.00 \\
\hline Satisfaction with life & 25.22 & 5.46 & 24.75 & 5.48 & 0.75 & 0.46 \\
\hline
\end{tabular}

$M$, mean; SD, standard deviation; $p$, statistical significance of parameter $F$. The measures highlighted in gray had significant differences between males and females.

in transformation of time between musicians and athletes maybe emerges only at a certain level of expert skills and by the most outstanding performers. It is relevant to note that the autotelic experience is more pronounced in elite musicians than in top athletes, and represents a pleasant experience which is intrinsically rewarded, since the gratification is directly connected with the activity performed. Satisfaction with life seems to be higher in elite musicians when compared to top athletes. In this case the level of significance is not high and this data needs to be confirmed in subsequent research.

Regarding the second hypothesis (There would be differences in flow regarding group versus individual performance setting), when we compared performers in team settings (athletes who participate in team sports and musicians who perform mainly in choirs or ensembles) to those who mainly perform individually (individual sports and solo performers in music) we found many differences, with higher scores for team performers.

Team performers have a better balance between challenge and skill, their awareness merges with what they are doing more frequently, they have clearer goals and more frequently experience total concentration than individual performers. They also have more sense of control, experience more transformation of time, have a more autotelic experience and the global flow score is higher in team performers (see Table 2). Conversely, the differences in satisfaction with life between team and individual performers are not statistically significant.

Table 3 shows several differences in experiencing flow between athletes and musicians in team and individual performances. The previously described differences correspond fully to the subsample of athletes, while in musicians only the transformation of time corresponds to those differences between individual and team performers. The differences in experiencing flow in athletes according to our results are not consistent with previous studies, which showed no significant differences in experiencing flow between individual and team sport performance (Fossmo, 2006; Elbe et al., 2010). The differences in our research may be a consequence of the selected population - top athletes - which have very developed performance skills. Conversely, previous studies confirmed the differences in musicians experiencing flow, showing that flow is experienced more frequently in group performing than in individual performing (Bloom and Skutnick-Henley, 2005; Berkopec, 2016). There were significant differences only for the transformation of time in elite musicians in our study.

Transformation of time is one phenomenon that characterizes music. A different perception of time could be induced when people are deeply involved during listening music. Also performing music or singing in a group enhances the transformation of time. In ancient civilizations group music making was one of the forms of accessing spiritual dimensions (Dewhurst-Maddock, 1999) and a different perception of time

TABLE 5 | Comparison of male and female musicians and athletes in flow dimensions and satisfaction with life.

\begin{tabular}{|c|c|c|c|c|c|c|c|c|c|c|}
\hline \multirow[t]{3}{*}{ Measures } & \multicolumn{5}{|c|}{ Athletes } & \multicolumn{5}{|c|}{ Musicians } \\
\hline & \multicolumn{2}{|c|}{ Male } & \multicolumn{2}{|c|}{ Female } & \multirow[b]{2}{*}{$t$} & \multicolumn{2}{|c|}{ Male } & \multicolumn{2}{|c|}{ Female } & \multirow[b]{2}{*}{$t$} \\
\hline & $M$ & $S D$ & $M$ & $S D$ & & $M$ & $S D$ & $M$ & $S D$ & \\
\hline Challenge-skill balance & 16.10 & 2.10 & 15.34 & 2.35 & $3.14^{* *}$ & 15.47 & 2.08 & 15.78 & 2.15 & -0.77 \\
\hline Merging of action and awareness & 14.48 & 2.77 & 14.04 & 2.89 & 1.44 & 14.39 & 2.55 & 14.68 & 2.13 & -0.66 \\
\hline Clear goals & 17.43 & 2.07 & 17.02 & 2.40 & 1.66 & 16.76 & 2.16 & 16.48 & 2.58 & 0.64 \\
\hline Unambiguous feedback & 16.28 & 2.31 & 15.46 & 2.53 & $3.11^{* *}$ & 14.90 & 2.48 & 14.57 & 2.77 & 0.66 \\
\hline Total concentration & 16.15 & 2.20 & 15.53 & 2.54 & $2.41^{*}$ & 15.84 & 2.38 & 15.46 & 2.27 & 0.88 \\
\hline Sense of control & 15.46 & 2.22 & 14.75 & 2.65 & $2.69 *$ & 15.47 & 2.32 & 14.86 & 2.28 & 1.42 \\
\hline Loss of self-consciousness & 14.50 & 3.39 & 12.91 & 3.86 & $4.01^{* *}$ & 14.22 & 3.65 & 13.21 & 3.98 & 1.40 \\
\hline Transformation of time & 14.02 & 3.94 & 14.14 & 3.69 & -0.29 & 16.00 & 2.95 & 16.05 & 2.91 & -0.09 \\
\hline Autotelic experience & 16.41 & 2.46 & 15.98 & 2.80 & 1.51 & 17.20 & 1.94 & 16.56 & 2.36 & 1.56 \\
\hline Global flow score & 140.84 & 14.63 & 135.17 & 18.16 & $3.14^{* *}$ & 140.25 & 14.18 & 137.63 & 15.32 & 0.94 \\
\hline Satisfaction with life & 24.74 & 5.36 & 24.17 & 5.77 & 0.71 & 26.06 & 5.57 & 25.63 & 4.91 & 0.43 \\
\hline
\end{tabular}

M, mean; SD, standard deviation; *t significant at 0.05 level; **t significant at 0.00 level. Highlighted in gray are the differences that were significant. 
TABLE 6 | Pearson correlation coefficients between flow dimensions and satisfaction with life.

\begin{tabular}{lc}
\hline Flow subscales & Satisfaction with life \\
\hline Challenge-skill balance & $0.36^{* *}$ \\
Merging of action and awareness & $0.26^{* *}$ \\
Clear goals & $0.22^{* *}$ \\
Unambiguous feedback & $0.27^{* *}$ \\
Total concentration & $0.29^{* *}$ \\
Sense of control & $0.28^{* *}$ \\
Loss of self-consciousness & $0.23^{* *}$ \\
Transformation of time & $0.24^{* *}$ \\
Autotelic experience & $0.29^{* *}$ \\
Global flow score & $0.41^{* *}$ \\
\hline
\end{tabular}

**Correlation is significant at the 0.01 level (2-tailed).

was induced. For musicians, time perception is the core of their flow experience and happens when all the levels of existence (body, emotions, mind, and spirit) merge into one whole during the performance. Elite musicians had a different trend than top athletes for satisfaction with life, which was higher for musicians who mainly perform in group settings.

When we compared male and female participants regarding the third hypothesis (There would be gender differences in flow with female top/elite performers experiencing flow more often than male), we found several differences in flow dimensions but no difference in satisfaction with life (as reported in Table 4). Males experienced flow more frequently, both in general, as the global flow score shows us, as well as in individual dimensions of flow. Males experienced a higher balance between the challenge of the situation and their skills, feeling that they had clearer feedback about the situation and more control when they are performing than did females. Males also experienced total concentration and loss of self-consciousness more frequently. These results are rather surprising because in the general population, dimensions of well-being and flow are usually more pronounced in females than in males. Gender differences can be partially explained by the fact that women tend to experience positive emotional states in a more intensive and vivid way than do men (Fujita et al., 1991).

As in the previous set of comparisons, we found that when we compared male and female musicians and athletes separately, the differences shown in the entire sample of participants correspond to the differences in athletes, while there were no differences between male and female musicians in the experience of flow (see Table 5). These findings are coherent with the assumption that musicians are more androgynous than the general population (Kemp, 2000). According to Bem's theory of psychological androgyny (1974, in Kemp, 2000), gender roles may involve a four-fold typology; masculine, feminine, androgynous and undifferentiated. In general, musicians appear to be able to extricate themselves from the major influences of the gender stereotypes. They may minimize differences in gender-related traits (Kemp, 2000). However, in the current study musicians and athletes were compared rather than musicians and general population. These findings could also be explained because the physical differences between male and female athletes have a larger effect in sport than in music performers.

Regarding the fourth hypothesis (Flow in top/elite performers would positively correlate with satisfaction with life) upon correlating dimensions of flow to satisfaction with life, we found significant correlations with all sub-dimensions and the global flow score, but they were quite low (see Table 6). Those that were correlated highly enough for serious discussion were the global flow score and the challenge-skill balance. Our results are consistent with the outcomes of previous studies (Smolej Fritz and Avsec, 2007; Chirico et al., 2015). The challenge-skill balance is important for satisfaction with life in top/elite performers, because this is a dimension over which a performer can have the most control. This sense of control could lower a performer's stress, resulting in higher satisfaction with life.

The participants of this study were all individuals who were highly successful in their area and who perform in competitions at the highest level. In the last analysis we verify whether the experience of flow influenced how satisfied they were with their

TABLE 7 | Prediction of satisfaction with life on the basis of flow disposition.

\begin{tabular}{|c|c|c|c|c|c|c|c|c|c|c|}
\hline & Dimension of flow & $B$ & $\beta$ & $T$ & $p(t)$ & $R$ & $R^{2}$ & $\operatorname{adj} R^{2}$ & $\boldsymbol{F}$ & $p(t)$ \\
\hline 1 & Group & 1.40 & 0.12 & 2.16 & 0.03 & & & & & \\
\hline Step & Constant & 4.05 & & 1.34 & 0.18 & 0.44 & 0.19 & 0.16 & 6.25 & 0.00 \\
\hline \multirow[t]{8}{*}{2} & Challenge-skill balance & 0.54 & 0.22 & 2.94 & 0.00 & & & & & \\
\hline & Merging of action and awareness & 0.08 & 0.04 & 0.63 & 0.53 & & & & & \\
\hline & Unambiguous feedback & 0.26 & 0.12 & 1.73 & 0.08 & & & & & \\
\hline & Total concentration & 0.20 & 0.09 & 1.23 & 0.22 & & & & & \\
\hline & Sense of control & -0.13 & -0.06 & -0.71 & 0.48 & & & & & \\
\hline & Loss of self-consciousness & 0.15 & 0.10 & 1.61 & 0.11 & & & & & \\
\hline & Transformation of time & 0.17 & 0.11 & 1.74 & 0.08 & & & & & \\
\hline & Autotelic experience & -0.05 & -0.03 & -0.32 & 0.75 & & & & & \\
\hline
\end{tabular}

B, Unstandardized regression coefficient; $\beta$, standardized regression coefficient. The significant dimension is highlighted in gray. 
lives. We calculated a linear regression of flow dimensions on satisfaction with life, but in the first step we excluded the effect of gender and the group (athletes and musicians), as we found some differences between those subgroups in the entire group of participants. As the model is statistically significant (see Table 7), we can argue that the experience of flow influences satisfaction with life in high-performing individuals in music or sports. The main effect is due to the perceived equilibrium between the challenge the situation presents and the perceived set of skills that make a believe they are able to face this particular situation.

\section{STUDY STRENGTHS, LIMITATIONS AND IMPLICATIONS}

There are several study strengths. First, it is to our knowledge the first study to date that has empirically explored differences in experiencing flow between elite musicians and top athletes. It highlights some important issues of accessing flow in different performance settings (individuals versus groups). It further investigated the relationship between flow and satisfaction with life, which has been rarely explored, finding that one dimension of flow is relevant as a predictor for satisfaction with life.

Our study has some limitations. The principal limitation concerns sampling. The sample might be biased because the more intrinsically motivated performers may have been more likely to take part in the research (Habe and Tement, 2016). The distribution of athletes and musicians differed, because it was harder to get access to elite musicians in comparison to top athletes who are organized through the Olympic Committee. The second limitation is connected to flow assessment and the use of retrospective self-report data which is biased because of the different response styles, personality characteristics, and affective states (Delle Fave et al., 2011b). It would be advisable to use an experience sampling procedure, but according to our experience it is very difficult to collect data from elite musicians and top athletes because top performers are focused on their optimal performance, trying to avoid any kind of distraction.

Future studies should consider applying a longitudinal design to examine stability and change in flow over time

\section{REFERENCES}

Abolghasemi, A., and Taklavi Varaniyab, S. (2010). Resilience and perceived stress: predictors of life satisfaction in the students of success and failure. Procedia Soc. Behav. Sci. 5, 748-752. doi: 10.1016/j.sbspro.2010.07.178

Aherne, C., Moran, A. P., and Lonsdale, C. (2011). The effect of mindfulness training on athletes' flow: an initial investigation. Sport Psychol. 25, 177-189. doi: $10.1123 /$ tsp.25.2.177

Altenmüller, E., and Ioannou, C. I. (2016). "Music performance: expectations, failures, and prevention," in Performance Psychology: Perception, Action, Cognition, and Emotion, eds M. Raab, B. Lobinger, S. Hoffmann, A. Pizzera, and S. Laborde (London: Academic Press), 103-119. doi: 10.1016/B978-0-12803377-7.00007-7

Arent, S., Landers, D. M., and Etnier, J. L. (2000). The effects of exercise on mood in older adults: a meta-analytic review. J. Aging Phys. Act. 8, 407-430. doi: 10.1123/japa.8.4.407
(Mäkikangas et al., 2010). It would also be advisable to highlight the relationship between flow and satisfaction with life from the perspective of job demands and job resources in top/elite professional performers. Flow could be associated also with constructs such as self-efficacy or resilience to verify other relevant aspects that could contribute to flow development.

The current study shows that several strategies related to different performance settings are required for accessing the optimal performing state of flow. For regulating satisfaction with life in top/elite performers the most important strategy is balancing skills and performing challenges, where not only physical but also psychological abilities should be taken into account. It would be advisable for more studies in top/elite performance settings to investigate interventions for gaining well-being in professional performers. In addition, participants such as students and trainees could be involved for assessing the differences between experts and novices and the developmental aspects of flow. A research focused on how flow develops could offer several ideas for education and the designing friendly flow environments.

\section{DATA AVAILABILITY}

All datasets generated for this study are included in the manuscript and/or the supplementary files.

\section{AUTHOR CONTRIBUTIONS}

$\mathrm{KH}$ and TK contributed conception and design of the study and organized the database. MB and TK performed the statistical analysis. $\mathrm{KH}$ wrote the first draft of the manuscript. $\mathrm{KH}, \mathrm{MB}$, and TK wrote sections of the manuscript, contributed to manuscript revision, and read and approved the submitted version.

\section{ACKNOWLEDGMENTS}

We would like to thank our students Betsabeja Berkopec and Domen Kralj for helping in collecting the data.

Baker, F. A., and MacDonald, R. A. R. (2013). Flow, identity, achievement, satisfaction and ownership during therapeutic songwriting experiences with university students and retirees. Music. Sci. 17, 131-146. doi: 10.1177/ 1029864913476287

Baker, F. A., Rickard, N., Tamplin, J., and Roddy, C. (2015). Flow and meaningfulness as mechanisms of change in self-concept and well-being following a songwriting intervention for people in the early phase of neurorehabilitation. Front. Hum. Neurosci. 9:299. doi: 10.3389/fnhum.2015. 00299

Bakker, A. B., Oerlemans, W., Demerouti, E., Bruins Slot, B., and Karamat Ali, D. (2011). Flow and performance: a study among talented Dutch soccer players. Psychol. Sport Exerc. 12, 442-450. doi: 10.1016/j.psychsport.2011. 02.003

Bassi, M., and Delle Fave, A. (2012). Optimal experience and self-determination at school: joining perspectives. Motiv. Emot. 36, 425-438. doi: 10.1007/s11031011-9268-z 
Bassi, M., Steca, P., Monzani, D., Greco, A., and Delle Fave, A. (2014). Personality and optimal experience in adolescence: implications for well-being and development. J. Happiness Stud. 15, 829-843. doi: 10.1007/s10902-013-9451-x

Berkopec, B. (2016). Doživljanje Zanosa pri Slovenskih Vrhunskih Glasbenikih [Experiencing Flow Among Slovenian Elite Musicians]. Master's thesis, University of Maribor, Maribor.

Bernier, M., Thienot, E., Codron, E., and Fournier, J. F. (2009). Mindfulness and acceptance approaches in sport performance. J. Clin. Sport Psychol. 3, 320-333. doi: $10.1123 /$ jcsp.3.4.320

Biasutti, M. (2015). Pedagogical applications of cognitive research on musical improvisation. Front. Psychol. 6:614. doi: 10.3389/fpsyg.2015.00614

Biasutti, M. (2017). "Flow and optimal experience," in Reference Module in Neuroscience and Biobehavioral Psychology, ed. J. P. Stein (New York, NY: Elsevier), doi: 10.1016/B978-0-12-809324-5.06191-5

Biasutti, M., and Concina, E. (2014). The role of coping strategy and experience in predicting music performance anxiety. Music. Sci. 18, 189-202. doi: 10.1177/ 1029864914523282

Biasutti, M., and Frezza, L. (2009). Dimensions of music improvisation. Creat. Res. J. 21, 232-242. doi: 10.1080/10400410902861240

Bloom, A. J., and Skutnick-Henley, P. (2005). Facilitating flow experiences among musicians. Am. Music Teach. 54, 24-28.

Bonaiuto, M., Mao, Y., Roberts, S., Psalti, A., Ariccio, S., Ganucci Cancellieri, U., et al. (2016). Optimal experience and personal growth: flow and the consolidation of place identity. Front. Psychol. 7:1654. doi: 10.3389/fpsyg.2016. 01654

Bradley, R., and Corwyn, R. (2004). Life satisfaction among European American, African American, Chinese American, Mexican American, and Dominican American adolescents. Int. J. Behav. Dev. 28, 385-400. doi: 10.1080/ 01650250444000072

Briegel-Jones, R. M. H., Knowles, Z., Eubank, M. R., Giannoulatos, K., and Elliot, D. (2013). A preliminary investigation into the effect of yoga practice on mindfulness and flow in élite youth swimmers. Sport Psychol. 27, 349-359. doi: $10.1123 /$ tsp.27.4.349

Byrne, C., MacDonald, R., and Carlton, L. (2003). Assessing creativity in musical compositions: flow as an assessment tool. Br. J. Music Educ. 20, 277-290. doi: 10.1017/S0265051703005448

Chirico, A., Serino, S., Cipresso, P., Gaggioli, A., and Riva, G. (2015). When music "flows". State and trait in musical performance, composition and listening: a systematic review. Front. Psychol. 6:906. doi: 10.3389/fpsyg.2015.00906

Croom, A. M. (2012). Music, neuroscience, and the psychology of well-being: a précis. Front. Psychol. 2:393. doi: 10.3389/fpsyg.2011.00393

Croom, A. M. (2015). Music practice and participation for psychological wellbeing: a review of how music influences positive emotion, engagement, relationships, meaning, and accomplishment. Music. Sci. 19, 44-64. doi: 10. $1177 / 1029864914561709$

Csikszentmihalyi, M. (1975). Beyond Boredom and Anxiety: Experiencing Flow in Work and Play. San Francisco, CA: Jossey-Bass.

Csikszentmihalyi, M. (1990). Flow: The Psychology of Optimal Performance. New York, NY: Harper and Row.

Csikszentmihalyi, M. (1993). The Evolving Self: A Psychology for the Third Millennium. New York, NY: HarperCollins.

Csikszentmihalyi, M., Larson, R., and Prescott, S. (1977). The ecology of adolescent activity and experience. J. Youth Adolesc. 6, 281-294. doi: 10.1007/BF02138940

Custodero, L. A. (2002). Seeking challenge, finding skill: flow experience and music education. Arts Educ. Policy Rev. 103, 3-9. doi: 10.1080/1063291020960028

Custodero, L. A. (2005). Observable indicators of flow experience: a developmental perspective on musical engagement in young children from infancy to school age. Music Educ. Res. 7, 185-209. doi: 10.1080/14613800500169431

de Manzano, Ö., Theorell, T., Harmat, L., and Ullén, F. (2010). The psychophysiology of flow during piano playing. Emotion 10, 301-311. doi: $10.1037 / \mathrm{a} 0018432$

Delle Fave, A., Massimini, F., and Bassi, M. (2011a). "Hedonism and eudaimonism in positive psychology," in Psychological Selection and Optimal Experience Across Cultures: Social Empowerment through Personal Growth, eds A. Delle Fave, F. Massimini, and M. Bassi (New York, NY: Springer Science+Business Media), 19-38. doi: 10.1007/978-90-481-9876-4

Delle Fave, A., Massimini, F., and Bassi, M. (2011b). "Instruments and methods in flow research," in Psychological Selection and Optimal Experience Across
Cultures: Social Empowerment through Personal Growth, eds A. Delle Fave, F. Massimini, and M. Bassi (New York, NY: Springer Science+Business Media), 19-38. doi: 10.1007/978-90-481-9876-4

Delle Fave, A., Massimini, F., and Bassi, M. (2011c). "Work: a paradox in flow research," in Psychological Selection and Optimal Experience Across Cultures: Social Empowerment through Personal Growth, eds A. Delle Fave, F. Massimini, and M. Bassi (New York, NY: Springer Science+Business Media), 155-175. doi: 10.1007/978-90-481-9876-4

Delle Fave, A., and Zager Kocjan, G. (2016). "Well-being in the arts and crafts sector," in Handbook of the Psychology of Positivity and Strengths-Based Approaches at Work, eds L. G. Oades, M. Steger, A. Delle Fave, and J. Passmore (Hoboken, NJ: John Wiley \& Sons), 508-526. doi: 10.1002/9781118977620.ch26

Dewhurst-Maddock, O. (1999). Zdravilna mocč Glasbe in Zvoka [The Healing Power of Music and Sound]. Ljubljana, SI: Tangram.

Diaz, F. M. (2013). Mindfulness, attention, and flow during music listening: an empirical investigation. Psychol. Music 41, 42-58. doi: 10.1177/0305735 611415144

Diener, E., Emmons, R. A., Larsen, R. J., and Griffin, S. (1985). The satisfaction with life scale. J. Pers. Assess. 49, 71-75. doi: 10.1207/s15327752jpa4901_13

Duncan, J., and West, R. E. (2018). Conceptualizing group flow: a framework. Educ. Res. Rev. 13, 1-11. doi: 10.5897/ERR2017.3313

Elbe, A. M., Strahler, K., Krustrup, P., Wikman, J., and Stelter, R. (2010). Experiencing flow in different types of physical activity intervention programs: three randomized studies. Scand. J. Med. Sci. Sports 20, 111-117. doi: 10.1111/j. 1600-0838.2010.01112.x

Fossmo, T. (2006). Age Matters. A Study on Motivation, Flow and Self-Esteem in Competing Athletes. Master's thesis, University of Tromsø, Tromsø.

Fujita, F., Diener, E., and Sandvik, E. (1991). Gender differences in negative affect and well-being: the case for emotional intensity. J. Pers. Soc. Psychol. 61, 427-434. doi: 10.1037/0022-3514.61.3.427

Fullagar, C. J., Knight, P. A., and Sovern, H. S. (2013). Challenge/skill balance, flow, and performance anxiety. Appl. Psychol. 62, 236-259. doi: 10.1111/j.1464-0597. 2012.00494.x

Gnezda, A. (2016). Doživljanje Delovnega Zanosa pri Profesorjih Inštrumenta in Petja (Experiencing Work-Flow in Instrumental and Vocal Professors) (Diplomsko Delo). Ljubljana, SI: Academy of music.

Habe, K., and Berkopec, B. (2016). Betsabeja. Tok Muzièkog Izvođenja kod Studenata Konzervatorijum i Muzièke Akademije = Flow in Conservatory and Academy Music Students. V: PETROVIF, Milena (ur.). Knjiga Sažetaka = Book of Abstracts: The 19th Pedagogical forum of Performing Arts, Belgrade Sept. 30 Okt. 2. 2016. Beograd: Rektorat Univerziteta umetnosti, 92-94.

Habe, K., and Tement, S. (2016). Flow among higher education teachers: a job demands-resources perspective. Horiz. Psychol. 25, 29-37. doi: 10.20419/2016. 25.442

Häkkinen, A., Rinne, M., Vasankari, T., Santtila, M., Häkkinen, K., and Kyröläinen, H. (2010). Association of physical fitness with health-related quality of life in Finnish young men. Health Qual. Life Outcomes 8:15. doi: 10.1186/ 1477-7525-8-15

Hart, E., and Di Blasi, Z. (2015). Combined flow in musical jam sessions: a pilot qualitative study. Psychol. Music 43, 275-290. doi: 10.1177/030573561350 2374

Hays, T., and Minichiello, V. (2005). The meaning of music in the lives of older people: a qualitative study. Psychol. Music 33, 437-451. doi: 10.1177/ 0305735605056160

Hodge, K., Henry, G., and Smith, W. (2014). A case study of excellence in élite sport: motivational climate in a world champion team. Sport Psychol. 28, 60-74. doi: 10.1123/tsp.2013-0037

Huebner, E. S., and Dew, T. (1996). The interrelationships of positive affect, negative affect, and life satisfaction in an adolescent sample. Soc. Indic. Res. 38, 129-137. doi: 10.1007/BF00300455

Jackson, S. A. (1992). Athletes in flow: a qualitative investigation of flow states in élite figure skaters. J. Appl. Sport Psychol. 4, 161-180. doi: 10.1080/ 10413209208406459

Jackson, S. A. (1996). Toward a conceptual understanding of the flow experience in élite athletes. Res. Q. Exerc. Sport 67, 76-90. doi: 10.1080/02701367.1996. 10607928

Jackson, S. A., and Csikszentmihalyi, M. (1999). Flow in Sports: The Keys to Optimal Experiences and Performances. Leeds: Human Kinetics. 
Jackson, S. A., and Eklund, R. C. (2002). Assessing flow in physical activity: the Flow State Scale-2 and Dispositional Flow Scale-2. J. Sport Exerc. Psychol. 24, 133-150. doi: 10.1123/jsep.24.2.133

Jackson, S. A., Ford, S. K., Kimiecik, J. C., and Marsh, H. W. (1998). Psychological correlates of flow in sport. J. Sport Exerc. Psychol. 20, 358-378. doi: 10.1123/jsep. 20.4.358

Jackson, S. A., and Marsh, H. W. (1996). Development and validation of a scale to measure optimal experience: the flow state scale. J. Sport Exerc. Psychol. 18, 17-35. doi: 10.1123/jsep.18.1.17

John, P. A. S. (2006). Finding and making meaning: young children as musical collaborators. Psychol. Music 34, 238-261. doi: 10.1177/0305735606061854

Juniu, S., Tedrick, T., and Boyd, R. (1996). Leisure or Work?: amateur and professional musicians' perception of rehearsal and performance. J. Leis. Res. 28, 44-56. doi: 10.1080/00222216.1996.11949760

Kajtna, T., and Jeromen, T. (2013). Šport z Bistro Glavo - Druga, Dopolnjena Izdaja [Sport with a Clear Mind - Second, Amended Edition]. Ljubljana: Self-Published.

Kee, Y. H., and Wang, C. K. J. (2008). Relationships between mindfulness, flow dispositions and mental skills adoption: a cluster analytic approach. Psychol. Sport Exerc. 9, 393-411. doi: 10.1016/j.psychsport.2007.07.001

Kemp, A. (2000). The education of the professional musician: its psychological demands and outcomes. Musical Perform. 2, 93-110.

Kenny, D. T., Davis, P., and Oates, J. (2003). Music performance anxiety and occupational stress amongst opera chorus artists and their relationship with state and trait anxiety and perfectionism. J. Anxiety Disord. 18, 757-777. doi: 10.1016/j.janxdis.2003.09.004

Kirchner, J. M. (2011). Incorporating flow into practice and performance. Work 40, 289-296. doi: 10.3233/WOR-2011-1232

Koehn, S., Morris, T., and Watt, A. P. (2014). Imagery intervention to increase flow state and performance in competition. Sport Psychol. 28, 48-59. doi: 10.1123/ tsp.2012-0106

Le Fevre, M., Matheny, J., and Kolt, G. (2003). Eustress, distress, and interpretation in occupational stress. J. Manag. Psychol. 18, 726-744. doi: 10. $1108 / 02683940310502412$

Lindsay, P., Maynard, I., and Thomas, O. (2005). Effects of hypnosis on flow states and cycling performance. Sport Psychol. 19, 164-177. doi: 10.1123/tsp.19.2.164

Lowis, M. J. (2002). Music as a trigger for peak experiences among a college staff population. Creat. Res. J. 14, 351-359. doi: 10.1207/S15326934CRJ1434_6

MacDonald, R., Byrne, C., and Carlton, L. (2006). Creativity and flow in musical composition: an empirical investigation. Psychol. Music 34, 292-306. doi: 10. 1177/0305735606064838

Mäkikangas, A., Bakker, A. B., Aunola, K., and Demerouti, E. (2010). Job resources and flow at work: modelling the relationship via latent growth curve and mixture model methodology. J. Occup. Organ. Psychol. 83, 795-814. doi: 10. 1348/096317909X476333

Marin, M. M., and Bhattacharya, J. (2013). Getting into the musical zone: trait emotional intelligence and amount of practice predict flow in pianists. Front. Psychol. 4:853. doi: 10.3389/fpsyg.2013.00853

Martin, A. J. (2008). Motivation and engagement in music and sport: testing a multidimensional framework in diverse performance settings. J. Pers. 76, 135-170. doi: 10.1111/j.1467-6494.2007.00482.x

Massimini, F., and Carli, M. (1988). "The systematic assessment of flow in daily experience," in Optimal Experience: Psychological Studies of Flow in Consciousness, eds M. Csikszentmihalyi and I. S. Csikszentmihalyi (New York, NY: Cambridge University Press), 266-287. doi: 10.1017/CBO9780511621 956.016

Moneta, G. B. (2012). "On the measurement and conceptualization of flow," in Advances in Flow Research, ed. S. Engeser (New York, NY: Springer Science+Business Media), 23-50. doi: 10.1007/978-1-4614-2359-1_2

Moreno, J. A., Cervelló, E., and González-Cutre, D. (2008). Relationships among goal orientations, motivational climate and flow in adolescent athletes: differences by gender. Span. J. Psychol. 11, 181-191. doi: 10.1017/ S1138741600004224

Murko Feguš, N. (2016). Samoporoèani Uèinki Petja na Psihièno Blagostanje pri Profesionalnih Solistiènih Pevcih [Self- Reported Effects of Singing on Psychological Well-Being at Professional Solo Singers]. Master's thesis, University of Maribor, Maribor.

Muzio, M., Riva, G., and Argenton, N. (2012). Flow, Benessere e Prestazione Eccellente. Dai Modelli Teorici alle Applicazioni Nello Sport e in Azienda. Milano: Franco Angeli.
Nakamura, J., and Csikszentmihalyi, M. (2002). "The concept of flow," in Handbook of Positive Psychology, eds C. R. Snyder and S. J. Lopez (New York, NY: Oxford University Press), 89-105.

Nicholls, A. R., Polman, R. C. J., and Holt, N. L. (2005). The effects of an individualized imagery interventions on flow states and golf performance. Athl. Insight 7, 43-66.

Nitsch, J. R., and Hackfort, D. (2016). "Theoretical framework of performance psychology: an action theory perspective," in Performance Psychology: Perception, Action, Cognition, and Emotion, eds M. Raab, B. Lobinger, S. Hoffmann, A. Pizzera, and S. Laborde (London: Academic Press), 11-29. doi: 10.1016/C2014-0-03104-8

Norsworthy, C., Thelwell, R., Weston, N., and Jackson, S. A. (2017). Flow training, flow states, and performance in élite athletes. Int. J. Sport Psychol. 49, 134-152.

O'Neill, S. (1999). Flow theory and the development of musical performance skills. Bull. Counc. Res. Music Educ. 141, 129-134. doi: 10.3389/fpsyg.2017.00911

O'Sullivan, G. (2011). The relationship between hope, eustress, self-efficacy, and life satisfaction among undergraduates. Soc. Indic. Res. 101, 155-172. doi: 10. 1007/s11205-010-9662-z

Pain, M. A., Harwood, C., and Anderson, R. (2011). Pre-competition imagery and music: the impact on flow and performance in competitive soccer. Sport Psychol. 25, 212-232. doi: 10.1123/tsp.25.2.212

Pates, J., and Cowen, A. (2013). The effect of a hypnosis intervention on performance and flow state of an élite golfer: a single subject design. Int. J. Golf Sci. 2, 43-53. doi: 10.1123/ijgs.2.1.43

Pates, J., Cowen, A. P., and Karageorghis, C. I. (2012). The effect of a client-centered approach on flow states and the performance of three élite golfers. Int. J. Golf Sci. 1, 113-126. doi: 10.1123/ijgs.1.2.113

Pates, J., Karageorghis, C. I., Fryer, R., and Maynard, I. (2003). Effects of asynchronous music on flow states and shooting performance among netball players. Psychol. Sport Exerc. 4, 415-427. doi: 10.1016/S1469-0292(02) 00039-0

Pavot, W., and Diener, E. (1993). The affective and cognitive context of selfreported measures of subjective well-being. Soc. Indic. Res. 28, 1-20. doi: 10. 1007/BF01086714

Peluso, M. A. M., and de Andrade, L. H. S. G. (2005). Physical activity and mental health: the association between exercise and mood. Clinics 60, 61-70. doi: 10.1590/S1807-59322005000100012

Phillips, L. L. (2005). Examining Flow States and Motivational Perspectives of Ashtanga Yoga Practitioners. Doctoral thesis, University of Kentucky, Lexington, KY, 336.

Russell, W. D. (2001). An examination of flow state occurrence in college athletes. J. Sport Behav. 24, 83-107.

Ryff, C. D. (1989). Happiness is everything, or is it? Explorations on the meaning of psychological well-being. J. Pers. Soc. Psychol. 57, 1069-1081. doi: 10.1037/ 0022-3514.57.6.1069

Sawyer, K. (2003). Group Creativity: Music, Theater, Collaboration. Mahwah, NJ: Lawrence Erlbaum Associates, Inc.

Sawyer, K. (2007). Group Genius: The Creative Power of Collaboration. New York, NY: Basic Books.

Sawyer, R. K. (2006). Group creativity: musical performance and collaboration. Psychol. Music 34, 148-165. doi: 10.1177/0305735606061850

Sedlár, M. (2014). Relationships between flow experience, life meaningfulness and subjective well-being in music students. Psychol. Contexts 5, 89-104.

Selye, H. (1975). Stress Without Distress. Philadelphia, PA: Lippincott Publishing.

Sinnamon, S., Moran, A., and O'Connell, M. (2012). Flow among musicians: measuring peak experiences of student performers. J. Res. Music Educ. 60, 6-25. doi: 10.1177/0022429411434931

Smolej Fritz, B., and Avsec, A. (2007). The experience of flow and subjective well-being of music students. Horiz. Psychol. 16, 5-17.

Ströhle, A. (2009). Physical activity, exercise, depression and anxiety disorders. J. Neural Transm. 116, 777-784. doi: 10.1007/s00702-0080092-x

Sugiyama, T., and Inomata, K. (2005). Qualitative examination of flow experience among top Japanese athletes. Percept. Motor Skills 100, 969-982. doi: 10.2466/ pms.100.3c.969-982

Swann, C., Keegan, R. J., Piggott, D., and Crust, L. (2012). A systematic review of the experience, occurrence, and controllability of flow states in élite sport. Psychol. Sport Exerc. 13, 807-819. doi: 10.1016/j.psychsport.2012. 05.006 
Thomson, P., and Jaque, S. V. (2016). Overexcitability and optimal flow in talented dancers, singers and athletes. Roeper Rev. 38, 32-39. doi: 10.1080/02783193. 2015.1112865

Thorgaard, B., Brøndsted Henriksen, B., Pedersbæk, G., and Thomsen, I. (2004). Specially selected music in the cardiac laboratory-an important tool for improvement of the wellbeing of patients. Eur. J. Cardiovasc. Nurs. 3, 21-26. doi: 10.1016/j.ejcnurse.2003.10.001

Waddell, G., Perkins, R., and Williamon, A. (2018). Making an impression: error location and repertoire features affect performance quality rating processes. Music Percept. 36, 60-76. doi: 10.1525/mp.2018. 36.1.60

Walker, C. J. (2010). Experiencing flow: is doing it together better than doing it alone? J. Posit. Psychol. 5, 3-11. doi: 10.1080/1743976090327 1116
Wrigley, W. J., and Emmerson, S. B. (2013). The experience of the flow state in live music performance. Psychol. Music 41, 292-305. doi: 10.1177/03057 35611425903

Conflict of Interest Statement: The authors declare that the research was conducted in the absence of any commercial or financial relationships that could be construed as a potential conflict of interest.

Copyright (c) 2019 Habe, Biasutti and Kajtna. This is an open-access article distributed under the terms of the Creative Commons Attribution License (CC BY). The use, distribution or reproduction in other forums is permitted, provided the original author(s) and the copyright owner(s) are credited and that the original publication in this journal is cited, in accordance with accepted academic practice. No use, distribution or reproduction is permitted which does not comply with these terms. 
OPEN ACCESS

Edited by:

Michele Biasutti,

University of Padova, Italy

Reviewed by:

Ioulia Papageorgi,

University of Nicosia, Cyprus

Claude Ferrand,

Université de Tours, France

${ }^{*}$ Correspondence:

Amanda E. Krause

Amanda.Krause@unimelb.edu.au

Specialty section:

This article was submitted to

Performance Science,

a section of the journal

Frontiers in Psychology

Received: 30 November 2018

Accepted: 11 February 2019

Published: 01 March 2019

Citation:

Krause $A E$, North $A C$ and

Davidson JW (2019) Using Self-Determination Theory to Examine Musical Participation and Well-Being.

Front. Psychol. 10:405

doi: 10.3389/fpsyg.2019.00405

\section{Using Self-Determination Theory to Examine Musical Participation and Well-Being}

\author{
Amanda E. Krause ${ }^{1,2 *}$, Adrian C. North ${ }^{2}$ and Jane W. Davidson ${ }^{1}$ \\ ${ }^{1}$ The Melbourne Conservatorium of Music, The University of Melbourne, Parkville, VIC, Australia, ${ }^{2}$ School of Psychology, \\ Curtin University, Perth, WA, Australia
}

A recent surge of research has begun to examine music participation and wellbeing; however, a particular challenge with this work concerns theorizing around the associated well-being benefits of musical participation. Thus, the current research used Self-Determination Theory to consider the potential associations between basic psychological needs (competence, relatedness, and autonomy), self-determined autonomous motivation, and the perceived benefits to well-being controlling for demographic variables and the musical activity parameters. A sample of 192 Australian residents (17-85, $\left.M_{\text {age }}=36.95\right)$, who were currently participating in a musical activity at the time, completed an online questionnaire. Results indicated that females were more likely to perceive benefits to their well-being; and that how important an individual considers music in their life was positively related to perceived well-being. Importantly, the analyses also revealed that the basic needs of competency and relatedness were related to overall perceived well-being as well as specifically social, cognitive, and esteem dimensions of well-being. Autonomous motivation demonstrated significant associations with both an overall well-being score as well as four of five specific wellbeing subscales measured. Collectively, the findings indicate that Self-Determination Theory offers a useful theoretical framework to understanding the relationship between musical participation and well-being. Further, the pattern of findings reiterates the positive associations between musical participation and one's psychosocial well-being, with broad implications for people involved in the facilitation of musical activity.

Keywords: musical participation, well-being, Self-Determination Theory, psychological needs, autonomous motivation

\section{INTRODUCTION}

There is a growing interest in researching the possible relationship between music and well-being (Clift et al., 2008; Skingley et al., 2011; MacDonald et al., 2012; MacDonald, 2013). However, research on the subject faces a number of challenges. One, in particular, concerns the challenge of producing systematic, empirical evidence to support claims that are sometimes taken to be self-evident (Skingley et al., 2011). Relatedly, much of the relevant work can be challenged on the grounds of a lack of theoretical grounding (Clift and Hancox, 2010). Recently, researchers (e.g., Küpers et al., 2014; Evans, 2015; Krause and Davidson, 2018) have suggested the utility of Self-Determination Theory as a framework to consider musical participation and well-being. 
Self-Determination Theory has been applied to a wide range of social psychological behaviors, spanning health, education, and social relationships, and is supported by a growing body of research (Evans, 2015). Indeed, Self-Determination Theory has been used to explain a range of behaviors that involve motivation over extended periods of time (e.g., Georgiadis et al., 2006; Reinboth and Duda, 2006; Sheldon and Krieger, 2007; Alivernini and Lucidi, 2011; Jang et al., 2012). Therefore, the present research examined perceived well-being associated with active musical participation using Self-Determination Theory as a theoretical framework.

\section{Self-Determination Theory}

Self-Determination Theory is concerned with human motivation, development, and wellness: it outlines how the concept of motivation relates to individuals' affect, behavior, and well-being (Deci and Ryan, 2000, 2008). Self-Determination Theory is a macro theory, encompassing several mini-theories. Two of these theories, Basic Psychological Needs and Organismic Integration Theory, are particularly relevant to musical participation and its perceived well-being benefits. Self-Determination Theory argues that internal, external, and contextual factors, combine to influence the fulfillment of needs by either increasing or decreasing one's motivation to participate (Ryan and Deci, 2000). In this way, Self-Determination Theory can be used to understand how engagement, such as musical participation, can be fostered. Importantly, motivation, an important element to starting and continuing in musical activities (O'Neill and McPherson, 2002; McPherson and O'Neill, 2016), is central to Self-Determination Theory, and both Basic Psychological Needs and Organismic Integration Theory in particular. In this context we note that participation in music, for the majority, takes place during leisure time and/or or an elective basis: given that Self-Determination Theory has been used to study ongoing engagement with other leisure and elective activities, it is a suitable candidate for explaining ongoing engagement with music.

Basic psychological needs theory states that people strive to satisfy three innate needs, namely competence, relatedness, and autonomy (Ryan and Deci, 2002; Hagger et al., 2006). Competence refers to the need to be effective in one's efforts; relatedness concerns being connected socially, and integrated into a social group; and autonomy concerns the need to feel that one's pursuits are self-governed and self-endorsed (Ryan and Deci, 2002). Deci and Ryan assert that needs for competence, relatedness, and autonomy are universal - they are essential, regardless of culture and life domain (Deci and Ryan, 1985b, 2000; Ryan and Deci, 2002). Both internal, personal factors and the social environment influence the degree to which the three needs are met (Ryan and Deci, 2000; Quested et al., 2018). Meeting these needs leads to personal growth, vitality, and wellbeing (Deci and Ryan, 2000; Ryan and Deci, 2002).

Organismic integration theory (Deci and Ryan, 1985a, 1991; Ryan and Deci, 2000) differentiates types of motivation (Ryan and Connell, 1989; McLachlan et al., 2011). It places particular emphasis on the quality of motivation, rather than merely the quantity (Niven and Markland, 2016). Six different types of motivation exist, and are often conceptualized as lying on a continuum (Gagné and Deci, 2005; McLachlan et al., 2011; Wilson et al., 2012; MacIntyre et al., 2018). Intrinsic motivation lies at the internal end of this continuum, and represents selfdetermined, internalized motivation. External motivation lies at the other end of the continuum, and refers to motivation that is characterized by engagement for reasons completely external to oneself. Three additional types of extrinsic behavioral regulation which differ in terms of the degree to which the motivation is internalized are positioned between these two poles. The three different types of external motivation are termed integrated, identified, and introjected (McLachlan et al., 2011). Integrated regulation is the most autonomous form of extrinsic regulation, followed by identified, and introjected (the least autonomous form of extrinsic motivation). Integrated regulation concerns behavior that is fully assimilated and consistent with one's self; identified regulation refers to behavior based on attaining "personally valued outcomes rather than for enjoyment or interest"; and introjected regulation concerns enhancing selfworth by avoiding negative affective states, such as shame and guilt (McLachlan et al., 2011, p. 724; Wilson et al., 2012). Amotivation refers to the absence of clear motivation or intentions (Markland and Tobin, 2004; McLachlan et al., 2011). The theory recognizes that people's actions within a given domain are simultaneously the product of several different motivations along the continuum; these can be accounted for by calculating a relative autonomy index (RAI). This index score, for which a higher score indicates greater autonomy, serves as an aggregate representation of how intrinsically or extrinsically motivated someone is Seymour and Peterman (2018). Organismic integration theory states that the fulfillment of psychological needs will lead to internalized motivation (Gagné and Deci, 2005).

The fulfillment of the basic psychological needs is fostered in environments that are autonomy-supportive and hindered in environments that are controlling (Bonneville-Roussy et al., 2013). Clear evidence of this comes from previous education research using Self-Determination Theory. Studies have found that students are more engaged and persistent in autonomy supportive activities and environments (e.g., Reeve et al., 2004; Hagger et al., 2015). In contrast, students suffer in environments in which teachers are more controlling (e.g., Soenens et al., 2012; Bonneville-Roussy et al., 2013). In short, in educational settings, autonomy support is associated with students having more self-determined forms of motivation and higher perceptions of competence (e.g., Williams and Deci, 1998) as well as higher levels of enjoyment, engagement, performance, and persistence (e.g., Black and Deci, 2000; Vansteenkiste et al., 2004; Chatzisarantis and Hagger, 2009; Niemiec and Ryan, 2009; Jang et al., 2010; Bonneville-Roussy et al., 2013; Oga-Baldwin et al., 2017; Ulstad et al., 2018).

Another claim of Self-Determination Theory is that satisfaction of needs is associated with well-being (Deci and Ryan, 2000; Milyavskaya and Koestner, 2011; Lombas and Esteban, 2018). Research has shown this in work settings (e.g., Ilardi et al., 1993; Deci et al., 2001; Baard et al., 2004; Van den Broeck et al., 2016) and healthcare settings, such as aged-care, 
where an autonomy-supportive environment has been associated with better well-being (Deci and Ryan, 1987; Ferrand et al., 2014). Research also links satisfying needs to perceived wellbeing with regard to leisure pursuits (Coleman and Iso-Ahola, 1993), including predominantly exercise (e.g., Chatzisarantis and Hagger, 2009; Lovell et al., 2016; Niven and Markland, 2016; Sebire et al., 2016), but also other pursuits such as community gardening (e.g, Quested et al., 2018) and relationship functioning (e.g., Patrick et al., 2007). As Coleman and Iso-Ahola (1993) found, leisure activities which promote fulfilling participants' basic needs, therefore, promote self-determination and are beneficial to well-being (see also Deci and Ryan, 2000; Ferrand et al., 2014). Indeed, Kuykendall et al. (2015) recent meta-analysis provided strong evidence that leisure engagement is consistently associated with subjective well-being as well as evidence that leisure satisfaction mediates the relationship between leisure engagement and well-being. It is therefore surprising that little research has considered musical participation and well-being using self-determination theory as a theoretical framework, given the prevalence of music participation as a leisure time activity (Laukka, 2007).

\section{Self-Determination Theory in Music Research}

Self-determination theory has recently been used in work concerning both music education and music therapy (Douglas, 2011; Evans and Bonneville-Roussy, 2016; Lee et al., 2016; Valenzuela et al., 2018). However, as Evans (2015, p. 7) referenced in his conceptual overview concerning how selfdetermination theory might be used to consider motivation in music education, there have only been "a small number of studies." In particular, music education researchers have focused on practice, both at the university and conservatoire level (Evans and Bonneville-Roussy, 2016; Valenzuela et al., 2018) and middleschool level (Schatt, 2018). Findings indicate that the fulfillment of psychological needs and autonomous motivation were associated with practicing more frequently and a higher quality of practice (Evans and Bonneville-Roussy, 2016). However, Schatt's (2018) findings indicated differences by instrument and grade level with regard to levels of self-determination to practice, suggesting that personal and contextual factors pertaining to the musical activity can influence one's motivation. Moreover, feelings of autonomy and competence are linked to intrinsic motivation and the experience of flow (Valenzuela et al., 2018) in practice; and autonomy support is also related to passion and persistence in music education (BonnevilleRoussy et al., 2013). Additional work has considered musical play at recess (Countryman, 2014) and singing games in and out of the classroom (Roberts, 2018), providing further support for applying self-determination theory to understand musical behaviors.

Given that motivation, broadly, has been recognized as an important feature with regard to both starting and continuing to participate in musical activities (O'Neill and McPherson, 2002; McPherson and O'Neill, 2016), researchers have also used basic psychological needs to consider who plays versus ceases playing (Evans et al., 2013; Freer and Evans, 2018). Evidence suggests that when the three basic psychological needs are met, people are more likely to continue participating in musical activities (Douglas, 2011; Evans et al., 2013). Findings concerning community band participation pointed to associations with autonomy and competence, specifically (Douglas, 2011). Indeed, students are more likely to continue participating in music as an elective subject when their psychological needs are met (Freer and Evans, 2018).

Further, importantly, research findings support positive associations between feelings of subjective well-being and the three psychological needs in the context of musical participation (Creech et al., 2013b). In Creech et al.'s (2013b, p. 40) study, "subjective well-being was found to be underpinned by a sense of purpose, feeling in control and autonomous, and receiving affirmation through positive social relationships that accord individuals with respect and status." Autonomous motivation was also related to coping strategies by university music students (Bonneville-Roussy et al., 2017).

\section{Present Research}

The current study aimed to examine musical participation and well-being relative to self-determination theory. In doing so, it addressed particular limitations of past research in this area. Firstly, while researchers, such as Evans (2015), have provided conceptual overviews applying self-determination theory to musical behaviors, "there is the need to test the ideas empirically" (MacIntyre et al., 2018, p. 702). Moreover, while limited prior research has considered self-determination theory and motivation within a musical context, very little of this work has considered the associated well-being benefits that might follow. Although the previous research on well-being and musical participation has demonstrated many perceived well-being benefits, the Krause et al. (2018) review highlighted the need to systematically and comprehensively consider these. In response to identifying a small number of broad categories to which these benefits align (such as social, emotional, and cognitive), Krause et al.'s measure was designed to measure perceived well-being holistically and employed in the present study.

Secondly, much of the previous work has been limited in scope and/or size by focusing on a particular, specific wellbeing benefit or a tightly-defined sample of participants (Krause et al., 2018). Therefore, the present study considered adult musical participation more broadly. It was not limited to only formal music education settings or to university and conservatory students; rather in spirit of life-long engagement, the present study considers musical participation in various contexts among a community sample. In turn, the present analyses included demographic variables and the context of the musical activity as potential covariates within the analyses.

Consequently, the present study aimed to identify associations between psychological needs, motivation, and well-being in the context of musical participation. In line with past research, it was hypothesized that the three basic psychological needs outlined by self-determination theory (autonomy, competence, and relatedness) would be positively associated with perceived 
well-being. It was also hypothesized that RAI scores would be positively associated with perceived well-being.

\section{MATERIALS AND METHODS}

\section{Sample}

An online questionnaire was completed by a sample of 192 Australian residents. Data were collected as a part of a larger study considering musical engagement (see also Krause et al., unpublished); the present research employed only those data concerning individuals who indicated that they were actively participating in a musical activity at the time, such that those individuals who had ceased participating or had never participated in a musical activity are excluded from the present study's analyses. The present research looks specifically at the variables concerning self-determination theory and perceived well-being, which are not reported in Krause et al., unpublished.

The sample was largely female $(63.5 \%$ female, $34.9 \%$ male, $1.6 \%$ declined to respond). Ages ranged from 17 to 85 ( $M=36.95$, $M d n=28.50, S D=19.28$ ); and $52.10 \%$ of the sample reported having a university qualification. Regarding the participants' primary musical activity, $49.7 \%$ reported that they played an instrument, $35.1 \%$ sung, and $15.2 \%$ indicated they were a facilitator (i.e., leading the activity for other people).

Participation in the study was voluntary. Recruitment included the use of online tools, including University student research participation programs, dedicated online study websites, social media postings, and the first author's/project's website. Those individuals who participated via a student research participation scheme received course credit.

\section{Design and Procedure}

All participants completed an online questionnaire (using Qualtrics); they were provided with information and consented to participate prior to accessing the questionnaire. Individuals completed the questionnaire as a series of webpages and were thanked and debriefed upon completion.

Individuals stated their age, gender, and country of residence, and were asked to rate the importance of music in their life using a seven-point scale $(1=$ not at all important, $7=$ extremely important). A direct question asked if the participants were currently participating in a musical activity, had previously participated in a musical activity but were no longer currently participating in a musical activity, or had never participated in a musical activity. After indicating that they were actively participating in a musical activity at the time of completing the questionnaire, participants were asked a series of questions about their current musical participation. In recognition that some individuals may be participating in more than one musical activity, participants were asked to report on their primary activity (e.g., the one concerning which most time is spent) and asked to indicate whether their involvement would be classified as mainly singing, playing an instrument, or facilitating (i.e., leading others in) the activity. Individuals also reported the length of time they had been participating in this particular activity (in years), rated their frequency of involvement using a five-point scale (where $1=$ daily, $2=2-3$ times per week, 3 = weekly, $4=$ fortnightly, $5=$ monthly), and indicated where the activity took place (by selecting either a domestic setting, community setting, or educational setting). They also estimated the number of other people with whom they regularly participate. While this response was open-ended, the responses were coded as (where $1=0,2=1-5,3=6-15,4=16-35,5=36-75$, and $6=76$ or more people).

Participants were asked to complete a Basic Psychological Needs measure concerning music participation. In particular, it was important to address basic psychological needs pertaining to music participation rather than in general. Many selfdetermination scales have been developed with regard to the context of exercise given the prominence of the theory's application to this domain (Ryan and Deci, 2007; Hagger and Chatzisarantis, 2008; Wilson et al., 2008); however, musicfocused Self-determination scales are scarce. Therefore, an amended version of the Basic Psychological Needs in Exercise Scale (BPNES; Vlachopoulos et al., 2010) was used. Previous research that has employed the BPNES in a variety of domains (e.g., Douglas, 2011; Evans et al., 2013; Niven and Markland, 2016). Following accepted practice, some of the item wording was amended to address musical participation specifically (e.g., 'I am able to meet the requirements of my music activity's program,' 'My relationships with the people I participate with are close'). Responses were made on a five-point scale $(1=I$ don't agree at all; 5 = I completely agree). Following Vlachopoulos et al.'s (2010) subscale coding, items were averaged in order to compute three scores (one for each of Autonomy, Competency and Relatedness) per participant. The BPNES has demonstrated good reliability and validity (e.g., Vlachopoulos et al., 2010; Lovell et al., 2016; Arrogi et al., 2017). Cronbach's alpha values were $0.804,0.855$, and 0.831 , for autonomy, competency, and relatedness, respectively.

An amended version of the BREQ-2 (Behavioural Regulation in Exercise Questionnaire-2, Markland and Tobin, 2004) which included questions concerning the integrated regulation form (Wilson et al., 2006; McLachlan et al., 2011) was used to measure the quality of each participant's motivation with regard to their musical activity. Again, in the interest of domain specificity (as previously done, e.g., Niven and Markland, 2016; Teques et al., 2017), amendments to this established, exercise-focused measure were made such that re-phrased items addressed participating in a musical activity specifically. Individuals were asked to respond to the set of 37 items (e.g., 'I think it is important to make the effort to participate regularly,' 'I will feel guilty if I do not participate in my musical activity') using a five-point scale $(0=$ not true for me; $4=$ very true for me $)$. Previous research has demonstrated the reliability of this measure (Markland and Tobin, 2004; Lovell et al., 2016; Ntoumanis et al., 2017). Cronbach's alpha values for the different forms of regulation were as follows: 0.905 for amotivation, 0.821 for external regulation, 0.650 for introjected regulation, 0.775 for identified regulation, 0.854 for integrated regulation, and 0.809 for intrinsic regulation. Adopting the approach used in previous research (e.g., Ryan and Connell, 1989; Niemiec et al., 2006; Wilson et al., 2012; Evans and Bonneville-Roussy, 2016), a RAI score was computed 
TABLE 1 | Results of the first step of the GLMM analyses concerning well-being score.

\begin{tabular}{|c|c|c|c|c|c|}
\hline Variable & $\boldsymbol{F}$ & $D F$ & DF & $p$ & $\eta_{\mathrm{p}}^{2}$ \\
\hline \multicolumn{6}{|l|}{ Total well-being score } \\
\hline Gender & 11.025 & 1 & 187 & 0.001 & 0.056 \\
\hline University degree & 7.042 & 1 & 189 & 0.009 & 0.036 \\
\hline Age & 17.504 & 1 & 190 & $<0.001$ & 0.084 \\
\hline Music importance rating $(1-7)$ & 31.808 & 1 & 188 & $<0.001$ & 0.145 \\
\hline Activity type & 0.981 & 2 & 188 & 0.377 & 0.010 \\
\hline Length of time participating & 8.873 & 1 & 186 & 0.003 & 0.046 \\
\hline Frequency of participation rating & 5.350 & 1 & 190 & 0.022 & 0.027 \\
\hline Location type & 2.285 & 2 & 188 & 0.105 & 0.024 \\
\hline Number of other participants (grouping) & 12.890 & 1 & 186 & $<0.001$ & 0.065 \\
\hline Autonomy score & 52.448 & 1 & 189 & $<0.001$ & 0.217 \\
\hline Competency score & 84.585 & 1 & 187 & $<0.001$ & 0.311 \\
\hline Relatedness score & 36.224 & 1 & 187 & $<0.001$ & 0.162 \\
\hline Relative autonomy index score & 160.531 & 1 & 173 & $<0.001$ & 0.481 \\
\hline \multicolumn{6}{|l|}{ Mood and coping well-being score } \\
\hline Gender & 5.839 & 1 & 187 & 0.017 & 0.030 \\
\hline University degree & 0.738 & 1 & 189 & 0.391 & 0.004 \\
\hline Age & 6.295 & 1 & 190 & 0.013 & 0.032 \\
\hline Music importance rating (1-7) & 25.988 & 1 & 188 & $<0.001$ & 0.121 \\
\hline Activity type & 0.862 & 2 & 188 & 0.424 & 0.009 \\
\hline Length of time participating & 6.419 & 1 & 186 & 0.012 & 0.033 \\
\hline Frequency of participation rating & 8.894 & 1 & 190 & 0.003 & 0.045 \\
\hline Location type & 0.074 & 2 & 188 & 0.929 & 0.001 \\
\hline Number of other participants (grouping) & 4.190 & 1 & 186 & 0.042 & 0.022 \\
\hline Autonomy score & 36.935 & 1 & 189 & $<0.001$ & 0.163 \\
\hline Competency score & 46.159 & 1 & 187 & $<0.001$ & 0.198 \\
\hline Relatedness score & 18.942 & 1 & 187 & $<0.001$ & 0.092 \\
\hline Relative autonomy index score & 61.847 & 1 & 173 & $<0.001$ & 0.263 \\
\hline \multicolumn{6}{|l|}{ Esteem and worth well-being score } \\
\hline Gender & 6.284 & 1 & 187 & 0.013 & 0.033 \\
\hline University degree & 10.900 & 1 & 189 & 0.001 & 0.055 \\
\hline Age & 19.955 & 1 & 190 & $<0.001$ & 0.095 \\
\hline Music importance rating (1-7) & 17.985 & 1 & 188 & $<0.001$ & 0.087 \\
\hline Activity type & 0.400 & 2 & 188 & 0.671 & 0.004 \\
\hline Length of time participating & 7.914 & 1 & 186 & 0.005 & 0.041 \\
\hline Frequency of participation rating & 5.435 & 1 & 190 & 0.021 & 0.028 \\
\hline Location type & 1.912 & 2 & 188 & 0.151 & 0.020 \\
\hline Number of other participants (grouping) & 6.531 & 1 & 186 & 0.011 & 0.034 \\
\hline Autonomy score & 39.117 & 1 & 189 & $<0.001$ & 0.171 \\
\hline Competency score & 61.492 & 1 & 187 & $<0.001$ & 0.247 \\
\hline Relatedness score & 18.888 & 1 & 187 & $<0.001$ & 0.092 \\
\hline Relative autonomy index score & 90.985 & 1 & 173 & $<0.001$ & 0.345 \\
\hline \multicolumn{6}{|l|}{ Socializing well-being score } \\
\hline Gender & 12.223 & 1 & 187 & 0.001 & 0.061 \\
\hline University degree & 1.552 & 1 & 189 & 0.214 & 0.008 \\
\hline Age & 9.442 & 1 & 190 & 0.002 & 0.047 \\
\hline Music importance rating (1-7) & 22.109 & 1 & 188 & $<0.001$ & 0.105 \\
\hline Activity type & 5.868 & 2 & 188 & 0.003 & 0.059 \\
\hline Length of time participating & 3.037 & 1 & 186 & 0.083 & 0.016 \\
\hline Frequency of participation rating & 0.191 & 1 & 190 & 0.662 & 0.001 \\
\hline Location type & 14.692 & 2 & 188 & $<0.001$ & 0.135 \\
\hline
\end{tabular}

(Continued)
TABLE 1 | Continued

\begin{tabular}{|c|c|c|c|c|c|}
\hline Variable & $\boldsymbol{F}$ & $D F$ & DF $F_{\text {error }}$ & $p$ & $\eta_{\mathrm{p}}^{2}$ \\
\hline Number of other participants (grouping) & 40.123 & 1 & 186 & $<0.001$ & 0.177 \\
\hline Autonomy score & 27.146 & 1 & 189 & $<0.001$ & 0.126 \\
\hline Competency score & 46.532 & 1 & 187 & $<0.001$ & 0.199 \\
\hline Relatedness score & 79.911 & 1 & 187 & $<0.001$ & 0.299 \\
\hline Relative autonomy index score & 9.118 & 1 & 173 & 0.003 & 0.050 \\
\hline \multicolumn{6}{|l|}{ Cognitive well-being score } \\
\hline Gender & 10.332 & 1 & 187 & 0.002 & 0.052 \\
\hline University degree & 6.200 & 1 & 189 & 0.014 & 0.032 \\
\hline Age & 12.256 & 1 & 190 & 0.001 & 0.061 \\
\hline Music importance rating (1-7) & 19.086 & 1 & 188 & $<0.001$ & 0.092 \\
\hline Activity type & 0.254 & 2 & 188 & 0.776 & 0.003 \\
\hline Length of time participating & 6.709 & 1 & 186 & 0.010 & 0.035 \\
\hline Frequency of participation rating & 1.291 & 1 & 190 & 0.257 & 0.007 \\
\hline Location type & 1.853 & 2 & 188 & 0.160 & 0.019 \\
\hline Number of other participants (grouping) & 5.523 & 1 & 186 & 0.020 & 0.029 \\
\hline Autonomy score & 32.452 & 1 & 189 & $<0.001$ & 0.147 \\
\hline Competency score & 60.820 & 1 & 187 & $<0.001$ & 0.245 \\
\hline Relatedness score & 20.376 & 1 & 187 & $<0.001$ & 0.098 \\
\hline Relative autonomy index score & 106.864 & 1 & 173 & $<0.001$ & 0.382 \\
\hline \multicolumn{6}{|l|}{ Self-actualization well-being score } \\
\hline Gender & 5.891 & 1 & 187 & 0.016 & 0.031 \\
\hline University degree & 12.113 & 1 & 189 & 0.001 & 0.060 \\
\hline Age & 13.781 & 1 & 190 & $<0.001$ & 0.068 \\
\hline Music importance rating (1-7) & 18.295 & 1 & 188 & $<0.001$ & 0.089 \\
\hline Activity type & 0.122 & 2 & 188 & 0.885 & 0.001 \\
\hline Length of time participating & 4.733 & 1 & 186 & 0.031 & 0.025 \\
\hline Frequency of participation rating & 1.575 & 1 & 190 & 0.211 & 0.008 \\
\hline Location type & 3.698 & 2 & 188 & 0.027 & 0.038 \\
\hline Number of other participants (grouping) & 10.735 & 1 & 186 & 0.001 & 0.055 \\
\hline Autonomy score & 31.551 & 1 & 189 & $<0.001$ & 0.143 \\
\hline Competency score & 48.503 & 1 & 187 & $<0.001$ & 0.206 \\
\hline Relatedness score & 15.762 & 1 & 187 & $<0.001$ & 0.078 \\
\hline Relative autonomy index score & 64.889 & 1 & 173 & $<0.001$ & 0.273 \\
\hline
\end{tabular}

DF, degrees of freedom.

for each participant. To create the RAI score in the present study, the formula employed was: RAI $=3 \times$ Intrinsic + $2 \times$ Integrated + Identified - Introjected $-2 \times$ External $3 \times$ Amotivation (Vallerand et al., 2008; Wilson et al., 2012). The participant's single RAI score was used in subsequent analyses.

Lastly, participants completed Krause et al.'s (2018) measure of the social-psychological well-being benefits of musical participation. Participants responded to the 36 items (e.g., 'It adds purpose/meaning to my life,' 'It does not help me to think about who I am) using a seven-point scale $(1=$ Disagree completely, $7=$ Agree completely). The total score (for which the 19 negative items were reverse-coded) and five sub-scale scores (addressing the dimensions of mood and coping, esteem and worth, socialization, cognition, and self-actualization respectively) were calculated by averaging the participants' responses as per Krause et al.'s (2018) sub-scale coding. Cronbach's alpha values were as follows: 0.951 for the total score, 0.903 for mood and coping, 
0.900 for esteem and worth, 0.851 for socializing, 0.771 for cognitive, and 0.747 for self-actualization.

\section{RESULTS AND DISCUSSION}

A two-step generalized linear mixed model (GLMM) analysis procedure was used, implemented through SPSS's (Version 24) GENLINMIXED procedure. In the first step, each of the predictor variables was entered separately with the wellbeing score as the criterion variable (see Table $\mathbf{1}$ for the results of the step 1 analyses). The predictor variables were: demographic variables (age, gender, music importance rating, university degree), activity parameters (participation type, length of participation, frequency of participation, location, number of other participants), psychological needs scores (autonomy, competency, relatedness), and RAI score. At step two, the predictor variables that demonstrated a significant relationship with the criterion variable $(\alpha<0.05)$ were entered together in a single GLMM analysis $(\alpha<0.008)$.

This process was repeated in order to conduct six separate analyses, in which each of the total well-being score and five wellbeing subscale scores served as the respective dependent variable. Tables 2-7 detail the results of these analyses.
As evident in Tables 2-7 and summarized in Figure 1, the individual models displayed similar patterns of results across the set of analyses. In particular, with regard to gender, the results indicate that females reported experiencing greater perceived well-being benefits on five of the six measures than males (all except for the mood and coping sub-scale score). The music importance rating was positively associated with the total well-being score and socializing sub-scale sore. Indeed, the evidence for positive associations between musical participation and perceived social well-being is growing (e.g., von Lob et al., 2010; Jutras, 2011; Rohwer and Rohwer, 2012; Creech et al., 2013b; McQueen et al., 2013; Krause et al., 2018).

The RAI score demonstrated a significant, positive association in five of the six analyses (all except for the socializing wellbeing score). More simply, greater well-being was associated with internalized motivation to participate in music (see Figure 1). Indeed, when the RAI score demonstrated a significant association, it accounted for the largest percentage of variance in each analysis, suggesting the particular importance of selfregulated motivation. The strong, positive associations between internalized motivation and perceived well-being support SelfDetermination Theory's links between autonomous motivation and well-being (e.g., Deci and Ryan, 2000).

TABLE 2 | Total well-being score model.

\begin{tabular}{|c|c|c|c|c|c|c|c|}
\hline Variable & $\boldsymbol{F}$ & $p$ & Beta & $t$ & & & $\eta^{2}$ \\
\hline University degree & 1.834 & 0.178 & 0.140 & 1.354 & -0.064 & 0.344 & 0.012 \\
\hline Music importance rating (1-7) & 4.183 & 0.043 & 0.154 & 2.045 & 0.005 & 0.302 & 0.028 \\
\hline Length of time participating & 1.584 & 0.210 & -0.004 & -1.259 & -0.011 & 0.002 & 0.011 \\
\hline Frequency of participation rating & 2.058 & 0.153 & 0.048 & 1.435 & -0.018 & 0.114 & 0.014 \\
\hline Autonomy score & 1.529 & 0.218 & 0.252 & 2.736 & 0.070 & 0.434 & 0.048 \\
\hline Competency score & 7.484 & 0.007 & 0.252 & 2.736 & 0.070 & 0.434 & 0.048 \\
\hline Relatedness score & 4.791 & 0.030 & 0.131 & 2.189 & 0.013 & 0.249 & 0.032 \\
\hline Relative autonomy index score & 84.114 & $<0.001$ & 0.086 & 9.171 & 0.067 & 0.104 & 0.364 \\
\hline
\end{tabular}

Full model: $F(11,147)=32.798, p<0.001, \eta_{p}^{2}=0.711$. Degrees of freedom $=1,147$ for each variable. $\mathrm{Cl}$, confidence interval.

TABLE 3 | Mood and coping well-being score model.

\begin{tabular}{|c|c|c|c|c|c|c|c|}
\hline $\begin{array}{l}\text { Variable } \\
\text { Gender }\end{array}$ & $\begin{array}{c}\boldsymbol{F} \\
3.871\end{array}$ & $\begin{array}{c}p \\
0.051\end{array}$ & $\begin{array}{l}\text { Beta } \\
0.255\end{array}$ & $\begin{array}{c}\boldsymbol{t} \\
1.968\end{array}$ & \multicolumn{2}{|c|}{$95 \% \mathrm{Cl}$} & $\frac{\eta^{2}}{0.026}$ \\
\hline Age & 0.248 & 0.619 & -0.002 & -0.498 & -0.009 & 0.006 & 0.002 \\
\hline Length of time participating & 0.109 & 0.742 & -0.001 & -0.330 & -0.010 & 0.007 & 0.001 \\
\hline Frequency of participation rating & 0.067 & 0.797 & 0.013 & 0.258 & -0.086 & 0.112 & 0.000 \\
\hline Number of other participants (grouping) & 0.081 & 0.776 & -0.011 & -0.285 & -0.086 & 0.064 & 0.001 \\
\hline Competency score & 3.407 & 0.067 & 0.208 & 1.846 & -0.015 & 0.430 & 0.023 \\
\hline Relatedness score & 0.851 & 0.358 & 0.063 & 0.922 & -0.072 & 0.198 & 0.006 \\
\hline Relative autonomy index score & 27.165 & $<0.001$ & 0.083 & 5.212 & 0.051 & 0.114 & 0.155 \\
\hline
\end{tabular}

Full model: $F(10,148)=13.379, p<0.001, \eta_{p}^{2}=0.475$. Degrees of freedom $=1,148$ for each variable. $\mathrm{Cl}$, confidence interval. 
TABLE 4 | Esteem and worth well-being score model.

\begin{tabular}{|c|c|c|c|c|c|c|c|}
\hline Variable & $\boldsymbol{F}$ & $p$ & Beta & $t$ & \multicolumn{2}{|c|}{$95 \% \mathrm{Cl}$} & $\eta^{2}$ \\
\hline University degree & 0.337 & 0.563 & 0.074 & 0.580 & -0.177 & 0.324 & 0.002 \\
\hline Music importance rating $(1-7)$ & 3.003 & 0.085 & 0.214 & 1.733 & -0.030 & 0.458 & 0.020 \\
\hline Length of time participating & 2.022 & 0.157 & -0.006 & -1.422 & -0.015 & 0.002 & 0.014 \\
\hline Frequency of participation rating & 1.593 & 0.209 & 0.057 & 1.262 & -0.032 & 0.147 & 0.011 \\
\hline Autonomy score & 2.001 & 0.159 & -0.179 & -1.414 & -0.429 & 0.071 & 0.013 \\
\hline Competency score & 4.550 & 0.035 & 0.248 & 2.133 & 0.018 & 0.478 & 0.030 \\
\hline Relatedness score & 0.604 & 0.438 & 0.055 & 0.777 & -0.085 & 0.195 & 0.004 \\
\hline Relative autonomy index score & 44.319 & $<0.001$ & 0.109 & 6.657 & 0.077 & 0.142 & 0.232 \\
\hline
\end{tabular}

Full model: $F(11,147)=12.054, p<0.001, \eta_{p}^{2}=0.474$. Degrees of freedom $=1,147$ for each variable. $C l$, confidence interval.

TABLE 5 | Socializing well-being score model.

\begin{tabular}{|c|c|c|c|c|c|c|c|c|}
\hline $\begin{array}{l}\text { Variable } \\
\text { Gender }\end{array}$ & $\begin{array}{c}\boldsymbol{F} \\
19.001\end{array}$ & $\begin{array}{c}\boldsymbol{p} \\
<0.001\end{array}$ & & $\begin{array}{l}\text { Beta } \\
0.559\end{array}$ & $\begin{array}{c}\boldsymbol{t} \\
4.359\end{array}$ & \multicolumn{2}{|c|}{$95 \% \mathrm{Cl}$} & $\frac{\eta^{2}}{0.114}$ \\
\hline Age & 0.710 & 0.401 & & 0.003 & 0.843 & -0.004 & 0.011 & 0.005 \\
\hline \multirow[t]{3}{*}{ Activity type } & 1.142 & 0.322 & Instrument - Sing & -0.172 & $-1.218, p=0.225$ & -0.451 & 0.107 & 0.010 \\
\hline & & & Instrument - Facilitate & 0.006 & $0.044, p=0.965$ & -0.277 & 0.289 & 0.000 \\
\hline & & & Sing - Facilitate & 0.178 & $1.348, p=0.180$ & -0.083 & 0.439 & 0.012 \\
\hline & & & $\begin{array}{l}\text { Domestic - Educational } \\
\text { establishment }\end{array}$ & -0.126 & $-0.725, p=0.469$ & -0.469 & 0.217 & 0.004 \\
\hline & & & $\begin{array}{l}\text { Community venue - } \\
\text { Educational } \\
\text { establishment }\end{array}$ & 0.214 & $1.532, p=0.128$ & -0.062 & 0.490 & 0.016 \\
\hline Relative autonomy index score & 0.079 & 0.779 & & 0.004 & 0.281 & -0.023 & 0.031 & 0.001 \\
\hline
\end{tabular}

Full model: $F(12,147)=18.307, p<0.001, \eta_{p}^{2}=0.599$. Degrees of freedom $=1,147$ for each variable; except for Activity type and Location type, where Degrees of freedom $=2,147 . \mathrm{Cl}$, confidence interval.

TABLE 6 | Cognitive well-being score model.

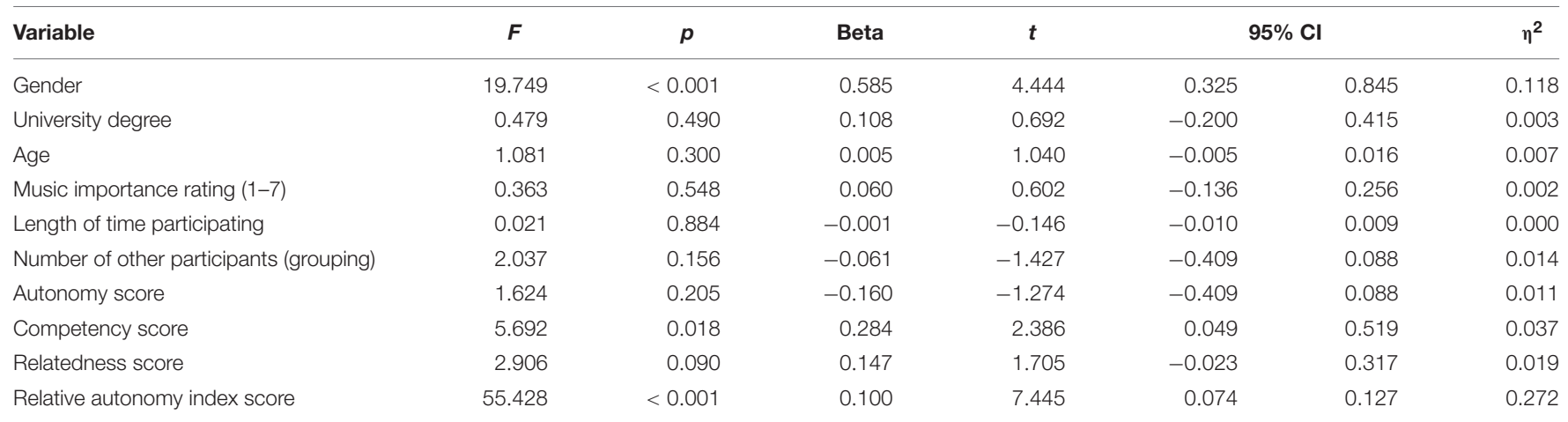

Full model: $F(10,148)=20.583, p<0.001, \eta_{p}^{2}=0.582$. Degrees of freedom $=1,148$ for each variable. $C l$, confidence interval. 
TABLE 7 | Self-actualization well-being score model.

\begin{tabular}{|c|c|c|c|c|c|c|c|c|}
\hline \multirow{2}{*}{$\begin{array}{l}\text { Variable } \\
\text { Gender }\end{array}$} & \multirow{2}{*}{$\begin{array}{c}\boldsymbol{F} \\
6.645\end{array}$} & \multirow{2}{*}{$\begin{array}{c}p \\
0.011\end{array}$} & & \multirow{2}{*}{$\begin{array}{l}\text { Beta } \\
0.414\end{array}$} & \multirow{2}{*}{$\begin{array}{c}\boldsymbol{t} \\
2.578\end{array}$} & \multicolumn{2}{|c|}{$95 \% \mathrm{Cl}$} & \multirow{2}{*}{$\frac{\eta^{2}}{0.044}$} \\
\hline & & & & & & 0.097 & 0.731 & \\
\hline University degree & 0.382 & 0.537 & & -0.101 & -0.618 & -0.424 & 0.222 & 0.003 \\
\hline Age & 0.509 & 0.477 & & 0.003 & 0.714 & -0.006 & 0.012 & 0.004 \\
\hline Music importance rating (1-7) & 1.271 & 0.261 & & 0.159 & 1.127 & -0.120 & 0.438 & 0.009 \\
\hline Length of time participating & 1.939 & 0.166 & & -0.008 & -1.392 & -0.018 & 0.003 & 0.013 \\
\hline \multirow[t]{3}{*}{ Location type } & 0.184 & 0.832 & $\begin{array}{l}\text { Domestic setting - } \\
\text { Community venue }\end{array}$ & -0.035 & $-0.180, p=0.857$ & -0.422 & 0.351 & 0.000 \\
\hline & & & $\begin{array}{l}\text { Domestic - Educational } \\
\text { establishment }\end{array}$ & -0.119 & $-0.552, p=0.582$ & -0.545 & 0.307 & 0.002 \\
\hline & & & $\begin{array}{l}\text { Community venue - } \\
\text { Educational } \\
\text { establishment }\end{array}$ & -0.084 & $-0.495, p=0.621$ & -0.418 & 0.250 & 0.002 \\
\hline Number of other participants (grouping) & 0.002 & 0.966 & & -0.002 & -0.043 & -0.117 & 0.112 & 0.000 \\
\hline Autonomy score & 1.427 & 0.234 & & -0.171 & -1.195 & -0.453 & 0.112 & 0.010 \\
\hline Competency score & 2.143 & 0.145 & & 0.234 & 1.464 & -0.082 & 0.550 & 0.015 \\
\hline Relatedness score & 1.169 & 0.281 & & 0.116 & 1.081 & -0.096 & 0.329 & 0.008 \\
\hline Relative autonomy index score & 20.921 & $<0.001$ & & 0.097 & 4.574 & 0.055 & 0.139 & 0.126 \\
\hline
\end{tabular}

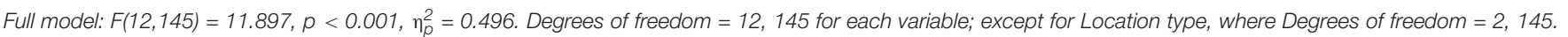
Cl, confidence interval.

Summary of Study Findings

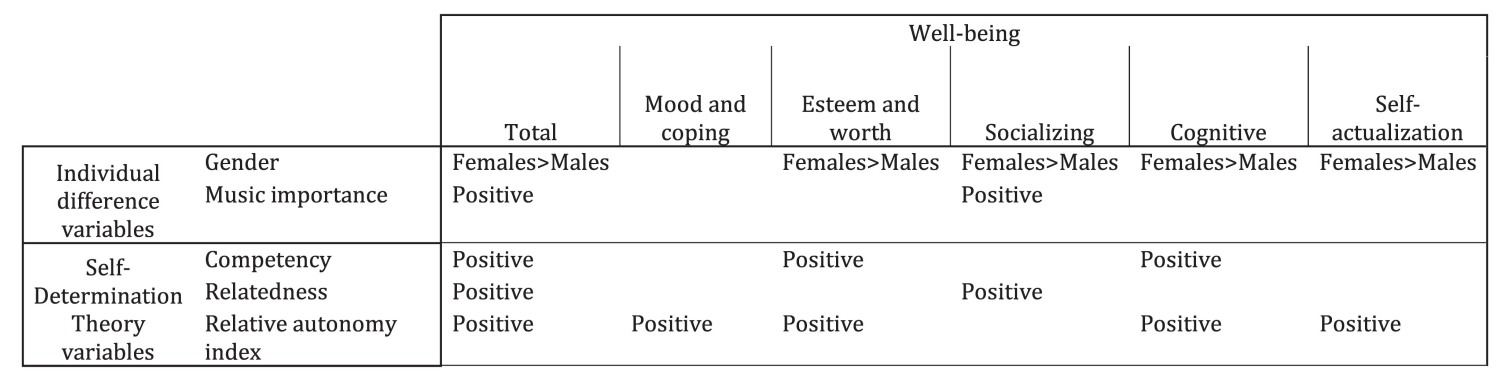

FIGURE 1 | Summary of study findings.

As seen in Figure 1, with regard to the three basic psychological needs, competency (defined as the need to be effective in one's efforts) was positively associated with the total, esteem and self-worth, and cognitive well-being sub-scale scores. Relatedness (the need to be socially connected) was positively associated with the total and socializing well-being sub-scale score. These findings demonstrate logical associations between those two types of psychological needs and the wellbeing types: while obviously not indicative of a causal effect, these findings indicate, within the context of specifically musical participation, a relationship between feeling related to other people and experiencing social well-being benefits, as has been reported in much research (e.g., von Lob et al., 2010; Jutras, 2011; Rohwer and Rohwer, 2012; Creech et al., 2013b; McQueen et al., 2013; Krause et al., 2018); and the positive relationship between competency and various aspects of both social and cognitive well-being supports prior research that also demonstrates links between musical participation and cognitive well-being (e.g., Gick, 2011; Kokotsaki and Hallam, 2011; Creech et al., 2013a). Indeed, relatedness was particularly important in Quested et al.'s (2018) community gardening investigation, as well as Sebire et al.'s (2016) dance research. Autonomy (the need to feel that one's activities are self-endorsed and volitional) did not demonstrate any significant associations with well-being: the absence of significant findings concerning autonomy per se is interesting, given that previous research findings have linked autonomy to motivation and engagement (e.g., Williams and Deci, 1998; Reeve et al., 2004; Valenzuela et al., 2018), as well as well-being (e.g., Ilardi et al., 1993).

Additionally, in the full models (Tables 2-7), the specific nature of musical participation (i.e., instrument versus singing versus facilitation) was not associated with well-being. It is also slightly surprising, but nonetheless encouraging, that wellbeing correlates of musical participation were not related to length of time and frequency of participating when considering overt measures of competence, relatedness, and autonomy as well. The lack of any such associations can be interpreted positively: experiencing well-being benefits in the context 
of musical participation does not appear to hinge on the particulars of the musical activity itself. Rather, people can select musical activities aligned with their personal preferences and which fit within their lifestyles without implications for their probability of experiencing greater well-being. Of course, it would not be fruitful to simply force people to participate in musical activities (e.g., at school, via private lessons, or in community spaces) without striving to also increase feelings of competence, relatedness, and autonomy. Our results suggest that self-determination is considerably more important than simple attendance. Similarly, the only demographic variable related to well-being was gender, so that age and education level are also unrelated to the relationship between musical participation and well-being. Thus, these findings have broad implications for music educators, community musicians, music therapists, and others who are involved in facilitating participation opportunities.

In particular, rather than the activity itself or individual differences (such as age and education level), the present findings suggest that it is competence, relatedness, and autonomy (expressed in terms of the RAI) that predict well-being in the context of musical participation, as predicted by self-determination theory. Indeed, the present findings (as summarized in Figure 1) suggest that musical participation opportunities should be interesting, challenging, and offered in contexts that support autonomous motivation. Clearly then, the challenge for music educators, community musicians, and music therapists that arises from these results concerns specifically how they might foster self-perceptions of competence, relatedness, and autonomy via a number of specific and more general approaches to musical participation.

Some of the arguments here also suggest interesting directions for future research. The role of the facilitator (i.e., the person who leads the activity) was not examined here, which is particularly unfortunate given that he/she may have an important role to play in shaping competence, relatedness, and autonomy. In the context of specifically musical participation, and particularly music education, the role of the facilitator is clearly crucial (e.g., Delano and Royse, 1987; Corenblum and Marshall, 1998; Evans et al., 2013). There exists, of course, an extensive literature from outside music that specifically addresses the form that these approaches might take (e.g., Gagné and Deci, 2005; Jang et al., 2010; Hagger et al., 2015). Facilitators can draw on the findings indicating how, in educational settings, autonomy supportive teachers positively influence students' psychological needs, motivation, and engagement (e.g., Niemiec and Ryan, 2009; Jang et al., 2010; Hagger et al., 2015). Specific to music education, Evans (2015) offered suggestions as to pedagogical strategies to promote the fulfillment of the psychological needs. It will be important for future research to empirically consider which particular pedagogical techniques are perceived by students as needs-supporting and needs-thwarting.

Further, longitudinal research could also consider these ideas with emphasis on continuation versus cessation of participation. For example, such work might consider associations between the fulfillment of psychological needs and the issues surrounding continued participation, such as potential barriers. It may be that musical participation occupies varying levels of importance at different points in someone's life. Participation must 'fit' within a person's lifestyle: examining the issues related to age and life-stage may illuminate how some people are able to prioritize/continue/cease their musical participation.

Additionally, while Self-Determination Theory has been applied to many phenomena involving motivation across multiple domains, its application to musical behaviors is nascent. Thus, the present research makes a novel contribution to knowledge by providing empirical evidence that supports the application of Self-Determination Theory to explain musical participation. However, the exploratory nature of this research should be noted. Thus, additional research is needed to refine its application as well as further explore particular aspects of well-being. For instance, future research could consider specifically eudemonic well-being with regard to the motivations and functions of continued participation (Groarke and Hogan, 2016).

In summary, the present study used Self-Determination Theory to examine musical participation and well-being. The pattern of results reiterates the positive associations between musical participation and benefits to one's emotional and social well-being; and makes clear that that feeling competent, a sense of relatedness to others, and autonomous motivation should be prioritized in music making opportunities. The findings indicate that Self-Determination Theory (including the mini theories of Basic Psychological Needs and Organismic Integration Theory in particular) offers a useful theoretical framework to understanding musical participation with regard to well-being.

\section{DATA AVAILABILITY}

The datasets for this study will not be made publicly available because the ethics permissions require that the data be kept and destroyed.

\section{ETHICS STATEMENT}

This project received ethical approval from Curtin University.

\section{AUTHOR CONTRIBUTIONS}

AK, JD, and AN jointly developed the conceptual and methodological approach and co-wrote the discussion. AK oversaw the ethics, participant recruitment, data collection, and developed the literature review and sketched the shape of the final article. AK and AN undertook the data analysis.

\section{FUNDING}

The funding for this work was obtained by JD. This research was funded by the Australian Research Council, Discovery Project, DP 140102679. 


\section{REFERENCES}

Alivernini, F., and Lucidi, F. (2011). Relationship between social context, selfefficacy, motivation, academic achievement, and intention to drop out of high school: a longitudinal study. J. Educ. Res. 104, 241-252. doi: 10.1080/ 00220671003728062

Arrogi, A., Schotte, A., Bogaerts, A., Boen, F., and Seghers, J. (2017). Shortand long-term effectiveness of a three-month individualized need- supportive physical activity counseling intervention at the workplace. BMC Publ. Health 17:52. doi: 10.1186/s12889-016-3965-1

Baard, P. P., Deci, E. L., and Ryan, R. M. (2004). Intrinsic need satisfaction: a motivational basis of performance and well-being in two work settings. J. Appl. Soc. Psychol. 34, 2045-2068. doi: 10.1111/j.1559-1816.2004.tb02690.x

Black, A. E., and Deci, E. L. (2000). The effects of instructors' autonomy support and students' autonomous motivation on learning organic chemistry: a selfdetermination theory perspective. Sci. Educ. 84, 740-756. doi: 10.1002/1098237X(200011)84:6<740::AID-SCE4>3.0.CO;2-3

Bonneville-Roussy, A., Evans, P., Verner-Filion, J., Vallerand, R. J., and Bouffard, T. (2017). Motivation and coping with the stress of assessment: gender differences in outcomes for university students. Contemp. Educ. Psychol. 48, 28-42. doi: 10.1016/j.cedpsych.2016.08.003

Bonneville-Roussy, A., Vallerand, R. J., and Bouffard, T. (2013). The roles of autonomy support and harmonious and obsessive passions in educational persistence. Learn. Individ. Diff. 24, 22-31. doi: 10.1016/j.lindif.2012.12.015

Chatzisarantis, N. L. D., and Hagger, M. S. (2009). Effects of an intervention based on self-determination theory on self-reported leisure-time physical activity participation. Psychol. Health 24, 29-48. doi: 10.1080/08870440701809533

Clift, S., and Hancox, G. (2010). The significance of choral singing for sustaining psychological wellbeing: Findings from a survey of choristers in england. Australia and Germany. Music Perform. Res. 3, 79-96.

Clift, S., Hancox, G., Staricoff, R., and Whitmore, C. (2008). Singing and Health: Summary of A Systematic Mapping and Review of Non-Clinical Research. Canterbury: Canterbury Christ Church University.

Coleman, D., and Iso-Ahola, S. E. (1993). Leisure and health: The role of social support and self-determination. J. Leis. Res. 25, 111-128. doi: 10.1080/ 00222216.1993.11969913

Corenblum, B., and Marshall, E. (1998). The band played on: predicting students' intentions to continue studying music. J. Res. Music Educ. 46, 128-140. doi: $10.2307 / 3345765$

Countryman, J. (2014). Missteps, flaws, and morphings in children's musical play: snapshots from school playgrounds. Res. Stud. Music Educ. 36, 3-18. doi: 10. 1177/1321103X14528456

Creech, A., Hallam, S., Gaunt, H., McQueen, H., Pincas, A., and Varvarigou, M. (2013a). The power of music in the lives of older adults. Res. Stud. Music Educ. 35, 87-102. doi: 10.1177/1321103X13478862

Creech, A., Hallam, S., Varvarigou, M., McQueen, H., and Gaunt, H. (2013b). Active music making: a route to enhanced subjective well-being among older people. Pers. Publ. Health 133, 36-43. doi: 10.1177/1757913912466950

Deci, E. L., and Ryan, R. M. (1985a). Intrinsic Motivation and Self-Determination in Human Behavior: Perspectives in Social Psychology. New York, NY: Plenum. doi: 10.1007/978-1-4899-2271-7

Deci, E. L., and Ryan, R. M. (1985b). The general causality orientations scale: self-determination in personality. J. Res. Pers. 19, 109-134. doi: 10.1016/00926566(85)90023-6

Deci, E. L., and Ryan, R. M. (1987). The support of autonomy and the control of behavior. J. Pers. Soc. Psychol. 53, 1024-1037. doi: 10.1037/0022-3514.53.6.1024

Deci, E. L., and Ryan, R. M. (1991). "A motivational approach to self: Integration in personality," in Current Theory and Research in Motivation, Nebraska Symposium on Motivation, 1990: Perspectives on Motivation, ed. R. A. Dienstbier (Lincoln, NE: University of Nebraska Press).

Deci, E. L., and Ryan, R. M. (2000). The "what" and "why" of goal pursuits: human needs and the self-determination of behavior. Psychol. Inq. 11, 227-268. doi: 10.1207/S15327965PLI1104_01

Deci, E. L., and Ryan, R. M. (2008). Facilitating optimal motivation and psychological well-being across life's domains. Can. Psychol. 49, 14-23. doi: 10.1037/0708-5591.49.1.14

Deci, E. L., Ryan, R. M., Gagné, M., Leone, D. R., Usunov, J., and Kornazheva, B. P. (2001). Need satisfaction, motivation, and well-being in the work organizations of a former eastern bloc country. Pers. Soc. Psychol. Bull. 27, 930-942. doi: 10.1177/0146167201278002

Delano, A., and Royse, D. (1987). Factors influencing the decision of college freshmen to participate or not to participate in kent state university music ensembles. Contrib. Music Educ. 14, 9-18.

Douglas, K. A. (2011). A Descriptive Analysis of The Psychological Needs of Adults Participating in Music Ensembles: A Survey of the New Horizons International Music Association Ensemble Participants (Doctoral Dissertation). Greensboro: The University of North Carolina.

Evans, P. (2015). Self-determination theory: An approach to motivation in music education. Music Sci. 19, 65-83. doi: 10.1177/1029864914568044

Evans, P., and Bonneville-Roussy, A. (2016). Self-determined motivation for practice in university music students. Psychol. Music 44, 1095-1110. doi: 10. 1177/0305735615610926

Evans, P., McPherson, G. E., and Davidson, J. W. (2013). The role of psychological needs in ceasing music and music learning activities. Psychol. Music 41, 600619. doi: 10.1177/0305735612441736

Ferrand, C., Martinent, G., and Durmaz, N. (2014). Psychological need satisfaction and well-being in adults aged 80 years and older living in residential homes: using a self-determination theory perspective. J. Aging Stud. 30, 104-111. doi: 10.1016/j.jaging.2014.04.004

Freer, E., and Evans, P. (2018). Psychological needs satisfaction and value in students' intentions to study music in high school. Psychol. Music 46, 881-895. doi: 10.1177/0305735617731613

Gagné, M., and Deci, E. L. (2005). Self-determination theory and work motivation. J. Organ. Behav. 26, 331-362. doi: 10.1002/job.322

Georgiadis, M. M., Biddle, S. J. H., and Stavrou, N. A. (2006). Motivation for weight-loss diets: a clustering, longitudinal field study using self-esteem and self-determination theory perspectives. Health Educ. J. 65, 53-72. doi: 10.1177/ 0017896906066067

Gick, M. L. (2011). Singing, health and well being: a health psychologist's review. Psychomusicology 21, 176-207. doi: 10.1037/h0094011

Groarke, J. M., and Hogan, M. J. (2016). Enhancing wellbeing: an emerging model of the adaptive functions of music listening. Psychol. Music 44, 769-791. doi: $10.1177 / 0305735615591844$

Hagger, M. S., and Chatzisarantis, N. L. D. (2008). Self-determination theory and the psychology of exercise. Int. Rev. Sport Exer. Psychol. 1, 79-103. doi: 10.1080/ 17509840701827437

Hagger, M. S., Chatzisarantis, N. L. D., and Harris, J. (2006). From psychological need satisfaction to intentional behavior: testing a motivational sequence in two behavioral contexts. PSPB 32, 131-148. doi: 10.1177/0146167205279905

Hagger, M. S., Sultan, S., Hardcastle, S. J., and Chatzisarantis, N. L. D. (2015). Perceived autonomy support and autonomous motivation toward mathematics activities in educational and out-of-school contexts is related to mathematics homework behavior and attainment. Contemp. Educ. Psychol. 41, 111-123. doi: 10.1016/j.cedpsych.2014.12.002

Ilardi, B. C., Leone, D., Kasser, T., and Ryan, R. M. (1993). Employee and supervisor ratings of motivation: main effects and discrepancies associated with job satisfaction and adjustment in a factory setting. J. Appl. Soc. Psychol. 23, 1789-1805. doi: 10.1111/j.1559-1816.1993.tb01066.x

Jang, H., Kim, E. J., and Reeve, J. (2012). Longitudinal test of self-determination theory's motivation mediation model in a naturally occurring classroom context. J. Educ. Psychol. 104, 1175-1188. doi: 10.1037/a0028089

Jang, H., Reeve, J., and Deci, E. L. (2010). Engaging students in learning activities: It is not autonomy support or structure, but autonomy support and structure. J. Educ. Psychol. 102, 588-600. doi: 10.1037/a0019682

Jutras, P. J. (2011). The benefits of new horizons band participation as self-reported by selected new horizons band members. Bull. Council Res. Music Educ. 187, 65-84.

Kokotsaki, D., and Hallam, S. (2011). The perceived benefits of participative music making for non-music university students: a comparison with music students. Music Educ. Res. 13, 149-172. doi: 10.1080/14613808.2011.577768

Krause, A. E., and Davidson, J. W. (2018). Effective educational strategies to promote life-long musical investment: perceptions of educators. Front. Psychol. 9:1977. doi: 10.3389/fpsyg.2018.01977

Krause, A. E., Davidson, J. W., and North, A. C. (2018). Musical activity and wellbeing: a new quantitative measurement instrument. Music Perc. 35, 454-474. doi: $10.1525 / \mathrm{mp} \cdot 2018.35 .4 .454$ 
Küpers, E., van Dijk, M., McPherson, G. E., and van Geert, P. (2014). A dynamic model that links skill acquisition with self-determination in instrumental music lessons. Music. Sci. 18, 17-34. doi: 10.1177/1029864913499181

Kuykendall, L., Tay, L., and Ng, V. (2015). Leisure engagement and subjective well-being: a meta-analysis. Psychol. Bull. 141, 364-403. doi: 10.1037/a0038508

Laukka, P. (2007). Uses of music and psychological well-being among the elderly. J. Happ. Stud. 8, 215-341. doi: 10.1007/s10902-006-9024-3

Lee, J., Davidson, J. W., and McFerran, K. S. (2016). Registered music therapists' motivations and perceptions of the impact of their practices on the well-being of clients and themselves. Aus. J. Music Ther. 27, 27-43.

Lombas, A. S., and Esteban, M. Á (2018). The confounding role of basic needs satisfaction between self-determined motivation and well-being. J. Happ. Stud. 19, 1305-1327. doi: 10.1007/s10902-017-9874-x

Lovell, G. P., Gordon, J. A. R., Mueller, M. B., Mulgrew, K., and Sharman, R. (2016). Satisfaction of basic psychological needs, self-determined exercise motivation, and psychological well-being in mothers exercising in group-based versus individual-based contexts. Health Care Women Int. 37, 568-582. doi: 10.1080/ 07399332.2015.1078333

MacDonald, R. A. R. (2013). Music, health, and well-being: A review. Int. J. Qual. Stud. Health Well-Being 8:20635. doi: 10.3402/qhw.v8i0.20635

MacDonald, R. A. R., Kreutz, G., and Mitchell, L. A. (2012). "What is music, health, and wellbeing and why is it important?" in Music, Health, and Wellbeing, eds R. A. R. MacDonald, G. Kreutz, and L. A. Mitchell (Oxford, UK: Oxford University Press), 3-11.

MacIntyre, P. D., Schnare, B., and Ross, J. (2018). Self-determination theory and motivation for music. Psychol. Music 46, 699-715. doi: 10.1177/ 0305735617721637

Markland, D., and Tobin, V. (2004). A modification to the behavioural regulation in exercise questionnaire to include an assessment of amotivation. J. Sport Exer. Psychol. 26, 191-196. doi: 10.1123/jsep.26.2.191

McLachlan, S., Spray, C., and Hagger, M. S. (2011). The development of a scale measuring integrated regulation in exercise. Br. J. Health Psychol. 16, 722-743. doi: 10.1348/2044-8287.002009

McPherson, G. E., and O'Neill, S. A. (2016). "Musical potential," in The Oxford Handbook of Music Psychology, eds S. Hallam, I. Cross, and M. Thaut (Oxford, UK: Oxford University Press), 433-448.

McQueen, H., Hallam, S., Creech, A., and Varvarigou, M. (2013). A philosophical perspective on leading music activities for the over 50s. Int. J. Lifelong Educ. 32, 353-377. doi: 10.1080/02601370.2012.738432

Milyavskaya, M., and Koestner, R. (2011). Psychological needs, motivation, and well-being: a test of self-determination theory across multiple domains. Pers. Individ. Diff. 50, 387-391. doi: 10.1016/j.paid.2010.10.029

Niemiec, C. P., Lynch, M. F., Vansteenkiste, M., Bernstein, J., Deci, E. L., and Ryan, R. M. (2006). The antecedents and consequences of autonomous self-regulation for college: a self-determination theory perspective on socialization. J. Adolesc. 29, 761-775. doi: 10.1016/j.adolescence.2005.11.009

Niemiec, C. P., and Ryan, R. M. (2009). Autonomy, competence, and relatedness in the classroom: applying self-determination theory to educational practice. Theory Res. Educ. 7, 133-144. doi: 10.1177/1477878509104318

Niven, A. G., and Markland, D. (2016). Using self-determination theory to understand motivation for walking: instrument development and model testing using Bayesian structural equation modeling. Psychol. Sport Exer. 23, 90-100. doi: 10.1016/j.psychsport.2015.11.004

Ntoumanis, N., Thøgersen-Ntoumani, C., Quested, E., and Hancox, J. (2017). The effects of training group exercise class instructors to adopt a motivationally adaptive communication style. Scand. J. Med. Sci. Sports 27, 1026-1034. doi: $10.1111 / \mathrm{sms} .12713$

Oga-Baldwin, W. L. Q., Nakata, Y., Parker, P., and Ryan, R. M. (2017). Motivating young language learners: a longitudinal model of self-determined motivation in elementary school foreign language classes. Contemp. Educ. Psychol. 49, 140-150. doi: 10.1016/j.cedpsych.2017.01.010

O’Neill, S. A., and McPherson, G. E. (2002). "Motivation," in The Science \& Psychology of Music Performance: Creative Strategies for Teaching and Learning, eds R. Parncutt and G. E. McPherson (Oxford, UK: Oxford University Press), 31-46. doi: 10.1093/acprof:oso/9780195138108.003.0003

Patrick, H., Canevello, A., Knee, C. R., and Lonsbary, C. (2007). The role of need fulfillment in relationship functioning and well-being: a self-determination theory perspective. J. Pers. Soc. Psychol. 92, 434-457. doi: 10.1037/0022-3514. 92.3.434
Quested, E., Thøgersen-Ntoumani, C., Uren, H., Hardcastle, S. J., and Ryan, R. M. (2018). Community gardening: basic psychological needs as mechanisms to enhance individual and community well-being. Ecopsychology 10, 173-180. doi: 10.1089/eco.2018.0002

Reeve, J., Jang, H., Carrell, D., Jeon, S., and Barch, J. (2004). Enhancing students' engagement by increasing teachers' autonomy support. Motiv. Emot. 28, 147169. doi: 10.1023/B:MOEM.0000032312.95499.6f

Reinboth, M., and Duda, J. L. (2006). Perceived motivational climate, need satisfaction and indices of well-being in team sports: a longitudinal perspective. Psychol. Sport Exer. 7, 269-286. doi: 10.1016/j.psychsport.2005. 06.002

Roberts, J. C. (2018). Self-determination theory and children's singing games in and out of the classroom: a literature review. Update 36, 12-19. doi: 10.1177/ 8755123317741488

Rohwer, D., and Rohwer, M. (2012). How participants envision community music in welsh men's choirs. Res. Issues Music Educ. 10:3. Available at: http://ir. sthomas.edu/rime/vol10/iss $1 / 3$

Ryan, R. M., and Connell, J. P. (1989). Perceived locus of causality and internalization: examining reasons for acting in two domains. J. Pers. Soc. Psychol. 57, 749-761. doi: 10.1037/0022-3514.57.5.749

Ryan, R. M., and Deci, E. L. (2000). Self-determination theory and the facilitation of intrinsic motivation, social development, and well-being. Am. Psychol. 55, 68-78. doi: 10.1037/0003-066X.55.1.68

Ryan, R. M., and Deci, E. L. (2002). "An overview of Self-determination Theory: An organismic-dialectical perspective," in Handbook of Self-Determination Research, eds E. L. Deci and R. M. Ryan (Rochester, NY: The University of Rochester Press).

Ryan, R. M., and Deci, E. L. (2007). "Active human nature: Self-determination theory and the promotion and maintenance of sport, exercise, and health," in Intrinsic Motivation And Self-Determination in Exercise and Sport, eds M. S. Hagger and N. L. D. Chatzisarantis (Champaign, IL: Human Kinetics), 1-20.

Schatt, M. D. (2018). Middle school band students' self-determination to practice. Psychol. Music 46, 208-221. doi: 10.1177/0305735617705008

Sebire, S. J., Kesten, J. M., Edwards, M. J., May, T., Banfield, K., Tomkinson, K., et al. (2016). Using self-determination theory to promote adolescent girls' physical activity: exploring the theoretical fidelity of the bristol girls dance project. Psychol. Sport Exer. 24, 100-110. doi: 10.1016/j.psychsport.2016.01.009

Seymour, G., and Peterman, A. (2018). Context and measurement: an analysis of the relationship between intrahousehold decision making and autonomy. World Dev. 111, 97-112. doi: 10.1016/j.worlddev.2018.06.027

Sheldon, K. M., and Krieger, L. S. (2007). Understanding the negative effects of legal education on law students: a longitudinal test of self-determination theory. Pers. Soc. Psychol. Bull. 33, 883-897. doi: 10.1177/0146167207301014

Skingley, A., Bungay, H., and Clift, S. (2011). Researching participatory arts, wellbeing and health: some methodological issues. J. Arts Commun. 3, 73-87. doi: 10.1386/jaac.3.1.73_1

Soenens, B., Sierens, E., Vansteenkiste, M., Dochy, F., and Goossens, L. (2012). Psychologically controlling teaching: Examining outcomes, antecedents, and mediators. J. Educ. Psychol.104, 108-120. doi: 10.1037/a0025742

Teques, P., Calmeiro, L., Silva, C., and Borrego, C. (2017). Validation and adaptation of the physical activity enjoyment scale (PACES) in fitness group exercisers. J. Sport Health Sci. 1-6. doi: 10.1016/j.jshs.2017.09.010

Ulstad, S. O., Halvari, H., and Deci, E. L. (2018). The role of students' and teachers' ratings of autonomous motivation in a self-determination theory model predicting participation in physical education. Scand. J. Educ. Res. 1-16. doi: 10.1080/00313831.2018.1476917

Valenzuela, R., Codina, N., and Pestana, J. V. (2018). Self-determination theory applied to flow in conservatoire music practice: the roles of perceived autonomy and competence, and autonomous and controlled motivation. Psychol. Music 46, 33-48. doi: 10.1177/0305735617694502

Vallerand, R. J., Pelletier, L. G., and Koestner, R. (2008). Reflections on selfdetermination theory. Can. Psychol. 49, 257-262. doi: 10.1037/a0012804

Van den Broeck, A., Ferris, D. L., Chang, C.-H., and Rosen, C. C. (2016). A review of self-determination theory's basic psychological needs at work. J. Manag. 42, 1195-1229. doi: 10.1177/0149206316632058

Vansteenkiste, M., Simons, J., Lens, W., Sheldon, K. M., and Deci, E. L. (2004). Motivating learning, performance, and persistence: the synergistic effects of intrinsic goal contents and autonomy-supportive contexts. J. Pers. Soc. Psychol. 87, 246-260. doi: 10.1037/0022-3514.87.2.246 
Vlachopoulos, S. P., Ntoumanis, N., and Smith, A. L. (2010). The basic psychological needs in exercise scale: translation and evidence for cross-cultural validity. Int. J. Sport Exer. Psychol. 8, 394-412. doi: 10.1080/1612197X.2010. 9671960

von Lob, G., Camic, P., and Clift, S. (2010). The use of singing in a group as a response to adverse life events. Int. J. Ment. Health Prom. 12, 45-53. doi: $10.1080 / 14623730.2010 .9721818$

Williams, G. C., and Deci, E. L. (1998). The importance of supporting autonomy in medical education. Ann. Inter. Med. 129, 303-308. doi: 10.7326/0003-4819129-4-199808150-00007

Wilson, P. M., Mack, D. E., and Grattan, K. P. (2008). Understanding motivation for exercise: a self-determination theory perspective. Can. Psychol. 49, 250-256. doi: 10.1037/a0012762

Wilson, P. M., Rodgers, W. M., Loitz, C. C., and Scime, G. (2006). It's who i am really! the importance of integrated regulation in exercise contexts. J. Appl. Biobehav. Res. 11, 79-104. doi: 10.1111/j.1751-9861.2006.tb00021.x
Wilson, P. M., Sabiston, C. M., Mack, D. E., and Blanchard, C. M. (2012). On the nature and function of scoring protocols used in exercise motivation research: an empirical study of the behavioral regulation in exercise questionnaire. Psychol. Sport Exer. 13, 614-622. doi: 10.1016/j.psychsport.2012. 03.009

Conflict of Interest Statement: The authors declare that the research was conducted in the absence of any commercial or financial relationships that could be construed as a potential conflict of interest.

Copyright (c) 2019 Krause, North and Davidson. This is an open-access article distributed under the terms of the Creative Commons Attribution License (CC BY). The use, distribution or reproduction in other forums is permitted, provided the original author(s) and the copyright owner(s) are credited and that the original publication in this journal is cited, in accordance with accepted academic practice. No use, distribution or reproduction is permitted which does not comply with these terms. 
OPEN ACCESS

Edited by: Michele Biasutti, University of Padova, Italy

Reviewed by: Catherine Alexandra Lebel, University of Calgary, Canada

Floris Tijmen Van Vugt, McGill University, Canada

*Correspondence: Psyche Lou p.loui@northeastern.edu

Specialty section: This article was submitted to Performance Science, a section of the journa Frontiers in Psychology

Received: 30 January 2019 Accepted: 07 May 2019

Published: 24 May 2019

Citation:

Loui P, Raine LB, Chaddock-Heyman L, Kramer AF and Hillman CH (2019)

Musical Instrument Practice Predicts White Matter Microstructure and Cognitive Abilities in Childhood.

Front. Psychol. 10:1198. doi: 10.3389/fpsyg.2019.01198

\section{Musical Instrument Practice Predicts White Matter Microstructure and Cognitive Abilities in Childhood}

\author{
Psyche Loui ${ }^{* *}$, Lauren B. Raine', Laura Chaddock-Heyman ${ }^{2}$, Arthur F. Kramer ${ }^{1}$ and \\ Charles H. Hillman ${ }^{1}$
}

'Department of Music, Department of Psychology, Northeastern University, Boston, MA, United States, ${ }^{2}$ Beckman Institute, University of Illinois, Champaign, IL, United States

Musical training has been associated with advantages in cognitive measures of IQ and verbal ability, as well as neural measures including white matter microstructural properties in the corpus callosum (CC) and the superior longitudinal fasciculus (SLF). We hypothesized that children who have musical training will have different microstructural properties in the SLF and CC. One hundred children aged 7.9-9.9 years (mean age 8.7) were surveyed for their musical activities, completed neuropsychological testing for general cognitive abilities, and underwent diffusion tensor imaging (DTI) as part of a larger study. Children who play a musical instrument for more than $0.5 \mathrm{~h}$ per week $(n=34)$ had higher scores on verbal ability and intellectual ability (standardized scores from the Woodcock-Johnson Tests of Cognitive Abilities), as well as higher axial diffusivity (AD) in the left SLF than those who did not play a musical instrument $(n=66)$. Furthermore, the intensity of musical practice, quantified as the number of hours of music practice per week, was correlated with axial diffusivity (AD) in the left SLF. Results are not explained by age, sex, socioeconomic status, or physical fitness of the participants. The results suggest that the relationship between musical practice and intellectual ability is related to the maturation of white matter pathways in the auditory-motor system. The findings suggest that musical training may be a means of improving cognitive and brain health during development.

Keywords: music, language, cognition, neuroimaging, brain structure, intelligence

\section{INTRODUCTION}

The impact of music training on human brain and cognitive development has been a topic of intense interest in recent years (Kraus and Chandrasekaran, 2010). Music perception skills in children are correlated with performance on phonological awareness and reading tests (Lamb and Gregory, 1993; Anvari et al., 2002), as well as on general performance on IQ tests (Lynn et al., 1989). Children who take music lessons outperform their musically untrained counterparts in tests of verbal memory and reading ability (Hurwitz et al., 1975; Ho et al., 2003). Children who initially perform below the mean in academic achievement tests, after a year of musical training, catch up with their musically untrained counterparts in academic achievement (Gardiner et al., 1996). While these results suggest a relationship between music training and cognitive abilities, the direction of causality is unclear. 
Randomized controlled trials provide a stronger test of the direction of causality, but their results are more mixed. Positive evidence comes from a randomized controlled trial in 144 six-year-olds, comparing 36 weeks of lessons in keyboard, voice, and drama against no-training controls, which found that children in the music groups exhibited greater increases in full-scale IQ than no-training controls (Schellenberg, 2004). Another randomized controlled trial comparing children in music and visual art training showed that children in the music group performed better on verbal intelligence measures after only 20 days of training, compared to no changes in the visual art group (Moreno et al., 2011). Furthermore, children in a school-based music program with weekly 45-min instrumental lessons showed greater improvements in verbal memory than children in science-education and no-training control groups, even after controlling for socio-economic status (SES), age, and IQ; in contrast, no differences between groups were found in the visual memory tests (Roden et al., 2012). Another longitudinal randomized controlled trial showed that children who underwent instrumental music training outperformed visual art training and no-intervention controls on neuropsychological tasks of inhibition, planning, and verbal IQ (Jaschke et al., 2018). In a further mediation analysis, the authors showed that performance on these neuropsychological tasks explained music training-related increases in standardized academic achievement scores, suggesting a far transfer effect from music education to academic achievement mediated by executive functions (Jaschke et al., 2018).

On the other hand, there are also training studies that have not observed transfer effects from musical training to non-musical cognitive tasks including receptive vocabulary, numerical discrimination, visual form analysis, and spatial navigation (Mehr et al., 2013). A meta-analysis of the effects of music training on children's cognitive and academic skills showed small effect sizes in transfer to intelligence, memory, mathematics, phonological processing, and spatial processing, with effect sizes being affected by methodological considerations: smaller effect sizes were generally observed in studies with randomized designs and in studies that compared music training against active control groups (Sala and Gobet, 2017). However, this meta-analysis did not take into account the intensity of musical training, which strongly influences the relationship between music training and IQ (Schellenberg, 2006). A fuller understanding of the relationship between music training and cognition may come from including information about the intensity of musical practice, as well as characterization of the underlying neural mechanisms.

Functional neuroimaging studies have examined the mechanisms underlying musical training effects on verbal and executive function tests in children. Musically trained children perform better on behavioral measures of verbal fluency using timed neuropsychological tests and fMRI measures during executive function tests such as task switching and rule representation (Zuk et al., 2014). Children with musical training and children with physical activity training both showed a stronger Stroop effect than no-training controls, coupled with more activity in the inferior frontal gyrus, supplementary motor area, and anterior cingulate cortex in the music group compared to the no-treatment group, with the physical activity group falling in between the music and no-treatment groups (Sachs et al., 2017). The results suggest that music training may transfer to executive functions by acting on the auditory-motor and cognitive control networks in the brain.

Longitudinal as well as cross-sectional studies have identified a network of areas associated with music training in auditorymotor regions that are shared with other functions such as language and dance (Sluming et al., 2002; Bermudez et al., 2009; Hyde et al., 2009; Karpati et al., 2017). A longitudinal study found that cumulative hours of music practice in children and adults was correlated with functional activation in the left supramarginal gyrus, part of the auditory-motor network, during music listening (Ellis et al., 2013). Early onset of musical training in childhood is associated with larger gray matter volume and higher cortical surface area in auditorymotor areas including the superior temporal lobe and inferior frontal lobe (Bailey et al., 2014) as well as increased fractional anisotropy (FA) in the temporal lobe and the corpus callosum (Steele et al., 2013).

A prominent white matter pathway that connects the temporal lobe and the frontal lobe is the superior longitudinal fasciculus (SLF), which includes the arcuate fasciculus. Microstructural properties of the SLF are associated with reading skills in children (Yeatman et al., 2011; Saygin et al., 2013). Fractional Anisotropy arcuate fasciculus is higher among people who excel at learning languages and grammatical structures (Floel et al., 2009; Qi et al., 2015) as well as new musical structures (Loui et al., 2011; Vaquero et al., 2018). People with congenital amusia, who have difficulty perceiving and producing pitch and melody, show reduced connectivity in the arcuate fasciculus (Loui et al., 2009), and diffusion properties in the arcuate and other frontal white matter pathways predict recovery from acquired amusia for stroke patients (Sihvonen et al., 2016, 2017). People with music training have shown both increases and decreases in FA and volume in the SLF (Oechslin et al., 2010; Halwani et al., 2011), as supported by cross-sectional comparisons and randomized training studies (Moore et al., 2017). FA of the SLF is also related to language ability and exposure (Romeo et al., 2018), as well as to age (Lebel et al., 2008; Krogsrud et al., 2016) and socio-economic status (Gullick et al., 2016).

In addition to the SLF, the corpus callosum (CC) is a white matter pathway that has shown differences as a result of early musical training. The anterior half of the CC is larger in musically trained adults, especially in those who started musical training before the age of 7 (Schlaug et al., 1995). The age of onset of musical training is correlated with microstructural properties of the CC, with musicians who began training earlier showing higher FA and lower radial diffusivity (RD) especially in the midpoint of the CC (Steele et al., 2013). More specific evidence for the effect of early musical training comes from a longitudinal study comparing children after 2 years of training in music, in physical activity, and in a no-training control group, which showed highest FA in the CC of the music group, specifically in the crossing pathways connecting superior frontal, sensory, and motor segments (Habibi et al., 2017). 
Taken together, mounting evidence suggests that musical training affects verbal ability, with more limited effects on general intellectual ability, and the underlying neural substrates most likely involve connectivity between frontal and temporal lobe regions and between left and right midline hemispheres, with white matter effects centering around the SLF and the CC.

Studies reviewed thus far have controlled for multiple possible sources of confounds in assessing the effects of musical training on brain and cognitive development. All of the studies reviewed herein controlled for age, sex, and socioeconomic status, and most also controlled or specifically examined the duration, intensity, and/or age of onset of musical training. Interestingly, studies on effects of musical training thus far have not controlled for physical activity. The effects of physical activity on brain and cognitive function have become increasingly clear in recent years (Hillman et al., 2008; Khan and Hillman, 2014). Aerobic fitness is specifically related to executive control, with more fit individuals showing stronger neural and cognitive indices of attention and executive function (Kramer et al., 1999; Donnelly et al., 2016). Since music making is a mild form of physical activity, persistent musical practice may require or enhance aerobic fitness, thus moderating the relationship between music training and brain and cognitive measures. Here, we assess the relationship between musical training and standardized measures of verbal ability and general intellectual ability and relate these to diffusion measures of the SLF and CC in a large sample of children, while controlling for possible sources of variability from aerobic fitness as well as age, sex, and socioeconomic status.

\section{MATERIALS AND METHODS}

\section{Subjects}

All behavioral and neuroimaging data from the present sample were collected as part of a larger study on the effects of physical activity on children's cognitive performance and brain structure and function (Chaddock-Heyman et al., 2014, 2018; Hillman et al., 2014). One hundred children aged 7.9-9.9 provided informed assent as approved by the Institutional Review Board (IRB) of the University of Illinois at Urbana-Champaign (UIUC), and their legal guardians provided written informed consent in accordance with the IRB of UIUC. Children were tested in general reading achievement and neuropsychological tests of cognitive ability. The legal guardians also answered simple questions about their musical experience and training, as detailed below.

\section{Stimuli and Procedures Musical Experience Measures}

Musical experience was assessed by a questionnaire to the parents. Questions included:

1. Does your child participate in musical activities? (Yes vs. No)

2. If yes: Does your child play an instrument? (Yes vs. No)

3. If so, what instrument(s)?

4. Does your child participate in choir? (Yes vs. No)

5. How many hours a week does your child spend participating in musical activities? (Numerical response)

\section{Verbal and Intellectual Ability}

Intellectual abilities were assessed using the Woodcock-Johnson Tests of Cognitive Abilities (Woodcock et al., 2001). These included measures of Brief Intellectual Ability (BIA standard score) and Verbal Ability (standard score) (Woodcock et al., 2001; Schrank, 2011). Participants completed the WoodcockJohnson III (WJ III) to assess a range of cognitive abilities. Administration of the WJ III was conducted individually by trained researchers. Various subtests of the WJ III were completed to assess cognitive abilities, and BIA was used to screen for below normal intelligence. A combination of individual subtests was completed to form the Verbal Ability cluster, which can be used for interpretive purposes. The Verbal Ability cluster was computed using the manufacturer's software, which provides measures of standard scores and percentiles. The WJ III is based on a standard score with a mean of 100 and a standard deviation of 15 .

\section{Age and Socio-Economic Status}

Age was recorded as years on the date of participation. SES was scored as three categories: participants received a " 1 " if they receive a free or reduced lunch ${ }^{1}$, if both parents have less than a high school education, or if they live in a one-parent household and that parent has less than a high school education. Participants received a " 3 " if one or both parents work and have a college education. All other participants received a "2." Table 1 shows demographics of participants with and without musical instrument training.

\section{Aerobic Fitness Testing}

As the present data were collected as part of a larger study on the effects of physical activity training, children also completed a test of cardiorespiratory fitness as described in ChaddockHeyman et al. (2018). Cardiorespiratory fitness was measured as maximal oxygen consumption $\left(\mathrm{VO}_{2} \mathrm{max}\right)$ during a graded exercise test, which employed a modified Balke protocol and was administered on a motor-driven treadmill (LifeFitness, Schiller Park, IL). Expired gases were analyzed using a TrueOne2400 Metabolic Measurement System (ParvoMedics, Sandy, Utah). Children walked and/or ran on the treadmill at a constant speed, with increasing grade increments of $2.5 \%$ every $2 \mathrm{~min}$, until volitional exhaustion. Oxygen consumption was measured using a computerized indirect calorimetry system (ParvoMedics True Max 2,400), and averages for $\mathrm{VO}_{2}$ and respiratory exchange ratio (RER) were assessed every $20 \mathrm{~s}$. Heart rate (HR) was measured using a polar HR monitor (Polar WearLink+31; Polar Electro, Finland) throughout the test, and ratings of perceived exertion (RPE) were assessed every 2 min using the children's OMNI scale (Utter et al., 2002). Maximal oxygen consumption was expressed in $\mathrm{ml} / \mathrm{kg} / \mathrm{min}$, and $\mathrm{VO}_{2}$ max was based upon maximal effort which was

${ }^{1}$ Free or reduced lunch is a national meal program that operates in public and nonprofit private schools. Eligibility is based on the national poverty line: students whose family income is at or below $130 \%$ of the poverty line are eligible for free lunch; students whose family income is at or below $180 \%$ of the poverty line are eligible for reduced price lunch. 
TABLE 1 | Demographic variables comparing participants with and without musical instrument training.

\begin{tabular}{|c|c|c|c|}
\hline & \multicolumn{2}{|c|}{ Musical instrument training } & \\
\hline & No & Yes & \\
\hline & Mean (SD) (range) & Mean (SD) (range) & \\
\hline$N$ & 66 & 34 & \\
\hline Age (years) & $8.677(0.5534)(7.9-9.9)$ & $8.733(0.54)(7.9-9.8)$ & $t=0.49, p=0.63$ \\
\hline $\operatorname{Sex}(n)$ & $33^{\circ} \mathrm{F} 33 \mathrm{M}$ & $21^{\circ} \mathrm{F} 13 \mathrm{M}$ & $X^{2}=1.25, p=0.26$ \\
\hline SES (score) & $1.92(0.81)(1-3)$ & $2.15(0.66)(1-3)$ & $t=1.48, p=0.14$ \\
\hline Music practice intensity (h/week) & $0.738(1.33)(0-5)$ & $2.111(1.19)(0.5-6)$ & $t=4.48, p<0.01^{\star}$ \\
\hline WJ III Brief Intellectual Ability (standard score) & $107.7(12.3)(79-132)$ & 117.35 (9.24) (89-133) & $t=4.4, p<0.01^{\star}$ \\
\hline WJ III Verbal Ability (standard score) & 107.68 (11.31) (74-132) & 116.24 (10.6) (85-151) & $t=2.97, p=0.004^{\star}$ \\
\hline $\mathrm{VO}_{2} \max (\mathrm{ml} / \mathrm{kg} / \mathrm{min})$ & 41.9 (7.14) (26.9-57.9) & 44.47 (9.22) (24.4-61.6) & $t=-1.40, p=0.17$ \\
\hline
\end{tabular}

${ }^{*} p<0.05$.

evidenced by four criteria: (1) a plateau in oxygen consumption with an increase of $<2 \mathrm{ml} / \mathrm{kg} / \mathrm{min}$ despite an increase in workload, (2) a peak HR $\geq 185$ beats per minute and a plateau in HR (Freedson and Goodman, 1993), (3) RER $\geq 1.0$ (BarOr, 1983), and/or (4) a rating of $\geq 8$ on the children's OMNI scale of perceived exertion (Utter et al., 2002). Greater $\mathrm{VO}_{2} \max$ reflects superior cardiovascular fitness.

\section{Magnetic Resonance Imaging Acquisition}

Diffusion-weighted images were acquired on a Siemens Magnetom Trio Allegra $3 \mathrm{~T}$ whole-body MRI scanner with a 12-channel receiver head coil, with repetition time $(\mathrm{TR})=4.8 \mathrm{~s}$, echo time $(\mathrm{TE})=100.4 \mathrm{~ms}$, and $3.44 \mathrm{~mm}^{2}$ in-plane resolution with $4 \mathrm{~mm}$ slice thickness. Thirty-two slices were collected parallel to the anterior-posterior commissure plane to obtain wholehead coverage with no gap. One 30-direction diffusion-weighted echo planar imaging scan $\left(b=1,000 \mathrm{~s} / \mathrm{mm}^{2}\right)$ and four T2-weighted b0 images $\left(b=0 \mathrm{~s} / \mathrm{mm}^{2}\right)$ were collected.

\section{Diffusion Data Analysis}

Image analyses were performed using FSL 5.0.1 (FMRIB Software Library) as part of a larger study on the effects of exercise activity on white matter microstructure in children (ChaddockHeyman et al., 2018). Preprocessing of each participant's data consisted of (1) motion and eddy current correction, (2) removal of non-brain tissue using the Brain Extraction Tool (Smith, 2002), and (3) local fitting of the diffusion tensor model at each voxel using FMRIB's Diffusion Toolbox v2.0 (FDT). These steps yielded fractional anisotropy (FA) and first, second, and third eigenvalue (L1, L2, L3) maps. The first eigenvalue map was used as the axial diffusivity (AD) image, whereas the mean of the second and third eigenvalue maps was used as the radial diffusivity (RD) image (Song et al., 2002).

Having obtained whole-brain FA, AD, and RD images, tractbased diffusion maps were defined using TBSS v1.2 [TractBased Spatial Statistics (Smith et al., 2006)]. Each participant's FA map was aligned into the $1 \mathrm{~mm} \times 1 \mathrm{~mm} \times 1 \mathrm{~mm}$ standard Montreal Neurological Institute (MNI152) space via the FMRIB58_FA template using the FMRIB's non-linear registration tool (Andersson et al., 2007a,b), and a mean diffusion image was created. The mean FA image was then thinned to create an average skeleton representing the centers of the tracts shared by all participants, and the skeleton was thresholded at FA $>0.20$. Each participant's aligned FA data were projected onto the skeleton to obtain FA skeleton values for each individual. RD and $\mathrm{AD}$ skeletons for each participant were formed in a similar manner by projecting the $\mathrm{RD}$ and $\mathrm{AD}$ maps onto the mean skeleton.

Tract ROIs were created from the JHU ICBM-DTI-81 white matter labels atlas (Mori and van Zijl, 2007; Wakana et al., 2007 ) in the left and right superior longitudinal fasciculus, as well as the genu, body, and splenium of the corpus callosum. Diffusion values (FA, RD, $\mathrm{AD}$ ) were calculated for each participant within each of the tract ROIs. FA, $\mathrm{AD}$, and $\mathrm{RD}$ values of each tract ROI were exported to SPSS for analysis.

\section{Statistical Analyses}

Our primary behavioral hypothesis was that children who played a musical instrument would show higher scores on verbal ability and intellectual ability as assessed by the WoodcockJohnson tests of cognitive abilities, even after accounting for the possible effects of age, sex, socio-economic status, and fitness. This was assessed using ANCOVAs comparing the standardized scores on verbal ability and intellectual ability between children who do and do not play a musical instrument, while incorporating age, sex SES, and $\mathrm{VO}_{2} \mathrm{max}$ as covariates.

Our primary neuroimaging hypothesis was that children who played a musical instrument would show differences in microstructural properties of the SLF and CC, as indexed by FA, $\mathrm{AD}$, and $\mathrm{RD}$ in these regions, compared to children who did not play a musical instrument. This was assessed using a multivariate general linear model (GLM) with the FA, $\mathrm{AD}$, and $\mathrm{RD}$ of left and right SLF and the genu, body, and splenium of the corpus callosum as dependent variables, playing an instrument as a fixed factor, and age, sex, SES, and aerobic fitness $\left(\mathrm{VO}_{2} \mathrm{max}\right)$ as covariates.

Our secondary hypothesis was that the same microstructural properties identified above would correlate with the intensity of musical practice. This was assessed by partial correlations between the number of hours of music practice per week and diffusion parameters that showed significant between-subject effects in the multivariate analysis above, with age, sex, SES, and $\mathrm{VO}_{2} \max$ as covariates. 


\section{RESULTS}

\section{Behavioral Results}

Participants who were reported as participating in musical activities $(n=47)$ performed significantly higher than their counterparts $(n=53)$ on verbal ability (with musical activities: mean $=114.6, \mathrm{SD}=10.05$; without musical activities: mean $=106.00, \mathrm{SD}=12.38$ ) and intellectual ability (with musical activities: mean $=114.77, \mathrm{SD}=11.56$; without musical activities: mean $=107.16, \mathrm{SD}=11.97)$. A one-way ANCOVA on the dependent variable of verbal ability, with the independent variables of musical activities and the covariates of age, sex, SES, and $\mathrm{VO}_{2}$ max, showed a significant effect of musical activities $\left[F(1,94)=12.56, p=0.001\right.$, partial $\left.\eta^{2}=0.12\right]$ and no significant effect of age, sex, SES, and $\mathrm{VO}_{2} \max$ [age: $F(1,94)=0.083$, $p=0.77$, partial $\eta^{2}=0.001$; sex: $F(1,92)=0.46, p=0.50$, partial $\eta^{2}=0.005$; SES: $F(1,94)=0.036, p=0.85$, partial $\eta^{2}<0.001 ; \mathrm{VO}_{2} \max : F(1,94)=3.72, p=0.057$, partial $\left.\eta^{2}=0.12\right]$. A one-way ANCOVA on the dependent variable of intellectual ability, with the independent variables of musical activities and the same covariates, showed a significant effect of musical activities $\left(F(1,94)=7.42, p=0.008\right.$, partial $\left.\eta^{2}=0.075\right)$, while age sex, SES, and $\mathrm{VO}_{2} \max$ were all not significant [age: $F(1,94)=1.89$, $p=0.17$, partial $\eta^{2}=0.020$; sex: $F(1,94)=2.09, p=0.15$, partial $\eta^{2}=0.022$; SES: $F(1,94)=0.51, p=0.48$, partial $\eta^{2}=0.005$; $\mathrm{VO}_{2} \max : F(1,94)=3.43, p=0.067$, partial $\left.\eta^{2}=0.036\right]$.

Within the group that were reported as participating in musical activities, those who were reported to play a musical instrument $(n=34)$ had a range of $0.5-6 \mathrm{~h}$ of practice per week, with a mean of $1.275(\mathrm{SD}=1.434) \mathrm{h}$ of practice per week. The instruments they play included piano $(n=20)$, violin $(n=5)$, guitar $(n=5)$, recorder $(n=3)$, and drums $(n=2)$. Participants who play a musical instrument scored higher on verbal ability compared to those who reported not playing a musical instrument (instrument players mean $=116$, $\mathrm{SD}=10.67$; non-players mean $=107.63, \mathrm{SD}=11.44$; see Figure 1). This was confirmed with a one-way ANCOVA comparing standard scores of verbal ability between children who do and do not report playing a musical instrument, with age, sex, SES, and $\mathrm{VO}_{2}$ max as covariates: the effect of playing an instrument was highly significant $[F(1,94)=9.10, p=0.003$, partial $\left.\eta^{2}=0.091\right]$. The covariates of age, sex, SES, and $\mathrm{VO}_{2} \max$ were all not significant [age: $F(1,94)=0.721, p=0.40$, partial $\eta^{2}=0.008$; sex: $F(1,94)=0.59, p=0.44$, partial $\eta^{2}=0.006$; SES: $F(1,94)=0.09, p=0.76$, partial $\eta^{2}=0.001 ; \mathrm{VO}_{2} \max$ : $F(1,94)=1.58, p=0.21$, partial $\left.\eta^{2}=0.017\right]$.

Participants who play a musical instrument also scored higher on the overall Brief Intellectual Ability test (instrument players mean $=117.27, \mathrm{SD}=9.37$; non-players mean $=107.69$, $\mathrm{SD}=12.46$; Figure 1). This was again confirmed with an ANCOVA comparing children who do and do not report playing a musical instrument, with age, sex, SES, and $\mathrm{VO}_{2} \max$ as covariates: the effect of playing an instrument was highly significant $\left(F(1,94)=14.88, p<0.001\right.$, partial $\left.\eta^{2}=0.138\right)$. The covariates of age, sex, SES, and $\mathrm{VO}_{2}$ max were not significant [age: $F(1,94)=4.648, p=0.033$, partial $\eta^{2}=0.033$; sex: $F(1,94)=1.23, p=0.27$, partial $\eta^{2}=0.009$; SES: $F(1,94)=1.019$,

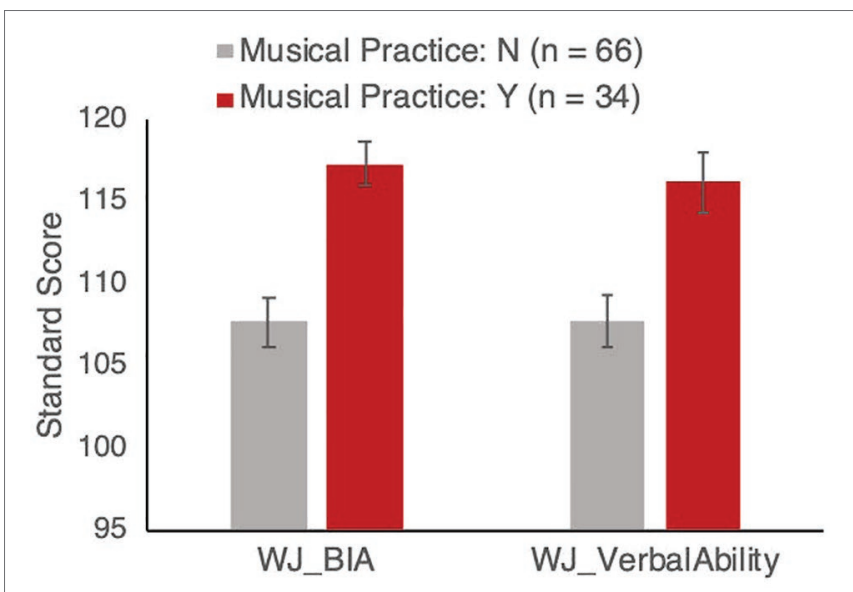

FIGURE 1 | Woodcock-Johnson Brief Intellectual Ability (BIA) and Verbal Ability standardized scores for groups with and without musical instrument training. Results remain significant after controlling for age, sex, socioeconomic status, and aerobic fitness, as described in the text. Error bars show between-subject standard error of the mean.

$p=0.315$, partial $\eta^{2}=0.007 ; \mathrm{VO}_{2} \max : F(1,94)=3.01, p=0.085$, partial $\left.\eta^{2}=0.022\right]$.

In contrast to playing a musical instrument, participants who report singing in choir $(n=15)$ did not score higher on verbal ability or on intellectual ability than those who did not report singing in choir $(n=85)$ (intellectual ability: choir mean $=110.13, \mathrm{SD}=13.68 ;$ non-choir mean $=110.64$, $\mathrm{SD}=12.13$. Verbal ability: choir mean $=110.33, \mathrm{SD}=10.675$; non-choir mean $=109.90, \mathrm{SD}=11.781$; all $p>0.2$; all partial $\left.\eta^{2}<0.05\right)$.

We further examined the relationship between the intensity of musical practice, quantified as number of hours of reported music practice per week, and verbal ability and intellectual ability in a partial correlation controlling for age, sex, SES, and $\mathrm{VO}_{2}$ max. Practice intensity was significantly correlated with verbal ability $\left(r_{p}=0.260, p_{p}=0.041\right)$, but not significantly correlated with intellectual ability $\left(r_{p}=0.21, p_{p}=0.099\right)$.

\section{Neuroimaging Results}

Since the behavioral results showed that whether or not children played a musical instrument was most predictive of verbal and intellectual abilities, we first compared children who did and did not play a musical instrument in a multivariate ANCOVA with all the diffusion measures (FA, $\mathrm{AD}$, and $\mathrm{RD}$ ) in the left and right SLF and CC (genus, body, splenium) as outcomes variables, while controlling for age, sex, SES, and $\mathrm{VO}_{2} \mathrm{max}$, the same four covariates as in behavioral analyses. The effect of playing a musical instrument on diffusion measures (considering $\mathrm{FA}, \mathrm{AD}$, and $\mathrm{RD}$ together) was highly significant $\left[F(15,75)=3.84, p<0.001\right.$, partial $\left.\eta^{2}=0.44\right]$ (Figure 2). The covariate of age on the diffusion measures was also significant $\left[F(15,75)=2.059, p=0.022\right.$, partial $\left.\eta^{2}=0.29\right]$. The effects of SES and $\mathrm{VO}_{2} \max$ were not significant at the 0.05 level [SES: $F(15,75)=1.64, p=0.083$, partial $\eta^{2}=0.25 ; \mathrm{VO}_{2} \max$ : $F(15,75)=1.06, p=0.40$, partial $\left.\eta^{2}=0.18\right)$. 


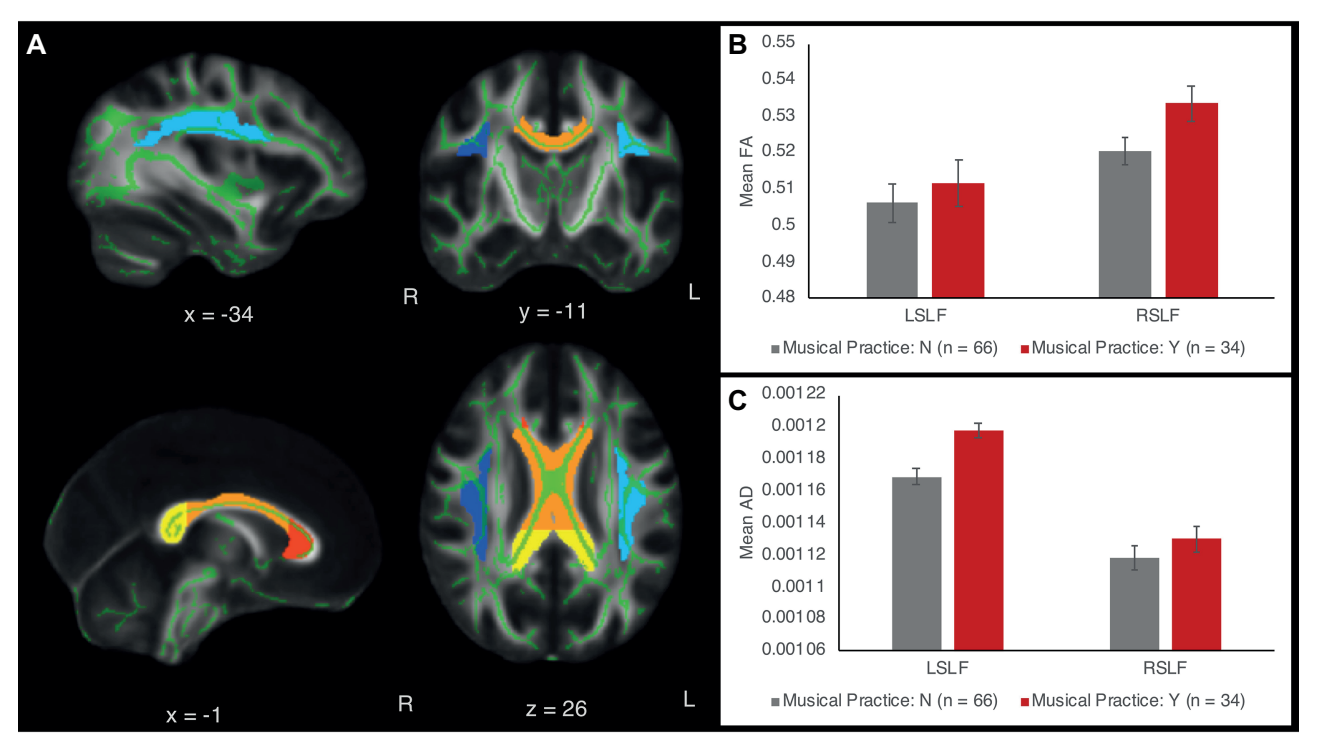

FIGURE 2 | (A) White matter tract ROls in the left and right superior longitudinal fasciculus (SLF) and the corpus callosum (CC). Light blue = left SLF; dark blue = right SLF; red = genu of CC; orange = body of CC; yellow = splenium of CC. The ROls are overlaid on the standard white matter skeleton (green) and $1 \mathrm{~mm}$ template FA image (grayscale). Green voxels inside the ROls are averaged across the ROI to obtain mean FA, AD, and RD values across the ROI. (B) FA of the left and right SLF as a function of musical expertise. (C) AD of left and right SLF as a function of musical expertise. Error bars show between-subject standard error of the mean.

Follow-up univariate ANCOVAs were conducted to test for between-subject differences in each diffusion measure for each tract ROI. Since we were testing three types of diffusion measures (FA, $\mathrm{AD}$, and $\mathrm{RD}$ ) and five tract ROIs (right SLF, left SLF, CC genu, CC body, and CC splenium), Bonferroni correction at the $p<0.05$ level was applied for $3 \times 5=15$ statistical tests. The results showed that instrument players had higher $\mathrm{AD}$ values in the left $\operatorname{SLF}[F(1,91)=15.64, p<0.001$, partial $\left.\eta^{2}=0.149\right]$, surviving Bonferroni correction at the $p<0.05$ level. No other FA, AD, or RD values in the left or right SLF were significant at the Bonferroni-corrected level. $\mathrm{FA}, \mathrm{AD}$, and $\mathrm{RD}$ values in the corpus callosum were similar between children with and without musical instrument training (all $p$ 's >0.1). Table 2 shows means and standard deviations of each diffusion measure for musically trained and untrained participants.

The covariate of age was also significant in FA of the right $\operatorname{SLF}\left[F(1,91)=11.562, p=0.001\right.$, partial $\left.\eta^{2}=0.115\right]$ and in $\mathrm{RD}$ of the left and right SLF [left SLF: $F(1,91)=8.956$, $p=0.004$, partial $\eta^{2}=0.091$; right SLF: $F(1,91)=6.557, p=0.012$, partial $\eta^{2}=0.069$ ]. Older children had higher FA and lower $\mathrm{RD}$, consistent with previous reports (Krogsrud et al., 2016).

Using participation in music activities (instead of playing a musical instrument) as a predictor in the multivariate test yielded the same significant effect of participation in musical activities $\left[F(15,75)=2.58, p=0.004\right.$, partial $\left.\eta^{2}=0.34\right]$, but the covariates of age, sex, SES, and $\mathrm{VO}_{2}$ max were not significant (all $p>0.1$ ). Follow-up ANCOVA again showed that AD in the left SLF was significantly higher among children who participated in music activities $[F(1,91)=8.955, p=0.004$, partial $\left.\eta^{2}=0.090\right]$; this was significant at the $p<0.05$ level but not at the Bonferroni-corrected level. Substituting singing
TABLE 2 | Means and standard deviations of diffusion parameters for participants with and without musical instrument training in each region of interest in the superior longitudinal fasciculus (SLF) and corpus callosum (CC).

\begin{tabular}{|c|c|c|c|c|c|c|}
\hline \multirow[b]{3}{*}{ Tract } & \multirow[b]{3}{*}{$\begin{array}{l}\text { Diffusion } \\
\text { statistic }\end{array}$} & \multirow[b]{3}{*}{ ROI } & \multicolumn{4}{|c|}{ Musical instrument training } \\
\hline & & & \multicolumn{2}{|c|}{ No $(n=66)$} & \multicolumn{2}{|c|}{ Yes $(n=34)$} \\
\hline & & & Mean & SD & Mean & SD \\
\hline \multirow[t]{6}{*}{ SLF } & FA & SLF right & 0.521 & 0.031 & 0.534 & 0.028 \\
\hline & & SLF left & 0.506 & 0.044 & 0.512 & 0.037 \\
\hline & $A D$ & SLF right & 1.12E-03 & 3.51E-05 & 1.13E-03 & 4.68E-05 \\
\hline & & SLF left* & 1.17E-03 & 3.90E-05 & 1.20E-03 & 4.22E-05 \\
\hline & $\mathrm{RD}$ & SLF right & 4.77E-04 & 3.42E-05 & 4.68E-04 & 2.98E-05 \\
\hline & & SLF left & 5.32E-04 & 3.64E-05 & 5.22E-04 & 2.35E-05 \\
\hline \multirow[t]{9}{*}{$\mathrm{CC}$} & FA & CC genu & 0.752 & 0.022 & 0.755 & 0.017 \\
\hline & & CC body & 0.698 & 0.033 & 0.697 & 0.027 \\
\hline & & CC splenium & 0.794 & 0.019 & 0.792 & 0.021 \\
\hline & $A D$ & CC genu & $1.48 \mathrm{E}-03$ & 5.42E-05 & 1.49E-03 & 5.03E-05 \\
\hline & & CC body & 1.54E-03 & 4.58E-05 & 1.55E-03 & 6.39E-05 \\
\hline & & CC splenium & 1.56E-03 & 4.75E-05 & 1.57E-03 & $9.85 \mathrm{E}-05$ \\
\hline & $\mathrm{RD}$ & CC genu & 3.17E-04 & 3.09E-05 & 3.11E-04 & 2.37E-05 \\
\hline & & CC body & 3.94E-04 & 4.68E-05 & 3.98E-04 & 4.03E-05 \\
\hline & & CC splenium & $2.76 \mathrm{E}-04$ & 2.81E-05 & 2.81E-04 & 3.47E-05 \\
\hline
\end{tabular}

Effects of musical instrument training, controlling for covariates of age, sex, SES, and fitness: * $p<0.001$, surviving Bonferroni correction at $p<0.05$ level for 15 comparisons.

in choir as a predictor in the same multivariate analysis yielded no significant effect of choir singing $[F(15,73)=0.79, p=0.68$, partial $\eta^{2}=0.14$ ) and no significant covariates (all $p$ 's $>0.1$ ).

We further examined the relationship between the intensity of musical practice and $\mathrm{FA}, \mathrm{AD}$, and $\mathrm{RD}$ in the left and right SLF. FA and RD were not significantly correlated with practice 
intensity; however, a significant positive correlation was found between practice intensity and axial diffusivity (AD) in the left SLF (Spearman rank-order correlation $r_{s}=0.305, p_{s}=0.011$ ). The association between $\mathrm{AD}$ and practice intensity remained significant after controlling for differences in age, sex, socioeconomic status, and $\mathrm{VO}_{2} \max$ (partial correlation: $\left.r_{p}=0.319, p_{p}=0.012\right)$. AD of the left SLF was also correlated with intellectual ability (Spearman rank-order correlation $\left.r_{s}=0.165, p_{s}=0.04\right)$ but not significantly with verbal ability (Spearman rank-order correlation $r_{s}=0.140, p_{s}=0.095$ ).

\section{DISCUSSION}

Here, we show that children who participate in musical activities, specifically by playing one or more musical instruments for at least $0.5 \mathrm{~h}$ per week, have higher verbal ability and better general intellectual ability, as assessed by standardized neuropsychological measures of cognitive performance. Furthermore, music training and cognitive outcome variables are associated with measures of white matter in the superior longitudinal fasciculus, a major white matter pathway in the brain. Effects are significant even after controlling for differences in age, sex, socio-economic status, and aerobic fitness. As early to middle childhood are periods of rapid cognitive and brain development, participation in cultural and/or artistic activities during these periods may have lasting effects on brain and cognitive health.

The present study relates music training and cognitive performance to white matter structure in a large population of children. In this sample, music training also predicted intellectual ability, corresponding to previous findings from randomized controlled trials on music learning (Moreno et al., 2011). The behavioral results are consistent with a meta-analysis on effects of musical training on literacy skills, which found support for the hypothesis that music training leads to gains in phonological awareness skills, albeit with a small effect size relative to the large variance in these skills across different ages and different family backgrounds (Gordon et al., 2015). Recent work has shown that children who have musical training also possess enhanced phonological processing abilities, with fMRI results showing more left-lateralized temporoparietal activity during phonological processing in musically trained children (Zuk et al., 2018). Our findings are broadly consistent with these results as we show that while right SLF is sensitive to differences between children with and without musical instrumental training, these differences could also be explained by age and SES; in contrast, AD of the left SLF is sensitive to training and practice intensity.

Diffusion properties of white matter in the SLF has been related to musical training and musical pitch identification abilities (Oechslin et al., 2010; Halwani et al., 2011; Moore et al., 2017), as well as to the ability to learn language (Floel et al., 2009; Qi et al., 2015) and music (Loui et al., 2011; Vaquero et al., 2018). FA in the left SLF predicts reading ability as defined by phonological awareness tests in childhood (Yeatman et al., 2011; Saygin et al., 2013); the same variable is also positively correlated with language exposure in childhood as defined by conversational turns in children's environment (Romeo et al., 2018). In the right hemisphere, FA in the right SLF is positively correlated with Mandarin Chinese learning success (Qi et al., 2015) and with pitch-related grammar learning (Loui et al., 2011). Here, we show that while $\mathrm{FA}$ and $\mathrm{RD}$ are related to age, $\mathrm{AD}$ of the left SLF is most strongly correlated with the intensity of musical practice. One limitation is that the resolution of the diffusion images is relatively low $(3.44 \mathrm{~mm} \times 3.44 \mathrm{~mm} \times 4 \mathrm{~mm})$, which could affect useful indices such as FA (Barrio-Arranz et al., 2015). FA measures anisotropy of the diffusion tensor and is useful as a general index of white matter as it is sensitive to several properties such as myelination, axonal diameter, and coherence of axonal fibers (but see Jones et al., 2013). RD is the average of the second and third eigenvalues of the diffusion tensor and may be related to myelination (Song et al., 2002); however, cautious interpretations are necessary due to issues with crossing fibers (Wheeler-Kingshott and Cercignani, 2009). In contrast, $\mathrm{AD}$ describes water mobility along the axis of the main fiber orientation (Jones et al., 2013). While RD increase has been linked to demyelination in animal models (Song et al., 2002), changes in $\mathrm{AD}$ tend to be more variable. Decreases in $\mathrm{AD}$ have been observed in axonal injury in animal models, specifically in mice inflicted with retinal ischemia, which in the early stage shows axonal damage without myelin damage (Song et al., 2003; Sun et al., 2006). Budde et al. (2009) also linked $\mathrm{AD}$ decrease to axonal damage, rather than myelination, in the mouse spinal cord. While these animal studies link axonal injury to $\mathrm{AD}$ decrease, $\mathrm{AD}$ of white matter tracts has been reported to increase during brain maturation (Alexander et al., 2011); specifically $\mathrm{AD}$ in the SLF has been shown to increase with age during adolescence (Ashtari et al., 2007). However, other studies have observed decreases, or little to no changes, in $\mathrm{AD}$ throughout the course of development (Lebel et al., 2008, 2012; Lebel and Beaulieu, 2011; Simmonds et al., 2014; Krogsrud et al., 2016; Moura et al., 2016; Seunarine et al., 2016). Based on these findings, one possible interpretation is that the observed increases in $\mathrm{AD}$ among children who practice musical instruments reflect greater coherence (movement along the same direction) of the mobility of water molecules along the principal direction of orientation of the SLF, likely linked to increased maturation or coherence of axons; this maturation or coherence is also associated with the intensity of musical practice. This is qualified by the fact that the relatively low spatial resolution in the present imaging parameters may limit our interpretations due to possible partial voluming. Future studies (e.g., using newer sequences and more detailed image analysis) will further disentangle the diffusion properties that contribute to white matter during brain development.

Our current results are consistent with recent work showing that a perisylvian network is changed by music training (Habibi et al., 2017; Zuk et al., 2018). Interestingly, and contrary to recent findings (Steele et al., 2013; Habibi et al., 2017), our results did not show musical training-related differences in corpus callosum specifically as a result of musical training. Part of this difference may arise from the fact that the present sample involves children from a restricted age; furthermore, we did not collect data on the age of onset of musical training; thus, we could 
not compare early and late starting musicians as in other reports (Steele et al., 2013). Moreover, recent results from the same population found that children who engage in a 9-month physical activity program had changes in FA and $\mathrm{RD}$ of the corpus callosum, compared to an untrained (wait-listed) control group (Chaddock-Heyman et al., 2018). As playing a musical instrument is a mild form of physical activity, results from these different lines of work may suggest that any physical activity that increases oxygen consumption may change the corpus callosum, along with a multitude of other changes in brain health (Hillman et al., 2008; Chaddock et al., 2011), whereas playing a musical instrument may more specifically influence auditory-motor regions especially the SLF, with more rigorous forms of musical training extending toward effects in the corpus callosum. Some support for this comes from the observation that children with musical training in this sample have slightly higher $\mathrm{VO}_{2} \max$ (Table 1), indicating slightly higher fitness than their musically untrained counterparts. Although this was not statistically significant in the present sample, future work with a larger sample may specifically investigate the relationship between musical training and aerobic fitness. At present, by incorporating $\mathrm{VO}_{2} \max$ as a covariate in our main analyses relating music training to neuropsychological and neuroimaging measures, we rule out the alternative explanation that the effects of musical training might be explained by aerobic fitness.

Interestingly, participation in choir did not predict cognitive measures in this sample. This may be because only a small subset $(15 \%)$ of our sample participated in choir. Although previous reports have shown positive effects of music and singing on wellbeing in adults (Daykin et al., 2017), there remains a need for research on the cognitive effects of singing training with younger populations (Demorest and Pfordresher, 2015). Also unlike previous reports (Oechslin et al., 2010), we did not observe a reversal in hemispheric asymmetry of the SLF in musicians. This difference may arise from differences in DTI methodology: while other reports simultaneously investigated volume (number of voxels) and FA of the SLF (Oechslin et al., 2010; Halwani et al., 2011), we used the tractbased spatial statistics approach, which aligns all participants' white matter skeletons such that the number of voxels within each ROI is the same across participants; this allows for a robust comparison of diffusion statistics (FA, $\mathrm{AD}, \mathrm{RD}$ ) in the same voxels across participants. Continued investigation with the current dataset may involve probabilistic tractography to trace the SLF over individually defined ROIs and to enable more methodologically similar comparisons with previous reports.

\section{REFERENCES}

Alexander, A. L., Hurley, S. A., Samsonov, A. A., Adluru, N., Hosseinbor, A. P., Mossahebi, P., et al. (2011). Characterization of cerebral white matter properties using quantitative magnetic resonance imaging stains. Brain Connect. 1, 423-446. doi: 10.1089/brain.2011.0071

Andersson, J. L., Jenkinson, M., and Smith, S. (2007a). Non-linear optimisation. FMRIB technical report TR07JA1. (Oxford, UK: University of Oxford FMRIB Centre).

Andersson, J. L., Jenkinson, M., and Smith, S. (2007b). Non-linear registration, aka spatial normalisation FMRIB technical report TR07JA2. FMRIB Analysis Group of the University of Oxford. 2.
In the current study, we limit our neuroimaging analyses to white matter microstructural properties of SLF and CC, as they are regions for which we have a priori hypotheses from previous literature on white matter effects of musical training. Future work may additionally consider the effects of musical instrumental practice on other white matter pathways, as well as on other brain measures such as resting state or task-related fMRI and EEG/ERP indices of executive function and other cognitive processes.

\section{CONCLUSION}

The results suggest that the relationship between musical practice and intellectual ability is related to axonal fibers in white matter pathways in the auditory-motor system.

\section{ETHICS STATEMENT}

This study was carried out in accordance with the recommendations of IRB of UIUC with written informed consent from all subjects. All subjects gave written informed consent in accordance with the Declaration of Helsinki. The protocol was approved by the Institutional Review Board at the University of Illinois. Parents provided written informed consent, and participants provided written assent.

\section{AUTHOR CONTRIBUTIONS}

PL conceptualized the idea behind this manuscript, performed data analyses, and wrote the first draft. LR, LC-H, AK, and $\mathrm{CH}$ conceptualized and designed the larger study, for which the data were obtained. LR and LC-H acquired and preprocessed the behavioral and neuroimaging data. All authors revised the manuscript and approved the submission.

\section{FUNDING}

Funding was provided by grants from the National Institute on Aging at the National Institute of Health to AK (R01 AG25032 and R37 AG025667) and the National Institute of Child Health and Human Development (HD069381) to $\mathrm{CH}$ and $\mathrm{AK}$.

Anvari, S. H., Trainor, L. J., Woodside, J., and Levy, B. A. (2002). Relations among musical skills, phonological processing, and early reading ability in preschool children. J. Exp. Child Psychol. 83, 111-130. doi: 10.1016/S0022-0965(02)00124-8

Ashtari, M., Cervellione, K. L., Hasan, K. M., Wu, J., McIlree, C., Kester, H., et al. (2007). White matter development during late adolescence in healthy males: a cross-sectional diffusion tensor imaging study. NeuroImage 35, 501-510. doi: 10.1016/j.neuroimage.2006.10.047

Bailey, J. A., Zatorre, R. J., and Penhune, V. B. (2014). Early musical training is linked to gray matter structure in the ventral premotor cortex and auditory-motor rhythm synchronization performance. J. Cogn. Neurosci. 26, 755-767. doi: 10.1162/jocn_a_00527 
Bar-Or, O. (Ed.) (1983). "Physiologic responses to exercise of the healthy child" in Pediatric sports medicine for the practitioner. (New York, NY: Springer), 1-65.

Barrio-Arranz, G., de Luis-García, R., Tristán-Vega, A., Martín-Fernández, M., and Aja-Fernández, S. (2015). Impact of MR acquisition parameters on DTI scalar indexes: a tractography based approach. PLoS One 10:e0137905. doi: 10.1371/journal.pone.0137905

Bermudez, P., Lerch, J. P., Evans, A. C., and Zatorre, R. J. (2009). Neuroanatomical correlates of musicianship as revealed by cortical thickness and voxel-based morphometry. Cereb. Cortex 19, 1583-1596. doi: 10.1093/cercor/bhn196

Budde, M. D., Xie, M., Cross, A. H., and Song, S.-K. (2009). Axial diffusivity is the primary correlate of axonal injury in the experimental autoimmune encephalomyelitis spinal cord: a quantitative pixelwise analysis. J. Neurosci. 29, 2805-2813. doi: 10.1523/JNEUROSCI.4605-08.2009

Chaddock, L., Neider, M. B., Lutz, A., Hillman, C. H., and Kramer, A. F. (2011). Role of childhood aerobic fitness in successful street crossing. Med. Sci. Sports Exerc. 44, 749-753. doi: 10.1249/MSS.0b013e31823a90cb

Chaddock-Heyman, L., Erickson, K. I., Holtrop, J. L., Voss, M. W., Pontifex, M. B., Raine, L. B., et al. (2014). Aerobic fitness is associated with greater white matter integrity in children. Front. Hum. Neurosci. 8:584. doi: 10.3389/ fnhum.2014.00584

Chaddock-Heyman, L., Erickson, K. I., Kienzler, C., Drollette, E. S., Raine, L. B., Kao, S.-C., et al. (2018). Physical activity increases white matter microstructure in children. Front. Neurosci. 12:950. doi: 10.3389/fnins.2018.00950

Daykin, N., Mansfield, L., Meads, C., Julier, G., Tomlinson, A., Payne, A., et al. (2017). What works for wellbeing? A systematic review of wellbeing outcomes for music and singing in adults. Perspect. Public Health 138, 39-46. doi: 10.1177/1757913917740391

Demorest, S. M., and Pfordresher, P. Q. (2015). Singing accuracy development from k-adult: a comparative study. Music. Percept. 32, 293-302. doi: 10.1525/ mp.2015.32.3.293

Donnelly, J. E., Hillman, C. H., Castelli, D., Etnier, J. L., Lee, S., Tomporowski, P., et al. (2016). Physical activity, fitness, cognitive function, and academic achievement in children: a systematic review. Med. Sci. Sports Exerc. 48, 1197-1222. doi: 10.1249/MSS.0000000000000901

Ellis, R. J., Bruijn, B., Norton, A. C., Winner, E., and Schlaug, G. (2013). Training-mediated leftward asymmetries during music processing: a crosssectional and longitudinal fMRI analysis. NeuroImage 75, 97-107. doi: 10.1016/j.neuroimage.2013.02.045

Floel, A., de Vries, M. H., Scholz, J., Breitenstein, C., and Johansen-Berg, H. (2009). White matter integrity in the vicinity of Broca's area predicts grammar learning success. NeuroImage 47, 1974-1981. doi: 10.1016/j. neuroimage.2009.05.046

Freedson, P. S., and Goodman, T. L. (1993). "Measurement of oxygen consumption" in Pediatric laboratory exercise testing: clinical guidelines, ed. T. W. Rowland, (Champaign, Il: Human Kinestics), 91-113.

Gardiner, M. F., Fox, A., Knowles, F., and Jeffrey, D. (1996). Learning improved by arts training. Nature 381:284. doi: 10.1038/381284a0

Gordon, R. L., Fehd, H. M., and McCandliss, B. D. (2015). Does music training enhance literacy skills? A meta-analysis. Front. Psychol. 6:1777. doi: 10.3389/ fpsyg.2015.01777

Gullick, M. M., Demir-Lira, O. E., and Booth, J. R. (2016). Reading skill-fractional anisotropy relationships in visuospatial tracts diverge depending on socioeconomic status. Dev. Sci. 19, 673-685. doi: 10.1111/ desc. 12428

Habibi, A., Damasio, A., Ilari, B., Veiga, R., Joshi, A. A., Leahy, R. M., et al. (2017). Childhood music training induces change in micro and macroscopic brain structure: results from a longitudinal study. Cereb. Cortex 28, 4336-4347. doi: 10.1093/cercor/bhx286

Halwani, G. F., Loui, P., Rueber, T., and Schlaug, G. (2011). Effects of practice and experience on the arcuate fasciculus: comparing singers, instrumentalists, and non-musicians. Front. Psychol. 2. doi: 10.3389/fpsyg.2011.00156

Hillman, C. H., Erickson, K. I., and Kramer, A. F. (2008). Be smart, exercise your heart: exercise effects on brain and cognition. Nat. Rev. Neurosci. 9, 58-65. doi: 10.1038/nrn2298

Hillman, C. H., Pontifex, M. B., Castelli, D. M., Khan, N. A., Raine, L. B., Scudder, M. R., et al. (2014). Effects of the FITKids randomized controlled trial on executive control and brain function. Pediatrics 134:e1063. doi: 10.1542/peds.2013-3219
Ho, Y. C., Cheung, M. C., and Chan, A. S. (2003). Music training improves verbal but not visual memory: cross-sectional and longitudinal explorations in children. Neuropsychology 17, 439-450. doi: 10.1037/0894-4105.17.3.439

Hurwitz, I., Wolff, P. H., Bortnick, B. D., and Kokas, K. (1975). Nonmusical effects of the Kodaly music curriculum in primary grade children. J. Learn. Disabil. 8, 167-174.

Hyde, K. L., Lerch, J., Norton, A., Forgeard, M., Winner, E., Evans, A. C., et al. (2009). Musical training shapes structural brain development. J. Neurosci. 29, 3019-3025. doi: 10.1523/JNEUROSCI.5118-08.2009

Jaschke, A. C., Honing, H., and Scherder, E. J. A. (2018). Longitudinal analysis of music education on executive functions in primary school children. Front. Neurosci. 12:103. doi: 10.1371/journal.pone.0207265

Jones, D. K., Knosche, T. R., and Turner, R. (2013). White matter integrity, fiber count, and other fallacies: the do's and don'ts of diffusion MRI. NeuroImage 73, 239-254. doi: 10.1016/j.neuroimage.2012.06.081

Karpati, F. J., Giacosa, C., Foster, N. E. V., Penhune, V. B., and Hyde, K. L. (2017). Dance and music share gray matter structural correlates. Brain Res. 1657, 62-73. doi: 10.1016/j.brainres.2016.11.029

Khan, N. A., and Hillman, C. H. (2014). The relation of childhood physical activity and aerobic fitness to brain function and cognition: a review. Pediatr. Exerc. Sci. 26, 138-146. doi: 10.1123/pes.2013-0125

Kramer, A. F., Hahn, S., Cohen, N. J., Banich, M. T., McAuley, E., Harrison, C. R., et al. (1999). Ageing, fitness and neurocognitive function. Nature 400, 418-419. doi: $10.1038 / 22682$

Kraus, N., and Chandrasekaran, B. (2010). Music training for the development of auditory skills. Nat. Rev. Neurosci. 11, 599-605. doi: 10.1038/nrn2882

Krogsrud, S. K., Fjell, A. M., Tamnes, C. K., Grydeland, H., Mork, L., Due-Tønnessen, P., et al. (2016). Changes in white matter microstructure in the developing brain-a longitudinal diffusion tensor imaging study of children from 4 to 11years of age. NeuroImage 124, 473-486. doi: 10.1016/j. neuroimage.2015.09.017

Lamb, S. J., and Gregory, A. H. (1993). The relationship between music and reading in beginning readers. Educ. Psychol. 13, 19-27. doi: $10.1080 / 0144341930130103$

Lebel, C., and Beaulieu, C. (2011). Longitudinal development of human brain wiring continues from childhood into adulthood. J. Neurosci. 31, 10937-10947. doi: 10.1523/JNEUROSCI.5302-10.2011

Lebel, C., Gee, M., Camicioli, R., Wieler, M., Martin, W., and Beaulieu, C. (2012). Diffusion tensor imaging of white matter tract evolution over the lifespan. NeuroImage 60, 340-352. doi: 10.1016/j.neuroimage.2011.11.094

Lebel, C., Walker, L., Leemans, A., Phillips, L., and Beaulieu, C. (2008). Microstructural maturation of the human brain from childhood to adulthood. NeuroImage 40, 1044-1055. doi: 10.1016/j.neuroimage.2007.12.053

Loui, P., Alsop, D., and Schlaug, G. (2009). Tone deafness: a new disconnection syndrome? J. Neurosci. 29, 10215-10220. doi: 10.1523/JNEUROSCI.1701-09.2009

Loui, P., Li, H. C., and Schlaug, G. (2011). White matter integrity in right hemisphere predicts pitch-related grammar learning. NeuroImage 55, 500-507. doi: 10.1016/j.neuroimage.2010.12.022

Lynn, R., Wilson, R. G., and Gault, A. (1989). Simple musical tests as measures of Spearman's g. Vol. 10 (Netherlands: Elsevier Science), 25-28.

Mehr, S. A., Schachner, A., Katz, R. C., and Spelke, E. S. (2013). Two randomized trials provide no consistent evidence for nonmusical cognitive benefits of brief preschool music enrichment. PLoS One 8:e82007. doi: 10.1371/journal. pone. 0082007

Moore, E., Schaefer, R. S., Bastin, M. E., Roberts, N., and Overy, K. (2017). Diffusion tensor MRI tractography reveals increased fractional anisotropy (FA) in arcuate fasciculus following music-cued motor training. Brain Cogn. 116, 40-46. doi: 10.1016/j.bandc.2017.05.001

Moreno, S., Bialystok, E., Barac, R., Schellenberg, E. G., Cepeda, N. J., and Chau, T. (2011). Short-term music training enhances verbal intelligence and executive function. Psychol. Sci. 22, 1425-1433. doi: $10.1177 / 0956797611416999$

Mori, S., and van Zijl, P. (2007). Human white matter atlas. Am. J. Psychiatry 164:1005. doi: 10.1176/ajp.2007.164.7.1005

Moura, L. M., Kempton, M., Barker, G., Salum, G., Gadelha, A., Pan, P. M., et al. (2016). Age-effects in white matter using associated diffusion tensor imaging and magnetization transfer ratio during late childhood and early adolescence. Magn. Reson. Imaging 34, 529-534. doi: 10.1016/j. mri.2015.12.021 
Oechslin, M. S., Imfeld, A., Loenneker, T., Meyer, M., and Jancke, L. (2010). The plasticity of the superior longitudinal fasciculus as a function of musical expertise: a diffusion tensor imaging study. Front. Hum. Neurosci. 3, 1-12. doi: 10.3389/neuro.09.076.2009

Qi, Z., Han, M., Garel, K., San Chen, E., and Gabrieli, J. D. E. (2015). Whitematter structure in the right hemisphere predicts mandarin Chinese learning success. J. Neurolinguistics 33, 14-28. doi: 10.1016/j.jneuroling.2014.08.004

Roden, I., Kreutz, G., and Bongard, S. (2012). Effects of a school-based instrumental music program on verbal and visual memory in primary school children: a longitudinal study. Front. Neurosci. 6:572. doi: 10.3389/ fpsyg.2012.00572

Romeo, R. R., Segaran, J., Leonard, J. A., Robinson, S. T., West, M. R., Mackey, A. P., et al. (2018). Language exposure relates to structural neural connectivity in childhood. J. Neurosci. 38, 7870-7877. doi: 10.1523/ JNEUROSCI.0484-18.2018

Sachs, M., Kaplan, J., Der Sarkissian, A., and Habibi, A. (2017). Increased engagement of the cognitive control network associated with music training in children during an fMRI Stroop task. PLoS One 12:e0187254. doi: 10.1371/ journal.pone.0187254

Sala, G., and Gobet, F. (2017). Does far transfer exist? Negative evidence from chess, music, and working memory training. Curr. Dir. Psychol. Sci. 26, 515-520. doi: 10.1177/0963721417712760

Saygin, Z. M., Norton, E. S., Osher, D. E., Beach, S. D., Cyr, A. B., OzernovPalchik, O., et al. (2013). Tracking the roots of reading ability: white matter volume and integrity correlate with phonological awareness in prereading and early-reading kindergarten children. J. Neurosci. 33, 13251-13258. doi: 10.1523/JNEUROSCI.4383-12.2013

Schellenberg, E. G. (2004). Music lessons enhance IQ. Psychol. Sci. 15, 511-514. doi: 10.1111/j.0956-7976.2004.00711.x

Schellenberg, E. G. (2006). Long-term positive associations between music lessons and IQ. J. Educ. Psychol. 98, 457-468. doi: 10.1037/0022-0663.98.2.457

Schlaug, G., Jancke, L., Huang, Y., Staiger, J. F., and Steinmetz, H. (1995). Increased corpus callosum size in musicians. Neuropsychologia 33, 1047-1055. doi: 10.1016/0028-3932(95)00045-5

Schrank, F. A. (2011). "Woodcock-Johnson III tests of cognitive abilities" in Handbook of pediatric neuropsychology. ed. A. S. Davis (New York, NY, US: Springer Publishing Co.), 415-434.

Seunarine, K. K., Clayden, J. D., Jentschke, S., Munoz, M., Cooper, J. M., Chadwick, M. J., et al. (2016). Sexual dimorphism in white matter developmental trajectories using tract-based spatial statistics. Brain Connect. 6, 37-47. doi: 10.1089/brain.2015.0340

Sihvonen, A. J., Ripollés, P., Leo, V., Rodríguez-Fornells, A., Soinila, S., and Särkämö, T. (2016). Neural basis of acquired amusia and its recovery after stroke. J. Neurosci. 36, 8872-8881. doi: 10.1523/JNEUROSCI.0709-16.2016

Sihvonen, A. J., Ripolles, P., Sarkamo, T., Leo, V., Rodriguez-Fornells, A., Saunavaara, J., et al. (2017). Tracting the neural basis of music: deficient structural connectivity underlying acquired amusia. Cortex 97, 255-273. doi: 10.1016/j.cortex.2017.09.028

Simmonds, D. J., Hallquist, M. N., Asato, M., and Luna, B. (2014). Developmental stages and sex differences of white matter and behavioral development through adolescence: a longitudinal diffusion tensor imaging (DTI) study. NeuroImage 92, 356-368. doi: 10.1016/j.neuroimage.2013.12.044

Sluming, V., Barrick, T., Howard, M., Cezayirli, E., Mayes, A., and Roberts, N. (2002). Voxel-based morphometry reveals increased gray matter density in Broca's area in male symphony orchestra musicians. NeuroImage 17, 1613-1622. doi: $10.1006 /$ nimg.2002.1288

Smith, S. M. (2002). Fast robust automated brain extraction. Hum. Brain Mapp. 17, 143-155. doi: 10.1002/hbm.10062
Smith, S. M., Jenkinson, M., Johansen-Berg, H., Rueckert, D., Nichols, T. E., Mackay, C. E., et al. (2006). Tract-based spatial statistics: voxelwise analysis of multi-subject diffusion data. NeuroImage 31, 1487-1505. doi: 10.1016/j. neuroimage.2006.02.024

Song, S. K., Sun, S. W., Ju, W. K., Lin, S. J., Cross, A. H., and Neufeld, A. H. (2003). Diffusion tensor imaging detects and differentiates axon and myelin degeneration in mouse optic nerve after retinal ischemia. NeuroImage 20, 1714-1722. doi: 10.1016/j.neuroimage.2003.07.005

Song, S. K., Sun, S. W., Ramsbottom, M. J., Chang, C., Russell, J., and Cross, A. H. (2002). Dysmyelination revealed through MRI as increased radial (but unchanged axial) diffusion of water. NeuroImage 17, 1429-1436. doi: 10.1006/nimg.2002.1267

Steele, C. J., Bailey, J. A., Zatorre, R. J., and Penhune, V. B. (2013). Early musical training and white-matter plasticity in the corpus callosum: evidence for a sensitive period. J. Neurosci. 33, 1282-1290. doi: 10.1523/JNEUROSCI.3578-12.2013

Sun, S. W., Liang, H. F., Le, T. Q., Armstrong, R. C., Cross, A. H., and Song, S. K. (2006). Differential sensitivity of in vivo and ex vivo diffusion tensor imaging to evolving optic nerve injury in mice with retinal ischemia. NeuroImage 32, 1195-1204. doi: 10.1016/j.neuroimage.2006.04.212

Utter, A. C., Robertson, R. J., Nieman, D. C., and Kang, J. (2002). Children's OMNI scale of perceived exertion: walking/running evaluation. Med. Sci. Sports Exerc. 34, 139-144. doi: 10.1097/00005768-200201000-00021

Vaquero, L., Ramos-Escobar, N., Francois, C., Penhune, V., and RodriguezFornells, A. (2018). White-matter structural connectivity predicts short-term melody and rhythm learning in non-musicians. NeuroImage. 181, 252-262. doi: 10.1016/j.neuroimage.2018.06.054

Wakana, S., Caprihan, A., Panzenboeck, M. M., Fallon, J. H., Perry, M., Gollub, R. L., et al. (2007). Reproducibility of quantitative tractography methods applied to cerebral white matter. NeuroImage 36, 630-644. doi: 10.1016/j. neuroimage.2007.02.049

Wheeler-Kingshott, C. A., and Cercignani, M. (2009). About "axial" and "radial" diffusivities. Magn. Reson. Med. 61, 1255-1260. doi: 10.1002/mrm.21965

Woodcock, R. W., Mather, N., McGrew, K. S., and Wendling, B. J. (2001). Woodcock-Johnson III tests of cognitive abilities. (Itasca, IL: Riverside Publishing Company).

Yeatman, J. D., Dougherty, R. F., Rykhlevskaia, E., Sherbondy, A. J., Deutsch, G. K., Wandell, B. A., et al. (2011). Anatomical properties of the arcuate fasciculus predict phonological and reading skills in children. J. Cogn. Neurosci. 23, 3304-3317. doi: 10.1162/jocn_a_00061

Zuk, J., Benjamin, C., Kenyon, A., and Gaab, N. (2014). Behavioral and neural correlates of executive functioning in musicians and non-musicians. PLoS One 9:e99868. doi: 10.1371/journal.pone.0099868

Zuk, J., Perdue, M. V., Becker, B., Yu, X., Chang, M., Raschle, N. M., et al. (2018). Neural correlates of phonological processing: disrupted in children with dyslexia and enhanced in musically trained children. Dev. Cogn. Neurosci. 34, 82-91. doi: 10.1016/j.dcn.2018.07.001

Conflict of Interest Statement: The authors declare that the research was conducted in the absence of any commercial or financial relationships that could be construed as a potential conflict of interest.

Copyright (c) 2019 Loui, Raine, Chaddock-Heyman, Kramer and Hillman. This is an open-access article distributed under the terms of the Creative Commons Attribution License (CC BY). The use, distribution or reproduction in other forums is permitted, provided the original author(s) and the copyright owner(s) are credited and that the original publication in this journal is cited, in accordance with accepted academic practice. No use, distribution or reproduction is permitted which does not comply with these terms. 


\title{
Orff-Based Music Training Enhances Children's Manual Dexterity and Bimanual Coordination
}

\author{
Marta Martins ${ }^{1}$, Leonor Neves ${ }^{1}$, Paula Rodrigues ${ }^{2,3}$, Olga Vasconcelos ${ }^{2}$ and \\ São Luís Castro ${ }^{1 *}$ \\ ${ }^{1}$ Center for Psychology at University of Porto, Faculty of Psychology and Education Sciences, University of Porto, Porto, \\ Portugal, ${ }^{2}$ Centre of Research, Education, Innovation and Intervention in Sport, Faculty of Sport, University of Porto, Porto, \\ Portugal, ${ }^{3}$ Research in Education and Community Intervention, Piaget Institute, Almada, Portugal
}

\section{OPEN ACCESS}

Edited by:

Graham Frederick Welch, UCL Institute of Education, United Kingdom

Reviewed by: Beatriz Senoi llari, University of Southern California, United States James Henry Byrne Humberstone

University of Sydney, Australia

*Correspondence:

São Luís Castro

slcastro@fpce.up.pt

Specialty section:

This article was submitted to Performance Science, a section of the journal

Frontiers in Psychology

Received: 10 August 2018 Accepted: 05 December 2018 Published: 21 December 2018

Citation:

Martins $M$, Neves L, Rodrigues $P$, Vasconcelos O and Castro SL (2018) Orff-Based Music Training Enhances Children's Manual Dexterity and Bimanual Coordination.

Front. Psychol. 9:2616.

doi: 10.3389/fpsyg.2018.02616
How music training and expertise influence non-musical abilities is a widely researched topic. Most studies focus on the differences between adult professional musicians and non-musicians, or examine the effects of intensive instrumental training in childhood. However, the impact of music programs developed in regular school contexts for children from low-income communities is poorly explored. We conducted a longitudinal training study in such communities to examine if collective (Orff-based) music training enhances fine motor abilities, when compared to a homologous training program in sports (basketball), and to no specific training. The training programs in music and sports had the same duration, 24 weeks, and were homologous in structure. A pretest, training, post-test and follow-up design was adopted. Children attending the 3rd grade ( $n=74$, 40 girls; mean age 8.31 years) were pseudorandomly divided into three groups, music, sports and control that were matched on demographic and intellectual characteristics. Fine motor abilities were assessed with the Purdue pegboard test (eyehand coordination and motor speed, both subsumed under manual dexterity, and bimanual coordination) and with the Grooved pegboard (manipulative dexterity) test. All groups improved in manipulative dexterity that was not affected by type of training. On bimanual coordination and manual dexterity, however, a robust and stable advantage of music training emerged. At the end of training (post-test), children from the music group significantly outperformed children from the sports and control groups, an advantage that persisted at follow-up 4 months after training at the start of the following school year. Also, at follow-up none of the children from the music group were performing below the 20th percentile in the Purdue pegboard subtests and more than half were performing at the high end level (>80th percentile). Children from the sports group also improved significantly from pre- to post-test but their performance was not significantly different from that of the control group. These results show that an affordable, collectivebased music practice impacts positively on fine-motor abilities, a finding that is relevant for a better understanding of the impact of music in childhood development, and that may have implications for education at the primary grade.

Keywords: music training, sports training, fine motor abilities, manual dexterity, bimanual coordination, children 


\section{INTRODUCTION}

Music training is a powerful tool to study human behavior (Schellenberg, 2004). The effects of music training in school-aged children have deserved a lot of research attention, as childhood is a period of major developmental changes in cognitive, social and motor areas, and it is also when music training typically begins. When compared to control groups without music training, musically trained children have enhanced music-related skills, such as pitch discrimination (Ilari et al., 2016) and rhythm perception/production abilities (Matthews et al., 2016), and also advantages in non-musical domains, such as verbal abilities (Moreno et al., 2008, 2011; Degé and Schwarzer, 2011; Roden et al., 2012), executive functions (Degé et al., 2011; Moreno et al., 2011; Zuk et al., 2014), and even IQ (Schellenberg, 2004; Moreno et al., 2008; Degé et al., 2011). Less explored is how music training affects motor abilities. Of course, music practice involves motor actions whose characteristics are shaped, at least in part, by the instrument being played. However, there is presently no account that specifically addresses the impact of music training on the development of fine motor abilities.

Fine motor abilities allow us to make coordinated hand and finger movements to, for example, grasp and handle objects. They can be assessed with tasks that require one hand (unimanual; e.g., pencil grasp) or the coordinated activity of both hands (bimanual; e.g., knitting). Less effort is required performing with the preferred vs. the non-preferred hand, which is usually associated with slower and less accurate movements (Rodrigues et al., 2009; Serrien et al., 2014). Differences between hands are determined by hand preference and brain hemisphere specialization; using functional neuroimaging, Lavrysen et al. (2008, 2012) have shown that some left-right hand asymmetries depend on functional specialization of the left and right hemispheres that are independent from hand preference. In bimanual tasks both hands must work together even when the movements of each hand, or their role in the overall movement, differ, and so they are typically more demanding than unimanual tasks (Serrien et al., 2014). Interestingly, bimanual coordination can be strongly modulated by attentional focus. Deliberate attention to the nonpreferred hand reduces left-right asymmetry (De Poel et al., 2008) and improves bimanual coordination (Pellegrini et al., 2004).

Fine motor abilities evolve over childhood. A developmental landmark occurs around 8 years, when a performance discontinuity related to changes in information processing capacities temporarily disturbs motor output (Serrien et al., 2014). Thus, the development of fine motor abilities interplays with the cognitive domain. An important instantiation of this is literacy acquisition. Writing requires eye-hand coordination and fine control of hand movements (Grissmer et al., 2010), and this motor component plays a non-negligible role in learning how to read and write. For example, Dinehart and Manfra (2013) have shown that object manipulation and motor writing ability were a strong predictor of 2nd-grade math and reading achievement of low-income children, even when controlling for demographic and cognitive characteristics.

It is a common belief that learning to play an instrument improves motor abilities. Indeed, continued practice of coordinated hand and finger movements together with attention to auditory feedback and online recalibration is bound to improve motor coordination and to fine-tune the integration of audio-visual information with motor control. However, studies addressing this issue are scarce and those that are relevant tend to compare adult musicians and non-musicians, leaving open the question of whether expertise-related differences reflect predispositions and/or result from training. An advantage of adult musicians when compared to non-musicians has been observed in finger-tapping tasks (Jäncke et al., 1997) and in unimanual and bimanual reaction times (Chang et al., 2014). Professional string players have a larger cortical representation of the fingers of the left-hand than non-musicians, early training associated with larger finger representation (Elbert et al., 1995); and professional musicians have larger anterior corpus callosum (indicative of better interhemispheric communication) than non-musicians, with more marked differences if training started before 7 years of age (Schlaug et al., 1995).

Extant studies with children have examined the effects of individual lessons of instrumental learning using longitudinal pre- and post-test designs. Costa-Giomi (2005) showed that some fine motor abilities of children who had 2 years of individual piano lessons improved significantly more than those of a control group of children with no training. The improvement was observed on the fine motor component of the BruininksOseretsky Test of Motor Proficiency (BOT), specifically on the total score of this component and on the response speed subtest; no differences between groups were found in the visuo-motor coordination subtest nor in the upper-limb speed and dexterity subtest.

A positive effect of music training in fine motor abilities was also observed by Forgeard et al. (2008) in a study of 9year-old children who had had three or more years of Suzuki or traditional instrumental instruction. Compared to a control group of children without music instrumental training, the musically trained 9-year-olds performed better in a four-finger sequence tapping task (completing three 4-finger sequences within $30 \mathrm{~s}$ ). Note that both groups had 30- to 40-min weekly music classes in school, but these did not include instrumental training nor one-to-one tutoring. In a more recent study, Lampe et al. (2015) reported that 18 months of piano instruction, 3045 min twice a week, significantly improved the uniformity of keystrokes of children and youths with hand motor disorders resulting from early brain damage. Converging evidence comes from a neuroimaging study by Schlaug et al. (2005) showing that musically trained children had significantly more gray matter in brain regions associated with skills learned during instrumental practice, namely independent fine motor control of both hands and auditory discrimination. Other behavioral studies suggest that early music instruction improves performance in domains associated with fine motor abilities, such as visuo-motor integration (Orsmond and Miller, 1999) and attentional capacity and reaction times (Patston et al., 2007).

Sports is another well-known activity that, as playing a musical instrument, is associated with improved motor abilities. The belief that those who practice sports or play a music instrument have enhanced motor abilities is widespread, and a 
few studies have approached this issue empirically. Correlational evidence has been collected in such diverse domains as movement timing, mental imagery, educational achievement, motivation and wellbeing. On timing skills, a comparison of adult athletes, musicians and controls revealed that although athletes outperformed musicians and controls in a circle-drawing task (emergent-based or continuous timing), in finger-tapping (eventbased or discrete timing) both groups had similar precision but only musicians outperformed controls (Braun Janzen et al., 2014). On mental imagery, more specifically, left/right judgment, Dey et al. (2012) found no differences in accuracy and reaction times between children participating in music, sports, both or neither activity. With respect to impact on education, results from a survey conducted with teenagers from the German SocioEconomic Panel (Cabane et al., 2016) showed that, in comparison with doing sports, playing music correlated more with ambition and better academic performance, particularly for girls and for children coming from highly educated families; on the other hand, doing sports correlated more with better perceived health than playing music. Similarities between music and sports may extend to motivation and socio-affective dimensions (e.g., Martin, 2008). For example, children who spent more time in sports or music in elementary school were likely to have higher motivation to practice the same activity 4 years later (in adolescence), whereas children who had not participated in neither sports nor music were less likely to engage in these activities during adolescence (Simpkins et al., 2010). Importantly, socio-emotional wellbeing can benefit from practicing sports (Lubans et al., 2012), as well as from practicing music (Welch et al., 2014).

In the present study, our goal is to further investigate how fine motor abilities are influenced by music training, and sport will use the active control condition. We consider a different type of music training from the ones reviewed above: shortterm (less than a year) Orff-based training in collective classes instead of relatively intensive individual lessons on how to play a musical instrument. In addition to a passive control group who will follow the regular school curriculum, our active control group will have sports training (basketball) of similar duration to the music training. Both training programs, music and sports, are conceived to be homologous and similarly challenging and attractive to the children; this allows to control for potential confounds due to motivational factors that would hinder an unambiguous interpretation of results (Moreno et al., 2008; Benz et al., 2016). Furthermore, the training programs will be conducted in a mostly low-income community as part of curricular and enrichment activities within the children's regular school schedule. This provides an excellent model to study the potential impact of widespread music training, especially for under-privileged children (Kraus et al., 2014). The design of the study is longitudinal, with pre-test, training, post-test and follow-up phases. In light of the evidence reviewed above, we expect that music training leads to improved fine motor abilities, and specifically that Orff-based training impacts on bimanual coordination more than sports training or no training. Additionally, as both types of training, music and sports, involve visuo-motor components, such as eye-hand coordination and manual dexterity, we expect that children from those groups will have more gains in motor abilities than the control group.

\section{MATERIALS AND METHODS}

\section{Participants}

Third graders were selected for this study because according to the Portuguese national curriculum collective sports and instrumental music are introduced as systematic school activities at this grade.

Eighty-six children were initially recruited to participate in the study. They were all Portuguese 3rd graders from five elementary public schools in the same greater Porto area in Northern Portugal. Children attending these schools come mostly from low-income communities: at the time of the study, more than $50 \%$ of them received social support from the Portuguese social security system and more than $70 \%$ of their parents had less than secondary education, a situation that was reflected in the SES of the children who participated in the study (see Table 1). The parents or legal guardians of the children completed a short questionnaire (see Procedure) from which we could gather that none of the children who were recruited had had prior experience in instrumental music practice nor in basketball practice. Twelve of them had to be excluded because of atypically low full-scale IQ (below 70; World Health Organization, 1992; $n=7$ ), neurological problems $(n=1)$, school transfer $(n=2)$ and incomplete data records $(n=2)$. The final sample consisted of 74 children (34 boys and 40 girls, $M$ age $=8.31$ years, range $=7.75-9.50, S D=0.35$ ) . Four were left handed, according to the Edinburgh Handedness Inventory (Oldfield, 1971), and 42 were involved in some kind of extra-curricular activity outside of the school, mostly sports other than basketball (either football or swimming). The children were assigned to the music, sports (active control) or no training (standard control) groups such that these were matched on age, full-scale IQ, verbal IQ and performance IQ (all Fs < 1). The groups also did not differ on sex $\left[\chi^{2}{ }_{(2)}=0.26, p=0.88\right]$, socioeconomic status $\left[\chi^{2}(2)=2.55, p=0.28\right]$ and handedness $(F<1)$. One of the left-handed girls was in the music group, the other in the control group, and one of the left-handed boys was in the sports group, the other in the control group. The groups were also similar regarding participation in extra-curricular activities not related to the study: 10 in the music group, 16 in the sports group and 16 in the control group $\left[\chi^{2}{ }_{(2)}=4.36, p=0.11\right]$.

The study was approved by the ethics committee of the Faculty of Psychology and Education Sciences at University of Porto and the school boards. Written informed consent was obtained from parents or legal guardians of the children, who gave their verbal assent before data collection started.

\section{Design and Materials}

This is a longitudinal training study that consisted of a pre-test, training for 24 weeks, a post-test and a follow-up 4 months after the end of training. In the pre-test phase, children completed an assessment protocol including handedness, general intellectual ability and fine motor abilities. In the post-test and follow-up phases, their motor abilities were again tested. 
Handedness was assessed with the Edinburgh Handedness Inventory (Oldfield, 1971), and general intellectual ability with the Portuguese version of the Wechsler Intelligence Scale for Children - 3rd Edition (WISC-III; Wechsler, 2003). All of the WISC-III subtests required to compute verbal IQ, performance IQ and full-scale IQ were used, viz. the Information, Similarities, Arithmetic, Vocabulary, and Comprehension subtests (verbal IQ) and the Picture Completion, Coding, Picture Arrangement, Block Design, and Object Assembly subtests (performance IQ). Fine motor abilities were assessed with two well established tests, the Purdue Pegboard test (Tiffin, 1968) and the Grooved Pegboard test (Trites, 1989).

The Purdue Pegboard test provides a measure of manual dexterity and bimanual coordination that relies on eye-hand coordination and motor speed. It consists of a board with two vertically aligned series of 25 holes where as many pegs as possible have to be inserted within a time limit of $30 \mathrm{~s}$. This task is performed with the preferred hand and with the non-preferred hand (unimanual subtests) and with both hands simultaneously (bimanual subtest). In the unimanual subtests, the score is the number of correctly inserted pegs, and in the bimanual subtest it is the number of pairs of pegs. In order to make results directly comparable across the two pegboard

TABLE 1 | Demographic, cognitive and motor characteristics of children in the music, sports, and control groups prior to training.

\begin{tabular}{|c|c|c|c|}
\hline Characteristics & $\begin{array}{c}\text { Music group } \\
n=25\end{array}$ & $\begin{array}{c}\text { Sports group } \\
n=25\end{array}$ & $\begin{array}{c}\text { Control group } \\
n=24\end{array}$ \\
\hline Sex & $13 \mathrm{~F} / 12 \mathrm{M}$ & $13 \mathrm{~F} / 12 \mathrm{M}$ & $15 \mathrm{~F} / 9 \mathrm{M}$ \\
\hline SES ${ }^{a}$ & $14 \mathrm{LM}-/ 11 \mathrm{M}+$ & 11 LM/14 M+ & $8 \mathrm{LM} / 16 \mathrm{M}+$ \\
\hline Age in years & $\begin{array}{r}8.35 \pm 0.31 \\
(7.83-9.00)\end{array}$ & $\begin{array}{c}8.29 \pm 0.42 \\
(7.75-9.50)\end{array}$ & $\begin{array}{l}8.29 \pm 0.31 \\
(7.83-8.75)\end{array}$ \\
\hline Handedness ${ }^{b}$ & $\begin{array}{c}84.80 \pm 29.53 \\
(-35-100)\end{array}$ & $\begin{array}{c}83.80 \pm 30.25 \\
(-40-100)\end{array}$ & $\begin{array}{c}71.25 \pm 55.37 \\
(-100-100)\end{array}$ \\
\hline Full-scale $I^{c}$ & $\begin{array}{c}95.44 \pm 12.32 \\
(74-113)\end{array}$ & $\begin{array}{c}93.60 \pm 13.40 \\
(74-120)\end{array}$ & $\begin{array}{c}96.29 \pm 14.73 \\
(74-125)\end{array}$ \\
\hline Verbal IQ & $\begin{array}{c}97.00 \pm 14.89 \\
(69-124)\end{array}$ & $\begin{array}{c}94.72 \pm 11.96 \\
(72-116)\end{array}$ & $\begin{array}{c}95.58 \pm 13.43 \\
(70-120)\end{array}$ \\
\hline Performance IQ & $\begin{array}{c}97.16 \pm 11.59 \\
(75-118)\end{array}$ & $\begin{array}{c}96.08 \pm 13.89 \\
(70-119)\end{array}$ & $\begin{array}{c}99.08 \pm 15.28 \\
(74-132)\end{array}$ \\
\hline \multicolumn{4}{|c|}{ Purdue pegboard test $^{d}$} \\
\hline Preferred hand & $\begin{array}{c}24.80 \pm 3.37 \\
(18-30)\end{array}$ & $\begin{array}{c}23.60 \pm 3.92 \\
(16-30)\end{array}$ & $\begin{array}{c}25.17 \pm 3.38 \\
(20-32)\end{array}$ \\
\hline Non-preferred hand & $\begin{array}{c}22.64 \pm 2.50 \\
\quad(16-26)\end{array}$ & $\begin{array}{c}21.92 \pm 2.97 \\
(16-28)\end{array}$ & $\begin{array}{c}23.08 \pm 4.41 \\
(14-32)\end{array}$ \\
\hline Both hands & $\begin{array}{c}18.08 \pm 3.19 \\
\quad(12-24)\end{array}$ & $\begin{array}{c}16.88 \pm 3.32 \\
(10-24)\end{array}$ & $\begin{array}{c}18.67 \pm 3.85 \\
(8-28)\end{array}$ \\
\hline \multicolumn{4}{|c|}{ Grooved pegboard test ${ }^{d}$} \\
\hline Preferred hand & $\begin{array}{c}18.16 \pm 3.60 \\
(9-25)\end{array}$ & $\begin{array}{c}16.62 \pm 3.87 \\
(10-26)\end{array}$ & $\begin{array}{c}18.37 \pm 3.72 \\
(10-26)\end{array}$ \\
\hline Non-preferred hand & $\begin{array}{c}16.78 \pm 3.79 \\
(10-25)\end{array}$ & $\begin{array}{c}14.57 \pm 3.48 \\
(8-22)\end{array}$ & $\begin{array}{c}16.36 \pm 4.11 \\
(7-24)\end{array}$ \\
\hline
\end{tabular}

Range in parentheses. ${ }^{a}$ SES as used in the Portuguese public school system: low or middle low ( $L M-)$ if children get free or price-reduced school meals, and middle or higher $(\mathrm{M}+)$ if no price reduction in school meals; 'b Edinburgh Handedness Inventory; ' Wechsler Intelligence Scale for Children - WISC-III, Portuguese version; dpegs/min. tasks (see below), we considered the ratio of pegs over time and used the number of pegs (or peg pairs) per minute as the measure of motor performance. The Grooved Pegboard test also requires participants to insert pegs on a board, but the holes are shaped in different orientations (key holes) and the pegs must be rotated accordingly in order to enter the key hole. This test provides a measure of manipulative dexterity that taxes visuo-spatial processing in addition to eye-hand coordination and motor speed. The board consists of a $5 \times 5$ matrix of key holes with different orientations, and the task is to be performed unimanually with the preferred hand and with the non-preferred one. The instruction is that the pegs should be inserted as quickly as possible. Based on age-related norms (Trites, 1989), we required children to fill the two first rows of the pegboard $(2 \times 5$ matrix; total of 10 pegs) with each hand, as quickly as possible. As the dependent measure, we took the number of pegs inserted per minute.

\section{Training}

The music and basketball training programs were prepared specifically for this study with the collaboration of two professional age-appropriate teachers, one specialized in music and the other in sports/basketball. Both programs consisted of structured groups of learning activities adapted to elementary school children with no prior systematic music or basketball instruction, and were organized to fit into the schedule of regular and extra-curricular (enrichment) school activities as two 90min collective sessions per week. They were conceived to be analogous regarding difficulty, expected progression along time and motivational aspects; for example, both programs included public presentations (musical performance/basketball game) for school and local communities. Thanks to an agreement protocol with local school authorities, the programs were provided to the children in the context of their school attendance and no extra fee was required.

The music training program used an Orff-based approach to initiate children into music knowledge and skill. The program was structured into four main areas: music awareness, elementary music concepts, rhythm and pitch skills, and instrumental and vocal performance (see Supplementary Table S1). Auditory and conceptual work was combined with collective Orff-type music practice such that music concepts were taught and practiced through instrumental playing, singing, or movement. Activities consisted mainly of collective instrumental practice using descant recorder, drums, xylophones and metallophones; singing, body percussion and unpitched percussion instruments were also included, though less frequently. In order to create challenging sound environments, all activities were planned to include at least three different sound sources. Children tried out various Orff instruments for a given music piece, and more complex melodic and rhythmic patterns were introduced according to their progress.

The sports training program consisted of basketball practice, including technical knowledge and skill. Activities were also organized into four areas: physical fitness, game relevant motor coordination (upper and lower limbs, eye-hand coordination), 
team sports concepts and schemes, and tactical planning (see Supplementary Table S2). Physical fitness activities and coordination exercises progressed from general to basketballoriented. Training emphasized the development of coordination skills and visuo-spatial performance as an individual player, but also at the collective level as member of a team.

Each of the training programs was conducted by the same teacher throughout its entire duration. The music teacher was graduated in Music Education and a regular chamber orchestra practitioner. The sports teacher had a degree in Physical Education and Sports and was a professional basketball team coach. Both of them had more than 10 years of professional experience with elementary school children.

\section{Procedure}

Prior to the start of data collection, the children's parents completed a questionnaire on demographic characteristics, on their children's previous experience regarding music and sports, namely basketball, training, and also on other current extra-curricular activities they might have been engaged with. Information regarding support received from the national social security system was also gathered, that is, whether children had the right to free or price-reduced school meals, or if no such reduction was applicable. This was used as a proxy for socioeconomic status that was classified as low or middle-low in the former case, and middle or higher in the second case.

At pre-test, post-test and follow-up, children were individually assessed in a quiet room of their school. The WISC-III battery was administered in one session by an experienced child psychologist. The handedness and motor tests were completed in different sessions and were administered by a trained research assistant. In each of the study phases, half of the children of each group started with the Purdue pegboard test and the other half with the Grooved pegboard test. The order of the hand (preferred vs. non-preferred) was counterbalanced in each group and test. Bimanual performance of the Purdue pegboard test was always assessed after both Purdue unimanual subtests. To ensure that the instructions had been properly understood, children were given a training trial of each test and subtest. Also, to exclude potential artifacts due to stress, time only started counting when the child picked up the first peg (Hermsdörfer et al., 1999). During unimanual tasks, the unused hand was placed over the table to the side of the pegboard.

Both training groups started their music and basketball sessions after pre-test assessment at the start of the school year (October). Training took place twice a week in 90-min sessions and lasted for almost a school year, from October until May with interruptions for school holidays - so in practice, there were 24 weeks of training. Children in the music group gave a public performance at the end of the school year, and children in the sports group participated in a basketball tournament. Children in the standard control group were engaged in different types of extra-curricular school activities not including systematic music or basketball training. Both types of training were planned to be available in the following year so that interested children from the standard control group could participate in them. Finally, children from the three groups completed a follow-up assessment 4 months later, at the beginning of the next school year.

\section{RESULTS}

The results obtained for each group in the motor performance tests before and after training including follow-up are shown in Figure 1. In a set of preliminary analyses, we checked if there were no group differences in motor skills prior to training, and confirmed that there were none: for the Purdue pegboard test with the preferred hand, $F_{(2,71)}=1.30, p=0.28$, with the nonpreferred hand, $F<1$, and with both hands, $F_{(2,71)}=1.71$, $p=0.19$; for the Grooved pegboard test with the preferred hand, $F_{(2,71)}=1.62, p=0.21$, and the non-preferred hand, $F_{(2,71)}=2.38, p=0.10$. We then analyzed the effects of training by calculating repeated measures ANOVAs with Group (music, sports and control) as between-subjects factor and Time (pre-test, post-test and follow-up) as within-subjects factor. Differences between groups at each time point and progression across time points were tested using post hoc pairwise-comparisons with the Bonferroni correction. For each pairwise comparison we report the mean difference $M$, standard error $S E$, $p$-value and Cohen's $d$ effect size.

Performance on the Purdue pegboard test with the preferred hand improved across time (main effect of Time: $F_{(2,142)}=44.45$, $\left.p<0.001, \eta_{p}^{2}=0.39\right)$, with a significant increase from pre- to post-test $(M=2.67 \mathrm{pegs} / \mathrm{min}, S E=0.44, p<0.001, d=0.75)$ and from post-test to follow-up $(M=1.21 \mathrm{pegs} / \mathrm{min}, S E=0.39$, $p<0.01, d=0.35)$. The main effect of Group was also significant, $F_{(2,71)}=8.69, p<0.001, \eta_{p}^{2}=0.20$ : the music group outperformed both the sports $(M=2.93$ pegs $/ \mathrm{min}, S E=0.80$, $p=0.001, d=1.03)$ and the control $(M=2.86 \mathrm{pegs} / \mathrm{min}, S E=0.81$, $p<0.01, d=1.01)$ groups. More important to the present study, the interaction Time $x$ Group was also significant, $F_{(4,142)}=7.35$, $p<0.001, \eta_{p}{ }^{2}=0.17$. As is clear from Figure 1A, the greatest increase from pre-test to post-test occurred in the music group, $M=5.04 \mathrm{pegs} / \mathrm{min}, S E=0.76, p<0.001, d=1.45$. The sports group also improved significantly from pre- to post-test, but not so markedly: $M=2.56$ pegs $/ \mathrm{min}, S E=0.76, p<0.01$, $d=0.66$. In the control group, the increase was not significant $(M=0.42 \mathrm{pegs} / \mathrm{min}, S E=0.77, p=1.00, d=0.13)$. Differences from post-test to follow-up were not significant in either group $\left(p_{s}>0.05\right)$. Looking at differences between groups, the same pattern of superiority of music training emerges: the music group significantly outperformed the sports group at post-test, $M=3.68$ pegs $/ \mathrm{min}, S E=0.99, p=0.001, d=0.99$, and follow-up, $M=3.92$ pegs $/ \mathrm{min}, S E=0.99, p=0.001, d=1.17$, and the control group at post-test, $M=4.26$ pegs $/ \mathrm{min}, S E=1.00, p<0.001, d=1.29$, and follow-up, $M=4.70$ pegs $/ \mathrm{min}, S E=1.00, p<0.001, d=1.33$.

With the non-preferred hand, performance on the Purdue pegboard test showed a significant main effect of Time, $F_{(2,142)}=34.85, p<0.001, \eta_{p}^{2}=0.33$, and an interaction of Group with Time, $F_{(4,142)}=3.98, p=0.004, \eta_{p}^{2}=0.10$; the main effect of Group was barely significant $\left[F_{(2,71)}=3.14\right.$, $\left.p=0.05, \eta_{p}{ }^{2}=0.08\right]$. More specifically, performance improved from pre- to post-test, $M=2.63$ pegs $/ \mathrm{min}, S E=0.41, p<0.001$, 


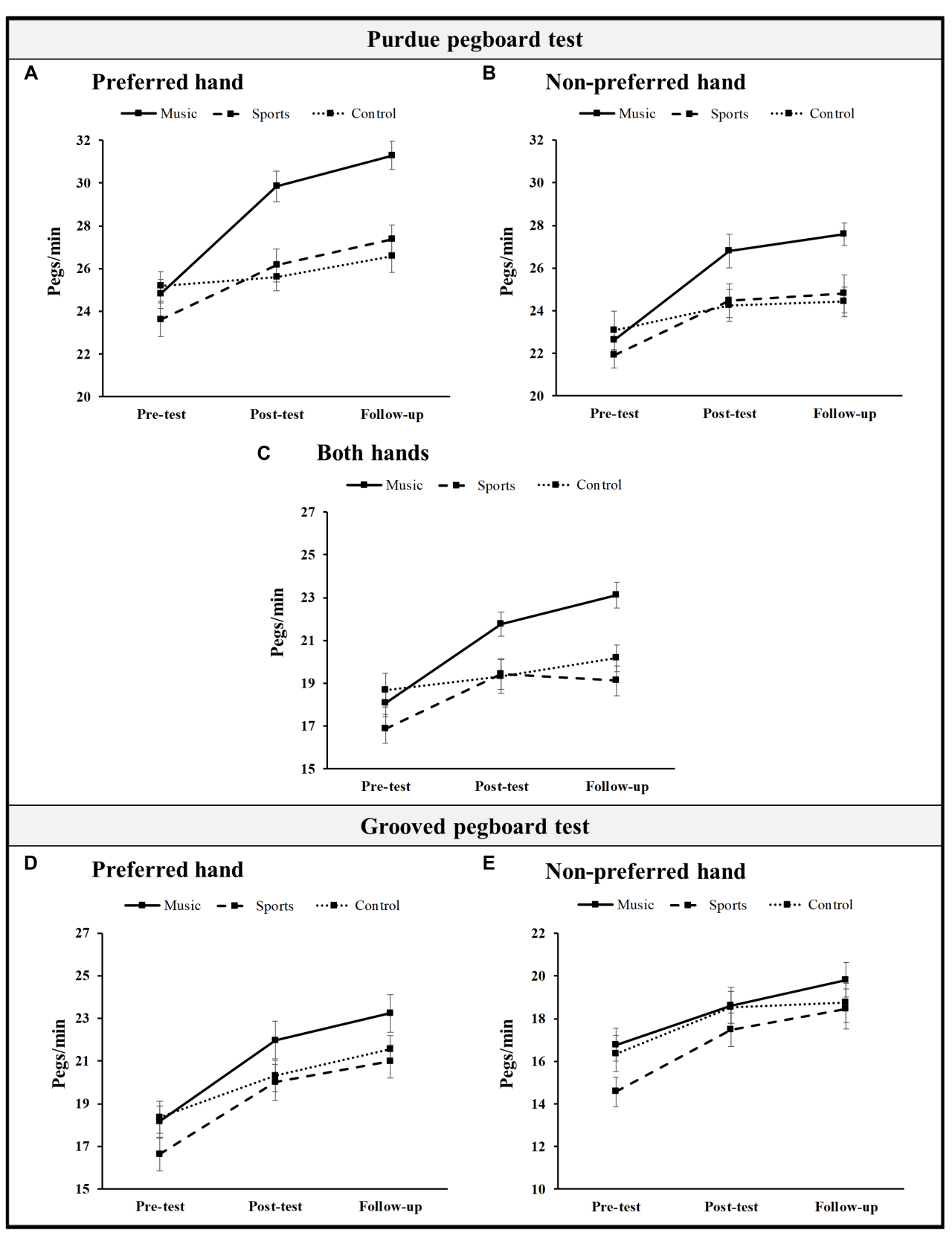

FIGURE 1 | Mean scores (pegs per minute) in the Purdue Pegboard test with the preferred hand (A), non-preferred hand (B), or both hands (C), and in the Grooved pegboard test with the preferred hand (D), and non-preferred hand (E), for the music, sports, and control groups at pre-test, post-test, and follow-up. Error bars indicate SEM

$d=0.73$, but not from post-test to follow-up, $M=0.43 \mathrm{pegs} / \mathrm{min}$, $S E=0.38, p=0.77, d=0.11$; both the music group and the sports group improved significantly from pre-test to post-test, but the improvement was greater in the music group, $M=4.16 \mathrm{pegs} / \mathrm{min}$, $S E=0.71, p<0.001, d=1.27$, than in the sports group, $M=2.56$ pegs $/ \mathrm{min}, S E=0.71, p<0.01, d=0.73$. There were no significant differences in the control group from pre- to post-test $(M=1.17$ pegs $/ \mathrm{min}, S E=0.72, p=0.33, d=0.29)$. Neither group had significant improvements from post-test to follow-up $\left(p_{s}>0.05\right)$.
Turning now to differences between groups, the only significant ones were at follow-up, where the music group outperformed the sports group, $M=2.80$ pegs $/ \mathrm{min}, S E=1.01, p=0.02, d=0.77$, and the control group, $M=3.18$ pegs $/ \mathrm{min}, S E=1.02, p<0.01$, $d=1.04$.

Bimanual performance on the Purdue pegboard test showed significant main effects of Time, $F_{(2,142)}=30.60, p<0.001$, $\eta_{p}^{2}=0.30$, Group, $F_{(2,71)}=5.50, p=0.006, \eta_{p}{ }^{2}=0.13$, and the double interaction, $F_{(4,142)}=4.68, p=0.001, \eta_{p}^{2}=0.12$. As 
in the unimanual subtests, improvements from pre- to post-test occurred in the music group, $M=3.68 \mathrm{pegs} / \mathrm{min}, S E=0.69$, $p<0.001, d=1.24$, and in the sports group, $M=2.56 \mathrm{pegs} / \mathrm{min}$, $S E$ 0.69, $p<0.01, d=0.75$, but not in the control group $(M=0.67 \mathrm{pegs} / \mathrm{min}, S E=0.71, p=1.00, d=0.17)$; between post-test to follow-up, there were no improvements $\left(p_{s}>0.05\right)$. Between-group comparisons again showed a superiority of the music group, that outperformed the sports group at follow-up, $M=4.00 \mathrm{pegs} / \mathrm{min}, S E=0.89, p<0.001, d=1.24$, and the control group at post-test, $M=2.43 \mathrm{pegs} / \mathrm{min}, S E=0.97, p=0.04$, $d=0.73$, and follow-up, $M=2.95$ pegs $/ \mathrm{min}, S E=0.90, p=0.005$, $d=0.99$. A barely significant advantage of the music group when compared to the sports group was also observed at post-test, $M=2.32 \mathrm{pegs} / \mathrm{min}, S E=0.96, p=0.06, d=0.73$.

Performance on the Grooved pegboard test had a different pattern of results. In the subtest with the preferred hand, only Time had a significant effect, $F_{(2,142)}=80.92, p<0.001, \eta_{p}^{2}=0.53$; neither Group $\left[F_{(2,71)}=1.88, p=0.16, \eta_{p}^{2}=0.05\right]$ nor the interaction Time $\mathrm{x}$ Group $\left[F_{(4,142)}=1.81, p=0.13, \eta_{p}^{2}=0.05\right]$ reached significance. There was an increase from pre- to posttest, $M=3.04$ pegs $/ \mathrm{min}, S E=0.34, p<0.001, d=0.78$, and from post-test to follow-up, $M=1.17 \mathrm{pegs} / \mathrm{min}, S E=0.34, p<0.01$, $d=0.29$. With the non-preferred hand, again only the effect of Time was significant, $F_{(2,142)}=41.82, p<0.001, \eta_{p}^{2}=0.37$; performance increased from pre- to post-test, $M=2.29 \mathrm{pegs} / \mathrm{min}$, $S E=0.32, p<0.001, d=0.59$, and, barely significantly, from post-test to follow-up, $M=0.80$ pegs $/ \mathrm{min}, S E=0.35, p=0.07$, $d=0.19$. Neither Group $\left[F_{(4,142)}=1.18 ; p=0.31 ; \eta_{p}^{2}=0.03\right]$ nor the interaction reached significance $\left[F_{(4,142)}=1.02, p=0.40\right.$, $\left.\eta_{p}^{2}=0.03\right]$.

In order to take a closer look at how training might have affected performance, we examined the distribution of individual results across performance levels. This analysis only considered the subtests showing the interaction of Time with Group (the Purdue subtests). Furthermore, we compared directly pre-test with follow-up as there was no evidence of performance changes from post-test to follow-up. Performance levels were defined in accordance with the normative values provided by Gardner and Broman (1979): below the 20th percentile (low), between the 20th and the 80th percentiles (middle), and above the 80th percentile (high). The distribution of children from the music, sports and control groups as a function of performance level at pre-test and follow-up is illustrated in Figure 2. At pretest, there were no significant differences between groups on the proportion of children in each performance level in the Purdue preferred hand $\left[\chi^{2}{ }_{(2)}=0.83, p=0.93, V=0.11\right]$ and bimanual $\left[\chi^{2}(2)=1.63, p=0.80, V=0.15\right]$ subtests; with the non-preferred hand, the control group had a greater proportion of high-performing children than the music group, $\chi^{2}{ }_{(2)}=10.28$, $p=0.04, V=0.37$. At follow-up, a significantly higher proportion of children from music group reached a high performance level when compared to sports and control groups, both in the preferred hand, $\chi^{2}{ }_{(2)}=17.90, p=0.001, V=0.49$, and bimanual subtests, $\chi^{2}{ }_{(2)}=21.23, p<0.001, V=0.54$. Although no significant differences were observed in the proportion of children from each group in the Purdue non-preferred hand subtest $\left[\chi^{2}{ }_{(2)}=5.00, p=0.29, V=0.26\right]$, almost thirty percent (28\%) of the children from the music group reached the highperforming level, compared to $16 \%$ from the sports group and $8 \%$ from the control group. None of the children from the music group scored at a low performance level in any of the Purdue subtests.

\section{DISCUSSION}

The present study investigated how children's fine motor abilities are influenced by music training. Unlike previous studies that relied on instrumental music instruction for at least 2 years, we focused on collective Orff-based music practice for a relatively short duration (24 weeks), and included an active control group undertaking basketball training in addition to a standard control group. Children were 8 -year-olds attending the 3 rd grade of public schools from mostly low-income communities. We measured eye-hand coordination, motor speed and bimanual coordination with the Purdue pegboard test, and manipulative dexterity with the Grooved pegboard test, before training, immediately after training finished at the end of the school year, and at the start of the following school year (follow-up). Overall, we found that children from the three groups improved in their motor abilities from pre-test to follow-up, but progress was not of the same magnitude for all of them: it depended on type of training and on the type of test. Critical to the goals of this study, there was an advantage of music training over sports training or no-training in enhancing bimanual coordination and manual dexterity that persisted at follow-up 4 months later. Sports training was also associated with significant progress across test points. These main findings (music training advantage; the case of sports training; overall progress) will be discussed below.

The advantage of music training showed up not only in bimanual coordination, as we had expected on the basis of the characteristics of Orff-based music practice, but also in manual dexterity. Musically trained children outperformed children from the sports and control groups at post-test and follow-up in the Purdue pegboard test with both hands (bimanual coordination) and with the dominant hand (manual dexterity). In both cases, $d$ effect sizes ranged from 0.73 to 1.29 at post-test and were slightly greater at follow-up, from 0.99 to 1.33 . Furthermore, at followup most children from the music group were performing at the upper level (80th percentile and up), and none in the lowest 20th percentile. With the non-dominant hand, progress from pre-test to post-test was also more marked in the music group $(d=1.27)$ than in the sports $(d=0.73)$ and the control $(d=0.29, n s)$ groups, but interestingly it was only at follow-up that significant between-groups differences emerged, with an advantage of the music over the sports group with a $d$ of 0.77 and over the control group with a $d$ of 1.04 . These findings clearly indicate that the effect of music training in enhancing manual dexterity and bimanual coordination were not a short-lived consequence of having played Orff instruments in the previous month or so. It is particularly revealing that the superiority of music training was maintained after 4 months, namely because this included summer 


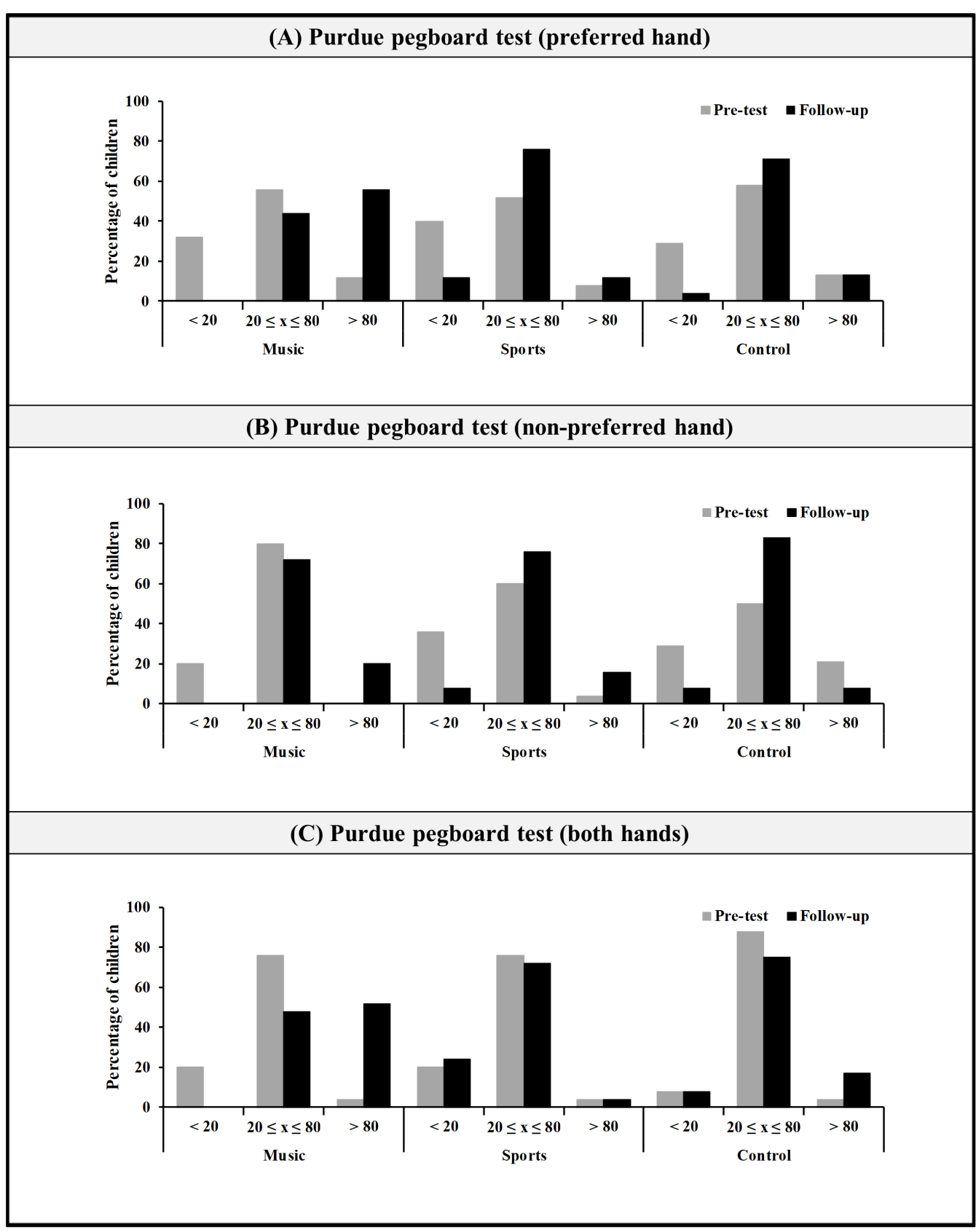

FIGURE 2 | Distribution of children from the three groups according to performance levels: low ( $<20$ th percentile), middle (20th-80th percentile), and high ( $>80$ th percentile), in the Purdue pegboard test with the preferred hand (A), non-preferred hand (B), and both hands (C).

holidays and thus an interval of almost 2 months from the typical school-like activities involving fine motor activities such as writing. We interpret this finding as indicating the stability of the improvement in fine motor abilities elicited by music training.

The findings described above come from the Purdue pegboard test and do not extend to the Grooved pegboard test that has a strong component of manipulative dexterity. Indeed, both tests involve different movement subcomponents: picking up the peg, moving the peg to the hole, placing the peg in the hole, and moving the hand to pick up the next peg (Roy et al., 2003), but critical to the Grooved pegboard test is visuo-spatial processing and manipulative ability that as such are not required, or are secondary, in the Purdue pegboard test. In the Purdue pegboard test, the third sub-component accounts for the largest amount of motor task time, and is probably the one that most aptly captures a developmental parameter (e.g., Annett et al., 1979). It is also likely that it is the one that accounts for our results as it is most directly related to Orff-based training. Apart from 
bimanual coordination, Orff-based music practice involves eyehand coordination and the ability to integrate speed and precision with controlled discrete movements, that is, event-based timing mechanisms (Baer et al., 2013; Braun Janzen et al., 2014). For instance, drumming, playing the xylophone or playing drones ${ }^{1}$, all three afford training opportunities to hit a target, maintain timing and movement accuracy, and coordinate both hands or upper limbs (in drone execution, both arms move synergistically for a relatively extended period of time). The placement of the peg may be considered homologous to 'hit the target' in Orff practices where, as in the Purdue pegboard test, it is not preceded by a manipulative movement. So it is possible that the effects that we have found on the Purdue pegboard test are an expression of near transfer from Orff-based music training to bimanual coordination, eye-hand coordination, and motor speed.

Another possibility is that the music advantage is not driven solely by movement-related processes, and that the benefits to fine motor skills arise via attentional mechanisms that are an important facet of music training (Duke et al., 2011; Strait et al., 2015). Music practice is a multisensory motor experience in which the player integrates sequential movements into a rhythmic and expressive context. The refinement of the movement may be achieved not only through repetition, or practice, proper, but also as a result of (or concomitantly with) the auditory feedback that allows for precise timing of motor control and gradually shapes performance (Schneider et al., 2010; Bevilacqua et al., 2016). The notion that music training is associated with enhanced control of attention has received empirical support from behavioral and neuroimaging studies (e.g., Gaser and Schlaug, 2003). For example, in comparison with non-musicians, orchestra musicians have better performance on selective, divided, and sustained attention tasks (Rodrigues et al., 2014), and a more balanced visual attentional capacity (Patston et al., 2007). However, as we did not manipulate attention our findings do not allow us to disentangle between potential motorbased or attention-based processes as the primary mechanism leading to the advantage of music training for fine motor abilities. This is a question to be addressed in future studies.

Irrespective of the mechanisms subtending the observed effects, our finding of an improvement in children's fine motor abilities following music practice agrees with previous ones from Costa-Giomi's (2005) and Forgeard et al.'s (2008) studies. Interestingly, fine motor abilities were measured differently in each of these studies and the improvement that was observed had some degree of specificity. In Costa-Giomi's study, it was response speed subtests, and not visuo-motor coordination or dexterity subtests, that carried the effect of the piano lessons; and in Forgeard et al.'s study, children had been learning how to play keyboard and/or string instruments and what was measured was finger independence and coordination, a certainly trained component during this sort of instrumental practice. In our study, the improvement that was specific to the music group included bimanual coordination, and eye-hand coordination and response speed, but not manipulative dexterity. So music training

\footnotetext{
${ }^{1}$ A drone is a harmonic or monophonic effect or accompaniment where a note, a set of notes or a chord is continuously sounded throughout most or all of a piece.
}

appears to induce improvement of fine motor abilities whose characteristics are either basic and general (motor speed) or linked to the music practiced (finger independence, bimanual coordination). Another important conclusion that may be drawn from the comparison of these studies and ours is that music training does not need to be of long duration nor in the form of individual classes of instrumental instruction to impact on fine motor abilities: collective Orff-based music practice for 24 weeks was sufficient to bring about positive effects that were stable for at least 4 months after training.

The second set of results worth mentioning concerns sports training, which in this study consisted of basketball. This sport involves gross motor activities like running and jumping that hardly bear any similarity with music training, but it also includes eye-hand coordination, bimanual coordination and manual dexterity (e.g., Park et al., 2011; Tsang et al., 2014) that resemble some of the aspects of music training. Additionally, both sports and music are bound to have a positive influence in a variety of domains (e.g., Martin, 2008; Cabane et al., 2016) including well-being (Lubans et al., 2012; Welch et al., 2014). So it is not surprising that, as we had expected, the sports group improved significantly from pre-test to post-test in the same abilities as the music group. However, the effect sizes were smaller ( $>1$ in the music group, around 0.7 in the sports group). In between-group comparisons the advantages of the sports group over the control group were not significant, whereas the ones from the music group were. These results show that basketball training was not as effective as music training in improving children's fine motor abilities. It is possible that in order to elicit substantial, or more marked, training effects sports and music require different amounts of practice, and/or have different time courses. If that is the case, longer or more intensive training in basketball might be necessary to achieve similar effects as music training. That music and sports/movement training may have homologous effects, with an advantage to music in some cases, is indeed what has been recently found in studies of science-based music rehabilitation of motor impairment (Moumdjian et al., 2017). For example, Schneider et al. (2010) have shown that music training was more effective than a functional motor program for the recovery of motor impairments in stroke patients. Findings from timing entrainment also suggest that music might have a more a powerful effect than sports, at least in the sense that music expertise boosts both event- and emergent-timing whereas the benefits from sports expertise appear to be confined to emergent timing (Braun Janzen et al., 2014). This might be related to different weightings that movement and flow, on the one hand, and finer-grained vs. coarser-grained events, on the other hand, might have in doing sports in comparison with playing music: maybe timing and control of fast paced and finegrained events (such as key presses in playing piano) are more critical to music than to sports. However, level of expertise and specific characteristics of the type of sport and music under comparison would also have to be examined in order to move from speculation to established finding. Our study focused on a relatively narrow set of fine-motor abilities, and thus it does not allow us to make that step. Indeed, a limitation of this study is to not have included the assessment of gross motor abilities that 
might be closer to basketball training than the fine motor abilities examined.

The final and third set of results is progress from pre-test to post-test and follow-up. In all subtests of the Purdue and Grooved pegboard tests we found a significant effect of time that suggests a pattern of general improvement in fine motor abilities. This was particularly marked in the tests of manipulative dexterity (Grooved pegboard test), where no interaction with group and no specific effects of music or sports training were found. Although direct comparisons of effects sizes from different tests should be interpreted with caution, it is interesting that the improvement from pre-test to post-test in manipulative dexterity was greater than that of the control group in manual dexterity (Purdue pegboard test). This apparently stronger progress in manipulative dexterity across time is consistent with Serrien et al.'s (2014) suggestion of heterogeneity in the development of motor abilities, where certain of them have a plateau at around 8 years of age, whereas others continue to improve. This would be the case of our findings in the Grooved pegboard test, where the progress from pre- to post-test and follow-up was not linked to specific training and is likely due to the development in manipulative ability that occurs at this age. However, the design of our study does not allow to firmly conclude on this issue. It should also be addressed in future studies.

Summing up, the present study has shown that collective Orffbased music training improves children's fine motor abilities, namely bimanual coordination and manual dexterity. To the best of our knowledge, this is the first time that this type of music training effect was given a stringent test by adopting a longitudinal approach with an active control group of basketball training as well as a standard passive control group. Importantly, training was embedded in regular school activities, and children came from mostly low-income communities. As such, the findings from the present study open an exciting prospect for educational contexts. Music-related benefits can be achieved at a good cost/benefit ratio, and may be especially relevant for children from low-income communities who often do not have the opportunity to be part of these activities outside of school. School-based programs may thus be an important means for

\section{REFERENCES}

Annett, J., Annett, M., Hudson, P. T., and Turner, A. (1979). The control of movement in the preferred and non-preferred hands. Q. J. Exp. Psychol. 31, 641-652. doi: 10.1080/14640747908400755

Baer, L., Thibodeau, J., Gralnick, T., Li, K., and Penhune, V. (2013). The role of musical training in emergent and event-based timing. Front. Hum. Neurosci. 7:191. doi: 10.3389/fnhum.2013.00191

Benz, S., Sellaro, R., Hommel, B., and Colzato, L. S. (2016). Music makes the world go round: the impact of musical training on non-musical cognitive functions a review. Front. Psychol. 6:2023. doi: 10.3389/fpsyg.2015.02023

Bevilacqua, F., Boyer, E. O., Françoise, J., Houix, O., Susini, P., Roby-Brami, A., et al. (2016). Sensori-motor learning with movement sonification: perspectives from recent interdisciplinary studies. Front. Neurosci. 1:385. doi: 10.3389/fnins. 2016.00385

Braun Janzen, T., Thompson, W. F., Ammirante, P., and Ranvaud, R. (2014). Timing skills and expertise: discrete and continuous timed movements among musicians and athletes. Front. Psychol. 5:1482. doi: 10.3389/fpsyg.2014.01482 foster the academic trajectory and the quality of life of all children (e.g., Eerola and Eerola, 2014; Kraus et al., 2014; Welch et al., 2014). As stressed by Valencia (2010), structural measures are especially valuable to reduce social inequality without falling prey to a deficit model of development where low-income might be inadvertently equated with at-risk. Training programs such as the one presented in this study may thus contribute to the ongoing discussion about educational practices suitable for all.

\section{AUTHOR CONTRIBUTIONS}

SLC and MM designed the study. MM and LN collected the data. $\mathrm{PR}, \mathrm{OV}$, and SLC supervised data-analysis and interpreted the data. MM analyzed and interpreted the data. SLC, MM, and LN wrote the manuscript. All authors read and approved the final version of the manuscript.

\section{FUNDING}

This work was supported by grants from BIAL Foundation (304/2014) and Portuguese Foundation for Science and Technology (CPUP UID/PSI/00050/2013 and SFRH/BD/99622/2014). The training programs were funded by Câmara Municipal de Matosinhos.

\section{ACKNOWLEDGMENTS}

We thank the school administration, teachers, parents, and very specially all the children who took part in the study.

\section{SUPPLEMENTARY MATERIAL}

The Supplementary Material for this article can be found online at: https://www.frontiersin.org/articles/10.3389/fpsyg. 2018.02616/full\#supplementary-material

Cabane, C., Hille, A., and Lechner, M. (2016). Mozart or pele? The effects of adolescents' participation in music and sports. Labour Econ. 41, 90-103. doi: 10.1016/j.labeco.2016.05.012

Chang, X., Wang, P., Zhang, Q., Feng, X., Zhang, C., and Zhou, P. (2014). The effect of music training on unimanual and bimanual responses. Music. Sci. 18, 464-472. doi: 10.1177/10298649145 47147

Costa-Giomi, E. (2005). Does music instruction improve fine motor abilities? Ann. N. Y. Acad. Sci. 1060, 262-264. doi: 10.1196/annals.1360.053

De Poel, H. J., Peper, C. L. E., and Beek, P. J. (2008). Laterally focused attention modulates asymmetric coupling in rhythmic interlimb coordination. Psychol. Res. 72, 123-137. doi: 10.1007/s00426-006-0096-9

Degé, F., and Schwarzer, G. (2011). The effect of a music program on phonological awareness in preschoolers. Front. Psychol. 2:124. doi: 10.3389/fpsyg.2011.00124

Degé, F., Wehrum, S., Stark, R., and Schwarzer, G. (2011). The influence of two years of school music training in secondary school on visual and auditory memory. Eur. J. Dev. Psychol. 8, 608-623. doi: 10.1080/17405629.2011. 590668 
Dey, A., Barnsley, N., Mohan, R., McCormick, M., McAuley, J. H., and Moseley, G. L. (2012). Are children who play a sport or a musical instrument better at motor imagery than children who do not? Br. J. Sports Med. 46, 923-926. doi: 10.1136/bjsports-2011-090525

Dinehart, L., and Manfra, L. (2013). Associations between low-income children's fine motor skills in preschool and academic performance in second grade. Early Educ. Dev. 24, 138-161. doi: 10.1080/10409289.2011.63 6729

Duke, R. A., Cash, C. D., and Allen, S. E. (2011). Focus of attention affects performance of motor skills in music. J. Res. Music Educ. 59, 44-55. doi: 10. $1177 / 0022429410396093$

Eerola, P. S., and Eerola, T. (2014). Extended music education enhances the quality of school life. Music Educ. Res. 16, 88-104. doi: 10.1080/14613808.2013.829428

Elbert, T., Pantev, C., Wienbruch, C., Rockstroh, B., and Taub, E. (1995). Increased cortical representation of the fingers of the left hand in string players. Science 270, 305-307. doi: 10.1126/science.270.5234.305

Forgeard, M., Winner, E., Norton, A., and Schlaug, G. (2008). Practicing a musical instrument in childhood is associated with enhanced verbal ability and nonverbal reasoning. PLoS One 3:e3566. doi: 10.1371/journal.pone.000 3566

Gardner, R. A., and Broman, M. (1979). The purdue pegboard: normative data on 1334 school children. J. Clin. Child Adolesc. Psychol. 8, 156-162. doi: 10.1080/ 15374417909532912

Gaser, C., and Schlaug, G. (2003). Brain structures differ between musicians and non-musicians. J. Neurosci. 23, 9240-9245. doi: 10.1523/JNEUROSCI.23-2709240.2003

Grissmer, D., Grimm, K. J., Aiyer, S. M., Murrah, W. M., and Steele, J. S. (2010). Fine motor skills and early comprehension of the world: two new school readiness indicators. Dev. Psychol. 46, 1008-1017. doi: 10.1037/a0020104

Hermsdörfer, J., Laimgruber, K., Kerkhoff, G., Mai, N., and Goldenberg, G. (1999). Effects of unilateral brain damage on grip selection, coordination, and kinematics of ipsilesional prehension. Exp. Brain Res. 128, 41-51. doi: 10.1007/ s002210050815

Ilari, B. S., Keller, P., Damasio, H., and Habibi, A. (2016). The development of musical skills of underprivileged children over the course of 1 year: a study in the context of an El Sistema-inspired program. Front. Psychol. 7:62. doi: 10.3389/fpsyg.2016.00062

Jäncke, L., Schlaug, G., and Steinmetz, H. (1997). Hand skill asymmetry in professional musicians. Brain Cogn. 34, 424-432. doi: 10.1006/brcg.1997.0922

Kraus, N., Slater, J., Thompson, E. C., Hornickel, J., Strait, D. L., Nicol, T., et al. (2014). Music enrichment programs improve the neural encoding of speech in at-risk children. J. Neurosci. 34, 11913-11918. doi: 10.1523/JNEUROSCI.188114.2014

Lampe, R., Thienel, A., Mitternacht, J., Blumenstein, T., Turova, V., and AlvesPinto, A. (2015). Piano training in youths with hand motor impairments after damage to the developing brain. Neuropsychiatr. Dis. Treat. 11, 1929-1938. doi: 10.2147/NDT.S84090

Lavrysen, A., Heremans, E., Peeters, R., Wenderoth, N., Feys, P., Swinnen, S. P., et al. (2012). Hemispheric asymmetries in goal-directed hand movements are independent of hand preference. NeuroImage 62, 1815-1824. doi: 10.1016/j.neuroimage.2012.05.033

Lavrysen, A., Heremans, E., Peeters, R., Wenderoth, N., Helsen, W. F., Feys, P., et al. (2008). Hemispheric asymmetries in eye-hand coordination. NeuroImage 39, 1938-1949. doi: 10.1016/j.neuroimage.2007.10.007

Lubans, D. R., Plotnikoff, R. C., and Lubans, N. J. (2012). A systematic review of the impact of physical activity programmes on social and emotional well-being in at-risk youth. Child Adolesc. Mental Health 17, 2-13. doi: 10.1111/j.1475-3588. 2011.00623.x

Martin, A. J. (2008). Motivation and engagement in music and sport: testing a multidimensional framework in diverse performance settings. J. Pers. 76, 135-170. doi: 10.1111/j.1467-6494.2007.00482.x

Matthews, T. E., Thibodeau, J. N., Gunther, B. P., and Penhune, V. B. (2016). The impact of instrument-specific musical training on rhythm perception and production. Front. Psychol. 7:69. doi: 10.3389/fpsyg.2016.00069

Moreno, S., Bialystok, E., Barac, R., Schellenberg, E. G., Cepeda, N. J., and Chau, T. (2011). Short-term music training enhances verbal intelligence and executive function. Psychol. Sci. 22, 1425-1433. doi: 10.1177/09567976114 16999
Moreno, S., Marques, C., Santos, A., Santos, M., Castro, S. L., and Besson, M. (2008). Musical training influences linguistic abilities in 8-year-old children: more evidence for brain plasticity. Cereb. Cortex 19, 712-723. doi: 10.1093/ cercor/bhn 120

Moumdjian, L., Sarkamo, T., Leone, C., Leman, M., and Feys, P. (2017). Effectiveness of music-based interventions on motricity or cognitive functioning in neurological populations: a systematic review. Eur. J. Phys. Rehabil. Med. 53, 466-482. doi: 10.23736/S1973-9087.16.04429-4

Oldfield, R. C. (1971). The assessment and analysis of handedness: the Edinburgh inventory. Neuropsychologia 9, 97-113. doi: 10.1016/0028-3932(71) 90067-4

Orsmond, G. I., and Miller, L. K. (1999). Cognitive, musical and environmental correlates of early music instruction. Psychol. Music 27, 18-37. doi: 10.1177/ 0305735699271003

Park, I. S., Lee, K. J., Han, J. W., Lee, N. J., Lee, W. T., and Park, K. A. (2011). Basketball training increases striatum volume. Hum. Mov. Sci. 30, 56-62. doi: 10.1016/j.humov.2010.09.001

Patston, L. L., Hogg, S. L., and Tippett, L. J. (2007). Attention in musicians is more bilateral than in non-musicians. Laterality 12, 262-272. doi: 10.1080/ 13576500701251981

Pellegrini, A. M., Andrade, E. C., and Teixeira, L. A. (2004). Attending to the nonpreferred hand improves bimanual coordination in children. Hum. Mov. Sci. 23, 447-460. doi: 10.1016/j.humov.2004.08.017

Roden, I., Kreutz, G., and Bongard, S. (2012). Effects of a school-based instrumental music program on verbal and visual memory in primary school children: a longitudinal study. Front. Neurosci. 6:572. doi: 10.3389/fpsyg.2012. 00572

Rodrigues, A. C., Loureiro, M., and Caramelli, P. (2014). Visual memory in musicians and non-musicians. Front. Hum. Neurosci. 8:424. doi: 10.3389/ fnhum.2014.00424

Rodrigues, P. C., Vasconcelos, O., Barreiros, J., and Barbosa, R. (2009). Manual asymmetry in a complex coincidence-anticipation task: handedness and gender effects. Laterality 14, 395-412. doi: 10.1080/13576500802469607

Roy, E. A., Bryden, P., and Cavill, S. (2003). Hand differences in pegboard performance through development. Brain Cogn. 53, 315-317. doi: 10.1016/ S0278-2626(03)00133-7

Schellenberg, E. G. (2004). Music lessons enhance IQ. Psychol. Sci. 15, 511-514. doi: 10.1111/j.0956-7976.2004.00711.x

Schlaug, G., Jäncke, L., Huang, Y., Staiger, J. F., and Steinmetz, H. (1995). Increased corpus callosum size in musicians. Neuropsychologia 33, 1047-1055. doi: 10. 1016/0028-3932(95)00045-5

Schlaug, G., Norton, A., Overy, K., and Winner, E. (2005). Effects of music training on the child's brain and cognitive development. Ann. N. Y. Acad. Sci. 1060, 219-230. doi: 10.1196/annals.1360.015

Schneider, S., Münte, T., Rodriguez-Fornells, A., Sailer, M., and Altenmüller, E. (2010). Music-supported training is more efficient than functional motor training for recovery of fine motor skills in stroke patients. Music Percept. 27, 271-280. doi: 10.1525/mp.2010.27.4.271

Serrien, D. J., Sovijärvi-Spapé, M. M., and Rana, G. (2014). Developmental changes in motor control: insights from bimanual coordination. Dev. Psychol. 50, 316-323. doi: 10.1037/a0032996

Simpkins, S. D., Vest, A. E., and Becnel, J. N. (2010). Participating in sport and music activities in adolescence: the role of activity participation and motivational beliefs during elementary school. J. Youth Adolesc. 39, 1368-1386. doi: 10.1007/s10964-009-9448-2

Strait, D. L., Slater, J., O’Connell, S., and Kraus, N. (2015). Music training relates to the development of neural mechanisms of selective auditory attention. Dev. Cogn. Neurosci. 12, 94-104. doi: 10.1016/j.dcn.2015.01.001

Tiffin, J. (1968). Purdue Pegboard Examiner Manual. Chicago, IL: Science Research Associates.

Trites, R. (1989). Grooved Pegboard Instruction Manual. Lafayette, LA: Lafayette Instrument.

Tsang, W. W., Fong, S. S., Cheng, Y. T., Daswani, D. D., Lau, H. Y., Lun, C. K., et al. (2014). The effect of vestibular stimulation on eye-hand coordination and postural control in elite basketball players. Am. J. Sports Sci. 2, 17-22. doi: 10.11648/j.ajss.20140202.12

Valencia, R. R. (2010). Dismantling Contemporary Deficit Thinking: Educational Thought and Practice. New York, NY: Routledge. 
Wechsler, D. (2003). Escala de Inteligência de Wechsler Para Crianças-3. ${ }^{a}$ Edição (WISC-III). Lisboa: Cegoc.

Welch, G. F., Himonides, E., Saunders, J., Papageorgi, I., and Sarazin, M. (2014). Singing and social inclusion. Front. Psychol. 5:803. doi: 10.3389/fpsyg.2014. 00803

World Health Organization (1992). The ICD-10 Classification of Mental and Behavioural Disorders: Clinical Descriptions and Diagnostic Guidelines. Geneva: World Health Organization.

Zuk, J., Benjamin, C., Kenyon, A., and Gaab, N. (2014). Behavioral and neural correlates of executive functioning in musicians and non-musicians. PLoS One 9:e99868. doi: 10.1371/journal.pone.0099868
Conflict of Interest Statement: The authors declare that the research was conducted in the absence of any commercial or financial relationships that could be construed as a potential conflict of interest.

Copyright (๑) 2018 Martins, Neves, Rodrigues, Vasconcelos and Castro. This is an open-access article distributed under the terms of the Creative Commons Attribution License (CC BY). The use, distribution or reproduction in other forums is permitted, provided the original author(s) and the copyright owner(s) are credited and that the original publication in this journal is cited, in accordance with accepted academic practice. No use, distribution or reproduction is permitted which does not comply with these terms. 


\section{OPEN ACCESS}

Edited by:

Graham Frederick Welch University College London, United Kingdom

Reviewed by: Claire M. Ghetti,

University of Bergen, Norway Andrea Schiavio,

University of Graz, Austria

*Correspondence: Katrina Skewes McFerran k.mcferran@unimelb.edu.au

Specialty section:

This article was submitted to Performance Science,

a section of the journal

Frontiers in Psychology

Received: 16 January 2020 Accepted: 11 February 2020 Published: 27 February 2020

Citation: McFerran KS, Lai HIC, Chang W-H, Acquaro D, Chin TC, Stokes $H$ and Crooke AHD (2020) Music, Rhythm and Trauma: A Critical Interpretive Synthesis of Research Literature. Front. Psychol. 11:324 doi: 10.3389/fpsyg.2020.00324

\section{Music, Rhythm and Trauma: A Critical Interpretive Synthesis of Research Literature}

\begin{abstract}
Katrina Skewes McFerran ${ }^{1 *}$, Hsin I. Cindy Lai ${ }^{1}$, Wei-Han Chang ${ }^{1}$, Daniela Acquaro ${ }^{2}$, Tan Chyuan Chin ${ }^{2}$, Helen Stokes ${ }^{2}$ and Alexander Hew Dale Crooke ${ }^{1}$

${ }^{1}$ Creative Arts and Music Therapy Research Unit, Faculty of Fine Arts and Music, The University of Melbourne, Melbourne, VIC, Australia, ${ }^{2}$ Melbourne School of Graduate Education, The University of Melbourne, Melbourne, VIC, Australia
\end{abstract}

Recent theorizing about the connection between the brain and trauma (Perry, 2009; Porges, 2011; van der Kolk, 2015) has led to a burgeoning of interest in the provision of music-based programs with people who have had adverse experiences. Although there has been critique of the lack of scientific basis of these theories and their implications for practice (McLean, 2016), they remain popular with practitioners who are keen to introduce innovative and potentially beneficial approaches to the people with whom they work. Music therapists have a long tradition of working with traumatized clients, however, the brain-based rationales did not seem congruent with the less predictable and more idiosyncratic benefits reported, which seem to occur through more psychodynamic mechanisms of action. In order to unravel what seemed to be a body of literature plagued by the conflation of theories, we undertook a critical interpretive synthesis of literature in the past 10 years to cross-examine the ways that music and trauma have been connected. To do this we extracted data from 36 identified articles to distinguish what music methods were used, what claims were made about benefits, what theoretical justifications were provided and how much research basis there was for the claims being made. Having systematically disentangled the various dimensions, we then constructed a spectrum of approaches that offers a logical categorization of four different ways of using music with people who have had adverse life experiences. These included using music for stabilizing, entrainment, expressive and performative purposes. Specific music-based methods were proposed for those associated with brain-based rationales, and more responsive, multi-method approaches were congruent with recovery and social change models. Future research would benefit from a more clearly articulated connection between theoretical rationale, music-based methods, benefits and research approaches. The resultant spectrum may provide useful guidance for both practice and research design.

Keywords: music, rhythm, trauma, adverse experiences, critical

\section{RATIONALE}

International interest in the relationship between rhythm and trauma has burgeoned in response to recent endorsement through popular trauma discourse that relies on neurological mechanisms of action. A number of popular theorists' ideas have been used to suggest that rhythm-based activities are beneficial for people who have had adverse experiences because it bypasses higher cognitive 
functioning and allows connections to form via more primitive, undamaged regions of the brain. One of these theories has been posed by psychiatrist Perry (2009), who uses brain studies to argue that since trauma has a visible impact on the primitive brain, interventions which also operate at a pre-conscious level should logically be more effective than cognitively mediated strategies. Porges (2011) has developed a more complex theoretical explanation, labeled Polyvagal theory, to propose that trauma symptoms are mediated by an amygdala that has become hyper-vigilant to threat-related cues, and therefore activities which regulate physiological arousal will be helpful in stimulating the vagally modulated social engagement system in positive ways. van der Kolk et al. (2007) adds that rhythmic activities can fulfill this function by reawakening feelings of pleasure and engagement dulled by prolonged trauma exposure, and that rhythm stimulates patterned, repetitive neural brainstem activity necessary for restoration of brain functioning. These three men have had an enormous influence on music practitioners in the field of trauma, with an anecdotal increase in rhythm-based programs being noticed through social media posts and requests for information from music therapists by the public and by students and practitioners in trauma related fields.

Some critique has emerged of these perspectives and the practice implications that have results from them, often categorized as "trauma-informed" practices. McLean (2016) summarizes the precarious assumptions underpinning this discourse as follows.

- "The way in which brain development in the context of early adversity and trauma is represented may be oversimplifying the science;

- Claims regarding the plasticity of the brain and what it might mean for therapeutic intervention are not justified by the available science; and

- Therapeutic interventions that are based on these assumptions (e.g., song, rhythmic drumming, spinning), although popular, have not yet been subject to the systematic evaluation that other trauma-specific therapies have." (p. 3).

We agree that there is a great deal of optimism in these theories and note that our practice experiences as therapists and teachers with young people who have had adverse childhood experiences suggests that the role of rhythm is less reliably mechanical, and more complexly woven into a fabric of relationship, musical encounters, creativity and safety. Because of our shared interest in the practical application of music/rhythm with young people, the final point made by McLean is our focus in this systematic review of the literature. Meticulous and systematic evaluation of these popular ideas is needed in order to ensure that they are effective, and prior to that, some of the key theoretical constructs need to be disentangled and clarified. To begin this process, we undertook a careful search of the literature that connects music with trauma, abuse and foster care. Our aim was to cross-examine the ways that music and trauma are connected in the literature. To do this, we extracted data to answer four questions from which we could draw conclusions in relation to the aim.
1. Which music methods are used by practitioners describing work with people who have experienced trauma, and to what degree is rhythm emphasized?

2. What claims are made about the benefits of using music to work with people who have experienced trauma?

3. What theoretical justifications are utilized for using music with people who have experienced trauma?

4. How much research basis is there for the claims being made?

\section{METHOD}

\section{Critical Interpretive Synthesis}

Systematic literature reviews have become an increasingly relevant method for scholars to interrogate a burgeoning literature that makes it difficult for readers to be fully cognizant of the breadth of theories and results that are constantly emerging. This notion was most popularly posed by Archie Cochrane, who recognized the divide between how clinicians were practicing in the field and the most recent findings being produced through medical research (Bero and Rennie, 1995). His intention was to increase awareness of findings in order to improve practice. The "Cochrane Reviews" then became a dominant and sometimes oppressive force within medical and educational practice (Aigen, 2015), accompanied by a demand for "evidence-based practice" which has been adopted by insurance companies and government agencies charged with distributing funds to service providers. The evidence-based movement is hierarchical, and privileges quantitative data over qualitative knowing because it supports the application of tenets of objectivism such as randomization and controlled conditions. The implications of this uneven emphasis have been critiqued, but this view continues to dominate in many cultures.

One alternative approach that has grown from the same desire to organize knowledge in ways that can inform readers about emerging ideas (Sackett et al., 1996), is the critical interpretive synthesis. Rather than summarizing knowledge, this critical approach recognizes that some knowledge is considered more credible than others, and that trends in the literature often emerge from a culture of privilege (Baines and Edwards, 2015). Therefore, rather than reinforce dominant ideas, a critical approach seeks to reveal the forces that have been previously rendered invisible, as articulated by Bourdieu (Navarro, 2006). There is an existing tradition of critical literature reviews such as these in the medical (Dixon-Woods et al., 2006) and ethnographic (Noblit and Hare, 1988) fields which provides a precedent for adopting a similar approach in the current investigation. By adopting a recursive but systematic approach it becomes possible to interrogate the literature, rather than to accept it, thereby challenging assumptions and recognizing forces at play that underpin knowledge generation (McFerran et al., 2017). This approach is well-suited to our aim of cross-examining the ways that music and trauma are connected in the literature.

\section{Search Strategy}

We undertook a number of searches to identify relevant literature, including a combination of words for "music" + "trauma." The alternate search terms used for music included: 
music, drum, drumming, hip hop, rhythm, improvisation, rap, beat making, songwriting, sing, singing, song, GIM (Guided Imagery and Music), and audio. Two additional terms were used as alternatives to trauma: abuse and foster care. Excluded topics were more focused on the event rather than the response and included: surgical trauma, medical trauma, cultural or historical trauma, immigration, political trauma, traumatic injury, physical trauma, traumatic event (not specifying the result of trauma), musician's trauma and substance abuse.

Our search focused on articles published in the last 10 years, which was from 2009 to 2018 . Ninety-one sources were identified through this search, including a range of manuscript types. We decided to focus on peer-reviewed literature including journal articles and dissertations, and excluded chapters and books from the analysis, we also removed repetition of research projects that were reported in multiple articles. This reduced the number to 36 sources which are summarized in Table 1 . Twenty-six of these were research studies and ten were case studies that did not ultimately qualify as research but which provided in-depth, detailed descriptions.

\section{Data Extraction}

Once the articles were identified, data was extracted from each against a set of questions as reported above. The intention was to generate a concise answer to the question, and not to rely on direct quotations which could require entire paragraphs. In some cases, interpretation was required if the information was not explicit. For example, not all authors distinguished between aims and outcomes, or gave details about the specific music method being used. The research assistant worked closely with the primary author to determine answers in these cases, using a combination of logical and reflexive questioning to determine if the information could be interpreted or if doing so was beyond our abilities to assume. For example, limited information about the setting of the program might be supplemented through online searching, or making assumptions based on the language used to describe the participants which may be more typical of medical or community contexts. We relied on the information published and did not make contact with authors if data was missing. The data were extracted in answer to the following prompts.

- How the participants are described-e.g., their behaviors and their presenting issues

- How the participants' trauma is described/diagnosed-e.g., complex, PTSD, etc.

- The reported cause of the trauma-e.g., events

- Age

- Setting

- Race/ ethnicity

- Group or individual program

- Number of people

- Research or not? If so, chosen design

- Claims about program aims

- Claims about program findings/results

- Music methods used

- Music genres referenced

- Any specific references to rhythm
- Citations used as justification for using music with traumatized people.

\section{Data Analysis}

Simple descriptive analysis was used to analyze the data in regard to the four research questions as a beginning point. This process required some interpretation when answers were not readily available, but our intention was to remain close to what the authors were describing in their articles, defined by our extractions being "recognizable." The over-arching question about how music and trauma are connected within the literature was then examined via meta-synthesis-exploring patterns that presented across the data, often examined through the intersections between multiple categories. This was undertaken with particular attention to critical questions about assumptions underpinning decisions, what was missing as well as what was dominant, who seems to benefit from the findings, and also attending to any emotional responses (frustration, sadness, surprise) to emerging patterns (McFerran et al., 2017).

\section{FINDINGS}

\section{Music Methods}

Music has a long history of application in the mental health field and practitioners have been using an array of music-based methods with people who have had adverse life experiences for many decades (e.g., Rogers, 1993). For example, the profession of music therapy was established in the 1950s in the U.S.A. in response to the high number of veterans who returned from fighting in the war with post-traumatic stress disorder, as well as in response to later wars (Slotoroff, 1994). Some of the authors are qualified music therapists, and therefore have a particular interest in, and knowledge of this discourse. This potentially influenced the balance of articles with just under half being music therapy articles $(n=17), 9$ being from psychology, and 10 coming from other disciplines including other creative arts and expressive arts therapies, guided imagery and music, education, occupational therapy, nursing and arts backgrounds. In categorizing the methods used however, we have not distinguished between music therapists uses of particular methods, such as song writing.

We began our investigation expecting to find an emphasis on rhythm in the literature given the prominence of reference to this musical element in the social discourse emerging from the neurological theories about trauma. In the initial search, the term "rhythm" did not result in any identified sources, nor did "beat making" or "audio." We reviewed our conception of "rhythm" and chose to expand our searching to recognize that rhythm is inherent in music and that we were interested in the degree to which it was made prominent by different authors. As noted above, we then used 14 terms for music in our searches of the peer-reviewed literature of which rhythm was the primary focus of 5 of the 37 manuscripts identified, with group drumming being the sole method described in all but one of these. In addition to the four using group drumming exclusively, 9 of the 37 articles in total included drumming as one of their methods and it was the fourth most common method described along with those that described playing on other instruments. Songwriting was the 
TABLE 1 | Included studies.

\begin{tabular}{|c|c|c|c|}
\hline References & Title & $\begin{array}{l}\text { Country of } \\
\text { study }\end{array}$ & Search terms \\
\hline Alanne (2010) & $\begin{array}{l}\text { Music psychotherapy with refugee survivors of torture: interpretations of three clinical case } \\
\text { studies }\end{array}$ & Finland & $\begin{array}{l}\text { Guided Imagery and } \\
\text { Music + trauma }\end{array}$ \\
\hline Beck et al. (2018) & $\begin{array}{l}\text { Feasibility of trauma-focused Guided Imagery and Music with adult refugees diagnosed } \\
\text { with PTSD: a pilot study }\end{array}$ & Denmark & $\begin{array}{l}\text { Guided Imagery and } \\
\text { Music + trauma }\end{array}$ \\
\hline Bensimon et al. (2012) & A pendulum between trauma and life: group music therapy with post-traumatized soldiers & Israel & Music + trauma \\
\hline Blanaru et al. (2012) & $\begin{array}{l}\text { The effects of music relaxation and muscle relaxation techniques on sleep quality and } \\
\text { emotional measures among individuals with posttraumatic stress disorder }\end{array}$ & Israel & $\begin{array}{l}\text { Guided Imagery and } \\
\text { Music + trauma }\end{array}$ \\
\hline Bolger (2015) & $\begin{array}{l}\text { Being a player: understanding collaboration in participatory music projects with } \\
\text { communities supporting marginalized young people }\end{array}$ & Australia & $\begin{array}{l}\text { Songwriting }+ \text { Out of } \\
\text { home care }\end{array}$ \\
\hline Carr et al. (2012) & $\begin{array}{l}\text { Group music therapy for patients with persistent post-traumatic stress disorder - an } \\
\text { exploratory randomized controlled trial with mixed methods evaluation }\end{array}$ & U.K. & Music + trauma \\
\hline Day et al. (2009) & $\begin{array}{l}\text { Experiences of song writing in a group programme for mothers who had experienced } \\
\text { childhood abuse }\end{array}$ & Australia & Songwriting + trauma \\
\hline der Heyde and Christine (2012) & $\begin{array}{l}\text { Interpersonal rhythms disrupted by a history of trauma: an in-depth case study of } \\
\text { analytical music therapy }\end{array}$ & U.S.A. & Improvisation + trauma \\
\hline Fairchild (2018) & $\begin{array}{l}\text { Collaborative songwriting with children experiencing homelessness and family violence to } \\
\text { understand their resources }\end{array}$ & Australia & Songwriting + abuse \\
\hline Faulkner (2017) & $\begin{array}{l}\text { Rhythm2Recovery: a model of practice combining rhythmic music with cognitive reflection } \\
\text { for social and emotional health within trauma recovery }\end{array}$ & Australia & Music + trauma \\
\hline Felsenstein (2013) & From uprooting to replanting: on post-trauma group music therapy for pre-school children & Israel & Music + trauma \\
\hline Flores (2013) & $\begin{array}{l}\text { African drumming as a medium to promote emotional and social well-being of children } \\
\text { aged } 7 \text { to } 12 \text { in residential care }\end{array}$ & & Drum + foster care \\
\hline Jespersen and Vuust (2012b) & $\begin{array}{l}\text { The effect of relaxation music listening on sleep quality in traumatized refugees: a pilot } \\
\text { study }\end{array}$ & Denmark & Music + trauma \\
\hline Jespersen and Vuust (2012a) & Music for improvement of trauma-related sleep problems & Denmark & Music + trauma \\
\hline Kim (2013) & $\begin{array}{l}\text { Music therapy with children who have been exposed to ongoing child abuse and poverty: } \\
\text { a pilot study }\end{array}$ & South Korea & Music + abuse \\
\hline Neupane and Taylor (2011) & Music therapy for incarcerated women recovering from trauma and abuse & U.S.A. & Music + trauma \\
\hline Osborne (2012) & $\begin{array}{l}\text { Neuroscience and "real world" practice: music as a therapeutic resource for children in } \\
\text { zones of conflict }\end{array}$ & U.K. & Music + PTSD \\
\hline Palidofsky and Stolbach (2012) & $\begin{array}{l}\text { Dramatic healing: the evolution of a trauma-informed musical theater program for } \\
\text { incarcerated girls }\end{array}$ & U.S.A. & Songs + trauma \\
\hline Precin (2011) & Occupation as therapy for trauma recovery: a case study & U.S.A. & Songs + trauma \\
\hline Reeves (2013) & $\begin{array}{l}\text { How music and lyrics protect and heal the souls of African women who have experienced } \\
\text { domestic-violence trauma, sexual abuse, or depression: a phenomenological study }\end{array}$ & & Music + trauma \\
\hline Rudstam et al. (2017) & $\begin{array}{l}\text { Trauma-focused group music and imagery with women suffering from PTSD/complex } \\
\text { PTSD: a feasibility study }\end{array}$ & Sweden & Music + trauma \\
\hline Salmon and Rickaby (2014) & $\begin{array}{l}\text { City of one: a qualitative study examining the participation of young people in care in a } \\
\text { theater and music initiative }\end{array}$ & U.K. & Music + foster care \\
\hline Schrader and Wendland (2012) & $\begin{array}{l}\text { Music therapy programming at an aftercare center in cambodia for survivors of child } \\
\text { sexual exploitation and rape and their caregivers }\end{array}$ & U.S.A. & Songwriting + trauma \\
\hline
\end{tabular}


TABLE 1 | Continued

\begin{tabular}{|c|c|c|c|}
\hline References & Title & $\begin{array}{l}\text { Country of } \\
\text { study }\end{array}$ & Search terms \\
\hline Story and Beck (2017) & $\begin{array}{l}\text { Guided Imagery and Music with female military veterans: an intervention development } \\
\text { study }\end{array}$ & Denmark & Music + PTSD \\
\hline Strehlow (2009) & The use of music therapy in treating sexually abused children & Germany & Music + trauma \\
\hline Sutton and De Backer (2009) & Music, trauma and silence: the state of the art & $\begin{array}{l}\text { U.K. and } \\
\text { Belgium }\end{array}$ & Music + trauma \\
\hline Wellman and Pinkerton (2015) & $\begin{array}{l}\text { The development of a music therapy protocol: a music } 4 \text { life }{ }^{\circledR} \text { case report of a veteran with } \\
\text { PTSD }\end{array}$ & U.S.A. & Drumming + trauma \\
\hline Zanders (2015) & $\begin{array}{l}\text { Music therapy practices and processes with foster-care youth: formulating an approach to } \\
\text { clinical work }\end{array}$ & U.S.A. & Music + foster care \\
\hline
\end{tabular}

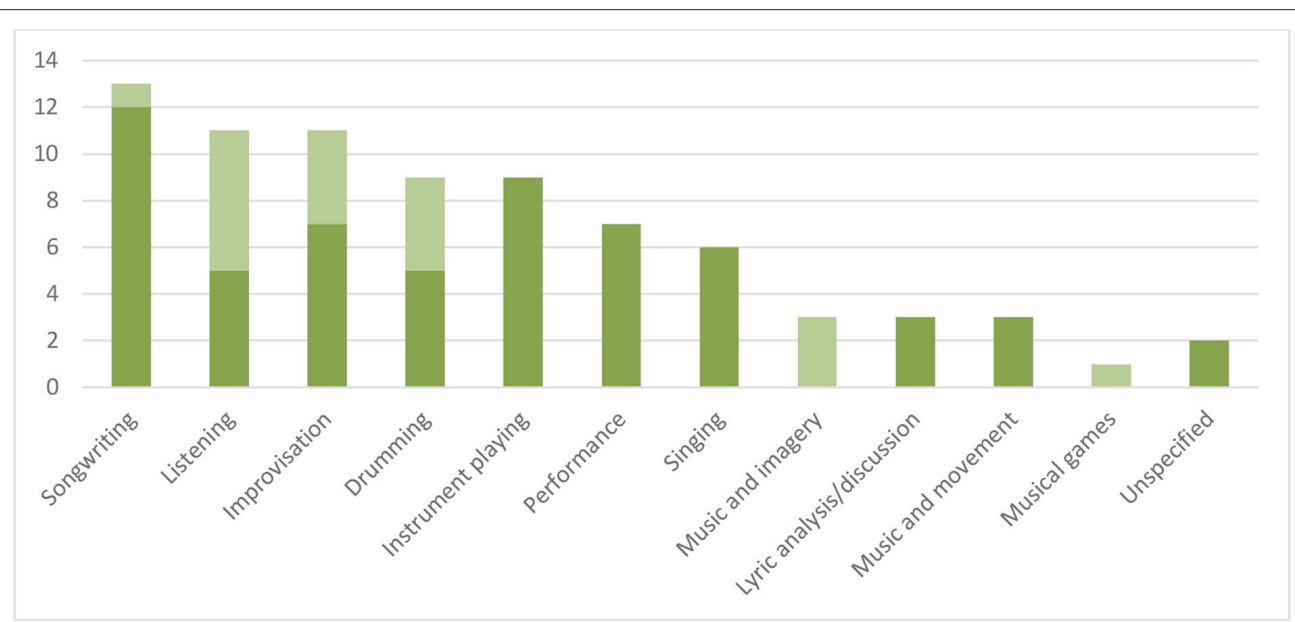

(only method=light; one of multiple methods = dark)

FIGURE 1 | Frequency of reference to music-based methods (only method = light; one of multiple methods = dark).

most common method referenced and both music listening and the music therapy technique of improvisation was reported in 11 articles. There was also reference to performances, singing, and others represented in Figure 1.

In further interrogating the representation of different methods within the literature, it became clear that there was an even split between those focusing on the use of one method only and those using multiple methods, with 18 manuscripts describing between 2 and 6 methods (average 3.6). Of those focusing on a single method, half relied on receptive music experiences, including music listening for analysis of lyrics, imagery, relaxation, and other meaning making processes that relied on associations being explored.

Although we only focused on literature in the past 10 years, we were interested to examine how many different approaches were being described in contemporary discourse, and to consider what trends may have impacted these findings. Only one study made reference to Hip Hop, despite our combined experience suggesting that it is a popular method in current practice, particularly in the provision of services for youth. This suggests that the research is lagging behind practice in this space, as McLean (2016) has suggested. It would be a mistake to assume this means Hip Hop is not relevant and/or helpful, however. A strong theoretical rationale exists for the value of culturally relevant music practices (Stige, 2002), whether that is related to black American culture, or youth culture more generally. Similarly, the prominence of songwriting does not imply this method is most useful, but rather, that it is most published.

\section{Theoretical Basis}

One important part of the rationale for this investigation was the influx of interest in the connection between music, trauma and the brain. In our view, this has largely been at the instigation of three popular authorities in the field of trauma-Perry (2009), van der Kolk et al. (2007), and Porges (2011). In addition, music therapy theorist, Thaut (2005), has long posited that the benefits of music therapy are best explained by studying brain based reactions, and has been particularly interested in the role of rhythm in this context. Our first interrogation of the theoretical underpinnings of the 37 studies was therefore to determine the degree to which a brain-based rationale was provided for musicbased interventions (see Figure 2). Ten of the articles made reference to either Porges or van der Kolk, with van der Kolk being the most referenced theorist ( 8 articles), while Porges 
was only noted in three and Thaut in one. The second most prominent theorist was Herman (2015), whose work on "Trauma and Recovery" transformed the discourse in the early 1990s and is probably still the most influential trauma text of our time. Herman does not directly address music, but rather it is her threestage model of recovery that many practitioners use as a guide to their work. Perry was not referenced in any of the articles, perhaps because he is more well-known as a presenter than a published author.

Music therapy theory and theory from other Creative Arts Therapies (CAT), including the specific field of Guided Imagery and Music which requires advanced training, was also referenced frequently in the included manuscripts, once again reflecting the professional training of some of the authors. No single CAT authors were referenced in particular, and many music therapists also integrate theoretical approaches dominant in psychology and psychiatry. This was true of the rationales used across many articles, including psychoanalytic and psychodynamic theorists, cognitive behavioral therapy, attachment theory, gestalt theory, as well as some scholars from music psychology and creative arts therapies more broadly. Resilience was referenced by a significant number of authors, although no particular resilience theorists were dominant.

\section{Ascribed Benefits}

Two sets of data were included in this analysis. The primary data was the findings/results that were described for participants in the music-based programs. However, we also felt it was important to critically analyze the authors' aims, since assumptions are often embedded in the intentions for a project, and therefore interrogating them may be revealing. This was certainly the case in our investigation, with 22 of the authors describing aims that included demonstrating how music could serve as a useful medium for people who had adverse experiences. This focus reveals a high degree of investment in positive outcomes; however, minimal reflexivity was presented in the qualitative research, and only two of the quantitative studies included a control condition so that an objective comparison could be made. Either of these approaches to confirming how findings may have been influenced by the expectations of the researchers would have enhanced the quality of the literature. Whilst it is natural that practitioner-researchers should believe in their approach, and certainly, it would make no sense to investigate something considered to be unhelpful, scientific standards demand that some effort is therefore made to ensure findings are trustworthy. Further development is needed in this area.

Working in the field of trauma is complex and unlike some other fields of practice where music is used (for example, rehabilitation), specific improvements cannot always be readily targeted and measured. It can be difficult for people with lived experience, their carers, and their workers to know exactly what part of the complex psychological ramifications of adverse experiences should be focused upon-behavior, memories, meaning-making, and emotions are all intertwined and difficult to separate. Herman (2015) popular model recognizes this by focusing on different program aims for each stage rather than focusing on benefits or expected outcomes. She does not make generalizable predictions about universal benefits but focuses on recovery as a unique and ongoing journey. However, there is a strong (reductive) trend in the cognitive and psychological sciences more generally that brain-based explanations appear to solve because it provides elegant explanations that could explain the complexity. However, the reality of the complex terrain of living with trauma is reflected in the kinds of benefits reported by authors in the literature. A focus on intrusive trauma symptoms that could be paired with measurable outcomes appeared adequate only for those living with the most severe behavioral responses. For example, symptom reduction was a target in 11 manuscripts of which seven were describing trauma experienced in military contexts, and six of this sub-set used

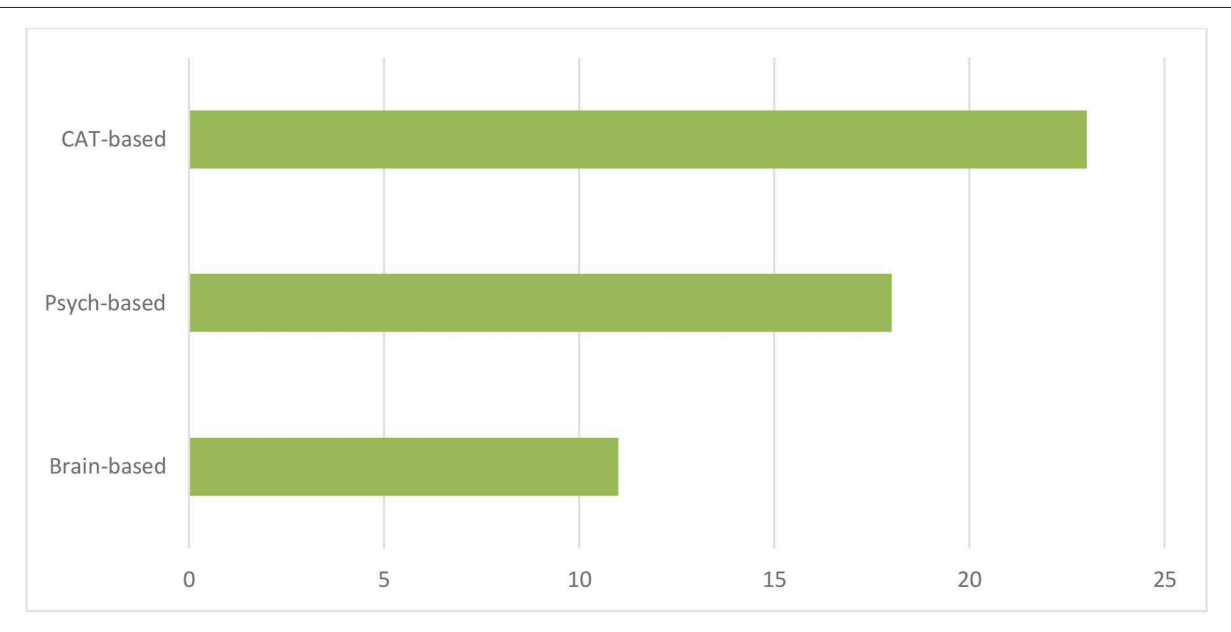

Note: CAT is abbreviated from Creative Arts Therapies

FIGURE 2 | Theoretical rationale provided in included manuscripts. CAT is abbreviated from Creative Arts Therapies. 
quantitative designs and two used mixed methods. Another behavioral outcome noted in the analysis was a focus was on sleep and/or relaxation, represented in five articles.

However, for those with adverse life experiences beyond the military it seemed more difficult for authors to nominate specific benefits and there was a great deal of variety in the ways that authors couched the outcomes they were reporting. For example, an emphasis on resilience and/or empowerment was present in five articles that were set in community-based contexts, including a kindergarten and youth center. Social benefits were posited in a different set of seven manuscripts, and all of these were related to programs provided in groups, rather than individual therapy where trust in the therapist might be more relevant, although not usually described as a benefit. Self-worth was another important benefit posited in a different set of eight manuscripts, with all of these being programs for marginalized young people in non-acute contexts. Some kind of emotional outcome was referenced by eight of the authors, but these were difficult to categorize as benefits, and were mostly described more generally as having opportunities for emotional expression and processing rather than improvements per se (i.e., emotional responsiveness, emotional balance, emotional expression, work through emotional aspects, understanding of emotion, expressing musical emotions, regulate and express emotions). The term affect was used by some quantitative researchers (i.e., affect and mood stabilization, fewer affective and cognitive disturbances), and feelings were also referenced by some authors (express repressed feelings, increased expression of non-traumatic feelings). Although the benefit of working with emotions was difficult to articulate, emotional challenges are central to many traumatized peoples' experience and the opportunity to participate seems justifiable as a benefit of therapeutic work with music.

There was a suite of 11 articles in this review from which it was more difficult to extrapolate specific well-being benefits to participants and that were more exploratory in nature. Many of these were focused on exploring whether music-based programs were useful in the diverse contexts where they had been introduced, from inpatient and outpatient mental health programs, private practice, and a range of community-based programs for survivors of torture, sexual exploitation, refugee detention and those in the foster care or family violence systems. Authors described aims such as:

1. Exploring the ways in which music can speak directly to the trauma, and how music therapy offers a unique means to understand the traumatized patient

2. Exploring whether and how rhythmic interactions within musical improvisations facilitate the repair of ruptures in such rhythms

3. Describing women's experiences of song writing to support parenting

4. Describing the evolution of an innovative program for incarcerated adolescent girls in which youth work collaboratively with theater professionals to create, develop, and perform musicals based on their experiences
5. Understanding to what extent, and in what ways, the mental health condition of the research subjects changes during music psychotherapy treatment

6. Exploring the ways in which music therapy programming can support and enhance care systems already in place for survivors of sex trafficking and their care staff

7. Presenting a new way of combining songwriting and visual art to facilitate emotional expression

8. Balancing the representation of children in this context by using music to explore their resources and what helps them to "do well" throughout their experiences of homelessness and family violence

9. Presenting a framework for music therapy processes and practices with foster-care youth

10. Describing how a performing arts troupe program supports vulnerable youth

11. Helping to develop musical aspects of a local program that contributes to the welfare of children and to use the social power of music to raise awareness of their situation in a wider world.

\section{Research Approaches}

This critical interpretive synthesis does not aim to synthesize all the literature on the topic of rhythm and trauma, but rather, to interrogate the ways that music and trauma are connected in the peer reviewed literature. Our decision to focus on peer-reviewed literature was intentional and we aimed to avoid the inclusion of anecdotal report and to focus on what McLean (2016) calls science, but what we would call researchbased reports. Despite this intention, of the 36 manuscripts included in our investigation, 10 were not research articles but were descriptive program reports without a clear research methodology. They did contain useful information in answer to the remaining questions however, and, were included in the analysis of other sections. Of the 26 manuscripts that had a clear methodology, the research approaches were dispersed across data types, with qualitative research being most common, and although the case studies are displayed separately, these all used qualitative approaches. Those studies that used quantitative data included two randomized controlled trials, while the rest of the studies did not have control conditions or randomization (see Figure 3).

The studies relying on quantitative data came from a range of regions, including Israel (3) and the Middle East (2), U.K. and Denmark, as well as Australia and the U.S.A. The qualitative studies were more likely to come from Australia (4) or the U.S.A. (3), with one each from the U.K. and Denmark, and the case studies were similarly dominated by north American authors, with one set of three case studies from Finland. There are a number of possible explanations for the limited number of objectivist studies. It may suggest that the interface between trauma and music is considered to be challenging by many to quantify, or that ethics approval for conducting studies with people considered to be so vulnerable is difficult in western countries with stringent systems for monitoring and approving research projects. Pragmatically, it 
can be difficult to adequately power experimental research studies with this population due to limited sample sizes. Alternatively, it might be that authors' felt quantitative data was incongruent with the importance of narrative that is often emphasized in therapeutic works with people who have had adverse experiences.

\section{META SYNTHESIS}

The notion of meta-analysis in quantitative systematic review refers to the use of statistical methods to combine the data from a large number of studies. In qualitative syntheses, the "meta" does not refer to aggregating, but rather to broadening understanding of a particular phenomenon (Grant and Booth, 2009), in this case through identifying more nuanced and qualitative distinctions between strands of data. After immersion in the results of the analysis above, we constructed a spectrum of approaches that could be used to distinguish four categories aligning with a set of proposed musical purposes relevant for working with people who have lived experience of trauma (see Table 2). The spectrum transpired after grappling with how theories that posit primitive neural activity as key mechanisms of action (such as

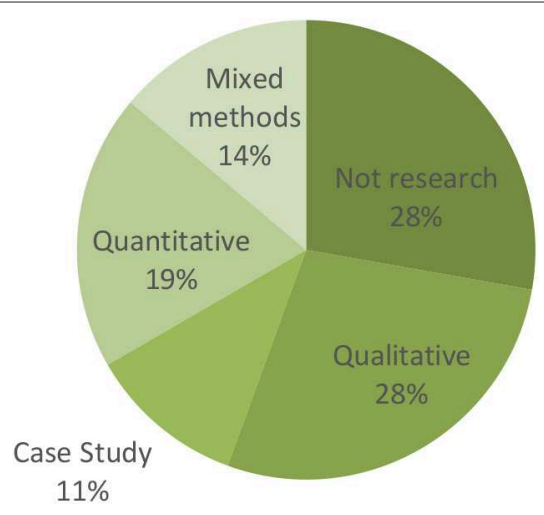

FIGURE 3 | Research approaches used in 26 manuscripts.
Porges, 2011; van der Kolk, 2015) were related to specific types of music methods suggested and this led us to conceptualize the first two categories. What remained were approaches that required higher level brain activity such as emotion processing and social awareness and these were then connected to different music methods proposed. Benefits were also an important consideration in constructing the emerging spectrum, since we were endeavoring to untangle various strands of discourse in order to identify whether there could be cogent links between theorized mechanisms of action, music methods and expected benefits. We used abductive logic to explore this possibility in the meta-synthesis and as a result, speculate that there are four ways of understanding how music and rhythm can afford a range of benefits for those who have had adverse life experiences resulting in trauma.

\section{Stabilizing}

The term stabilizing has been used to label this group of manuscripts because there was no suggestion of the music being used in a responsive way. Rather, listening to recorded music was the selected method for individuals with a PTSD diagnosis because it provided a safe structure affording a stable pulse lasting for the duration of each musical piece. This allows for predictability when used in a repeated way and emphasizes tempo rather than rhythm per se. Importantly, recorded music is reliable as a variable within quantitative research designs and music listening was found to be effective in influencing physiological regulation, resulting in improved sleep, increased relaxation and the reduction of negative PTSD symptoms.

The theoretical basis for this category relies on the premise that physiological processes can be modulated via brain-based processing that bypass areas of the brain damaged by trauma. For example, Perry's neurosequential model (Perry, 2006, 2009) suggests that regular and repetitive rhythmic activities can regulate these primitive areas of the brain, even when early adversity has led to chronic hyper-arousal. Porges' theorizing also implicates rhythm as a way to calm an over-sensitive central nervous system by drawing on the autonomic nervous system (Porges, 2011). He introduced the term neuroception

TABLE 2 | Spectrum of approaches to music and trauma.

\begin{tabular}{|c|c|c|c|c|}
\hline Musical purpose & Stabilizing & Entrainment & Exploratory & Performative \\
\hline Music methods & Focused music listening & $\begin{array}{l}\text { Group drumming, structured } \\
\text { improvisation, shared singing }\end{array}$ & $\begin{array}{l}\text { Music psychotherapy -improvisation, } \\
\text { guided imagery, song writing }\end{array}$ & $\begin{array}{l}\text { Song writing and } \\
\text { performance }\end{array}$ \\
\hline Relevant theories & Porges, Perry & Siegel, Ogden & Herman, Van der Kolk & Herman, Butler \\
\hline Form & Individual & Group & Individual or Group & Group \\
\hline Regulatory & Physiological regulation & Co-regulation & Emotion regulation & Self-regulation \\
\hline Reported benefits & $\begin{array}{l}\text { Reduced negative symptoms, sleep, } \\
\text { relaxation }\end{array}$ & Varied & Emotional work, self-worth & $\begin{array}{l}\text { Social connections, } \\
\text { resilience }\end{array}$ \\
\hline Research & Quant & Varied & Varied & $\begin{array}{l}\text { Participatory/Program } \\
\text { description }\end{array}$ \\
\hline
\end{tabular}


(Porges, 2004) to refer to the ways the autonomic nervous system evaluates risk in the environment, and suggests that prosodic acoustic stimulation signals safety. These theories are precisely the ones criticized by McLean (2016) because of the lack of scientific evidence. Whilst we believe her critique is well-founded, these studies do demonstrate that music listening can improve physiological markers of distress in people who have had adverse experiences. These are likely to be temporary, rather than leading to healing in the brain, as suggested by these popular theorists, however they may still be appreciated by those benefiting from greater amounts of sleep and relaxation.

\section{Entrainment}

The term entrainment has a long history in musicological and music psychology discourse and is usually paired with the notion of synchronization. Some scholars distinguish entrainment as the purpose and as "a process that governs the alignment of the auditory and motor domain," whilst synchronization is the action, being "a dynamic attraction point where the timing differences between music and person are stabilized" (Moumdjian et al., 2018). In the music psychology literature, it is often about pairing human behavior with an external auditory source, as described by Cameron and Grahn (2016): “The synchronization of internal rhythm processes (such as neuronal oscillations) or behavior (such as tapping or dancing) to external, periodic events (e.g., the beats in a rhythm)" (p. 363). However, in the ethnomusicology literature, the freedom to oscillate rather than be mechanically steady is emphasized (Clayton et al., 2005), which is critical to its use in this meta-synthesis since keeping perfectly in time is neither necessary nor likely when working with non-musicians.

The term entrainment has been chosen here because it is a logical extension of an approach that relies on primitive neural activity as a mechanism for bypassing more complex trauma related behaviors such as hyper- and hypo-arousal-bringing people into a more optimal "window of tolerance," as described by Siegel (1999). This kind of sensorimotor approach has been advocated by much quoted practitioner-researcher, Pat Ogden (Ogden et al., 2006), who suggests connecting with people via basic functions such as rhythm when cognitive processing and self-regulation are considered to be unavailable. Hence music methods that rely on clearly guided musical experiences emphasizing basic pulses and synchronized activity are a good match for this theorizing.

Drumming is the most obvious music method that can be classified as a sensorimotor activity in music with basic research suggesting that this should result in improvements through coregulation. Drumming groups often imply high levels of structure and rule-following, as distinct from the more emergent and responsive approaches described in the Expressive category. However, drumming appears to have been integrated into a range of approaches in the literature and used in both dynamics and more ordered ways. This distinction is critical for facilitators to consider, since the way drumming is used would logically lead to quite different outcomes. This point has been made in Michael Thaut's rhythm-based approach to music therapy (Thaut et al., 2015) which has been tested in rehabilitative contexts, but for which there is less evidence in mental health domains. Critical to this approach is focused and repetitive music activities that are reliably delivered with strict adherence to musical parameters, similar to a behavioral approach. This does involve live music making, so that the therapist can make adjustments to timing where necessary, however, it is very different from a psychodynamic approach that may use drumming experiences as the basis of interpretation and fostering insight. In the literature reviewed, it was not always possible to understand how rhythmbased methods were being offered.

\section{Expressive}

This category takes expression in its title because it encompasses an array of personally expressive dimensions-including emotions and feelings as one component, but also the broader constructs of personal expression, identity work, and a range of other well-being benefits. Rather than bypassing damaged regions of the brain, as posed in the previous two categories, the expressive emphasis is about acknowledgment and integration of adverse experiences. This requires higher cognitive functioning and the ability to actively reflect and make meaning and is classically associated with psychotherapy, where uncovering repressed experiences is considered critical to recovery. However, following on from the seminal work of Herman (2015), contemporary trauma work emphasizes the importance of establishing safety and stability before progressing to reconstructing the trauma narrative. This may explain the mixture of methods used in this category, where song writing can be used for reconstructing the trauma narrative, and improvisation for exploring less conscious reactions, and movement through a range of other activities can provide opportunities for moving back into relationship building and re-establishing safety.

van der Kolk's (2015) work provides a good theoretical framework for this category, with an emphasis on finding ways to be fully alive and engaged. Self-exploration within a safe therapeutically contained relationship is critical to this grouping, which is all contributed by trained professional psychotherapists specializing in music-music therapists. Carefully managed, expressive arts-based activities can be useful in re-establishing ownership of body and mind (van der Kolk et al., 2007) through becoming more familiar with ways of expressing the self-one's history, aspirations, relational capacities and hopes and dreams. This incorporates the emotional work noted by Herman (2015) in the second stage of remembrance and mourning, and having multiple creative methods available means that the therapist can circle back into safety and self-soothing, or forward into reconnection as required.

\section{Performative}

The distinction between personal work and recognition that war and abuse occur in a social context that condones or tolerates these adverse experiences is critical to the distinction between this and the previous category. The word performative has been selected because it encompasses this critical positioning [influenced strongly by Butler (2010)], where identity is bought to life through words and actions rather than being an expression 
of something that is already fixed. This upends the notion that people's lives have been determined by their adverse experiences, and focuses on how certain affordances invite agency (Withagen et al., 2017), in this case, how public, creative affordances offered through musical performances can invite reconstruction of identity and also, advocate for social change.

As a critical scholar, Herman's (2015) final stage of recovery is aligned with the integration of private and public self that is demanded by the performative category. Other renowned critical scholars from outside the trauma field also provide an important framework for understanding. Freire's (1993) pedagogical theories for education are often applied in community, and emphasizes the importance of political performative acts to bring about social change. Similarly, arts-based approaches have been theorized with social justice agendas, such as Community Music Therapy (Stige et al., 2010) and Community Music more broadly (Bartleet and Higgins, 2018). Although not specifically theorized for those who have had adverse life experiences, these discourses do have oppressed and marginalized persons at their center, with an emphasis on society's responsibilities for the conditions that allowed abuse, rather than centralizing the individual's experiences of it and certainly not the neural pathways in the brain.

This category is therefore not restricted to contributions by qualified therapists, but also includes community artists, public health researchers, and other allied health practitioners working in community contexts. Groups are privileged because of the opportunities they afford for internal as well as publicfacing performances, and the benefits are linked to these social possibilities. Interestingly, they are reported most often by privileged practitioners in first world economies working with people from marginalized backgrounds, including people traumatized through experiences of war in other countries.

\section{CONCLUSION}

This critical interpretive synthesis grew from a shared frustration with a set of literature that seemed to conflate a range of theories about trauma and music-based practices. Ideas about how rhythm influences brain activity were used as a rationale for a wide range of music-based experiences that placed little emphasis on rhythm. The possibilities of bypassing damaged areas of the brain through musical engagement seemed to draw attention away from critical therapeutic processes built through safety and containment. Beliefs about what music could do to the brain seemed to override fundamental therapeutic tenets such as responsiveness, compassion and careful exploration of repressed material through music. This enthusiasm for a scientific rationale seemed to bear little relevance to our shared experiences of working with young people who had adverse childhood experiences as teachers, therapists and researchers.

By first asking specific questions of the literature and extracting data into discrete categories, we were able to identify where patterns existed and where no patterns could be discerned.
For example, the theoretical rationales provided for most programs did not bear direct relevance to the specific type of music-based methods being used. We hope that this synthesis will encourage authors to articulate more clear lines of argument in the future. In addition, it became evident that there were high levels of bias in the research of all types, with little reflexivity, use of control groups, or any other strategies that might support a more scientific assertion of why music might be helpful for traumatized individuals and groups. Sometimes this was overt, such as when people's explicit intentions were to demonstrate how valuable their program had been, and other times it was covert but still missing. There was also little explanation for why specific music methods were used or how that decision related to the intended outcome. The inclusion of this information would benefit readers and scientists alike.

In order to make the discourse more amenable to scientific standards, a more coherent approach may be helpful that involves congruent connections between intentions, rationales, music methods, benefits and research methodologies. This is relatively straight forward when designing programs that target entrainment or stabilization-specific music methods should be linked to predictable outcomes if it is proposed that they will be activated through neural mechanisms, and these can be measured and compared to control conditions. Michael Thaut has modeled this approach in rehabilitation (2015), and appropriately advocates for strict application in order for this to be effective. By contrast, programs that have expressive or performative purposes do not rely on understanding music as a variable but instead draw more on sociological notions of music affording possibilities for constructing self-understandings and enacting social critique. In this context, multiple methods may lead to benefits at different times depending on what is needed by the individual of group in a given moment, with particular attention being paid to safety in the context of trauma. Subjective and emancipatory research is more congruent with these values and would benefit the field. One approach that might address the need for greater scientific basis would be to systematically compare different approaches to determine if there are differences in outcomes, which would confirm the necessary connections between inputs and outcomes. This would suit the first two categories of stabilization and entrainment, but might not capture the complexity of the second two, which would demand the inclusion of more subjective views collected through qualitative data, reflexively analyzed.

In concluding, it is important to recognize the complexity of the lived experience of trauma. For people to have presented to services for support that are then documented in the manuscripts analyzed, we can assume that adverse experiences have had a profound experience on their lives. There was clearly a need for helpful, and perhaps evidence-based support. However, given the statistics available at a population level on the high level of abuse that is perpetrated in most societies, we must also recognize that the response to adverse experiences is idiosyncratic, not universal. Some people survive without need for expert support, some people develop inspiring resilience and thrive in the same society that allowed abuse to occur. 
Therefore, it is logical that not all people benefit from the same supports, for a range of reasons including personal readiness, trust in therapist and services, suitability of services etc. A spectrum of approaches is therefore necessary, and it would be inappropriate to advocate for one over the other, but rather to attend to the individual in context and determine what musicbased approach would meet their needs and desires at a given moment in time.

\section{DATA AVAILABILITY STATEMENT}

The datasets generated for this study are available on request to the corresponding author.

\section{REFERENCES}

Aigen, K. (2015). A critique of evidence-based practice in music therapy. Music Ther. Perspect. 33, 12-24. doi: 10.1093/mtp/miv013

Alanne, S. (2010). Music Psychotherapy With Refugee Survivors Of Torture: Interpretations Of Three Clinical Case Studies. Helsinki: University of the Arts Helsinki.

Baines, S., and Edwards, J. (2015). Considering the ways in which anti-oppressive practice principles can inform health research. Arts. Psychother. 42, 28-34. doi: 10.1016/j.aip.2015.01.001

Bartleet, B.-L., and Higgins, L. (2018). "An overview of community music in the twenty-first century," in The Oxford Handbook of Community Music, eds B.-L. Bartleet and L. Higgins (Oxford, UK: Oxford University Press), 1-20. doi: 10.1093/oxfordhb/9780190219505.001.0001

Beck, B. D., Messel, C., Meyer, S. L., Cordtz, T. O., Søgaard, U., Simonsen, E., et al. (2018). Feasibility of trauma-focused guided imagery and music with adult refugees diagnosed with PTSD: a pilot study. Nord. J. Music Ther. 27, 67-86. doi: $10.1080 / 08098131.2017 .1286368$

Bensimon, M., Amir, D., and Wolf, Y. (2012). A pendulum between trauma and life: group music therapy with post-traumatized soldiers. Art. Psychother. 39, 223-233. doi: 10.1016/j.aip.2012.03.005

Bensimon, M., Bodner, E., and Shrira, A. (2017). The emotional impact of national music on young and older adults differing in posttraumatic stress disorder symptoms. Aging Ment. Health 21, 1090-1098. doi: $10.1080 / 13607863.2016 .1196338$

Bero, L., and Rennie, D. (1995). The Cochrane Collaboration: preparing, maintaining, and disseminating systematic reviews of the effects of health care. J. Am. Med. Assoc. 274, 1935-1938. doi: 10.1001/jama.1995.03530240 045039

Blanaru, M., Bloch, B., Vadas, L., Arnon, Z., Ziv, N., Kremer, I., et al. (2012). The effects of music relaxation and muscle relaxation techniques on sleep quality and emotional measures among individuals with posttraumatic stress disorder. Mental Illness 4:e13. doi: 10.4081/mi.2012.e13

Bolger, L. (2015). Being a player: understanding collaboration in participatory music projects with communities supporting marginalised young people. Qual. Inquiries Music Ther. 10, 77-126.

Butler, J. (2010). Performative agency. J. Cult. Econ. 3, 147-161. doi: $10.1080 / 17530350.2010 .494117$

Cameron, D. J., and Grahn, J. A. (2016). "The neuroscience of rhythm," in The Oxford Handbook of Music Psychology, 2nd Edn, eds S. Hallam, I. Cross, and M. Thaut (Oxford, UK: Oxford University Press), 357-368.

Carr, C., d'Ardenne, P., Sloboda, A., Scott, C., Wang, D., and Priebe, S. (2012). Group musictherapy for patients with persistent posttraumatic stress disorder-an exploratory randomized controlled trial with mixed methods evaluation. Psychol. Psychother. 85, 179-202. doi: 10.1111/j.2044-8341.2011.02026.x

Christenbury, K. R. (2017). I will follow you: the combined use of songwriting and art to promote healing in a child who has been traumatized. Music Ther. Perspect. 35, 1-12. doi: 10.1093/mtp/miv005

\section{AUTHOR CONTRIBUTIONS}

KM conceived the project, conducted the analysis, and drafted the article. W-HC undertook the initial data extraction, supported by HL. AC contributed to conceptualization and offered feedback. HS, DA, and TC contributed to analysis and editing.

\section{FUNDING}

This research was funded by internal grants from the Melbourne Social Equity Institute and Melbourne Engagement Grants at The University of Melbourne, Australia.

Clayton, M., Sager, R., and Will, U. (2005). "In time with the music: the concept of entrainment and its significance for ethnomusicology," Paper Presented at the European Meetings in Ethnomusicology. Rauland.

Colegrove, V. M., Havighurst, S. S., Kehoe, C. E., and Jacobsen, S. L. (2018). Pilot randomized controlled trial of tuning relationships with music: intervention for parents with a trauma history and their adolescent. Child Abuse Negl. 79, 259-268. doi: 10.1016/j.chiabu.2018.02.017

Day, T., Baker, F., and Darlington, Y. (2009). Experiences of song writing in a group programme for mothers who had experienced childhood abuse. Nord. J. Music Ther. 18, 133-149. doi: 10.1080/08098130903062405

der Heyde, A., and Christine, T. M. (2012). Interpersonal Rhythms Disrupted by a History of Trauma: an In-Depth Case Study of Analytical Music Therapy. New York, NY: City University of New York.

Dixon-Woods, M., Cavers, D., Agarwal, S., Annandale, E., Arthur, A., Harvey, J., et al. (2006). Conducting a critical interpretive synthesis of the literature on access to healthcare by vulnerable groups. BMC Med. Res. Methdol. 6:35. doi: 10.1186/1471-2288-6-35

Fairchild, R. E. (2018). Collaborative Songwriting with Children Experiencing Homelessness and Family Violence to Understand Their Resources. Melbourne, VIC: The University of Melbourne.

Faulkner, S. (2017). Rhythm2Recovery: a model of practice combining rhythmic music with cognitive reflection for social and emotional health within trauma recovery. Aust. N. Z. J. Fam. Ther. 38, 627-636. doi: 10.1002/anzf.1268

Felsenstein, R. (2013). From uprooting to replanting: on post-trauma group music therapy for pre-school children. Nord. J. Music Ther. 22, 69-85. doi: 10.1080/08098131.2012.667824

Flores, K. (2013). African Drumming as a Medium to Promote Emotional and Social Well-Being of Children Aged 7 to 12 in Residential Care (Doctoral dissertation), University of Pretoria, Pretoria, South Africa.

Freire, P. (1993). Pedagogy of the Oppressed. London: Penguin Books.

Gerber, M. M., Hogan, L. R., Maxwell, K., Callahan, J. L., Ruggero, C. J., and Sundberg, T. (2014). Children after war: a novel approach to promoting resilience through music. Traumatol. Int. J. 20, 112-118. doi: 10.1037/h0099396

Graham, S. (2011). Effect of Music Therapy on the Emotional Expressivity of Children and Adolescents Who Have Experienced Abuse or Neglect. Tallahassee, FL: Florida State University.

Grant, M. J., and Booth, A. (2009). A typology of reviews: an analysis of 14 review types and associated methodologies. Health Info. Libr. J. 26, 91-108. doi: 10.1111/j.1471-1842.2009.00848.x

Greene, A. H., Goldenberg, L., and Freundlich, M. (2009). The use of expressive therapies and social support with youth in foster care: the performing arts troupe. J. Youth Develop. 4, 60-77. doi: 10.5195/JYD.2009.275

Hannigan, P. D., and McBride, D. L. (2011). Drumming with intimate partner violence clients: getting into the beat; therapists' views on the use of drumming in family violence treatment groups. Can. Art Ther. Assoc. J. 24, 2-9. doi: 10.1080/08322473.2011.11434787

Herman, J. L. (2015). Trauma and Recovery. New York, NY: Basic Books.

Hunter, S. V., and Rosevear, S. (2011). Evaluating a creative arts program designed for children who have been sexually abused. Aust. N. Z. J. Art Ther. 6, 39-50. 
Jespersen, K. V., and Vuust, P. (2012a). Music for improvement of trauma-related sleep problems. Group 2:5.

Jespersen, K. V., and Vuust, P. (2012b). The effect of relaxation music listening on sleep quality in traumatized refugees: a pilot study. J. Music Ther. 49, 205-229. doi: $10.1093 /$ jmt/49.2.205

Kim, J. (2013). Music therapy with children who have been exposed to ongoing child abuse and poverty: a pilot study. Nord. J. Music Ther. 24, 27-43. doi: 10.1080/08098131.2013.872696

McFerran, K. S., Hense, C., Medcalf, L., Murphy, M., and Fairchild, R. (2017). Integrating emotions into the critical interpretive synthesis. Qual. Health Res. 27, 13-23. doi: 10.1177/1049732316639284

McLean, S. (2016). The Effect Of Trauma On The Brain Development of Children: Evidence-Based Principles for Supporting the Recovery of Children in Care. Melbourne: Australian Institute of Family Studies. Retrieved from: https://aifs.gov.au/cfca/publications/effect-trauma-brain-developmentchildren (accessed December 12, 2019).

Moumdjian, L., Buhmann, J., Willems, I., Feys, P., and Leman, M. (2018). Entrainment and synchronization to auditory stimuli during walking in healthy and neurological populations: a methodological systematic review. Front. Hum. Neurosci. 12:263 doi: 10.3389/fnhum.2018.00263

Navarro, Z. (2006). In search of a cultural interpretation of power: the contribution of Pierre Bourdieu. IDS Bull. 37, 11-22. doi: 10.1111/j.1759-5436.2006.tb00319.x

Neupane, S., and Taylor, J. Y. (2011). Music Therapy for Incarcerated Women Recovering from Trauma and Abuse. Iowa City, IA: The University of Iowa.

Noblit, G. W., and Hare, R. D. (1988). Meta-Ethnography. Thousand Oaks, CA: SAGE Research Methods.

Ogden, P., Minton, K., and Pain, C. (eds.). (2006). Trauma and the Body: A Sensorimotor Approach to Psychotherapy (Norton Series on Interpersonal Neurobiology). New York, NY: W.W. Norton \& Co.

Osborne, N. (2012). Neuroscience and "real world" practice: music as a therapeutic resource for children in zones of conflict. Ann. N. Y. Acad. Sci. 1252, 69-76. doi: 10.1111/j.1749-6632.2012.06473.x

Palidofsky, M., and Stolbach, B. C. (2012). Dramatic healing: the evolution of a trauma-informed musical theatre program for incarcerated girls. J. Child Adolesc. Trauma. 5, 239-256. doi: 10.1080/19361521.2012.697102

Perry, B. (2006). "Applying principles of neurodevelopment to clinical work with maltreated and traumatized children: the neurosequential model of therapeutics," in Working with Traumatized Youth in Child Welfare: Social Work Practice with Children and Families, ed N. B. Webb (New York, NY: Guilford Press), 27-52.

Perry, B. D. (2009). Examining child maltreatment through a neurodevelopmental lens: Clinical applications of the neurosequential model of therapeutics. J. Loss Trauma 14, 240-255. doi: 10.1080/15325020903004350

Porges, S. (2011). The Polyvagal Theory. New York, NY: W. W. Norton and Co.

Porges, S. (2004). Neuroception: a subconscious system for detecting threats and safety. Zero Three 24, 19-24.

Precin, P. (2011). Occupation as therapy for trauma recovery: a case study. Work 38, 77-81. doi: 10.3233/WOR-2011-1106

Reeves, P. D. (2013). How Music and Lyrics Protect and Heal the Souls of African American Women who have Experienced Domestic-Violence Trauma, Sexual Abuse, or Depression: A Phenomenological Study. Carpinteria, CA: Pacifica Graduate Institute.

Rogers, P. (1993). "Research in music therapy with sexually abused clients," in Handbook of Inquiry in the Arts Therapies, ed H. Payne (London: Jessica Kingsley), 197-217.
Rudstam, G., Elofsson, U., Søndergaard, H. P., Bonde, L. O., and Beck, B. D. (2017). Trauma-focused group music and imagery with women suffering from PTSD/complex PTSD: a feasibility study. Approach 9, 202-216.

Sackett, D. L., Rosenberg, W. M., Gray, J. M., Haynes, R. B., and Richardson, W. S. (1996). Evidence based medicine: what it is and what it isn't. Br. Med. J. 312, 71-72. doi: 10.1136/bmj.312.7023.71

Salmon, D., and Rickaby, C. (2014). City of one: a qualitative study examining the participation of young people in care in a theatre and music initiative. Child. Soc. 28, 30-41. doi: 10.1111/j.1099-0860.2012.00444.x

Schrader, E. M., and Wendland, J. M. (2012). Music therapy programming at an aftercare center in Cambodia for survivors of child sexual exploitation and rape and their caregivers. Soc. Work Christ. 39, 390-406.

Siegel, D. (1999). The Developing Mind. New York, NY: Guilford.

Slotoroff, C. (1994). Drumming technique for assertiveness and anger management in the short-term psychiatry setting for adult and adolescent survivors of trauma. Music Ther. Perspect. 12, 111-116.doi: 10.1093/mtp/12.2.111

Stige, B. (2002). Culture-Centered Music Therapy. Gilsum, NH: Barcelona Publishers.

Stige, B., Ansdell, G., Elefant, C., and Pavlicevic, M. (2010). Where Music Helps: Community Music Therapy in Action and Reflection. Surrey, UK: Ashgate.

Story, K. M., and Beck, B. D. (2017). Guided Imagery and Music with female military veterans: an intervention development study. Art. Psychother. 55, 93-102. doi: 10.1016/j.aip.2017.05.003

Strehlow, G. (2009). The use of music therapy in treating sexually abused children. Nord. J. Music Ther. 18, 167-183. doi: 10.1080/08098130903062397

Sutton, J., and De Backer, J. (2009). Music, trauma and silence: the state of the art. Art. Psychother. 36, 75-83. doi: 10.1016/j.aip.2009.01.009

Thaut, M. (2005). Rhythm, Music, and the Brain. New York, NY: Routledge.

Thaut, M., McIntosh, G. C., and Hoemberg, V. (2015). Neurobiological foundations of neurologic music therapy: rhythmic entrainment and the motor system. Front. Psychol. 5:1185. doi: 10.3389/fpsyg.2014.01185

van der Kolk, B., McFarlane, A., and Weisaeth, W. (2007). Traumatic Stress: The Effects of Overwhelming Experience on Mind, Body and Society. New York, NY: The Guilford Press.

van der Kolk, B. A. (2015). The Body Keeps the Score: Brain, Mind, and Body in the Healing of Trauma. London: Penguin Books.

Wellman, R., and Pinkerton, J. (2015). The development of a music therapy protocol: a music 4 life ${ }^{\circledR}$ case report of a veteran with PTSD. Music Med. 7, 24-39.

Withagen, R., Araújo, D., and de Poel, H. J. (2017). Inviting affordances and agency. New Ideas Psychol. 45, 11-18. doi: 10.1016/j.newideapsych.2016.12.002

Zanders, M. L. (2015). Music therapy practices and processes with foster-care youth: formulating an approach to clinical work. Music Ther. Perspect. 33, 97-107. doi: 10.1093/mtp/miv028

Conflict of Interest: The authors declare that the research was conducted in the absence of any commercial or financial relationships that could be construed as a potential conflict of interest.

Copyright (c) 2020 McFerran, Lai, Chang, Acquaro, Chin, Stokes and Crooke. This is an open-access article distributed under the terms of the Creative Commons Attribution License (CC BY). The use, distribution or reproduction in other forums is permitted, provided the original author(s) and the copyright owner(s) are credited and that the original publication in this journal is cited, in accordance with accepted academic practice. No use, distribution or reproduction is permitted which does not comply with these terms. 


\section{OPEN ACCESS}

Edited by: Rosie Perkins,

Royal College of Music, United Kingdom

Reviewed by:

Kai Johannes Lorenz, Bundeswehrkrankenhaus, Germany Rob Van Son,

The Netherlands Cancer Institute (NKI), Netherlands

${ }^{*}$ Correspondence: Evangelos Himonides e.himonides@ucl.ac.uk

Specialty section: This article was submitted to Performance Science, a section of the journal Frontiers in Psychology

Received: 01 June 2019 Accepted: 02 December 2019 Published: 14 January 2020

Citation:

Moors T, Silva S, Maraschin D, Young D, Quinn JM, de Carpentier J, Allouche $J$ and Himonides E (2020)

Using Beatboxing for Creative Rehabilitation After Laryngectomy: Experiences From a Public Engagement Project.

Front. Psychol. 10:2854. doi: 10.3389/fpsyg.2019.02854

\section{Using Beatboxing for Creative Rehabilitation After Laryngectomy: Experiences From a Public Engagement Project}

\author{
Thomas Moors' ${ }^{1}$ Sanjeev Silva', Donatella Maraschin ${ }^{2}$, David Young ${ }^{3}$, John M. Quinn ${ }^{4}$, \\ John de Carpentier ${ }^{5}$, Johan Allouche ${ }^{6}$ and Evangelos Himonides ${ }^{7 *}$ \\ ${ }^{1}$ Shout at Cancer, London, United Kingdom, ${ }^{2}$ School of Arts and Creative Industries, London South Bank University, \\ London, United Kingdom, ${ }^{3}$ School of Science and Engineering, University of Dundee, Dundee, United Kingdom, ${ }^{4}$ First \\ Faculty of Medicine, Institute of Hygiene and Epidemiology, Charles University, Prague, Czechia, ${ }^{5}$ Royal Preston Hospital, \\ Preston, United Kingdom, ${ }^{6}$ Hôpital Saint-Pierre, Brussels, Belgium, ${ }^{7}$ UCL Institute of Education, University College London, \\ London, United Kingdom
}

Laryngectomy is the surgical removal of the larynx (voice box), usually performed in patients with advanced stages of throat cancer. The psychosocial impact of losing the voice is significant, affecting a person's professional and social life in a devastating way, and a proportion of this patient group subsequently must overcome depression (22-30\%) and social isolation (40\%). The profound changes to anatomical structures involved in voicing and articulation, as a result of surgery, radiotherapy or chemotherapy (separately or in combination with one another), introduce challenges faced in speech rehabilitation and voice production that complicate social reintegration and quality of life. After laryngectomy, breathing, voicing, articulation and tongue movement are major components in restoring communication. Regular exercise of the chest, neck and oropharyngeal muscles, in particular, is important in controlling these components and keeping the involved structures supple. It is, however, a difficult task for a speech therapist to keep the patient engaged and motivated to practice these exercises. We have adopted a multidisciplinary approach to explore the use of basic beatboxing techniques to create a wide variety of exercises that are seen as fun and interactive and that maximize the use of the structures important in alaryngeal phonation. We herein report on our empirical work in developing patients' skills, particularly relating to voiced and unvoiced consonants to improve intelligibility. In collaboration with a professional beatboxing performer, we produced instructional online video materials to support patients working on their own and/or with support from speech therapists. Although the present paper is focused predominantly on introducing the structure of the conducted workshops, the rationale for their design and the final public engagement performance, we also include feedback from participants to commence the critical discourse about 
whether this type of activity could lead to systematic underlying research and robustly assessed interventions in the future. Based on this exploratory work, we conclude that the innovative approach that we employed was found to be engaging, useful, informative and motivating. We conclude by offering our views regarding the limitations of our work and the implications for future empirical research.

Keywords: head and neck cancer, throat cancer, voice rehabilitation, laryngectomy, beatboxing

\section{INTRODUCTION}

Head and neck cancers (HNC) are a burgeoning public health burden worldwide, causing significant mortality and morbidity despite clinical advances enabling early diagnosis and treatment (Gupta et al., 2016, 2017). The estimated incidence rates of HNC are shifting toward predominance in the less and least developed regions of the world, where health-care offerings may be inadequately equipped to diagnose and appropriately treat HNC and, thus, health-care outcomes may be much worse as a result (Gupta et al., 2016). Surgical intervention for HNC requires significant expertise and can often have a serious impact on the quality of daily life; therefore, exploring novel support strategies and activities as part of a holistic approach in rehabilitation might ensure improved quality of life among those affected.

\section{BACKGROUND}

\section{Epidemiology}

Head and neck cancers include cancer of the lips, oropharynx, hypopharynx, pharynx, major salivary glands, larynx and sinuses according to the WHO classification of head and neck tumors and series on histological and genetic typing of human tumors (Gatta and Botta, 2017; Wright and Vered, 2017). HNC are considered a rare cancer with reported annual incidence rates of less than six per 100,000 individuals and a prevalence of less than five per 10,000 individuals in some populations (Gatta and Botta, 2017).

A large proportion (35\%) of cases of HNC is laryngeal cancer (Cancer Research United Kingdom, 2019). A laryngectomy is only suggested in the advanced stages of throat cancer in an effort to mitigate metastasis and mortality in this patient group. In developed countries, the number of laryngectomies performed is low because of early presentation and ease of access to health care involving combinations of radiotherapy and chemotherapy. In the United Kingdom (UK), for example, each year there are approximately 500-600 laryngectomies performed, with 542 conducted in 2016-2017 (NHS, 2018). In the developing world, however, many laryngeal cancers are diagnosed at more advanced stages and require total laryngectomy (Staffieri et al., 2006).

Patients with HNC, in common with other 'rare cancers', face difficulties in diagnosis, treatment planning, power of

Abbreviations: E, oesophageal; EL, electrolaryngeal; HNC, head and neck cancers; HPV, human papillomavirus; IPA, International Phonetic Alphabet; PE, pharyngoesophageal; SBN, Standard Beatbox Notation; SCC, squamous cell carcinoma; TE, tracheoesophageal; UCL, University College London; WHO, World Health Organization. research and organization of the disease management approach (Gatta and Botta, 2017). In developing countries, this problem may be worse due to economic reasons limiting both access to health-care provision that might allow early diagnosis and perhaps lead to non-surgical therapies, thus avoiding the need for laryngectomy (Staffieri et al., 2006; Stevens and Huys, 2017).

\section{Laryngectomy}

Laryngectomy is usually performed in patients at later stages of throat cancer. Cancer definitions from the new WHO update divide tumors of the oral cavity and oropharynx into separate chapters, classify SCCs of the oropharynx on the basis of HPV status, abandon the practice of histologic grading for oropharyngeal SCCs that are HPV-positive, recognize small cell carcinoma of the oropharynx, and combine polymorphous low-grade adenocarcinoma and cribriform adenocarcinoma of the tongue and minor salivary glands under the single term 'polymorphous adenocarcinoma' (Westra and Lewis, 2017).

In the presurgical situation, air is expelled from the lungs and passes from the trachea (the windpipe) through the larynx, where vibrations of the mucosa over the vocal folds (the glottis) create a mucosal wave; this is known as the fundamental frequency. In contrast, everything else above the laryngeal assembly that participates in the shaping, amplification, dampening and branding of the sound produced by the glottis is customarily called the vocal tract (including the soft tissue, cartilage, nasal cavity, tongue, teeth and lips; Welch et al., 2019).

When performing a laryngectomy, the surgeon preserves as much pharyngeal mucosa as possible to limit the defect created and make the reconstruction of the neopharynx easier, avoid or minimize flap reconstruction, and facilitate voice recovery (Elmiyeh et al., 2010). The trachea is detached from the larynx and brought forward to be attached to the skin, creating a stoma (opening) in the front of the neck. It is important to note that, upon laryngectomy, the patients' airway is not directly connected with the mouth anymore: instead, they are breathing in and out of the neck (Elmiyeh et al., 2010; Figure 1).

After removal of the larynx, a PE segment is reconstructed with a resonating pharyngeal segment above it (Elmiyeh et al., 2010). The resonating segment will act as the main source of vibration of the air expelled from the lungs and diverted into this segment, resulting in sound production; it is therefore called the neoglottis (Elmiyeh et al., 2010).

The procedure is, in effect, a substantial amputation of the anatomy responsible for sound production. It affects both the afferent and efferent pathways for voice production and control. The whole speech production process and co-ordination, often 

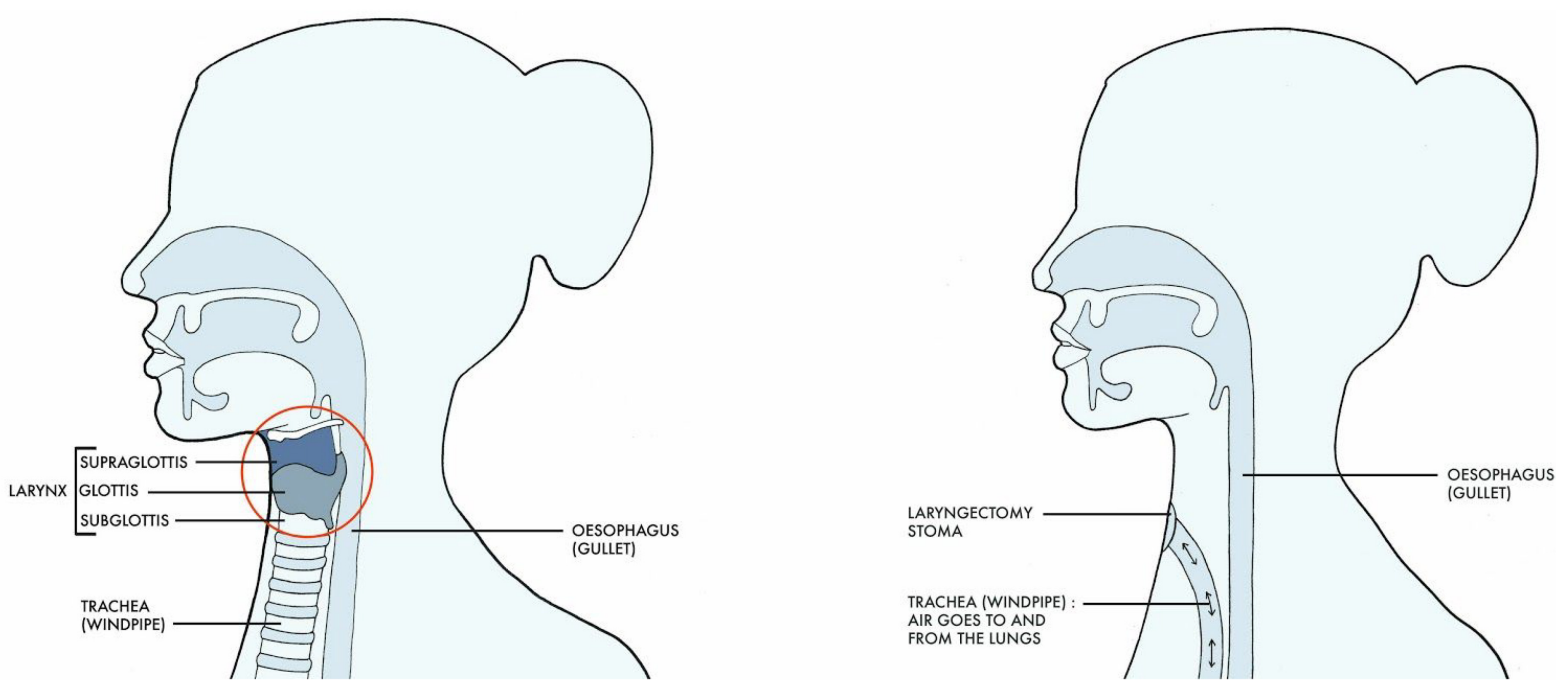

FIGURE 1 | Anatomical changes seen before and after laryngectomy (Drawing Claire Holmes).

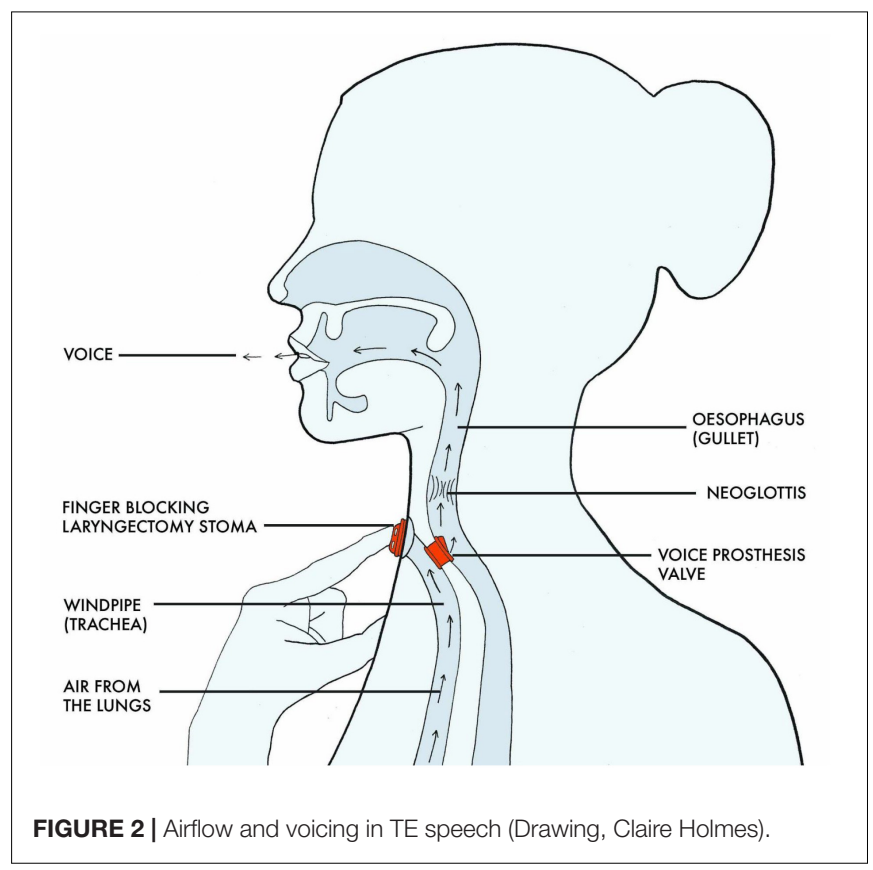

including hand movements, need to be relearned or adjusted depending on which type of voice restoration has been employed.

There are four different ways to restore speech, with TE voice among them nowadays considered to be the gold standard (Jongmans et al., 2006; Elmiyeh et al., 2010; Bohnenkamp et al., 2011).

\section{TE Voice}

In this approach, a surgical puncture allows the placement of a replaceable unidirectional valve that diverts air from the trachea into the esophagus (Figure 2).
When the stoma is occluded, the air has to escape and is therefore pushed through the valve, causing vibration of the neoglottis. Because of the small diameter of this valve, it takes approximately 7.5 times more pressure to achieve voicing when compared with normal laryngeal phonation (Bohnenkamp et al., 2011).

To recommence speaking after laryngectomy, a new coordination strategy is required to prepare and control breathing and for manually occluding the stoma at precisely the right moment so that sufficient pressure can build up to open the valve and divert air through the valve into the neopharynx, where it forms a vibrating column of air as it passes upwards through the neo-glottis. This column of vibrating air can be articulated into sounds and speech similar to the way described earlier (Bohnenkamp et al., 2011).

Overall, this approach produces a more natural sound, requires greater power from the lungs, facilitates more volume control (van As et al., 1998; Jongmans et al., 2006; Kaye et al., 2017), makes it easier to differentiate between voiced and unvoiced consonants (Jongmans et al., 2006), is expensive and necessitates that the valve be replaced every 3-6 months (Staffieri et al., 2006), and offers patients a higher degree of voice satisfaction (Kaye et al., 2017).

\section{Electrolarynx (EL) Voice}

With this solution, there is no valve connecting the trachea with the neopharynx; instead, an artificial vibrating device is held against the user's throat or cheek. When switched on, controlled by its user's thumb, the sound vibration is transmitted to the oral cavity, where it is articulated into speech (Elmiyeh et al., 2010; Kaye et al., 2017).

This approach produces a monotonous, electronic sound; facilitates the speaking of unlimited words; is easy to learn; requires an expensive first purchase but subsequent maintenance is cheap; and makes it difficult to differentiate voiced from 
unvoiced consonants, depending on hand coordination (Kaye et al., 2017).

\section{Esophageal (E) Voice}

E speakers don't have a valve connecting the trachea with the esophagus. Instead, air is drawn into the upper esophagus and then released into the mouth, producing vibrations in the pharyngeal and PE wall that generates a sound (Elmiyeh et al., 2010).

Here, the sounds, volume and number of words are limited; the sound produced is monotonous; the technique is harder to learn; there is no extra cost or need for regular valve replacements; and it is difficult to differentiate voiced from unvoiced consonants (Kaye et al., 2017).

\section{Mouthing or Lip Speech}

When all other options fail or are not available, patients can over-articulate and make limited use of sounds solely using their mouths. With this approach, no sound is produced, there is difficulty differentiating voiced and unvoiced consonants, intelligibility is limited and there is no need for medical devices.

One of the difficulties experienced in speech after laryngectomy, in addition to the issues of volume control and pitch range, is the differentiation between voiced and unvoiced consonants (Jongmans et al., 2006). The variation between these consonants simply depends on whether or not the voice is used to support the articulation. TE phonation demands a controlled expiration through the valve that causes the PE segment to vibrate. For E speech, this is even more difficult, as the phonation comes from swallowed air, while, in the context of EL voice, it depends on the digital control of the device. Therefore for both $\mathrm{E}$ and EL phonation, success comes down to the combination of lip speech and their voicing techniques. Lip speech is hardly practiced in the West, but we assume that it is more frequent in developing countries where patients face difficulty in accessing facilities and medical devices (Staffieri et al., 2006). Nevertheless, evidence suggests that it will add to the clarity of both $\mathrm{E}$ and EL voice.

The psychosocial impact of losing the voice is significant, affecting a person's professional and social life in a devastating way (Dooks et al., 2012; Keszte et al., 2013). A high percentage of this group suffers from social withdrawal (40\%; Danker et al., 2010) and depression (22-30\%; Bussian et al., 2010; Danker et al., 2010; Dooks et al., 2012; Keszte et al., 2013; Perry et al., 2015).

\section{Speech Production}

Normal speech (and singing) requires the involvement of more than 100 muscles. It is remarkable that this process takes place entirely within the body without visual control over movement (Kleber et al., 2010).

The interaction between the activity of the vocal folds, larynx, respiration and articulators is fine-tuned (Dejonckere and Lebacq, 1981) and performed at a fast rate, necessitating the presence of a control system that mainly depends on an intrinsic reflex system (Abo-El-Enein and Wyke, 1966).
This complex process is thought to become automatic in speech once development is complete (Smith and Zelaznik, 2004). It is obvious that a laryngectomy or the removal of the anatomical part responsible for initiating the vibration that results in voicing affects this vocal motor system.

Cohen et al. (1991) showed that lesions, like amputations, cause a neuroplastic reorganization of motor outputs in the brain targeting the muscles proximal to the injury, allowing for a rapid reallocation of the available neural networking. We can expect a similar response in the affected vocal motor system after laryngectomy because of the suggested specific cortical area for the larynx and articulators (Kleber et al., 2010), even though the laryngeal and orofacial muscle fibers are distinct from peripheral muscles (Kent, 2004).

It is suggested that regular practice with great attention to auditory and kinesthetic feedback (e.g., from laryngeal mechanoreceptors) for vocal control helps voice professionals optimize the co-ordination of the vocal motor system, including the articulators and larynx (Sundberg, 1987; Mürbe et al., 2004).

Kleber et al. (2010) also suggest that vocal training increases the involvement of implicit memories of movement control, while Mürbe et al. (2004) postulated that the auditory feedback is most important in the early stages of vocal training, with a fundamental role in pitch control, and that the kinesthetic feedback circuit seems to be particularly improved (e.g., in classical singing) after years of training (Mürbe et al., 2004).

Therefore we suggest that, during the (initial) relearning phases for controlling the new vocal instrument in order to speak, patients who underwent laryngectomy need to be supported whilst exploring their voices and practicing phonation effectively and systematically. Any effective means to foster, maintain, and/or increase the motivation for patients to practice and try to improve would be welcome.

\section{Beatboxing}

At its base nature, beatboxing is the art of vocal percussion; more recently, it has been linked to what is now described as 'hip-hop' culture and is popular amongst younger audiences and artists (Stowell and Plumbley, 2008). Beatboxing employs multiple beat modalities, including vocal instruments, to produce both rhythmic and melodic sounds. These sounds are often perceived as overlapping (occurring in synchrony) in time. The majority of beatboxing sounds imitate percussion instruments like drums and cymbals yet are also seen as similar to speech sounds and can be described using symbols from the International Phonetic Alphabet (IPA; Stowell and Plumbley, 2008; Stowell and Plumbley, 2010; Proctor et al., 2013) ${ }^{1}$ or with the use of characters from a standard English computer keyboard as in the SBN (Splinter and TyTe, 2002).

Despite this similarity with sounds used in speech, beatboxers explore their instruments continually and have been 'inventing' and introducing novel sounds that are non-native to them (Proctor et al., 2013) or even extralinguistic (Proctor et al., 2013; de Torcy et al., 2014).

\footnotetext{
${ }^{1}$ http://www.mcld.co.uk/beatboxalphabet/
} 
Beatboxing is inexpensive, as no purchase of instruments or technical equipment is required to start learning the basics. Beatboxing is also presented as a pluralistic and democratic artform (Himonides et al., 2018) where 'every sound is valid.' We felt that it would be worth investigating whether this inexpensive and easily accessible activity could be of use in speech pathology and, particularly, in rehabilitation after laryngectomy.

\section{Why Beatboxing and Laryngectomy?}

Proctor et al. (2013) showed that beatboxers, like other voice professionals, display an increase in the sensorimotor areas specific for voicing but that this fine-tuned control is 'exploited' to obtain a musical effect.

Due to the surgical changes in anatomy after laryngectomy, other interesting and potentially beneficial aspects of beatboxing include the skill of detaching laryngeal from pharyngeal activity (de Torcy et al., 2014) using the hypopharynx as an individual resonator (Kitamura et al., 2005) and the ability to create plosive sounds with a closed glottis independent of the airflow used for breathing support (de Torcy et al., 2014). An analysis of imaging (Proctor et al., 2013) showed a diversity existed in tongue movement, supporting the possible benefits of beatboxing techniques in promoting suppleness and linking articulation to breathing control and voicing.

Overall, all of these factors support the usefulness of beatboxing in TE, EL, E and lip speech. The rhythmic, playful and explorative approach makes it a useful tool to motivate people to practice co-ordination in voicing and improve intelligibility.

Here, we used beatboxing to explore the alaryngeal voice, breathing control and vocal pitch, paying particular attention to unvoiced and voiced sounds. An additional reason for exploring the use of beatboxing was because it is perceived to be a fun activity, is simple and cheap to conduct, and can be readily adapted for online participation, thus improving accessibility.

\section{MATERIALS AND METHODS}

This project involved Shout at Cancer (a non-profit organization specializing in post-laryngectomy voice), Marv Radio (a beatboxer), UCL music education researchers/facilitators, a group of cancer survivors with laryngectomy coming from across the United Kingdom, local East London youth, and an audience (for the final public performance) that involved families and guests from across London and the United Kingdom.

There were nine laryngectomy patients, including six males and three females, with a mean age of 65 years. Seven used TE voice, one voiced with an EL, and one relied on lip speech or mouthing.

Inclusion criteria were total laryngectomy using TE, E, EL or lip speech. There were no exclusion criteria.

The present study did not require research ethics approval as confirmed by the joint Medical Research Council (MRC) and United Kingdom National Health Service (NHS) Health Research Authority online ethics assessment tool ${ }^{2}$. Nevertheless, written

${ }^{2}$ http://www.hra-decisiontools.org.uk/ethics/index.html informed consent was obtained from all participants and, in the case of the young performers, written informed consent was obtained from their legal guardians.

\section{Workshops}

We organized five workshops, each lasting $2 \mathrm{~h}$, that were held weekly. The defined goals of the workshops were:

(1) To engage a vulnerable group of individuals in collaborative music-making using novel techniques (i.e., beatboxing),

(2) To engage a wider group of local youth in East London in artistic expression and collaboration with cancer patients, and

(3) To engage a wider public audience in an open showcase of masterclass outcomes/concert to explore the use of beatboxing techniques in laryngectomy.

During the workshops, we explored whether beatboxing techniques are applicable in speech rehabilitation after laryngectomy. Patients, clinicians and speech-language pathologists were invited to participate with the beatboxer in developing vocal and breathing skills. We focused on unvoiced and voiced consonants in lip speech, EL and TE voice. We followed the basic beatboxing sounds described in Tables $\mathbf{1}$, 2. We approached the exploration of the consonants, used in the English language, as beatboxing sounds (Table 3). Participants practiced the sounds separately at first and then in different rhythms and combinations to refine and improve the hand, breathing and voicing co-ordination.

In the last two sessions, we included local youth, specifically four boys and two girls between six and 13 years old, who were introduced to basic beatboxing. They practiced together with the patients after an introduction and explanatory talks about laryngectomy. We prepared the songs of the program for the concert 3 weeks later. They were encouraged to interact with the patients and to ask questions.

At first, we introduced three basic beatboxing sounds (see Figure 3): the classic kick drum $\left(\{b\},\left[p^{\prime} I\right]\right)$, the basic or closed hi-hat $\left(\{\mathrm{t}\}\right.$, [țs $\left.\left.\mathrm{t}^{\urcorner}\right]\right)$and the rimshot $\left(\{\mathrm{k}\}\left[\mathrm{k}^{\prime}\right]\right)$. We started to work on each sound separately to focus on pronunciation, controlling volume and tempo. To practice the control of volume, we pronounced the same sound at different levels of loudness, from soft to loud and then at random (e.g., for the kick drum: b,b,b; $\mathrm{B}, \mathrm{B}, \mathrm{B} ; \mathrm{B}, \mathrm{B}, \mathrm{B}$; or $\mathrm{bBb})$.

We worked on the co-ordination of breathing and voicing by repeating the same sound in different tempos, increasing the speed of pronouncing the same sound and progressing from slowly to as fast as possible. During the exercise, it was important to pronounce the sound properly.

For example: b, b, b b,b,b b,b,b

We combined both exercises to work on a better control of speed and volume at the same time For example: $b, B$, B, bbb, B, B, bbb.

The next step, to make it more playful and to help participants understand how easily these exercises can be built into their daily routine, we exercised with rhythms from familiar dance styles or famous songs (e.g., waltz, samba, tango, salsa, 'We Will Rock 
TABLE 1 | Musical classification and the phonetic description of the basic beatboxing sounds used during the workshops based on the description used in prior research (Proctor et al., 2013; IPA, 2015), the SBN (Splinter and TyTe, 2002) and the phonological description of consonants (O'Grady et al., 2017).

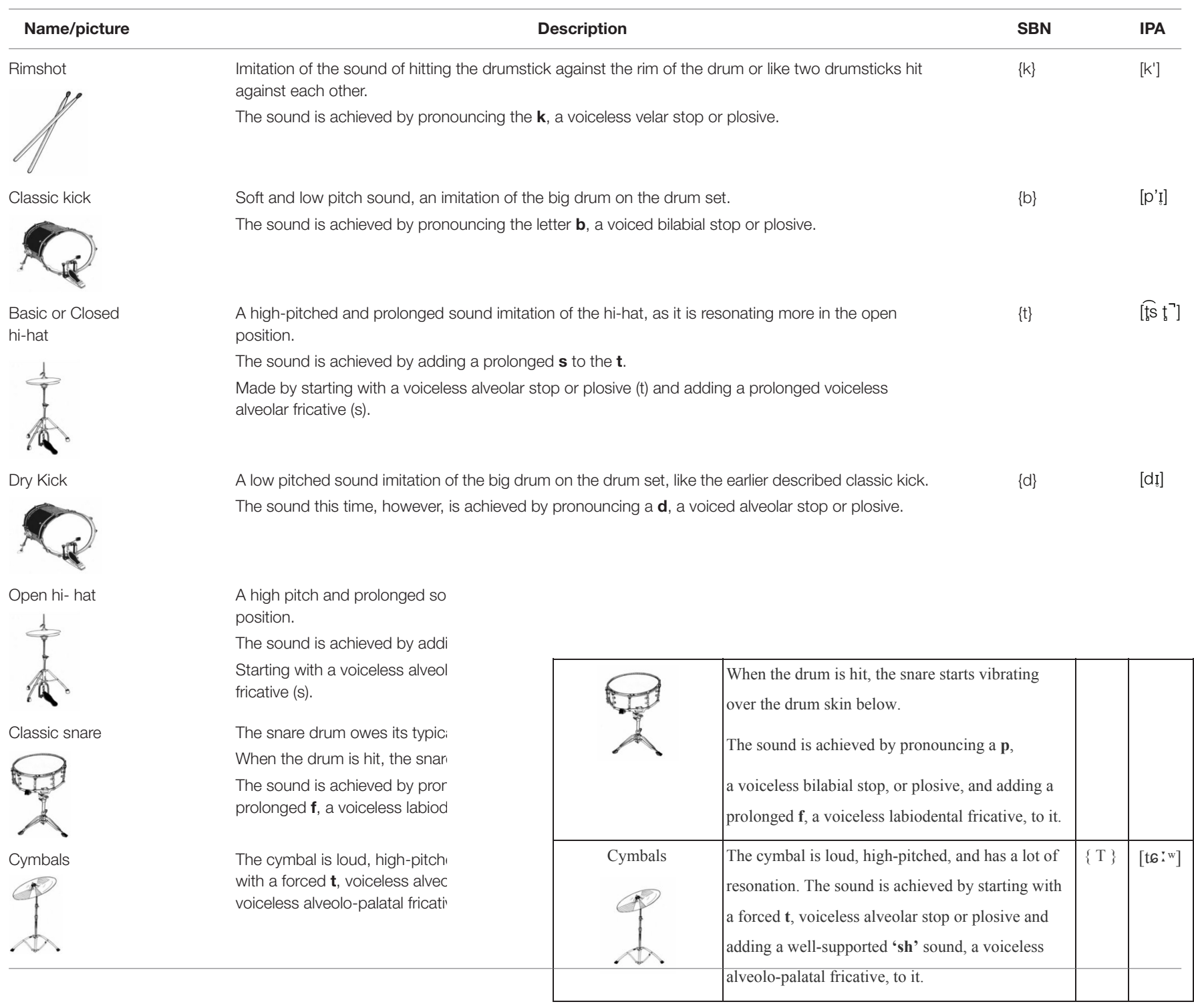

You' by Queen). The integration of these exercises into music facilitated practicing individually.

The workshops were structured in a repetitive way with a variety of actions with an increasing level of difficulty.

The next step focused on developing the participants' controf of the volume of a sequence of different sounds, allowing them to link breathing and voicing control to a limited set of different positioning of the articulators and paying careful attention to its pronunciation.

For example: b,t,k B,T,K $\quad$ B,T,K or bTk.

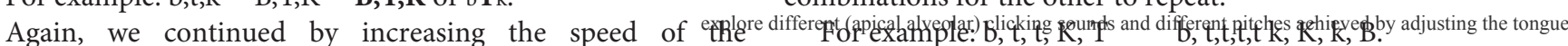
pronounced sounds.

For example: $b, \quad \mathrm{t}, \quad \mathrm{k} \quad \mathrm{b}, \mathrm{t}, \mathrm{k} \mathrm{b}, \mathrm{t}, \mathrm{k}$,

We ended up going really fast and practiced using rhythms from familiar dance styles or famous songs. The changes in tempo and in rhythm add a gradient of fun to the exercise; they also make it a cognitive exercise as it demands more concentration.
Once the participants understood basic beatboxing - that is, the idea of repeating sounds in different levels of volume and rhythms-we started to introduce more sounds and helped them interact more with the beatboxer and each other.

- The beatboxer demonstrated challenging combinations that they had to repeat all together, in pairs or individually.

Several times during the workshops, we conducted battles where the beatboxer challenged the participants or

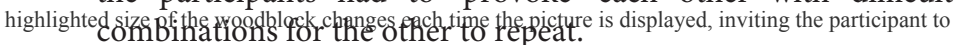

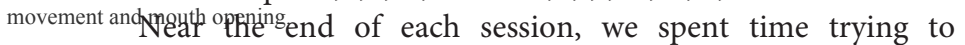
come up with new sounds. Initially, this idea appeared difficult to understand and was met with some hesitation. To spport the participarts with the challenge, §we invited them to imitate different sounds that we are likely to be fainjiar such dog barking, a hellegpter, 飞a car

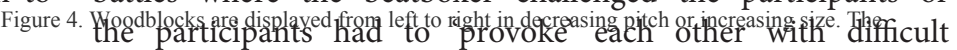


TABLE 2 | Additional sounds (IPA, 2015).

\begin{tabular}{clc}
\hline Name & \multicolumn{1}{c}{ Sound description } \\
\hline IPA block & $\begin{array}{l}\text { A woodblock is a percussion instrument } \\
\text { that has a warm and hollow sound; it } \\
\text { comes in different sizes, each with a } \\
\text { different pitch. } \\
\text { The sound is achieved by a tongue click, } \\
\text { apical (post)alveolar click and changes in } \\
\text { the shape of the mouth cavity to change } \\
\text { pitch. } \\
\text { The whip, slapstick, or clapper creates a } \\
\text { cracking noise. } \\
\text { The sound is achieved by a tongue click, a } \\
\text { laminal postalveolar click and a } \\
\text { simultaneous mouth opening. }\end{array}$ \\
&
\end{tabular}

passing by, a car braking or honking, a phone ringing or a bee buzzing.

When the group felt more confident, we let the participants and the children challenge others in the group with different vas a playful way to improve the ach other explore sounds.

workshops, we had built up a rcal tricks and integrated these in ormance. In one of the songs, we , building up the chaos gradually We then stopped abruptly and struggling with your voice, living

1s we included in the further

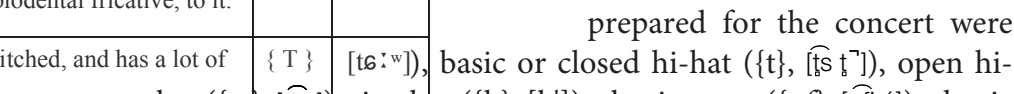

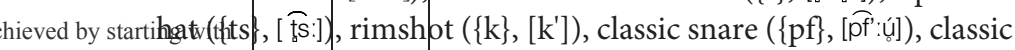
ar stop or plosivkififk drum $\left(\{\phi\},\left[p^{\prime} I\right]\right)$ and dry kick drum $\left.(\{d\},[d]]\right)$.

', sound, a voiceless Tongue-click sounds were a way to do warm-up exercises for it the jaw and the tonque (e.g., whip or slapstick [†] or wood block it. [!]) (Figures 4, 5). These also represent an easier way to practice rhythms within a group, as they do not require co-ordination among breathing, hand movement, voicing and articulation.
Furthermore, it showed to the patients that sound can be created without voicing.

\section{Lip Speech or Mouthing}

To explain how beatboxing could possibly make lip speech or mouthing more clear, we focused on tongue clicks at first. The beatboxer demonstrated how changing the shape and opening of the mouth influences the pitch of the sound. We then practiced pitch control of the clicking sounds in the group and individually. The next step was to shape the vocal tract to obtain the vowels $\mathrm{A}, \mathrm{E}, \mathrm{I}, \mathrm{O}$, and $\mathrm{U}$ in combination with the clicking sounds. We repeated the vowels obtained with clicking sounds, changing the volume, rhythm and pitch.

After the group felt more confident in being creative enough to give shape to vowels without using voice, we explained what the difference is between voiced and unvoiced consonants. Table 3 shows the protocol/set of sounds that we tried to follow systematically. We focused on tackling the production of the paired sounds $\mathrm{t}$ and $\mathrm{d}, \mathrm{p}$ and $\mathrm{b}, \mathrm{f}$ and $\mathrm{v}, \mathrm{s}$ and $\mathrm{z}$, and $\mathrm{k}$ and $\mathrm{g}$ because these were close to the basic beatboxing sounds we had covered already.

For each pair, we repeated each one four times (e.g., $p$ p p p b b b b, p p p p b b b b). To make it slightly harder, we included variations in tempo and volume. Then, we encouraged the participants to pronounce the voiced and non-voiced paired consonants alternatively (e.g., $\mathrm{p} \mathrm{b} \mathrm{p} \mathrm{b} \mathrm{p}$ b p b), again continuing with changing the required volume and tempo. We finished these series of exercises by combining all the sounds randomly where the group had to imitate the voice coach or each other when we divided them up in pairs to practice.

At this point, the participants were paying more attention to the co-ordination in using or not using voice in articulation.

We then explored in a similar way as above the more subtle paired consonants $\theta$ and $\partial, \int$ and 3 , and $t y$ and $\delta$. The nasal sounds $\mathrm{m}, \mathrm{n}$ and $\eta$ are difficult to pronounce in mouthing or lip speech and we did not find beatboxing sounds to make these consonants easier to differentiate.

We approached the consonant $\mathrm{L}$ in a similar way as the tongue clicks, changing the pitch of the sound by adjusting the mouth

\begin{tabular}{|c|c|c|c|c|c|c|c|}
\hline \multirow[t]{2}{*}{ Manner of articulation } & \multirow[t]{2}{*}{ Unvoiced/ voiced } & \multicolumn{6}{|c|}{ Anatomical placement of the articulated consonant } \\
\hline & & Bilabial & Labiodental & Interdental & Alveolar & Palatal & Velar \\
\hline yed, 9 strtititeng the participtspto & Unvoiced & $p$ & & & $\mathrm{t}$ & & k \\
\hline hieved by adjusting the tongue & Voiced & $\mathrm{b}$ & & & $d$ & & $g$ \\
\hline \multirow[t]{2}{*}{ Fricative } & Unvoiced & & $f$ & $\theta$ & s & $\int$ & \\
\hline & Voiced & & v & $\partial$ & z & 3 & \\
\hline \multirow[t]{2}{*}{ Affricate } & Unvoiced & & & & & tf & \\
\hline & Voiced & & & & & d & \\
\hline \multirow{3}{*}{ sonorant 1 Nasal } & Voiced & $\mathrm{m}$ & & & $\mathrm{n}$ & & $\eta$ \\
\hline & Voiced & & & & 1 & $x$ & \\
\hline & Voiced & w & & & j & & \\
\hline
\end{tabular}

Note that the glottal sounds have been left out because of the level of difficulty for the laryngectomy patient. 
opening. The exercise here was to make the sound of pouring water from a bottle in a glass, in a slow or fast way.

\section{TE Speech}

The exercises for TE speech focused on linking breathing to hand co-ordination, phonation and articulation and establishing a clear distinction between the voiced and unvoiced consonants.

We aimed to begin each workshop with breathing exercises as a warm-up, paying a lot of attention to control the expiration through the speech valve and out of the mouth without causing the neoglottis to vibrate or phonate.

We encouraged the participants to prolong the unvoiced consonants $\mathrm{f}$ and $\mathrm{s}$ for $5 \mathrm{~s}$, building up to $10 \mathrm{~s}$. The next step was to steadily grow louder or the other way around. Following this, they were helped to pronounce an $\mathrm{f}$ or s sound five times, each lasting approximately for a second, followed by a 1-s pause. Once they were able to control the $\mathrm{f}$ and s sounds, they were asked to flow over from a prolonged $\mathrm{f}$ into a $\mathrm{v}$ sound or an $\mathrm{s}$ into a $\mathrm{z}$. Then, we started to practice series of five times repeating $f$ and $\mathrm{v}$ alternatively (e.g., f v f v f v f v f v or s z s z s z s z s z).

These exercises seemed to help the participants focus on voiced and unvoiced sounds and, from then on, we systematically followed the consonants presented in Table 3. We tackled the following paired sounds - $\mathrm{t}$ and $\mathrm{d}, \mathrm{p}$ and $\mathrm{b}, \mathrm{f}$ and $\mathrm{v}, \mathrm{s}$ and $\mathrm{z}$, and $\mathrm{k}$ and $g$-because they are close to the basic beatboxing sounds we had covered already.

Next, we made them pronounce the voiced and unvoiced paired consonants alternatively (e.g., $\mathrm{p} \mathrm{b} \mathrm{p} \mathrm{b} \mathrm{p} \mathrm{b} \mathrm{p} \mathrm{b),} \mathrm{again}$ continuing with changing the required volume and tempo. We finished these series of exercises by combining all of the sounds randomly in a scenario where the group had to imitate the voice coach or each other when we divided them into pairs to practice.

We then explored in a similar way as above the more subtle paired consonants $\theta$ and $\partial, 3$ and $t y$, and $t f$ and $\delta$. Unlike in lip speech or mouthing, the nasal sounds $m, n$ and $\eta$ are easy to pronounce in TE speech. In fact, we used these sounds to work on resonance and made the group imitate car or bike engines that were accelerating, slowing down, or hitting their brakes.

The advantage of TE speech, unlike the other voice restoration possibilities, is the airflow that supports voicing. An interesting exercise to control airflow with or without voicing is rolling the $r$ sound. To make it engaging, we imitated growling dogs.

\section{The Concert}

These workshops culminated in a public performance, the world premiere of Beatboxing Without a Voice, at the Olympic Village, Stratford, East London on 8 April 2017. This concert was an interactive session involving local people and families across London, the patients, an opera singer and the beatboxer. The research team also offered brief explanatory talks presenting the layered impact of throat cancer and laryngectomy. There were over 130 people in the audience (this included confirmed bookings as well as Olympic Village visitors who tagged along without prior registration).

Those at the public performance were invited to provide feedback not only about their experience but also about the knowledge that they gained/acquired regarding throat cancer.

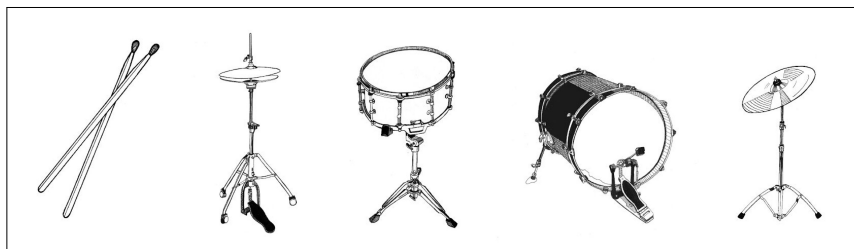

FIGURE 3 | From left to right: Rimshot, Hi-hat, snare drum, kick drum, and cymbal. When the picture is displayed, the patient is invited to imitate his/her interpretation of the sound made by the instrument. Thanks to a visual input only, the patient is freer to explore his or her expression without having been influenced by someone else's performance.

\section{RESULTS}

\section{Pictures}

We translated the basic sounds of beatboxing (Tables 1, 2), into pictures and invited artist Claire Holmes to create bespoke graphics for our learning materials (Figure 3).

\section{Video}

We created a series of video exercises in which the beatboxer first demonstrates the basic beatboxing sounds one by one, followed by a variety of different combinations of these basic beatboxing sounds in different levels of difficulty and tempo. Participants are invited to repeat each exercise.

\section{TE Consonants}

Thanks to breathing control, it is possible to make a difference between voiced and unvoiced consonants in the TE voice. We had the patients practice different rhythms and sound combinations in which there is a change from voiced to unvoiced consonants (Table 2).

\section{Lip Speech Consonants}

We explored how to make the differences between voiced and unvoiced consonants used in English more clear by adding sounds supporting the voiced consonants (Table 3; also, a supporting video on the Shout at Cancer website ${ }^{3}$ is available).

\section{Electrolarynx}

We provided an EL to our beatboxer, who explored and demonstrated the beatboxing possibilities with the device.

With our laryngectomy participants, we worked on the combination of lip speech techniques to obtain unvoiced consonants without the use of the electrolarynx and a smooth coordination of the use of the device to obtain vowels and voiced consonants. These demand a high amount of attention, initially is frustrating to use, and is hard to maintain ${ }^{4}$.

\section{Workshop With Local Youth}

The workshop allowed the patients to be more comfortable and to explore sounds without judgement from outside the group.

\footnotetext{
${ }^{3}$ http://www.shoutatcancer.org/beatboxing

${ }^{4}$ http://bit.ly/2Sl5Ye7
} 
It also helped us in the preparation of the patients and to get the youth familiarized with the aftereffects of laryngectomy. We included the young participants in talks about laryngectomy during the performance.

\section{Working With Voice Professionals}

For some of the voice professionals, it was the first time they had been involved in a beatboxing program. Although focused on the alaryngeal voice, it was interesting for the health-care professional to explore the voice in a different way, determine overlapping skills and be able to explain sounds in a different way. The team reported to have benefited from the techniques learned in the project and will be able to implement these in their own clinical or client-based activities.

\section{Beatboxer}

The beatboxing artist involved in the project faced the incredibly challenging task of having to learn to take other people's physical constraints and limitations into account and also being required to form an understanding about the pathology and social impact on patients after laryngectomy. He worked hard in trying to explore a voice with so many restrictions. This was an incredibly challenging process for a freestyle artist, and the research team witnessed a professional with incredible talent. The interaction with both patients and health-care professionals was challenging, and the learning curve was steep and required continual adjustment and critical thinking.

\section{Final Performance and Feedback}

The project exceeded the aims set out in the initial proposal. Beatboxing after laryngectomy had an impact at several levels, including on both the individuals and the partners involved and potentially on future research.

However, this type of activity was not viewed enthusiastically by all laryngectomees. One participant was particularly negative about beatboxing as an artform and reported that they did not enjoy the workshops, the music or working with the beatboxing expert. Somewhat paradoxically, though, even that particular participant reported that the umbrella of activities leading to the final public engagement concert seemed to offer some benefits for developing breathing control as well as for exercising the different structures for alaryngeal phonation. This was particularly due to beatboxing's strong reliance on rhythmic precision and adhering to strict rhythmical patterns.

Although the present work was primarily centred on public engagement and was not intended to form a clinical research study or intervention study, the team nonetheless decided to record some feedback from the participants and the participating audience. This was seen as essential to gauge the potential or value for similar work to play a key role in a future, systematically researched project.

Throughout the span of this work, the laryngectomees, the core team, the artist, and the collaborating speech and language pathologists/therapists worked in synergy to tweak the beatboxing exercises/tasks to a level where there was a good balance achieved between task-appropriateness for the patients and artistic value for a beatboxing performance. This proved to
TABLE 4 | Laryngectomees' short evaluation of their beatboxing experiences.

\begin{tabular}{lccccccc}
\hline Participant & Q1 & Q2 & Q3 & Q4 & Q5 & Q6 & average \\
\hline p1 & 7 & 7 & 7 & 7 & 7 & 7 & 7.0 \\
p2 & 7 & 7 & 7 & 7 & 7 & 7 & 7.0 \\
p3 & 7 & 7 & 7 & 7 & 7 & 7 & 7.0 \\
p4 & 6 & 4 & 3 & 5 & 5 & 5 & 4.7 \\
p5 & 7 & 6 & 7 & 6 & 7 & 7 & 6.7 \\
p6 & 7 & 4 & 6 & 4 & 5 & 4 & 5.0 \\
p7 & 3 & 3 & 3 & 3 & 3 & 3 & 3.0 \\
p8 & 7 & 3 & 7 & 5 & - & 7 & 5.8 \\
Average & 6.4 & 5.1 & 5.9 & 5.5 & 5.9 & 5.9 & 5.8 \\
\hline
\end{tabular}

be a very meaningful exploratory process where not only key challenges but also useful methods were identified.

All participant laryngectomees were invited to offer feedback about their beatboxing experience using SMS messaging (for convenience) and/or email. Participants were asked to rate the extent to which they agreed or disagreed with the following six statements:

(1) I enjoyed participating in the project,

(2) I benefited psychologically from participating in the project,

(3) My voice production ability has benefited from participating in the project,

(4) I felt more confident about myself after participating in the project,

(5) I would recommend beatboxing to other laryngectomees,

(6) I would participate in a beatboxing project again in the future.

All responses appeared to be positive but not overwhelmingly so (Table 4). Nevertheless, as hinted above, only one laryngectomee appeared to have an overall negative view about their participation in the project; all other respondents offered ratings with a strong sense of positivity (average score $=6.2$ points, standard deviation $=1.01$ points).

Out of the 130+ final concert participants, 58 individuals offered feedback on an online survey instrument, a link to which was made available post-concert using registered participants' email addresses. Participants were allowed to offer ratings about the extent to which they agreed or disagreed with three statements. They were also offered the chance to provide free text feedback in a dedicated textbox. Ratings were performed on a seven-point Likert-type scale, and the available scores ranged from one point (completely disagree) to seven points (completely agree), with four points denoting neutrality (neither agree nor disagree). The three statements that participants were invited to rate were: 'I enjoyed participating in this event,' 'I feel that my understanding about laryngectomy is greater because of this event' and 'I would like to attend a similar event in the future.' Responses were overwhelmingly positive, therefore negating the need for the identification of commonality or diversity in response between different age or sex groups. Table 5 summarizes participants' responses to the three statements.

In addition to the rating of the three statements, 18 participants offered further comments in the available textbox. 
TABLE 5 | Final performance participants' online evaluation.

\begin{tabular}{|c|c|c|c|c|c|c|c|c|c|}
\hline \multirow[t]{2}{*}{ Question } & \multicolumn{7}{|c|}{ Number of responses per scale item } & \multirow[t]{2}{*}{ Mean rating } & \multirow[t]{2}{*}{ Total } \\
\hline & 1 & 2 & 3 & 4 & 5 & 6 & 7 & & \\
\hline $\begin{array}{l}\text { I enjoyed participating } \\
\text { in this event }\end{array}$ & 0 & 0 & 0 & 0 & 0 & 8 & 50 & 6.86 & 58 \\
\hline $\begin{array}{l}\text { I feel that my } \\
\text { understanding about } \\
\text { laryngectomy is greater } \\
\text { because of this event }\end{array}$ & 0 & 0 & 0 & 1 & 7 & 9 & 41 & 6.55 & 58 \\
\hline $\begin{array}{l}\text { I would like to attend a } \\
\text { similar event in the } \\
\text { future }\end{array}$ & 0 & 0 & 0 & 2 & 3 & 10 & 43 & 6.62 & 58 \\
\hline
\end{tabular}

In line with the inordinate positivity shown in the ratings, participants offered optimistic commentaries. Some examples are as follows:

- 'It was a great experience. I felt at ease and look forward to many more similar events in the future.'

- 'A thrill to have been there, so inspiring, keep up the amazing work.'

- 'Amazing effort! Sentences like "I cannot" look ridiculous to be said by anyone about anything after this event! Congratulations!'

- 'This event was truly inspirational. To hear the stories of the larynx group, accompanied by the beautiful words spoken by the children and then the great music really touched my heart. I think the work of all those involved should be applauded and supported. I hope in the future similar events can happen to raise awareness and get the needs of this condition more in the public eye.'

- 'Really interesting to see the work done and the progress made by the alaryngeal individuals, and learn more about the challenges they face and what can be done.'

- 'The speeches delivered by some of the participants were moving and thought-provoking. Understanding that the operation not only removes the voice box but also make the act of breathing so much harder gave me a new sense of respect and appreciation for what these people are going through. The courage and physical stamina they have shown in the face of their situation is a massive inspiration.'

- 'This was absolutely brilliant! I still feel blessed. Thank you for this experience.'

- 'A unique experience and approach to vocal development and requires lateral thinking and helps as a result and not only the feeling of rhythm but also understanding of the mechanics, aiding breathing control.'

- 'I was very impressed, particularly by the kids. The audience participation was a good idea-perhaps more of that in future.'

- 'This was an inspirational and informative event. The concept was so simple yet so uplifting. Thank you for the opportunity to hear patients, professionals, young people, and the public share in making amazing music together.'

- 'This charity gives patients such hope that where they are now, does not always have to be where they stay, they are

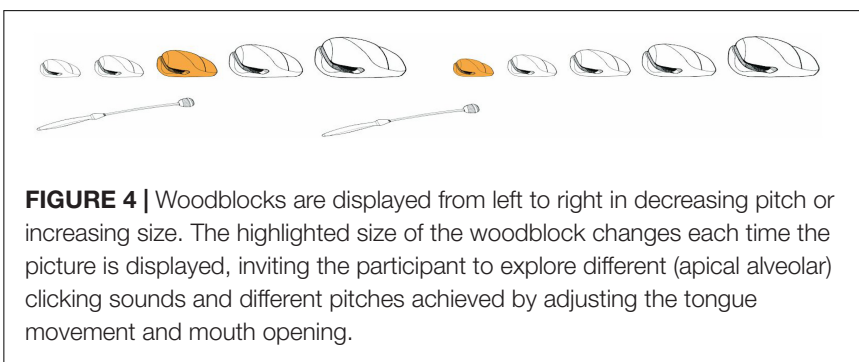

not alone, and that they can achieve anything that they put their mind to. There is life after laryngectomy! And events like this will educate the public on these forgotten patients and their condition.'

- 'Fantastic!! Great community feel to the event, very entertaining and thought-provoking.'

- 'It was a wonderful and inspiring afternoon. What a fabulous project. I thought everyone involved especially the participants were amazing. It was also educational to hear details about the effects of not having a larynx. Profoundly moving experience.'

One comment in particular seemed to capture the ethos of this work and the importance of public engagement, stating 'This event was an amazing celebration of why we need this type of public engagement. What a waste of time would it have been for all these wonderful people to practice beatboxing inside a hospital, in front of researchers!!! You just had to feel the energy in the room in order to understand how powerful this experience was for everybody, patients, children performers and audience. Many congratulations to all involved. .'

\section{DISCUSSION}

This work involved a small group of laryngectomees and a novel approach to creative voice rehabilitation and development within a supportive and intimate environment. This reinforced the work of patients with voice professionals and encouraged an exploration of the patients' voices through beatboxing. It also involved a final public performance in 


\section{$\approx$
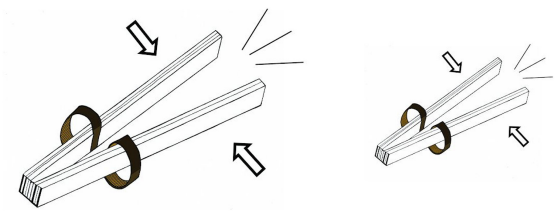

FIGURE 5 | A whip or slapstick. These pictures invite the participant to explore a laminal postalveolar click. By changing the size of the displayed picture, they must react accordingly with volume control. This way, they learn to control the tongue pressure and release in combination with mouth opening in order to influence the volume of the sound. Such helps to practice using the involved articulators, including mainly the tongue.

front of a broad audience. A major challenge for an activity that involves such an eclectic group of individuals is the vulnerability to absences and, unfortunately, three of our patient group became very ill and were unable to participate during the period in which the described workshops took place. For the same reason, we were unable to include someone with $\mathrm{E}$ speech in this group, although we had planned to do so.

Beatboxing is a vocal art that is popular amongst a younger audience, and to introduce it to an older patient group (mean age of 65 years) was initially, as expected, welcomed with some reservation. This is perhaps also reflected in the somewhat conservative scoring that some laryngectomees offered when asked to rate their experiences. However, the laryngectomy group responded well, overall, to the proposed exploration and exercises during the workshops. The recorded feedback was positive; the participants liked it, and it made them approach their voice in a different way, improving voice awareness and work on their pronunciation. All participants reported their engagement in this project has had beneficial effects on their phonation as well as their breathing and the control of support mechanisms.

In order to allow the patient group to have more time for exploring possible sounds systematically and to start developing their basic skills, we only introduced the young coparticipants in the last two sessions. The young participants picked up skills very quickly, with some exceeding the expected level of basic beatboxing techniques.

Although none of the young participants had been exposed to the laryngectomy voice or even heard of the condition before, they were intrigued and not intimidated, exhibiting an openness toward the patients. They were attentive during the introduction and the more detailed explanations about the layered impact after laryngectomy. During the workshops, both patients and participants interacted smoothly with each other. The young people asked questions freely and reacted in a collegial and respectful manner when the laryngectomy group was struggling or had to do a certain exercise at a slower pace. We included the young participants in introducing the small topics presented during the performance. This motivated them to interact with the patient group even more closely and also to prepare and perform background information searches. The societal awareness benefit to this project was not a prime objective, but such a secondary and tertiary gain for all participants anecdotally is of significant note.

The audience in the final public performance was very receptive to the interaction of the young participants with the laryngectomees, the background information and introductions, and the performances. This is also echoed in the written feedback offered, some of which is presented above.

The youth and their parents involved in the project not only found their participation to be rewarding but also reported enjoying being active participants in and contributors to public education and engagement; they reported learning a lot about the actual condition and also commented about being happy and proud to share their experience with the friends and family they had invited. Further, some offered to become more involved with charitable work. This approach of workshops, wherein the patient and target groups are interacting, seemed to be an effective way to explain and experience the impact of a condition. It certainly has inspired the research group and non-profit organization Shout at Cancer to identify an interest in the development of this model further and to organize workshops in different locations and/or with different target groups. This model is likely to be suitable for different types of conditions and these outcomes may be transferable to a wider patient and/or participant group; thus, we believe that it is worthy of further exploration. We note this is obviously something that requires future systematic scrutiny.

The patients seemed to have a stimulating effect on each other; as soon as one of them appeared to have mastered a certain technique or sound, the others found it easier to imitate. Even so, there was variability in the produced sounds and skills. We acknowledge that this was a very limited group of patients ( $\underline{n}=9$ in total), but the positive outcomes and constructive interactions between patients suggest the need to further explore the use of workshops within a group context/environment. This experience suggests further research into the use of more experienced laryngectomy speakers in the voice recovery of patients who have recently had a laryngectomy would be worthwhile.

Furthermore, we discovered that these workshops demanded great concentration and that the exercises could often be tiring. In future workshops we will need to factor in additional time for resting, relaxation and recovery.

The alaryngeal participants were encouraged to allow some time for practice at home between workshops and in preparation for the final public performance. In reality, some of the participants reported they had not performed any alone. When asked why, they stated that not only are the group sessions a social activity that they enjoyed but also these sessions made it easier for them to perform these exercises. They reported that they did not feel incentivized to practice on their own, as it was the group experience that acted as the motivation catalyst. Meanwhile, others reflected that they did find time to do the rhythmical exercises because they were easily built into any type of music they are listening to, and they found themselves beatboxing along to many songs on numerous occasions. 
To facilitate individuals' exercising, we developed and made available relevant demonstration videos and pictures, based on the outcomes of the workshops. We aim to create more exercises at different levels of difficulty and interaction and integrate these in an online platform. The effectiveness of such a design is currently being assessed with further empirical research. This research will hopefully allow the team to offer support to individuals and groups and is hoped to also involve sessions for patients with varying levels of experience but also voice professionals that wish to support laryngectomees. Similar online services could help to improve accessibility to health care (in this case, voice recovery) in both developed and developing countries (Guo and Li, 2018).

\section{CONCLUSION}

Laryngectomy affects a relatively small and scattered population with a higher incidence in the developing world, where there may be limited access to health care and support. The operation has a negative psychosocial impact, leading to high percentages of depression and social isolation among the affected population, where communication issues play an important role.

This public engagement project of beatboxing after laryngectomy used a series of workshops and a final public performance not only to increase awareness about throat cancer and its impact on people's lives but also to explore the potential benefits of creative group participation of laryngectomees and to rehearse whether this has the potential to inform future research and practice.

We explored the implementation of beatboxing techniques into speech rehabilitation after laryngectomy. Both the research evidence from the literature and our somewhat limited empirical findings during the explorative workshops are supportive of the inclusion of beatboxing techniques in rehabilitation of the voice after laryngectomy. The overwhelmingly positive feedback that we have received suggests there is value in presenting how we structured our work and what methods we employed so that future research can build upon the significant effort invested in developing the essential materials, methods and media needed for this work. We adopted and translated some of our findings into educational materials and exercises supported by pictures and video tutorials. These materials are already being accessed by patients, carers and practitioners and are assets of a currently under development interactive technology platform that is intended to increase accessibility in voice recovery.

Clearly, there is a need for future systematic research within this context if a musico-therapeutic curriculum were to be established and/or if a battery were to be developed for the assessment of the effectiveness of such work as 'intervention.' Given the particular demographic and specific challenges that laryngectomees face on a daily basis, a future research design will need to be context sensitive, and we do not foresee a randomized control trial as applicable within the present paradigm.

Albeit a small number of participant laryngectomees, as a willing subset of what we believe to be the world's only organized and systematically practicing and performing alaryngeal vocal ensemble, we found that beatboxing is an exciting, pluralistic, inclusive and very engaging way to introduce a safe synergistic environment within which laryngectomees engaged in creative activity using their novel vocal instruments. These activities were reported to offer greater benefits in allowing the laryngectomees to further develop their breathing as well as support-structures control whilst engaging in meaningful music-making.

\section{DATA AVAILABILITY STATEMENT}

The datasets generated for this study are available on request to the corresponding author.

\section{ETHICS STATEMENT}

An ethics approval was not required as per the authors' Institutions' guidelines and national regulations. This was also confirmed by the joint Medical Research Council (MRC) and United Kingdom National Health System (NHS) Health Research Authority online ethics assessment tool (http:// www.hra-decisiontools.org.uk/ethics/index.html). Nevertheless, written informed consent was obtained from all adult participants and from the parents/legal guardians of non-adult participants/performers.

\section{AUTHOR CONTRIBUTIONS}

TM and EH designed the research project, and conducted the workshops and public engagement performance. TM developed the materials and drafted the manuscript. $\mathrm{EH}$ designed the feedback instrument, collected and analyzed the feedback data, performed the further edits, and finalized the manuscript. All other authors offered feedback and comments for the completion of the final manuscript.

\section{FUNDING}

This study was supported by a UCL Culture Beacon Bursary.

\section{ACKNOWLEDGMENTS}

The authors are grateful to UCL Culture, Shout at Cancer, Marv Radio (beatboxer), Laverne Williams (soprano), Claire Holmes (illustrator), Edoardo Brighenti (video production) and all project and final event participants. 


\section{REFERENCES}

Abo-El-Enein, M. A., and Wyke, B. (1966). Laryngeal myotatic reflexes. Nature 209, 682-686. doi: 10.1038/209682a0

Bohnenkamp, T. A., Forrest, K. M., Klaben, B. K., and Stager, J. M. (2011). Lung volumes used during speech breathing in tracheoesophageal speakers. Ann. Otol. Rhinol. Laryngol. 120, 550-558. doi: 10.1177/000348941112000811

Bussian, C., Wollbrück, D., Danker, H., Herrmann, E., Thiele, A., Dietz, A., et al. (2010). Mental health after laryngectomy and partial laryngectomy: a comparative study. Eur. Arch. Otorhinolaryngol. 267, 261-266. doi: 10.1007/ s00405-009-1068-7

Cancer Research United Kingdom, (2019). Head and Neck Cancer. Available at: https://about-cancer.cancerresearchuk.org/about-cancer/head-neck-cancer (accessed June 12, 2019).

Cohen, L. G., Bandinelli, S., Findley, T. W., and Hallett, M. (1991). Motor reorganization after upper limb amputation in man: a study with focal magnetic stimulation. Brain 114, 615-627. doi: 10.1093/brain/114.1.615

Danker, H., Wollbrück, D., Singer, S., Fuchs, M., Brähler, E., and Meyer, A. (2010). Social withdrawal after laryngectomy. Eur. Arch. Otorhinolaryngol. 267, 593-600. doi: 10.1007/s00405-009-1087-4

de Torcy, T., Clouet, A., Pillot-Loiseau, C., Vaissière, J., Brasnu, D., and CrevierBuchman, L. (2014). A video-fiberscopic study of laryngopharyngeal behaviour in the human beatbox. Logoped. Phoniatr. Vocol. 39, 38-48. doi: 10.3109/ 14015439.2013.784801

Dejonckere, P., and Lebacq, J. (1981). Mechanism of initiation of oscillatory motion in human glottis. Arch. Int. Physiol. Biochim. 89, 127-136. doi: 10.3109/ 13813458109073992

Dooks, P., McQuestion, M., Goldstein, D., and Molassiotis, A. (2012). Experiences of patients with laryngectomies as they reintegrate into their community. Support. Care Cancer 20, 489-498. doi: 10.1007/s00520-0111101-4

Elmiyeh, B., Dwivedi, R., Jallali, N., Chisholm, E., Kazi, R., Clarke, P., et al (2010). Surgical voice restoration after total laryngectomy: an overview. Indian J. Cancer 47, 239-247. doi: 10.4103/0019-509X.64707

Gatta, G., and Botta, L. (2017). "New epidemiologic aspects in head and neck cancers," in Critical Issues in Head and Neck Oncology, eds J. B. Vermorken, V. Budach, C. R. Leemans, J.-P. Machiels, P. Nicolai, and B. O'Sullivan, (Cham: Springer International Publishing), 31-50.

Guo, J., and Li, B. (2018). The application of medical artificial intelligence technology in rural areas of developing Countries. Health Equity 2, 174-181. doi: 10.1089/heq.2018.0037

Gupta, B., Bray, F., Kumar, N., and Johnson, N. W. (2017). Associations between oral hygiene habits, diet, tobacco and alcohol and risk of oral cancer: a casecontrol study from India. Cancer Epidemiol. 51, 7-14. doi: 10.1016/j.canep. 2017.09.003

Gupta, B., Johnson, N. W., and Kumar, N. (2016). Global epidemiology of head and neck cancers: a continuing challenge. Oncology 91, 13-23. doi: 10.1159/ 000446117

Himonides, E., Moors, T., Maraschin, D., and Radio, M. (2018). "Is there potential for using beatboxing in supprting laryngectomees? Findings from a public engagement project," in Proceedings of the Sempre MET2018: Researching Music, Education, Technology, eds E. Himonides, A. King, and F. Cuadrado, (London: Senate House, University of London), 165-168.

IPA, (2015). The International Phonetic Alphabet (revised to 2015). Available at: https://www.internationalphoneticassociation.org/sites/default/files/IPA_Kiel_ 2015.pdf (accessed June 12, 2019).

Jongmans, P., Hilgers, F. J. M., Pols, L. C. W., and van As-Brooks, C. J. (2006) The intelligibility of tracheoesophageal speech, with an emphasis on the voicedvoiceless distinction. Logoped. Phoniatr. Vocol. 31, 172-181. doi: 10.1080/ 14015430500515732

Kaye, R., Tang, C. G., and Sinclair, C. F. (2017). The electrolarynx: voice restoration after total laryngectomy. Med. Devices 10, 133-140. doi: 10.2147/MDER S133225

Kent, R. D. (2004). The uniqueness of speech among motor systems. Clin. Linguist. Phon. 18, 495-505, doi: 10.1080/02699200410001703600

Keszte, J., Danker, H., Dietz, A., Meister, E., Pabst, F., Vogel, H.-J., et al. (2013). Mental disorders and psychosocial support during the first year after total laryngectomy: a prospective cohort study. Clin. Otolaryngol. 38, 494-501. doi: $10.1111 /$ coa. 12194

Kitamura, T., Honda, K., and Takemoto, H. (2005). Individual variation of the hypopharyngeal cavities and its acoustic effects. Acoust. Sci. Technol. 26, 16-26. doi: 10.1250/ast.26.16

Kleber, B., Veit, R., Birbaumer, N., Gruzelier, J., and Lotze, M. (2010). The brain of opera singers: experience-dependent changes in functional activation. Cereb. Cortex 20, 1144-1152. doi: 10.1093/cercor/bhp177

Mürbe, D., Pabst, F., Hofmann, G., and Sundberg, J. (2004). Effects of a professional solo singer education on auditory and kinesthetic feedback-a longitudinal study of singers' pitch control. J. Voice 18, 236-241. doi: 10.1016/j.jvoice.2003. 05.001

NHS, (2018). Laryngeal (larynx) Cancer. London: NHS.

O'Grady, W., Archibald, J., Aronoff, M., and Rees-Miller, J. (2017). Contemporary Linguistics: An Introduction, 7th edn, Boston, NY: Bedford Books.

Perry, A., Casey, E., and Cotton, S. (2015). Quality of life after total laryngectomy: functioning, psychological well-being and self-efficacy. Int. J. Lang. Commun. Disord. 50, 467-475. doi: 10.1111/1460-6984.12148

Proctor, M., Bresch, E., Byrd, D., Nayak, K., and Narayanan, S. (2013). Paralinguistic mechanisms of production in human "beatboxing": a real-time magnetic resonance imaging study. J. Acoust. Soc. Am. 133, 1043-1054. doi: $10.1121 / 1.4773865$

Smith, A., and Zelaznik, H. N. (2004). Development of functional synergies for speech motor coordination in childhood and adolescence. Dev. Psychobiol. 45, 22-33. doi: 10.1002/dev.20009

Splinter, M., and TyTe, G. (2002). Standard Beatbox Notation (SBN). Available at: https://www.humanbeatbox.com/articles/standard-beatbox-notation-sbn/ (accessed June 12, 2019).

Staffieri, A., Mostafea, B. E., Varghese, B. T., Kitcher, E. D., Jalisi, M., Fagan, J. J., et al. (2006). Cost of tracheoesophageal prostheses in developing countries. Facing the problem from an internal perspective. Acta Otolaryngol. 126, 4-9. doi: 10.1080/00016480500265935

Stevens, H., and Huys, I. (2017). Innovative approaches to increase access to medicines in developing countries. Front. Med. 4:218. doi: 10.3389/fmed.2017. 00218

Stowell, D., and Plumbley, M. D. (2008). Characteristics of the beatboxing vocal style (No. C4DM-TR-08-01) (London: Department of Electronic Engineering, Queen Mary), 1-4.

Stowell, D., and Plumbley, M. D. (2010). Delayed decision-making in real-time beatbox percussion classification. J. New Music Res. 39, 203-213. doi: 10.1080/ 09298215.2010.512979

Sundberg, J. (1987). The Science of the Singing Voice. Dekalb: Northern Illinois Univ. Pr.

van As, C. J., Hilgers, F. J. M., Verdonck-de Leeuw, I. M., and Beinum, F. J. K. (1998). Acoustical analysis and perceptual evaluation of tracheoesophageal prosthetic voice. J. Voice 12, 239-248. doi: 10.1016/S0892-1997(98)80044-1

Welch, G., Howard, D. M., and Nix, J. (2019). The Oxford Handbook of Singing 1st edn, Oxford: Oxford University Press.

Westra, W. H., and Lewis, J. S. Jr. (2017). Update from the 4th edition of the World Health Organization classification of head and neck tumours: oropharynx. Head Neck Pathol. 11, 41-47. doi: 10.1007/s12105-017-0793-2

Wright, J. M., and Vered, M. (2017). Update from the 4th Edition of the World Health Organization classification of head and neck tumours: odontogenic and maxillofacial bone tumors. Head Neck Pathol. 11, 68-77. doi: 10.1007/s12105017-0794-

Conflict of Interest: The authors declare that the research was conducted in the absence of any commercial or financial relationships that could be construed as a potential conflict of interest.

Copyright (c) 2020 Moors, Silva, Maraschin, Young, Quinn, de Carpentier, Allouche and Himonides. This is an open-access article distributed under the terms of the Creative Commons Attribution License (CC BY). The use, distribution or reproduction in other forums is permitted, provided the original author(s) and the copyright owner(s) are credited and that the original publication in this journal is cited, in accordance with accepted academic practice. No use, distribution or reproduction is permitted which does not comply with these terms. 


\section{OPEN ACCESS}

Edited by: Michele Biasutti, Università degli Studi di Padova, Italy

Reviewed by:

Margaret S. Osborne,

University of Melbourne, Australia Alexander Refsum Jensenius, University of Oslo, Norway

${ }^{*}$ Correspondence: Garry Kuan garry@usm.my

Specialty section: This article was submitted to Performance Science, a section of the journa Frontiers in Psychology

Received: 07 March 2018 Accepted: 11 June 2018 Published: 10 July 2018

Citation: Nikol L, Kuan G, Ong M, Chang Y-K and Terry PC (2018) The Heat Is On: Effects of Synchronous Music on Psychophysiological Parameters and Running Performance in Hot and Humid Conditions. Front. Psychol. 9:1114. doi: 10.3389/fpsyg.2018.01114

\section{The Heat Is On: Effects of Synchronous Music on Psychophysiological Parameters and Running Performance in Hot and Humid Conditions}

\author{
Luke Nikol1,2, Garry Kuan ${ }^{1,2 \star}$, Marilyn Ong ${ }^{1}$, Yu-Kai Chang ${ }^{3}$ and Peter C. Terry ${ }^{4}$ \\ ${ }^{1}$ Exercise and Sports Science, School of Health Sciences, Universiti Sains Malaysia, Kota Bharu, Malaysia, ${ }^{2}$ Sports Science \\ Unit, School of Medical Sciences, Universiti Sains Malaysia, Kota Bharu, Malaysia, ${ }^{3}$ Graduate Institute of Athletics and \\ Coaching Science, National Taiwan Sport University, Taoyuan City, Taiwan, ${ }^{4}$ Division of Research and Innovation, University \\ of Southern Queensland, Toowoomba, QLD, Australia
}

Running in high heat and humidity increases psychophysiological strain, which typically impairs running performance. Listening to synchronous music has been shown to provide psychophysiological benefits, which may enhance running performance. The present randomized, crossover study examined effects of listening to synchronous music on psychophysiological parameters and running performance in hot and humid conditions. Twelve male runners $(21.7 \pm 2.2$ y; $166.17 \pm 7.18 \mathrm{~cm} ; 60.32 \pm 9.52 \mathrm{~kg}$; $59.29 \pm 5.95 \mathrm{ml} \mathrm{kg}^{-1} \mathrm{~min}^{-1}$ ) completed two running trials in simulated conditions $\left(31^{\circ} \mathrm{C}\right.$ and $70 \%$ humidity) with and without synchronous music. Participants ran on a treadmill inside a climatic chamber for 60 min at $60 \% \dot{\mathrm{V}}_{2}$ max and continued to run to exhaustion at $80 \% \dot{\mathrm{V}}_{2}$ max. Time-to-exhaustion under the synchronous music condition was $66.59 \%$ longer ( mean $=376.5 \mathrm{~s}$ vs. $226.0 \mathrm{~s}, p=0.02, d=0.63$ ) compared to the no music condition. Ratings of perceived exertion were significantly lower for the synchronous music condition at each time point $(15,30,45$, and $60 \mathrm{~min}$ ) of the steady state portion of the running trials. Small differences in heart rate were detected between conditions. No significant between-condition differences were found in urine specific gravity, percentage of body weight loss, thermal comfort, and blood lactate. Findings suggest that listening to synchronous music is beneficial to running performance and perceived exertion in hot and humid conditions.

Keywords: synchronous music, psychophysiology, running, heat, humidity

\section{INTRODUCTION}

Listening to music while engaging in physical activity is a common practice for legions of athletes and exercise participants. A substantial body of empirical evidence has shown that music has the potential to produce a range of beneficial effects in the sport and exercise domain (Terry and Karageorghis, 2011). Benefits of music include positive emotional responses, such as feeling energized (e.g., Karageorghis and Jones, 2014; Hutchinson et al., 2018), reduced perceived 
exertion (e.g., Lim et al., 2014; Ruscello et al., 2014), improved performance (e.g., Terry et al., 2012; Bood et al., 2013), and greater physiological efficiency (e.g., Szmedra and Bacharach, 1998; Bacon et al., 2012).

Several studies have investigated the effects on endurance performance of synchronous music, where participants perform repetitive movements (e.g., walking, running, cycling) in time with the rhythmical elements of the music such as the beat or tempo. For example, Terry et al. (2012) tested synchronous music effects on treadmill running among elite triathletes. Time-toexhaustion was 18.1 and $19.7 \%$ longer when running in time to motivational and neutral music, respectively, compared to no music. Motivational music typically has a fast tempo ( $>120$ beats per minute $[\mathrm{bpm}])$, a strong rhythm, inspiring lyrics and an uplifting harmonic structure, which collectively tend to increase energy and induce bodily action. By contrast, neutral music does not have these characteristics but is not regarded as demotivational. Karageorghis et al. (2009) similarly compared motivational synchronous music, neutral synchronous music and no music during treadmill walking to exhaustion. Music condition accounted for $38 \%$ of the variance in endurance time, with motivational music associated with $15 \%$ longer endurance time over no music and $6 \%$ over neutral music. Bood et al. (2013) also showed that time-to-exhaustion during treadmill running was significantly longer with synchronous motivational music than without. Indeed, use of a simple metronome was associated with significantly better performance than no music, suggesting that any acoustic stimuli with a consistent beat that matches the rhythm of the activity may assist participants to synchronize their running stride to the tempo of the music, which seemingly increases effort and improves running economy.

In tropical countries such as Malaysia, athletes and exercisers are physically active for prolonged periods in hot, humid conditions, typically resulting in impaired physical performance (Tatterson et al., 2000; Saat et al., 2005). Hydration status is an important factor in detecting hypohydration and preventing performance deficits, such as increased perceived exertion, decreased time to fatigue, and increased thermal and cardiovascular strain (Minton et al., 2015). Saat et al. (2005) assessed the challenges to thermoregulation and blood parameters of running in tropical conditions, demonstrating significantly increased dehydration rate and decreased resting hemoglobin, hematocrit and red blood cells among runners completing a daily 60 -min run over a 14 -day period. Further, a study of the Australian National Road Cycling Squad, comparing performance in a 30 -min time-trial, with $\left(32^{\circ} \mathrm{C}\right)$ and without $\left(23^{\circ} \mathrm{C}\right)$ heat stress, reported $6.5 \%$ reduced power output, higher skin temperature and higher sweat rate in the heat condition overall, with lower blood lactate and higher $\mathrm{pH}$ in the latter stages of the trial (Tatterson et al., 2000).

The effects of listening to synchronous music while exercising in the heat have not yet been established empirically. Therefore, in the present study, we assessed running performance in hot, humid conditions with synchronous music compared to no music, and examined whether listening to synchronous music affected blood lactate, heart rate (HR), thermal comfort, perceived exertion, and hydration status of recreational athletes.

\section{MATERIALS AND METHODS}

\section{Participants}

Twelve healthy male participants (mean age $=21.7 \pm 2.2 \mathrm{y}$ ) who ran at least 3 days per week were recruited as participants. Smokers and those with respiratory infections were excluded. Body mass index (BMI) of participants was $21.83 \pm 2.96 \mathrm{~kg}$ $\mathrm{m}^{-2}$. Maximal oxygen uptake $\left(\mathrm{V}_{2} \mathrm{max}\right)$ of participants assessed at baseline was $59.29 \pm 5.95 \mathrm{ml} \mathrm{kg}{ }^{-1} \mathrm{~min}^{-1}$. Baseline $\dot{\mathrm{V}} \mathrm{O}_{2}$ max data were used to calculate running intensity for each participant during the experimental trials. The Physical Activity Readiness Questionnaire (PAR-Q; Gledhill, 2002), informed consent and participant demographic sheet were completed prior to participation. The required sample size was estimated using G-Power Version 3.1 (Faul et al., 2007). Based on a repeatedmeasures ANOVA with 2 running conditions (synchronous music and no music) x 4 time points (baseline, mid [30 and $60 \mathrm{~min}]$, post), statistical power set at $80 \%$ with a $95 \%$ confidence interval, and an effect size of 0.64 (Terry et al., 2012; $\dot{\mathrm{VO}}_{2} \mathrm{max}$ ), a sample of 12 participants was judged to be sufficient to detect the hypothesized between-condition differences.

\section{Measures and Materials}

\section{Rating of Perceived Exertion and Thermal Comfort}

The Rating of Perceived Exertion (RPE) scale (Borg, 1998) was used to assess the intensity of physical work as perceived by participants during the two trials, on a scale from 6 (no exertion) to 20 (maximal exertion). RPE was recorded pre-task, in-task (30 and $60 \mathrm{~min}$ ), and immediately after the run to exhaustion. Thermal comfort was recorded pre-task and in-task (30 and $60 \mathrm{~min}$ ) using the American Society of Heating, Refrigerating and Air-Conditioning Engineers (1966) (ASRHAE) Standard 55 (see also Epstein and Moran, 2006) which ranges from -3 (very cold) to +3 (very hot).

\section{Music Selection}

A shortlist of 20 music tracks with potential to be synchronized to individual running stride ( 1 or 2 strides per beat) was established using the protocol recommended by Karageorghis (2008) and rated for motivational qualities (rhythm, style, melody, tempo, sound and beat) by 10 health science undergraduate students using the Brunel Music Rating Inventory-3 (BMRI-3; Karageorghis, 2008). Music tracks with BMRI-3 ratings of 36-42, indicating motivational qualities, were shortlisted. Participants chose their preferred music selection from the shortlist, which were then assessed for synchronicity with running stride and small adjustments made to tempo ( $\leq 4 \mathrm{bpm})$ using the Virtual DJ software. Table 1 includes the 20 music tracks used in this study. As an example, if a participant with a running cadence of 156 strides per minute chose Pump It by the Black Eyed Peas $(\mathrm{bpm}=77)$ as preferred music, then the music tempo was raised to $78 \mathrm{bpm}$ to allow the participant to run in synchrony with the music at two strides per beat. 
TABLE 1 | List of the 20 music tracks used.

\begin{tabular}{|c|c|c|}
\hline Track Title & Artist & BPM \\
\hline We Will Rock You & Five & 91 \\
\hline Pump It & The Black Eyed Peas & 77 \\
\hline Hurricane & Scorpions and Berlin Philharmonic & 120 \\
\hline Gemuruh & Faizal Tahir & 79 \\
\hline $\begin{array}{l}\text { Gonna Fly Now (Theme from } \\
\text { Rocky) }\end{array}$ & Bill Conti & 81 \\
\hline Extravaganza & Bunkface & 162 \\
\hline Hall Of Fame & William feat. The Script & 85 \\
\hline Mentera Semerah Padi & M.Nasir and Spider & 77 \\
\hline Gemuruh Suara & Team Malaysia's Theme Song & 148 \\
\hline Fikirkan Boleh & Metropolitan & 154 \\
\hline $\begin{array}{l}\text { Standing In The Eyes Of The } \\
\text { World }\end{array}$ & Ella & 84 \\
\hline Stronger & Kanye West & 104 \\
\hline $\mathrm{Na} \mathrm{NaNa}$ & My Chemical Romance & 166 \\
\hline Smells Like Teen Spirit & Nirvana & 116 \\
\hline Afterlife & Avenged Sevenfold & 110 \\
\hline All I Do Is Win & $\begin{array}{l}\text { DJ Khaled ft. Ludacris, Rick Ross, } \\
\text { Snoop Dogg, and T-Pain }\end{array}$ & 150 \\
\hline Battle Scars & Guy Sebastian ft. Lupe Fiasco & 84 \\
\hline Worth It & Fifth Harmony ft. Kid Ink & 100 \\
\hline Unstoppable & Sia & 89 \\
\hline Bang Bang & $\begin{array}{l}\text { Jessie J, Ariana Grande, and Nicki } \\
\text { Minaj }\end{array}$ & 150 \\
\hline
\end{tabular}

\section{Ambient Temperature, Relative Humidity and Music Volume}

Figure 1 shows a schematic of the experimental set up. An hour prior to exercise testing, the exercise laboratory was heated to $31^{\circ} \mathrm{C}$ using halogen lamps (Philips-500 W, France). Humidity was established at 70\% using a water-bath (Memment W350t, Germany). A standing fan was used to mimic airflow in an open environment. Ambient room temperature and relative humidity were monitored continuously using a digital psychrometer kit (Extech Instrument RH305, United States). Music was played via a laptop computer using Virtual DJ software with one speaker (Sony GTK-XB90) placed $1 \mathrm{~m}$ in front of participants at a $45^{\circ}$ angle, although in retrospect use of stereo headphones to deliver the music may have provided a more ecologically valid set-up. Music volume was set at $75 \mathrm{~dB}$, assessed adjacent participants' ears, as recommended by Alessio and Hutchinson (1991). No filtering of the sound occurred to, for example, control how much bass was present in the sound.

\section{Procedure}

The study received approval from the Universiti Sains Malaysia (USM) Human Research Ethics Committee (USM/JEPeM/16020085) and was conducted in accordance with the guidelines of the International Declaration of Helsinki. The test protocol involved five sessions each of $1 \mathrm{~h}$ duration over a 3-5 week period. During sessions 1-3, music selection, anthropometric assessment, submaximal testing, a maximum oxygen uptake $\left(\dot{\mathrm{V}}_{2} \max \right)$ test and a familiarization trial were conducted. During sessions 4-5, participants completed two counterbalanced, experimental trials running in the heat with either synchronous music or no music.

The submaximal test involved running on a motorized treadmill (Track Master TMX425CP, United States) to determine the relationship between speed and oxygen consumption. Each participant completed a 1-2 min warm-up at 5.0-6.0 $\mathrm{km} \mathrm{h}^{-1}$ followed by four, 4-min periods of running at $6,7,8$, and $9 \mathrm{~km}$

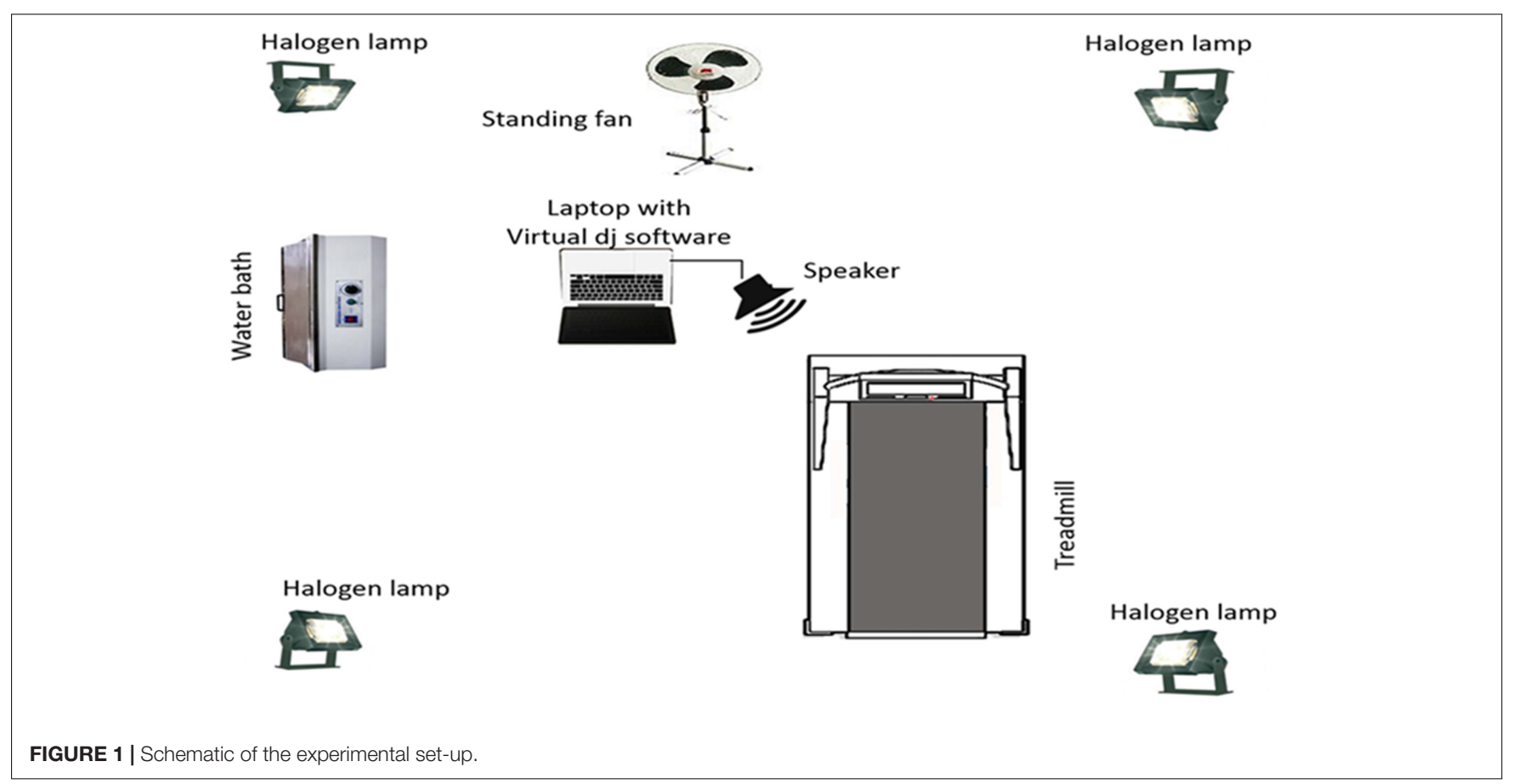


$\mathrm{h}^{-1}$. Expired air was measured at $20 \mathrm{~s}$ intervals using a gas analyzer (Metamax 3B, Germany). Heart rate (HR) and rate of perceived exertion (RPE) were assessed during the final min of each 4-min running period, as recommended by Minetti et al. (1994).

To assess $\dot{\mathrm{VO}}_{2}$ max, participants completed a 2-min warm-up at $5.0-6.0 \mathrm{~km} \mathrm{~h}^{-1}$ and then rested for $1 \mathrm{~min}$ while headgear, mouthpiece, and nose clip were fitted. An appropriate speed was selected that allowed participants to run for at least $12 \mathrm{~min}$ based on previous calculations of individual speed- $\dot{\mathrm{V}} \mathrm{O}_{2}$ correlations. The incremental test began with a gradient of $3.5^{\circ}$, which was increased by $2.5^{\circ}$ at the end of each 3 -min period $\left(3.5^{\circ}, 6.0^{\circ}\right.$, $\left.8.5^{\circ}, 11.0^{\circ}\right)$. Expired air was measured at $20 \mathrm{~s}$ intervals. HR and RPE were recorded in the last $30 \mathrm{~s}$ of each gradient increment. Data collection continued until participants raised one finger to signal the final 1 min before exhaustion. Treadmill speed was reduced rapidly and progressively after the final 1 min was completed.

During the familiarization trial, participants ran at $60 \%$ $\dot{\mathrm{V}} \mathrm{O}_{2}$ max for $60 \mathrm{~min}$ and then continued running at $80 \%$ of their $\dot{\mathrm{V}} \mathrm{O}_{2} \max$ until exhaustion. The familiarization trial was completed to ensure that the prescribed intensity was sustainable for participants without risking injury. Running cadence (step/min) of participants was established during this trial using a GPS-enabled watch (Suunto, Ambit2S, Finland). The familiarization trial was carried out in the heat $\left(31^{\circ} \mathrm{C}\right.$, $70 \%$ relative humidity). After the familiarization, the first experimental trial was conducted 1 week later.

Two experimental trials (synchronous music and no music) were completed in the climatic chamber. During the synchronous music condition, music was played for 60 min while running in the heat chamber at $60 \% \dot{\mathrm{VO}}_{2} \max$ and throughout the run to exhaustion at $80 \% \dot{\mathrm{V}} \mathrm{O}_{2} \max$, terminating upon exhaustion. A 1-week recovery period occurred between the two trials. Participants reported to the laboratory at $8.00 \mathrm{am}$, having refrained from food consumption for at least eight hours. Participants consumed a standardized breakfast of 1 piece of white bread (60 calories) and $250 \mathrm{ml}$ of plain water $2 \mathrm{~h}$ prior to the experiment trial.

Each experimental trial was conducted on a motorized treadmill (Track Master TMX425CP, United States) and running time-to-exhaustion time was recorded using a stopwatch. Tympanic temperature was monitored every $10 \mathrm{~min}$. Heart rate was recorded at pre-, $15 \mathrm{~min}, 30 \mathrm{~min}, 45 \mathrm{~min}, 60 \mathrm{~min}$, exhaustion and $1 \mathrm{~h}$ recovery. For hydration status, urine was sampled and body weight measured (Tanita, Japan) at pre, exhaustion and $1 \mathrm{~h}$ recovery to obtain percentage changes of body weight (McDermott et al., 2017) and urine specific gravity (Sper Scientific 300003C). Blood samples $(\sim 8 \mathrm{ml})$ were taken at pre-, $60 \mathrm{~min}$ during exercise, exhaustion and $1 \mathrm{~h}$ recovery using cannulation via the antecubital veins into preservative-free tubes. Whole blood was centrifuged at $1300 \mathrm{RCF}$ for $10 \mathrm{~min}$, and extracted plasma was stored at $-20^{\circ} \mathrm{C}$ for later analysis. A portion of plasma was used to analyze lactate using an enzymatic calorimetric method (EnzyChrom, BioAssays, United States). A $85 \mu \mathrm{l}$ plasma was mixed with $15 \mu \mathrm{l}$ buffer assay enzyme containing lactate dehydrogenase and a color-producing reagent before the absorbance was read at $565 \mathrm{~nm}$ at $0 \mathrm{~min}$ and $25 \mathrm{~min}$ following the start of incubation. The intra- and inter-assay coefficient variation were 4.0 and $7.2 \%$, respectively.

\section{Data Analyses}

Data analysis was conducted using the Statistical Package for the Social Sciences (SPSS version 22.0). Variables were described as means $(M) \pm$ standard deviation $(S D)$, and distributions of all variables were assessed for normality. No non-normal distributions were identified. Mixed factorial ANOVAs and pairwise comparisons were conducted on HR, RPE, blood lactate, and thermal comfort data. A paired samples $t$ - test was used to compare time to exhaustion between the two experimental conditions. A flow chart of the study design is shown in Figure 2.

\section{RESULTS}

\section{Heart Rate}

Table 2 shows the HR comparisons for 12 runners during the synchronous music and no music conditions. Mixed factorial ANOVA identified a significant difference in HR over time, $F(6,17)=432.64, p=0.001, \eta^{2}=0.993$ but no significant overall difference between groups $F(1,22)=0.056, p=0.816, \eta^{2}=0.003$, nor interaction between time and group, $F(6,17)=0.183$, $p=0.978, \eta^{2}=0.061$. Pairwise comparisons showed that HR was 1-3 bpm lower for the synchronous music condition compared to the no music condition at each stage of the running trial. This difference was statistically significant at the 15 min mark $(t=2.20$, $p=0.05, d=0.16)$.

\section{Blood Lactate}

Table 3 shows blood lactate comparisons for 12 runners during the synchronous music and no music conditions. Mixed factorial ANOVA identified a significant difference in blood lactate over time, $F(3,20)=30.51, p=0.001, \eta^{2}=0.821$ but no significant difference between groups $F(1,22)=0.015, p=0.903, \eta^{2}=0.001$ nor interaction between time and group, $F(3,20)=0.796$, $p=0.510, \eta^{2}=0.107$.

\section{Hydration Status}

Table 4 shows urine specific gravity and \% body weight change for 12 runners during the synchronous music and no music conditions. Mixed factorial ANOVA identified no significant differences in hydration status over time, $F(1,22)=1.195$, $p=0.286, \eta^{2}=0.052$, no significant difference between groups, $F(1,22)=0.171, p=0.683, \eta^{2}=0.008$, and no significant interaction between time and group, $F(1,22)=0.531, p=0.474$, $\eta^{2}=0.024$.

\section{Rating of Perceived Exertion (RPE)}

Table 5 shows RPE values for 12 runners during the synchronous music and no music conditions. Mixed factorial ANOVA identified a significant difference in perceived exertion over time, $F(5,18)=63.234, p=0.001, \eta^{2}=0.946$, and between groups, $F(1,22)=4.690, p=0.041, \eta^{2}=0.176$, but no significant 


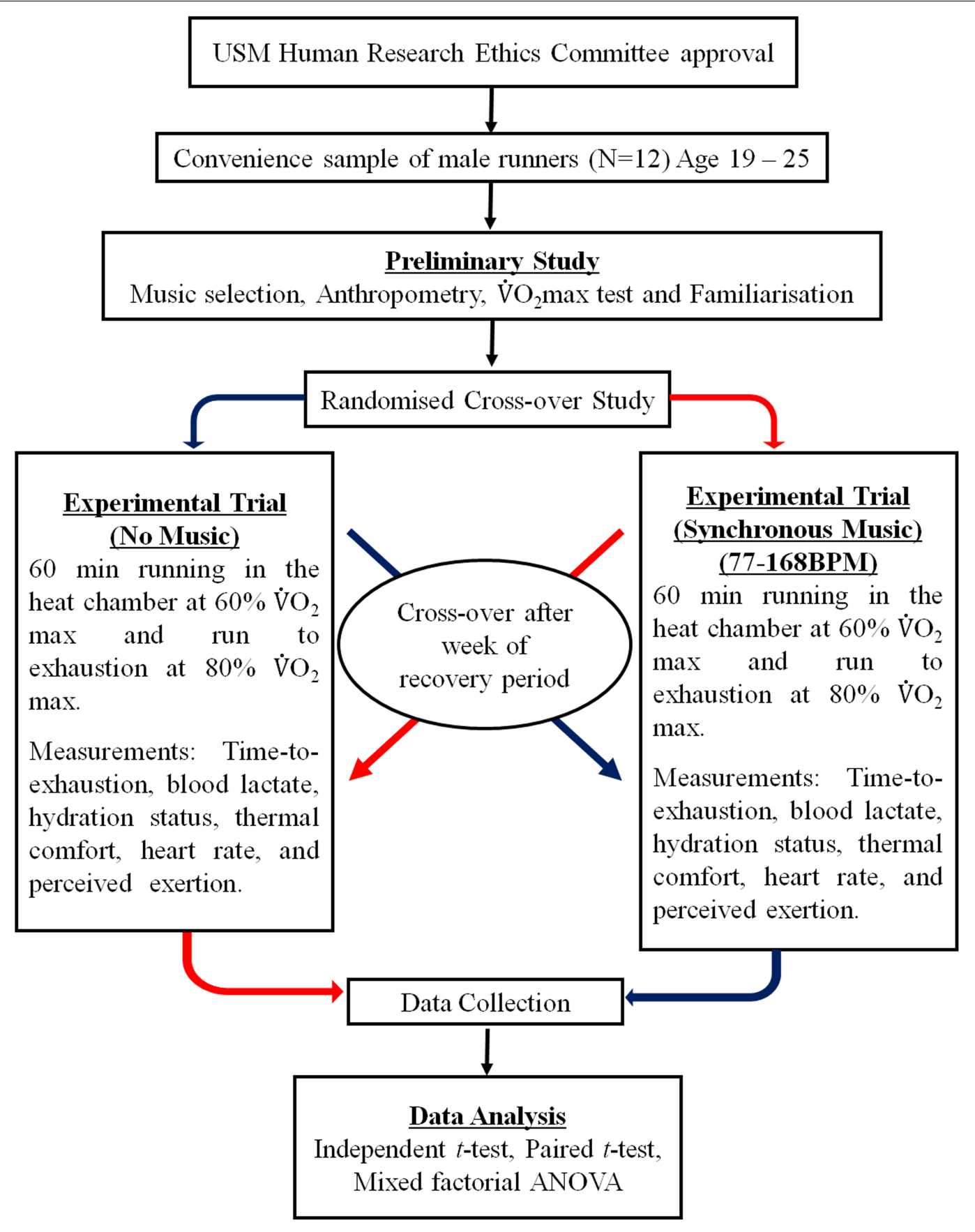

FIGURE 2 | Flow chart of the study design.

interaction between time and group, $F(5,18)=1.117, p=0.386$, $\eta^{2}=0.237$. RPE was significantly lower in the synchronous music condition throughout the steady state portions of the running trial, $(d=0.72-1.05)$ even though the workload was objectively the same. During the first $60 \mathrm{~min}$ of the two trials, when participants completed the same workload, RPE was on average $22 \%$ lower for the synchronous music condition. Perceived exertion at the end of the run-to-exhaustion was $5 \%$ lower $(d=0.34)$ for the synchronous music condition even though participants had run for significantly longer at the same intensity.

\section{Thermal Comfort}

Table 6 shows thermal comfort scores for 12 runners during the synchronous music and no music conditions. Mixed factorial ANOVA identified no significant difference in thermal comfort over time, $F(4,19)=18.680, p=0.001, \eta^{2}=0.797$, no significant difference between groups, $F(1,22)=0.007, p=0.934$, 
TABLE 2 | Heart rate data for 12 runners under synchronous music and no music conditions.

\begin{tabular}{|c|c|c|c|c|c|}
\hline & No music & Synchronous music & $t$ & $p$-value & Effect size (d) \\
\hline Heart Rate - pre-test (bpm) & $78.08 \pm 14.92$ & $78.50 \pm 9.61$ & -0.011 & 0.92 & 0.03 \\
\hline Heart Rate - 15 min (bpm) & $152.67 \pm 11.56$ & $150.58 \pm 13.16^{*}$ & 2.20 & 0.05 & 0.16 \\
\hline Heart Rate - 30 min (bpm) & $161.58 \pm 12.25$ & $158.75 \pm 15.20$ & 1.41 & 0.19 & 0.21 \\
\hline Heart Rate - 45 min (bpm) & $167.17 \pm 12.66$ & $165.83 \pm 18.42$ & 0.56 & 0.59 & 0.08 \\
\hline Heart Rate - 60 min (bpm) & $171.17 \pm 13.69$ & $170.67 \pm 18.12$ & 0.24 & 0.82 & 0.03 \\
\hline Heart Rate - exhaustion (bpm) & $186.75 \pm 11.50$ & $185.50 \pm 12.58$ & 0.41 & 0.69 & 0.10 \\
\hline Heart Rate $-1 \mathrm{~h}$ recovery & $85.83 \pm 14.07$ & $85.50 \pm 12.95$ & 0.11 & 0.92 & 0.02 \\
\hline
\end{tabular}

Data expressed as mean \pm standard deviation. ${ }^{*} p \leq 0.05$.

TABLE 3 | Blood lactate data for 12 runners under synchronous music and no music conditions.

\begin{tabular}{|c|c|c|c|c|c|}
\hline & No music & Synchronous music & $t$ & $p$-value & Effect size (d) \\
\hline Lactate - pre-test (mmol/L) & $2.98 \pm 1.43$ & $2.21 \pm 1.32$ & 1.36 & 0.20 & 0.56 \\
\hline Lactate - 60min (mmol/L) & $3.54 \pm 1.93$ & $3.89 \pm 2.64$ & -0.53 & 0.60 & 0.15 \\
\hline Lactate - exhaustion (mmol/L) & $7.80 \pm 2.67$ & $8.33 \pm 1.83$ & -0.56 & 0.59 & 0.23 \\
\hline Lactate recovery (mmol/L) & $2.50 \pm 2.71$ & $2.58 \pm 0.47$ & 0.09 & 0.93 & 0.04 \\
\hline
\end{tabular}

Data expressed as mean \pm standard deviation.

TABLE 4 | Urine specific gravity and \% body weight change for 12 runners.

\begin{tabular}{|c|c|c|c|c|c|}
\hline & No Music & Synchronous Music & $t$ & $p$-value & Effect size (d) \\
\hline \multicolumn{6}{|l|}{ Urine Specific Gravity } \\
\hline Pre & $1.019 \pm 0.008$ & $1.021 \pm 0.009$ & 0.76 & 0.46 & 0.23 \\
\hline Post & $1.021 \pm 0.007$ & $1.022 \pm 0.002$ & 0.36 & 0.73 & 0.19 \\
\hline Pre-Post & $-0.001 \pm 0.003$ & $-0.000 \pm 0.004$ & 0.83 & 0.43 & 0.28 \\
\hline Body weight change (\%) & $1.77 \pm 0.57$ & $1.82 \pm 0.64$ & -0.55 & 0.59 & 0.08 \\
\hline
\end{tabular}

Data expressed as mean \pm standard deviation. Body weight change $(\%)=[($ pre-exercise bodyweight - post-exercise body weight)/pre-exercise body weight $\times 100$.

$\eta^{2}=0.918$, and no significant interaction between time and group, $F(4,19)=1.579, p=0.221, \eta^{2}=0.249$.

\section{Running Time-to-Exhaustion}

Table 7 shows running time-to-exhaustion for 12 runners during the synchronous music and no music conditions. Participants ran for significantly longer before reaching exhaustion while listening to synchronous music $(376.50 \pm 304.97 \mathrm{~s})$ compared to the no music condition ( $226 \pm 150.32 \mathrm{~s}), t=2.63, p=0.02$. The performance benefit of running to synchronous music represented a moderate-to-large effect $(d=0.63)$, with a mean gain of $150.50 \mathrm{~s}$.

\section{DISCUSSION}

The present study investigated effects of listening to synchronous music on physiological, psychophysiological and performance measures under heat stress conditions. The greatest benefit of synchronous music was apparent for time-to-exhaustion, where participants ran 2.5 min longer on average, equating to a $66.59 \%$ improvement over the no music condition. This performance benefit is consistent with previous findings (e.g., Karageorghis et al., 2009; Terry et al., 2012) but of a much greater magnitude. In addition, perceived exertion was significantly lower, by an average of $22 \%$, during the synchronous music condition compared to the no music condition. This benefit is also consistent with previous findings (e.g., Dyrlund and Wininger, 2008; Hutchinson and Karageorghis, 2013) but again is of a greater magnitude.

The mechanisms by which listening to music could improve endurance performance by such a substantial margin are not clearly established, although several factors may have contributed in combination. Firstly, a benefit may accrue via attentional processes, wherein limited processing capacity causes signals of fatigue to be masked by attending to music, thereby reducing perceived exertion and encouraging participants to work harder and/or for longer (Hardy and Rejeski, 1989). This effect would tend to be nullified toward the latter stages of a run-to-exhaustion because the intensity of the fatigue symptoms, particularly the sharp rise in respiration rate and blood lactate, diverts attention away from the music (Ekkekakis, 2003).

Secondly, from an evolutionary perspective, humans appear to have gained a genetic predisposition to synchronize movement to musical rhythms (Patel, 2008; Phillips-Silver and Keller, 2012). A central pattern generator or pacemaker in the brain has been proposed (Schneider et al., 2010), which regulates temporal functioning and governs the human rhythm response, meaning that enhanced rhythm in the movement pattern created 
TABLE 5 | RPE for 12 runners under synchronous music and no music conditions.

\begin{tabular}{|c|c|c|c|c|c|}
\hline & No Music & Synchronous Music & $t$ & $p$-value & Effect size (d) \\
\hline RPE - pre-test & $6.83 \pm 1.59$ & $6.50 \pm 0.90$ & 1.30 & 0.22 & 0.26 \\
\hline $\mathrm{RPE}-15 \mathrm{~min}$ & $9.92 \pm 2.57$ & $7.67 \pm 1.61^{* *}$ & 3.04 & 0.01 & 1.05 \\
\hline $\mathrm{RPE}-30 \mathrm{~min}$ & $11.17 \pm 2.48$ & $8.83 \pm 2.55^{* *}$ & 3.39 & 0.01 & 0.93 \\
\hline $\mathrm{RPE}-45 \mathrm{~min}$ & $13.08 \pm 2.15$ & $11.00 \pm 3.05^{* *}$ & 3.49 & 0.01 & 0.79 \\
\hline RPE - 60 min & $14.58 \pm 2.23$ & $12.83 \pm 2.59^{* *}$ & 3.17 & 0.01 & 0.72 \\
\hline RPE - exhaustion & $17.83 \pm 2.08$ & $17.00 \pm 2.80$ & 1.36 & 0.20 & 0.34 \\
\hline
\end{tabular}

Data expressed as mean \pm standard deviation. $* * 0.01$.

TABLE 6 | Thermal comfort scores for 12 runners under synchronous music and no music conditions.

\begin{tabular}{|c|c|c|c|c|c|}
\hline & No Music & Synchronous Music & $t$ & $p$-value & Effect size (d) \\
\hline Thermal Comfort - pre-test & $0.42 \pm 0.67$ & $0.25 \pm 0.97$ & 1.00 & 0.34 & 0.20 \\
\hline Thermal Comfort - 15 min & $1.33 \pm 0.65$ & $1.33 \pm 0.49$ & 0.01 & 1.00 & 0.0 \\
\hline Thermal Comfort - 30 min & $1.58 \pm 0.79$ & $1.75 \pm 0.75$ & -0.80 & 0.44 & 0.22 \\
\hline Thermal Comfort - 45 min & $2.17 \pm 0.72$ & $1.92 \pm 0.79$ & 1.39 & 0.19 & 0.33 \\
\hline Thermal Comfort - 60 min & $2.33 \pm 0.98$ & $2.5 \pm 0.80$ & -0.69 & 0.50 & 0.19 \\
\hline
\end{tabular}

Data expressed as mean \pm standard deviation.

TABLE 7 | Time-to-exhaustion for 12 runners under synchronous music and no music conditions.

\begin{tabular}{|c|c|c|c|c|c|}
\hline & No music & Synchronous music & $t$ & $p$-value & Effect size (d) \\
\hline Time-to-exhaustion (s) & $226 \pm 150.32$ & $376.50 \pm 304.97^{*}$ & -2.63 & 0.02 & 0.63 \\
\hline
\end{tabular}

Data expressed as mean \pm standard deviation. ${ }^{*} p \leq 0.05$.

by running synchronously to music helps to coordinate nerve signal processes that regulate locomotion, neurovascular control and sensory integration more efficiently. Finally, exercisers have reported that running in synchrony with the prominent beat of a music track can create feelings that border on a spiritual experience, which appear to inspire them to additional effort (Juslin, 2013).

Perceived exertion was significantly lower during the synchronous music condition at each stage of the running trials, which is consistent with previous research where participants completed the same workload with and without music (e.g., Dyrlund and Wininger, 2008; Hutchinson and Karageorghis, 2013). In the absence of the external stimulation of the music, participants may have focused more on their own efforts, detecting increased signals of fatigue in the process (Edworthy and Waring, 2006). Unsolicited feedback from participants indicated that thoughts of the time remaining and the required exertion prevailed during the no music trial, whereas during the synchronous music condition the beat of the music distracted them from the effort of running, and assisted continuation due to the motivational qualities of the songs.

Heart rate was 1-3 bpm lower for synchronous music at each time point during the running trial and at exhaustion. This difference was significant at the 15-min mark. It is sometimes unclear whether decreased HR represents an advantage or disadvantage of music. With consistent workload across conditions, such as during the first $60 \mathrm{~min}$ of the running trials in our study, lower HR represents a benefit of music, albeit small in this case. However, where participants attempt to produce maximal power (Stork et al., 2015), go faster (Tate et al., 2012), or maintain effort for longer (Bood et al., 2013), interpretation is more challenging. A benefit ensues if $\mathrm{HR}$ is lower despite equivalent or greater workload, whereas a disadvantage ensues if $\mathrm{HR}$ is higher despite equivalent or lesser workload. However, where HR is higher with a greater workload (Eliakim et al., 2007; Sanchez et al., 2014), it is unclear whether this is indicative of an advantage, given that increased HR may be a function of additional work rather than the effect of music. Researchers should consider this potential confounding effect when designing studies to test the influence of music on HR and other physiological parameters.

In our study, the lower HR values for the synchronous music condition compared to no music during the first $60 \mathrm{~min}$ of the run, despite running speed being objectively the same for both conditions, suggests that music had some capacity to improve running economy. This may have occurred because running in synchrony with music required fewer micro adjustments to running stride resulting in a more biomechanically efficient run. Alternatively, music may have promoted a generalized relaxation response in participants that marginally enhanced blood flow efficiency. Another possibility is that music may have facilitated the entrainment process (Clayton, 2012) whereby the runners entrained their breathing pattern to their stride rate.

No significant between-group differences were found for blood lactate, hydration status, or thermal comfort. Previous studies assessing blood lactate accumulation during steady state 
endurance performance with and without music (Schwartz et al., 1990; Szmedra and Bacharach, 1998; Terry et al., 2012) provided equivocal findings. Our results suggest that synchronous music offers no benefit to lactate production during endurance activity in hot and humid conditions. Hydration status, assessed in terms of urine specific gravity and percentage body weight change, also did not vary between conditions, indicating that synchronizing running stride to music tempo offered no benefit in terms of ameliorating hypohydration. Thermal comfort was assessed in the present study primarily for safety reasons. Given that the run-to-exhaustion was completed under heat stress, monitoring thermal comfort was a condition of ethics approval. Unsurprisingly, results showed no difference in thermal comfort ratings because temperature and humidity were identical for both the synchronous music and no music conditions. This finding indicated that synchronous music did not distract participants from sensations of heat and humidity.

\section{CONCLUSION}

Results showed that running in hot and humid conditions while listening to synchronous music significantly improved running time-to-exhaustion, lowered perceived exertion and, to a

\section{REFERENCES}

Alessio, H. M., and Hutchinson, K. M. (1991). Effects of submaximal exercise and noise exposure on hearing loss. Res. Q. Exerc. Sport 62, 413-419. doi: 10.1080/02701367.1991.10607542

American Society of Heating, Refrigerating and Air-Conditioning Engineers (1966). Thermal Comfort Conditions, Standard 55. New York, NY: ASRHAE.

Bacon, C., Myers, T., and Karageorghis, C. I. (2012). Effect of music-movement synchrony on exercise oxygen consumption. J. Sports Med. Phys. Fit. 52, 359-365.

Bood, R. J., Nijssen, M., Van Der Kamp, J., and Roerdink, M. (2013). The power of auditory-motor synchronization in sports: enhancing running performance by coupling cadence with the right beats. PLoS One 8:e70758. doi: 10.1371/journal. pone. 0070758

Borg, G. (1998). The Borg RPE Scale. Borg's Perceived Exertion and Pain Scales. Champaign, IL: Human Kinetics, 29-38.

Clayton, M. (2012). What is entrainment? Definition and applications in musical research. Empir. Musicol. Rev. 7, 49-56. doi: 10.18061/1811/52979

Dyrlund, A. K., and Wininger, S. R. (2008). The effects of music preference and exercise intensity on psychological variables. J. Music Ther. 45, 114-134. doi: $10.1093 / \mathrm{jmt} / 45.2 .114$

Edworthy, J., and Waring, H. (2006). The effects of music tempo and loudness level on treadmill exercise. Ergonomics 49, 1597-1610. doi: 10.1080/ 00140130600899104

Ekkekakis, P. (2003). Pleasure and displeasure from the body: perspectives from exercise. Cogn. Emot. 17, 213-239. doi: 10.1080/02699930302292

Eliakim, M., Meckel, Y., Nemet, D., and Eliakim, A. (2007). The effect of music during warm-up on consecutive anaerobic performance in elite adolescent volleyball players. Int. J. Sports Med. 28, 321-325. doi: 10.1055/s-2006-92 4360

Epstein, Y., and Moran, D. S. (2006). Thermal comfort and the heat stress indices. Ind. Health 44, 388-398. doi: 10.2486/indhealth.44.388

Faul, F., Erdfelder, E., Lang, A., and Buchner, A. (2007). G*Power 3: a flexible statistical power analysis program for the social, behavioral, and biomedical sciences. Behav. Res. Methods 39, 175-191. doi: 10.3758/bf03193146

Gledhill, N. (2002). Physical Activity Readiness Questionnaire (PAR-Q) - Revised. Ottawa, ON: Canadian Society for Exercise Physiology. lesser extent, reduced HR. Findings have application for runners performing in tropical conditions.

\section{AUTHOR CONTRIBUTIONS}

All authors listed have made a substantial, direct and intellectual contribution to the work, and approved it for publication.

\section{FUNDING}

This present study was supported by Short-term Grant (USM304.PPSP.61313041) and Research University's Individual Grant (1001/PPSP/812149) from the Universiti Sains Malaysia.

\section{ACKNOWLEDGMENTS}

We grateful acknowledge all the participants for their commitment and cooperation in the process of data collection, the staff of the Sports Science Laboratory and lecturers of the Sports Science Unit, School of Medical Sciences, Universiti Sains Malaysia, Kubang Kerian, Malaysia.

Hardy, C. J., and Rejeski, W. J. (1989). Not what, but how one feels: the measurement of affect during exercise. J. Sport Exerc. Psychol. 11, 304-317. doi: 10.1123/jsep.11.3.304

Hutchinson, J. C., Jones, L., Vitti, S. N., Moore, A., Dalton, P. C., and O’Neill, B. J. (2018). The influence of self-selected music on affectregulated exercise intensity and remembered pleasure during treadmill running. Sport Exerc. Perform. Psychol. 7, 80-92. doi: 10.1037/spy000 0115

Hutchinson, J. C., and Karageorghis, C. I. (2013). Moderating influence of dominant attentional style and exercise intensity on psychological and psychophysical responses to asynchronous music. J. Sport Exerc. Psychol. 35, 625-643. doi: 10.1123/jsep.35.6.625

Juslin, P. N. (2013). From everyday emotions to aesthetic emotions: towards a unifying theory of musical emotions. Phys. Life Rev. 10, 235-266. doi: 10.1016/ j.plrev.2013.05.008

Karageorghis, C. I. (2008). "The scientific application of music in sport and exercise," in Sport and Exercise Psychology, ed. A. M. Lane (London: Hodder Education), 109-137.

Karageorghis, C. I., and Jones, L. (2014). On the stability and applicability of the exercise heart rate-music-tempo preference relationship. Psychol. Sport Exerc. 15, 299-310. doi: 10.1016/j.psychsport.2013.08.004

Karageorghis, C. I., Mouzourides, D. A., Priest, D. L., Sasso, T. A., Morrish, D. J., and Walley, C. L. (2009). Psychophysical and ergogenic effects of synchronous music during treadmill walking. J. Sport Exerc. Psychol. 31, 18-36. doi: 10.1123/ jsep.31.1.18

Lim, H. B. T., Karageorghis, C. I., Romer, L. M., and Bishop, D. T. (2014). Psychophysiological effects of synchronous versus asynchronous music during cycling. Med. Sci. Sports Exerc. 46, 407-413. doi: 10.1249/MSS. 0b013e3182a6378c

McDermott, B. P., Anderson, S. A., Armstrong, L. E., Casa, D. J., Cheuvront, S. N., Cooper, L., et al. (2017). National athletic trainers' association position statement: fluid replacement for the physically active. J. Athl. Train. 52, 877-895. doi: 10.4085/1062-6050-52.9.02

Minetti, A. E., Ardigo, L. P., and Saibene, F. (1994). The transition between walking and running in humans: metabolic and mechanical aspects at different gradients. Acta Physiol. Scand. 150, 315-323. doi: 10.1111/j.1748-1716.1994. tb09692.x 
Minton, D. M., O’Neal, E. K., and Torres-McGehee, T. M. (2015). Agreement of urine specific gravity measurements between manual and digital refractometers. J. Athl. Train. 50, 59-64. doi: 10.4085/1062-6050-49.3.47

Patel, A. D. (2008). Music, Language, and the Brain. New York, NY: Oxford University Press.

Phillips-Silver, J., and Keller, P. E. (2012). Searching for roots of entrainment and joint action in early musical interactions. Front. Hum. Neurosci. 6:26. doi: 10.3389/fnhum.2012.00026

Ruscello, B., D’Ottavio, S., Padua, E., Tonnelli, C., and Pantanella, L. (2014). The influence of music on exercise in a group of sedentary elderly women: an important tool to help the elderly to stay active. J. Sports Med. Phys. Fit. 54, 536-544.

Saat, M., Sirisinghe, R. G., Singh, R., and Tochihara, Y. (2005). Effects of short-term exercise in the heat on thermoregulation, blood parameters, sweat secretion and sweat composition of tropic-dwelling subjects. J. Physiol. Anthropol. Appl. Hum. Sci. 24, 541-549. doi: 10.2114/jpa.24.541

Sanchez, X., Moss, S. L., Twist, C., and Karageorghis, C. I. (2014). On the role of lyrics in the music-exercise performance relationship. Psychol. Sport Exerc. 15, 132-138. doi: 10.1016/j.psychsport.2013.10.007

Schneider, S., Askew, C. D., Abel, T., and Strüder, H. K. (2010). Exercise, music, and the brain: is there a central pattern generator? J. Sports Sci. 28, 1337-1343. doi: 10.1080/02640414.2010.507252

Schwartz, S. E., Fernhall, B., and Plowman, S. A. (1990). Effects of music on exercise performance. J. Cardiopulm. Rehabil. Prev. 10, 312-316. doi: 10.1097/00008483199009000-00002

Stork, M. J., Kwan, M., Gibala, M. J., and Martin Ginis, K. A. (2015). Music enhances performance and perceived enjoyment of sprint interval exercise. Med. Sci. Sports Exerc. 47, 1052-1060. doi: 10.1249/MSS.0000000000000494
Szmedra, L., and Bacharach, D. W. (1998). Effect of music on perceived exertion, plasma lactate, norepinephrine and cardiovascular hemodynamics during treadmill running. Int. J. Sports Med. 19, 32-37. doi: 10.1055/s-2007-971876

Tate, A. R., Gennings, C., Hoffman, R. A., Strittmatter, A. P., and Retchin, S. M. (2012). Effects of bone-conducted music on swimming performance. J. Strength Cond. Res. 26, 982-988. doi: 10.1519/JSC.0b013e31822dcdaf

Tatterson, A. J., Hahn, A. G., Martin, D. T., and Febbraio, M. A. (2000). Effects of heat stress on physiological responses and exercise performance in elite cyclists. J. Sci. Med. Sport 3, 186-193. doi: 10.1016/S1440-2440(00)80 080-8

Terry, P. C., and Karageorghis, C. I. (2011). "Music in sport and exercise," in The New Sport and Exercise Psychology Companion, eds T. Morris and P. C. Terry (Morgantown, WV: Fitness Information Technology), 359-380.

Terry, P. C., Karageorghis, C. I., Saha, A. M., and D'Auria, S. (2012). Effects of synchronous music on treadmill running among elite triathletes. J. Sci. Med. Sport 15, 52-57. doi: 10.1016/j.jsams.2011.06.003

Conflict of Interest Statement: The authors declare that the research was conducted in the absence of any commercial or financial relationships that could be construed as a potential conflict of interest.

Copyright (c) 2018 Nikol, Kuan, Ong, Chang and Terry. This is an open-access article distributed under the terms of the Creative Commons Attribution License (CC BY). The use, distribution or reproduction in other forums is permitted, provided the original author(s) and the copyright owner(s) are credited and that the original publication in this journal is cited, in accordance with accepted academic practice. No use, distribution or reproduction is permitted which does not comply with these terms. 


\title{
Born to Speak and Sing: Musical Predictors of Language Development in Pre-schoolers
}

\author{
Nina Politimou ${ }^{1 *}$, Simone Dalla Bella ${ }^{2,3,4,5 *}$, Nicolas Farrugia ${ }^{6}$ and Fabia Franco ${ }^{1}$ \\ ${ }^{1}$ Department of Psychology, Middlesex University, London, United Kingdom, ${ }^{2}$ International Laboratory for Brain, Music \\ and Sound Research (BRAMS), Montreal, QC, Canada, ${ }^{3}$ Department of Psychology, University of Montreal, Montreal, QC, \\ Canada, ${ }^{4}$ Centre for Research on Brain, Language and Music (CRBLM), Montreal, QC, Canada, ${ }^{5}$ Department of Cognitive \\ Psychology, University of Economics and Human Sciences in Warsaw, Warsaw, Poland, ${ }^{6}$ Lab-STICC, Department \\ of Electronics, IMT Atlantique, Brest, France
}

OPEN ACCESS

Edited by:

Evangelos Himonides,

University College London,

United Kingdom

Reviewed by:

Andrea Schiavio,

University of Graz, Austria Katarina Habe,

University of Ljubljana, Slovenia

*Correspondence: Nina Politimou

a.politimou@mdx.ac.uk

Simone Dalla Bella

simone.dalla.bella@umontreal.ca

Specialty section:

This article was submitted to Performance Science, a section of the journal

Frontiers in Psychology

Received: 27 January 2019

Accepted: 09 April 2019

Published: 24 May 2019

Citation:

Politimou N, Dalla Bella S,

Farrugia N and Franco F (2019) Born

to Speak and Sing: Musical

Predictors of Language Development in Pre-schoolers.

Front. Psychol. 10:948. doi: 10.3389/fpsyg.2019.00948
The relationship between musical and linguistic skills has received particular attention in infants and school-aged children. However, very little is known about pre-schoolers. This leaves a gap in our understanding of the concurrent development of these skills during development. Moreover, attention has been focused on the effects of formal musical training, while neglecting the influence of informal musical activities at home. To address these gaps, in Study 1, 3- and 4-year-old children $(n=40)$ performed novel musical tasks (perception and production) adapted for young children in order to examine the link between musical skills and the development of key language capacities, namely grammar and phonological awareness. In Study 2, we investigated the influence of informal musical experience at home on musical and linguistic skills of young pre-schoolers, using the same evaluation tools. We found systematic associations between distinct musical and linguistic skills. Rhythm perception and production were the best predictors of phonological awareness, while melody perception was the best predictor of grammar acquisition, a novel association not previously observed in developmental research. These associations could not be explained by variability in general cognitive functioning, such as verbal memory and non-verbal abilities. Thus, selective music-related auditory and motor skills are likely to underpin different aspects of language development and can be dissociated in pre-schoolers. We also found that informal musical experience at home contributes to the development of grammar. An effect of musical skills on both phonological awareness and language grammar is mediated by home musical experience. These findings pave the way for the development of dedicated musical activities for pre-schoolers to support specific areas of language development.

Keywords: musical skills, language development, pre-school children, informal musical experience, home environment 


\section{INTRODUCTION}

Music, like language, is a highly complex system. In language smaller units such as phonemes and morphemes are combined to form higher-order structures, namely words and sentences. Similarly, in music, separate units (pitches and durations) are combined to form higher-order sequences such as musical phrases and compositions. Musical and linguistic sequences both contain melodic and rhythmic patterns: melody and rhythm in music, and prosody in language (Patel, 2010).

Commonalities between music and speech are evident when looking at infant perception and production of sounds and mother-infant communication. The remarkable sensitivity of infants to melodic features like contour and pitch changes (e.g., Trehub et al., 1985) may arise from the early auditory, in utero experience of maternal speech (Kisilevsky et al., 2004; Mampe et al., 2009). Notably, important musical elements of infant vocalizations during the first year of life, namely melodic and temporal patterns (D'Odorico et al., 1985; D'Odorico and Franco, 1991), and manner of phonation (Franco, 1984) are produced in specific communicative contexts (see also Papaeliou and Trevarthen, 2006). The distinctive way adults speak when addressing infants, also known as Infant Directed (ID) speech or "motherese" (Fernald, 1985; Fernald and Kuhl, 1987), is characterized by higher pitch and exaggerated rhythmic and melodic patterns (Fernald, 1985; Trehub et al., 1993). This richly intonated type of speech that efficiently conveys the prosodic features of one's native language may shape infant vocal production in the first year of life. Indeed it has been argued that not only infants' early vocal "musical" behaviors are directly linked to the prosodic characteristics of their native language (Ruzza et al., 2003; Welch, 2006) but also that newborn cry vocalizations imitate their surrounding native speech prosody (Mampe et al., 2009). Interestingly, the capacity to process speech prosody and to perceive intonational contours in melody is an area where music and language overlap in the brain (Patel, 2010).

The discovery of early musical abilities in infants has also provided fruitful ground for the study of music and language from a phylogenetic perspective (Cross, 2003; Tomlinson, 2013). Similar to language, infants appear to be immediately sensitive to music (e.g., Trehub et al., 1985). This inclination is later shaped by human interaction in the context of culture-specific forms, i.e., different languages and different musical systems. Shared features between language and music have led to the hypothesis that a song-like communication system may be the phylogenetic precursor of modern language (Darwin, 1871; Livingstone, 1973; Brown, 2001; Merker, 2002; Mithen, 2005). Although there is still much debate about the adaptive function of musical skills in evolutionary terms (Fitch, 2006, for a discussion), some interesting observations of human infants also point to deep connections between musicality and early communication development. For example, childdirected singing has been shown to play an important role in regulating infant arousal and in establishing the motherinfant emotional bond (Trehub and Trainor, 1998; Trevarthen, 1999; Shenfield et al., 2003). Moreover, infants display superior attention to sung than spoken infant-directed communication
(Nakata and Trehub, 2004; Tsang et al., 2017). Similarly, musical characteristics of mother-infant vocal interactions are critical in promoting the development of socio-emotional regulation (Trevarthen, 1999; Dissanayake, 2000; Van Puyvelde et al., 2014). Finally, the acquisition of musical and linguistic features such as rhythm entrainment and vocal learning may rely on common learning mechanisms with adaptive value for social development, such as imitation (Carpenter, 2006; Fitch, 2013; Laland et al., 2016). In sum, interesting comparisons can be drawn between the phylogeny of language and music when focusing on their role for early communication development.

Accordingly, linguistic and musical skills are expected to emerge in parallel and to be linked across early development. Evidence from previous studies points in this direction. Randomized controlled trials (RCTs) with school-aged children have revealed language-related advantages for children participating in music groups (Moreno et al., 2009; François et al., 2013; Kraus et al., 2014). For example, after 2 years of participation in music versus painting classes, a music group outperformed controls on both electrophysiological and behavioral measures of speech segmentation (i.e., ability to extract pseudo-words from a continuous stream of nonsense syllables) (François et al., 2013). In another study, 8-year-old children receiving conventional musical training for 24 weeks showed enhanced performance in both reading measures and pitch discrimination abilities in speech (Moreno et al., 2009). Notably, these differences cannot be accounted for by preexisting traits in musicians, like general IQ (Schellenberg, 2004), as such variables were controlled in these experiments.

Pertinent research with pre-schoolers has been scarce. Two RCTs with 4- to 6-year-old children comparing the effects of musical training to other types of training reported enhancements in phonological awareness skills (Degé and Schwarzer, 2011, with 5- and 6-year-olds), and vocabulary (Barac et al., 2011, with 4- and 6-year-olds). These findings suggest that features of music instruction may strengthen aspects of linguistic development in young children. In a correlational study Anvari et al. (2002) showed that both rhythmic and melodic aspects of musical ability were associated with phonological awareness and early reading ability (early identification of letters and reading small phrases) in 4-year-old children. Other researchers have shown that pitch discrimination contributes to phonological awareness in 4.5- to 6-year-old children (Lamb and Gregory, 1993; Forgeard et al., 2008), while a link between phonological awareness and rhythmic abilities was observed in 5-year-old children (Anvari et al., 2002; Verney, 2013). Only one study so far has investigated a link between rhythmic abilities and phonological awareness in children younger than 4 years (Woodruff Carr et al., 2014). In this study, synchronization to an external beat was linked to speech encoding and phonological awareness in 3- and 4-year-old children. Taken together the results from correlational and longitudinal studies indicate that specific associations between musical and linguistic abilities may be present in pre-school children.

The potential involvement of pitch and melody perception in the language development of children younger than 4 years old remains unclear. Melody and pitch are critical elements of 
speech prosody, influencing language learning mechanisms such as statistical learning (Saffran et al., 1996; Thiessen et al., 2005), and conveying crucial information that aids speech segmentation and linguistic pattern extraction (Brooks and Kempe, 2012; Xie, 2012). Furthermore, aspects of linguistic development other than phonology, like the development of morphological rules and grammar have been neglected in studies with younger age groups. Only one study of typical children shows that rhythm perception skills and language structure and morphology are related in 6year-old children (Gordon et al., 2015). Yet, infants and children are likely to rely on both rhythmic and melodic prosodic cues to extract grammatical structures. For example, changes in pitch tend to correspond to boundaries between different syntactic clauses and phrases (Brooks and Kempe, 2012) aiding the extraction of grammatical information from continuous speech.

In sum, associations between linguistic and musical skills are observed quite systematically in children older than 4 years. Findings show that formal musical training generates advantages for auditory processing and language skills. Commonalities in the perception of musical and linguistic sounds have also been researched in infancy. However, a comprehensive account of the relationship between music and language across the full developmental spectrum is lacking. There is a significant knowledge gap for children between 1 and 4 years of age. Filling this gap is paramount to examine the effects of specific musicoriented training on early years education.

The first goal of this study is therefore to shed light on the developmental trajectory of the relationship between musical skills and different aspects of language development in 3and 4-year-old children. To this aim, in Study 1, children were submitted to tests of both phonological awareness and grammar, two areas of language development that have been linked to the successful acquisition of literacy skills and academic attainment (Deacon and Kirby, 2004; Melby-Lervåg et al., 2012; Cunningham and Carroll, 2015). Notably, these abilities have been treated as prerequisites for the development of fine-grained aspects of language production and understanding, such as pragmatics (Hoff and Marilyn, 2009). To pinpoint the musical skills relating to these key areas of language development we used a range of musical measures, assessing pitch, melody, rhythm, and tempo perception, singing and synchronization to the beat. In line with previous studies in older children we hypothesized that musical and linguistic skills would be linked in young pre-schoolers.

Testing musical skills in young pre-schoolers (3- and 4-yearolds), so far mainly neglected, poses methodological challenges. The typical testing environment combined with the auditory nature of the stimuli requires a level of attention that is difficult for such young children to maintain. Musical testing in this age group has for a long time relied on Audie's test (Gordon, 1989), which measures musical perception abilities but only includes two subtests, melody and rhythm discrimination, excluding other aspects of music perception important for musical expression and performance such as tempo (Law and Zentner, 2012) or basic auditory perceptual abilities such as pitch discrimination. Furthermore, it does not include any music production tasks, which can be rich sources of information for measuring a child's musical ability. To achieve the goals of Study 1 we developed a set of novel musical tests of melody and rhythm perception/production adapted specifically for this age group.

Another largely unexplored area of research is the influence of informal musical activities at home on musical and linguistic development. Notably, most pre-school children experience music through informal interaction at home rather than receiving formal music lessons. Only two studies so far (Putkinen et al., 2013; Williams et al., 2015) have directly assessed the effect of informal home musical experience on language development. They reported enhanced language and music-related auditory processing (Putkinen et al., 2013) and improved vocabulary (Williams et al., 2015) in young pre-schoolers as a function of informal musical experience at home. Although these findings are important, they remain fragmentary and a more systematic examination of the effect of this type of experience on key musical and linguistic skills is lacking. Such findings can inform early childcare practice both in the family and in educational contexts. They also have theoretical implications for the role of environmental factors in the development of children's abilities.

The objective of Study 2 was therefore to examine whether informal musical interactions and experience within the family might have an impact on children's musical and linguistic skills as assessed in Study 1. Based on previous findings we hypothesized that this aspect of environmental input would have a specific influence on the development of key linguistic areas (i.e., phonological awareness and grammar) and musical abilities, as assessed in Study 1.

\section{STUDY 1: LINKS BETWEEN MUSICAL AND LINGUISTIC SKILLS}

\section{Materials and Methods Participants}

Forty pre-school children (21 boys) between the ages of 3 years and 5 months and 4 years and 9 months $\left(M_{\text {age }}=4\right.$ years, $S D=4.7$ months) were recruited from nursery classes. Twenty-eight children were monolingual English speakers and 12 children were bilingual but had English as their first language (as reported by the parents). A language comprehension test (British Picture Vocabulary Scale; Dunn et al., 1997) was administered to all participants to ensure that they possessed an adequate level of English comprehension for their age. None of the participants had hearing difficulties or had been diagnosed with developmental delays. All of the children experienced comparable musical activities in their nursery as reported by their teachers. Five children also received music-related training (dancing or singing) outside the home.

\section{Tests of Musical Abilities}

An original battery of age-appropriate musical tests was designed for this study. The battery included both music perception and music production tasks. Music perception tasks assessed pitch, melody, rhythm, and tempo perception. Music production tasks tested singing and tapping to a beat. Extensive piloting served to evaluate the feasibility of these tasks for young children. 


\section{Music perception}

Music perception tasks (pitch, melody, rhythm, and tempo perception) used a $2+1$ oddity paradigm (e.g., Jensen and Neff, 1993). This method is well suited for non-verbal assessment of auditory discrimination in young children (White et al., 1990; Jensen and Neff, 1993), as confirmed in our pilot study. In each trial the child listened to a musical stimulus (i.e., melody or pitch) corresponding to the drawing of a little girl named Maggie that appeared at the top of the computer screen (see Figure 1). Two identical shapes would then appear successively on the lower left and right sides of the screen corresponding to two musical stimuli. One was the same as Maggie's stimulus, the other was different. The child was asked to point to the shape that sounded the same as Maggie's stimulus. Stimulus position on the screen (left or right) and order of appearance (whether the same item would be heard first or second) was counterbalanced. All stimuli were presented at $75 \mathrm{db}$ (Jensen and Neff, 1993). Stimuli in each trial were separated by 1 -s silent intervals. Inter-trial intervals varied in order to ensure that the child was attentive before each trial. Order of trials in all tasks was randomized across participants. Positive and negative feedback was provided to increase motivation. All tasks were designed and run using E-prime software.

To ensure that the children fully understood the music perception task format, in the first session they received four practice trials including easily identifiable sound stimuli (e.g., a dog barking). The child had to complete three out of four trials correctly. Participants were excluded from the study if they did not meet this criterion. Only one child failed to reach the criterion. Three practice trials also preceded each music perception task.

Single pitch perception. The stimuli $(n=10)$ in the pitch perception task were pure tones (duration $=400 \mathrm{~ms}$; with 25 ms linear onset and offset ramps), generated with Audacity software. The pitch of the standard stimulus $(1,000 \mathrm{~Hz})$ was chosen as infants' discrimination ability is superior for high rather than low frequencies (Olsho, 1984), and it has been used as a reference frequency in a number of experiments both with children (e.g., Thompson et al., 1999; Agnew et al., 2004; Bobin-Bègue and Provasi, 2005) and with adults (e.g., Tillmann et al., 2003; Paraskevopoulos et al., 2012; Weiss et al., 2014). Comparison stimuli differed in frequency (lower or higher) and hovered around 1,000 Hz. Fifty percent of the comparison

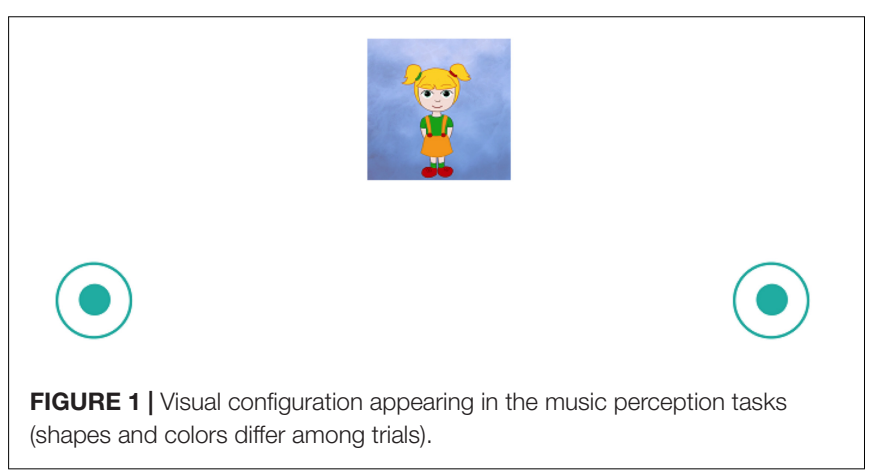

stimuli had a lower pitch, while the remaining $50 \%$ had a higher pitch compared to the standard stimulus. The first comparison stimulus represented the easiest trial and differed from the standard by $120 \mathrm{~Hz}$ while the difference between comparison and standard stimuli in the remaining nine trials ranged from 12 to $60 \mathrm{~Hz}$.

Melody perception. Six 3-tone melodies and six 5-tone melodies (1-3 s) were composed for the melody perception task. Each melody was originally composed in C major but was then transposed into a different musical key (all major scales were used). Melodies were played at a tempo of 140 BPM, which is close to the spontaneous motor tempo of children in this age group (Provasi and Bobin-Bègue, 2003).

Differences to be detected in the comparison stimuli consisted of one-tone changes. Difficulty was manipulated across two levels: (a) the length of the melodies (stimuli included six 3- and six 5 -note melodies) and (b) changes in comparison stimuli were either contour-violating or contour-preserving. This was based on previous work showing that 4- to 6-year-old children can more readily identify contour-violating compared to contourpreserving transformations in short melodies (Morrongiello et al., 1985). All changes in comparison melodies were limited to one tone in the middle of the melody (i.e., second tone in 3-note melodies and second, third, or fourth tone in the 5note melodies). Changes did not violate the key of the standard melody. Pitch interval changes ranged from 3 to 12 semitones and included both upward and downward changes. The average pitch interval changes were equivalent across 3- and 5-note melodies with a mean of 6.5 (range: $3-12$ ) and 6.2 (range: $3-$ 12) semitones, respectively. Average pitch interval changes were, however, different between contour-violating (mean of 9.3 and range of 8-12 semitones) and contour-preserving stimuli (mean of 3.8 and range of 3-5 semitones). Figure 2 shows the musical notation for standard and comparison versions of one example 3-note melody and one example 5-note melody.

Rhythm perception. The same melodies from the melody discrimination task were used in this task. Differences to be detected in the comparison stimuli consisted of changes in the duration of adjacent tones. This manipulation altered the rhythmic grouping of the comparison melody while preserving the number of notes and the overall duration and meter of the standard melody (see also Peretz et al., 2013). Difficulty of the stimuli was manipulated (a) by varying the length of the melodies (as in the melody discrimination task), (b) by changing duration of either two or three tones in a sequence, and (c) by introducing changes in duration occurring either on the downbeat (easy trials, $50 \%$ of stimuli) or on the upbeat (more difficult trials, $50 \%$ of stimuli) of the melody's meter.

Tempo perception. Ten 4-note melodies were used in the tempo perception task. Four, rather than 3-note melodies (as in part of the rhythm and melody discrimination tasks) were used to ensure that the children adequately recognized the tempo of each melody. All melodies were composed in the key of $\mathrm{C}$ major and were then transposed into different musical keys (10 major scales). The tempo of all standard stimuli was 100 BPM. 


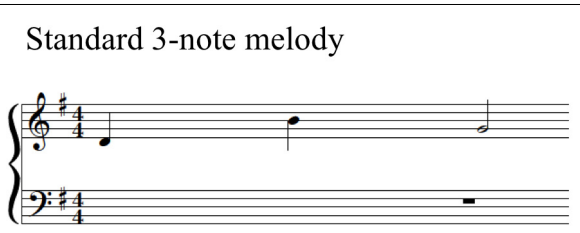

Comparison 3-note melody

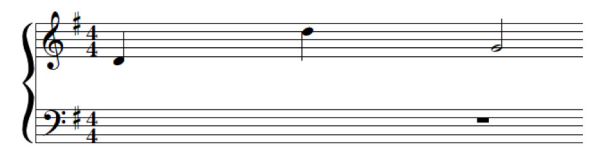

Standard 5-note melody

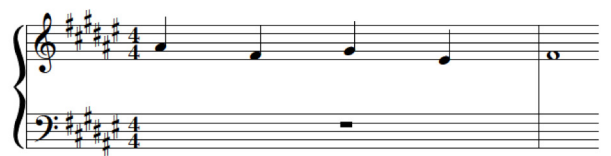

Comparison 5-note melody

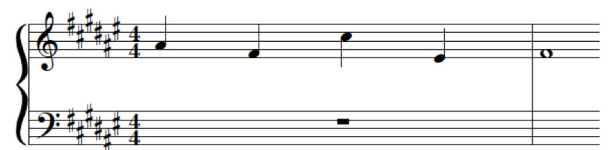

FIGURE 2 | Musical notation for standard and comparison versions of one example 3-note melody and one example 5-note melody.

The rate of the standard stimuli was chosen based on previous experiments showing that this is the optimal sensitivity zone for tempo discrimination in both infants (Baruch and Drake, 1997) and adults (Baruch et al., 2004). Furthermore, the only study that has so far examined tempo discrimination thresholds in children of 3- and 4-years of age has used $100 \mathrm{BPM}$ as a the standard tempo (Bobin-Bègue and Provasi, 2005). Based on the above study and on pilot observations, the differences in tempo in the final set of stimuli ranged from 25 to 70 BPM (around the standard tempo).

\section{Music production}

Song production. For the evaluation of song production children were asked to sing a popular children's song ("Twinkle Twinkle Little Star") along with the voice of the experimenter that was prerecorded and played through a portable speaker at a tempo of 100 BPM [the instrumental part of the recording was taken from Verney (2013)]. Using Audacity software and a Zoom H4n audio interface the child's voice was recorded on a MacBook Pro laptop.

Two musically trained independent raters evaluated the performance of the children. Evaluation was based on two rating scales developed by Rutkowski (1997; Singing Voice Development Measure) and Welch (1998, 2006). These rating scales were based on two longitudinal studies of children's singing development starting from pre-school years, which led to models featuring different phases of early vocal development. Taken together, these models suggest that children's performance progresses from singing that is centered on the words of the song (chant-like singing) to singing within a limited pitch range that follows the contour of the target melody.

Rating of the songs involved identifying the specific pitch range produced by the child and comparing it to the recorded song. Pitch range was identified using Audacity, which provides an accurate estimation of the range in fundamental frequency. The Rutkowski's (1997) scale was slightly adjusted to include an extra category (i.e., "Melodic shape exists and follows the contour of the original. There is some variability in pitch not necessarily accurate but following the correct contour") that proved to be useful in the evaluation of the sample. A mean score of the two evaluations was calculated (range 0-10) and entered in all subsequent analyses. Inter-observer agreement was very high as shown by the high correlation between observers' ratings $(r=0.91, p<0.001)$.

Synchronization to the beat. The ability to move to a beat was assessed with a synchronized tapping task. In this task, children were asked to tap along to the sounds of a metronome, played at two tempi (120 BPM and $100 \mathrm{BPM}$ ) in two separate trials. The beat (metronome clicks) was played by an animated avatar on a computer screen. The specific tempi were chosen based on previous work suggesting that pre-school children synchronize with stimuli presented at fast tempi ranging from 100 to $150 \mathrm{BPM}$ (Provasi and Bobin-Bègue, 2003). As each child has a unique preferred tempo, this is unlikely to be captured by one of the two tapping rates; calculating the mean of the two tapping rates seemed more appropriate to reflect the participants' performance (see Woodruff Carr et al., 2014). Children's tapping was recorded using a custom contact microphone that was inserted inside a toy drum. A sampling rate of $44,100 \mathrm{~Hz}$ was used to record the audio from the contact microphone.

The onsets of the taps $(n=20)$ were identified using the audio signal from the contact microphone, processed using a semi-automatic method. Each sample was squared and compared to a default threshold set to four times the standard deviation of the squared trial, and was marked as a candidate tap onset. A refractory window preventing the detection of a new tap onset in the $200 \mathrm{~ms}$ following each candidate tap onset was applied in order to only detect the first sample of each tap. This procedure was run on all trials and all subjects, and the results were visually inspected to ensure that each tap is detected only once, and that all taps were detected. Next, the two parameters (threshold for candidate taps and length refractory window) were manually adjusted relative to each participant's tapping volume, and a final visual inspection was performed to validate all tap onsets. Deviations of each tap time from the corresponding metronome times (i.e., asynchronies) were calculated; the standard error of these asynchronies (SEA) indicated the variability of synchronization performance. Smaller SEA values indicate better synchronization performance (Table 1 shows a summary of music perception and production tasks). 


\section{Language}

The Language Structure Index (LSI) from the Clinical Evaluation of Language Fundamentals - Preschool-2 (CELF-Preschool-2; Wiig et al., 2004) was administered to assess language grammar. The LSI consists of three subtests: Sentence Structure, Word Structure, and Recalling Sentences. Cronbach's $\alpha$ reliability coefficients for the LSI and all the relevant subtests range from 0.78 to 0.94 (age range: $3.5-4.5$ years). Standardized scores for the three subtests and the LSI were computed and used in all analyses.

Two subtests from the CELF-Preschool-2 were used to assess Word/Syllable blending and Sentence/Syllable segmentation. Two tests of rhyme and alliteration awareness - the Phonological Oddity - Rhyme and the Phonological Oddity - Alliteration task (Maclean et al., 1987) - replaced the rest of the phonological awareness subtests of the CELF-Preschool-2 (Rhyme perception and Rhyme generation), because during pilot testing these were deemed to be inappropriate for the younger, as well as some of the older children.

In order to derive a reliable composite score for phonological awareness and in absence of standardized scores for 3year-old children, principal component analysis (PCA) was conducted on the four phonological awareness subtests. The Kaiser-Meyer-Olkin measure verified the sampling adequacy for the analysis: $\mathrm{KMO}=0.68$ (acceptable according to Kaiser, 1974). Bartlett's test of sphericity, $\chi^{2}(6)=33.1, p<0.001$, indicated that correlations between variables were sufficiently large for PCA. One factor had an eigenvalue exceeding Kaiser's criterion of 1 . All four subtests (Phonological Oddity Rhyme, Phonological Oddity - Alliteration and Word-Syllable Blending and Sentence/Syllable Segmentation) loaded adequately onto that factor, which explained $55.89 \%$ of the variance (loadings $\geq 0.39$ ). Therefore, factor scores for phonological awareness were used in subsequent analyses in addition to the separate subtests.

\section{Memory and General Ability}

The Digit Span subtest from the British Ability Scales II (BAS, Elliott et al., 1996) was used to assess verbal memory. Reliability coefficients for the Digit Span were calculated using the Hoyt (1941) formula and were 93 and 83 for 3- and 4-year-olds, respectively. The Block Design from the Wechsler Preschool and
Primary Scale of Intelligence IV (WPPSI-IV, Wechsler, 2013) was used as a proxy for non-verbal ability (split-half reliability coefficient for 3- and 4-year-olds $=0.84$ ).

\section{Procedure}

All participants were tested during six or seven individual sessions spread across 6-7 days, each lasting approximately $20 \mathrm{~min}$. Testing for each participant was completed within 2-3 weeks of their first session. Individual sessions took place in specified quiet rooms in the participating nurseries. The first session included four practice trials of the music perception task format in order to determine whether participants understood the instructions and were able to perform the task. One child failed to meet the criterion (see section "Music Perception"). Order of administration was held constant for all children, alternating music and language tasks. However, the number of tasks completed in each session varied depending on the child's attention span and mood on the given day. Before the start of the sessions, the experimenter who later conducted the testing spent approximately 2 days in classrooms playing with the children, in order for them to become acquainted with her and to feel comfortable during testing.

\section{Data Analysis}

All data were analyzed using SPSS version 20.0 and R software environment ( $\mathrm{R}$ Core Team, 2012). Bivariate correlations were performed between musical, linguistic and cognitive skills. To investigate potential predictive relationships between musical and linguistic abilities, data were entered into two separate linear regression models with phonological awareness and language grammar as the dependent variables. Measures of musical ability (Pitch, Rhythm, Tempo and Melody Perception, Song Production and Synchronization) were treated as predictors. Age and gender were also entered into the models in order to examine whether they contribute to the variance in linguistic abilities. The drop1() function in $\mathrm{R}$ was used to gradually eliminate variables with no significant contribution to the model. The reported models are those which are the most explanatory and parsimonious after progressively removing the different predictors (backward).

TABLE 1 | Summary of music perception and production tasks.

\begin{tabular}{|c|c|c|c|c|c|}
\hline Musical tasks & Equipment & Stimuli & Participant task & No. of trials & Scoring \\
\hline Pitch Perception & Computer & Sinusoids & $\begin{array}{l}\text { Identify which of two pitches is the } \\
\text { same as the standard }\end{array}$ & 10 & $\%$ correct \\
\hline Melody Perception & Computer & Melodies & $\begin{array}{l}\text { Identify which of two melodies is the } \\
\text { same as the standard }\end{array}$ & 12 & $\%$ correct \\
\hline Tempo Perception & Computer & Melodies & $\begin{array}{l}\text { Identify which of two melodies is the } \\
\text { same as the standard }\end{array}$ & 10 & $\%$ correct \\
\hline Rhythm Perception & Computer & Melodies & $\begin{array}{l}\text { Identify which of two melodies is the } \\
\text { same as the standard }\end{array}$ & 12 & $\%$ correct \\
\hline Song Production & Computer, microphone & Song recording & $\begin{array}{l}\text { Sing along to recording of Twinkle } \\
\text { Twinkle Little Star }\end{array}$ & 1 & $\begin{array}{l}\text { Scored by two independent } \\
\text { raters }\end{array}$ \\
\hline Synchronization & $\begin{array}{l}\text { Computer, drum, } \\
\text { contact mic }\end{array}$ & Metronome clicks & Tap along to metronome clicks & $100,120 \mathrm{bpm}$ & Standard error of asynchronies \\
\hline
\end{tabular}


TABLE 2 | Participants' performance in cognitive, linguistic, and musical tasks.

\begin{tabular}{|c|c|c|c|c|c|c|}
\hline & Method of scoring & $N^{1}$ & Min & $\operatorname{Max}$ & Mean & $\begin{array}{l}\text { Standard } \\
\text { deviation }\end{array}$ \\
\hline BPVS & $M=100, S D=15$ & 40 & 86 & 129 & 107.8 & 10.03 \\
\hline CELF-LSI & $M=100, S D=15$ & 37 & 21 & 95 & 66.81 & 19.73 \\
\hline Phon Aw & $\begin{array}{l}\text { Range of } \\
\text { scores }=1-100\end{array}$ & 35 & 16.2 & 81.4 & 51.56 & 21.38 \\
\hline Block Des & $\begin{array}{l}\text { Range of } \\
\text { scores }=1-19\end{array}$ & 39 & 8 & 14 & 10.87 & 1.73 \\
\hline Digit Span & $M=50, S D=10$ & 38 & 22 & 92 & 69.63 & 20.16 \\
\hline $\begin{array}{l}\text { Musical } \\
\text { tasks }\end{array}$ & Range of scores & $N^{1}$ & Min & $\operatorname{Max}$ & Mean & $\begin{array}{l}\text { Standard } \\
\text { deviation }\end{array}$ \\
\hline \multicolumn{7}{|l|}{ Perception } \\
\hline Pitch & $1-10$ & 37 & 3 & 10 & 6.35 & 1.60 \\
\hline Melody & $1-12$ & 38 & 4 & 11 & 7.81 & 1.99 \\
\hline Tempo & $1-10$ & 37 & 3 & 10 & 7.18 & 1.74 \\
\hline Rhythm & $1-12$ & 38 & 5 & 12 & 8.60 & 2.19 \\
\hline \multicolumn{7}{|l|}{ Production } \\
\hline Song & $1-10$ & 36 & 2.5 & 10 & 7.06 & 2.18 \\
\hline Synch & $1.28-6.64$ & 38 & 1.28 & 6.64 & 3.79 & 1.63 \\
\hline
\end{tabular}

BPVS, British Picture Vocabulary Scale; CELF-LSI, Clinical Evaluation of Language Fundamentals - Preschool - Language Structure Index; Phon Aw, phonological awareness composite score; Block Des, Block Design. ${ }^{1}$ Please note that $N$ sizes differ, as 7 out of 40 children did not complete all the tasks included in the assessment.

Residual plots were visually inspected for all models to test for the assumptions of normal distribution, linearity, and heteroscedasticity of the data. No obvious patterns were observed and residuals did not appear to deviate from a straight line in any of the models, therefore meeting the assumptions.

\section{Results}

Means and standard deviations of participants' group performance in all linguistic, baseline cognitive, and musical tasks are presented in Table 2.

A series of non-parametric comparisons (Mann-Whitney and Kolmogorov-Smirnoff) were first performed between monolingual $(n=28)$ and bilingual children $(n=12)$ to identify any differences in linguistic or cognitive performance resulting from bilingualism. As there were no statistically significant differences between monolingual and bilingual children in any of the tasks, data from both monolingual and bilingual children were pooled for all subsequent analyses.

To examine the relations between musical and linguistic skills, correlations between the measures in the respective tasks were calculated (see Table 3). As can be seen, a number of significant relationships were observed between musical skills and subtests and composite scores of phonological awareness and grammar. Different musical abilities (rhythm and synchronization vs. melody) were associated with different linguistic tasks. Performance in temporal or rhythmic tasks was mostly linked with phonological awareness, whereas performance in the melody task was associated with grammar. Rhythm perception was also significantly associated with one of the language grammar subtests (Recalling Sentences) while
TABLE 3 | Correlations between musical tasks and subtests of phonological awareness and language structure.

\begin{tabular}{lcrlllll}
\hline $\begin{array}{l}\text { Language } \\
\text { tasks }\end{array}$ & & Pitch & Tempo & Melody & Rhythm & Song & Synch \\
\hline Grammar & SS & 0.16 & 0.17 & 0.27 & 0.10 & 0.19 & 0.13 \\
& WS & 0.09 & 0.17 & $0.35^{*}$ & 0.02 & 0.17 & -0.17 \\
& RS & 0.25 & 0.12 & $0.37^{*}$ & $0.33^{*}$ & 0.12 & -0.15 \\
& Comp & 0.09 & 0.23 & $0.43^{* * *}$ & 0.24 & $0.29^{1}$ & -0.21 \\
Phon Aw & W/S & 0.15 & $0.40^{*}$ & 0.26 & $0.36^{*}$ & -0.09 & -0.20 \\
& S/S & 0.10 & $0.41^{*}$ & $0.41^{*}$ & $0.31^{*}$ & 0.04 & $-0.36^{*}$ \\
& Rh & -0.27 & 0.26 & 0.17 & $0.45^{* *}$ & 0.19 & $-0.32^{*}$ \\
& Alit & 0.09 & $0.35^{*}$ & 0.25 & $0.67^{* * *}$ & 0.21 & $-0.38^{*}$ \\
& Comp & 0.04 & $0.38^{*}$ & $0.29^{1}$ & $0.63^{* * *}$ & 0.20 & $-0.47^{* *}$ \\
\hline
\end{tabular}

${ }^{*} p<0.05,{ }^{* *} p<0.01,{ }^{* * *} p<0.001 .{ }^{1}$ Marginally significant $(p<0.1)$. SS, Sentence Structure, WS, Word Structure, RS, Recalling Sentences, W/S, Word/Syllable Blending, S/S, Sentence/Syllable Segmentation; Rh, Phonological Oddity Rhyme; Alit, Phonological Oddity Alliteration; Comp, Composite Score.

TABLE 4 | Correlations between musical tasks and tests of non-verbal ability (WPPSI-Block Design) and verbal memory (Digit Span).

\begin{tabular}{lcc}
\hline Tests & WPPSI-Block Design & Digit Span \\
\hline Musical tasks & & \\
Pitch Perception & 0.14 & $0.41^{* *}$ \\
Tempo Perception & 0.27 & 0.16 \\
Melody Perception & 0.18 & 0.22 \\
Rhythm Perception & $0.36^{*}$ & $0.35^{*}$ \\
Song Production & 0.23 & 0.05 \\
Synchronization & -0.14 & -0.04 \\
Language tasks & & \\
Language Grammar & $0.54^{* * *}$ & $0.58^{* * *}$ \\
Phonological Awareness & 0.25 & $0.56^{* * *}$ \\
\hline$* p<0.05, * * p<0.01, * * *<0.001$. & &
\end{tabular}

melody perception was associated with one of the phonological awareness subtests (Sentence/Syllable Segmentation).

Moderate to strong associations were also observed between cognitive ability and some musical and linguistic measures (see Table 4). Therefore, to ensure that any links between musical and linguistic skills cannot be attributed to underlying cognitive factors, scores in both cognitive tests were controlled for in subsequent regression analyses.

\section{Predicting Linguistic Abilities Based on Musical Skills Musical abilities and phonological awareness}

A linear model was built, in which all measures of musical abilities, as well as age and gender, were entered as predictors and phonological awareness was treated as the dependent variable. As can be seen in Table 5 (Model 2a) Rhythm Perception and Synchronization were significant predictors of phonological awareness. An increase in the performance of rhythm perception and lower error in synchronization were associated with better phonological awareness. Given that Synchronization had a lower beta and $t$-value we tested whether a model where Synchronization is included (Model 2a) would show a better fit than a more parsimonious model where only Rhythm Perception 
is included (Model 1a). Results indicated that the models differed significantly (see Table 5), suggesting that the Synchronization variable significantly contributes to the model.

The strong association between rhythmic and synchronization abilities and phonological awareness was further examined to determine whether this could be driven by latent cognitive factors accounting for better performance in both linguistic and musical tasks. Non-verbal ability and verbal memory were entered into the previous model (2a). The resulting model was highly significant (Model 3a in Table 5) and showed that Rhythm Perception, Synchronization and verbal memory significantly predicted phonological awareness, while non-verbal ability did not $[$ Beta $=-0.04, t(27)=-0.03$, n.s. $]$ and hence it was dropped. An increase in verbal memory predicted higher phonological awareness. Finally, the model that included verbal memory (Model 3a) was compared to Model 2a to ensure that verbal memory adds explanatory value to phonological awareness. Results showed that the two models differed significantly, thus proving the superiority of Model 3a over Model 2a in explaining the variance in phonological awareness.

\section{Musical abilities and language grammar}

In the final model (Model 1b), only Melody Perception significantly predicted language grammar (see Table 6), suggesting that better perceptual processing of melodies is associated with higher grammar scores. As shown in Table 6, Model $1 \mathrm{~b}$ explained a notable amount of variance in language grammar scores.

Next, non-verbal ability and verbal memory were entered into the regression model (Model 2b). Results indicated that all variables significantly predicted language grammar (see Table 6). Finally, to ensure that Melody Perception adds explanatory value to language grammar, Model $2 \mathrm{~b}$ was compared to a further model (Model 3b) where only non-verbal ability and verbal memory were included. Results showed that the two models differed significantly, suggesting that Melody Perception predicts language grammar, over and above general cognitive abilities. As shown in Table 6, Model 2b explained a significant amount of variance in language grammar scores and showed a better model fit compared to Model 3b.

Overall, results showed that there are strong associations between musical and linguistic skills in 3- and 4-year-old children. Crucially, we found unique associations between distinct musical and linguistic skills that remained significant even when cognitive skills, such as verbal memory and nonverbal ability were accounted for. Specifically, rhythmic measures (perceptual and sensorimotor) were the best predictors of phonological awareness while melody perception was the best

TABLE 5 | Summary and comparisons between Models 1a, 2a, and 3a predicting phonological awareness.

\begin{tabular}{|c|c|c|c|c|c|c|c|c|}
\hline & $\beta$ & $t$ & $p$ & $R^{2}$ & $A I C$ & $\boldsymbol{F}$ & $p$ & Model comparisons \\
\hline Model 1a & & & & 0.40 & -24.57 & 20.56 & $<0.001$ & \\
\hline \multirow[t]{2}{*}{ Rhythm Perception } & 0.63 & 4.53 & $<0.001$ & & & & & \\
\hline & & & & & & & & Model 2a vs. Model 1a \\
\hline Model 2a & & & & 0.53 & -31.03 & 16.29 & $<0.001$ & $F(1,29)=8.72, p<0.01$ \\
\hline Rhythm Perception & 0.57 & 4.40 & $<0.001$ & & & & & \\
\hline \multirow[t]{2}{*}{ Synchronization } & -0.38 & -2.95 & $<0.01$ & & & & & \\
\hline & & & & & & & & Model 2a vs. Model 3a \\
\hline Model 3a & & & & 0.65 & -38.58 & 17.38 & $<0.001$ & $F(1,29)=9.74, p<0.01$ \\
\hline Rhythm Perception & 0.44 & 3.77 & $<0.001$ & & & & & \\
\hline Synchronization & -0.38 & -3.41 & $<0.01$ & & & & & \\
\hline Verbal Memory & 0.36 & 3.12 & $<0.01$ & & & & & \\
\hline
\end{tabular}

TABLE 6 | Summary and comparisons between Models 1b, 2b and 3b predicting language grammar.

\begin{tabular}{|c|c|c|c|c|c|c|c|c|}
\hline & $\beta$ & $t$ & $p$ & $R^{2}$ & $A I C$ & $\boldsymbol{F}$ & $p$ & Model comparisons \\
\hline Model $1 b$ & & & & 0.19 & 205.64 & 7.71 & $<0.01$ & \\
\hline Melody & 0.43 & 2.77 & $<0.01$ & & & & & \\
\hline Model 2b & & & & 0.56 & 188.42 & 13.04 & $<0.001$ & \\
\hline Melody & 0.28 & 2.32 & $<0.05$ & & & & & \\
\hline NVA & 0.38 & 2.97 & $<0.01$ & & & & & \\
\hline \multirow[t]{2}{*}{ VM } & 0.35 & 2.71 & $<0.05$ & & & & & \\
\hline & & & & & & & & Model 2b vs. Model 3b \\
\hline Model 3b & & & & 0.48 & 196.77 & 15.26 & $<0.001$ & $F(1,32)=5.38, p<0.05$ \\
\hline NVA & 0.39 & 2.92 & $<0.01$ & & & & & \\
\hline VM & 0.44 & 3.26 & $<0.01$ & & & & & \\
\hline
\end{tabular}

Melody, Melody Perception; NVA, Non-Verbal Ability; VM, Verbal Memory. 
predictor of grammar. This suggests that the mechanisms underlying these distinct music-language associations might be, at least in part, dissociable.

\section{STUDY 2: EFFECT OF INFORMAL MUSICAL EXPERIENCE AT HOME ON MUSICAL AND LINGUISTIC SKILLS}

Study 2 was designed to examine whether informal musical interactions and experience at home (within the family) influence the children's musical and linguistic skills as assessed in Study 1.

\section{Materials and Methods}

\section{Participants}

Thirty-four parents of children who participated in Study 1 completed two self-report questionnaires on informal musical experience in the family and personal experience with music. Mean age of the parents was 36.4 years and in $44 \%$ of the families at least one parent had received a Bachelor's degree or above.

\section{Materials}

The Musical Experience in the Family Questionnaire (henceforth MEF; provided by co-author of this manuscript FF) was used to assess frequency and type of musical engagement in the child's home environment. The MEF included questions about frequency of musical engagement in the child's home environment (singing and music making) as well as richness of musical exposure. The Goldsmith's Musical Sophistication Index (henceforth Gold-MSI; Müllensiefen et al., 2014) was used to assess the parents' degree of musicality. The average of maternal and paternal scores on Gold-MSI was entered in all analyses, together with the MEF score.

\section{Procedure}

At the launch of Study 1, parents of participating children were informed of the objectives of the project and the research procedure and a questionnaire including the materials described above was given to them. Completed questionnaires were collected at different times during the course of Study 1.

\section{Results}

Associations of Children's Musical and Linguistic Abilities With Musical Experience in the Family, Musical Sophistication of Parents and

\section{Parental Education}

Bivariate correlations were performed between children's musical perception and production tasks, composite scores of children's language grammar and phonological awareness and the parental scores on Gold-MSI, MEF, and educational level. As can be seen in Table 7, a significant association was observed between the MEF scores and Song Production. Associations were also found between Gold-MSI and MEF scores indicating that parents' engagement with music, combined with the amount of musical training they had received, can be reflected in the way they interact musically with their children. No significant associations were found between parental education and any of the music perception or production tasks, the MEF or the Gold-MSI.

Interestingly, apart from the link between the MEF and song, and a non-significant trend for an association between the GoldMSI and song $(p=0.062)$, no relationships were found between home musical environment variables and the development of musical abilities.

Both the MEF and the Gold-MSI were associated with language grammar scores (see Table 7), indicating that an enriched home musical environment may contribute to supporting the development of young children's complex language skills. Interestingly, no associations were found between level of parental education and scores on the linguistic abilities tasks. Therefore, parental education was not included in subsequent regression analyses.

\section{The Role of Informal Musical Experience in the Family in the Relationship Between Musical and Linguistic Abilities}

Two linear regression models were built with language grammar and phonological awareness as dependent variables. Interactions between the musical abilities found to be the strongest predictors of these linguistic abilities and MEF were entered as predictors in each model separately.

Informal musical experience in the family, musical abilities, and phonological awareness

A linear model was built using phonological awareness as the dependent variable, and two interactions as predictors $($ MEF $\times$ Rhythm Perception, MEF $\times$ Synchronization).

TABLE 7 | Correlations between MEF, Gold-MSI-Musical Sophistication, parental education, and musical tasks.

\begin{tabular}{lccc}
\hline & MEF & Gold-MSI & Parental education \\
\hline MEF & - & $0.44^{* *}$ & -0.05 \\
Gold-MSI & $0.44^{* *}$ & - & 0.07 \\
Parental Education & -0.05 & 0.07 & - \\
Pitch Perception & 0.13 & -0.11 & -0.17 \\
Tempo Perception & 0.17 & -0.03 & -0.06 \\
Melody Perception & 0.02 & -0.19 & -0.02 \\
Rhythm Perception & -0.03 & 0.16 & 0.18 \\
Song Production & $0.41^{*}$ & $0.33^{1}$ & 0.01 \\
Synchronization & -0.17 & 0.23 & -0.14 \\
Grammar & $0.36^{*}$ & $0.37^{*}$ & 0.14 \\
Phonological Awareness & 0.18 & 0.30 & 0.26
\end{tabular}

${ }^{*} p<0.05,{ }^{* *} p<0.01 .{ }^{1}$ Marginally significant $(p<0.1) .{ }^{2}$ Composite scores of grammar and phonological awareness have been included. ${ }^{3}$ Since phonological awareness scores were not age-normed, age in months was controlled for in all relevant correlations. ${ }^{4}$ Preliminary non-parametric comparisons indicated that there were no significant differences between children receiving musical or dancing training outside the nursery/home $(n=5)$ and the rest of the sample $(n=29)$ in the linguistic composite scores, the MEF or the Gold-MSI. No significant differences were found for any of the musical tasks apart from Rhythm Perception where non-musically trained children performed higher than their counterparts $(U=26.5, p=0.031)$. 
Results indicated that both interactions significantly predicted phonological awareness (see Table 8). To ensure that the model where both interactions are included (Model 2c) shows a better fit than a more parsimonious model where only the strongest predictor is included (Model 1c), an ANOVA comparison was performed. Results indicated that the models differed significantly, suggesting that both interactions add notable explanatory value to the model (see Table 8).

To examine whether these interactions would still predict phonological awareness above and beyond other important environmental factors linked to musical experience at home, the parents' musical sophistication scores were entered into Model 2c. Results indicated that both interactions as well as the GoldMSI significantly predicted phonological awareness (see Table 8). The model that included the Gold-MSI (Model 3c) was then compared to Model 2c. Results showed that the two models differed significantly, suggesting that all variables significantly and independently predict phonological awareness (see Table 8).

To plot the effects of the interactions, two separate models were built with phonological awareness as the dependent variable and Rhythm $\times$ MEF interaction (Model 1d) and Synchronization $\times$ MEF interaction (Model 2d), respectively, as predictors. Both models significantly predicted phonological awareness [Model 1d: $F(1,25)=7.38, p<0.05, R^{2}=0.22$, Model 2d: $\left.F(1,27)=4.32, p<0.05, R^{2}=0.13\right]$. As can be seen in Figure 3, higher levels of MEF contribute toward a stronger link between the child's musical abilities and phonological awareness.

Informal musical experience in the family, musical abilities, and language grammar

A linear model was built using language grammar as the dependent variable and the MEF $\times$ Melody perception as a predictor.

Results showed that the interaction significantly predicted language grammar (Model 1e) and explained a significant amount of the variance in language grammar (see Table 9). A plot of the effect of the MEF $\times$ Melody perception interaction on language grammar is presented in Figure 4. In line with what was observed for phonological awareness, the association between Melody perception and language grammar is stronger in children with higher levels of MEF.
The next step was to add Gold-MSI scores to the model. Results indicated that the interaction between MEF and Melody Perception still predicted language grammar significantly while the Gold-MSI did not (Model 2e, see Table 9). A comparison between Model 1e and Model 2e where all predictors were present showed that the two models did not differ significantly, confirming that the Gold-MSI did not explain a significant amount of variance in language grammar (see Table 9).

\section{GENERAL DISCUSSION}

The goal of the present study was to shed light on the relationship between language and musical skills in pre-school children. To this end, in Study 1 we tested the early links between distinct musical and linguistic skills. Three- and 4-year-old children underwent a thorough assessment of their musical and linguistic abilities. In Study 2, we examined associations between informal musical experience at home and early musical and linguistic skills as assessed in Study 1, aiming to test whether this type of experience mediates the relation between musical and linguistic skills.

\section{Strong Link Between Musical and Linguistic Skills in Early Development}

We provide compelling evidence that both melodic and timing aspects of musical processing are associated with measures of phonological awareness and grammar in children as young as 3-4 years of age. Timing abilities and specifically tempo and rhythm perception and synchronization to a beat were consistently associated with phonological awareness. In contrast, melodic processing was particularly associated with language grammar.

Regression analyses revealed that synchronization to the beat (its variability) and rhythm perception were the most significant predictors of phonological awareness. These musical predictors contributed to phonological awareness above and beyond cognitive skills, namely, verbal memory, and non-verbal ability. These findings demonstrate that there are strong links between timing skills and phonological awareness as early as at 3 years of age, as previously reported for older children (5-year olds:

TABLE 8 | Summary and comparisons between Models 1c, 2c, and 3c predicting phonological awareness.

\begin{tabular}{|c|c|c|c|c|c|c|c|c|}
\hline & Beta & $t$ & $p$ & $R^{2}$ & AIC & $\boldsymbol{F}$ & $p$ & Model comparisons \\
\hline Model 1c & & & & 0.22 & -14.15 & 7.38 & $<0.01$ & \\
\hline \multirow[t]{2}{*}{ Rhythm:MEF } & 0.006 & 2.71 & $<0.05$ & & & & & \\
\hline & & & & & & & & Model 1c vs. Model 2c \\
\hline Model 2c & & & & 0.45 & -21.37 & 9.87 & $<0.001$ & $F(1,24)=9.76, p<0.01$ \\
\hline Rhythm:MEF & 0.007 & 3.64 & $<0.01$ & & & & & \\
\hline \multirow[t]{2}{*}{ Synch:MEF } & -0.012 & -3.12 & $<0.01$ & & & & & \\
\hline & & & & & & & & Model 2c vs. Model 3c \\
\hline Model 3c & & & & 0.59 & -27.84 & 11.46 & $<0.001$ & $F(1,23)=8.48, p<0.01$ \\
\hline Rhythm:MEF & 0.005 & 2.79 & $<0.05$ & & & & & \\
\hline Synch:MEF & -0.017 & -4.48 & $<0.001$ & & & & & \\
\hline Gold-MSI & & 2.91 & $<0.01$ & & & & & \\
\hline
\end{tabular}

Rhythm:MEF, interaction between Rhythm Perception and MEF; Synch:MEF, interaction between Synchronization and MEF. 

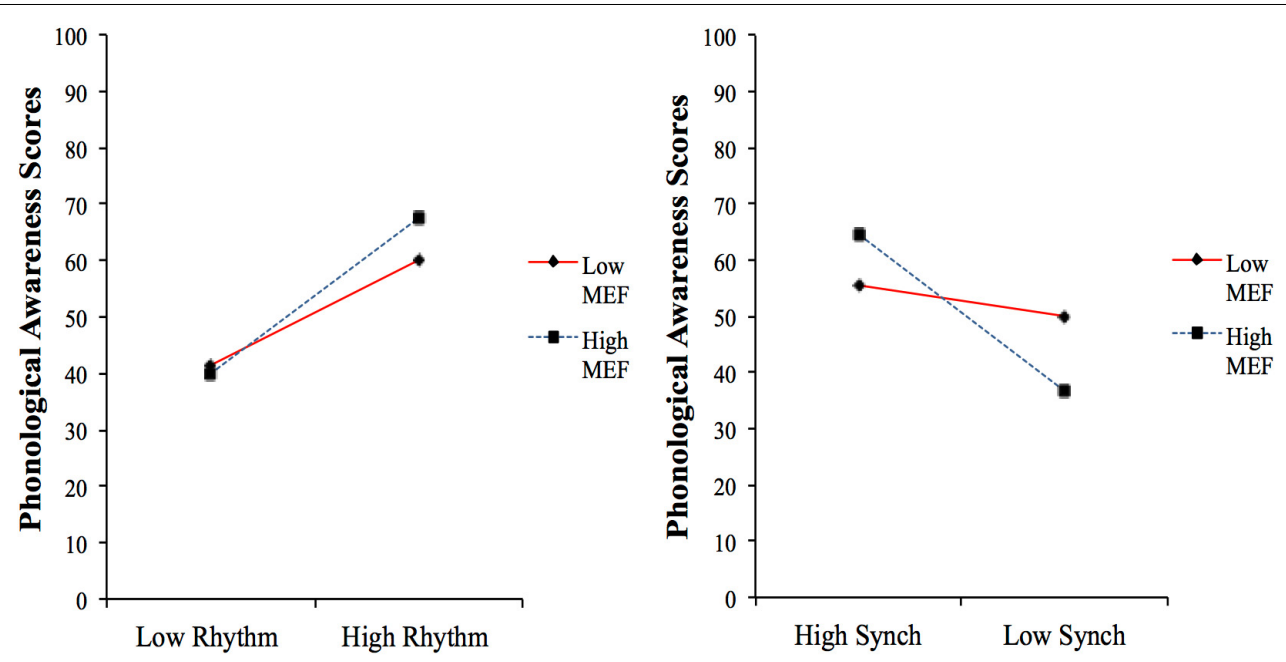

FIGURE 3 | Interactions between MEF and musical abilities in predicting phonological awareness scores; Rhythm, Rhythm Perception; Synch, Synchronization (N.B. smaller scores in the Synchronization task indicate better performance). Means and standard deviations of all variables and unstandardized regression coefficients of both direct and interaction effects were used to generate points in the graphs that represent high and low performance in each of the independent variables.

TABLE 9 | Summary and comparisons between Models 1e and 2e predicting grammar.

\begin{tabular}{|c|c|c|c|c|c|c|c|c|}
\hline & Beta & $T$ & $p$ & $R^{2}$ & $A / C$ & $\boldsymbol{F}$ & $P$ & Model comparison \\
\hline Model 1e & & & & 0.31 & 167.59 & 12.41 & $<0.001$ & \\
\hline \multirow[t]{2}{*}{ Melody:MEF } & 0.19 & 3.52 & $<0.001$ & & & & & Model 1e vs. Model 2e \\
\hline & & & & & & & & $F(1,27)=2.65, p=$ n.s. \\
\hline Model 2e & & & & 0.37 & 166.76 & 7.91 & $<0.01$ & \\
\hline Melody:MEF & 0.17 & 3.12 & $<0.01$ & & & & & \\
\hline Gold-MSI & 0.33 & 1.63 & n.s. & & & & & \\
\hline
\end{tabular}

Melody:MEF, interaction between Melody Perception and MEF.

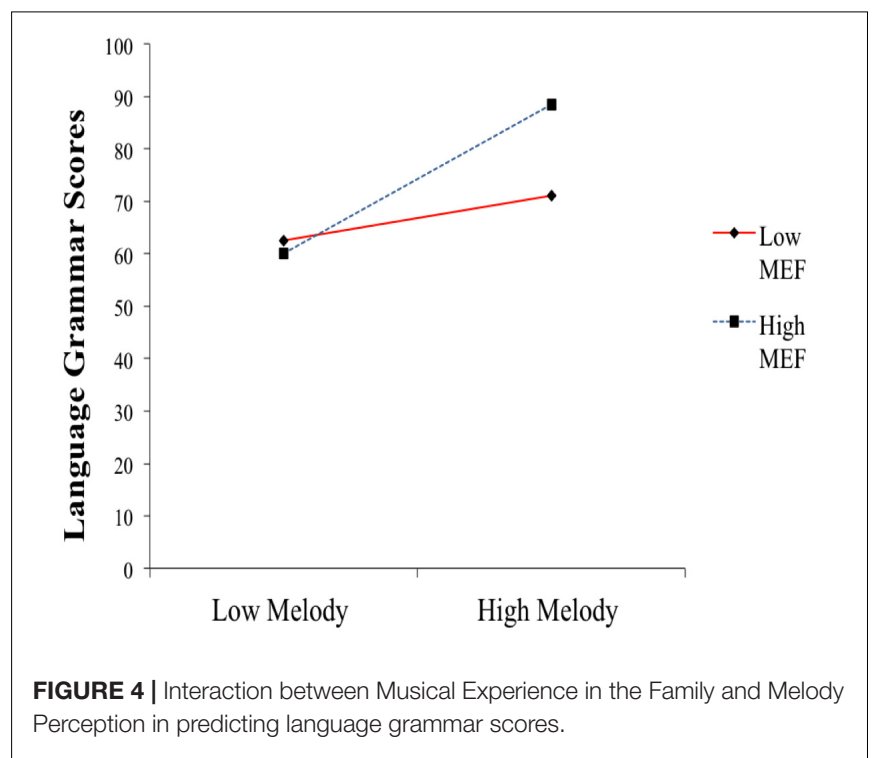

Anvari et al., 2002; Verney, 2013; 8- to 13-year-olds: Huss et al., 2011). This is in line with the only study to date that showed an association between mutual synchronization in 3-and 4-year-old children and early reading skills (Woodruff Carr et al., 2014). The present study provides the first evidence that this link also extends to rhythmic pattern perception in children younger than 4 years.

A specific mechanism implying direct links between the perception of metrical structure in language and in music has been proposed by Goswami (2011; Temporal Sampling Framework) to explain specific phonological deficits in developmental dyslexia. According to the Temporal Sampling Framework, speech perception relies on the encoding of temporal modulations across different frequencies relevant for speech. Poor speech segmentation skills in dyslexic children are thought to arise from a specific difficulty in tracking the sound "rise time" (i.e., the time taken for the sound to reach its peak amplitude). Rise times are critical for segmenting the speech signal into syllables, as they reflect the patterns of amplitude modulation marking the passage from one sound to another. According to Goswami (2011), networks of neurons might entrain to an input rhythm marked by syllable rise times in speech, which form patterns of strong and weak beats alternating to avoid stress clashes (see also Giraud and Poeppel, 2012 for insights on the role of neuronal oscillations in speech processing). Temporal periodicities and meter are much more regular in music than in language. However, a similar neural mechanism might be at work with metrical structure in music as this also relates to 
strong and weak beat patterns (Goswami et al., 2013). This idea implies a specific and unique association between the processing of metrical structure in language and music and phonological awareness (Goswami, 2011). Our findings are consistent with the above framework, showing that timing skills such as the perception of rhythmic patterns within a melody and the ability to synchronize to an external beat are strong predictors of phonological awareness.

Interestingly, rhythm perception and synchronization ability predicted phonological awareness independently from each other, suggesting that these timing skills might be dissociable, at least in part. The idea that synchronization to the beat, and rhythm/beat perception can be separated and potentially under the control of partly different mechanisms has received some support from recent single-case studies of beat deafness and poor synchronization, (Sowiński and Dalla Bella, 2013; Dalla Bella and Sowiński, 2015; Bégel et al., 2017a), and in a patient with brain damage (Fries and Swihart, 1990). The intriguing possibility that perception and action may represent a double dissociation in the timing domain suggests that separate pathways might underlie perceptual and sensorimotor timing skills (Bégel et al., 2017a). These abilities might relate to phonological awareness via distinct neural mechanisms. For example, Goswami (2011) proposed that the tracking of the amplitude envelope in speech that is crucial for perceiving individual phonemes relies on phaselocking of neurons to slow oscillations $(1.5-7 \mathrm{~Hz})$ in the auditory cortex, while the same neural mechanism has been proposed for perceiving rhythmic structure in music (Large, 2008). On the other hand, it has been argued that both synchronization and phonological skills depend on the accurate representation of timing in the subcortical auditory system (Tierney and Kraus, 2014). In support of this idea, both individuals who exhibit variability in moving to a metronome (Tierney and Kraus, 2013) and children with developmental dyslexia (Hornickel and Kraus, 2013) show poor subcortical processing of timing information as reflected in delayed responses to sound and greater trial-by-trial timing variability in the auditory brainstem. Therefore, the possibility of separate timing skills impacting language through different (cortical versus subcortical) pathways is well-documented.

Crucially, we have provided new evidence of a relationship between melody perception and language grammar, an association not previously observed in pre-schoolers or other developmental groups. This relationship persisted even when verbal memory and non-verbal ability were controlled. These results strongly suggest that similar auditory perceptual mechanisms may be responsible for both melody perception and language grammar, at least at this stage in development. In addition, these mechanisms might be partly independent from those underlying the association between rhythmic abilities and phonological awareness.

With regards to the mechanism underlying the unique association between melody perception and grammar, one possibility is that melodic aspects of prosody are particularly important for the acquisition and development of grammar. Indeed, it has been suggested that pitch changes in continuous speech appear to mark boundaries between different syntactic units (e.g., clauses and phrases), thus assisting the extraction of grammatical information (Speer and Ito, 2009; Brooks and Kempe, 2012; Xie, 2012). Consistent with this view, Cohrdes et al. (2016) showed that melodic discrimination in 5- to 7-yearold children was associated with the processing of emotional prosody in linguistic phrases, suggesting that this type of basic auditory skill may work to strengthen more fine-grained aspects of language.

Another possibility is that statistical learning, a mechanism thought to underlie the internalization of melodic patterns of one's musical culture (François and Schön, 2014) as well as the acquisition of grammar (Saffran, 2003; Saffran and Wilson, 2003; Gómez and Lakusta, 2004), may play a crucial role for both melody perception and grammar at this specific stage of development. With respect to the role of statistical learning, Saffran (2002) showed that both school-aged children and adults rely on distributional information in the sequencing of words (i.e., type A words always preceding type B words) to learn an artificial grammar, while Gómez and Lakusta (2004) found that even 12-month-old infants can categorize words based on distributional information. As suggested by Saffran and Wilson (2003), infants and children may use statistical regularities to acquire different levels of structure in language and this can occur in a cascaded manner. In their experiment, 12-month-old infants were exposed to multi-word sentences organized according to a finite-state grammar where transitional probabilities of syllables were lower between words compared to within words. Results showed that infants learned to distinguish between grammatical and ungrammatical sentences, suggesting that they were able to segment words from continuous speech and then use this knowledge to acquire grammatical rules in an artificial language (Saffran and Wilson, 2003). Given that the acquisition of grammar is a long and complex process that continues into the late pre-school years (Brown, 1973; Brooks and Kempe, 2012), it is possible that 3- and 4-yearold children continue to rely, among other mechanisms, on the distributional properties of input speech to internalize the structures of their native grammar. At the same time, between the third and fourth year of age, children are in the process of internalizing melodic and harmonic structures from their musical environment (Corrigall and Trainor, 2009, 2013), a process that has been argued to rely on statistical learning, that is, extracting regularities from musical input (Tillmann et al., 2000).

Given that developmental theories have supported the idea that the acquisition of language is a gradual process moving from the awareness of language phonemes in infancy (Jusczyk and Aslin, 1995) to more complex skills such as recognizing the meaning and functions of words in sentences (Cohrdes et al., 2016), it seems plausible that distinct musical skills that rely on common learning mechanisms will develop in parallel and in a similar fashion. Indeed, accounts in which musical skill acquisition develops in a gradual manner have also been proposed (Welch, 1985; Dowling, 1999). The findings of the present study bring together these accounts to suggest that specific mechanisms may operate in different manners across development to underlie both the acquisition of distinct language 
and musical skills but also the connections between them. This is compatible with emergentist models of language development which suggest that infants recruit a number of mechanisms to acquire language while the weight of reliance on different mechanisms changes over time (Hollich et al., 2000; HirshPasek et al., 2004; O'Grady, 2005). Specifically, we propose that sensitivity to metrical structure in 3- and 4-year-old children may contribute both to the acquisition of phonological awareness in language and to skills necessary for perceiving and producing rhythm structures in music. On the other hand, as demonstrated above, pre-schoolers may make use of sensitivity to statistical regularities to process melodies as well as grammatical structures at this specific point in development. Although the extraction of statistical regularities from speech may also play an important role in phonological awareness, evidence has shown that infants as young as $6-8$ months already track statistical distributions of sounds in continuous speech to extract phonetic categories (6-month-olds; Maye et al., 2002) and sequences of syllables (8-month-olds; Saffran et al., 1996). Therefore, 3- and 4-yearolds may primarily rely on other auditory and cognitive skills to refine their knowledge of phonological structure. Similarly, the internalization of regularities and enculturation in rhythmic patterns may take place earlier than in melodic sequence (Hannon and Trehub, 2005b; see also Corrigall and Trainor, 2009, 2013). For instance, 6-month-old North-American infants can detect variations in both Western and Balkan music meters equally well (Hannon and Trehub, 2005a,b), whilst 12-monthold infants are already facilitated by the isochronous meter typical in Western music when detecting rhythmic changes in musical sequences (Hannon and Trehub, 2005b). Thus, infants at 12 months may already be displaying a form of musical enculturation for rhythmic aspects. Broadly in support of these findings, it has been suggested that rhythmic skills such as rhythm discrimination (Anvari et al., 2002) and production of rhythmic structures may develop earlier than melodic skills (see also Tafuri and Villa, 2002). This may explain the unique association between melody perception and grammar at 3- and 4-years.

The notion of specific intersections between distinct language and musical skills is also consistent with theoretical accounts according to which there are shared resources but also dissociable features in the cognitive processing of speech and music (Patel, 1998, 2003; Saffran, 2003; Peretz, 2006; see also Asaridou and McQueen, 2013 for a review of this issue). For example, in his highly influential theory, Patel has proposed that the online memory process of integrating new items in unfolding sequences in language (i.e., sentences) and in music (i.e., chord progressions) may rely on common cognitive and neural resources (Patel, 1998, 2003) while linguistic and musical longterm memory systems may be independent (Peretz et al., 1994; Brown et al., 2006; Slevc et al., 2009). Indeed, a number of behavioral and neuroscientific studies in adults and children have supported the idea of shared online processing (Koelsch et al., 2002; Jentschke et al., 2008; Fedorenko et al., 2009; Jentschke and Koelsch, 2009; Sammler et al., 2009; Schön et al., 2010; Slevc et al., 2013; Kunert et al., 2015). Studies of brain damage has offered findings sometimes consistent with the shared resources approach (e.g., agrammatic Broca's aphasia associated with failure to process musical syntax: Patel et al., 2008) but has also found dissociations (e.g., impaired processing of harmonic relations with linguistic syntactic processing intact, Peretz et al., 1994, or impaired grammatical processing of language with preserved musical syntax, Slevc et al., 2016).

Overall, although there is evidence suggesting that some aspects of musical skills are domain-specific, the use of domaingeneral mechanisms for the processing of music and the acquisition of musical skills is generally supported (Saffran, 2003; Peretz, 2006). The present study informs influential accounts such as the above, first by identifying specific connections between distinct language and musical skills in a younger age than previously studied, and second by proposing domain-general learning mechanisms responsible for these links.

\section{Informal Musical Experience at Home Contributes to Language Development}

The results of this study provide compelling evidence that informal musical experience is associated with children's development of language grammar. This suggests that higher levels of home engagement with singing, music making and greater exposure to music can serve as scaffolding for the acquisition of verbal skills, greatly extending previous suggestions (Putkinen et al., 2013; Williams et al., 2015). Perhaps the rhythmic and melodic properties of music when combined with speech in everyday interactions offer additional cues for children to successfully extract and internalize linguistic structures and information from their environment. Indeed, infants as young as 6- to 8-months old appear to benefit from complex input (i.e., melody and lyrics) with information from one modality facilitating learning in the other (Thiessen and Saffran, 2009). Furthermore, given that musical interactions among groups in early childhood have been linked to pro-social attitudes and socio-emotional bonding (Custodero, 2006; Kirschner and Tomasello, 2010; Cirelli et al., 2014), music making in the home might facilitate emotion regulation and cooperation in the context of learning complex information. Indeed, it has been argued that affective and social aspects in the environment affect cognitive performance and learning in young children as well as infants (Kuhl, 2011; Franco et al., 2014). For instance, 9-monthold English-learning infants exposed to Mandarin native speakers who vividly interacted with them across 12 sessions learned to discriminate Mandarin phonemes, as opposed to a control group who was exposed to the same amount of foreign language sounds only via audio-visual and audio recordings (Kuhl et al., 2003). These findings emphasize the role of interpersonal interaction in language learning and cognition and suggest that the prosocial function of musical interactions may play an important role in promoting the acquisition of language. Whether it is predominantly socio-emotional functions of music-making that contribute to language learning or simply the perceptionfacilitating (e.g., rhythmic and melodic) properties of music, remains a crucial question for future studies.

When considering the influence of informal musical interactions at home on children's musical skills, only the ability of children to sing in tune was significantly affected. 
This finding complements evidence on how immersion (i.e., contexts in which a child hears songs several times without being required to perform them), can facilitate song learning (Klinger et al., 1998). It may also be the case that children who often engage in singing activities at home might feel more comfortable about performing in a testing environment. Alternatively, the development of a range of musical skills might be influenced by additional variables that were not considered in this study, for instance personal engagement of the child with music, or quality of parent-child singing and music-making (e.g., singing in or out of tune, keeping a steady beat). It is also highly likely that unlike more focused music-making contexts (Gerry et al., 2012), the focus of these spontaneous interactions in the home is not to practice music in a consistent manner, but rather it is an opportunity for pleasant joint activities between parents and children (Custodero and Johnson-Green, 2003; Custodero, 2006). Not surprisingly, home musical interactions have been shown to play a supportive role to other learning goals (e.g., counting songs to learn the numbers), accompany everyday activities to make them enjoyable (Custodero and Johnson-Green, 2006; Barrett, 2009) or serve other purposes such as soothing, providing distraction and regulating behavior (Custodero and Johnson-Green, 2006; Young, 2008; Barrett, 2009).

Besides the direct effects of informal musical experience at home on either linguistic or musical skills in young pre-schoolers, the most intriguing and novel finding in the present study was that the interaction between home music experience and musical skills was predictive of language development. Specifically, the observed predictive relationships between children's musical and linguistic skills, (namely the rhythm - phonological awareness and melody perception - grammar links), varied as a function of musical experience in the home, with children from more musically active families showing a stronger connection between musical and linguistic skills. This is consistent with findings by Forgeard et al. (2008) who found that predictive relationships between music perception skills and reading competence in 6year-old children were stronger for musically trained compared to untrained peers. In our study, the fact that informal musical play often brings together speech and music within a context of positive interpersonal interaction may enhance interconnectivity between the areas of cognition that are engaged during this process. Such interconnections may later facilitate music to language transfer if children receive formal musical training. This finding complements insights provided by studies that have highlighted the role of active music making in promoting neural changes in the processing of language (Moreno et al., 2009; François et al., 2013; Kraus et al., 2014). Indeed, although actively engaging with music making appears to be important, it is also the unstructured and informal nature of home musical interactions that may have unique potential for cognitive transfer in the early years. Although this study was not designed to directly address these possibilities, the present findings generate critical questions for future exploration.

Finally, the level of parental education did not affect the relationships explored above, and the interactions between MEF scores and musical skills significantly predicted both grammar and phonological awareness skills even when the parents' level of musical sophistication was taken into account. This means that it is specifically an active parent-child musical engagement that is mediating the music-language link. It is important to note, however, that the Gold-MSI had a contribution in predicting phonological awareness independent of the interaction between MEF score and musical ability, suggesting that the parents' musical sophistication might also reflect a level of musical engagement with their children at home possibly not captured by the MEF Questionnaire.

\section{CONCLUSION}

There are three conclusions from the research presented here. First, we have shown that distinct musical and linguistic skills are linked in young pre-schoolers, independently of individual differences in non-verbal ability and verbal memory. Thus, timing and melodic skills are not by-products of other cognitive skills, but rather they are independently associated with language development. Rhythmic and melodic aspects of musical ability differentially predict, respectively, phonological awareness and language grammar, revealing part of the developmental trend of the music-language relationships. This finding brings together theories of musical and linguistic development that support a step-by-step acquisition of skills in both modalities (Welch, 1985; Dowling, 1999; Cohrdes et al., 2016). Such distinct abilities rely on common learning mechanisms, yet partly dissociable domain-specific aspects. The task for future research is to identify the mechanisms underlying the connections between specific musical and linguistic skills along the developmental trajectory.

Second, we have provided evidence that informal musical input at home can have an impact on the development of complex language skills in 3- and 4-year-old children. This finding complements a growing body of research focusing on the potential of formal musical training for language development (e.g., Moreno et al., 2009; François et al., 2013; Kraus et al., 2014) and extends such promise to the informal home environment. It also suggests that informal and unstructured musical interactions may scaffold the development of other skills. More research is needed to delineate possible pathways through which engagement with music may support language, communication and social skills in the early years.

Third, above and beyond parents' musical sophistication musical experience in the family interacts with musical abilities in predicting language development, thus suggesting that this type of experience may help to strengthen connections between musical and linguistic skills at a crucial stage when children develop school-readiness. This opens new areas of inquiry about the mechanisms through which musical experience may work to strengthen the developing brain. It also informs studies demonstrating the potential of musical training to promote changes in neurophysiological and cognitive functioning: it may be the case that a musically enriched home environment holds promise for enabling the transfer of skills from one domain to the other. 
Together these results provide transferable insights by paving the way for using music-based training for strengthening language development in the early years. This is relevant for both early years practitioners and parenting practices, by suggesting musical enrichment as a powerful tool alongside other activities such as book reading, in supporting children's development. Based on the present findings, musical programs focusing on specific skills could be designed to address language development targets at an age where the brain is still highly plastic. Such music-based interventions may have the potential to prevent language-learning difficulties, facilitate language development in challenging contexts (e.g., L2 acquisition in migrant children) and support language development in disadvantaged groups. For instance, training rhythmic skills may work to strengthen brain networks underlying the segmentation of syllables and words from the speech stream. Therefore, rhythm-based musical interventions could potentially improve phonological awareness and reading readiness in children at risk of developmental language disorders such as dyslexia (Overy, 2000, 2003; Goswami, 2011) or those being schooled in a new language. Such forms of rhythmic training can potentially take advantage of mobile technology and be developed into games or applications on portable devices, e.g., tablets or smartphones (Bégel et al., 2017b, 2018). In an age of rapid technological advance this can constitute a pleasant and highly motivating means for children to engage with interventions with promising potential for language development.

\section{ETHICS STATEMENT}

This study was carried out in accordance with the recommendations of the Middlesex University Psychology

\section{REFERENCES}

Agnew, J. A., Dorn, C., and Eden, G. F. (2004). Effect of intensive training on auditory processing and reading skills. Brain Lang. 88, 21-25. doi: 10.1016/S0093-934X(03)00157-3

Anvari, S. H., Trainor, L. J., Woodside, J., and Levy, B. A. (2002). Relations among musical skills, phonological processing, and early reading ability in preschool children. J. Exp. Child Psychol. 83, 111-130. doi: 10.1016/s0022-0965(02) 00124-8

Asaridou, S. S., and McQueen, J. M. (2013). Speech and music shape the listening brain: evidence for shared domain-general mechanisms. Front. Psychol. 4:321. doi: 10.3389/fpsyg.2013.00321

Barac, R., Moreno, S., Chau, T., Schellenberg, E. G., Cepeda, N. J., and Bialystok, E. (2011). Short-term music training enhances verbal intelligence and executive function. Psychol. Sci. 22, 1425-1433. doi: 10.1177/09567976114 16999

Barrett, M. S. (2009). Sounding lives in and through music: a narrative inquiry of the everyday musical engagement of a young child. J. Early Child. Res. 7, 115-134. doi: 10.1177/1476718X09102645

Baruch, C., and Drake, C. (1997). Tempo discrimination in infants. Infant Behav. Dev. 20, 573-577. doi: 10.1016/S0163-6383(97)90049-7

Baruch, C., Panissal-Vieu, N., and Drake, C. (2004). Preferred perceptual tempo for sound sequences: comparison of adults, children, and infants. Percept. Mot. Skills 98, 325-339. doi: 10.2466/pms.98.1.325-339

Bégel, V., Benoit, C. E., Correa, A., Cutanda, D., Kotz, S. A., and Dalla Bella, S. (2017a). "Lost in time" but still moving to the beat. Neuropsychologia 94, 129-138. doi: 10.1016/j.neuropsychologia.2016.11.022
Department's Research Ethics Committee, with informed consent from all participants. All participants gave informed consent in accordance with the Declaration of Helsinki. The protocol was approved by the Middlesex University Psychology Department's Research Ethics Committee.

\section{AUTHOR CONTRIBUTIONS}

NP conceived and designed the project, performed the experiments, analyzed the data, and drafted the manuscript. FF was involved in the design and planning of the research and supervised the project. SDB supervised statistical analyses. FF and SDB provided significant input that helped to shape the manuscript. NF performed the analysis of the synchronization data and contributed to the final version of the manuscript.

\section{FUNDING}

NP was supported by a Middlesex University Ph.D. scholarship.

\section{ACKNOWLEDGMENTS}

We would like to thank sound engineer Peter Williams and animator Eleonora Quario for their contributions in building musical tasks. We are also thankful to John Verney and Smaragda Lekkou for their assistance and feedback with regards to the musical tasks. We finally wish to thank Jason Musil who provided the initial version of the algorithm for tap onset detection and Paul De Mornay Davies for reviewing the final version of this manuscript.

Bégel, V., Di Loreto, I., Seilles, A., and Dalla Bella, S. (2017b). Music games: potential application and considerations for rhythmic training. Front. Hum. Neurosci. 11:273. doi: 10.3389/fnhum.2017.00273

Bégel, V., Seilles, A., and Dalla Bella, S. (2018). Rhythm Workers: a music-based serious game for training rhythm skills. Music Sci. 1, 1-16. doi: 10.1177/ 2059204318794369

Bobin-Bègue, A., and Provasi, J. (2005). Tempo Discrimination in 3- and 4- year-old children: performances and threshold. Curr. Psychol. Lett. 2, 1-14.

Brooks, P., and Kempe, V. (2012). Language Development. Chichester: John Wiley \& Sons.

Brown, R. (1973). A First Language: The Early Stages. London: George Allen \& Unwin.

Brown, S. (2001). Are music and language homologues? Ann. N. Y. Acad. Sci. 930, 372-374. doi: 10.1111/j.1749-6632.2001.tb05745.x

Brown, S., Martinez, M. J., and Parsons, L. M. (2006). Music and language side by side in the brain: a PET study of the generation of melodies and sentences. Eur. J. Neurosci. 23, 2791-2803. doi: 10.1111/j.1460-9568.2006.04785.x

Carpenter, M. (2006). "Instrumental, social and shared goals and intentions in imitation," in Imitation and the Social Mind: Autism and Typical Development, eds S. J. Rogers and J. H. G. Williams (New York, NY: Guilford Press).

Cirelli, L. K., Einarson, K. M., and Trainor, L. J. (2014). Interpersonal synchrony increases prosocial behavior in infants. Dev. Sci. 17, 1003-1011. doi: 10.1111/ desc. 12193

Cohrdes, C., Grolig, L., and Schroeder, S. (2016). Relating language and music skills in young children: a first approach to systemize and compare distinct competencies on different levels. Front. Psychol. 7:1616. doi: 10.3389/fpsyg. 2016.01616 
Corrigall, K., and Trainor, L. J. (2009). Effects of musical training on key and harmony perception. Ann. N. Y. Acad. Sci. 1169, 164-168. doi: 10.1111/j.17496632.2009.04769.x

Corrigall, K. A., and Trainor, L. J. (2013). Enculturation to musical pitch structure in young children: evidence from behavioral and electrophysiological methods. Dev. Sci. 17, 142-158. doi: 10.1111/desc.12100

Cross, I. (2003). "Music and biocultural evolution," in The Cultural Study of Music, eds M. Clayton, T. Herbert, and R. Middleton (London: Routledge), 19-30.

Cunningham, A. J., and Carroll, J. M. (2015). Early predictors of phonological and morphological awareness and the link with reading: evidence from children with different patterns of early deficit. Appl. Psycholinguist. 36, 509-531. doi $10.1017 /$ S0142716413000295

Custodero, L., and Johnson-Green, E. (2006). Caregiving in counterpoint: reciprocal influences in the musical parenting of younger and older infants. Early Child. Dev. Care 178, 15-39. doi: 10.1080/03004430600601115

Custodero, L. A. (2006). Singing practices in 10 families with young children. J. Res. Music Educ. 54, 37-56. doi: 10.1177/002242940605400104

Custodero, L. A., and Johnson-Green, E. A. (2003). Passing the cultural torch: musical experience and musical parenting of infants. J. Res. Music Educ. 51, 102-114. doi: $10.2307 / 3345844$

Dalla Bella, S., and Sowiński, J. (2015). Uncovering beat deafness: detecting rhythm disorders with synchronized finger tapping and perceptual timing tasks. J. Vis. Exp. 97:e51761. doi: 10.3791/51761

Darwin, C. (1871). The Descent of Man and Selection in Relation to Sex. London: John Murray.

Deacon, S. H., and Kirby, J. R. (2004). Morphological awareness: just "more phonological"? The roles of morphological and phonological awareness in reading development. Appl. Psycholinguist. 25, 223-238. doi: 10.1017/ S0142716404001110

Degé, F., and Schwarzer, G. (2011). The effect of a music program on phonological awareness in preschoolers. Front. Psychol. 2:124. doi: 10.3389/fpsyg.2011.00124

Dissanayake, E. (2000). "Antecedents of the temporal arts in early mother-infant interaction," in The Origins of Music, eds N. L. Wallin, B. Merker, and S. Brown (Cambridge, MA: The MIT Press), 389-410.

D'Odorico, L., and Franco, F. (1991). Selective production of vocalisations in different communication contexts. J. Child Lang. 18, 475-499. doi: 10.1017/ S0305000900011211

D’Odorico, L., Franco, F., and Vidotto, G. (1985). Temporal characteristics in infant cry and non-cry vocalisations. Lang. Speech 28, 29-46. doi: 10.1177/ 002383098502800102

Dowling, W. J. (1999). “The development of music perception and cognition," in The Psychology of Music, 2nd Edn, ed. D. Deutsch (London: Academic Press), 603-625. doi: 10.1016/b978-012213564-4/50016-0

Dunn, L. M., Dunn, L. M., Whetton, C., and Burley, J. (1997). British Picture Vocabulary Scale. Windsor: NFER-Nelson.

Elliott, C. D., Smith, P., and McCulloch, K. (1996). British Ability Scales (BAS II): Full Age Range. Windsor: NFER-Nelson.

Fedorenko, E., Patel, A., Casasanto, D., Winawer, J., and Gibson, E. (2009). Structural integration in language and music: evidence for a shared system. Mem. Cogn. 37, 1-9. doi: 10.3758/MC.37.1.1

Fernald, A. (1985). Four-month-old infants prefer to listen to motherese. Infant Behav. Dev. 8, 181-195. doi: 10.1016/j.cogpsych.2010.01.002

Fernald, A., and Kuhl, P. (1987). Acoustic determinants of infant preference for motherese speech. Infant Behav. Dev. 10, 279-293. doi: 10.1016/0163-6383(87) 90017-8

Fitch, W. (2013). Rhythmic cognition in humans and animals: distinguishing meter and pulse perception. Front. Syst. Neurosci. 7:68. doi: 10.3389/fnsys.2013.00068

Fitch, W. T. (2006). The biology and evolution of music: a comparative perspective. Cognition 100, 173-215. doi: 10.1016/j.cognition.2005.11.009

Forgeard, M., Schlaug, G., Norton, A., Rosam, C., Iyengar, U., and Winner, E. (2008). The relation between music and phonological processing in normalreading children and children with dyslexia. Music Percept. 25, 383-390. doi: 10.1525/mp.2008.25.4.383

Franco, F. (1984). Differences in manner of phonation of infant cries: relationship to communicative context. Lang. Speech 27, 59-78. doi: 10.1177/ 002383098402700105

Franco, F., Swaine, J. S., Israni, S., Zaborowska, K. A., Kaloko, F., Kesavarajan, I. et al. (2014). Affect-matching music improves cognitive performance in adults and young children for both positive and negative emotions. Psychol. Music 42, 869-887. doi: 10.1177/0305735614548500

François, C., Chobert, J., Besson, M., and Schön, D. (2013). Music training for the development of speech segmentation. Cereb. Cortex 23, 2038-2043. doi: $10.1093 /$ cercor/bhs 180

François, C., and Schön, D. (2014). Neural sensitivity to statistical regularities as a fundamental biological process that underlies auditory learning: the role of musical practice. Hear. Res. 308, 122-128. doi: 10.1016/j.heares.2013.08.018

Fries, W., and Swihart, A. A. (1990). Disturbance of rhythm sense following right hemisphere damage. Neuropsychologia 28, 1317-1323. doi: 10.1016/00283932(90)90047-R

Gerry, D., Unrau, A., and Trainor, L. J. (2012). Active music classes in infancy enhance musical, communicative and social development. Dev. Sci. 15, 398407. doi: 10.1111/j.1467-7687.2012.01142.x

Giraud, A. L., and Poeppel, D. (2012). Cortical oscillations and speech processing: emerging computational principles and operations. Nat. Neurosci. 15, 511-517. doi: 10.1038/nn.3063

Gómez, R. L., and Lakusta, L. (2004). A first step in form-based category abstraction by 12-month-old infants. Dev. Sci. 7, 567-580. doi: 10.1111/j.1467-7687.2004. 00381.x

Gordon, E. (1989). Audie: A Game for Understanding and Analyzing your Child's Music Potential. Chicago, IL: GIA Publications.

Gordon, R. L., Shivers, C. M., Wieland, E. A., Kotz, S. A., Yoder, P. J., and Devin McAuley, J. (2015). Musical rhythm discrimination explains individual differences in grammar skills in children. Dev. Sci. 18, 635-644. doi: 10.1111/ desc. 12230

Goswami, U. (2011). A temporal sampling framework for developmental dyslexia. Trends Cogn. Sci. 15, 3-10. doi: 10.1016/j.tics.2010.10.001

Goswami, U., Huss, M., Mead, N., Fosker, T., and Verney, J. P. (2013). Perception of patterns of musical beat distribution in phonological developmental dyslexia: significant longitudinal relations with word reading and reading comprehension. Cortex 49, 1363-1376. doi: 10.1016/j.cortex.2012.05.005

Hannon, E. E., and Trehub, S. E. (2005a). Metrical categories in infancy and adulthood. Psychol. Sci. 16, 48-55. doi: 10.1111/j.0956-7976.2005.00779.x

Hannon, E. E., and Trehub, S. E. (2005b). Tuning in to musical rhythms: infants learn more readily than adults. Proc. Natl. Acad. Sci. U.S.A. 102, 12639-12643. doi: 10.1073/pnas.0504254102

Hirsh-Pasek, K., Golinkoff, R. M., Hennon, E. A., and Maguire, M. J. (2004). "Hybrid theories at the frontier of developmental psychology: the emergentist coalition model of word learning as a case," in Weaving a Lexicon, eds D. G. Hall and S. R. Waxman (Cambridge, MA: MIT Press), 173-204.

Hoff, E., and Marilyn, S. (2009). Blackwell Handbook of Language Development. Hoboken, NJ: John Wiley \& Sons.

Hollich, G., Hirsh-Pasek, K., and Golinkoff, R. M. (2000). II. The emergentist coalition model. Monogr. Soc. Res. Child Dev. 65, 17-29. doi: 10.1111/15405834.00092

Hornickel, J., and Kraus, N. (2013). Unstable representation of sound: a biological marker of dyslexia. J. Neurosci. 33, 3500-3504. doi: 10.1523/JNEUROSCI.420512.2013

Hoyt, C. (1941). Test reliability estimated by analysis of variance. Psychometrika 6, 153-160. doi: 10.1007/BF02289270

Huss, M., Verney, J. P., Fosker, T., Mead, N., and Goswami, U. (2011). Music, rhythm, rise time perception and developmental dyslexia: perception of musical meter predicts reading and phonology. Cortex 47, 674-689. doi: 10.1016/j. cortex.2010.07.010

Jensen, J. K., and Neff, D. L. (1993). Development of basic auditory discrimination in preschool children. Psychol. Sci. 4, 104-107. doi: 10.1111/j.1467-9280.1993. tb00469.x

Jentschke, S., and Koelsch, S. (2009). Musical training modulates the development of syntax processing in children. Neuroimage 47, 735-744. doi: 10.1016/j. neuroimage.2009.04.090

Jentschke, S., Koelsch, S., Sallat, S., and Friederici, A. D. (2008). Children with specific language impairment also show impairment of music-syntactic processing. J. Cogn. Neurosci. 20, 1940-1951. doi: 10.1162/jocn.2008.20135

Jusczyk, P. W., and Aslin, R. N. (1995). Infants' detection of the sound patterns of words in fluent speech. Cogn. Psychol. 29, 1-23. doi: 10.1006/cogp.1995.1010

Kaiser, H. F. (1974). An index of factorial simplicity. Psychometrika 39, 31-36. doi: $10.1007 / \mathrm{BF} 02291575$ 
Kirschner, S., and Tomasello, M. (2010). Joint music making promotes prosocial behavior in 4- year-old children. Evol. Hum. Behav. 31, 354-364. doi: 10.1016/j. evolhumbehav.2010.04.004

Kisilevsky, S., Hains, S. M., Jacquet, A.-Y., Granier-Deferre, C., and Lecanuet, J. P. (2004). Maturation of fetal responses to music. Dev. Sci. 7, 550-559. doi: 10.1111/j.1467-7687.2004.00379.x

Klinger, R., Campbell, P., and Goolsby, T. (1998). Approaches to children's song acquisition: immersion and phrase-by-phrase. J. Res. Music Educ. 46, 24-34. doi: $10.2307 / 3345757$

Koelsch, S., Gunter, T. C., Cramon, D. Y. V., Zysset, S., Lohmann, G., and Friederici, A. D. (2002). Bach speaks: a cortical "language-network" serves the processing of music. Neuroimage 17, 956-966. doi: 10.1006/nimg.2002.1154

Kraus, N., Hornickel, J., Strait, D. L., Slater, J., and Thompson, E. (2014). Engagement in community music classes sparks neuroplasticity and language development in children from disadvantaged backgrounds. Front. Psychol. 5:1403. doi: 10.3389/fpsyg.2014.01403

Kuhl, P. K. (2011). "Social mechanisms in early language acquisition: understanding integrated brain systems supporting language," in Oxford Handbook of Social Neuroscience, eds J. Decety and J. T. Cacioppo (New York, NY: Oxford University Press), 649-667.

Kuhl, P. K., Tsao, F.-M., and Liu, H. M. (2003). Foreign-language experience in infancy: effects of short-term exposure and social interaction on phonetic learning. Proc. Natl. Acad. Sci. U.S.A. 100, 9096-9101. doi: 10.1073/pnas. 1532872100

Kunert, R., Willems, R. M., Casasanto, D., Patel, A. D., and Hagoort, P. (2015). Music and language syntax interact in Broca's area: an fMRI study. PLoS One 10:e0141069. doi: 10.1371/journal.pone.0141069

Laland, K., Wilkins, C., and Clayton, N. (2016). The evolution of dance. Curr. Biol. 26, R5-R9. doi: 10.1016/j.cub.2015.11.031

Lamb, S. J., and Gregory, A. H. (1993). The relationship between music and reading in beginning readers. Educ. Psychol. 13, 19-27. doi: 10.1080/0144341930130103

Large, E. W. (2008). "Resonating to musical rhythm: theory and experiment," in The Psychology of Time, ed. S. Grondin (Bingley: Emerald Group), 189-232.

Law, L. N., and Zentner, M. (2012). Assessing musical abilities objectively: construction and validation of the Profile of Music Perception Skills. PLoS One 7:e52508. doi: 10.1371/journal.pone.0052508

Livingstone, F. B. (1973). Did the Australopithecines sing? Curr. Anthropol. 14, 25-29. doi: 10.1086/201402

Maclean, M., Bryant, P., and Bradley, L. (1987). Rhymes, nursery rhymes, and reading in early childhood. Merrill Palmer Q. 33, 255-281.

Mampe, B., Friederici, A. D., Christophe, A., and Wermke, K. (2009). Newborns' cry melody is shaped by their native language. Curr. Biol. 19, 1994-1997. doi: 10.1016/j.cub.2009.09.064

Maye, J., Werker, J. F., and Gerken, L. (2002). Infant sensitivity to distributional information can affect phonetic discrimination. Cognition 82, B101-B111. doi: 10.1016/S0010-0277(01)00157-3

Melby-Lervåg, M., Lyster, S. A. H., and Hulme, C. (2012). Phonological skills and their role in learning to read: a meta-analytic review. Psychol. Bull. 138, 322-352. doi: $10.1037 / \mathrm{a} 0026744$

Merker, B. (2002). Music: the missing Humboldt system. Music. Sci. 6, 3-21. doi: $10.1177 / 102986490200600101$

Mithen, S. (2005). The Singing Neanderthals: The Origins of Music, Language, Mind, and Body. London: Weidenfeld Nicolson.

Moreno, S., Marques, C., Santos, A., Santos, M., Castro, S. L., and Besson, M. (2009). Musical training influences linguistic abilities in 8-year-old children: more evidence for brain plasticity. Cereb. Cortex 19, 712-723. doi: 10.1093/ cercor/bhn 120

Morrongiello, B. A., Trehub, S. E., Thorpe, L. A., and Capodilupo, S. (1985). Children's perception of melodies: the role of contour, frequency, and rate of presentation. J. Exp. Child Psychol. 40, 279-292. doi: 10.1016/0022-0965(85) 90090-6

Müllensiefen, D., Gingras, B., Musil, J., and Stewart, L. (2014). The musicality of non- musicians: an index for assessing musical sophistication in the general population. PLoS One 9:e89642. doi: 10.1371/journal.pone.0089642

Nakata, T., and Trehub, S. E. (2004). Infants' responsiveness to maternal speech and singing. Infant Behav. Dev. 27, 455-464. doi: 10.1016/j.infbeh.2004.03.002

O'Grady, W. (2005). Syntactic Carpentry. New York, NY: Routledge. doi: 10.4324/ 9781410612571
Olsho, L. W. (1984). Infant frequency discrimination. Infant Behav. Dev. 7, 27-35. doi: 10.1016/S0163-6383(84)80020-X

Overy, K. (2000). Dyslexia, temporal processing and music: the potential of music as an early learning aid for dyslexic children. Psychol. Music 28, 218-229. doi: $10.1177 / 0305735600282010$

Overy, K. (2003). Dyslexia and music. Ann. N. Y. Acad. Sci. 999, 497-505. doi: 10.1196/annals. 1284.060

Papaeliou, C. F., and Trevarthen, C. (2006). Prelinguistic pitch patterns expressing 'communication' and 'apprehension'. J. Child Lang. 33, 163-178. doi: 10.1017/ S0305000905007300

Paraskevopoulos, E., Kuchenbuch, A., Herholz, S. C., and Pantev, C. (2012). Musical expertise induces audiovisual integration of abstract congruency rules. J. Neurosci. 32, 18196-18203. doi: 10.1523/JNEUROSCI.1947-12.2012

Patel, A. (2003). Language, music, syntax and the brain. Nat. Neurosci. 6, 674-681. doi: $10.1038 / \mathrm{nn} 1082$

Patel, A. D. (1998). Syntactic processing in language and music: different cognitive operations, similar neural resources? Music Percept. 16, 27-42. doi: 10.2307/ 40285775

Patel, A. D. (2010). Music, Language, and the Brain. Oxford: Oxford University Press.

Patel, A. D., Iversen, J. R., Wassenaar, M., and Hagoort, P. (2008). Musical syntactic processing in agrammatic Broca's aphasia. Aphasiology 22, 776-789. doi: 10. 1080/02687030701803804

Peretz, I. (2006). The nature of music from a biological perspective. Cognition 100, 1-32. doi: 10.1016/j.cognition.2005.11.004

Peretz, I., Gosselin, N., Nan, Y., Caron-Caplette, E., Trehub, S. E., and Béland, R. (2013). A novel tool for evaluating children's musical abilities across age and culture. Front. Syst. Neurosci. 7:30. doi: 10.3389/fnsys.2013.00030

Peretz, I., Kolinsky, R., Tramo, M., Labrecque, R., Hublet, C., Demeurisse, G., et al. (1994). Functional dissociations following bilateral lesions of auditory cortex. Brain 117, 1283-1301. doi: 10.1093/brain/117. 6.1283

Provasi, J., and Bobin-Bègue, A. (2003). Spontaneous motor tempo and rhythmical synchronisation in 21/2-and 4-year-old children. Int. J. Behav. Dev. 27, 220-231. doi: 10.1080/01650250244000290

Putkinen, V., Tervaniemi, M., and Huotilainen, M. (2013). Informal musical activities are linked to auditory discrimination and attention in 2-3-year-old children: an event-related potential study. Eur. J. Neurosci. 37, 654-661. doi: 10.1111/ejn.12049

R Core Team (2012). R: A Language and Environment for Statistical Computing. Vienna: R Foundation for Statistical Computing.

Rutkowski, J. (1997). “The nature of children's singing voices: characteristics and assessment," in The Phenomenon of Singing, ed. B. A. Roberts (St. John's: Memorial University Press), 201-209.

Ruzza, B., Rocca, F., Boero, D. L., and Lenti, C. (2003). "Investigating the musical qualities of early infant sounds," in The Neurosciences and Music, Vol. 999, eds G. Avanzini, C. Faienza, D. Minciacchi, L. Lopez, and M. Majno (NewYork, NY: Annals of the NewYork Academy of Sciences), 527-529. doi: 10.1196/annals. 1284.066

Saffran, J. R. (2002). Constraints on statistical language learning. J. Mem. Lang. 47, 172-196. doi: 10.1006/jmla.2001.2839

Saffran, J. R. (2003). Statistical language learning: mechanisms and constraints. Curr. Dir. Psychol. Sci. 12, 110-114. doi: 10.1111/1467-8721.01243

Saffran, J. R., Aslin, R. N., and Newport, E. L. (1996). Statistical learning by 8-month-old infants. Science 274, 1926-1928. doi: 10.1126/science.274.5294. 1926

Saffran, J. R., and Wilson, D. P. (2003). From syllables to syntax: multilevel statistical learning by 12-month-old infants. Infancy 4, 273-284. doi: 10.1207/ S15327078IN0402_07

Sammler, D., Koelsch, S., Ball, T., Brandt, A., Elger, C. E., Friederici, A. D., et al. (2009). Overlap of musical and linguistic syntax processing: intracranial ERP evidence. Ann. N. Y. Acad. Sci. 1169, 494-498. doi: 10.1111/j.1749-6632.2009. 04792.x

Schellenberg, E. G. (2004). Music lessons enhance IQ. Psychol. Sci. 15, 511-514. doi: 10.1111/j.0956-7976.2004.00711.x

Schön, D., Gordon, R., Campagne, A., Magne, C., Astésano, C., Anton, J. L., et al. (2010). Similar cerebral networks in language, music and song perception. Neuroimage 51, 450-461. doi: 10.1016/j.neuroimage.2010.02.023 
Shenfield, T., Trehub, S. E., and Nakata, T. (2003). Maternal singing modulates infant arousal. Psychol. Music 31, 365-375. doi: 10.1177/03057356030314002

Slevc, L. R., Faroqi-Shah, Y., Saxena, S., and Okada, B. M. (2016). Preserved processing of musical structure in a person with agrammatic aphasia. Neurocase 22, 505-511. doi: 10.1080/13554794.2016.1177090

Slevc, L. R., Reitman, J. G., and Okada, B. M. (2013). "Syntax in music and language: the role of cognitive control," in Proceedings of the 35th Annual Conference of the Cognitive Science Society, (Austin, TX: Cognitive Science Society), 3414-3419.

Slevc, L. R., Rosenberg, J. C., and Patel, A. D. (2009). Making psycholinguistics musical: self-paced reading time evidence for shared processing of linguistic and musical syntax. Psychon. Bull. Rev. 16, 374-381. doi: 10.3758/16.2.374

Sowiński, J., and Dalla Bella, S. (2013). Poor synchronization to the beat may result from deficient auditory-motor mapping. Neuropsychologia 51, 1952-1963. doi: 10.1016/j.neuropsychologia.2013.06.027

Speer, S. R., and Ito, K. (2009). Prosody in first language acquisition-Acquiring intonation as a tool to organize information in conversation. Lang. Linguist. Compass 3, 90-110. doi: 10.1111/j.1749-818x.2008.00103.x

Tafuri, J., and Villa, D. (2002). Musical elements in the vocalisations of infants aged 2- 8 months. Br. J. Music Educ. 19, 73-88. doi: 10.1017/S0265051702000153

Thiessen, E. D., Hill, E. A., and Saffran, J. R. (2005). Infant-directed speech facilitates word segmentation. Infancy 7, 53-71. doi: 10.1207/s15327078in 0701_5

Thiessen, E. D., and Saffran, J. R. (2009). How the melody facilitates the message and vice versa in infant learning and memory. Ann. N. Y. Acad. Sci. 1169, 225-233. doi: 10.1111/j.1749-6632.2009.04547.x

Thompson, N. C., Cranford, J. L., and Hoyer, E. (1999). Brief-tone frequency discrimination by children. J. Speech Lang. Hear. Res. 42, 1061-1068. doi: 10. 1044/jshr.4205.1061

Tierney, A., and Kraus, N. (2013). The ability to move to a beat is linked to the consistency of neural responses to sound. J. Neurosci. 33, 14981-14988. doi: 10.1523/JNEUROSCI.0612-13.2013

Tierney, A., and Kraus, N. (2014). Auditory-motor entrainment and phonological skills: precise auditory timing hypothesis (PATH). Front. Hum. Neurosci. 8:949. doi: 10.3389/fnhum.2014.00949

Tillmann, B., Bharucha, J. J., and Bigand, E. (2000). Implicit learning of tonality: a self-organizing approach. Psychol. Rev. 107, 885-913. doi: 10.1037//0033-295x. 107.4.885

Tillmann, B., Janata, P., and Bharucha, J. J. (2003). Activation of the inferior frontal cortex in musical priming. Cogn. Brain Res. 16, 145-161. doi: 10.1016/S09266410(02)00245-8

Tomlinson, G. (2013). Evolutionary studies in the humanities: the case of music. Crit. Inq. 39, 647-675. doi: 10.1086/671351

Trehub, S. E., Thorpe, L. A., and Morrongiello, B. A. (1985). Infants' perception of melodies: changes in a single tone. Infant Behav. Dev. 8, 213-223. doi: 10.1016/S0163-6383(85)80007-2

Trehub, S. E., and Trainor, L. (1998). Singing to infants: lullabies and play songs. Adv. Infancy Res. 12, 43-78.

Trehub, S. E., Trainor, L. J., and Unyk, A. M. (1993). "Music and speech processing in the first year of life," in Advances in Child Development and Behavior, Vol. 24, ed. H. W. Reese (New York, NY: Academic Press), 1-35. doi: 10.1016/s00652407(08)60298-0

Trevarthen, C. (1999). Musicality and the intrinsic motive pulse: evidence from human psychobiology and infant communication. Music. Sci. 3, 155-215. doi: $10.1177 / 10298649000030 \$ 109$
Tsang, C. D., Falk, S., and Hessel, A. (2017). Infants prefer infant-directed song over speech. Child Dev. 88, 1207-1215. doi: 10.1111/cdev.12647

Van Puyvelde, M., Loots, G., Vanfleteren, P., Meys, J., Simcock, D., and Pattyn, N. (2014). Do you hear the same? Cardiorespiratory responses between mothers and infants during tonal and atonal music. PLoS One 9:e106920. doi: 10.1371/ journal.pone. 0106920

Verney, J. P. (2013). Rhythmic Perception and Entrainment in 5-Year-Old Children. An Exploration of the Relationship between Temporal Accuracy at Four Isochronous Rates and its Impact on Phonological Awareness and Reading Development. Doctoral dissertation, University of Cambridge, Cambridge.

Wechsler, D. (2013). Wechsler Preschool \& Primary Scale of Intelligence - (WPPSIIV UK), 4th Edn. London: Pearson Assessment.

Weiss, A. H., Granot, R. Y., and Ahissar, M. (2014). The enigma of dyslexic musicians. Neuropsychologia 54, 28-40. doi: 10.1016/j.neuropsychologia.2013. 12.009

Welch, G. F. (1985). A schema theory of how children learn to sing in tune. Psychol. Music 13, 3-18. doi: 10.1177/03057356 85131001

Welch, G. F. (1998). Early childhood musical development. Res. Stud. Music Educ. 11, 27-41. doi: 10.1177/1321103X9801100104

Welch, G. F. (2006). "Singing and vocal development," in The Child as Musician: A Handbook of Musical Development, ed. G. McPherson (Oxford: Oxford University Press), 311-329.

White, D. J., Dale, P. S., and Carlsen, J. C. (1990). Discrimination and categorization of pitch direction by young children. Psychomusicology 9, 39-58. doi: $10.1037 / \mathrm{h} 0094159$

Wiig, E., Secord, W., and Semel, E. (2004). Clinical Evaluation of Language Fundamentals: Preschool 2. San Antonio, TX: Psychological Corporation.

Williams, K. E., Barrett, M. S., Welch, G. F., Abad, V., and Broughton, M. (2015). Early Childhood Research Quarterly Associations between early shared music activities in the home and later child outcomes: findings from the Longitudinal Study of Australian Children. Early Child. Res. Q. 31, 113-124. doi: 10.1016/j. ecresq.2015.01.004

Woodruff Carr, K., White-Schwoch, T., Tierney, A. T., Strait, D. L., and Kraus, N. (2014). Beat synchronization predicts neural speech encoding and reading readiness in preschoolers. Proc. Natl. Acad. Sci. 111, 14559-14564. doi: 10.1073/ pnas. 1406219111

Xie, Y. (2012). Transitional probability and word segmentation. Int. J. Engl. Linguist. 2:27.

Young, S. (2008). Lullaby light shows: everyday musical experience among under-two year-olds. Int. J. Music Educ. 26, 33-46. doi: 10.1177/02557614070 85648

Conflict of Interest Statement: The authors declare that the research was conducted in the absence of any commercial or financial relationships that could be construed as a potential conflict of interest.

Copyright (c) 2019 Politimou, Dalla Bella, Farrugia and Franco. This is an openaccess article distributed under the terms of the Creative Commons Attribution License (CC BY). The use, distribution or reproduction in other forums is permitted, provided the original author(s) and the copyright owner(s) are credited and that the original publication in this journal is cited, in accordance with accepted academic practice. No use, distribution or reproduction is permitted which does not comply with these terms. 


\title{
Neural Encoding of Pitch Direction Is Enhanced in Musically Trained Children and Is Related to Reading Skills
}

\author{
Vesa Putkinen ${ }^{1,2 *}$, Minna Huotilainen ${ }^{1,3}$ and Mari Tervaniemi ${ }^{1,3}$ \\ 'Cognitive Brain Research Unit, Faculty of Medicine, University of Helsinki, Helsinki, Finland, ${ }^{2}$ TURKU PET Centre, University \\ of Turku, Turku, Finland, ${ }^{3}$ Faculty of Educational Sciences, University of Helsinki, Helsinki, Finland
}

Musical training in childhood has been linked to enhanced sound encoding at different stages of the auditory processing. In the current study, we used auditory event-related potentials to investigate cortical sound processing in 9 - to 15-year-old children $(N=88)$ with and without musical training. Specifically, we recorded the mismatch negativity (MMN) and $\mathrm{P} 3 \mathrm{a}$ responses in an oddball paradigm consisting of standard tone pairs with ascending pitch and deviant tone pairs with descending pitch. A subsample of the children

OPEN ACCESS

Edited by:

Graham Frederick Welch, UCL Institute of Education, United Kingdom

Reviewed by: Erich Schröger, Leipzig University, Germany Chia-Ying Lee, Academia Sinica, Taiwan

*Correspondence: Vesa Putkinen vesa.putkinen@utu.fi

Specialty section: This article was submitted to Performance Science, a section of the journal Frontiers in Psychology

Received: 04 February 2019 Accepted: 11 June 2019

Published: 24 July 2019

Citation:

Putkinen V, Huotilainen M and Tervaniemi M (2019) Neural Encoding of Pitch Direction Is Enhanced in Musically Trained Children and Is Related to Reading Skills.

Front. Psychol. 10:1475.

doi: 10.3389/fpsyg.2019.01475
$(N=44)$ also completed a standardized test of reading ability. The musically trained children showed a larger P3a response to the deviant sound pairs. Furthermore, the amplitude of the P3a correlated with a pseudo-word reading test score. These results corroborate previous findings on enhanced sound encoding in musically trained children and are in line with studies suggesting that neural discrimination of spectrotemporal sound patterns is predictive of reading ability.

Keywords: mismatch negativity, musical training, P3a, reading, brain development

\section{INTRODUCTION}

A vast number of event-related potential (ERP) studies have found evidence for enhanced neural sound processing in musicians. One of the most frequently used EPR components in this framework is the mismatch negativity (MMN) (for a review, see Putkinen and Tervaniemi, 2018). The MMN is elicited by infrequent "deviant" sounds that differ from the preceding frequent "standard" sounds in some way (Kujala et al., 2007; Näätänen et al., 2007). According to influential theoretical accounts, the MMN is a cortical correlate of a prediction error that occurs when an incoming sound (the deviant) disconfirms predictions that the auditory system has automatically created on the basis of the preceding input (the standards) (Näätänen et al., 2007; Winkler et al., 2009). MMN studies have shown that, when compared to non-musicians, musicians show earlier or larger MMN responses to violations of different spectral, temporal, and spatial regularities (Koelsch et al., 1999; Tervaniemi et al., 2001; Fujioka et al., 2004; van Zuijen et al., 2005).

During the last decade, MMN studies have also begun to explore how such differences between musically trained and untrained individuals emerge with accumulation of musical experience in childhood. Cross-sectional MMN studies indicate that musically trained children show enhanced neural discrimination of pitch of violin tones (i.e., their main instrument; Meyer et al., 2011) 
and major vs. minor chords (Virtala et al., 2012) as well as pitch and voice-onset time changes in speech sounds (Chobert et al., 2011). A longitudinal study in 8- to 10-year-old children who were randomly assigned to music or painting classes (Chobert et al., 2014) found that MMNs to changes in syllable frequency, duration, and voice-onset time increased in amplitude after 12 months of training in the music group but not in the painting group. We have conducted a longitudinal study where we recorded the MMN and other neural markers of sensory processing and higher order cognitive functions in children who play a musical instrument and have attended a public elementary school with heavy emphasis on music in the curriculum, and children who do not play a musical instrument and are matched to the music group with regard to age and socioeconomic status (Putkinen et al., 2014a,b, 2015; Saarikivi et al., 2016; Putkinen and Saarikivi, 2018). In one of these studies (Putkinen et al., 2014b), the amplitude of the MMN elicited by minor chord deviants presented among major chord standards increased more in the music group than in the control group between the ages of 7 and 13 years. In another study (Putkinen et al., 2014a), we found that the MMNs obtained to melody, rhythm, timbre, and tuning deviants increased more in amplitude during the follow-up period. Neither study found evidence of pre-training differences between the groups in neural sound discrimination.

In addition to the enhanced $M M N$, we found that a P3a-like response elicited by minor chord deviants increased in amplitude with age in the music group but not in the control group (Putkinen et al., 2014a,b) in line with studies in adults that have reported enhanced $\mathrm{P} 3 \mathrm{a}$ responses in adult musicians (Nikjeh et al., 2008; Vuust et al., 2009; Seppänen et al., 2012). The P3a is typically interpreted as a marker of involuntary attention capture triggered by salient deviant sounds (Escera et al., 1998; Escera and Corral, 2007). Thus, it appears that certain acoustic changes are more salient for musicians than non-musicians and trigger the attentional orienting more readily in musicians.

Here we report new data from a cross-sectional study conducted partly in the same children and adolescents who participated in our longitudinal studies described above. In the current study, we employed an oddball paradigm consisting of standard tone pairs with ascending pitch and deviant tone pairs with descending pitch $(500-750 \mathrm{~Hz}$ vs. $750-500 \mathrm{~Hz}$ ). The aim was to test whether the enhanced processing of changes in musical chords and melodies that we observed previously in the music group would generalize to more basic-level sound processing of pitch order reversal. As a secondary aim, we also tested whether the neural discrimination of the pitch order reversal was associated with reading ability. The rationale was based on the findings that (1) that low-level processing of pitch changes predicts reading abilities (Ahissar et al., 2000; Kujala et al., 2001) and (2) musical training is linked with improved reading skills (Anvari et al., 2002; Moreno et al., 2009) perhaps because musical training improves auditory skills that are necessary for linking speech with its written form. Thereby, we administered standardized test of reading skills in a subset of the children and tested whether the MMN and the P3a elicited would predict their test performance.

\section{MATERIALS AND METHODS}

\section{Subjects}

Eighty-eight children and adolescents participated in the ERP experiment. The music group ( $N=41$, age range: $8.75-15.92$, 25 girls) consisted of children and adolescents who had started playing a musical instrument approximately at age 7 and had attended a public elementary school that integrates musical training (instrument lessons, orchestra practice, music theory studies) in the daily curriculum. The control group $(N=47$, age range: $8.91-15.83,18$ girls) consisted of children and adolescents without formal musical training who had attended a standard elementary school.

A written informed consent for participation in the study was obtained from the children and their parents or guardians before the experiment. The children were rewarded with two movie tickets for their participation. The experiment protocol was approved by the Ethical Committee of the Department of Psychology (currently the Department of Psychology and Logopedics), University of Helsinki, Finland.

\section{Stimuli}

The stimuli in the ERP experiment consisted of tone pairs ( $p \approx 85 \%$ ) with ascending pitch and deviant tones pairs with descending pitch $(p \approx 15 \%)$. For the standard pairs, the fundamental frequencies of the first and the second tone were 500 and $750 \mathrm{~Hz}$, respectively. For the deviant pairs, the order of the tones was reversed. The duration of each tone was $40 \mathrm{~ms}$ including 5-ms rise and fall times. The duration of the silent gap between the tones within a pair was $10 \mathrm{~ms}$. The tone pairs were presented with the stimulus onset asynchrony of $600 \mathrm{~ms}$. All tones were composed of the fundamental and two upper partials and were presented at an intensity of $\sim 60 \mathrm{~dB}$ (SPL) through headphones (Sony Dynamic Stereo Headphones, MDR-7506). The stimuli were presented in a pseudorandom order so that two deviant pairs were never presented in succession.

\section{Procedure and EEG Recording}

The duration of the experimental session was approximately $2 \mathrm{~h}$ and included five different electroencephalography (EEG) experiments conducted in a counterbalanced order, and a break midway through the session (the four additional experiments are reported elsewhere). EEG was recorded in an electrically shielded and soundproof room while the subjects watched a captioned movie with the sound turned off. They were asked to ignore the sounds and to avoid unnecessary movement.

EEG was recorded using a BioSemi Active-Two system with a sampling rate of $512 \mathrm{~Hz}$ from 64 active electrodes mounted on a BioSemi headcap. Additional electrodes were placed below and at the outer canthus of the right eye for monitoring eye movements and blinks, and at the right and left mastoids for offline re-referencing of the data.

\section{Tests of Reading Skills}

Reading ability was measured with a standardized test (Nevala et al., 2006) that involved reading aloud a list of 30 words and 
a list 30 pseudo-words. The subjects were instructed to read each list as fast and accurately as possible. A composite score incorporating both the completion time and the number of errors was computed separately for the words and pseudo-words for each child. Because of the long duration of the ERP experiment, we only tested the children who were 13 years or older out whom 44 completed both the EEG recording and the reading task (music group: $N=20,12$ girls; control group: $N=24,10$ girls).

\section{EEG Data Preprocessing}

The data were analyzed using BESA 5.1 software. Data obtained at noisy electrodes were interpolated and the automatic artifact correction system implemented in BESA was applied to remove artifacts related to eye blinks and saccades. The data were filtered with a bandwidth of $1-20 \mathrm{~Hz}$ and epoched from -100 to $500 \mathrm{~ms}$ relative to stimulus onset. Epochs with voltage changes exceeding $\pm 100 \mu \mathrm{V}$ were excluded. The epochs were averaged separately for the deviant and standard sounds and re-referenced to the average of the mastoid channels and baseline corrected ( -100 to $0 \mathrm{~ms})$.

\section{Statistical Analyses}

Mean response amplitudes were calculated for the deviants and standards over 80 -ms time windows centered at the latencies of $200 \mathrm{~ms}$ ("MMN") and $275 \mathrm{~ms}$ ("P3a"). Based on visual inspection of the scalp distribution, we chose the channels FC3, FCz, FC4, C3, Cz, and C4 for the analysis of the response amplitudes. The amplitudes were analyzed with a repeated measures ANOVA with Group (music vs. control) as a betweensubjects factor and Stimulus (deviant vs. standard) and LeftMiddle-Right (FC3 and $\mathrm{C} 3$ vs. FCz and $\mathrm{Cz}$ vs. F4 and $\mathrm{FC} 4$ ) and Frontal-Central (FC3 and $\mathrm{FCz}$ and $\mathrm{FC} 4$ vs. $\mathrm{C} 3$ and $\mathrm{Cz}$ and C4) as within-subjects factors. Greenhouse-Geisser correction was used when the sphericity assumption was violated.

Performance in the reading tasks was analyzed by fitting a linear model where reading score was predicted with age, gender, and group. Partial correlations (controlling for the effects of age and group) were calculated between the reading scores and the response amplitudes at the channels where the deviant-minus-standard amplitude was the largest for each response, i.e., at $\mathrm{FC} 4$ for the $\mathrm{MMN}$ and at $\mathrm{Cz}$ for the P3a.

\section{RESULTS}

The responses to the standard and deviant tone pairs and the deviant-minus-standard difference signals are shown in Figure 1. The deviant tone pairs elicited a two-peaked negative response, the latter of which was interpreted as the MMN based on its latency and was chosen for further analysis (Figure 1B). The MMN-like response was followed by a P3a-like positive deflection.

\section{Event-Related Potential Amplitudes}

The responses to the standard and deviant tone pairs, the deviant-minus-standard difference signals at $\mathrm{Cz}$ and the scalp distributions of the MMN and P3a are shown in Figure 1.
The deviant pairs elicited a significant $M M N$ response (main effect of stimulus: $F(1,86)=42.024, p<0.001)$ which was largest over the frontal channels and the right hemisphere (Stimulus $\times$ Frontal-Central $\times$ Left-Midline-Right interaction: $F(2,172)=8.419$, $p<0.001$, all pairwise comparisons of deviant-standard difference at channel F4 vs. other channels, $p<0.05$ ). There was no significant group difference MMN amplitude (main effect of Group and all interaction involving the Group factor, $p>0.05$ ).

The deviant pairs also elicited a significant P3a-like response (main effect of stimulus: $F(1,86)=6.500, p<0.05$ ) that was differentially distributed over the left, central and right channel locations (Stimulus $\times$ Left-Midline-Right interaction: $F(2,172)=4.876, p<0.05)$. Bonferroni corrected pairwise comparisons of the deviant-minus-standard amplitudes indicated that the P3a was smallest at the right channels (Figure 1).

There was also significant Group $\times$ Stimulus $\times$ Frontal-Central $\times$ Left-Midline-Right interaction $(F(2,172)=4.448, p<0.05)$ indicating that the $\mathrm{P} 3 \mathrm{a}$ amplitude differed between the groups at some electrodes included in the analysis. Bonferroni corrected pairwise group comparisons of the deviant-standard amplitudes indicated that the P3a was larger in the music group than in the control group at channels $\mathrm{Cz}, \mathrm{C} 3, \mathrm{Cz}$, and F4 $(p<0.05)$.

\section{Reading Test Performance}

Performance in the pseudo-word subtest improved with age $(b=0.504, t(41)=2.241, p<0.05)$ whereas no effect of age was found for the word subtest $(p>0.30)$. No group difference was found for either subtest (Figure 2) although there was a trend toward a slightly higher scores in the music group than the control group in the pseudo-word subtest $(p<0.08)$. There was no significant correlation between the amplitude of MMN and the performance in either subtest. In contrast, the P3a amplitude at $\mathrm{Cz}$ correlated negatively with the score in the pseudo-word subtest (partial $r(42)=-0.302, p<0.05$, controlling for the effects of age and group membership) (Figure 2).

\section{DISCUSSION}

The current study investigated whether musically trained children show facilitated preattentive neural discrimination of pitch order in tone pairs as indexed by the MMN and the P3a. Indeed, we found evidence for enhanced neural discrimination of ascending and descending tone pairs in musically trained children and adolescents. Namely, the music group showed a stronger P3a-like response to reversal of tone order. The MMN, in contrast, did not significantly differ between the groups. Interestingly, the amplitude of the P3a-like response correlated with performance in a pseudo-word reading task in line with the idea that elementary sound processing is linked with reading ability (Ahissar et al., 2000).

The lack of significant group difference in the MMN amplitude is in contrast with a large number of studies that have found enhanced MMN responses in musically trained adults and children (Putkinen and Tervaniemi, 2018). It is noteworthy that the same children who participated in the current study also took part in our previous studies that found stronger 
A

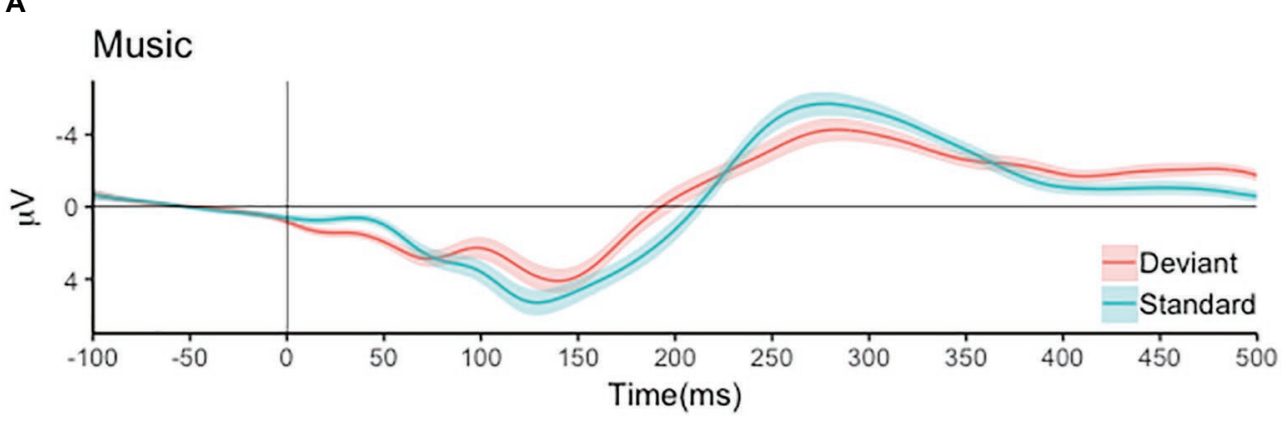

\section{Control}
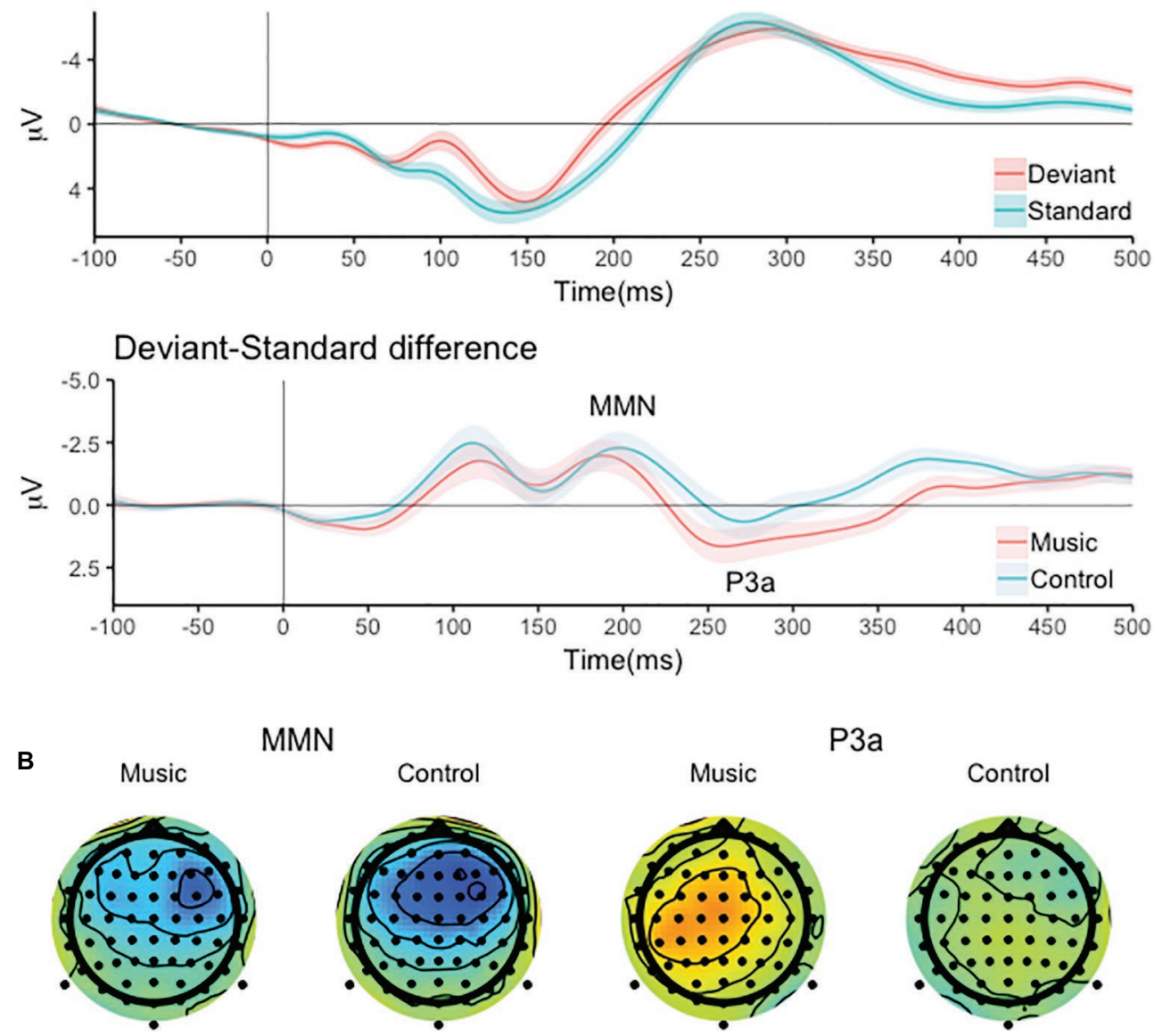

MMN
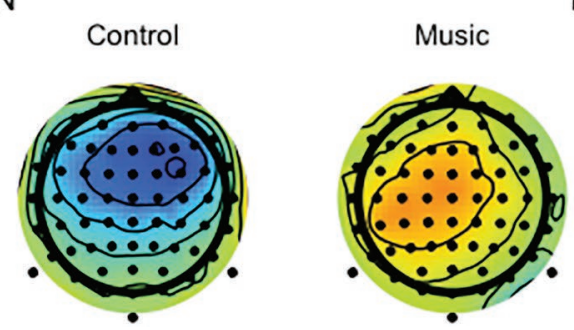

P3a

FIGURE 1 | (A) The responses to the standard and deviant tone pairs, the deviant-minus-standard difference signals and (B) the scalp distribution of the MMN and P3a-like responses for the music and control group.

MMNs in the musically trained children for musical chords (Putkinen et al., 2014b) and various musically relevant changes in piano melodies (Putkinen et al., 2014a). These results suggest that musical training is associated with enhanced discrimination, as indexed by the MMN, of changes in complex, musically relevant sounds whereas "lower level" sound discrimination investigated in the current study appears not to differentiate musically trained and non-trained children (although strictly speaking the statistical analyses employed in the current study cannot provide evidence for the null hypothesis that the groups do not differ in MMN amplitude). In line with this, our previous study found no evidence for enhanced MMN in the musically trained children for frequency, duration, intensity, and gap deviants of simple tone stimuli (Putkinen et al., 2014b).

The enhanced P3a-like response in the music group nonetheless indicates that the children in the music group were more sensitive to the deviant tone pairs than those in the control group. This result is in line with previous studies in adults and children that have reported enlarged P3a-like responses in musically trained adults and children (Nikjeh 

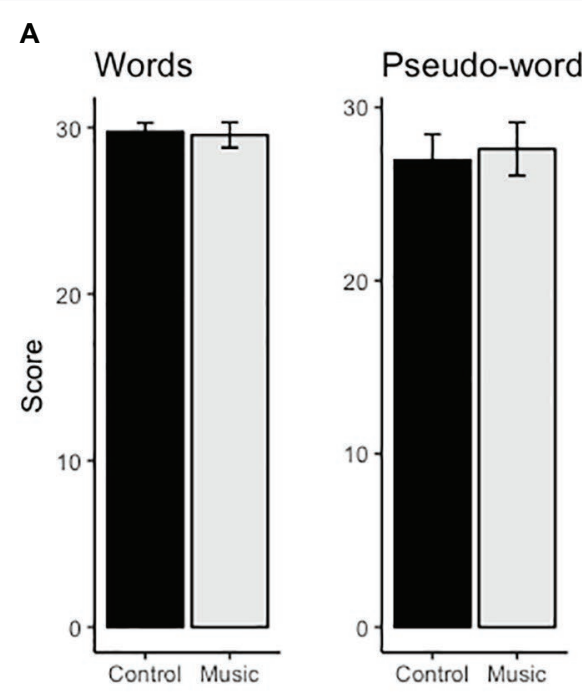

B

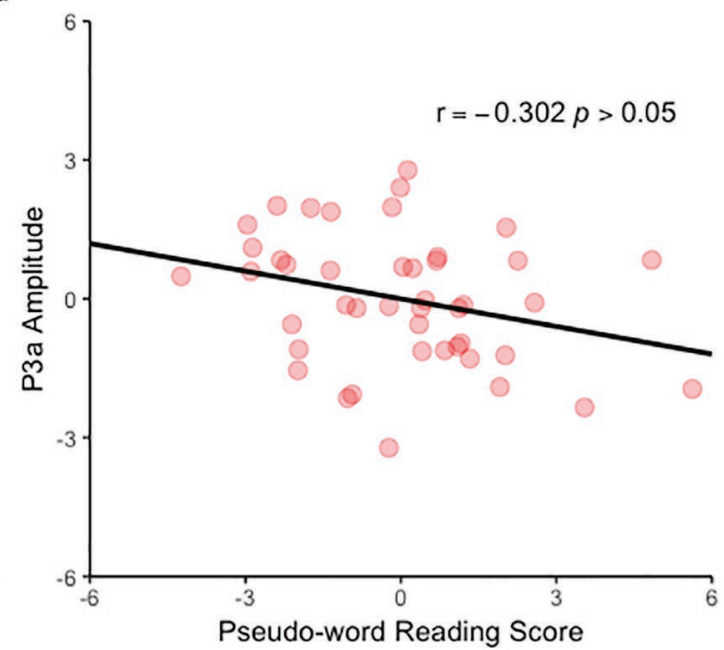

FIGURE 2 | (A) The bar charts illustrate the scores for the word and pseudo-word reading task for the music and control group. (B) The scatter plot illustrates the relationship between pseudo-word reading score and P3a amplitude (the effects of age and group membership has been controlled and therefore values have the mean 0 ).

et al., 2009; Vuust et al., 2009; Seppänen et al., 2012; Putkinen et al., 2014b). This result indicates that even though the initial change detection reflected by the MMN did not differentiate the groups, a subsequent sound processing stage - possibly related to attention capture - was triggered more strongly in the musically trained children than in the untrained ones.

Interestingly, the MMN was largest and the P3a smallest over the right hemisphere. Although the scalp distribution of ERPs is not related to the location of their neural sources in a straightforward manner, the rightward asymmetry in MMN distribution dovetails with studies showing a stronger involvement of right than left auditory cortical regions in processing of pitch direction (Johnsrude et al., 2000; for reviews see Tervaniemi and Hugdahl, 2003; Zatorre and Gandour, 2008). The leftward asymmetry of the P3a-like response is also intriguing given that this response correlated with reading performance.

The correlation between P3a-like response and reading performance was found for the pseudo-word subtest but not the word reading task. The link between neural sound processing and reading skills might be more robust for pseudo-words than for words because pseudo-word reading requires the conversions of previously unseen letter strings to speech and, arguably, taps into phonetic processing more directly than more automated reading of known words. Notably the participants in the reading tests were the oldest participants of the current study (13 and 15 years) and fully fluent in reading their native language. In fact, due to full orthographic transparency of Finnish alphabet, it is commonly considered as one of the easiest languages for learning to read. Thus, the pseudo-word test may be more sensitive than the word reading test to the processes that govern reading fluency in early adolescence.

Previous studies on examining the relationship between neural sound discrimination indexed by the $M M N$ and the
P3a have focused on comparing subjects with reading difficulties and readers in the typical skill range (Kujala et al., 2003; Corbera et al., 2006). It should be noted that these studies have reported smaller responses in adult dyslexic subjects and thereby suggest an opposite correlation between P3a amplitude and reading performance to the one found in the current study. Furthermore, the lack of statistically significant correlation between the MMN and reading skills is seemingly in contrast with previous studies that have reported smaller MMNs in subjects with reading difficulties when compared to those whose reading skills are in the typical range (e.g., Kujala et al., 2001). The reason for this discrepancy is unclear but may originate from differences in the subject samples: It may be more difficult to detect a significant relationship between the MMN and reading ability in typical readers than in a sample that includes a wider range of reading skills. Our P3a results nonetheless offer further support for the notion that elementary neural processing of pitch is associated with reading performance in childhood and adolescence even in typical readers. The negative correlation between the $\mathrm{P} 3 \mathrm{a}$ and reading performance could reflect the negative impact of heightened distractibility on reading skills.

Previous studies indicate that musical training is associated with improved readings skills (Butzlaff, 2000). It has been hypothesized that by improving basic auditory processing such as frequency and duration discrimination, musical training may also benefit higher order phonological processing that is critical for reading acquisition (Patel, 2011). The current study, however, did not find clear evidence for improved reading skills in the music group although there was a trend toward a higher score in the music group for the pseudo-word subtest. This suggests that the null result might be due to insufficient statistical power stemming from the relatively small sample size (note that, as 
mentioned above, only oldest children 13 and 15 years took part in the reading tests for practical reasons).

In conclusion, the current study indicates that pitch direction processing is enhanced in musically trained children and adolescents. However, this enhancement was not evident for the initial change detection reflected by the MMN but only at a later processing stage reflected by a P3a-like response. Furthermore, we found that the $\mathrm{P} 3 \mathrm{a}$-like response was correlated with performance in a reading task in line with the notion that elementary auditory processing is predictive of reading skills.

\section{ETHICS STATEMENT}

This study was carried out in accordance with the recommendations of Ethical Committee of the former Department

\section{REFERENCES}

Ahissar, M., Protopapas, A., Reid, M., and Merzenich, M. M. (2000). Auditory processing parallels reading abilities in adults. Proc. Natl. Acad. Sci. USA 97, 6832-6837. doi: 10.1073/pnas.97.12.6832

Anvari, S. H., Trainor, L. J., Woodside, J., and Levy, B. A. (2002). Relations among musical skills, phonological processing, and early reading ability in preschool children. J. Exp. Child Psychol. 83, 111-130. doi: 10.1016/ S0022-0965(02)00124-8

Butzlaff, R. (2000). Can music be used to teach reading? J. Aesthetic Educ. 34, 167-178.

Chobert, J., Francois, C., Velay, J.-L., and Besson, M. (2014). Twelve months of active musical training in 8 - to 10 -year-old children enhances the preattentive processing of syllabic duration and voice onset time. Cereb. Cortex 24, 956-967. doi: 10.1093/cercor/bhs377

Chobert, J., Marie, C., François, C., Schön, D., and Besson, M. (2011). Enhanced passive and active processing of syllables in musician children. J. Cogn. Neurosci. 23, 3874-3887. doi: 10.1162/jocn_a_00088

Corbera, S., Escera, C., and Artigas, J. (2006). Impaired duration mismatch negativity in developmental dyslexia. Neuroreport 17, 1051-1055. doi: 10.1097/01.wnr.0000221846.43126.a6

Escera, C., Alho, K., Winkler, I., and Näätänen, R. (1998). Neural mechanisms of involuntary attention to acoustic novelty and change. J. Cogn. Neurosci. 10, 590-604. doi: 10.1162/089892998562997

Escera, C., and Corral, M. J. (2007). Role of mismatch negativity and novelty-P3 in involuntary auditory attention. J. Psychophysiol. 21, 251-264. doi: 10.1027/02698803.21.34.251

Fujioka, T., Trainor, L. J., Ross, B., Kakigi, R., and Pantev, C. (2004). Musical training enhances automatic encoding of melodic contour and interval structure. J. Cogn. Neurosci. 16, 1010-1021. doi: 10.1162/0898929041502706

Johnsrude, I. S., Penhune, V. B., and Zatorre, R. J. (2000). Functional specificity in the right human auditory cortex for perceiving pitch direction. Brain 123, 155-163. doi: 10.1093/brain/123.1.155

Koelsch, S., Schröger, E., and Tervaniemi, M. (1999). Superior pre-attentive auditory processing in musicians. Neuroreport 10, 1309-1313. doi: 10.1097/ 00001756-199904260-00029

Kujala, T., Belitz, S., Tervaniemi, M., and Näätänen, R. (2003). Auditory sensory memory disorder in dyslexic adults as indexed by the mismatch negativity. Eur. J. Neurosci. 17, 1323-1327. doi: 10.1046/j.1460-9568.2003.02559.x

Kujala, T., Karma, K., Ceponiene, R., Belitz, S., Turkkila, P., Tervaniemi, M., et al. (2001). Plastic neural changes and reading improvement caused by audiovisual training in reading-impaired children. Proc. Natl. Acad. Sci. USA 98, 10509-10514. doi: 10.1073/pnas.181589198

Kujala, T., Tervaniemi, M., and Schröger, E. (2007). The mismatch negativity in cognitive and clinical neuroscience: theoretical and methodological considerations. Biol. Psychol. 74, 1-19. doi: 10.1016/j.biopsycho.2006.06.001 of Psychology, University of Helsinki, Finland, with written informed consent from all subjects. All subjects gave written informed consent in accordance with the Declaration of Helsinki. The protocol was approved by the Ethical Committee of the former Department of Psychology, University of Helsinki, Finland.

\section{AUTHOR CONTRIBUTIONS}

$\mathrm{MH}$ and MT designed the study. VP analyzed the data. VP, $\mathrm{MT}$, and MH wrote the manuscript.

\section{FUNDING}

This work was supported by the Academy of Finland.

Meyer, M., Elmer, S., Ringli, M., Oechslin, M. S., Baumann, S., and Jancke, L. (2011). Long-term exposure to music enhances the sensitivity of the auditory system in children: musical training changes childrens' auditory system. Eur. J. Neurosci. 34, 755-765. doi: 10.1111/j.1460-9568.2011.07795.x

Moreno, S., Marques, C., Santos, A., Santos, M., Castro, S. L., and Besson, M. (2009). Musical training influences linguistic abilities in 8-year-old children: more evidence for brain plasticity. Cereb. Cortex 19, 712-723. doi: 10.1093/ cercor/bhn120

Näätänen, R., Paavilainen, P., Rinne, T., and Alho, K. (2007). The mismatch negativity (MMN) in basic research of central auditory processing: a review. Clin. Neurophysiol. 118, 2544-2590. doi: 10.1016/j.clinph.2007.04.026

Nevala, J., Kairaluoma, L., Ahonen, T., Aro, M., and Holopainen, L. (2006). Lukemis- ja kirjoittamistaitojen yksilötestistö nuorille ja aikuisille. (Standardization version ed.). Jyväskylä: Niilo Mäki Instituutti.

Nikjeh, D. A., Lister, J. J., and Frisch, S. A. (2009). Preattentive cortical-evoked responses to pure tones harmonic tones, and speech: influence of music training. Ear Hear. 30, 432-446. doi: 10.1097/AUD.0b013e3181a61bf2

Nikjeh, D. A., Lister, J. J., and Frisch, S. A. (2008). Hearing of note: an electrophysiologic and psychoacoustic comparison of pitch discrimination between vocal and instrumental musicians. Psychophysiology 45, 994-1007.

Patel, A. D. (2011). Why would musical training benefit the neural encoding of speech? The OPERA hypothesis. Front. Psychol. 2:142. doi: 10.3389/fpsyg.2011.00142

Putkinen, V., and Saarikivi, K. (2018). Neural correlates of enhanced executive functions: is less more? Ann. N. Y. Acad. Sci. 1423, 117-125. doi: 10.1111/ nyas. 13645

Putkinen, V., and Tervaniemi, M. (2018). "Neuroplasticity in music learning" in The Oxford handbook of music and neuroscience, (Oxford, England: Oxford University Press).

Putkinen, V., Tervaniemi, M., Saarikivi, K., de Vent, N., and Huotilainen, M. (2014a). Investigating the effects of musical training on functional brain development with a novel melodic MMN paradigm. Neurobiol. Learn. Mem. 110, 8-15. doi: 10.1016/j.nlm.2014.01.007

Putkinen, V., Tervaniemi, M., Saarikivi, K., and Huotilainen, M. (2015). Promises of formal and informal musical activities in advancing neurocognitive development throughout childhood: musical activities in neurocognitive development. Ann. N. Y. Acad. Sci. 1337, 153-162. doi: 10.1111/nyas.12656

Putkinen, V., Tervaniemi, M., Saarikivi, K., Ojala, P., and Huotilainen, M. (2014b). Enhanced development of auditory change detection in musically trained school-aged children: a longitudinal event-related potential study. Dev. Sci. 17, 282-297. doi: 10.1111/desc.12109

Saarikivi, K., Putkinen, V., Tervaniemi, M., and Huotilainen, M. (2016). Cognitive flexibility modulates maturation and music-training-related changes in neural sound discrimination. Eur. J. Neurosci. 44, 1815-1825. doi: 10.1111/ejn.13176

Seppänen, M., Pesonen, A.-K., and Tervaniemi, M. (2012). Music training enhances the rapid plasticity of $\mathrm{P} 3 \mathrm{a} / \mathrm{P} 3 \mathrm{~b}$ event-related brain potentials for unattended and attended target sounds. Atten. Percept. Psychophys. 74, 600-612. doi: 10.3758/s13414-011-0257-9 
Tervaniemi, M., and Hugdahl, K. (2003). Lateralization of auditory-cortex functions. Brain Res. Rev. 43, 231-246. doi: 10.1016/j.brainresrev.2003.08.004

Tervaniemi, M., Rytkönen, M., Schröger, E., Ilmoniemi, R. J., and Näätänen, R. (2001). Superior formation of cortical memory traces for melodic patterns in musicians. Learn. Mem. 8, 295-300. doi: 10.1101/lm.39501

van Zuijen, T. L., Sussman, E., Winkler, I., Näätänen, R., and Tervaniemi, M. (2005). Auditory organization of sound sequences by a temporal or numerical regularity-A mismatch negativity study comparing musicians and non-musicians. Cogn. Brain Res. 23, 270-276. doi: 10.1016/j. cogbrainres.2004.10.007

Virtala, P., Huotilainen, M., Putkinen, V., Makkonen, T., and Tervaniemi, M. (2012). Musical training facilitates the neural discrimination of major versus minor chords in 13-year-old children: musical training facilitates neural discrimination of chords. Psychophysiology 49, 1125-1132. doi: 10.1111/ j.1469-8986.2012.01386.x

Vuust, P., Ostergaard, L., Pallesen, K. J., Bailey, C., and Roepstorff, A. (2009). Predictive coding of music - brain responses to rhythmic incongruity. Cortex 45, 80-92. doi: 10.1016/j.cortex.2008.05.014
Winkler, I., Denham, S. L., and Nelken, I. (2009). Modeling the auditory scene: predictive regularity representations and perceptual objects. Trends Cogn. Sci. 13, 532-540. doi: 10.1016/j.tics.2009.09.003

Zatorre, R. J., and Gandour, J. T. (2008). Neural specializations for speech and pitch: moving beyond the dichotomies. Philos. Trans. R. Soc. Lond. Ser. B Biol. Sci. 363, 1087-1104. doi: 10.1098/rstb.2007.2161

Conflict of Interest Statement: The authors declare that the research was conducted in the absence of any commercial or financial relationships that could be construed as a potential conflict of interest.

Copyright (C) 2019 Putkinen, Huotilainen and Tervaniemi. This is an open-access article distributed under the terms of the Creative Commons Attribution License (CC BY). The use, distribution or reproduction in other forums is permitted, provided the original author(s) and the copyright owner(s) are credited and that the original publication in this journal is cited, in accordance with accepted academic practice. No use, distribution or reproduction is permitted which does not comply with these terms. 


\title{
Music Listening for Supporting Adolescents' Sense of Agency in Daily Life
}

\author{
Suvi Helinä Saarikallio*, William M. Randall and Margarida Baltazar \\ Department of Music, Art and Culture Studies, University of Jyväskylä, Jyväskylä, Finland
}

Sense of agency refers to the ability to influence one's functioning and environment, relating to self-efficacy, and wellbeing. In youth, agency may be challenged by external demands or redefinition of self-image. Music, having heightened relevance for the young, has been argued to provide feelings of self-agency for them. Yet, there is little empirical research on how music impacts adolescents' daily sense of agency. The current study investigated whether music listening influences adolescents' perceived agency in everyday life and which contextual determinants would explain such an influence. Participants were 44 adolescents (48\% female, 36\% with training in music, mean age 14), recruited through local schools. The mobile Experience Sampling app

OPEN ACCESS

Edited by:

Evangelos Himonides, University College London,

United Kingdom

Reviewed by:

Graça Maria Boal-Palheiros, Instituto Politécnico do Porto,

Portugal

Makiko Sadakata, Radboud University Nijmegen,

Netherlands

*Correspondence:

Suvi Helinä Saarikallio suvi.saarikallio@jyu.fi

Specialty section:

This article was submitted to

Performance Science,

a section of the journal

Frontiers in Psychology

Received: 19 August 2019 Accepted: 09 December 2019 Published: 08 January 2020

Citation:

Saarikallio SH, Randall WM and Baltazar M (2020) Music Listening for Supporting Adolescents' Sense

of Agency in Daily Life.

Front. Psychol. 10:2911.

doi: 10.3389/fpsyg.2019.02911
MuPsych was used to collect brief self-reports of personal music listening experiences during daily life. This method assessed the change in the listener's perceived control over both their emotional states (internal agency), and their external environment (external agency), over 5 min of music listening. Also measured were changes in mood states, and contextual variables (social situation, concurrent activity, and reason for listening). The impact of music on the sense of agency was analyzed using multilevel structural equation modeling. There was no general increase of agency across all music episodes, but agency fluctuations were determined by specific contextual factors. External agency change was predicted negatively by changing environments, while internal agency change was predicted by initial mood and various reasons for listening, including for enjoyment, coping, and enhancing current mood state. Our findings confirmed the plasticity and situational embeddedness of the sense of agency. Music indeed can support agency, but the impact is dependent on a range of situational factors. Sense of agency can be seen as a health resource and significant part of youth development, and current findings provide new insight into when and by which conditions such affordance is likely to be employed.

Keywords: sense of agency, music listening, everyday life, adolescents, experience sampling

\section{INTRODUCTION}

\section{Agency: Concept and Definition}

Human agency is a core concept in social cognitive theory (Bandura, 1982, 1989, 2006), which posits that people are self-organizing, proactive, self-regulating, and self-reflecting (Bandura, 2006, p. 164). Agency is defined as the ability to influence one's environment and functioning (Bandura, 1982, 1989). Notwithstanding, individuals are not totally detached from their surroundings; 
instead, there is a reciprocal causation process, where personal and environmental determinants operate. Agency can, thus, be conceived as "embedded and situated, yet also emergent within historical sociocultural contexts, which in turn are nested in the biological and physical world" (Martin et al., 2003, p.133). Consequently, agency is a plastic, changing, and negotiable stated (as opposed to a trait or competence), reliant on context-bound, interdependent, and dynamic processes (Esser et al., 2016).

Importantly, how successfully people can influence, control, and negotiate events that affect their lives varies from moment to moment, but so do the beliefs in their capability of action (Bandura, 1995). These beliefs are commonly termed as "self-efficacy" (Bandura, 1982), and they influence behavior and its outcomes through several cognitive (e.g., prediction of success/failure), motivational (e.g., resilience), and affective processes (e.g., stress). According to the models of human agency and self-efficacy, every motivator of action and change has in its foundation the core belief that one has the power to effect changes by one's actions. Simply put, without beliefs of self-efficacy, people do not act on their surroundings or own states. Beliefs of efficacy act as vital forces supporting motivation, well-being, and personal accomplishment in all areas of life (Pajares, 2005, p.339). Indeed, several studies have consistently shown that efficacy beliefs contribute to, for example, smoke-cessation (Schnoll et al., 2011), adherence to healthy behavior (Strecher et al., 1986), recovery from traumatic situations (Bosmans and van der Velden, 2015), and lower levels of depression (Sawatzky et al., 2012).

In sum, agency reveals itself through intentional acts that can produce different outcomes. This can be the case of listening to music: the act of music listening in a given moment and context immediately changes the situation - be it in terms of soundscapes, affective states, connectedness, meanings... (DeNora, 1999, 2000, 2013; Lincoln, 2005; Krueger, 2011, 2018; Skånland, 2013; O'Neill, 2017). In this paper, we conceptualize musical agency as the use of music (in its different genres, behaviors, and contexts...) to capitalize, exercise, and regain agency and sense of agency (similar to "aesthetic agency," DeNora, 2000, 2001). ${ }^{1}$

\section{Agency in Adolescence}

In many aspects, adolescence is defined by the individuals' trajectory toward their empowerment as agents who can create and control several aspects of their world (Beyers et al., 2003; Gowers, 2005). As adolescents increase their independence, form their identity, and work on their emotional experiences, they are faced with the opportunity to become self-sufficient agents. During adolescence, agency is practiced throughout almost every dimension of life and a personal growth through mastery is observed. The adolescents' sense of efficacy is strengthened by learning how to successfully deal with pressing issues (such as vocational definition, sexuality, family, and affect regulation), accompanied by vicarious experiences (such as modeling of a peer's behavior), persuasive information from others (such as

\footnotetext{
${ }^{1}$ Different from "musical agency" used in the context of music education by Wiggins (2016) as a "sense that they can initiate and carry out their own musical ideas and ideas about music."
}

verbal encouragement), and positive physiological feedback (such as states of arousal appraised as confidence) (Bandura, 1977).

Importantly, the sense of agency has been pointed out as a key variable to understand the paths through adolescence into adulthood (Bandura, 1995; Pajares and Urdan, 2006). Thus, it closely relates to several aspects of adolescents' healthy development and adaptation. Agency is part of the development of behavioral regulation: adolescents' self-regulation has been for instance defined in terms of three agentic behaviors: selection (choosing opportunities that match with one's sense of self and personal potential), optimization (refining one's resources to reach goals) and compensation (being able to "change course" if resources fail) (Lerner et al., 2001). Agency also closely relates to identity formation: a composite measure of agency, consisting of self-esteem, purpose in life, ego strength, and internal locus of control, has been found to positively relate to identity achievement and negatively to identity diffusion (Côté and Schwartz, 2002). Similarly, internal locus of control has been shown to relate to identity achievement while external locus of control relates to identity statuses of moratorium, foreclosure, and diffusion (Lillevoll et al., 2013). It has been argued that the increased pressure in Western societies for each young person to find their own, personal, path to adulthood places agency, self-determination, and self-directedness in a heightened role: those who are able to capitalize on the breadth of opportunities are likely to flourish but those who are unable to exercise personal agency at this stage may find their developmental period particularly distressing (Schwartz et al., 2005). In line with these, the Self-Determination Theory stresses autonomy and competence as essential features for adaptive development and wellbeing (Ryan and Deci, 2000).

\section{Music, Agency, and Self-Efficacy}

A well-established body of literature documents the role of music in youth development (McFerran, 2011; ter Bogt et al., 2012; Miranda, 2013). There is evidence showing that musical behaviors foster the adolescent's ability to cope with the challenges that matter the most during this developmental stage: identity definition (North and Hargreaves, 1999; North et al., 2000; Hense and McFerran, 2017), connection with peers (Selfhout et al., 2009; Papinczak et al., 2015; ter Bogt et al., 2017), affect regulation (Saarikallio and Erkkilä, 2007; McFerran and Saarikallio, 2014; Leipold and Loepthien, 2015), and shaping of agency (Laiho, 2004; Gold et al., 2011). It has been argued that music occupies such an important place in adolescents' lives precisely because of the developmental functions it serves (Schwartz and Fouts, 2003; Laiho, 2004; Miranda, 2013). In terms of serving as a personal resource for agency music holds particular potential for the young, as Saarikallio (2019b) points out: "Music is the kids" own world, their playground and kingdom, in which they can shout and be silly, to be fragile and in search of themselves, and make their own, personal, choices."

As argued by Krueger (2011), music is perceived from birth as a structure that affords possibilities - for constructing and regulating emotions, for expressing and communicating, for shaping relationships and situations. Children's play, symbols, drawings, and music uses are not only contemplative, but mainly 
participatory, engaged, and active-i.e., agentic (Karlsen, 2011; Bonsdorff, 2017; Rissanen, 2017; Kuuse, 2018). Through the use of these esthetic resources, children develop from early ages their agency and sense of self-efficacy. In the case of music, it has been observed that there is a constant, informal learning in everyday life (Batt-Rawden and DeNora, 2005), supported by music listening, reflection, and narrative, that create memories, patterns, meanings, and gateways between the individual and the social surroundings. Learning how music can be used empowers the individual to act on their own affective states, wellbeing, health, and agency modes (DeNora, 2000, 2001; Skånland, 2013).

\section{Music-Fostered Agency and Wellbeing}

Seeking and exploring the sense of agency through music seems to be particularly relevant for individuals that are either developing their agency (i.e., youth) or experiencing a reduction in their ability to control their actions and/or environment (e.g., due to illness or challenging personal situations) (Magee, 2017). Batt-Rawden and colleagues have conducted studies with individuals dealing with chronic affective or physical illnesses (Batt-Rawden and DeNora, 2005; Batt-Rawden et al., 2005; BattRawden and Tellnes, 2011). Their data show that, by reflecting on their music uses and learning self-care, the participants increased their sense of control and agency. When using music to cope with pain or stressful medical interventions, control and agency have also appeared to matter significantly: the beneficial effects of music have been reported to be especially visible when the participants could choose their own music to listen to (Mitchell and Macdonald, 2006; Pothoulaki et al., 2008; Bernatzky et al., 2011; Jiang et al., 2013). In a feasibility study of a brief music-based intervention for adolescents, McFerran et al. (2018) observed that the sense of agency was key for the participants: after the intervention, the young participants reported an increased awareness of their position as agents who can utilize the affordances of music to reduce distress and promote their own development.

Sense of agency has been identified as a defining element in music usages and their role on social-emotional health (Saarikallio, 2017, 2019a,b; Saarikallio and Baltazar, 2018). In the conceptual model of health-relevant competencies in music, Saarikallio $(2017,2019$ a) argues that engaging in musical behaviors can foster emotional health when the emotions expressed, evoked, and regulated through music are surrounded by (a) self-reflective awareness and (b) sense of self-control and agency. Concerning musical self-enhancement, Elvers (2016) proposed a framework according to which there are three main musical experiences bolstering positive self-evaluations: induction of empathy (through identification with the music, self/other merging, and self-confirmation), promotion of social cohesion (through rhythmic entrainment and reinforcement of a cultural worldview and sense of social belonging), and pleasure (through the induction of positive affect, default mode networkactivation, and self-referential emotions). According to Elvers, this framework encompasses "the kind of musical experiences that elicit positive affect related to the self, that induce feelings of power and control, that promote positive self-evaluations, and ultimately promote self-esteem" (Elvers, 2016, p. 3). In this line of thought, the use of music to increase the sense of agency and beliefs of efficacy can be seen as one way of musical self-enhancement.

\section{Aims of the Current Study}

Despite the breadth of research arguing that music holds potential for serving as a resource for agency in adolescents' lives, there is little empirical work on the impacts of music listening to adolescents' sense of agency. This study was designed to investigate how music listening would relate to the everyday fluctuations of adolescents' sense of agency.

Following our definition of agency as a plastic and contextbound ability to influence one's environment and functioning, we placed particular focus on exploring the unfolding situational aspects of daily life as the context in which the impacts of music on agency would occur. We addressed agency both in terms of internal control of one's feelings and in terms of one's perceived sense of having control over the external environment (labeled in this paper as internal and external agency). We chose to focus on music listening in contrast to active music making, because that is an activity available practically for all adolescents in the multitude of their everyday life events and environments. Finally, as the majority of current research on music and agency is constituted of theoretical propositions, we decided to provide empirical evidence through measuring changes in agency as a function of daily music listening. Although it has not been observed empirically yet, we expected to find differences in perceived agency after an episode of music listening.

Firstly, we expected to observe a general increase in the self-perceived sense of agency (in terms of both internal and external control) across the episodes of music listening. Secondly, we explored whether the impacts of music listening on the sense of agency would depend on specific contextual factors including simultaneous activities, presence of other people, current mood and energy level, and reasons for music listening at the given moment. Thirdly, we expected changes in both internal and external agency to correlate with changes in valence, with increased sense of agency correlating positively with mood improvement.

\section{MATERIALS AND METHODS}

\section{Participants}

Participants were recruited through middle schools within the Jyväskylä sub-region of Finland. The research team first contacted the rectors and music teachers of the schools, visited the schools, and provided information about the study to the students as part of their music classes. Students who volunteered downloaded the app with assistance provided by a video instruction and/or the researchers directly. Participants were required to have a mobile phone with the Android mobile operating system, that they used for personal music listening. The sample consisted of 44 year 7 and 8 students (age $M=13.67, S D=1.87$ ), all native to Finland, with a mix of both girls and boys ( $47.7 \%$ female). The majority of participants reported no formal practical music training (36.4\% 
reported at least 1 year of training). Participation was incentivized through a draw to win Spotify subscriptions.

\section{Materials}

All research materials were presented through the MuPsych app, which was originally developed and tested by Randall and Rickard (2013), with an updated version developed at the University of Jyväskylä. The app collected data through two means: music experience sample reports (ESRs), and surveys. All items were presented in Finnish. A total of 327 music listening episodes were reported $(M=7.43, S D=3.66)$.

Music experience sample reports were presented immediately when the participant listened to any music on their phone. The initial screen assessed current affective state, with responses given on two 7-point slider scales: the first titled "Mood," with labels for "Negative," "Neutral" (center) and "Positive"; and the second titled "Energy," labeled from "Very low" to "Very high." These variables were recorded as "initial valence" and "Initial arousal," respectively. These two dimensions have been demonstrated to be efficient and reliable measures of music induced emotion, explaining a high proportion of variance (Vuoskoski and Eerola, 2011; Thoma et al., 2012). Participants additionally selected one distinct mood that best matched their current feelings and rated how intensely ("Not at all" to "Very") they felt that mood (named "Mood Intensity"). The following screen consisted of two 7-point slider scales, which were designed to assess the sense of agency: For the external component of agency - a sense of being in charge of the environment - a question was phrased: "Do you feel that the situation is in your control?" (from "Not at all" to "Completely"); and for the internal component of agency - focusing on sense of being in charge of one's feelings - a question was phrased "Is your mood the way you want it to be?" (from "Not at all" to "Completely"). The variables were labeled as "External Agency" and "internal agency," respectively. The following three screens used a list-response format to assess who the listener was with (Listeners), where they were (Location), and what they were doing while listening (Activity).

If music was still playing on the phone after a period of $5 \mathrm{~min}-$ as automatically determined by the app - a second series of screens was presented. The first of these screens assessed the same continuous variables measured at the start of listening (Valence, Arousal, Mood Intensity, External Agency, and internal agency), to determine how these changed over the listening episode. The second screen included questions related to the music, on five 7-point slider scales: Mood of the music (subjectively perceived emotion of the music, as distinct from the induced emotion of the listener; Negative - Neutral - Positive), the Energy of the music (Low Energy - High Energy); "How much attention are you paying to the music?" (Attention; None - Complete); "How much are you enjoying this music?" (Enjoyment; Not at all - Very much); and "How familiar are you with this music?" (Familiarity; Not at all - Very familiar). These five music questions were included to provide an overview about how the listener perceived the music at the given episode. The final screens of the music ESR used a branching list-response format to assess the main reason for listening. These reasons were categorized as: For current activity; For entertainment/enjoyment; To relax/calm down; To focus on the Music; For boredom/habit; To cope with a situation; For thinking/reflecting; To raise/boost energy; To enhance my current mood; To diminish my current mood; and To maintain my current mood.

The surveys presented within MuPsych were a series of psychometric scales to assess individual variables, which could be completed at any time. Participants were asked to report their gender and how many years of formal practical music training they had received. They also filled in the 44-item version of the Big Five personality Inventory (John and Srivastava, 1999). Previous research has demonstrated that scales presented within MuPsych produce similar Cronbach alpha scores to those published from the standard questionnaires (Randall and Rickard, 2013).

\section{Procedure}

Prior to the study, an ethics approval was applied for and granted by the University of Jyväskylä Ethical Committee. Information statements were sent to parents of all potential participants through an internal school system, before the participants themselves were contacted during their music classes. They were presented with details about the research and instructed to install the MuPsych app on their mobile phone. All elements of the study were presented through the app, including an information statement, music ESRs, and all surveys. Music ESRs were presented when any music was played on the phone, however, once one had been completed, no more were presented for a period of $4 \mathrm{~h}$, to avoid respondent fatigue. Surveys were presented within the app and could be completed at any time of convenience. This data collection continued for a period of 1 week, after which no more ESRs were presented.

\section{Data Analyses}

Two separate approaches were utilized to analyze the data: aggregate scores on the participant level were used to determine changes in agency overall and across different listening reasons and contexts; and a multilevel model was used to consider context variables, with musical experiences nested within listeners. For the first approach, aggregate scores were created for each individual participant, producing means for valence, arousal, mood intensity, internal, and external agency. This approach is recommended for ESM (Hektner et al., 2007), and has been utilized in previous ESM studies of music use (Randall et al., 2014). Paired-samples $t$-tests were then performed to determine if music listening produced any significant changes in these aggregate scores. The second approach involved the implementation of a multilevel structural equation model (SEM), using Mplus software (version 7.4: Muthén and Muthén, 2015). A total of 327 musical experiences were included in the model, clustered within 44 listeners. This multilevel approach accounted for both the variance in emotional outcomes due to variation amongst musical experiences, and the variance due to variation amongst individual listeners. Maximum likelihood estimation with robust standard errors (MLR) was used, with an accelerated expectation-maximization (EMA) optimization algorithm. Pearson correlation was used to test the relatedness of agency change with valence, arousal, and mood intensity change. 


\section{RESULTS}

\section{Aggregate Analysis}

To determine if any overall changes in External Agency, internal agency, Valence, Arousal, or Mood Intensity were produced from music listening, repeated-measures $t$-tests were performed on the aggregate participant scores. These tests revealed no significant changes, with the details reported in Table 1. Also on the aggregate level, no significant effect of gender was found on either external $(p=0.239)$ or INTERNAL AGENCY change $(p=0.371)$, and no significant correlations were observed between these agency measures and BFI personality scales or years of musical training. Aggregate level frequencies revealed that $91 \%$ of listening episodes were solitary, $58.11 \%$ were at home, $20.42 \%$ traveling, and $17.44 \%$ at school and the most frequent activity was "Nothing/waiting" $11.72 \%$, followed by $11.15 \%$ "In a car," $11.02 \%$ "Focussed listening," and 10.68\% "Studying." The most frequently reported reasons for listening were "For entertainment/enjoyment" (16.42\%), "For current activity" (15.32\%), "For boredom/habit" (14.03\%), and "To relax/calm down” (12.77\%).

\section{Multilevel Analysis}

Correlations between changes in External Agency, internal agency, Valence, Arousal, and Mood Intensity are displayed in Table 2. This shows significant positive correlations between all variables, with the strongest between internal agency change and Valence change. In line with the hypothesis, valence change and agency change were positively correlated.

A multilevel structural equation model was constructed to assess the interaction of all measured variables, with all significant predictors presented in Table 3 (with the effects of initial agency states on their respective changes in agency removed). This model produced a standardized root mean square residual (SRMR) of 0.038 on the musical experience level (with values of less than 0.08 generally considered a good fit: $\mathrm{Hu}$ and Bentler, 1999).

TABLE 1 | Overall changes in agency and affective variables during music listening.

\begin{tabular}{lccccc}
\hline & $\begin{array}{c}\text { Mean } \\
(\mathbf{0} \text { } \mathbf{i n})\end{array}$ & $\begin{array}{c}\text { Mean } \\
(\mathbf{5} \text { } \mathbf{i n})\end{array}$ & $\begin{array}{c}\text { Mean } \\
\text { difference }\end{array}$ & $\boldsymbol{t}$ & $\boldsymbol{p}$ \\
\hline External agency & 1.879 & 1.975 & 0.096 & 1.184 & 0.246 \\
Internal agency & 0.985 & 1.135 & 0.150 & 1.628 & 0.115 \\
Valence & 1.238 & 1.334 & 0.096 & 0.933 & 0.359 \\
Arousal & 0.213 & 0.429 & 0.217 & 1.901 & 0.068 \\
Mood intensity & 1.697 & 1.418 & -0.280 & -2.457 & $0.020^{*}$ \\
\hline
\end{tabular}

*is significant at point 0.05 .

TABLE 2 | Correlations between agency change variables and affective change variables.

\begin{tabular}{lcccc}
\hline & $\begin{array}{c}\text { Internal } \\
\text { agency } \\
\text { change }\end{array}$ & $\begin{array}{c}\text { Valence } \\
\text { change }\end{array}$ & $\begin{array}{c}\text { Arousal } \\
\text { change }\end{array}$ & $\begin{array}{c}\text { Mood } \\
\text { intensity } \\
\text { change }\end{array}$ \\
\hline External agency change & $0.197^{* *}$ & $0.186^{* *}$ & $0.147^{* *}$ & 0.017 \\
Internal agency change & - & $0.293^{* *}$ & $0.142^{* *}$ & $-0.105^{*}$ \\
\hline
\end{tabular}

*is significant at point 0.05 and **is significant at point 0.01 .
These results in Table 3 reveal two weak negative predictors for External Agency change: the activities of walking $(\beta=-0.112$; frequency of walking experiences $=5.11 \%$ ), and on transport ("On bus/train/plane"; $\beta=-0.080$; frequency $2.18 \%$ ). internal agency change was positively predicted by initial valence of the listener $(\beta=0.306)$, the reason "Enjoyment" ("For entertainment/enjoyment"; $\beta=0.200$; frequency $16.42 \%$ ), the reason "To cope" ("To cope with a situation"; $\beta=0.153$; frequency $4.35 \%$ ), the reason "Enhance mood" ["To feel more (of my selected mood state)"; $\beta=0.152$; frequency $2.75 \%$ ], listening alone ( $\beta=0.128$; frequency $91.00 \%)$, and negatively predicted by the activity of studying ( $\beta=-0.113$; frequency $10.68 \%)$.

\section{DISCUSSION}

The analysis of the music listening experiences as a whole revealed that changes in the sense of agency are not transversal to all episodes. However, fluctuations in agency were observed within the episodes and particular context factors and reasons for listening were identified as explaining the modulations in perceived agency. These results are in line with both music literature and agency literature. The former informs us that there is a plethora of contextual factors that shape the usages and related affective impact of music in everyday life listening (Randall and Rickard, 2017a,b), and the latter posits that the sense of agency is embedded, context-bound, plastic, and malleable (Martin et al., 2003; Esser et al., 2016). The findings of the current study confirm that the relationship of music and perceived agency is intrinsically embedded in the multifaceted nature of our daily experiences, dependent on specific contextual variables. Additionally, the current results confirm the feasibility of measuring fluctuations in perceived agency, even after a short period of music listening ( $5 \mathrm{~min}$ ).

The two components of agency - internal and external were predicted by a distinctively different set of contextual determinants. Significant predictors of external agency were relatively straightforward, with walking and transportation negatively predicting increases in external agency. This is illustrative of external agency being dependent on objective, environmentally defined, constraints, and activities rather than internal experiences. These effects may be less dependent on the music listening experience itself, as we would expect control over our external world to decrease when traveling through different environments, regardless of music-related variables. Previous studies have looked into the motivations and outcomes of listening to music in situations where there is little or no control over our surroundings, such as traveling (Heye and Lamont, 2010; Skånland, 2013), driving (Dibben and Williamson, 2007), and working in an office environment (Haake, 2011). It has been found that listeners envelop themselves in their personal music, with high levels of attention to the music itself, but also increased awareness of their external environment (cf. "auditory bubble," Bull, 2006; Heye and Lamont, 2010). Listening to music when the external sense of agency is low can be seen as an attempt at managing inner/outer spaces, avoiding stressors from the environment, and self-regulating. Indeed, our results confirm that when initial external agency was low, listeners were more 
TABLE 3 | Structural equation model predictors of agency change.

\begin{tabular}{|c|c|c|c|c|c|}
\hline Outcome & Predictor & $\beta$ & SE & $\beta / S E$ & $p$ \\
\hline \multirow[t]{2}{*}{ External agency change } & Activity: Walking & -0.112 & 0.051 & -2.20 & 0.028 \\
\hline & Activity: On transport & -0.080 & 0.040 & -2.04 & 0.042 \\
\hline \multirow[t]{7}{*}{ Internal agency change } & Initial valence & 0.306 & 0.084 & 3.62 & $<0.001$ \\
\hline & Reason: Enjoyment & 0.200 & 0.085 & 2.37 & 0.018 \\
\hline & Reason: To cope & 0.153 & 0.068 & 2.26 & 0.024 \\
\hline & Reason: Enhance mood & 0.152 & 0.053 & 2.87 & 0.004 \\
\hline & Listening alone & 0.128 & 0.062 & 2.06 & 0.040 \\
\hline & Activity: Studying & -0.113 & 0.046 & -2.49 & 0.013 \\
\hline & Reason: Improve mood & 0.085 & 0.041 & 2.07 & 0.039 \\
\hline \multirow[t]{5}{*}{ Music valence } & Initial valence & 0.310 & 0.105 & 2.95 & 0.003 \\
\hline & Location: Home & 0.160 & 0.072 & 2.23 & 0.026 \\
\hline & Activity: Walking & 0.124 & 0.058 & 2.15 & 0.031 \\
\hline & Activity: Housework & 0.114 & 0.040 & 2.85 & 0.004 \\
\hline & Activity: Studying & 0.108 & 0.049 & 2.22 & 0.026 \\
\hline \multirow[t]{3}{*}{ Music arousal } & Initial valence & 0.322 & 0.105 & 3.05 & 0.002 \\
\hline & Activity: Focussed listening & 0.144 & 0.070 & 2.07 & 0.039 \\
\hline & Activity: Housework & 0.124 & 0.045 & 2.76 & 0.006 \\
\hline \multirow[t]{4}{*}{ Attention to music } & Listening alone & 0.191 & 0.083 & 2.32 & 0.020 \\
\hline & Initial internal agency & 0.186 & 0.089 & 2.09 & 0.037 \\
\hline & Reason: Improve mood & 0.144 & 0.061 & 2.37 & 0.018 \\
\hline & Activity: Housework & 0.120 & 0.049 & 2.46 & 0.014 \\
\hline Familiarity of music & Listening alone & 0.193 & 0.088 & 2.19 & 0.028 \\
\hline \multirow[t]{2}{*}{ Reason: Enjoyment } & Initial arousal & -0.213 & 0.076 & -2.80 & 0.005 \\
\hline & Activity: Going to sleep & -0.146 & 0.043 & -3.42 & 0.001 \\
\hline Reason: Diminish mood & Initial internal agency & -0.210 & 0.095 & -2.21 & 0.027 \\
\hline \multirow[t]{3}{*}{ Reason: Enhance mood } & Initial valence & -0.312 & 0.089 & -3.49 & $<0.001$ \\
\hline & Initial external agency & 0.228 & 0.072 & 3.18 & 0.001 \\
\hline & Initial internal agency & 0.171 & 0.055 & 3.10 & 0.002 \\
\hline \multirow[t]{3}{*}{ Reason: Focus on the music } & Activity: In a car & -0.194 & 0.087 & -2.24 & 0.025 \\
\hline & Initial external agency & -0.169 & 0.085 & -1.99 & 0.047 \\
\hline & Listening alone & 0.163 & 0.065 & 2.49 & 0.013 \\
\hline \multirow[t]{3}{*}{ Reason: For activity } & Activity: Focussed listening & -0.168 & 0.054 & -3.10 & 0.002 \\
\hline & Activity: Eating & -0.126 & 0.032 & -3.92 & $<0.001$ \\
\hline & Activity: Doing nothing & -0.116 & 0.058 & -1.99 & 0.046 \\
\hline Reason: Improve mood & Location: Home & 0.216 & 0.082 & 2.62 & 0.009 \\
\hline Reason: Maintain mood & Activity: Eating & -0.055 & 0.024 & -2.33 & 0.020 \\
\hline \multirow[t]{6}{*}{ Reason: Raise energy } & Location: Traveling & -0.334 & 0.100 & -3.35 & 0.001 \\
\hline & Location: Home & -0.250 & 0.111 & -2.25 & 0.025 \\
\hline & Listening alone & -0.193 & 0.054 & -3.56 & $<0.001$ \\
\hline & Location: Work/school & -0.166 & 0.070 & -2.38 & 0.018 \\
\hline & Activity: On transport & 0.153 & 0.049 & 3.15 & 0.002 \\
\hline & Activity: In a car & 0.110 & 0.049 & 2.25 & 0.024 \\
\hline Reason: Thinking & Activity: Gaming & -0.061 & 0.025 & -2.47 & 0.014 \\
\hline \multirow[t]{5}{*}{ Reason: To cope } & Activity: Walking & 0.166 & 0.082 & 2.03 & 0.043 \\
\hline & Location: Work/ school & 0.145 & 0.057 & 2.53 & 0.011 \\
\hline & Activity: Studying & -0.113 & 0.055 & -2.06 & 0.039 \\
\hline & Activity: Going to sleep & -0.104 & 0.049 & -2.13 & 0.034 \\
\hline & Activity: Eating & -0.088 & 0.024 & -3.73 & $<0.001$ \\
\hline \multirow[t]{4}{*}{ Reason: To relax } & Listening alone & -0.252 & 0.071 & -3.53 & $<0.001$ \\
\hline & Activity: Going to sleep & 0.184 & 0.081 & 2.28 & 0.023 \\
\hline & Activity: Focussed listening & 0.134 & 0.049 & 2.72 & 0.006 \\
\hline & Activity: On transport & -0.128 & 0.038 & -3.32 & 0.001 \\
\hline
\end{tabular}

$\beta$, standardized parameter estimate; SE, standard error; $\beta / S E$, $t$ value associated with the parameter estimate; $p$, two-tailed $p$ value (only <0.05 shown). 
likely to be listening for the reason "to focus on the music." This suggests that if listeners have little control over their external environment, the music itself becomes important to them; they feel the need for the "auditory bubble." The reason for listening "to cope" is of particular relevance here, as it was utilized more frequently by those who were walking, and in turn positively predicted internal agency. Worded as "to cope with a situation," this suggests that listeners were using music to improve their internal mood state to deal with their lack of control over the external situation. Furthermore, previous research using mobile ESM found this specific reason to be related to low valence, low arousal moods, as well as poor emotional health and wellbeing of the listener (Randall and Rickard, 2017b). These external agency findings also resonate with a concept that Krueger calls the musically extended mind (2014). Music can be seen as an external resource that enhances internal abilities, and affords possibilities for action at the affective, physical, and social levels (Krueger, 2011, 2014). In a way, this kind of focused music listening has the potential to alter the external environment and the individual's position in it, thus creating a sense of agency (DeNora, 1999, 2000; Krueger, 2014).

In contrast, change of internal agency was significantly predicted by several mood-related experiences, reasons, and goals - in a word, internal experiences. High initial levels of internal agency also predicted greater attention to music, a direct contrast to external agency: engaged (focused, attentive) listening was predicted by low control of the situation but by high contentment of the internal experience. In general, the fact that internal agency change was positively predicted by specific internal reasons for listening suggests that people intend to use music in certain ways that will change their mood to the way they want it to be. This concept is strongly supported by the music psychology literature (Thayer et al., 1994; DeNora, 1999; Saarikallio, 2011; Van Goethem and Sloboda, 2011; Baltazar and Saarikallio, 2016; Baltazar, 2019), including previous mobile ESM research that has found that emotional reasons for listening on mobile phones are utilized to fulfill specific emotional needs (Randall and Rickard, 2017b). The model results show that when internal agency is low (mood state is not ideal), people more frequently intend to diminish the intensity of their current mood state while when internal agency is high (mood state is ideal), people more frequently intend to enhance the intensity of their current mood state, which in turn predicts an increase of internal agency. Overall, it seems that people deliberately use music to control their internal states and this successfully results in an increased sense of internal agency.

Internal agency change was particularly predicted by positive initial valence and reasons for listening that related to positive mood maintenance or mood repair: entertainment/enjoyment, mood enhancement, mood improvement, and coping. Prior research has identified entertainment and positive mood maintenance as one of the most common motivations for music listening for adolescents and young adults (North et al., 2000; Saarikallio, 2008; Randall and Rickard, 2017b). Entertainment has previously been linked to raising moods while listening to music alone (Saarikallio and Erkkilä, 2007) and to higher wellbeing and lower symptomatology (Gebhardt and von Georgi,
2007; Thomson et al., 2014), and the use of music as a coping resource in general has widely been acknowledged in the recent literature (e.g., Miranda and Claes, 2009; Skånland, 2011; Van Goethem and Sloboda, 2011; Miranda, 2019). Entertainment has been considered a regulation strategy of affect that is more embodied and pleasure-oriented, while coping has been seen more closely linked with mental contemplation and the use of cognitive resources: these two types of listening have been argued to serve as complementary components of affect self-regulation (Baltazar and Saarikallio, 2019). Our results show both aspects being predictive of internal agency change, demonstrating that internal agency can be achieved through both types of affectregulatory acts.

A noteworthy observation is that while mood enhancement was predicted by both high initial internal and external agency, the strongest predictor of mood enhancement actually was low initial valence. This indicates that adolescents sometimes want to enhance their current negative mood. This deliberate maintenance of a negative state through music listening has been identified in previous research and discussed in relation to the concept of "misery-sharing" (Gibson et al., 2000; Zillmann and Vorderer, 2000; Skånland, 2013). Whether the purposeful enhancement of negative mood increases internal agency remains a bit ambiguous: Mood enhancement did predict internal agency. However, the expected positive correlation between valence increase and internal agency increase would indicate that the increase of internal agency relates to mood improvement, not to the enhancement of negative mood. Another noteworthy observation was that internal agency change correlated negatively with mood intensity change. The correlation was relatively low but suggests that decrease of mood intensity may relate to increase of internal agency. Previous research has demonstrated emotional stability as a determinant of affective wellbeing (John and Srivastava, 1999; Tomyn and Cummins, 2011), while adolescence has been characterized by higher emotional intensity and lower stability (e.g., Larson et al., 2002). Due to the low effect size this finding should be interpreted with some caution, but future research on how internal agency may correlate with attempts toward emotional homeostasis might be fruitful for understanding music as agentic mood management.

Overall, our results on internal agency and mood changes might be partly explained by the general reciprocal connection between positive experiences, self-efficacy, and sense of agency. As mentioned previously, self-efficacy is the belief that one is able to achieve a certain goal. This belief is reinforced by positive experiences and, in turn, promotes a sense of agency and action-taking. Thus, a positive initial valence might have fostered the participants' sense of agency on their own feelings. This process is better captured by the correlations observed between agency (internal and external) and changes in mood (valence, especially, but arousal also). Feeling better or feeling more of a certain positive affective state might have been experienced as a successful event by the participants. Generally, experiences of mastery and accomplishment serve as a boost to the adolescents' perception of self-efficacy, which in turn inform the perception of agency in the short-term and foster the development of agency in the medium- and long-term (Bandura, 1982, 2005; 
Zimmermann and Cleary, 2005). Music has been proposed as one resource that successfully leads to feeling empowerment (i.e., the reinforcement of efficacy beliefs; Wallace-DiGarbo and Hill, 2006) and self-enhancement (i.e., the tendency to evaluate oneself positively; Elvers, 2016). Saarikallio et al. (2018) found that power-related emotions (self-determination, independence, daring, freedom, strength, empowerment, rebelliousness, and unconstrained feeling) were the second strongest factor out of six explaining music-related pleasure in the participants' everyday life. According to the authors, the factor power reflects healthfostering experiences and is closely related to the sense of agency given the self-determination and control it implies. The observed relatedness of agency increase with valence increase is a noteworthy addition to the broader literature on music and wellbeing, proposing that agency and mood regulation may serve as dialogical determinants for adaptive and health-fostering music engagement (Saarikallio, 2019a).

Internal agency also increased when the adolescents were listening to music alone. This further solidifies the linkage of internal agency to personal and engaged listening. Furthermore, the act of solitary listening can be seen as an important part of youth development in particular. During youth, solitude becomes a constructive part of daily life, supporting the development toward independence, with solitary music listening emerging as an opportunity for self-exploration and personal mood regulation (Larson, 1995). When listening to music on their own, adolescents often report that they create their own world where they can work their affective material (e.g., Saarikallio and Erkkilä, 2007; Van Goethem and Sloboda, 2011). Music also seems to afford precious possibilities for moving between separation and connectedness (DeNora, 2000; Karlsen, 2011). One might argue that this context (listening alone) fosters their autonomy and agency as important steps and outcomes of their development. In developmental psychology, it has been suggested that agency is the marking factor in healthy paths through adolescent, rather than independence, separation, and autonomy from parents (Beyers et al., 2003). Yet, according to Beyers and colleagues, separation might be a needed step at the onset of adolescence, with the goal of developing as a self-direct and, importantly, connected agent.

In line with the social cognitive theory and agency literature, the two aspects of agency measured in the current study internal and external sense of agency - were correlated, but with relatively low effect size. Both components are closely interconnected in the experience of agency, but, notwithstanding, may fluctuate somehow independently based on the context and on the relevance of internal or external factors in the particular situation (Bandura, 1982, 1989). At this point, it is not possible to discern whether the correlation between the internal and external sense of agency is situated at the goal or at the outcomes level (i.e., were the adolescents seeking to regulate their agency globally or did the change in one aspect of agency foster the change in the other aspect). Further investigation would be needed to explore the internal structure of self-perceived agency in adolescents' daily use of music. Nonetheless, it is noteworthy that the two aspects of agency were related to music listening behaviors in a differentiated manner in terms of the contextual determinants. Even though agency in the context of music education and art programs is commonly addressed as a homogeneous concept, at least in the case of music listening it might be useful to explore separately the sense of agency related to inner mood states and the sense of agency related to the surrounding context.

There are certain limitations to consider when interpreting the current results. Firstly, the study involved the subjective assessment of various internal and emotional states, which may be vulnerable to a range of confounding factors. These issues may be amplified for young adolescents, who may have individual sensitivity issues or difficulty in identifying and reporting their emotional states. This subjective self-reporting may also be susceptible to the somewhat intrusive nature of event-based sampling, which could influence the reported emotions. The concepts of internal and external agency were measured only with single items, thus reflecting a relatively narrow definition of each. While this was necessary in the current study in order to keep the episodes answerable when the same questions were answered several times a day, it would be important to encourage future research to take a more elaborate qualitative approach on the conceptual definition of music-based agency and its potential subcomponents. Furthermore, the timeframe over which changes in these variables were measured was limited to $5 \mathrm{~min}$. The results suggest that this was adequate time to elicit changes in agency, but a longer measurement period in future research would yield further insight into how agency fluctuates over time. Finally, the relatively small sample size of the current study was appropriate for exploring contextual variation between the episodes but did not allow modeling the larger interplay of the musical, individual, and contextual determinants. Collecting larger samples for the inclusion of individual differences or using objective measures for assessing musical content might be interesting avenues for future research.

\section{CONCLUSION}

In conclusion, it appears that music listening can indeed support adolescents' sense of agency in daily life, but this clearly is not a phenomenon that can be averaged across all situations and for all listeners, or even for all subcomponents of agency. Our ESM approach was able to identify particular contextual predictors for the impact of music listening on internal and external agency within the plethora of everyday experiences. As would be expected, External Agency was decreased when listeners were traveling through different environments, either on foot or in transport. In contrast, internal agency was increased when the initial mood of the listener was positive, and also when specific reasons for listening were used, suggesting that this outcome is more dependent on the deliberate use of music to influence mood. Overall, the findings provide pioneering insight into understanding music listening from the perspective of adolescent agency and wellbeing within the complexity of daily experiences.

\section{DATA AVAILABILITY STATEMENT}

The datasets generated for this study are available on request to the corresponding author. 


\section{ETHICS STATEMENT}

The studies involving human participants were reviewed and approved by the University of Jyväskylä Ethics Board. Informed consent from the participants' legal guardian/next of kin was obtained by pressing the accept button in the data collection interface of the mobile phone.

\section{AUTHOR CONTRIBUTIONS}

SS, WR, and MB conceived the study, developed the design, conducted the participant recruitment and data collection, compiled the literature, and wrote the manuscript. SS and MB drafted the items. WR set up the data collection app and

\section{REFERENCES}

Baltazar, M. (2019). "Musical affect regulation in adolescents: a conceptual model," in Handbook of Music and Adolescence, eds K. S. McFerran, P. Derrington, and S. Saarikallio, (Oxford: Oxford University Press).

Baltazar, M., and Saarikallio, S. (2016). Toward a better understanding and conceptualization of affect self-regulation through music: a critical, integrative literature review. Psychol. Music 44, 1500-1521. doi: 10.1177/ 0305735616663313

Baltazar, M., and Saarikallio, S. (2019). Strategies and mechanisms in musical affect self-regulation: a new model. Musicae Sci. 23, 177-195. doi: 10.1177/ 1029864917715061

Bandura, A. (1977). Self-efficacy: toward a unifying theory of behavioral change. Psychol. Rev. 84, 191-215. doi: 10.1037/0033-295X.84.2.191

Bandura, A. (1982). Self-efficacy mechanism in human agency. Am. Psychol. 37, 122-147. doi: 10.1016/0006-8993(86)91535-0

Bandura, A. (1989). Human agency in social cognitive theory. Am. Psychol. 44, 1175-1184. doi: 10.1037/0003-066X.44.9.1175

Bandura, A. (2005). "Adolescent development from an agentic perspective," in SelfEfficacy Beliefs of Adolescents ebook, eds T. C. Urdan, and F. Pajares, (Charlotte, NC: Information Age Publishing), 1-43. doi: 10.1016/j.jbusvent.2009.07.006

Bandura, A. (2006). Toward a psychology of human agency. Perspect. Psychol. Sci. 1, 164-180. doi: 10.1111/j.1745-6916.2006.00011.x

Bandura, A. Ed. (1995). Self-Efficacy in Changing Societies. Cambridge: Cambridge University Press.

Batt-Rawden, K., and DeNora, T. (2005). Music and informal learning in everyday life. Music Educ. Res. 7, 289-304. doi: 10.1080/14613800500324507

Batt-Rawden, K., DeNora, T., and Ruud, E. (2005). Music listening and empowerment in health promotion: a study of the role and significance of music in everyday life of the long-term III. Nordic J. Music Ther. 14, 120-136. doi: 10.1080/08098130509478134

Batt-Rawden, K., and Tellnes, G. (2011). How music may promote healthy behaviour. Scand. J. Public Health 39, 113-120. doi: 10.1177/1403494810393555

Bernatzky, G., Presch, M., Anderson, M., and Panksepp, J. (2011). Emotional foundations of music as a non-pharmacological pain management tool in modern medicine. Neurosci. Biobehav. Rev. 35, 1989-1999. doi: 10.1016/j. neubiorev.2011.06.005

Beyers, W., Goossens, L., Vansant, I., and Moors, E. (2003). A structural model of autonomy in middle and late adolescence: connectedness, separation, detachment, and agency. J. Youth Adolesc. 32, 351-365. doi: 10.1023/A: 1024922031510

Bonsdorff, P. V. (2017). "Transformations of the everyday: the social aesthetics of childhood," in Ästhetiken in Kindheit und Jugend : Sozialisation im Spannungsfeld von Kreativität, Konsum und Distinktion, eds S. Schinkel, and I. Herrmann, (Bielefeld: transcript Verlag), 319-334. doi: 10.14361/ 9783839434833-018

Bosmans, M. W. G., and van der Velden, P. G. (2015). Longitudinal interplay between posttraumatic stress symptoms and coping self-efficacy: a four-wave conducted the data analyses. SS and WR acquired the ethical clearance. All authors reviewed and approved the final version of the manuscript.

\section{FUNDING}

This study was funded by the Academy of Finland (project number 316912).

\section{ACKNOWLEDGMENTS}

The authors would like to thank Essi Asikainen and Reetta Honkavuori for helping in data collection.

prospective study. Soc. Sci. Med. 134, 23-29. doi: 10.1016/j.socscimed.2015. 04.007

Bull, M. (2006). "Investigating the culture of mobile listening: from Walkman to iPod," in Consuming Music Together: Social and Collaborative Aspects of Music Consumption Technologies, eds K. O’Hara, and B. Brown, (London: Springer), 131-149. doi: 10.1007/1-4020-4097-0_7

Côté, J. E., and Schwartz, S. J. (2002). Comparing psychological and sociological approaches to identity: identity status, identity capital, and the individualization process. J. Adolesc. 25, 571-586. doi: 10.1006/jado.2002.0511

DeNora, T. (1999). Music as a technology of the self. Poetics 27, 31-56. doi: 10. 1016/S0304-422X(99)00017-0

DeNora, T. (2000). Music in Everyday Life. Cambridge: Cambridge University Press.

DeNora, T. (2001). "Aesthetic agency and musical practice: new directions in the sociology of music and emotion," in Music and Emotion: Theory and Research, eds P. N. Juslin, and J. A. Sloboda, (Oxford: Oxford University Press), 161-180.

DeNora, T. (2013). Music Asylums: Wellbeing Through Music in Everyday Life. Farnham: Ashgate.

Dibben, N., and Williamson, V. J. (2007). An exploratory survey of in-vehicle music listening. Psychol. Music 35, 571-589. doi: 10.1177/030573560707 9725

Elvers, P. (2016). Songs for the ego: theorizing musical self-enhancement. Front. Psychol. 7:2. doi: 10.3389/fpsyg.2016.00002

Esser, F., Baader, M. S., Betz, T., and Hungerland, B. eds (2016). Reconceptualising Agency and Childhood: New Perspectives in Childhood Studies. Abingdon: Routledge, doi: 10.4324/9781315722245

Gebhardt, S., and von Georgi, R. (2007). Music, mental disorder and emotional reception behavior. Music Ther. Today 8, 419-445.

Gibson, R., Aust, C. F., and Zillmann, D. (2000). Loneliness of adolescents and their choice and enjoyment of love-celebrating versus love-lamenting popular music. Empir. Stud. Arts 18, 43-48. doi: 10.2190/b51g-8u0w-n0eq-mjuu

Gold, C., Saarikallio, S., and McFerran, K. S. (2011). "Music therapy," in Encyclopedia of Adolescence, ed. R. J. R. Levesque, (New York, NY: Springer), 1-12. doi: 10.1007/978-1-4419-1695-2

Gowers, S. (2005). Development in adolescence. Psychiatry 4, 6-9. doi: 10.1383/ psyt.4.6.6.66353

Haake, A. B. (2011). Individual music listening in workplace settings: an exploratory survey of offices in the UK. Musicae Sci. 15, 107-129. doi: 10.1177/ 1029864911398065

Hektner, J. M., Schmidt, J. A., and Csikszentmihalyi, M. (2007). Experience Sampling Method: Measuring the Quality of Everyday Life. Thousand Oaks, CA: Sage.

Hense, C., and McFerran, K. S. (2017). Promoting young people's musical identities to facilitate recovery from mental illness. J. Youth Stud. 20, 997-1012. doi: $10.1080 / 13676261.2017 .1287888$

Heye, A., and Lamont, A. (2010). Mobile listening situations in everyday life: the use of MP3 players while travelling. Musicae Sci. 14, 95-120. doi: 10.1177/ 102986491001400104 
Hu, L. T., and Bentler, P. M. (1999). Cutoff criteria for fit indexes in covariance structure analysis: conventional criteria versus new alternatives. Struc. Equ. Model. 6, 1-55. doi: 10.1080/10705519909540118

Jiang, J., Zhou, L., Rickson, D., and Jiang, C. (2013). The effects of sedative and stimulative music on stress reduction depend on music preference. Arts Psychother. 40, 201-205. doi: 10.1016/j.aip.2013. 02.002

John, O. P., and Srivastava, S. (1999). "The Big-Five trait taxonomy: history, measurement, and theoretical perspectives," in Handbook of Personality: Theory and Research, Vol. 2, eds L. A. Pervin, and O. P. John, (New York, NY: Guilford Press), 102-138.

Karlsen, S. (2011). Using musical agency as a lens: researching music education from the angle of experience. Res. Stud. Music Educ. 33, 107-121. doi: 10.1177/ $1321103 \times 11422005$

Krueger, J. (2011). Doing things with music. Phenomenol. Cogn. Sci. 10, 1-22. doi: 10.1007/s11097-010-9152-4

Krueger, J. (2014). Affordance and the musically extended mind. Front. Psychol. 4:1003. doi: 10.3389/fpsyg. 2013.01003

Krueger, J. (2018). "Music as affective scaffolding," in Music and Consciousness II: Philosophical, Psychological, and Cultural Perspectives, eds D. Clarke, R. Herbert, and E. Clarke, (Oxford: Oxford University Press).

Kuuse, A. (2018). "We will fight Goliath": negotiation of space for musical agency in children's music education. Res. Stud. Music Educ. 40, 140-156. doi: 10.1177/ $1321103 \times 18771796$

Laiho, S. (2004). The psychological functions of music in adolescence. Nordic J. Music Ther. 13, 47-63. doi: 10.1080/08098130409478097

Larson, R. (1995). Secrets in the bedroom: adolescents' private use of media. J. Youth Adolesc. 24, 535-549.

Larson, R. W., Moneta, G., Richards, M. H., and Wilson, S. (2002). Continuity, stability, and change in daily emotional experience across adolescence. Child Dev. 73, 1151-1165. doi: 10.1111/1467-8624.00464

Leipold, B., and Loepthien, T. (2015). Music reception and emotional regulation in adolescence and adulthood. Musicae Sci. 19, 111-128. doi: 10.1177/ 1029864915570354

Lerner, R. M., Freund, A. M., De Stefanis, I., and Habermas, T. (2001). Understanding developmental regulation in adolescence: the use of the selection, optimization, and compensation model. Hum. Dev. 44, 29-50. doi: $10.1159 / 000057039$

Lillevoll, K. R., Kroger, J., and Martinussen, M. (2013). Identity status and locus of control: a meta-analysis. Identity 13, 253-265. doi: 10.1080/15283488.2013. 799471

Lincoln, S. (2005). Feeling the noise: teenagers, bedrooms and music. Leisure Stud. 24, 399-414. doi: 10.1080/02614360500199544

Magee, W. L. (2017). "Music-making in therapeutic contexts: reframing identity following disruptions to health," in Handbook of Musical Identities, eds R. MacDonald, D. J. Hargreaves, and D. Miell, (New York, NY: Oxford University Press), 624-641. doi: 10.1093/acprof:oso/9780199679485.003. 0034

Martin, J., Sugarman, J., and Thompson, J. (2003). Psychology and the Question of Agency. Albany, NY: SUNY Press.

McFerran, K. S. (2011). "Music and adolescents," in Lifelong Engagement with Music: Benefits for Mental Health and Well-Being, eds N. S. Rickard, and K. S. McFerran, (Hauppauge, NY: Nova Science Publishers), 97-108.

McFerran, K. S., Hense, C., Koike, A., and Rickwood, D. (2018). Intentional music use to reduce psychological distress in adolescents accessing primary mental health care. Clin. Child Psychol. Psychiatry 23, 567-581. doi: 10.1177/ 1359104518767231

McFerran, K. S., and Saarikallio, S. (2014). Depending on music to feel better: being conscious of responsibility when appropriating the power of music. Arts Psychother. 41, 89-97. doi: 10.1016/j.aip.2013.11.007

Miranda, D. (2013). The role of music in adolescent development: much more than the same old song. Int. J. Adolesc. Youth 18, 5-22. doi: 10.1080/02673843.2011. 650182

Miranda, D. (2019). A review of research on music and coping in adolescence. Psychomusicology 29, 1-9. doi: 10.1037/pmu0000229

Miranda, D., and Claes, M. (2009). Music listening, coping, peer affiliation and depression in adolescence. Psychol. Music 37, 215-233. doi: 10.1177/ 0305735608097245
Mitchell, L. A., and Macdonald, R. A. R. (2006). An experimental investigation of the effects of preferred and relaxing music listening on pain perception. J. Music Ther. 43, 295-316. doi: 10.1093/jmt/43.4.295

Muthén, L. K., and Muthén, B. O. (2015). Mplus User's Guide, 7th Edn. Los Angeles, CA: Muthén \& Muthén.

North, A. C., and Hargreaves, D. J. (1999). Music and adolescent identity. Music Educ. Res. 1, 75-92. doi: 10.1080/1461380990010107

North, A. C., Hargreaves, D. J., and O'Neill, S. A. (2000). The importance of music to adolescents. Br. J. Educ. Psychol. 70, 255-272. doi: 10.1348/000709900158083

O'Neill, S. A. (2017). "Young people's musical lives: learning ecologies, identities, and connectedness," in Handbook of Musical Identities, eds R. MacDonald, D. J. Hargreaves, and D. Miell, (New York, NY: Oxford University Press), 79-104. doi: 10.1093/acprof:oso/9780199679485.003.0005

Pajares, F. (2005). "Self-efficacy during childhood and adolescent: implications for teachers and parents," in Self-Efficacy Beliefs of Adolescents ebook, eds T. C. Urdan, and F. Pajares, (Charlotte, NC: Information Age Publishing), 339-367.

Pajares, F., and Urdan, T. eds (2006). Self(-)Efficacy Beliefs of Adolescents (ebook). Greenwich: Information Age Publishing.

Papinczak, Z. E., Dingle, G. A., Stoyanov, S. R., Hides, L., and Zelenko, O. (2015). Young people's uses of music for well-being. J. Youth Stud. 18, 1119-1134. doi: 10.1080/13676261.2015.1020935

Pothoulaki, M., MacDonald, R. A. R., Flowers, P., Stamataki, E., Filiopoulos, V., Stamatiadis, D., et al. (2008). An investigation of the effects of music on anxiety and pain perception in patients undergoing haemodialysis treatment. J. Health Psychol. 13, 912-920. doi: 10.1177/1359105308095065

Randall, W. M., and Rickard, N. S. (2013). Development and trial of a music experience sampling method (m-ESM) for personal music listening. Music Percept. 31, 157-170. doi: 10.1525/mp.2013.31.2.157

Randall, W. M., and Rickard, N. S. (2017a). Personal music listening: a model of emotional outcomes developed through mobile experience sampling. Music Percept. 34, 501-514. doi: 10.1525/mp.2017.34.5.501

Randall, W. M., and Rickard, N. S. (2017b). Reasons for personal music listening: a mobile experience sampling study of emotional outcomes. Psychol. Music 45, 479-495. doi: 10.1177/0305735616666939

Randall, W. M., Rickard, N. S., and Vella-Brodrick, D. A. (2014). Emotional outcomes of regulation strategies used during personal music listening: a mobile experience sampling study. Musicae Sci. 18, 275-291. doi: 10.1177/ 1029864914536430

Rissanen, M. (2017). "It's as if..." Preschoolers encountering contemporary photography. Int. J. Educ. Arts 18, 2-31.

Ryan, R. M., and Deci, E. L. (2000). Self-determination theory and the facilitation of intrinsic motivation, social development, and well-being. Am. Psychol. 55, 68-78. doi: 10.1037/0003-066X.55.1.68

Saarikallio, S. (2008). Music in mood regulation: initial scale development. Musicae Sci. 12, 291-309. doi: 10.1371/journal.pone.0211362

Saarikallio, S. (2011). Music as emotional self-regulation throughout adulthood. Psychol. Music 39, 307-327. doi: 10.1177/0305735610374894

Saarikallio, S. (2017). "Musical identity in fostering emotional health," in Handbook of Musical Identities, eds R. Macdonald, D. J. Hargreaves, and D. Miell, (Oxford: Oxford University Press), 602-623. doi: 10.1093/acprof:oso/9780199679485. 003.0033

Saarikallio, S. (2019a). Access-Awareness-Agency (AAA) model of music-based social-emotional competence (MuSEC). Music Sci. 2, 1-16. doi: 10.1177/ 2059204318815421

Saarikallio, S. (2019b). "Music \& identity: agency \& empowerment," in Handbook of Music, Adolescents, and Wellbeing, eds K. McFerran, P. Derrington, and S. Saarikallio, (Oxford: Oxford University Press).

Saarikallio, S., and Baltazar, M. (2018). "Music as a forum for social-emotional health," in Music and Public Health: A Nordic Perspective, eds L. O. Bonde, and T. Theorell, (New York, NY: Springer International Publishing), 101-113. doi: 10.1007/978-3-319-76240-1

Saarikallio, S., and Erkkilä, J. (2007). The role of music in adolescents' mood regulation. Psychol. Music 35, 88-109. doi: 10.1177/0305735607068889

Saarikallio, S., Maksimainen, J., and Randall, W. M. (2018). Relaxed and connected: insights into the emotional-motivational constituents of musical pleasure. Psychol. Music 47, 644-662. doi: 10.1177/0305735618778768

Sawatzky, R. G., Ratner, P. A., Richardson, C. G., Washburn, C., Sudmant, W., and Mirwaldt, P. (2012). Stress and depression in students: the mediating role 
of stress management self-efficacy. Nurs. Res. 61, 13-21. doi: 10.1097/NNR. 0b013e31823b1440

Schnoll, R. A., Martinez, E., Tatum, K. L., Glass, M., Bernath, A., Ferris, D., et al. (2011). Increased self-efficacy to quit and perceived control over withdrawal symptoms predict smoking cessation following nicotine dependence treatment. Addict. Behav. 36, 144-147. doi: 10.1016/j.addbeh.2010.08.024

Schwartz, K. D., and Fouts, G. T. (2003). Music preferences, personality style, and developmental issues of adolescents. J. Youth Adolesc. 32, 205-213. doi: 10.1023/a:1022547520656

Schwartz, S. J., Cote, J. E., and Arnett, J. (2005). Identity and agency in emerging adulthood: two developmental routes in the individualization process. Youth Soc. 37, 201-229. doi: 10.1177/0044118x05275965

Selfhout, M. H. W., Branje, S. J. T., ter Bogt, T. F. M., and Meeus, W. H. J. (2009). The role of music preferences in early adolescents' friendship formation and stability. J. Adolesc. 32, 95-107. doi: 10.1016/j.adolescence.2007.11.004

Skånland, M. S. (2011). Use of mp3-players as a coping resource. Music Arts Act. 3, 15-33.

Skånland, M. S. (2013). Everyday music listening and affect regulation: the role of MP3 players. Int. J. Qual. Stud. Health Well-Being 8:20595. doi: 10.3402/qhw. v8i0.20595

Strecher, V. J., Mcevoy Devellis, B., Becker, M. H., and Rosenstock, I. M. (1986). The role of self-efficacy in achieving health behavior change. Health Educ. Q. 13, 73-92. doi: 10.1177/109019818601300108

ter Bogt, T. F. M., Soitos, S., and Delsing, M. (2012). "Music listening in adolescence," in Encyclopedia of Adolescence, Vol. 1, ed. R. J. R. Levesque, (Amsterdam: Elsevier), 240-250. doi: 10.1016/B978-0-12-37395-5.00 029-2

ter Bogt, T. F. M., Vieno, A., Doornwaard, S. M., Pastore, M., and van den Eijnden, R. J. J. M. (2017). "You're not alone": music as a source of consolation among adolescents and young adults. Psychol. Music 45, 155-171. doi: 10.1177/ 0305735616650029

Thayer, R. E., Newman, J. R., and McClain, T. M. (1994). Self-regulation of mood: strategies for changing a bad mood, raising energy, and reducing tension. J. Pers. Soc. Psychol. 67, 910-925. doi: 10.1037//0022-3514.67.5.910

Thoma, M. V., Ryf, S., Mohiyeddini, C., Ehlert, U., and Nater, U. M. (2012). Emotion regulation through listening to music in everyday situations. Cogn. Emot. 26, 550-560. doi: 10.1080/02699931.2011.595390
Thomson, C. J., Reece, J. E., and Di Benedetto, M. (2014). The relationship between music-related mood regulation and psychopathology in young people. Musicae Sci. 18, 150-165. doi: 10.1177/102986491452 1422

Tomyn, A. J., and Cummins, R. A. (2011). Subjective wellbeing and homeostatically protected mood: theory validation with adolescents. J. Happiness Stud. 12, 897-914. doi: 10.1007/s10902-010-9235-5

Van Goethem, A., and Sloboda, J. A. (2011). The functions of music for affect regulation. Musicae Sci. 15, 208-228. doi: 10.1177/102986491140 1174

Vuoskoski, J., and Eerola, T. (2011). Measuring music-induced emotion: a comparison of emotion models, personality biases, and intensity of experiences. Musicae Sci. 15, 159-173. doi: 10.1177/1029864911403367

Wallace-DiGarbo, A., and Hill, D. C. (2006). Art as agency: exploring empowerment of at-risk youth. J. Am. Art Ther. Assoc. 23, 119-125. doi: 10. 1080/07421656.2006.10129627

Wiggins, J. (2016). "Musical agency," in The Child as Musician: A Handbook of Musical Development, 2nd Edn, ed. G. E. McPherson, (St. Ives: Oxford University Press), 102-121. doi: 10.1093/acprof:oso/9780198744443.003. 0006

Zillmann, D., and Vorderer, P. (2000). Media Entertainment: The Psychology of Its Appeal. Mahwah, NJ: Lawrence Erlbaum.

Zimmermann, B. J., and Cleary, T. J. (2005). “Adolescents' development of personal agency: the role of self-efficacy beliefs and self-regulatory skill," in Self-Efficacy Beliefs of Adolescents ebook, eds T. C. Urdan, and F. Pajares, (Charlotte, NC: Information Age Publishing), 45-69.

Conflict of Interest: The authors declare that the research was conducted in the absence of any commercial or financial relationships that could be construed as a potential conflict of interest.

Copyright (C) 2020 Saarikallio, Randall and Baltazar. This is an open-access article distributed under the terms of the Creative Commons Attribution License (CC BY). The use, distribution or reproduction in other forums is permitted, provided the original author(s) and the copyright owner(s) are credited and that the original publication in this journal is cited, in accordance with accepted academic practice. No use, distribution or reproduction is permitted which does not comply with these terms. 


\section{OPEN ACCESS}

Edited by:

Michele Biasutti,

University of Padova, Italy

Reviewed by:

Graça Maria Boal-Palheiros, Escola Superior de Educação, Instituto Politécnico do Porto,

Portugal

Helen Phelan,

University of Limerick, Ireland

*Correspondence:

Sandra Garrido

s.garrido@westernsydney.edu.au

Specialty section:

This article was submitted to

Performance Science,

a section of the journal

Frontiers in Psychology

Received: 31 January 2019 Accepted: 07 May 2019

Published: 24 May 2019

Citation:

Stewart J, Garrido S, Hense C and McFerran K (2019) Music Use

for Mood Regulation: Self-Awareness and Conscious Listening Choices in Young People With Tendencies

to Depression

Front. Psychol. 10:1199. doi: 10.3389/fpsyg.2019.01199

\section{Music Use for Mood Regulation: Self-Awareness and Conscious Listening Choices in Young People With Tendencies to Depression}

\author{
Joanna Stewart ${ }^{1}$, Sandra Garrido ${ }^{1 *}$, Cherry Hense ${ }^{2}$ and Katrina McFerran ${ }^{2}$ \\ ${ }^{1}$ MARCS Institute for Brain, Behaviour and Development, Western Sydney University, Penrith, NSW, Australia, ${ }^{2}$ Department \\ of Music Therapy, Faculty of Fine Arts and Music, The University of Melbourne, Melbourne, VIC, Australia
}

The current study explored the circumstances in which seven young people with a tendency to depression chose different styles of music to listen to, and their level of awareness of the impact of their music listening habits on mood and wellbeing. A model of various pathways through music use was developed that may explain why music listening intentions in young people do not always align with their wellbeing outcomes. We suggest that the relationship between intentions and outcomes are mediated by differing levels of self-awareness and insight into the mood regulation processes occurring during music listening.

Keywords: young people, music, mood regulation, depression, self-awareness

\section{INTRODUCTION}

Depression is responsible for the deaths of many people globally each year, with suicide being the leading cause of death around the world in 15-29 year-olds (WHO, 2017). Many more young people experience depression at highly debilitating levels, around $8 \%$ in Australia meeting the DSM criteria for Major Depressive Disorder (MDD) (Lawrence et al., 2015), and 13\% in the United States (National Institute of Mental Health, 2017). The early onset of depression is a critical factor in terms of projected quality of life (Sullivan et al., 2012), and if left untreated, depression can become a lifelong disability (Meade and Dowsell, 2016). Depression also has an impact on the social and intellectual development of young people as well as reducing engagement with education at a crucial developmental stage. It is therefore imperative to address depression in young people before its impact on their lives increases.

\section{Depression and Media Use}

Access to online media has increased exponentially with the onset of digitisation and technological advancement (Brown and Bobkowski, 2011). Research has demonstrated that young people are even more likely to turn to media when they are in a negative mood (Dillman Carpentier et al., 2008). In fact, withdrawal from socialization and normal daily activity has been identified as a behavior consistent with clinical depression and this often involves an increase in general media use (O'Keeffe and Clarke-Pearson, 2011). This increased engagement with media includes music listening, with emotional dependency on music also tending to increase during periods of depression (McFerran, 2016).

However, research has demonstrated that this increased reliance on music during episodes of psychological distress does not always have positive mental health outcomes for the young people involved. For example, Garrido and Schubert $(2015 a, b)$ have demonstrated that people with 
a ruminative coping style, which is highly predictive of clinical depression, tend to be attracted to music that can intensify symptoms of depression. Similarly, in a study by McFerran et al. (2015) the authors discovered that having high levels of distress while listening to music was associated with more intense, negative moods afterward. Other studies confirm the fact that people with depression are not always able to effectively select music that helps them to feel better (Wilhelm et al., 2013; Hense et al., 2014). They may also use music as part of generally unhealthy coping strategies such as emotion-focused coping (Miranda et al., 2012), rumination (Garrido and Schubert, 2013), or social withdrawal (McFerran and Saarikallio, 2014).

\section{Self-Awareness and Depression}

Self-awareness can be described as clear awareness of one's own feelings, emotions, and behaviors (Blakemore and Frith, 2003). Such awareness is generally regarded as an adaptive function that can result in identification of aspects of the self that would benefit from modification. Experiencing feelings of sadness can often provide the motivation for self-scrutiny and behavioral modification, even increasing detail oriented thinking and realistic thinking that is useful for problem solving behaviors (Keedwell, 2008). However, in depression, the adaptive function of sadness tends to malfunction, with depression being associated with increased pessimism and reduced motivation to engage in problem solving (Bianco et al., 2013).

In general, individuals differ as to their levels of cognitive insight, or their capacity to understand their own thoughts, behaviors and affective states (Riggs et al., 2012). However, research has demonstrated that low emotional awareness is highly predictive of depression and anxiety in young people (Kranzler et al., 2016). Emotional awareness, or the ability to identify emotional experiences, can be a protective factor against psychopathology by allowing an individual to recognize the need to activate appropriate emotion regulation strategies (Barrett et al., 2001). On the other hand, young people with low emotional awareness tend to have reduced access to effective strategies for coping with negative affect and interpersonal difficulties (Flynn and Rudolph, 2014).

This lack of awareness may also play into music listening choices in young people with depression. This is implied by one study conducted by Garrido and Schubert (2015b) in which participants with high levels of rumination reported having benefited from listening to sad music while at the same time reporting an increase in depressive symptoms. Similarly, in a study on listening to nostalgic music, Garrido (2018) found that implicit mood measures (in which participants are unaware that their mood is being assessed) indicated a much higher level of negative mood responses after listening to nostalgic music than participants reported in response to direct questioning. The issue of the discrepancy between perceived and real mood changes was also discussed by McFerran et al. (2016) in a systematic review of 33 articles about music and mental health. Their review revealed that while direct questioning usually suggested positive mood effects from listening to music, non-direct mood indicators suggested results were not always so positive. At times this appeared to be because researchers worded questions in such a way as to suggest positive effects. In other cases study participants demonstrated a tendency to construe music listening positively regardless of its effect on their mood. This reveals issues both with demand characteristics in study design as well as a degree of positive bias in participants.

In exploring the concept of awareness further, McFerran and Saarikallio (2014) identified three different response styles with regards to music choices. They found that some people can recognize that the music they listen to is not beneficial to their mood and then be proactive in changing their listening habits. The second response style is when a person can be made aware of deteriorations in their mood by others and change their habits. This has been shown to be possible, for example, with young people who are seeking help for depression and who work with a music therapist to identify more helpful ways of listening to their preferred music (McFerran et al., 2018). The third response style is when a person may either recognize or be made aware of the negative impact but is not inclined to modify their listening behaviors. Alternatively, if an individual's mental health is very poor, they may not be able to focus on therapeutic interventions that demand high cognitive function such as this level of metareflection on intentional music listening (Hense et al., 2018). Thus, it appears that there is a need to develop nuanced strategies for increasing awareness of the effect that music listening can have on young people's mood and wellbeing. Given the central role that music plays in the lives of young people, increasing such awareness has the potential for positive benefits through increased understanding of adaptive and maladaptive behaviors more generally. There is a need to further understand how young people are enabled to increase their awareness about the effects of music on their wellbeing.

The current study uses a grounded theory approach to explore the following research question through interviews with seven young people: To what degree are young people with symptoms of depression aware of the effect their music-listening has on mood and wellbeing, and how do they reach a state of awareness?

\section{METHODS}

The research question lends itself to an inductive approach in which a topic is explored with no prior hypothesis. Grounded theory is one qualitative method that is often used to investigate the ways in which various conditions interact with an individual's experience of a given phenomenon, with an emphasis on analyzing people's actions and integrating what they do as well as what they say (Charmaz and Bryand, 2007) This method enables theoretical notions to be extrapolated from qualitative data, rather than generating a rich description (Dey, 2007). In grounded theory, the researchers endeavor to approach the data without being influenced by a priori knowledge. Through processes of coding, constant comparison and abstraction of concepts from data categories, a conceptual hypothesis or theory can be developed (Chun Tie et al., 2019).

\section{Participants}

Participants were recruited from among people who had taken part in an online survey and had indicated their interest in being involved in further research (Garrido et al., 2017). Initially, 
TABLE 1 | Demographic information of interview participants.

\begin{tabular}{lcccc}
\hline Participant \# & Age & Sex & $\begin{array}{c}\text { Previous treatment } \\
\text { for depression }\end{array}$ & Country \\
\hline 1 & 28 & Female & No & Australia \\
2 & 19 & Female & Yes & United States \\
3 & 24 & Female & No & United States \\
4 & 22 & Female & No & Brazil \\
5 & 19 & Female & No & United Kingdom \\
6 & 22 & Female & Yes & Canada \\
7 & 23 & Male & No & United Kingdom \\
\hline
\end{tabular}

615 people participated in the survey and were asked to complete the Depression and Anxiety Stress Scale (DASS; Henry and Crawford, 2005) and the Rumination-Reflection Questionnaire (RRQ; Trapnell, 1997). Purposive sampling was used, and potential participants with DASS scores above 15 and rumination scores above four were approached, as these are indicative of severe symptoms of depression and ongoing ruminative coping styles. This generated a list of 27 potential participants, 4 of whom were not approached because they had indicated being negatively affected by participating in the survey. The participants on the inclusion list were contacted by email, with 7 young people aged from 19 to 28 years (mostly female) responding and being interviewed. Participant demographics are included in Table 1. As the previous study had been conducted online the participants lived in a number of different countries.

\section{Materials and Procedure}

Ethics approval was granted by the Human Research Committee (\#1443393.1) at the University of Melbourne. Potential participants were contacted via email and if they responded with interest were sent a Plain Language Statement and Consent form. Written informed consent was obtained from all participants prior to conduct of the interviews. Due to the diverse locations of participants, all but one interview was conducted via the video call function on Skype using Version 7.39.0.102. Audio of the interviews was recorded using MP3 Skype Recorder 4.32 Free Edition. Since all participants were fluent English speakers interviews were conducted in English and took approximately $45 \mathrm{~min}$ to $1 \mathrm{~h}$. Participants were offered a \$15 iTunes voucher for participating in the study.

Interviews were conducted by the first two authors and memos were created after each interview to record the impressions of the interviewers about participants' demeanor, body language, tone of voice and time taken to respond as well as the interviewers' reflections on how their own personal biases may have influenced their conduct of the interview (Finlay, 2002). Discussions between the first two authors took place after each interview to determine the direction of the subsequent interview. An interview guide was used, however, interviews proceeded freely based on participant responses (see Appendix). In general, the interviewer sought to prompt participants to discuss their use of music to regulate their moods, and in particular negative moods. In order to determine the level of awareness and responsiveness to the idea that music does not always have positive effects on mood, and to avoid the positive bias that has limited some previous studies, the interviewers deliberately introduced the topic of negative effects toward the end of the interview if participants had not raised the issue themselves.

\section{Analysis}

Interviews were transcribed verbatim. A preliminary analysis of each interview was conducted using open coding in accordance with Strauss and Corbin (2008). The initial in vivo codes related primarily to the ways the young people described using music to regulate mood and included codes such as "for comfort", "music is used as a distraction" and "to keep fighting." This initial coding allowed the first author to immerse herself in the data and revealed new questions for consideration in subsequent interviews to enable deeper exploration of new issues. For example, themes of anxiety began to emerge as common around the fourth interview, and so additional questions in relation to this were added to the interview guide for subsequent interviews.

Once open coding of the interviews had been completed a second wave of analysis was conducted independently by two pairs of researchers (the first two and last two authors) who each identified themes and central categories that connected the various ideas that were emerging from the data. Axial coding as described by Charmaz (2006) was then used to discern possible relationships between the various categories and codes, with constant return to the data to better understand whether similar coding was representative of shared ideas. The codes were examined to assess the motivations participants gave for adopting particular mood regulation strategies with music and the factors influencing the relative success of these strategies. This informed the generation of sub-categories within the larger concepts that had been identified across participants. The properties and dimensions of the categories and sub-categories were then delineated with a focus on the central code of awareness and, where present, how this awareness was described as developing. Memos were used as a way of noting impressions from the data, with statements such as "It seems like..." The analysts would then return to the raw data to see whether these impressions could be sustained by what had actually been said. This process allowed for a constant attention to the possibility of the researchers' pre-assumptions influencing interpretation of the data, rather than allowing details about the phenomenon to emerge from the participants' experiences, which was the focus of this study. The two research teams then compared their analyses and used selective coding to integrate and further refine the central category, it's properties and dimensions and to develop a theoretical proposition.

\section{Reflexivity}

Since researchers who have worked in a particular field for some time may already be familiar with previous findings in this area and be somewhat influenced by their knowledge, reflexivity was considered an important part of the research process (Finlay, 2002). Qualitative analysis is an inherently subjective process and two of the authors (SG and KM) have undertaken a number of studies of this topic previously. It was therefore critical to ensure that analysis was not used 
to simply confirm our existing beliefs (confirmation bias). Undertaking two separate analyses was our primary strategy for testing our ability to focus on what was being said by the seven participants and we frequently returned to the data to scrutinize our emerging ideas and test how closely it matched the raw data. This allowed us to rigorously debate and refine our presentation of the findings. Throughout the interviews and analysis process, the researchers also engaged in personal and shared reflections and debate about potential meanings inherent in the data.

\section{FINDINGS}

\section{Strategies for Music Choice}

Our analysis suggested that the strategies participants described for using music to manage negative moods fell into two broad categories: (i) selecting music that differed from the negative mood in an effort to shift a negative mood, and (ii) selecting music that mirrored the negative mood in an effort to cope with negative feelings. These strategies are depicted in Figure 1 following the model of Biasutti (2013, 2018). Both strategies appeared to have negative outcomes at times and positive outcomes at other times.

\section{Music That Differed From Current Mood}

Many (5 of 7) of the participants described listening to music that differed from the mood they were experiencing in order to try to alter a negative mood. For example, Participant 3 described listening to classical music when angry to help her calm down: "If I'm listening to classical music because I'm trying to calm myself down, it's soothing. It helps me relax. It's more like trying to relax, that I'm feeling the music and trying to absorb every note." Similarly, several participants identified using calming music to reduce feelings of anxiety. Participant 5 stated "I have anxiety issues so I find it quite a nice way to settle myself," while Participant 7 described using music to "get out of my head" when feeling anxious. Participant 2 also reported sometimes using music to "block out things that are bothering me." Participant 5 also described successfully listening to upbeat music when feeling down. She stated: "If I'm feeling depressed I tend to put on happy music like cheesy pop and things to try and cheer myself up almost. Something with a fast tempo to kind of boost my mood." These strategies were considered to represent conscious processes adopted by participants to change their mood. In contrast, Participant 4 reported that listening to music that didn't match her negative mood gave her "the impression that everyone else is having fun except for me."

\section{Music That Mirrored Current Mood}

The other prevalent theme we perceived across most (6 of 7) participants was the use of music to mirror mood in an attempt to cope with feelings of sadness and depression. While the term 'cope' can cover a wide range of strategies for dealing with undesirable situations and affective states including problemsolving and attempting to change one's mood, in the context of this data the term is used to describe strategies designed to mitigate or lessen the intensity or unpleasantness of an undesirable mood without actually shifting it's valence. Different participants described selecting music that mirrored their current mood in relation to a diverse range of intentions or aims, and outcomes. However, our interpretation was that these strategies often appeared to be designed to help participants cope with affective states, rather than to change them.

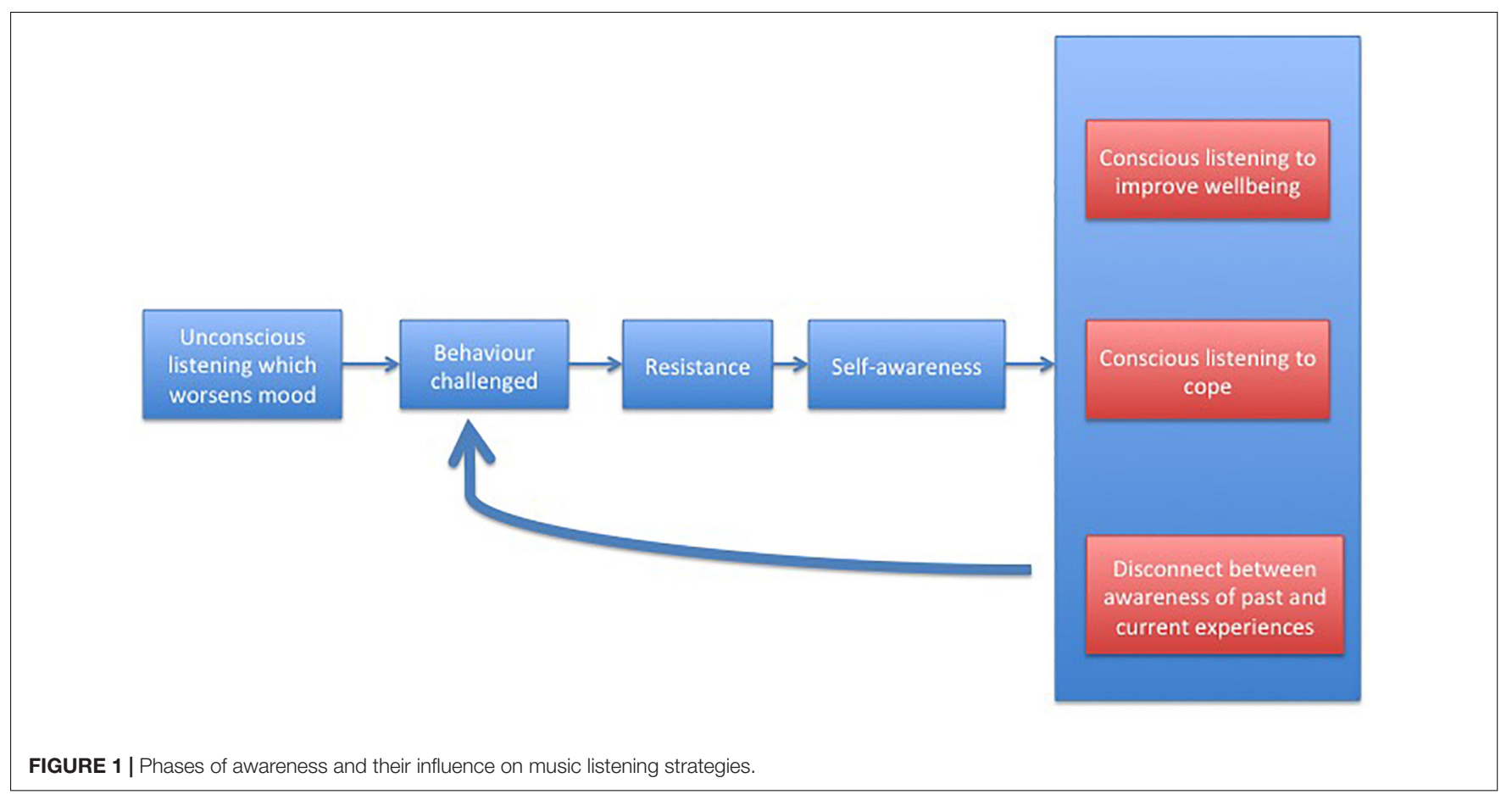


Some participants expressed the desire to be "comforted" when listening to music, gaining understanding that "I'm not the only one going through problems" (Participant 3). This strategy provided a feeling of "validation" of the experience for Participant 4, and Participant 6 described something similar saying, "I'm tired and I'm still sad but it's less heavy and it's like someone understands." Participant 3 also reported that sometimes it was just a matter of changing the degree of sadness being felt, describing how in the past she found it useful to listen to music that was "at a level that is just a little bit above what I'm feeling, to maybe bring me up a little bit but not so much that it would bother me." In this case, even a small improvement in mood was perceived as a positive change.

Others listened to mood-matching music with the express aim of intensifying their negative emotions. Several participants reported listening to slow, acoustic, classical pieces to reflect and emphasize a sad or low mood state. For example, Participant 1 described deliberately choosing songs that conveyed "extreme manifestations" of the sad mood she was in. Two participants described their motivation as being to "drown in" (Participant 3 ) or "wallow in" the negative emotions (Participant 4). Participant 3 described how this had a positive effect, and how it "gets me to the highest point and then I come down," suggesting that she experienced some relief once the more intense emotions diminished. For Participant 1, the effect was less clear and she reported listening to music with suicidal lyrics when depressed, stating that this "probably just intensified the emotion, which [may] or [may not] be beneficial." Participant 4 similarly reported that this could leave her not even feeling "motivated enough to change the music."

In contrast to the others, Participant 2 reported that she preferred to avoid music that could make her feel more depressed. This did not necessarily entail listening to music that was upbeat and happy, which she tended to listen to when having a "better day," but she said: "If I'm not feeling so good I'll listen to a classical piece, something slow." She also made the following statement.

"I try not to listen to depressing music if I'm already feeling down because it's not going to do anything to help really. Sometimes I kind of need it to know that other people feel the same way, but a lot of time its just going to make me feel worse and so then I don't want to do that. . Usually I listen to more positive things."

\section{Factors Influencing the Outcomes}

Our analysis of the properties and dimensions of the strategies presenting in the data revealed that both positive and negative effects were experienced from both strategies for managing moods with music. This was determined during axial coding, where we examined the circumstances surrounding these strategies in order to determine some of the factors that contributed to a positive or negative outcome. We identified three properties: (i) the messages conveyed by the lyrics, (ii) the frequency and duration of listening to certain music, (iii) the nature and intensity of the prior affective state of the listener.

\section{The Messages Conveyed by the Lyrics}

Some participants described particularly being attracted to music with lyrics that have special meaning for them when feeling down.
When Participant 1 described the kind of music she was drawn to in a depressed mood, she said "It's both the music and the lyrics as well, and I think that what the singer's expressing is a sort of frustration and I'll think, 'Oh yes, that's exactly what we feel here'." Participant 6 described how music with lyrics was especially important to her when she was feeling sad: "When I'm really, really sad that's the only time I'll listen to music where I care more about the lyrics."

However, the outcomes weren't necessarily positive for participants when they listened to music with lyrics that closely related to how they felt. Participant 1 stated: "I started thinking about the lyrics and stuff and it's not pleasant stuff and I began to think, well maybe it's listening to this stuff which is really contributing to my being in a very low mood." Participant 6 similarly said: "I was just getting really perturbed because I was listening to the lyrics too much and I could relate and then I could go and watch TV or something but I kept thinking about the song."

More positive effects were noted when listening to songs that were considered "emotional but" also inspired "some optimism," or had an "uplifting message" (Participant 6). Participant 2 stated that "If the music has a positive outlook on life, I'm likely to kind of adopt that somewhat." Thus, several participants demonstrated how the differing messages in music, even music that mirrored their mood, could have differing effects on their mood.

\section{Frequency and Duration of Music Listening}

Participant 1 explained how she had experienced a phase in her life when she was listening intensely to music with very negative, suicidal lyrics. She made the following statement.

"I was listening for hours a day. . .to be hearing people talking about drug problems, how much they don't like themselves, that they were locked up in the mental hospital etc. On a daily basis that probably isn't the world's best for mental health."

Participant 2 similarly described that the amount of time she spent listening to sad music needed to be limited. "Sometimes I kind of need it to know that other people feel the same way. . . But there does come a point when you are feeling bad enough and then that would make you feel worse and it's something you have to stay away from." Participant 6 also mentioned a time when she had been listening to songs about suicide "quite a few times. . too much" with negative results. Thus, there was a recognition that intense or frequent listening to music that reflected negative thinking was likely to have a more negative impact on wellbeing.

\section{Nature and Intensity of the Prior Affective State}

Being in a low negative mood state was frequently mentioned as a factor that would result in music listening having a negative or neutral effect on mood. For example, Participant 4 said: "If I am having a really bad day then nothing I do will really change that." She also stated that, "When I'm in a more neutral mood I can change my emotions according to what I'm listening to but when I'm really sad nothing helps." Similarly, Participant 7 commented that it's the strength of his mood that influences how easy it is to modify with music: "Some moods are harder to shake." 
A number of participants described using music to distract from, or mask unpleasant emotional states. For example, Participant 6 described herself as having "an anxiety disorder" and being able to use music to "distract" herself or "calm" herself down. Although this strategy was sometimes described as helpful in alleviating the intensity of emotional experiences, some participants also acknowledged the temporary nature of this solution. For example, Participant 7 stated that music could not remove anxious feelings altogether, but that it would just "temporarily mask the depression" and then he would "be back to square one" when the music finished. Similarly, Participant 6 stated that "on the spot it's useful because I'm just thinking about the music, I'm forcing myself not to think about what's making me anxious but when I stop everything comes back." Thus, some participants identified that 'covering up' emotions were a short-term and somewhat limited solution.

Strategies also appeared to differ depending on the individual's mood. Participant 3 said that when she was feeling sad she usually chose music that matched her mood because she found it "comforting" and "reassuring." However, when angry she would listen to music that she hoped would change her mood.

\section{Awareness}

In examining some of the factors that influenced the outcome of music listening for people with symptoms of depression, it seemed possible that a key factor was the level of awareness and consciousness with which individuals selected music. Selective coding allowed us to explore the data in order to further test this theory. In order to overcome previous study limitations which have demonstrated that individuals do not always directly report negative effects of listening to music, coding strategies here looked not only at clear statements relating to awareness, but at other indications such as inconsistent responses or signs of ambiguity or confusion. Upon direct request, most participants were able to list a song or a type of music that had previously caused a deterioration in mood for them. For example, Participant 4 reported that listening to "emo" music had previously had a negative effect on her mood. Participant 1 similarly described listening to Elliott Smith and realizing that her mood was "continually low."

It was evident that for several participants their insight into the potential for music to have negative effects was something they had gained over time, usually after some negative experiences. For example, Participant 5 stated:

\footnotetext{
"It's something I've developed over time. It's like a mechanism I've developed as I got more used to having mental health problems. . .I used to listen to a lot of punk rock stuff and all that kind of emo stuff nonsense and it just used to make me much more worked up because it's so intense that it does not help. But it took me quite a long time to realize what was happening."
}

Awareness was obtained in several ways. Some participants were made more aware by the comments of friends and family. Participant 5 made the following statement.

\footnotetext{
"My family members were like, 'This is too intense. Why are you listening to this? You are obviously struggling.' There is a history of mental illness in my family so they are quite good at knowing
}

how to deal with it. And I was like, 'No it is helping'... until I heard a few people my own age say that it's not working.

She similarly said: "Some of my friends had some of the same issues and they said how they listened to some music that made them feel worse and I was like "oh maybe they have figured out what's wrong with me too" and I sort of realized that."

Of note in Participant 5's comments is the fact that she had at first believed that the music was helping her, but she ultimately identified with the experience of friends who noted that it was not always as helpful as it could be.

For Participant 6 it was therapy that had helped her to become more aware of her listening habits. She made the following statement:

\begin{abstract}
"When I was younger I did have a tendency to just listen to songs more, especially if they made me sad and they emphasized my mood. Now, I would take a break from them and listen to something different because I'm trying, well not trying to control my mood but it was one of the things I discussed with my therapist. I was not trying not to hurt myself more, but trying to not feel worse about things."
\end{abstract}

For Participant 1, the realization appears to have come to her personally without prompting from other people. "I began to think, 'Well maybe it's listening to this stuff which is really contributing to my being in a very low mood, so I had to stop then." She reported realizing that listening to particular music with suicidal lyrics had "brought [her] into a more negative mood."

However, there was some evidence that despite a recognition of the negative effects of past experiences, this did not always translate into an awareness of the potential impact of current listening behaviors. Several participants seemed to lack clarity in their own mind about whether particular listening was useful or not. This was evidenced by some inconsistent statements within the interviews. For example, when Participant 4 was asked what she listens to when she is in a low mood she said: "emo music." However, when asked to describe a situation where music had made her feel worse she described how in high school emo music had made her "feel worse." When asked again whether she would do the same thing now, she said: "Probably not." In this case, the participant recognized some negative effects in the past but still reported the same listening choices. However, asking the participant to reflect on the past negative experience caused her to change her answer about current listening choices. Whether or not this reflected an actual change in opinion is unclear.

Participant 6 was similarly somewhat ambiguous about whether her listening choices made her feel worse. As cited above, this participant reported discussing with her therapist about avoiding music that made her feel "worse about things." She seemed to have some useful strategies for regulating her mood such as listening to music that is "sad" but that gives her "some optimism," or music that gave her some relief in that after listening she was "still sad but less heavy." However, when speaking about a previous experience she made the following statement. 


\begin{abstract}
"I don't know if it made me feel worse. . .but there was this one song about suicide that I remember listening to. . .the song itself kind of hit close to home at that moment... I was just getting really perturbed because I was listening to the lyrics too much."
\end{abstract}

When asked if she would listen to the same song again she said "sometimes I do," but preferred not to listen to it when in a good mood because it "brings back memories." Thus, while this participant appeared to have some awareness of her complex responses to certain music in the past, she sometimes seemed to revert to unhealthy patterns in periods of depression. Of note with this participant was a recognition that the message in the lyrics - whether suicidal or optimistic - was an important factor even when the music was "sad" overall, with the former tending to make her feel worse, while the latter helped her to feel "like someone understands."

Similarly, Participant 1 gave some conflicting statements in reference to her preference for listening to Elliott Smith and his music with suicidal themes when feeling depressed. At first when describing her response to this music she stated, "I just kind of think, 'Oh God, this is just so sad and so depressing.' I kind of sit there and think 'oh woe is me'." She also reported having had to stop listening to this music at one stage because she had been getting too depressed, and stated that the music "probably just intensified the emotion." However, when the interviewer introduced the question of whether some other listening choices might be more helpful, the participant was quick to justify her preferences stating, "If I listen to depressing music when I'm depressed it does have some benefits." Music was intensely important to this participant. She reported listening to music much of each day and spending a lot of time reading about musicians and their lives. The type of music she listened to appeared to be closely connected to her sense of identity, and her attraction to music that was meaningful and musically "masterful," something which she associated with singer-songwriters such as Elliott Smith. Thus, for this participant there appeared to be some resistance to the idea that such music choices could have a negative influence on mental health despite a recognition of past negative experiences.

Participant 2's approach contrasted with the other participants. She described herself as having been better in recent years, but even when going through a period of depression, her music listening choices did not necessarily have a negative outcome. She described using music as a temporary thing to escape from her difficulties. "I remember there were times when I would find a 12 min piece and just put my headphones on, turn it up, probably too loud, and then sit there and enjoy it for $12 \mathrm{~min}$ and that was 12 min that I didn't have to deal with everything else." This demonstrated some intentionality of music use, but not necessarily an ability to sustain the wellbeing benefits with music. Participant 4 similarly described her approach to music listening when depressed as "a way of soothing my emotions rather than solving them."

Thus the participants in general described unconscious listening that worsened their mood as a past way of using music when they had little to no insight about their emerging mental health problems. This suggests that when participants were not aware of how poor their mental health was becoming, they used music in ways that contributed to their deterioration and stopped once something challenged them - the realization of depression, comments by family or friends, or therapy. However, the same listening behaviors sometimes seemed to continue or re-occur in current circumstances especially when there was a deterioration in wellbeing.

\section{Pathways to More Conscious Music Use}

The emphasis placed by grounded theory analysts on actions and interactions encourages modeling of diverse pathways through a phenomenon (Strauss, 1987; Morse et al., 2016). For this study, the findings suggested a model of the pathways young people with symptoms of depression take through music use that reflect differing strategies for dealing with undesirable moods (see Figure 2). The model demonstrates how listeners may have the intention to either cope with or to change an undesirable mood, but outcomes vary depending on the strategy used, which in turn is influenced by the individuals' fluctuating level of awareness. In this model, individuals have the antecedent condition of depression and are influenced in their music listening selections by the central condition, which is the state of being in an undesirable mood. They then exercise their intention to either cope with their mood or change their mood through strategies that involve selecting either mood matching music or music that is different to their mood. These selections are influenced by their own changing levels of awareness which in turn, are influenced by intervening conditions such as negative experiences or discussions with family, friends, or a therapist. These differing strategies can have varying outcomes, with mood matching music generally leading to either maintenance of mood or feeling worse, and listening to music that is different to the initial undesirable mood generally resulting in mood repair or a temporary change to mood.

\section{DISCUSSION}

This study focused on exploring the degree to which young people with symptoms of depression are aware of the effect their music listening choices have on mood and wellbeing, and how they reach that state of awareness. Our findings demonstrated that most young people in our study reported past behaviors reflecting limited awareness and unconscious motivations, often with undesirable outcomes. However, intervening conditions including insights gained from friends, family, a therapist or through self-reflection, resulted in some increased awareness. Previous research has demonstrated that increased awareness of the effect of music listening choices can be deliberately influenced, such as through use of the Healthy-Unhealthy Music Scale as an awareness raising tool (Saarikallio et al., 2015; McFerran et al., 2018).

However, in the current study, some young people demonstrated an initial resistance to increased awareness, or a reversion to previous unhelpful patterns of music listening even after reaching a level of awareness, particularly during depressive 


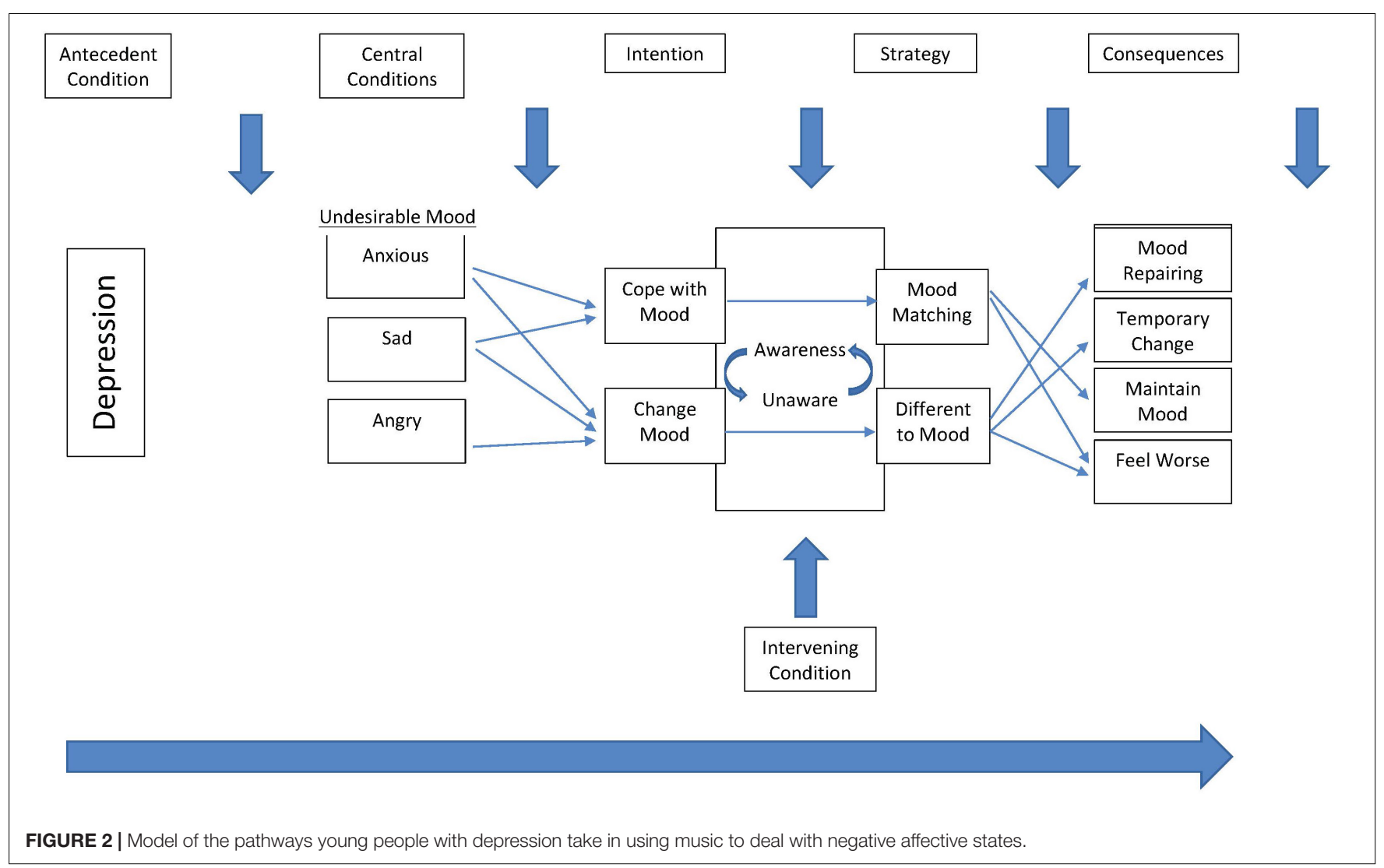

episodes. Thus, the pathways young people took through music listening and awareness of the effects of their music listening choices were not always linear. Skill building appeared to be a gradual process of discovery by continual cycling through varying intentions, strategies and outcomes, with new levels of consciousness being reached as new events and experiences challenged old behaviors.

Of note in the current study was that some participants described being more able to use music to change states such as anxiety or anger even when these were quite severe, but were less inclined to use this strategy when feeling depressed. When seeking to change an anxious mood, for example, participants reported listening to calming music - music that did not match their current mood. In contrast, when dealing with depression, many of the participants reported using music that maintained their current mood in order to feel validated and to have their feelings acknowledged.

While the intention of participants was to use music to help them cope with their depressed mood, this sometimes resulted in intensifying the state. It may be that the different interactional strategies used in each case contributed to the differing outcomes since research suggests that listening to mood shifting music is often more effective than listening to sad music when feeling depressed (Garrido and Schubert, 2015b). Alternately, it may be that anxiety is more amenable to influence by music listening. In a systematic review of studies relating to music and people with dementia, for example, it was found that music can reliably reduce agitation in patients, while the effects of music on symptoms of depression are less consistent (Garrido et al., 2018). Anxiety is often exacerbated by a fear of the symptoms of anxiety themselves (Dugas et al., 2012), but calming music can reduce physiological symptoms of anxiety thereby inducing a relaxation response (Hamel, 2001). On the other hand, depression is often closely related to thought patterns which may be less likely to be altered when listening to music, particularly if the music echoes the existing negative thoughts.

It may be this relationship between thought patterns and depression that can help explain why the benefits of listening to distracting music was sometimes time-limited, ceasing as soon as the song was over. This has further been noted in CheongClinch's study of adolescents with mental illness (Cheong-Clinch and McFerran, 2016), where music was found to mediate mood momentarily, but it was more difficult for young people to achieve sustained benefits. As reflected in our findings, the content of the lyrics often had an influence on whether positive benefits were achieved. When participants listened to music that mirrored their current circumstances this appeared to have less desirable mood outcomes, possibly because listening to such music is akin to ruminating. In contrast, outcomes were more positive for participants in the current study when they listened to music with optimistic messages. Previous research has similarly demonstrated that the thoughts triggered by music have a greater impact on mood outcomes than features of the music itself (Garrido et al., 2016). Thus, listening to music that is distracting 
as opposed to music that alters mood via shifting thought patterns, may be only of temporary benefit.

Nevertheless it is important not to ignore momentary benefits for people struggling with depression. Some theorists suggest that the cumulative benefits of positive moments can serve as protective factors that eventually lead to improved wellbeing (Rutter, 2012). More pragmatically, young people who are struggling with suicidal thoughts appreciate even small periods of escape (Cheong-Clinch and McFerran, 2016). Such brief distractions are helpful in that they reduce time spent ruminating and can reduce the incidence of self-harm and suicide attempts (Polanco-Roman et al., 2015). Furthermore, studies in music therapy have indicated that selecting music that matches one's mood as the beginning point of a process that gradually shifts toward more positive music - a strategy known as the iso-principle - can produce a more enduring repair of mood (Davis et al., 2008). The participants in the current study did report experiencing some lessening of the intensity of their negative moods after listening to moodmatching music. It is possible that for some, the reduced intensity of their negative moods was the beginning of a process of recovery. However, the data in this study did not reveal this clearly, and it is likely that the long-term outcomes of this process differ from individual to individual particularly in situations where the person has a high level of unawareness about the thinking patterns and emotions being triggered by the music.

\section{Clinical Implications}

While some participants in this study described reaching awareness of their strategies for music use on their own, external input such as from friends, family or a therapist was also described and has been categorized as intervening conditions in Figure 2. Although this may suggest that telling young people to be more careful about their music listening could be beneficial, a broader cultural context is also at play. Young people report feeling resentful of the judgments made about their music choices, and one function of music is often described as being to assert an independent identity, beyond parental authority (Laiho, 2004). In a previous study, we were able to encourage young people seeking support for depression to contemplate their music listening habits, but this occurred within a respectful conversation that involved both validating music preferences as well as dialoguing about consequences (McFerran et al., 2018).

It is also common for caring adults to mistake the mechanism of action in this scenario and to blame the qualities of the music itself, rather than focusing on how music choices reflect mental health. This has historically been a point of contention between fans of heavier genres, such as Rock and Rap, and correlations are frequently found with antisocial behaviors (Lozon and Bensimon, 2014). Nevertheless, a causal relationship between particular music genres and mental illness or problem behaviors has never been established (North and Hargreaves, 2006). Rather, complex interactions between an array of personal and social mechanisms underlie our emotional reactions to music (Juslin et al., 2015). Interventions that focus on self-reflection and raising awareness of the interaction between thoughts and feelings triggered by our music listening choices are likely to be more successful than those targeting particular music genres or styles.

\section{CONCLUSION}

There is ample evidence to demonstrate that people use music to improve their mood on a daily basis, both in everyday life (DeNora, 2000; Saarikallio, 2007; McFerran et al., 2015; Papinczak et al., 2015) and in music therapy (Maratos et al., 2009; CheongClinch, 2013; Bibb and Skwews McFerran, 2018). There is also an emerging body of research which seeks to qualify these findings, since it is clear that music is not a magic pill that can immediately resolve a negative mood and nor is it always helpful. This research contributes to this second discourse, highlighting how individual's uses of music can result in various outcomes depending on a range of factors. Individuals can use music listening to improve, maintain or intensify a mood, and may do any of these things at various times. Although it appears that people with depression are most likely to use music to intensify a negative mood, they are also the least aware of this tendency. This is further complicated by the finding that an individual can become aware of unhelpful listening habits, but can lose that awareness when in a depressive state and revert to intensifying strategies.

The current study is limited by the fact that the sample was primarily female. This gender imbalance is not unusual in studies relating to mental health (see for e.g., Lindner et al., 2016), and is likely a reflection of the higher rates of depression among females (Freeman and Freeman, 2013). Nevertheless, future studies could benefit from recruitment of a more balanced sample so as to explore gender differences in strategy selection and outcomes of music use. Future research should also consider the influence of cultural context. The current study included at least one participant from a non-English speaking background. While music tastes among young people are becoming increasingly globalized (Cicchelli and Octobre, 2017), culture nevertheless has an impact not only on music selections, but on the way individuals value particular emotional experiences (Oishi et al., 2007).

Using music to influence mood is likely to continue to be a popular strategy for many people, both in their everyday life and through music therapy or other therapeutic contexts. Therefore, our ability to predict when this is likely to be more or less helpful and to develop strategies for supporting people during the most difficult moods is critical. However, the nuances of the pathways through music listening and toward an improved mood are complex and need to be individually identified and negotiated. The findings from this research indicate that promoting awareness of the power of music to enhance any mood is helpful, but that we should be prepared for circuitous pathways and open to change in all directions when people engage with their preferred music. 


\section{ETHICS STATEMENT}

The study was approved by the Human Ethics Committee of the University of Melbourne. Written consent was provided by all participants.

\section{REFERENCES}

Barrett, L. F., Gross, J., Christensen, T. C., and Benvenuto, M. (2001). Knowing what you're feeling and knowing what to do about it: mapping the relation between emotion differentiation and emotion regulation. Cogn. Emot. 15, 713-724.

Bianco, N. J., Otto, A. R., Maddox, T. W., Beevers, C., and Love, B. (2013). The influence of depression symptoms on exploratory decision-making. Cognition 129, 563-568. doi: 10.1016/j.cognition.2013.08.018

Biasutti, M. (2013). Ochestra rehearsal strategies: conductor and performer views. Music. Sci. 17, 57-71.

Biasutti, M. (2018). Strategies adopted during collaborative online music composition. Int. J. Music Educ. 36, 473-490.

Bibb, J., and Skwews McFerran, K. (2018). Musical recovery: the role of group singing in regaining healthy relationships with music to promote mental health recovery. Nord. J. Music Ther. 27, 235-251. doi: 10.1080/08098131.2018. 1432676

Blakemore, S.-J., and Frith, C. (2003). Self-awareness and action. Curr. Opin. Neurobiol. 13, 219-224.

Brown, B. D., and Bobkowski, P. S. (2011). Older and newer media: patterns of use and effects on adolescents health and well-being. J. Res. Adolesc. 21, 95-113.

Charmaz, K. (2006). Constructing Grounded Theory: A Practical Guide Through Qualitative Analysis. London: SAGE.

Charmaz, K., and Bryand, A. (2007). "Grounded theory and credibility," in Handbook of Qualitative Psychology, eds C. Willig and W. Stainton-Rogers (London: SAGE), 291-306.

Cheong-Clinch, C. (2013). Musical Diaries : An Investigation of Preferred Music Listening by Young People with Mental Illness in Various Contexts and Conditions. Melbourne, AU: The University of Melbourne.

Cheong-Clinch, C., and McFerran, K. (2016). Musical diaries: examining the preferred music listening of australian young people with mental illness. J. Appl. Youth Stud. 1, 77-94.

Chun Tie, T., Birks, M., and Francis, K. (2019). Grounded theory research: a design framework for novice researchers. SAGE Open Med. 7:2050312118822927. doi: $10.1177 / 2050312118822927$

Cicchelli, V., and Octobre, S. (2017). Youth culture in the age of globalisation: an approach via cultural and aesthetic cosmopolitanism. Culture Etudes 1, $1-20$.

Davis, W. B., Gfeller, K. E., and Thaut, M. H. (2008). An Introduction to Music Therapy: Theory and Practice, 3rd Edn. Spring, MA: American Music Therapy Association.

DeNora, T. (2000). Music in Everyday life. Cambridge: Cambridge Press.

Dey, I. (2007). “Grounding categories," in The Sage Handbook of Grounded Theory, eds A. Bryant and K. Charmaz (London: Sage), 167-190.

Dillman Carpentier, F. R., Brown, J. D., Bertocci, M., Silk, J. S., Forbes, E. E., and Dahl, R. C. (2008). Sad kids, sad media? Applying mood management theory to depressed adolescents' use of media. Media Psychol. 11, 143-166.

Dugas, M. J., Laugesen, N., and Bukowski, W. M. (2012). Intolerance of uncertainty, fear of anxiety, and adolescent worry. J. Abnorm. Child Psychol. 40, 863-870. doi: 10.1007/s10802-012-9611-1

Finlay, L. (2002). "Outing the researcher: the provenance, process and practice of reflexivity. Qual. Health Res. 12, 531-545.

Flynn, M., and Rudolph, K. D. (2014). A prospective examination of emotional clarity, stress responses, and depressive symptoms during early adolescence. J. Early Adolesc. 34, 923-939. doi: 10.1177/0272431613513959

Freeman, D., and Freeman, J. (2013). The Stressed Sex: Uncovering the Truth About Men, Women and Mental Health. Oxford: Oxford University Press.

Garrido, S. (2018). The influence of personality and coping style on the affective outcomes of nostalgia: is nostalgia a healthy coping mechanism or rumination? Pers. Individ. Differ. 120, 259-264. doi: 10.1016/j.paid.2016.07.021

\section{AUTHOR CONTRIBUTIONS}

SG and KM developed the initial project design. JS and SG undertook the data collection. All authors contributed substantially to data analysis, write up, and development of the conceptual model.

Garrido, S., Bangert, D., and Schubert, E. (2016). Musical prescriptions for mood improvements: a mixed methods study. Arts Psychother. 51, 46-53.

Garrido, S., Eerola, T., and McFerran, K. (2017). Group rumination: social interactions around music in people with depression. Front. Psychol. 8:490. doi: $10.3389 /$ fpsyg. 2017.00490

Garrido, S., and Schubert, E. (2013). Adaptive and maladaptive attraction to negative emotion in music. Music. Sci.17, 145-164.

Garrido, S., and Schubert, E. (2015a). Moody melodies: do they cheer us up? A study of the effect of sad music on mood. Psychol. Music 43, 244-261. doi: $10.1177 / 0305735613501938$

Garrido, S., and Schubert, E. (2015b). Music and people with tendencies to depression. Music Percept. 32, 313-321. doi: 10.1525/MP.2015.32.4.313

Garrido, S., Stevens, C., Chang, E., Dunne, L., and Perz, J. (2018). Music and dementia: individual differences in response to personalized playlists. J. Alzheimer's Dis. 64, 933-941. doi: 10.3233/JAD-180084

Hamel, W. J. (2001). The effects of music intervention on anxiety in the patient waiting for cardiac catheterization. Intensiv. Crit. Care Nurs. 17, 279-285.

Henry, J. D., and Crawford, J. R. (2005). The short-form version of the depression anxiety stress scales (DASS-21): construct validity and normative data in a large non-clinical sample. Br. J. Clin. Psychol. 44, 227-239.

Hense, C., McFerran, K., and McGorry, P. (2014). Constructing a grounded theory of young people's recovery of musical identity in mental illness. Arts Psychother. 41, 594-603.

Hense, C., Silverman, M., and McFerran, K. (2018). Using the healthy-unhealthy uses of music scale as a single session music therapy intervention on an acute young mental health inpatient unit. Music Ther. Perspect. 36, 267-276. doi: 10.1093/mtp/miy013

Juslin, P. N., Barradas, G., and Eerola, T. (2015). From sound to significance: exploring the mechanisms underlying emotional reactions to music. Am. J. Psychol. 128, 281-304.

Keedwell, P. (2008). How Sadness Survived: The Evolutionary Basis of Depression. Milton Keynes: Radcliffe Publishing.

Kranzler, A., Young, J. F., Hankin, B. L., Abela, J. R. Z., Elias, M. J., and Selby, E. A. (2016). Emotional awareness: a transdiagnostic predictor of depression and anxiety for children and adolescents. J. Clin. Child Adolesc. Psychol. 45, 262-269. doi: 10.1080/15374416.2014.987379

Laiho, S. (2004). The psychological functions of music in adolescence. Nord. J. Music Ther. 13, 47-63.

Lawrence, D., Johnson, S., Hafekost, J., Boterhoven De Hann, K., Sawyer, M., Ainley, J., et al. (2015). The Mental Health of Children and Adolescents. Report on the Second Australian Child and Adolescent Survey of Mental Health and Wellbeing. Canberra: Department of Health.

Lindner, P., Carlbring, P., Flodman, E., Hebert, A., Poysti, S., Hagkvist, F., et al. (2016). Does cognitive flexibility predict treatment gains in Internet-delivered psychological treatment of social anxiety disorder, depression, or tinnitus? PeerJ 4:e1934. doi: 10.7717/peerj.1934

Lozon, J., and Bensimon, M. (2014). Music misuse: a review of the personal and collective roles of "problem music". Aggress. Violent Behav. 19, 207-218.

Maratos, A., Gold, C., Wang, X., and Crawford, M. (2009). Music Therapy for Depression. London: The Cochrane Library.

McFerran, K. (2016). Contextualising the relationship between music, emotions and the well-being of young people: a critical interpretive synthesis. Music. Sci. 20, 103-121.

McFerran, K., Garrido, S., O'Grady, L., Grocke, D., and Sawyer, S. M. (2015). Examining the relationship between self-reported mood management and music preferences in Australian teenagers. Nord. J. Music Ther. 24, 1-17. doi: 10.1080/08098131.2014.908942

McFerran, K., Garrido, S., and Saarikallio, S. (2016). A critical interpretive synthesis of the literature linking music and adolescent mental health. Youth Soc. 48, 521-538. 
McFerran, K., Hense, C., Koike, A., and Rickwood, D. (2018). Intentional music use to reduce psychological distress in adolescents accessing primary mental health care. Clin. Child Psychol. Psychiatry 23, 567-581. doi: 10.1177/ 1359104518767231

McFerran, K., and Saarikallio, S. (2014). Depending on music to feel better: being conscious of responsibility when appropriating the power of music. Arts Psychother. 41, 89-97.

Meade, T., and Dowsell, E. (2016). Adolescents health-related quality of life (HRQoL) changes over time: a three year longitudinal study. Health Qual. Life Outcomes 14:14. doi: 10.1186/s12955-016-0415-9

Miranda, D., Gaudrea, P., Debrosse, R., Morizot, J., and Kirmayer, L. (2012). "Music listening and mental health: variations on internalizing psychopathology," in Music, Health and Wellbeing, eds A. R. M. Raymond, G. Kreutz, and L. Mitchell (New York, NY: Oxford University Press), 513-530.

Morse, J., Stern, P. N., Corbin, J., Bowers, B., Charmaz, K., and Clarke, A. (2016). Developing Grounded Theory: The Second Generation. New York, NY: Routledge.

National Institute of Mental Health (2017). Major Depression. Bethesda, MD: National Institute of Mental Health.

North, A. C., and Hargreaves, D. J. (2006). Problem music and self-harming. Suicide Life Threat. Behav. 36, 582-590.

Oishi, S., Schimmack, U., Diener, E., Kim-Prieto, C., Scollon, C. N., and Choi, D.-W. (2007). The value-congruence model of memory for emotional experiences: an explanation for cultural differences in emotional self-reports. J. Pers. Soc. Psychol. 93, 897-905.

O'Keeffe, G. S., and Clarke-Pearson, K. (2011). The impact of social media on children, adolescents and families. Pediatrics 127, 800-804. doi: 10.1542/peds. 2011-0054

Papinczak, Z. E., Dingle, G. A., Stoyanov, S. R., Hides, L., and Zelenko, O. (2015). Young people's uses of music for well-being. J. Youth Stud. 18, 1119-1134. doi: 10.1080/13676261.2015.1020935

Polanco-Roman, L., Jurska, J., Quinones, V., and Miranda, R. (2015). Brooding, reflection and distraction: relation to non-suicidal self-injury versus suicide attempts. Arch. Suicide Res. 2015, 350-365. doi: 10.1080/13811118.2014.981623
Riggs, S. E., Grant, P. M., Perivoloitis, D., and Beck, A. T. (2012). Assessment of cognitive insight: a qualitative review. Schizophr. Bull. 38, 338-350. doi: $10.1093 / \mathrm{schbul} / \mathrm{sbq0} 05$

Rutter, M. (2012). Resilience as a dynamic concept. Dev. Psychopathol. 24, 335-344. doi: 10.1017/S0954579412000028

Saarikallio, S. (2007). Music as Mood Regulation in Adolescence. yväskylän yliopisto: University of Jyväskylä.

Saarikallio, S., Gold, C., and McFerran, K. (2015). Development and validation of the healthy-unhealthy music scale. Child Adolesc. Mental Health 20, 210-217.

Strauss, A. (1987). Qualitative Analysis for Social Scientists. Cambridge: Cambridge University Press.

Strauss, A., and Corbin, J. (2008). Basics of Qualitative Research: Techniques and Procedures for Developing Grounded Theory, 3rd Edn. Thousand Oaks, CA: SAGE.

Sullivan, M. D., O’Connor, O., and Feeney, P. (2012). Depression predicts all-cause mortality: epidemiological evaluation from the ACCORD HRQL substudy. Diabetes Care 35, 1708-1715. doi: 10.2337/dc11-1791

Trapnell, P. D. (1997). Reflection-Rumination Questionnaire Short Forms Data. Vancouver, BC: University of British Columbia.

WHO (2017). Depression and Other Common Mental Disorders: Global Health Estimates. Geneva: World Health Organization.

Wilhelm, K., Gilllis, I., Schubert, E., and Whittle, E. L. (2013). On a blue note: depressed people's reasons for listening to music. Music Med. 5, 76-83.

Conflict of Interest Statement: The authors declare that the research was conducted in the absence of any commercial or financial relationships that could be construed as a potential conflict of interest.

Copyright (c) 2019 Stewart, Garrido, Hense and McFerran. This is an open-access article distributed under the terms of the Creative Commons Attribution License (CC BY). The use, distribution or reproduction in other forums is permitted, provided the original author(s) and the copyright owner(s) are credited and that the original publication in this journal is cited, in accordance with accepted academic practice. No use, distribution or reproduction is permitted which does not comply with these terms. 


\section{APPENDIX: INTERVIEW GUIDE}

(1) Could you start by telling me what kind of music you like?

(2) How important is music to you in your life?

(3) Is it something you listen to every day?

(4) Do you tend to focus on it most of the time or is it just in the background?

(5) How important are the lyrics in the music?

(6) Do you ever find yourself listening to music to try to influence your mood one way or another?

(7) What sort of effect would it have on you when you do that?

(8) Would you find it annoying to listen to something upbeat when you are feeling low?

(9) Have you ever listened to music that made you feel worse?

(10) Do you think its possible that sometimes music could make you feel worse? If so, what kind? When and how? 
OPEN ACCESS

Edited by: Graham Frederick Welch, UCL Institute of Education, United Kingdom

Reviewed by:

Kimberly Sena Moore,

University of Miami, United States Lori Gooding,

Florida State University, United States

${ }^{*}$ Correspondence:

Rebecca Vaudreuil

rebecca_vaudreuil@mail.harvard.edu

Specialty section:

This article was submitted to Performance Science, a section of the journal

Frontiers in Psychology

Received: 07 September 2018

Accepted: 14 January 2019

Published: 05 February 2019

Citation:

Vaudreuil $\mathrm{R}$, Bronson $\mathrm{H}$ and Bradt J (2019) Bridging the Clinic to Community: Music Performance as

Social Transformation for Military Service Members.

Front. Psychol. 10:119. doi: 10.3389/fpsyg.2019.00119

\section{Bridging the Clinic to Community: Music Performance as Social Transformation for Military Service Members}

\author{
Rebecca Vaudreuill,2*, Hannah Bronson ${ }^{1}$ and Joke Bradt ${ }^{3}$ \\ ${ }^{1}$ National Endowment for the Arts, Washington, DC, United States, ${ }^{2}$ National Intrepid Center of Excellence, Bethesda, MD, \\ United States, ${ }^{3}$ Department of Creative Arts Therapies, Drexel University, Philadelphia, PA, United States
}

The use of music performance in music therapy with military service members is discussed as a vehicle for social transformation and reintegration. The use of performance in music therapy is not without controversy primarily because therapy is considered a process, not a product, and confidentiality and privacy are essential components of therapy. However, others have argued that public performances can validate therapeutic changes in clients, give voice to their experiences, raise awareness of social issues within their communities, transform perceptions of injury, or illness in audience members, and may result in the clients gaining support and validation from their communities. We discuss the potential of music performances to contribute to individual development, reinforce rehabilitation, enhance function, and facilitate change at the community level to support reintegration of military service members. We illustrate this through two brief case reports of service members who received music therapy as part of their treatment for post-traumatic stress disorder, traumatic brain injury, and other psychological health concerns at the National Intrepid Center of Excellence, a Directorate of the Walter Reed National Military Medical Center, Bethesda, MD, United States. The service members wrote, learned, and refined songs over multiple music therapy sessions and created song introductions to share with audiences the meanings and benefits gained from integrating performance in music therapy. The case reports also include excerpts of interviews conducted with these service members several months after treatment about their experiences of performing and the perceived impact of their performances on the audience and greater community.

Keywords: performance, music, music therapy, social transformation, military service members, traumatic barin injury, post traumatic stress disorder

\section{INTRODUCTION}

Music performance is an integral part of the creative process. Whereas the general public may associate the concept of performance with entertainment, performances are increasingly used to stimulate discourse related to socio-political and psychological issues (e.g., Yotis et al., 2017). In music therapy, performance is utilized to give voice to clients' experiences and facilitate greater understanding of their struggles Baker, 2013; O'Grady et al., 2015). Recently, media outlets 
have reported on music performances by military personnel who have sustained physical and/or psychological injuries (Public Broadcasting Service, 2017; KXTV, 2018). Little attention has been paid in the literature, however, to the unique benefits and challenges of the use of performance with military populations. The purpose of this perspective article is to discuss the potential for growth and social transformation for service members when integrating performance into music therapy. In addition, we present considerations to ensure that performance is an enriching experience for service members and audiences. We illustrate this through two brief case reports of service members who chose to integrate performance into music therapy treatment.

\section{THE USE OF PERFORMANCE IN MUSIC THERAPY}

Board-certified music therapists use a variety of music experiences within a therapeutic relationship to address individualized goal areas (e.g., emotional, cognitive, physical, social, spiritual) (American Music Therapy Association [AMTA], 1998-2018), which may include music-guided relaxation, expressive/(e.g., singing, instrument playing) and receptive/(e.g., listening to live/recorded music) music engagement, and songwriting. The focus of music therapy is on the therapeutic process, not the musical product (Ansdell, 2010); therefore, many music therapists are opposed to public performances by clients as this may blur boundaries of privacy and confidentiality (Ansdell, 2010). Additionally, music therapists have cautioned against incorporating performance because performance-related stress and potential adverse audience responses may put clients at risk (Turry, 2005). However, music therapists who practice within a community music therapy model (Ansdell, 2005; Soshensky, 2011; Stige and Aarø, 2012) have proposed that performance has significant potential for enhancing clients' health and psychosocial well-being. Ruud (2004) argued that performances in music therapy facilitate negotiation of "space between the private and the public, the client and the institution, or the client and the community." O'Grady et al. (2015) encourage music therapists to "shift the focus to when, not if, performance might be considered an appropriate method in music therapy" (p.124).

Performance integrated in music therapy offers opportunities for personal transformation and social change. First, performance provides a context for clients to creatively connect with audiences and share their experiences through artistic expression (Day et al., 2009). Through performance, clients can bring their experiences into a public space, which can raise awareness about personal struggles and societal (mis)perceptions, including mental health stigma (O'Grady, 2009). Second, it provides opportunities for clients to step outside of their comfort zones through structured risk-taking (Baker, 2013). Third, performance allows clients to demonstrate their skills and take pride in creativity (Baker, 2013). Fourth, positive feedback and public acknowledgment may enhance a client's self-confidence and motivation (O'Grady, 2009; Baker, 2013). Finally, performing can reciprocally impact the client-audience relationship: audiences can witness healthy parts of clients rather than deficits, and clients can feel supported by audiences, potentially resulting in transformative experiences (Soshensky, 2011; Baker, 2013).

\section{MUSIC PERFORMANCE BY MILITARY SERVICE MEMBERS}

\section{Music Therapy in Military Settings}

Music therapy is "a reflexive process wherein the therapist helps the client to optimize the client's health, using various facets of music experience and the relationships formed through them as the impetus for change" (Bruscia, 2014, Chapter 4, para. 5). Music therapy is utilized in the United States to treat service members recovering from service-related injuries, specifically traumatic brain injury (TBI) and posttraumatic stress disorder (PTSD). The National Endowment for the Arts (NEA) Creative Forces: NEA Military Healing Arts Network is a partnership with the United States. Departments of Defense and Veterans Affairs and the state and local arts agencies with administrative support provided by Americans for the Arts. Creative Forces programming places creative arts therapies at the core of treatment models in military healthcare (National Endowments for the Arts, n.d.), and is established at 11 military facilities throughout the United States (Bronson et al., 2018). Before considering performance in clinical work, music therapists address functional rehabilitation (e.g., cognition, speech/language, sensorimotor) and behavioral health needs (Bronson et al., 2018). For example, a United States Army Captain co-authored a case report citing music therapy cotreatment as integral to his interdisciplinary rehabilitation. It motivated him to surpass his functional and psychological goals, ultimately leading to performances on local, national, and international platforms (Vaudreuil et al., 2018).

\section{Performance as a Vehicle for Rehabilitation and Social Transformation}

Performance contributes to service members' agency in recovery processes and has the potential to facilitate change and enhance overall perception of self and others by mastering challenges of TBI and PTSD. We find it helpful to conceptualize the levels of transformation per Bronfenbrenner's Ecological Systems theory (Bronfenbrenner, 1977), specifically how people interact, adapt, and develop within their environments. Social transformation is a fluid process for the individual and occurs within the microsystem (e.g., installation, clinic) and in the macrosystem (e.g., culture, community). Figure 1 shows a model adapted from Bronfenbrenner to reflect the levels of environment that impact military personnel.

Performance provides opportunities for service members to be viewed in ways that enhance interactions within different environments (e.g., family, occupational, and community). It allows service members to increase awareness of selfperceptions, challenge belief systems, and view treatment as an evolving mechanism for change. Historically, the image of the warrior across many cultures has represented strength, power, and indestructibility, while demonstrating emotions and 


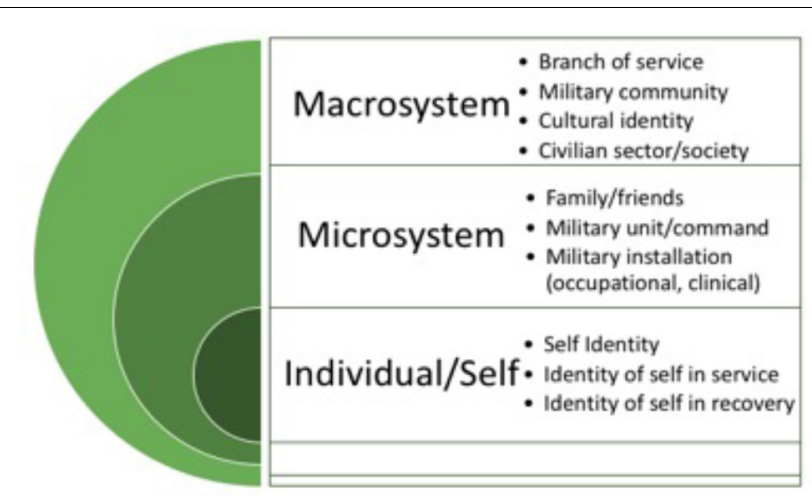

FIGURE 1 | Systems of social transformation for service members (based on Bronfenbrenner, 1977).

vulnerability has been interpreted as weakness (Peace et al., 2016). Performance allows reframing of the service members' and audiences' perspectives, transforming vulnerability to be recognized as resilience. A major transformation that service members ascribe to performance is a shift in perception from primarily internal to one that supports simultaneous awareness of internal and external processes. They describe public performances as validating and report that it allows expression of their inner-selves while feeling connected to audiences.

Through observing service members' performances, audiences, commonly comprised of family, peers, clinicians, and community members can: (1) increase awareness of service members' unique strengths by observing their products of music therapy, (2) comprehend service members' experiences in an innovative way, (3) shift perspective of service members as healthy and resilient, rather than viewing deficits, and (4) develop an understanding of how to construct supportive communities that better accommodate service member (re)integration.

\section{Clinical Considerations of Performance in Music Therapy}

Performance is not a requirement of music therapy, rather an optional component that can serve to cultivate tactical creativity and culminate therapeutic experiences. If service members decide to perform, they have complete autonomy over their experience including: (1) target audience, (2) arrangement/dynamics, (3) accompaniment/instrumentation, and (4) co-performance participation. Co-performances can occur between service member and peer(s), music therapists, providers from other disciplines, visiting musicians, or any combination of these. In some cases, performances are recorded and used as auditory feedback for service members to gain insight, further process experiences, and have as a tangible item to take with them.

It is important to consider how music therapists utilize the therapeutic relationship to support performance. Transparency regarding potential benefits and risks of performance must be addressed prior to client engagement in performance. Service members may be hesitant about performing personal content and therapists must remain acutely aware of this by placing emphasis on preparation, continuous evaluation of the experience, and offering emotional support throughout (Baker, 2013). There are many reasons why service members may feel uncomfortable sharing their experiences through performance, including stigma of injury and the perceived lack of understanding from the civilian sector. If the service member does not possess the internal resources to withstand increased vulnerability, performance can be counter-therapeutic or re-traumatizing; thus, the clinician must recognize the client's unique capacity for risk-taking (Baker, 2013). In addition, it is essential that informed consent is established prior to the client engaging in performance. This provides consistency of client autonomy and optimization of choice and control when introducing integration of performance in music therapy. Ethical safeguards were employed with the service members highlighted in the case report section of this article to mitigate risk of performance. Clear and continuous communication on a spectrum from intervention to performance was incorporated throughout preperformance preparation, support during the performance, and post-performance processing.

\section{PERFORMANCE INITIATIVES AND CASE EXAMPLES}

We present here two examples of performance initiatives integrated into military music therapy programs followed by two brief case reports of service members who received music therapy in intensive outpatient and longitudinal care at the NICoE and performed at the Creative Arts Café as part of their treatment. Case examples are based on clinical observations, service member interviews, and focus on how performance shaped social transformation. Both service members were interviewed about their experiences several months after treatment. A content analysis of the interviews focused on three areas: (1) music therapy treatment, (2) transformation through performance, and (3) post-music therapy transition. Please note that pseudonyms are used to maintain client confidentiality.

\section{Resounding Joy's Semper Sound Band}

The Semper Sound Band (est. 2011) is a component of Resounding Joy's Military Music Therapy program (Resounding Joy, n.d.). Performance was a natural progression for service members who first received individual music therapy and progressed to group sessions. Mutual interest amongst group members led to a 'jam group', which resulted in the formation of the band. Performance allows band members to showcase talents to family members, peers, clinicians, and communities through events, on and off base.

\section{Creative Forces Creative Arts Café}

Based on the concept of the Semper Sound Band, the Creative Arts Café is an initiative developed at the National Intrepid Center of Excellence (NICoE) in 2016 and replicated across Creative Forces sites (Bronson et al., 2018). The Creative Arts Café is a performance space where service members can highlight 
certain aspects of their treatment processes in creative arts therapies. It serves as a platform for service members, family members, and staff to share creativity through the arts. It supports the clinic to community continuum, as service members often first perform in music therapy and then may decide to perform on base and in the greater community. It is important to note that the Creative Arts Café initiative is open to all forms of artistry (music, art, dance, writing/spoken word, theatre, etc. ..), however, the case reports presented below focus exclusively on music performance.

\section{Case Report 1 Background}

"Greg" is a male in his twenties and was a Sergeant (SGT) in the United States Army. He was an unrestrained passenger in a Humvee rollover in 2014. He suffered intracranial hemorrhages, presented unresponsive, and was intubated. Greg was medevacked, stabilized, and returned to the United States for ongoing treatment. He was referred to music therapy by a nurse case manager for cognitive deficits due to severe TBI. Greg received a music therapy assessment in January 2016 and received weekly individual and small group sessions for 15-months as part of outpatient treatment.

\section{Music Therapy Treatment}

Upon initial music therapy assessment, it was determined that Greg was experiencing expressive speech issues in addition to memory and coordination deficits. The music therapist consulted with speech therapy to create integrative treatment approaches. Music therapy sessions were designed to assist his breathing, speech fluency, and rhythmic phrasing. A few months into music therapy, Greg and a fellow service member were working on a song that supported shared speech and cognition goals. The music therapist determined that it was appropriate to offer performance as an opportunity to: (1) encourage active participation in treatment, (2) enhance self-practice, and (3) engage with an audience while applying skills learned in music therapy. Greg and his peer were working on the song, "We Didn't Start the Fire" by Billy Joel and opted to perform it as a duet. In his interview "Greg" stated, "I chose that song, because I knew for a fact that it would help with my speech...and it helped with my memory."

Therapeutic singing, rhythmic cueing, and vocal prosody exercises were used to address hypernasality, slurred speech, fluidity, intonation, and articulation. Greg stated, "[Music therapy] helped my pronunciation of words, [performance] pushed me harder in music therapy, which in turn pushed my brain harder to focus on my words and my motor functions." He furthermore acknowledged how performance had helped him address vocal and cognitive changes experienced since his accident: "My voice changed since I got hurt...I didn't really want to talk to people... With music therapy, I learned not to be ashamed of my voice, and that it won't get better if I don't talk. It helped me with my memory because I had to remember lyrics, chord changes, and everything else...it made a great impact overall." Through performance in music therapy, Greg gained insight into personal struggles and was motivated to continue engaging in independent practice and performance. He shared, "Watching expressions of people I don't even know...seeing and feeling that someone you've never met before [is] showing happiness and joy from your performance. . that is what is really empowering."

\section{Performance as Motivation for Therapeutic Work}

Greg participated in monthly performances as a therapeutic tool to provide awareness of his successes in rehabilitation goals. Eventually, he was able to simultaneously sing and play, which is a high-level cognitive process and an ongoing goal. He recounts, "Between music therapy sessions, I was rehearsing. Every time I got stuck on a word, I went back to my room and [would] say it, and my speech got clearer. It made me feel amazing after [performing] because, honestly I noticed every time I did something, people would be amazed." Performance supported Greg in changing from an internal focus to being able to communicate with others using music. This was observed by a shift in song selections from those that solely challenged his speaking/singing ability to songs that he connected with on a deeper level. Intentional song selection eventually led to Greg writing original compositions that reflected his belief systems and included messages to inspire himself and others. He spoke about the emotional benefits and comfort that he experienced from performance in music therapy, "With music, that's your friend, your therapist, and everything beyond, and it doesn't have anything bad to say about you ever."

\section{Generalization of Music Therapy in Transition}

When Greg started music therapy, it was difficult and uncomfortable for him to speak on command or initiate conversations. Through the process of preparing for performances, which also included verbal song introductions, he continued to make clinical gains post-music therapy treatment, including speaking at his retirement ceremony and national public performances. Greg medically retired in May 2017 and is currently attending college with aspirations to become a motivational speaker. He reflected about his experiences performing in music therapy, "[Performance] worked my body harder. If I did not have music therapy, I would not be doing as well as I am today. I [still] incorporate what music expresses in my life."

\section{Case Report 2 Background}

"Chad" is a male in his thirties and was a Staff Sergeant (SSgt) in the United States Marine Corps. He served multiple combat deployments over his 13 years of service, during which he experienced blast exposure. Upon homecoming, Chad was diagnosed with mild TBI, chronic PTSD, and other combatrelated psychological health concerns. He initially engaged in individual and group music therapy through the intensive outpatient program. Chad continued music therapy for 1 year post-IOP, in weekly individual sessions.

\section{"Tuning in" to Treatment}

Chad's music therapy goals addressed pervasive symptoms of chronic PTSD, as well as mild TBI issues of cognition, emotional 
regulation, and expression. Music therapy sessions incorporated various interactive experiences (e.g., active music making, lyric analysis, songwriting) to assist him in processing his military service and positively reframing self-perception and identity. Chad often selected songs by preferred artists to express things that were difficult to communicate prior to music therapy. He recounts, "Something about music made it easier to talk about issues that were bothering me. We spoke about issues that I hadn't even discussed with my [psycho] therapist at that point." After 8 months of individual sessions, Chad opted to perform a song at a Creative Arts Café that he had been preparing for his wedding. The integration of personally meaningful music into music therapy motivated his engagement in performance. Lyric analysis and songwriting facilitated emotional expression, as Chad modified songs to reflect his values. He shared, "I was able to add a spin on the song from my perspective, with help from the music therapist, which involved changing lyrics at the end, "Mercy," look what's become of us/One by one we turned it around/Maybe carry on just a little bit longer/God's gonna give us what we need" (adapted from "Mercy" Dave Matthews Band, 2012).

\section{Transforming Trauma}

A song of particular significance that Chad subsequently performed was an arrangement of "Walls" by Kings of Leon. He reflected on the song's symbolism," 'When the walls come down,' that's how I felt music therapy was helping me...my walls coming down." Chad's musical engagement often inspired feelings of hope and reduction of personal guilt as reflected in his comment, "I feel like singing or even whistling means that there is some kind of happiness inside me, and it's not all dark...Music therapy is like God answering my prayers. A light in the darkness, and a reassurance that while I have experienced horror, I am not the cause of it all." He shared how performing helped him reduce isolation, "Performances can help to reconnect with people and even give others a chance to understand me more." The reciprocal experience of self-understanding while feeling "heard" by the audience allowed for continuous progression in treatment.

\section{Moving to the Next "Stage"}

As Chad began preparing for his transition from active duty, he transferred skills developed through performance to continue growing in his life and relationships. His evolving interpretations of songs he performed in music therapy reflected his progression in treatment. For example, when he first used "Mercy" in sessions, the meaning was focused on in-the-moment needs, specifically symptom management. It progressed to address broader areas, namely humanization and becoming a better partner. Ultimately, Chad sang and played bass and percussion in a multimedia project of "Mercy" that he presented this to his wife, family, and friends at his wedding. He speaks about how the spirituality of the song aligned with his transformation in recovery, "The first line in the song is, "Don't give up". Even though I felt like I had so many times, I didn't give up, and to this day I am alive and not giving up. It was a sad song for me when the demons were in control, but God transformed the song into healing when it took on a new meaning for my wife and I, when we listened to it together.
It transformed again as we grew as a couple, and we both went through really hard times along the path to recovery. [My wife] stayed with me through everything...there was no way I could let this song not be a part of our wedding day and music therapy was the reason it became a part of it." Chad medically retired in July 2017.

\section{CONCLUSION}

Performance is an impactful tool for music therapists to utilize in support of clients' transformative processes. When integrated with apt intention and appropriate oversight, performance can be clinically conducive, informative, and reciprocally beneficial for service members and audiences. Performance helps educate military leadership, clinical providers, and the general public about the power of incorporating creative expression into structured environments. Creative Arts Cafés are models replicated across multiple sites that serve to bridge the clinic to community, supporting the overall transformation of service members and their families by validating their experiences and allowing for expression of their inner-selves while feeling connected to audiences. Musical performance benefits our society as a whole, supporting personal, collective, and societal transformation. This shapes the performer as well the perception of audiences and builds stronger, healthier communities in which individuals thrive.

\section{DISCLOSURE STATEMENT}

The views expressed in this article are those of the author and do not reflect the official policy of the Department of the Army/Navy/Air Force, Department of Defense, or United States Government. The identification of specific products, scientific instrumentation, organizations, individuals or compositions is considered an integral part of the research endeavor and does not constitute endorsement or implied endorsement on the part of the author, DoD, or any component agency.

\section{ETHICAL DISCLOSURE}

The case studies presented in this article were determined exempt by the NICoE/WRNMMC institutional review committee. Written informed consent was obtained from the participants for the publication of the case reports.

\section{ETHICS STATEMENT}

This study was carried out in accordance with the recommendations of the National Intrepid Center of Excellence/Walter Reed National Military Medical Center $\mathrm{PAO}$ and designated as exempt from ethics committee review. All subjects gave written informed consent in accordance with the Declaration of Helsinki. The protocol was approved by the 
National Intrepid Center of Excellence/Walter Reed National Military Medical Center PAO.

\section{AUTHOR CONTRIBUTIONS}

$\mathrm{RV}$ is a board-certified music therapist and served as contract support to the NICoE through National Endowment for the Arts, Creative Forces: NEA Military Healing Arts Network. During her tenure at the NICoE, she was the primary music therapist of the service members who were highlighted in the case report section of this article. As lead author, she conceptualized this article and took the lead in writing the article. She was responsible for final edits of the manuscript. HB contributed to content analysis of the interviews and writing the manuscript. JB contributed to sections of the manuscript.

\section{FUNDING}

This work was supported by the National Endowment for the Arts, Creative Forces: NEA Military Healing Arts Network.

\section{REFERENCES}

American Music Therapy Association [AMTA] (1998-2018). What is Music Therapy?. Available at: https://www.musictherapy.org/

Ansdell, G. (2005). Being Who You Aren't; Doing What You Can't. Community Music Therapy \& The Paradoxes of Performances. Voices: A World Forum for Music Therapy. Available at: https:/voices.no/index.php/voices/article/view/ 1719/1479 doi: 10.15845/voices.v5i3.229

Ansdell, G. (2010). "Where performing helps: processes and affordances of performance in community music therapy," in Where Music Helps: Community Music Therapy in Action and Reflection, eds B. Stige, G. Ansdell, C. Elefant, and M. Pavlicevic (Aldershot: Ashgate), 161-188.

Baker, F. A. (2013). Front and center stage: participants performing songs created during musc therapy. Arts Psychother. 40, 20-28. doi: 10.1016/j.aip.2012.09.004

Bronfenbrenner, U. (1977). Toward an experimental ecology of human development. Am. Psychol. 32, 513-531. doi: 10.1037/0003-066X.32.7.513

Bronson, H., Vaudreuil, R., and Bradt, J. (2018). Music therapy programs for treatment of active duty military populations: an overview of long and shortterm care models. Music Ther. Perspect. 36, 195-206. doi: 10.1093/mtp/miy006

Bruscia, K. (2014). Defining Music Therapy, 3rd Edn. Oak Park, IL: Barcelona Publishers.

Dave Matthews Band (2012). Mercy. Away from the World.

Day, T., Baker, F., and Darlington, Y. (2009). Beyond the therapy room: Client experiences of 'going public' with song creations. Br. J. Music Ther. 23, 19-26. doi: $10.1177 / 135945750902300103$

KXTV (2018). Music Therapy Helping Camp Pendleton Marines with Traumatic Brain Injuries. Available at: https://www.youtube.com/watch?v=ytgLhg2MptE

National Endowments for the Arts (n.d.). Creative Forces: NEA Military Healing Arts Network. Available at: https://www.arts.gov/partnerships/creative-forces

O'Grady, L. (2009). The Therapeutic Potentials of Creating and Performing Music with Women in Prison: A Qualitative Case Study. Ph.D. thesis, The University of Melbourne, Melbourne.

O'Grady, L., Rolvsjord, R., and McFerran, K. (2015). Women performing music in prison: an exploration of the resources that come into play. Nord. J. Music Ther. 24, 123-147. doi: 10.1080/08098131.2013.877518

\section{ACKNOWLEDGMENTS}

The authors would like to express heartfelt thanks to the service members highlighted in this article for openly sharing their powerful music therapy and performance experiences. Special recognition to service members and veterans who share their creative artistry through performance and shed a light that cannot be replicated. Special gratitude is extended to all the men and women who bravely serve their country in the United States Armed Forces and the leadership, providers, and staff who assist in their rehabilitation processes. Special thanks to Kalli Pelaccio, MT-BC for her efforts in developing the Creative Arts Café model at NICoE. The authors of this paper would like to acknowledge the contributions of Creative Forces $^{\mathrm{TM}}$ : NEA Military Healing Arts Network, an initiative of the National Endowment for the Arts (NEA) in partnership with the United States Departments of Defense and Veterans Affairs and the state and local arts agencies for their extensive support of creative arts therapies clinical programming and research. Administrative support for the initiative is provided by Americans for the Arts.

Peace, J., Billera, M., and Gerard, G. (2016). Military culture and the transition to civilian life: Suicide risk and other considerations. Soc. Work 61, 83-86. doi: $10.1093 /$ sw/swv050

Public Broadcasting Service (2017). National Memorial Day Concert. Available at: http://www.pbs.org/national-memorial-day-concert/features/music-therapy/

Resounding Joy (n.d.). Semper Sound: Music Therapy. to Assist Military Rehabilitation. Available at: https://resoundingjoyinc.org/semper-sound/

Ruud, E. (2004). Debating the winds of change in community music therapy. Voices: A world forum for music therapy. Available at: http://www.voices.no/ discussions/discm4_04.html

Soshensky, R. (2011). Everybody is a star: Performing, recording and community music therapy. Music Ther. Perspect. 29, 23-30. doi: 10.1093/mtp/29.1.23

Stige, B., and Aarø, L. E. (2012). Invitation to Community Music Therapy. New York, NY: Routledge.

Turry, A. (2005). Music psychotherapy and community music therapy: Questions and considerations. Voices World Forum Music Ther. 5, doi: 10.15845/voices. v5i1.208

Vaudreuil, R., Avilla, L., Bradt, J., and Pasquina, P. (2018). Music therapy applied to complex blast injury in interdisciplinary care: a case report. Disabil. Rehabil. doi: 10.1080/09638288.2018.1462412. [Epub ahead of print].

Yotis, L., Theocharopoulos, C., Fragiadaki, C., and Begioglou, D. (2017). Using playback theatre to address the stigma of mental disorders. Arts Psychother. 55, 80-84. doi: 10.1016/j.aip.2017.04.009

Conflict of Interest Statement: The authors declare that the research was conducted in the absence of any commercial or financial relationships that could be construed as a potential conflict of interest.

Copyright (c) 2019 Vaudreuil, Bronson and Bradt. This is an open-access article distributed under the terms of the Creative Commons Attribution License (CC BY). The use, distribution or reproduction in other forums is permitted, provided the original author(s) and the copyright owner(s) are credited and that the original publication in this journal is cited, in accordance with accepted academic practice. No use, distribution or reproduction is permitted which does not comply with these terms. 


\section{OPEN ACCESS}

Edited by:

Evangelos Himonides,

University College London,

United Kingdom

Reviewed by:

Monica Esslin-Peard,

University of Liverpool, United Kingdom

Francisco Cuadrado,

Universidad Loyola Andalucía, Spain

*Correspondence:

Andrew King

a.king@hull.ac.uk

Specialty section:

This article was submitted to

Performance Science,

a section of the journal

Frontiers in Psychology

Received: 22 December 2018 Accepted: 26 February 2019

Published: 22 March 2019

Citation:

Waddington-Jones C, King A and Burnard $P$ (2019) Exploring

Wellbeing and Creativity Through Collaborative Composition as Part of Hull 2017 City of Culture. Front. Psychol. 10:548. doi: 10.3389/fpsyg.2019.00548

\section{Exploring Wellbeing and Creativity Through Collaborative Composition as Part of Hull 2017 City of Culture}

\author{
Caroline Waddington-Jones ${ }^{1}$, Andrew King ${ }^{1 \star}$ and Pamela Burnard ${ }^{2}$ \\ ${ }^{1}$ School of Music, University of Hull, Kingston upon Hull, United Kingdom, 2Faculty of Education, University of Cambridge, \\ Cambridge, United Kingdom
}

Several studies have highlighted the positive effects of group music-making and have suggested that it may be the creative and social aspects of such activities, which have a positive effect on participants' wellbeing. Collaborative composition offers strong examples of both aspects as participants work together to create new material. However, although it seems likely that participants' influence over and ownership of the creative material contributes to these positive effects, studies have yet to examine these elements in detail. Through analysis of video observations, pre- and post-project interviews, video recall interviews, and questionnaires, this article aims to: (1) evaluate the impact of participation in collaborative composition workshops on the subjective and psychological wellbeing of older adults and (2) identify skills and approaches employed by the composer-facilitators in order to understand more fully the approach and skills employed to engage participants effectively in the creative process. This second aim is of particular interest given the current movement toward social prescribing and arts and health interventions in the UK. Analysis revealed that all dimensions of the PERMA framework for subjective and psychological wellbeing were present in this collaborative composition project. The specific nature of collaborative composition is considered in comparison with other forms of group musical engagement. For older adults, collaborative composition has much to offer as an activity encouraging social interaction with others with shared interests, increasing positive affect, and enhancing self-esteem. Analysis of workshop videos and interviews with composers identified various facilitation skills employed by the composers to establish safe creative space and to encourage participants to engage in the process of collaborative composition.

Keywords: wellbeing, collaborative composition, older adults, creativity, Hull 2017, PERMA

\section{INTRODUCTION}

Since ancient times, humans have been fascinated with how to live a good life, with prominent thinkers such as Aristotle arguing that wellbeing is the overarching purpose of all human actions (Aristotle, 2004). In recent years, there has been increased research interest in wellbeing, as people seek to learn how they might lead healthier and happier lives. There has been much debate within the realm of positive psychology over how to define wellbeing, with researchers 
broadly conceptualizing two dimensions of wellbeing: subjective and psychological (e.g., Diener et al., 1999; Deci and Ryan, 2008; Proctor et al., 2009).

Our modern conceptualization of subjective wellbeing is rooted in the utilitarian hedonist philosophies of John Stuart Mill and Jeremy Bentham who both argued that pleasure is central to wellbeing. This dimension of wellbeing primarily concerns our subjective judgments of how satisfied we are with life (Diener, 1984) and emphasizes high positive affect and low negative affect (Bradburn, 1969; Kahneman et al., 1999). Subjective wellbeing involves the pursuit of happiness and is based on the principle that the more positive emotion we experience, the happier we will be (Seligman, 2002). By contrast, psychological wellbeing has emerged from Aristotle's concept of eudaimonia and accentuates positive psychological functioning and human development (e.g., Rogers, 1961; Waterman, 1993). Various dimensions of positive psychological functioning have been proposed, including how we relate to other people, how independent we are, how we engage in various experiences, and how we find meaning in our lives (e.g., Seligman et al., 2005; Ryan et al., 2008).

More recently, positive psychologists have suggested that in order for humans to flourish fully, a balance of the elements of both subjective and psychological dimensions of wellbeing is required (Peterson et al., 2005; Sirgy and Wu, 2009; Seligman, 2010). Seligman (2010) has proposed a framework that incorporates elements of both dimensions of wellbeing: PERMA (Positive emotions, Engagement, Relationships, Meaning, Accomplishment). Positive emotions are the subjective wellbeing element of the PERMA model. The more positive affect or pleasure we experience, the happier we will be. Engagement in this context is closely connected to flow theory (Csikszentmihalyi, 1975) and refers to the experience of total immersion in an activity. The relationships' dimension of the framework recognizes that humans are inherently social and that we thrive through our connections to and interactions with others. Having a clear sense of meaning or purpose in life seems to be a major component of human wellbeing and is the key to understanding motivation (De Muijnck, 2013). Finally, Seligman (2010) considers accomplishment to be a key motivation for many people. It is closely connected to goals and ambition, and it seems likely that gaining mastery or competence in a particular skill may increase self-esteem.

\section{Older Adults and Wellbeing}

As people are now living longer lives, more research is being dedicated to understanding how we can flourish later in life. A review of the existing literature on older adults and wellbeing suggests that the Engagement and Relationships dimensions of PERMA may be particularly relevant. Cumming and Henry (1961) have proposed that as adults age, they disengage from society physically and socially, and that society also withdraws from older adults. Empirical research has suggested that older adults who are most connected report greater satisfaction with themselves and their lives-or greater subjective wellbeing (Bjorklund, 2011). Research has found that social engagement, in the form of social activities, productive activities, helping activities, formal and informal learning, and leisure, is positively correlated with older adults' physical and mental health (Herzog et al., 2002), and that joint participation in leisure activities with members of one's social network has been positively associated with life satisfaction (Holman and Jacquart, 1988).

Elsewhere, studies have found that participation in adult education has positive impacts on older adults' psychological wellbeing (Schuller et al., 2002; Feinstein and Hammond, 2004; Hammond and Feinstein, 2006). This tended to take the form of increased self-esteem and seems likely to be related to the Accomplishment as well as the Engagement and Relationships dimensions of PERMA. Through mastering a new skill or acquiring knowledge, as well as engaging in a new activity and socializing with others, participants' psychological wellbeing increases.

\section{Musical Engagement and Wellbeing}

The field of Arts and Health in the UK is flourishing at present, particularly in light of the current emphasis on health promotion (see e.g., Coulter and Gordon-Nesbitt, 2016) and the formation of the All-Party Parliamentary Group on Arts for Health and Wellbeing in 2014. Most recently, in the UK, there has been a movement toward social prescribing, which advocates for engagement in arts-based activities to enhance wellbeing. Several studies have highlighted the positive relationship between musical engagement and both subjective and psychological dimensions of wellbeing (e.g., Hays and Minichiello, 2005; Ritchie and Williamon, 2011; Van Goethem and Sloboda, 2011; Croom, 2015). It has been argued that active rather than passive musical engagement tends to provide benefits for an individual's psychological as well as subjective wellbeing (e.g., Csikszentmihalyi, 2002; Hallam et al., 2012; Creech et al., 2013). It seems likely that this may be due to the higher levels of engagement, the opportunities for selfexpression, and the subsequent sense of accomplishment and clarity of identity inherent in active music-making.

Croom (2015) has proposed that the PERMA framework (Seligman, 2010) provides a useful lens through which to examine musical engagement and wellbeing. He cites evidence for musical engagement increasing positive affect (e.g., Laukka, 2007; Van Goethem and Sloboda, 2011; DeMarco et al., 2012), offering opportunities for deep engagement (e.g., Pates et al., 2003; Dietrich, 2004; Rogatko, 2009; de Manzano et al., 2010; Peifer, 2012), connecting with other people (e.g., Packer and Ballantyne, 2011; Procter, 2011; Schiepe-Tiska and Engeser, 2012; Koelsch, 2013; Rabinowitch et al., 2013; Ballantyne et al., 2014), bringing a sense of meaning or purpose to people's lives (Frith, 1996; DeNora, 2000; Hays and Minichiello, 2005), and accomplishment (e.g., Hylton, 1981; Edgerton, 1994; Lee and Nantais, 1996; Hiscock et al., 2013; Klaphajone et al., 2013). Musical engagement, therefore, has the potential to contribute positively to all dimensions of wellbeing.

\section{Older Adults, Musical Engagement, and Wellbeing}

Musicking in the lives of older people has begun to receive a lot of attention in recent years, with studies examining, for 
example, the benefits of everyday musical engagement for older adults (e.g., Hays and Minichiello, 2005), adults with dementia (e.g., Sixsmith and Gibson, 2007), and the impact learning a musical instrument can have on wellbeing for older adults (Perkins and Williamon, 2014).

\section{Group Musicking}

Wellbeing in relation to engagement in group music activities has been a particular focus of studies, as researchers seek to understand more fully this long-acknowledged positive impact of musicking with others (Small, 1999). Creech et al. (2013) investigated the relationship between engagement in group music-making activities and wellbeing according to the Basic Psychological Needs Scale (Deci and Ryan, 2008) and the CASP-12 measure of quality of life in later life (Higgs et al., 2003), finding that, even compared to other group activities, group music-making had a significant positive impact on participants' self-reported wellbeing for the "purpose," "control and autonomy," and "social affirmation" components of these scales. Elsewhere, Hallam and Creech (2016) combined questionnaires with interviews and observations to examine the effects of participation in community music activities can have on older adults' wellbeing. Again, compared to a control, the older adults participating in group music-making scored higher on quality of life instruments. Analysis of the qualitative data revealed benefits for health, emotional, and cognitive dimensions of wellbeing. Similarly, in a study characterizing collaborative and communal creativities in instrumental group learning and performance, Burnard and Dragovic (2014a,b) found the physicality of performance and levels of performance development to be sites for enhancing pupil wellbeing.

\section{Collaborative Composition}

Although such studies have considered that it may be the creative and social aspects of group music-making which particularly influence wellbeing, so far only one study (Habron et al., 2013) has explored the potential benefits of group music-making involving the collaborative creation of new musical material. The researchers found that the sense of control over musical materials, opportunities for creativity and identity formation, as well as social interaction with other participants and musicians were all beneficial for participants' wellbeing. This is supported by other studies on collaborative composition, which have highlighted its potential for inducing flow (Macdonald et al., 2006) and have considered the collaborative aspect of the activity both socially and musically (Miell and MacDonald, 2000; Burland and Davidson, 2001; Hopkins, 2015; Williams, 2018).

Collaborative composition has not been considered yet through the lens of PERMA. However, as a form of active group musical engagement, and based on the evidence from these existing studies of collaborative composition and group music-making, it seems reasonable to suggest that collaborative composition may influence all dimensions of PERMA. While the participants in Habron et al.'s (2013) investigation of the relationship between collaborative composition and wellbeing had creative control over the music realized by the professional musicians involved in the project, they did not create the music themselves. Furthermore, no study has yet examined the facilitative skills required by composers in order to engage participants in collaborative composition. The present research therefore sought to explore the perceived impact of collaborative composition and group performance on diverse groups of people and to identify skills and approaches employed by the composer-facilitators.

\section{Hull 2017: New Music Biennial}

As part of the program for Hull City of Culture 2017, five composer residencies took place over 4 months within various communities in the city as part of the PRS Foundation's New Music Biennial. Designed to spark interest in collaborative composition and new music, the composers ran workshops with different groups of participants, including refugees and asylum seekers, travelers, older adults, primary school children, formerly homeless people, and vulnerable young people. The residencies culminated in two public performances at Hull City Hall, involving performances from each composer's groups and a "supergroup" performance delivered by all the groups together.

Here, we present an evaluation of two of the composer residencies that sought to engage older people and primary school children: the first, a song-writing project bringing together older people living in retirement communities and children from a primary school in East Hull; the second, the creation of an ambisonic sound installation facilitated by a beatboxer and sound artist of international acclaim with members of choral group based in Hull. Two research questions will be addressed:

1. What was the perceived impact of engaging in collaborative composition in terms of psychological and subjective wellbeing?

2. What skills were required by the composers as workshop facilitators, engaging diverse groups of participants in collaborative composition?

\section{MATERIALS AND METHODS}

\section{Participants}

The participants were two male, non-Hull-based, professional composers (Composer A and Composer B). Neither composer had a teaching qualification, but both had extensive experience of facilitating composition workshops, and this was the main reason that these two residencies were chosen out of the five for data collection and evaluation. Composer A worked with 25 members of a local choral group, a community choir whose membership changes on a project-by-project basis. Some members of the chorus were new for this project. Composer B worked with nine residents of two Hull-based homes from an independent Housing Association for older people in Hull. These residents formed a new group for the project. Later in the residency, they were joined by 25 years four pupils from a primary school in East Hull to collaborate for a final performance. 


\section{Procedure}

The two composers were interviewed at the start and at the end of their four-month residencies. Interviews were semistructured and questions focused on the composers' intentions for their residencies, their perception of possible challenges, and what they hoped the impact of the residency might be on the participants. Post-residency interviews followed a similar question structure but sought to reveal what had actually happened during the residency, what the challenges had been, and what the composers perceived to have been the impact of their residencies.

Two-hour workshop sessions with each composer were observed, and three of these observed sessions were also videorecorded. In addition to this observational data, recall interviews were conducted with two participants in Composer A's residency using excerpts of the video data from one of the workshops. Finally, post-project questionnaires were administered to participants at one of the performance days at City Hall at the end of the project. These asked participants about their perception of the impact of the residency on them.

\section{Analysis}

Interview, observational, and questionnaire data were reviewed, transcribed, and collated using NVivo. Thematic analysis was undertaken using an inductive approach modeled on grounded theory in which the aim of the analysis was to describe the data and theorize the findings. Themes were developed by collapsing, combining, or extending initial codes.

\section{RESULTS AND DISCUSSION}

In terms of the perceived impact of participating in the collaborative composition workshops and group performances on wellbeing, an inductive thematic analysis of the video-recall interview and post-residency questionnaire data revealed various themes. These themes were then considered in relation to the dimensions of PERMA, and some close connections were identified. Table 1 shows the theme and sub-themes, which emerged from the analysis.

\section{Enjoyment}

Participants described or displayed "Enjoyment" of sessions. This theme seems to relate closely to the positive emotion dimension of PERMA. Examples of this theme from the postresidency questionnaires included comments such as "The project has been uplifting-I look forward to rehearsals" and "Uplifting-lifts the soul. Wonderful." Reflecting on the residency, one of the video-recall interview participants explained:

It was fun, and I wanted to do more of it. To be part of different things ... I have really enjoyed it and it definitely gave me a lot of energy and inspired me for the time in between the workshops and then afterwards. (Videorecall interview participant 1)
TABLE 1 | Themes and sub-themes for perceived impact of residencies according to participants.

\begin{tabular}{ll}
\hline Theme & Sub-theme \\
\hline Enjoyment & \\
Togetherness & Social cohesion \\
& Musical cohesion \\
Empowerment & Engagement \\
& Meaning \\
& Accomplishment \\
\hline
\end{tabular}

The positive emotion dimension of PERMA is connected to subjective wellbeing: the more positive affect we experience, the happier we will be. Since the participants found the workshops uplifting and fun, it seems likely that their participation in the workshops will have increased their subjective wellbeing.

\section{Togetherness}

The theme "Togetherness" variously described bonding between participants ("social cohesion") and coming together as a group musically ("musical cohesion") and seems to be closely related to the Relationships dimension of PERMA.

\section{Social Cohesion}

In reflecting on their experiences of the residency, many participants emphasized their pleasure at making new friends and being together on a regular basis as a group. Comments from the Big Elastic Band on the post-residency questionnaires included: "I have made some great friends"; "I have really enjoyed getting out and being together with others in the group"; "I have taken away a spirit of willingness: wanting to learn and participate with everybody-children and adults." Hull Freedom Chorus participants commented that they: "enjoyed being together"; "enjoyed meeting new people"; and "felt involved and happy with a changing group of singers."

Despite being a group formed especially for this residency, it was evident that the Big Elastic Band members grew to value the social dimension that the collaborative composition workshops provided. This seems to connect closely to the Relationships dimension of the PERMA framework. The collaborative composition workshops offered participants a means of bringing together people with a shared interest in creating music who would not otherwise meet. The Big Elastic Band had been formed especially for the collaborative composition residency, and the 25 members of Hull Freedom Chorus who came together for this residency included several individuals who had not been a part of the group previously.

\section{Musical Cohesion}

In the post-project questionnaires, participants commented on the impact of the residency in terms of being able to "working with and listening to others" and "feeling able to make a creative contribution to the group." While reviewing the video footage of a workshop, both video recall participants described their experiences of creating new sounds as a group: 
I did have my eyes closed and I could hear everyone else. It amplified everything. Well that's what the sea does, there's all sorts of different sounds in the sea. It's not just one sound, it's all sorts of different sounds, and that's what it sounded like to me. It just sounded like the sea. If you think of ripples of the sea going over pebbles. Each pebble is a different shape and making a different sound as it rushes back. That's what it felt like to me. Everybody was doing it, like the sea ebbing away. It was a big sound and that's what the sea is, it's a big sound. (Video-recall interview participant 2)

Here, the participant describes a moment of group vocal improvisation in which the group are exploring the sounds of the sea. Research in group music-making has suggested that creating music together involves communication, cooperation, empathy, and ultimately may facilitate increased social cohesion (King, 2006; Seddon and Biasutti, 2009; Koelsch, 2013; Waddington, 2017). Since many participants in the present study reported that their participation in the residencies led to the formation of new friendships and a sense of group togetherness, the findings of this analysis seem to support the existing evidence in this area. Since collaborative composition involves co-creating new musical material rather than reproducing existing musical material, the various social and musical processes underlying this co-creation may be more pronounced.

In music educational contexts, Rusinek (2012) has suggested that when engaging in collaborative composition, participants operate in the "zone of proximal development" (Vygotsky, 1978) in which they co-construct musical understanding and new musical material. It is possible that these shared experiences of musicking and co-creation of musical material also contribute positively toward strengthening social relationships and an emerging sense of group identity.

\section{Empowerment}

The theme of "Empowerment" broke down into sub-themes of "Engagement," "Meaning," and "Accomplishment": dimensions of PERMA.

\section{Engagement}

In the Engagement sub-theme, participants described moments of total absorption in the creative process. When reviewing some workshop footage, one of the video-recall participants explained: "I found the sounds provoke the images for me. That's what I liked about it. I was able not just to immerse myself in the sound which developed but the images came as well. I really appreciated that. I liked that" (video-recall interview participant 2). Such moments of absorption in these creative activities are characteristic of flow experiences (Csikszentmihalyi, 1975). Research has suggested that the more flow we experience, the happier we will be (Csikszentmihalyi, 2002). The finding of the present study that engaging in collaborative composition and group musicking may lead to flow experiences adds further weight to the existing evidence that group musical engagement in general and collaborative composition in particular can positively contribute to psychological wellbeing (e.g., Macdonald et al., 2006; Waddington, 2017).

\section{Meaning}

In their post-residency questionnaires, participants spoke about how the workshops had contributed to the structure of their weeks. One participant commented that the Big Elastic Band workshops were an important moment in their week to "get out and be together with others in the group." Many participants wrote that they were looking forward to continuing to sing and compose together as a group. It seems likely that they had gained a sense of group identity and purpose.

\section{Accomplishment}

The post-residency questionnaires recorded many examples of accomplishment. Participants described various musical achievements: "confidence in singing (especially in a smaller group, it has proved you can sing, even though you are in your 80s)"; "musically-I now know how to make a song from scratch"; "stepping out of my comfort zone"; and "opened my mind to a greater variety of options for experimenting with sound and my voice." One of the video-recall participants also noted that she "had a different feeling afterwards, like a sense of achievement because I did the drill and I got to hear myself being the drill." A few participants also noted that this sense of accomplishment had led to, e.g., "greater confidence on a personal level."

\section{Collaborative Composition and Wellbeing}

There are many similarities between collaborative composition and other forms of active group music-making, such as ensemble playing. Both activities involve musical interaction, potential for experiencing flow, and socioemotional bonding. However, unlike ensemble playing, collaborative composition also involves creating new musical material. Consequently, there is a sense of group and individual ownership over the creative process in collaborative composition that may not be so prominent in ensemble playing activities. This aspect of ownership in collaborative composition also differs somewhat from group improvization in which the musical material is recorded in some form to enable reproduction. Capturing group members' creative contributions in this way offers tangible evidence of accomplishment. This may enhance participants' sense of achievement and increase self-esteem.

Another facet of collaborative composition, which differs from other forms of group musical engagement, is the potential for facilitating interaction and strengthening relationships between group members. Collaborative composition in the residencies evaluated for the present paper involved participants drawing on their life experiences or their personal memories of Hull as inspiration for the creation of songs or vocal soundscapes. As such, there were many opportunities for sharing personal experiences in each of the groups as part of the workshops as part of group discussions at the start of the creative process or through sharing ideas on 
the shaping of the musical material once composition had begun. It is likely that the sharing of these personal experiences facilitated socioemotional bonding between the group members. Below are the lyrics to one of the songs composed by the Big Elastic Band.

\section{Whisper}

I whisper in the night to my lost love

To dance with romance

To dance with romance

I took a chance

Forever in my heart

The love of my life

I wasn't ready for you to go

Oh, how I loved you so

In the silence, you answer me

You say you needed to be free

Forever in my heart

The love of my life

Memories you left so clear

Will always keep you near

Through the heartache and pain

I know I will dance again

I know we will dance again some day.

(Lyrics to a song written by the Big Elastic Band)

This was one example of several sets of lyrics that members shared with the rest of the group. The lyrics to this song were written by one of the group members about a personal life experience. She shared the lyrics with the rest of the group who then all worked together, facilitated by Composer B, to create a melody for the song. The lyrics to this song are deeply personal to this group member. That she felt able to share her words with the rest of the group may be taken as evidence of the trust and the relationship that developed between members over the relatively short span of the residency. This adds further weight to the findings of the analysis of the interview and questionnaire data above that revealed that participants felt that their participation in the residencies had resulted in the development and strengthening of relationships with other members of the group.

These two elements-the recorded evidence of group or individual contributions and the opportunities for sharing personal experiences or ideas-seem to be unique to collaborative composition and relate closely to the Accomplishment and Relationships dimensions of PERMA. As with other forms of group music-making and as demonstrated in the analysis of the interview and questionnaire data here, collaborative composition can also contribute positively to the other dimensions of PERMA and, hence, to subjective and psychological wellbeing. For older adults in particular who may be less socially engaged or have lower self-esteem than younger adults (Orth et al., 2010), collaborative composition activities may positively contribute to the Relationships and Accomplishment dimensions of wellbeing.

Since there is compelling evidence in the rich, qualitative data analyzed here that collaborative composition had numerous benefits for older adults' subjective and psychological wellbeing, it is important that we also seek to understand how these participants can be engaged successfully in collaborative composition. Encouraging participants to share ideas and engage in the creative process requires a lot of skill from the composers as facilitators move beyond their musical expertise. They must be able to build a rapport with the group members and establish a safe, non-judgmental space within the group that promotes this kind of engagement.

\section{Composers as Facilitators}

These two composers were identified by the City of Culture managers in their post-project interviews as particularly good examples of composers working collaboratively to create material with participants. In their pre-project interviews, the composers identified two key challenges when considering how to approach engaging participants in collaborative composition during their residencies.

Composer A reflected on the challenge of coming in as an outsider and building trust with the participants in his residency in order to facilitate a creative process:

I think the first part of the challenge is building up a trust with people in Hull. I'm very conscious about being an artist that goes to a place where I'm not from ...So, the whole thing for me is about really working with the people from Hull, to produce something that will not only go out, but will also hopefully remain in Hull. So I see myself more as a catalyst to facilitate a process that will come from the people that are already there. (Composer A, pre-residency interview)

The challenges of encouraging participants to engage in the creative process were also highlighted by Composer $\mathrm{B}$, as he spoke about trying to bring people together:

And I suppose the challenge is always allowing people to be connected and easy with [the process of collaborative composition].... It's a very fractured community, you have people with all sorts of different abilities and they're coming in, they're coming out, so everything has to grow very organically. I think the challenges are really just about finding different ways that people can kind of connect to it, you know. As soon as they connect to it, it starts being part of what they are and they have a sort of sense of belonging to it and then that helps them grow from there. It's just finding different ways of doing that .... The whole residency is about allowing all of those people to write their own stuff essentially. (Composer B, pre-residency interview)

This composer recognized the additional challenge that at least one of the groups of participants that he was facilitating was formed just to participate in his residency. As a newly formed group, there would be a period of "forming" (Tuckman, 1965) as members settled into the pattern of working together as well as working with the composer-facilitator. This presents a further challenge from the facilitator's perspective in terms of engaging participants in a group creative process such as collaborative composition. 
TABLE 2 | Coding framework for facilitator skills.

\begin{tabular}{ll}
\hline Theme & Sub-themes \\
\hline Confidence and clarity & Demonstrating expertise \\
& Modeling \\
& Precise explanation \\
Facilitator-group dynamics & Intimacy vs. authority \\
& Group ownership \\
& Composer-directed \\
& Praise and feedback \\
& Humor \\
Comfort & Comfort level \\
& Environment and physical needs
\end{tabular}

Common to both of these accounts is a sense that facilitating group ownership rather than composer ownership over the creative process and material produced was a priority for both composers. Composer A described himself as "a catalyst to facilitate a process that will come from the people" and Composer B spoke about participants' sense of "belonging" and connection to the process. It was evident from these pre-residency interviews that these composers both set out to facilitate true collaborative composition rather than taking a more composer-directed approach to their residencies.

Analysis of the video data identified the facilitation skills and tools employed by the composers (see Table 2). The creation of safe space is critical for participants to feel comfortable engaging in a creative process (Rogers, 1993). Both composers used a range of skills to facilitate this engagement. Three themes were identified in the video data: "Confidence and Clarity," "Facilitator-Group Dynamic," and "Comfort." Each theme had various sub-themes.

\section{Confidence and Clarity}

Both composers were coded as "Demonstrating Expertise" at regular intervals. These were moments were the composerfacilitators demonstrated their musical skills and served to remind workshop participants of the composers' expertise and, consequently, encouraged a higher level of confidence in both the composer and the creative process. Both composers modeled techniques frequently rather than explaining verbally. The theme of "Modeling" often overlapped with "Demonstrating Expertise," suggesting that Modeling was a way of explaining exactly what the composer wanted the group to do while also reminding them of the composer's expertise. Ensuring a good understanding of instructions also contributed to the establishment of safe space. When verbal instructions were used, both composers were very clear in outlining ideas (coded as "Precise Explanation"). This seemed to minimize confusion and uncertainty and so contributed to establishing safe space and facilitating creative expression. This supports Macdonald et al.s (2006) finding that the clarity of instructions is crucial for engaging participants in collaborative composition.

\section{Facilitator-Group Dynamic}

There were examples in both composers' workshops of the composers taking on a more authoritative role, to guide the workshop, for example, and a more equal role with the participants, encouraging group members to contribute or take ownership. These moments were coded as "Intimacy vs. Authority." Within this sub-theme was two further sub-themes: "Group Ownership" and "Composer-Directed."

Group Ownership described moments when the composers placed control over the creative material into their group's hands. This was supported by the video-recall interviews in which one participant commented that the composer had "given us that freedom. He's given us that opening to say 'Yes, I want to know what your opinions are' not closed up and said, 'You'll do it my way."' This sub-theme was balanced at times with "Composer Direction," where composers would direct the creative process toward the requisite end product: the performances at City Hall.

To encourage contributions from the group, create a positive atmosphere, and offer reassurance and clarity, both composers offered "Praise and Feedback" to participants at regular intervals throughout the workshops. This seems to be in line with flow theory (Csikszentmihalyi, 1975), which suggests that frequent feedback is a condition for flow. Both composers offered encouragement, acknowledged effort, and praised participants at regular intervals during workshops.

Another tool both composers employed to cultivate a more egalitarian relationship with the group members and to encourage engagement was "Humor." In his pre-residency interview, Composer B explained:

The thing about writing music on any level is that it's like being in this brilliant playground where you are surrounded by lots of stuff, you know. So it's just a question of putting things on top of each other and connecting things together. And it's a real sense of play you know. And that's, I think, what we try to do, we enable this sense of just pure, ridiculous fun in sticking stuff together. And then that leads somewhere. (Composer B, pre-residency interview)

This concept of play for both composers was central to engaging participants. During one of his workshops, composer A explained this to his participants:

Underneath it all it's all about play. It's all about playing. It's like what happens when you are a kid and just sheer, just getting muddy just for the hell of it. Covered in dirt, wow. It's about playing, and what I am trying to say to you is just try to play with stuff and see what happens, you know. That's the most important thing is that we play. (Composer A, workshop 19 June 2017)

This finding supports existing research suggesting that overcoming participants' fear of failure is crucial to engaging them in the creative process and in flow (Macdonald et al., 2006). Taking a playful approach to composition seemed to be strategies employed by both composers in their workshops. Humor seemed to be a mechanism for encouraging play during both composers' workshops. 


\section{Comfort}

Both composers emphasized voluntary participation at individual group members' comfort levels: “Comfort Level.” By emphasizing the voluntary nature of participation, the composers empower the participants, giving them control over their level of engagement in the creative process. The composers also considered group members' comfort on a more practical level. Examples included reminders to rehydrate, asking whether participants would prefer to sit, checking energy levels and the temperature of the room. This was coded as "Environment and Physical Needs." This seems to relate to Maslow's (1987) Hierarchy of Needs, since if participants' basic needs in terms of physical comfort are not met, they will not be able to engage.

\section{The Role of the Composer-Facilitator}

For these residencies, there was a balance between working toward a final product, a public performance, and emphasizing engaging in the creative process. Reflecting this challenge, there was a balance between composer-directed moments, and moments where the composers encouraged the group members to take ownership over the creative process. This way of working draws upon the composers' expertise not only in composition but also in facilitating, and ultimately empowering, their participants.

\section{CONCLUSION}

This study has added further evidence to the existing literature on the relationship between collaborative composition and wellbeing. Analysis revealed that participants in the 4-month collaborative composition residencies for Hull 2017 perceived a positive impact on their subjective and psychological wellbeing, with participants' experiences being closely associated with all five dimensions of the PERMA framework (Seligman, 2010). It is interesting to note that the PERMA framework here emerged from an inductive analysis of the observational, videorecall interview, and questionnaire data rather than from a direct application of the framework to the data. The PERMA framework is beginning to be applied to highlight the benefits of musical engagement in various music educational and performance contexts (e.g., Lee et al., 2017; Ascenso et al., 2018). However, our work here suggests that there is much potential for this framework to be used as an evaluative tool for community music and music therapy projects as well.

The specific nature of collaborative composition has been considered in comparison with other forms of group musical engagement, and the potential for participants to experience a sense of achievement in identifying their own contribution to the group's work as well as the potential for group discussion, particularly focusing on personal experiences, and the impact that may have on strengthening relationships between group members have been highlighted. For older adults, collaborative composition has much to offer as an activity encouraging social interaction with others with shared interests, increasing positive affect, and enhancing self-esteem.
While there is strong evidence here for the value of collaborative composition as an activity that may enhance wellbeing, the intersubjective process of collaborative composition is complex and requires a range of skills from the composer-facilitator in order to engage participants effectively. With the current interest in arts and health interventions and social prescribing, it is vital that consideration be given to the quality of provision of projects such as this. This article has begun to consider some of the skills required to facilitate effective collaborative composition workshops with older adults. The City of Culture managers and the authors had identified the two composer residencies reported here as being the most successful in engaging participants in collaborative composition. Analysis of interview and video data identified the various techniques employed by the composerfacilitators to establish a safe space for participants to feel able to engage in the creative process.

Future work should engage in practices of reconfiguring and re-reading what counts as generative forces of wellbeing and the physicality of collaborative composition and performance practice/engagement and to evaluate the legacy of collaborative composition and performance projects such as this in terms of participants' continued engagement with music-making and the perceived impact on their wellbeing over time. The intention to keep engaging in music activities beyond this project was declared by most participants in post-project questionnaires and recall interviews. Many members of the Hull Freedom Chorus were keen to be involved in future projects exploring beatboxing, rap, or other less conventional styles. Funding has been awarded based on the success of this residency to support further work with the Big Elastic Band and continue their song-writing workshops. We intend to work with some of these participants over the coming months to evaluate their continued engagement in music-making beyond this project.

Nearly 20 years ago, in one of the earliest studies of children's group music compositions, Morgan et al. (2000) argued that the principal element of task activity within groups is the dialog among members. It is surely high time we recognized the importance of continued engagement with such music-making in terms of impact on wellbeing because it allows for ways to think differently and produce, juxtapose, contrast, combine, and communicate ideas through collaborative composition.

\section{DATA AVAILABILITY}

The datasets for this study will not be made publicly available because participants have not consented for their video and interview data to be made available.

\section{ETHICS STATEMENT}

This study was carried out in accordance with the recommendations of "The University Code of Good Research Practice," University of Hull Ethics Committee, with written informed consent from all subjects. All subjects gave written informed consent in 
accordance with the Declaration of Helsinki. The protocol was approved by the University of Hull Faculty of Arts, Culture, and Education Ethics Committee.

\section{AUTHOR CONTRIBUTIONS}

CW-J collected the data, analyzed, and drafted the manuscript. $\mathrm{AK}$ is a principal investigator and designed the study.

\section{REFERENCES}

Aristotle (2004). Nicomachean ethics. London: Penguin.

Ascenso, S., Perkins, R., and Williamon, A. (2018). Resounding meaning: a PERMA wellbeing profile of classical musicians. Front. Psychol. 9:1895. doi: 10.3389/fpsyg.2018.01895

Ballantyne, J., Ballantyne, R., and Packer, J. (2014). Designing and managing music festival experiences to enhance attendees' psychological and social benefits. Music. Sci. 18, 65-83. doi: 10.1177/1029864913511845

Bjorklund, B. R. (2011). The journey of adulthood. 7th edn. Boston, MA: Prentice Hall.

Bradburn, N. M. (1969). The structure of psychological well-being. Chicago: Aldine.

Burland, K., and Davidson, J. W. (2001). Investigating social processes in group musical composition. Res. Stud. Music Educ. 16, 46-56. doi: 10.1177/1321103X010160010901

Burnard, P., and Dragovic, T. (2014a). Collaborative creativity in instrumental group learning as a site for enhancing pupil wellbeing. Camb. J. Educ. 45, 371-392. doi: 10.1080/0305764X.2014.934204

Burnard, P., and Dragovic, T. (2014b). Characterising communal creativity in instrumental group learning. Depart. Crit. Qual. Res. 3, 336-362. doi: 10.1525/ dcqr.2014.3.3.336

Coulter, A., and Gordon-Nesbitt, R. (2016). All Party Parliamentary Group on Arts, Health and Wellbeing: submission to the DCMS white paper consultation. Available at: http://www.artshealthandwellbeing.org.uk/sites/default/files/ APPGAHW\%20submission\%20to\%20DCMS.pdf (Accessed May 11, 2018).

Creech, A., Hallam, S., Varvarigou, M., McQueen, H., and Gaunt, H. (2013). Active music-making: a route to enhanced subjective well-being among older people. Perspect. Public Health 133, 36-43. doi: 10.1177/1757913912466950

Croom, A. (2015). Music practice and participation for psychological wellbeing: a review of how music influences positive emotion, engagement, relationships, meaning, and accomplishment. Music. Sci. 15, 44-64. doi: 10.1177/1029864914561709

Csikszentmihalyi, M. (1975). Play and intrinsic rewards. J. Humanist. Psychol. 15, 41-63. doi: 10.1177/002216787501500306

Csikszentmihalyi, M. (2002). Flow: The classic work on how to achieve happiness. London: Rider.

Cumming, E., and Henry, W. E. (1961). Growing old: The process of disengagement. New York, NY: Basic Books.

De Manzano, O., Theorell, T., Harmat, L., and Ullen, F. (2010). The psychophysiology of flow during piano playing. Emotion 10, 301-311. doi: 10.1037/a0018432

De Muijnck, W. (2013). The meaning of lives and the meaning of things. J. Happiness Stud. 14, 1291-1307. doi: 10.1007/s10902-012-9382-y

Deci, E. L., and Ryan, R. M. (2008). Hedonia, eudaimonia and well-being: an introduction. J. Happiness Stud. 9, 1-11. doi: 10.1007/s10902-006-9018-1

DeMarco, J., Alexander, J. L., Nehrenz, G., and Gallagher, L. (2012). The benefit of music for the reduction of stress and anxiety in patients undergoing elective cosmetic surgery. Music Med. 4, 44-48. doi: 10.1177/1943862111424416

DeNora, T. (2000). Music in everyday life. Cambridge, UK: Cambridge University Press.

Diener, E. (1984). Subjective well-being. Psychol. Bull. 95, 542-575. doi: 10.1037/0033-2909.95.3.542

Diener, E., Suh, E. M., Lucas, R. E., and Smith, H. L. (1999). Subjective wellbeing: three decades of progress. Psychol. Bull. 125, 276-302. doi: 10.1037/0033-2909.125.2.276

Dietrich, A. (2004). Neurocognitive mechanisms underlying the experience of flow. Conscious. Cogn. 13, 746-761. doi: 10.1016/j.concog.2004.07.002
$\mathrm{AK}$ and $\mathrm{PB}$ revised the manuscript. $\mathrm{PB}$ consulted at each stage of project.

\section{FUNDING}

The funder for this research study was Paul Hamlyn, via the Performing Rights Society and Sound and Music. Grant account: YAE048.

Edgerton, C. L. (1994). The effect of improvisational music therapy on the communicative behaviors of autistic children. J. Music. Ther. 31, 31-62. doi: $10.1093 / \mathrm{jmt} / 31.1 .31$

Feinstein, L., and Hammond, C. (2004). The contribution of adult learning to health and social capital. Wider benefits of learning, Research Report No. 8. London: Institute of Education.

Frith, S. (1996). "Music and identity" in Questions of cultural identity. eds. S. Hall and P. Du Gay (London, UK: Sage), 108-127.

Habron, J., Butterly, F., Gordon, I., and Roebuck, A. (2013). Being well, being musical: music composition as a resource and occupation for older people. Br. J. Occup. Ther. 76, 308-316. doi: 10.4276/03080221 3X13729279114933

Hallam, S., Creech, A., Varvarigou, M., McQueen, H., and Gaunt, H. (2012). Perceived benefits of active engagement with making music in community settings. Int. J. Community Music 5, 155-174. doi: 10.1386/ ijcm.5.2.155

Hallam, S., and Creech, A. (2016). Can active music-making promote health and well-being in older citizens? London J. Prim. Care 8, 21-25. doi: 10.1080/17571472.2016.1152099

Hammond, C., and Feinstein, L. (2006). Are those who flourished at school healthier adults? What role for adult education. Wider benefits of learning Research Report No. 17. London: Centre for Research on the Wider Benefits of Learning: Institute of Education.

Hays, T., and Minichiello, V. (2005). The meaning of music in the lives of older people: A qualitative study. Psychol. Music 33, 437-451. doi: $10.1177 / 0305735605056160$

Herzog, A. R., Ofstedal, M. B., and Wheeler, L. M. (2002). Social engagement and its relationship to health. Clin. Geriatr. Med. 18, 595-609. doi: 10.1016/ S0749-0690(02)00025-3

Higgs, P., Hyde, M., Wiggins, R., and Blane, D. (2003). Researching quality of life in early old age: the importance of the sociological dimension. Soc. Policy Adm. 37, 239-252. doi: 10.1111/1467-9515.00336

Hiscock, N., O'Callaghan, C., Goodwin, M., and Wheeler, G. (2013). Music, intelligence, and the neurocognitive effects of childhood cancer treatment. Music Med. 5, 93-98. doi: 10.1177/1943862113479973

Holman, T. B., and Jacquart, M. (1988). Leisure-activity patterns and marital satisfaction: a further test. J. Marriage Fam. 50, 69-77. doi: 10.2307/352428

Hopkins, M. T. (2015). Collaborative composing in high school string chamber music ensembles. J. Res. Music Educ. 62, 405-424. doi: $10.1177 / 0022429414555135$

Hylton, J. B. (1981). Dimensionality in high school student participants' perceptions of the meaning of choral singing experience. J. Res. Music Educ. 29, 287.

Kahneman, D., Diener, E., and Schwarz, N. (eds.) (1999). Well-Being: The foundations of hedonic psychology. New York: Russell Sage Foundation.

King, E. C. (2006). The roles of student musicians in quartet rehearsals. Psychol. Music 34, 262-282. doi: 10.1177/0305735606061855

Klaphajone, J., Thaikruea, L., Boontrakulpoontawee, M., Vivatwongwana, P., Kanongnuch, S., and Tantong, A. (2013). Assessment of music therapy for rehabilitation among physically disabled people in Chiang Mai province: a pilot study. Music Med. 5, 23-30. doi: 10.1177/1943862112470462

Koelsch, S. (2013). From social contact to social cohesion-the 7 Cs. Music Med. 5, 204-209. doi: 10.1177/1943862113508588

Laukka, P. (2007). Uses of music and psychological well-being among the elderly. J. Happiness Stud. 8, 215-241. doi: 10.1007/s10902-006-9024-3 
Lee, B., and Nantais, T. (1996). Use of electronic music as an occupational therapy modality in spinal cord injury rehabilitation: an occupational performance model. Am. J. Occup. Ther. 50, 362-369. doi: 10.5014/ajot.50.5.362

Lee, J., Krause, A. E., and Davidson, J. W. (2017). The PERMA well-being model and music facilitation practice: preliminary documentation for wellbeing through music provision in Australian schools. Res. Stud. Music Educ. 39, 73-89. doi: 10.1177/1321103X17703131

Macdonald, R., Byrne, C., and Carlton, L. (2006). Creativity and flow in musical composition. Psychol. Music 34, 292-306. doi: 10.1177/0305735606064838

Maslow, A. H. (1987). Motivation and personality. 3rd edn. Delhi, India: Pearson Education.

Miell, D., and MacDonald, R. (2000). Children's creative collaborations: the importance of friendship when working together on a musical composition. Soc. Dev. 9, 348-369. doi: 10.1111/1467-9507.00130

Morgan, L., Hargreaves, D., and Joiner, R. (2000). Children's collaborative music composition: communication through music. ICMPS 2000 Proceedings paper. Available at: https://www.escom.org

Orth, U., Trzesniewski, K. H., and Robins, R. W. (2010). Self-esteem development from young adulthood to old age: a cohort-sequential longitudinal study. J. Pers. Soc. Psychol. 98, 645-658. doi: 10.1037/a0018769

Packer, J., and Ballantyne, J. (2011). The impact of music festival attendance on young people's psychological and social well-being. Psychol. Music 39, 164-181. doi: $10.1177 / 0305735610372611$

Pates, J., Karageorghis, C. I., Fryer, R., and Maynard, I. (2003). Effects of asynchronous music on flow states and shooting and performance among netball players. Psychol. Sport Exerc. 4, 415-427. doi: 10.1016/S1469-0292(02)00039-0

Peifer, C. (2012). "Psychophysiological correlates of flow-experience" in Advances in flow research. ed. S. Engeser (Dordrecht, the Netherlands: Springer), 139-164.

Perkins, R., and Williamon, A. (2014). Learning to make music in older adulthood: A mixed-methods exploration of impact on wellbeing. Psychol. Music 42, 550-567. doi: 10.1177/0305735613483668

Peterson, C., Park, N., and Seligman, M. E. P. (2005). Orientations to happiness and life satisfaction: the full life versus the empty life. J. Happiness Stud. 6, 24-41. doi: 10.1007/s10902-004-1278-Z

Procter, S. (2011). Reparative musicing: Thinking on the usefulness of social capital theory within music therapy. Nord. J. Music Ther. 20, 242-262. doi: 10.1080/08098131.2010.489998

Proctor, C. L., Linley, P. A., and Maltby, J. (2009). Youth life satisfaction: a review of the literature. J. Happiness Stud. 10, 583-630. doi: 10.1007/s10902-008-9110-9

Rabinowitch, T. C., Cross, I., and Burnard, P. (2013). Long-term musical group interaction has a positive influence on empathy in children. Psychol. Music 41, 484-498. doi: 10.1177/0305735612440609

Ritchie, L., and Williamon, A. (2011). Primary school children's self-efficacy for music learning. J. Res. Music. Educ. 59, 146-161. doi: 10.1177/0022429411405214

Rogatko, T. P. (2009). The influence of flow on positive affect in college students. J. Happiness Stud. 10, 133-148. doi: 10.1007/s10902-007-9069-y

Rogers, C. R. (1961). On becoming a person: A psychotherapists view of psychotherapy. Boston: Houghton Mifflin.

Rogers, N. (1993). The creative connection: Expressive arts as healing. Palo Alto, CA: Science and Behavior Books.

Rusinek, G. (2012). "Action-research on collaborative composition: an anlysis of research questions and designs" in Musical creativity: Insights from music education research. ed. O. Odean (Farnham: Ashgate), 185-200.
Ryan, R. M., Huta, V., and Deci, E. L. (2008). Living well: a self-determination theory perspective on eudaimonia. J. Happiness Stud. 9, 139-170. doi: 10.1007/ s10902-006-9023-4

Schiepe-Tiska, A., and Engeser, S. (2012). "Flow in nonachievement situations" in Advances in flow research. ed. S. Engeser (Dordrecht, the Netherlands: Springer), 87-107.

Schuller, T., Brassett-Grundy, A., Green, A., Hammond, C., and Preston, J. (2002). Learning, continuity and change in adult life. Wider Benefits of Learning Research Report No. 3. London: Centre for Research on the Wider Benefits of Learning, Institute of Education.

Seddon, F. A., and Biasutti, M. (2009). Modes of communication between members of a string quartet. Small Group Res. 40, 115-137. doi: 10.1177/1046496408329277

Seligman, M. E. P. (2002). Authentic happiness: Using the new positive psychology to realize your potential for lasting fulfillment. New York: Free Press.

Seligman, M. E. P. (2010). "Flourish: positive psychology and positive interventions" in The Tanner lectures on human values (Ann Arbor: University of Michigan Press), 1-243.

Seligman, M. E. P., Parks, A. C., and Steen, T. (2005). "A balanced psychology and a full life" in The science of well-being. eds. F. A. Huppert, N. Baylis, and B. Keverne (Oxford: Oxford University Press), 275-304.

Sirgy, M. J., and Wu, J. (2009). The pleasant life, the engaged life, and the meaningful life: what about the balanced life? J. Happiness Stud. 10, 183-196. doi: 10.1007/s10902-007-9074-1

Sixsmith, A., and Gibson, G. (2007). Music and the wellbeing of people with dementia. Ageing Soc. 27, 127-145. doi: 10.1017/S0144686X06005228

Small, C. (1999). Musicking: the meanings of performing and listening. A lecture. Music. Educ. Res. 1, 9-22. doi: 10.1080/1461380990010102

Tuckman, B. W. (1965). Developmental sequence in small groups. Psychol. Bull. 63, 384-399. doi: 10.1037/h0022100

Van Goethem, A., and Sloboda, J. (2011). The functions of music for affect regulation. Music. Sci. 15, 208-228. doi: 10.1177/1029864911401174

Vygotsky, L. S. (1978). Mind in society: The development of higher psychological processes. Cambridge, MA: Harvard University Press.

Waddington, C. (2017). "“When it clicks": co-performer empathy in ensemble playing" in Music and empathy. eds. E. King and C. Waddington (Farnham: Ashgate), 230-247.

Waterman, A. S. (1993). Two conceptions of happiness: contrasts of personal expressiveness (eudaemonia) and hedonic enjoyment. J. Pers. Soc. Psychol. 64, 678-691. doi: 10.1037/0022-3514.64.4.678

Williams, J. B. (2018). Creative departures from compositional principles: a collaborative case study of contemporary, theatrical minimalism with live electronics. Princ. Music Compos. 16, 99-106.

Conflict of Interest Statement: The authors declare that the research was conducted in the absence of any commercial or financial relationships that could be construed as a potential conflict of interest.

Copyright (c) 2019 Waddington-Jones, King and Burnard. This is an open-access article distributed under the terms of the Creative Commons Attribution License (CC BY). The use, distribution or reproduction in other forums is permitted, provided the original author(s) and the copyright owner(s) are credited and that the original publication in this journal is cited, in accordance with accepted academic practice. No use, distribution or reproduction is permitted which does not comply with these terms. 
OPEN ACCESS

Edited by:

Michele Biasutti,

University of Padova, Italy

Reviewed by:

Aillbhe Kenny,

Mary Immaculate College, Ireland

Karen Burland,

University of Leeds, United Kingdom

${ }^{*}$ Correspondence:

Graeme B. Wilson

graeme.wilson@ed.ac.uk

Specialty section:

This article was submitted to

Performance Science,

a section of the journal

Frontiers in Psychology

Received: 11 February 2019

Accepted: 17 May 2019

Published: 28 June 2019

Citation:

Wilson GB and MacDonald RAR

(2019) The Social Impact of Musical Engagement for Young Adults With Learning Difficulties: A Qualitative Study.

Front. Psychol. 10:1300.

doi: 10.3389/fpsyg.2019.01300

\section{The Social Impact of Musical Engagement for Young Adults With Learning Difficulties: A Qualitative Study}

\section{Graeme B. Wilson* and Raymond A. R. MacDonald}

Reid School of Music, Edinburgh College of Art, University of Edinburgh, Edinburgh, United Kingdom

There is evidence that music interventions can offer opportunities for creative, psychological, and social developments for individuals with mild to profound learning disabilities, addressing the disadvantages they face in respect of social outcomes. This paper reports on a qualitative study investigating a community music intervention for such a population. Thirty-seven adult service users ( 12 female, 25 male) took part in weekly music workshops for 10 weeks. Their learning difficulties ranged from mild to profound, and their levels of independence ranged from requiring constant one-to-one care to living alone in sheltered accommodation. Interviews were conducted at multiple time points with music and resource center staff as well as participants and members of their families and other center users; researchers also observed all workshops, taking field notes. Thematic analysis of the data informed understanding of the disadvantages facing participants, their experience of the workshop program and its immediate and wider social outcomes, as well as suggesting key mechanisms for effects. Disadvantages and barriers facing participants included: limited access to enjoying or learning music; boredom, isolation, and limited networks; lack of experience of new social contexts; and an associated lack of confidence, low mood or self-esteem. Participants were found to enjoy and sustain engagement with a program of dedicated group music workshops delivered by staff trained in an empathic and inclusive approach. Impacts included an ongoing enthusiasm to engage in music; wider recognition of musicality; increased self-confidence; being happier, more relaxed, and/or enthusiastic after the workshops; better ability to interact with unfamiliar situations and people; and participation in social activities for an unprecedented length of time. Key factors in achieving those impacts are that participants: had fun and interacted socially; felt secure, welcomed, and involved at all times; exercised choice; worked with others in nonverbal tasks; and encountered challenge while engaging and progressing at their own rate.

Keywords: music, learning difficulties, disability, inequality, interaction, psychology, social 


\section{INTRODUCTION}

Individuals with support needs in respect of physical or mental impairments are disproportionately affected by loneliness, isolation, and limited self-efficacy, with negative impacts upon them and their communities (de Jong Gierveld et al., 2006; Hawthorne, 2008; Cacioppo and Cacioppo, 2014). However, in Scotland, barriers to employment, activities, and supportive networks of meaningful relationships are such that adults with a long-term limiting condition are still less likely to experience good general health or be economically active at working age, and more likely to experience discrimination, than those without a disability (Scottish Government, 2016a,b,c).

Positive benefits can accrue from initiatives to increase opportunities accessible by people with impairments and change prevailing perceptions of their potential to lead full social lives (Shochet et al., 2016), particularly if participant-led (Milner and Kelly, 2009). Making music represents one such initiative. As a key creative field, music offers powerful scope for selfdefinition (Green, 2006) and therefore great potential to address disadvantages faced by individuals with impairments. Music is an important activity, sophisticatedly woven into all daily lives (DeNora, 2000). It can be considered a fundamental aspect of society, central to many social activities, while profoundly affecting our moods, emotions, and behavior (Becker, 1986). At the same time, music also helps in the construction, negotiation, and maintenance of identities (Frith, 1996; MacDonald et al., 2017). Music therefore has a unique sociological and psychological importance, both in terms of how it is embedded within societies around the world, and how it facilitates social interaction (Roy and Dowd, 2010). While everyday music listening can play an important role in health and well-being (DeNora, 2013), a substantial body of evidence now shows that the creative and expressive physical and mental processes of making music together offer a powerful and accessible means to maintain well-being and foster social relationships (MacDonald et al., 2012). Making music with others in community settings can moreover have value throughout adult life (Pitts, 2005; Kenny, 2016). Nevertheless, barriers to access affect music as much as other fields.

A growing literature on the unique potential of musical involvement to improve outcomes for children with autism is emerging (Simpson and Keen, 2011). However, there is scarce literature on the impacts of music in the community (nonclinical) setting. Although development for all individuals continues throughout life, albeit at a slower rate following childhood, there is also little research on the potential of music at different stages of adulthood for individuals with autism. Furthermore, studies tend to consider outcomes in terms of behaviors or symptoms associated with the condition, rather than considering music's potential to tackle the persistent exclusion and inequality of disabled people and build social capital for this sector (Bates and Davis, 2004).

One previous study considered the impacts of a community music program for adults with a range of disabilities, which provided participants with experiences of a wide range of musical genres and facilitated them in developing new rock music based on their own lives and ideas (MacDonald and Miell, 2002). Interviews with six longer-term participants indicated a broader range of impacts than those on musical proficiency and understanding alone. Forming a band and performing professionally had forged new social links and allowed interviewees to interact in ways they had not before. They described substantial positive effects on their confidence and the identities they perceived for themselves. Musical involvement was a powerful means of challenging negative identities around their conditions that others constructed for them; interviewees blamed a daily experience of stereotyping for negative impacts on their mental health. However this research produced a limited picture based on snapshot retrospective interviews with participants who had already been involved in the program for years in some cases, and for whom there was no baseline information.

This paper reports on a recent research project in Scotland to address these gaps in knowledge. The project involved development and delivery of a new program of innovative music workshops for a key population experiencing inequality: young adults with autism. Qualitative research around this initiative aimed to answer the following questions:

- What social needs associated with having learning impairments can interactive music workshops address?

- How do adults with such impairments experience interactive music workshops?

- What impacts on social needs do they and those around them perceive from taking part in music?

- What features of the music workshops might be effective, and why?

\section{MATERIALS AND METHODS}

\section{Design}

- Twenty music workshops for 37 individuals recruited via resource centers in an accessible rural area

- Interviews with music staff, resource center users, their families and staff

- Observation of workshops with co-researcher from service user group

- Focus group with stakeholders

\section{Intervention}

The workshops were designed and delivered by a registered charity (Limelight Music Ltd.) which, for over 25 years, has pioneered innovative access to high-quality musical education, qualifications, involvement, and careers for individuals of all ages and abilities. Their workforce constitutes Scotland's first generation of professional musicians with impairments, who work alongside established nondisabled artists to train the next generation in inclusive music-making, and support those with additional support needs toward full-time musical employment.

As well as facilitating participants' musical development and future musicality, the workshops were intended to encourage 
their social interaction, relationship skills, and empathy, enhancing opportunities for greater community integration and development of social capital. Sessions for example included group music exercises followed by instrumental training (e.g., guitars or singing) to build achievable musical skills. Coaching in composing original songs was seen as a means to develop participants' confidence in their own musicality and proficiency, while rehearsing and performing in smaller music ensembles built confidence in social interaction.

Participants were individually supported during workshops by Limelight and care staff.

\section{Participants}

Thirty-seven adult service users (12 female, 25 male) were recruited via four separate centers located around Renfrewshire (a local authority in central Scotland) to take part in workshop groups on either Mondays or Fridays. Their learning difficulties ranged from mild to profound, and their levels of independence ranged from requiring constant one-to-one care to living alone in sheltered accommodation.

\section{Interviews and Observations}

Regular interviews with music staff allowed them to describe and explain their decisions, and delineate key objectives. Researchers observed all workshops, taking field notes.

In an initial 6-week consultation, in-depth interviews were carried out with six service users and six staff at one resource center for young adults with a diagnosis of autism or related conditions, and with parents of three further service users whose views could not be accessed directly. The interviews explored disadvantages faced by individuals with learning difficulties and identified priorities that music workshops might best address. Learning was fed back to the music workshop team as they designed the music activities.

Interviews were also sought during and after the workshop program with carers and family members and (where appropriate) workshop participants themselves, to gather their views on the workshops and any objectives and impacts they perceived. Informed consent for research participation was gathered in advance from participants, or from an identified person with responsibility if they lacked capacity for this. To inform potential interviewees, accessible printed or recorded information sheets were prepared detailing the voluntary, confidential, and anonymous nature of participation and how data would be used.

\section{Procedure}

The partners arranged a 10-week program of music workshops for two separate groups between October and December 2017. One group was subdivided to accommodate, in separate halves, a group with profound disabilities, and a group with relatively high independent living. The other group brought together participants from three different centers. Seventeen 2-hour workshops were delivered from October to December 2017 in an accessible community rehearsal facility located separately from the resource centers, and another at one of the centers; two further workshops had to be rescheduled beyond the research period due to a conflict of activities. A lay researcher was recruited to contribute to observations and analysis; GW and/or the lay researcher observed all 18 workshops that took place during the research, taking notes, and conducted interviews with 10 participants, 4 parents of participants, and 11 resource center staff.

\section{Analysis}

Interviews and observation notes were analyzed using thematic analysis (Denzin and Lincoln, 2017). Transcripts and notes were repeatedly read as they were gathered and coded by one researcher for initial themes and discussed regularly by the research team to resolve divergent instances and arrive at a consistent coding of higher level themes. These were reviewed in two focus groups, one with stakeholders from the project and one with music staff, to ensure a consistent understanding between research team and the population being researched.

\section{Ethics Approval Statement}

This research received ethical approval from the Edinburgh College of Art Research Ethics Sub-Committee, University of Edinburgh, which includes consideration of any risk of disadvantaging individuals through the research.

\section{RESULTS}

\section{Disadvantages and Barriers Faced by Participants in Their Lives}

The initial interviews with resource center staff and users sought to understand what challenges center users faced in their lives, and therefore what inequalities the music intervention might have an impact on. These included limited options for activities, including music activities, difficulties in encountering public spaces, and associated mental health impacts.

Staff and family members perceived the center users as frequently bored or lacking resources or opportunities to keep themselves occupied, and some interviews with center users confirmed this:

\section{I: what would your life be like if you didn't come here once a week?}

\section{R: I don't know, just sit in the house mainly. [aye] Just do nothing, sit in the house, lying in bed, watch TV, tidy up, you know? (Malcolm)}

Some were concerned that their client or relative developed little if anything in the way of a social circle, only seeing family members and those who came to their house. Center users were seen as being particularly disadvantaged in social contexts outwith their homes because of difficulties in coping or interacting with people they did not know. Staff perceived many of the individuals they worked with as struggling to maintain concentration on activities or tasks, and to grasp the conventions of social interaction such as eye contact, or turn-taking. 
one of the deficits somebody may have in their life is difficulties with social communication. So they may lead not solitary lives, but they may not have friends. They may not have people to share their lives with, and may not be thinking along those lines. (staff)

It could be difficult for some to cope with a physical context with which they were not familiar; heightened sensitivity to noise among some of those with autism meant that they (or their families) avoided many public contexts where they might encounter other people. These challenges operated in a cycle with lack of confidence and low mood or self-esteem:

\section{We would have certain people on mood charts that would go into real depths of depression for three months (resource centre staff)}

Some staff and parents were concerned that center users experienced inequalities because of other people's assumptions about what they were able to do, or wanted to do.

Listening to music was described as extremely important to center users, both by themselves and those around them. For instance, the mother of participant Hilary observed that different aspects of the music her daughter listened to could command and retain her attention in ways that other communicative activities did not:

\begin{abstract}
So sometimes she'll take a wee notion where she's listening, I've noticed maybe it's more to do with the piano part of it that she's playing over and over again, sometimes it might be a wee drum bit that she's playing, sometimes it's the words. But I feel that there's something. It connects with her intrinsically, you know what I mean? There's something going on (Hilary's mother)
\end{abstract}

The center users were enthusiastic about the particular artists or genres they liked, and staff recognized that listening to music was a very popular activity or background to other activities. Pavel, one of the participants who was interviewed, attested to much time spent at home listening to a particular genre of pop music ("Britpop") not simply as a preference, but as a central aspect of his own identity ("my generation"):

I sing at home, mostly to Blur, Pulp, Oasis, Suede, and loads of other Britpop bands, those are the bands of my generation. (Pavel)

Center users enjoyed some opportunities to make music together. Staff at the centers sometimes ran musical activities such as group sing-alongs, with one center running a weekly drumming and chanting group for center users with profound disabilities. Karaoke was cited as a popular pastime for many individuals, and some had instruments at home, typically a keyboard.

Interviewees did not however describe other opportunities to develop an interest in playing music. Two of the youngest center users recalled attending classroom music lessons at school; otherwise, no formal music education or training was reported. Although staff and family members acknowledged center users' interests, they tended to prescribe a limited musical identity for them associated with perceived limits to their musical ability:

He doesn't necessarily know the finger work, but he can strum. He enjoys a good strum. But for the past year or so even that's not been happening for him. (Family 3)

In the quote above, the parent of one participant acknowledges that their son has, prior to the preceding year, shown the capacity and inclination to play guitar by "strumming." This is positioned by the parent as a limited extent of music-making ("even that") since, in their view, it is not coordinated with fretting using the other hand ("finger work") Some center users' accounts suggested they faced assumptions that musical opportunities would not be for them:

June: My dad can play guitars.

I: So, there's always been guitars in your house growing up?

June: Yes, yep.

I: Did you used to have a shot of his guitar?

June: No.

June was enthusiastic and committed in taking up guitar at the workshops, showing rapid development, and here and elsewhere attributes this partly to her familiarity with her father's guitar playing. Nevertheless, she stresses that until the workshops, she has not been given the opportunity to play herself. This could be because her family assumes she will not be interested in playing, or because of a protective attitude toward the instruments in the house, or because it is perceived that she will be less able to play due to her disability; June however does not speculate on the reasons herself.

The extracts above demonstrate that musical activities could provide center users with opportunities for meaningful cognitive and emotional engagement. As listening merges with playing, identity processes around musical preferences link with developing instrumental techniques involving complex motor coordination. As such, taking part in music has a strong potential to address the limited scope for purposeful activity and engagement in social environments that were perceived for individuals with learning disabilities. The next section describes how such an opportunity was engaged with.

\section{Taking Part in the Music Workshops}

Through observation and interviews, the research aimed to establish a detailed picture of how adults with a range of learning difficulties were facilitated in music-making through the workshop program, how they embraced the format and content of the workshops, and how this affected their behavior. 
Workshops took place with all participants and music and center staff seated in a circle. Sessions began with $10 \mathrm{~min}$ of physical and vocal warm-up exercises, then a series of musical activities involving drumming on djembes, and singing or drumming along to African chants, folk music, and pop tunes, played on instruments from BoomWhackers (pitched plastic pipes) to keyboards. Those who expressed an interest in guitars and a drumkit that were in the room were supported to try these out or use them in some of the activities. Each session also involved leader narrating a story, which was to be punctuated by musical interjections from the group. Participants learned melodies and lyrics, and as the weeks went by, they had the opportunity to express their own musical ideas either spontaneously through call-and-response within singing or drumming exercises, or through suggestions for songs that the group devised together. Participants came from three different resource centers in the area; they were encouraged to stay in the space and interact during the break for refreshments, with one of the workshop team leading a conversation on, for example, favorite films.

Workshop observations recorded a change over the program in how individuals took part. (A summary of observation notes for one participant is provided in Supplementary File A as an illustration of how changes in participation developed.) In the early weeks, many participants did not speak or join in with singing or spoken responses. Coordination on drumming was poor, and those who did join in vocally tended to do so haphazardly. Eye contact was limited during the workshops, with many participants staring into the distance or looking at the ground. Participants frequently got up or moved away from the circle and around the room during activities. Those from the group with profound disabilities often left the room or displayed repetitive behaviors; some frequently put their fingers to their ears during pieces.

Over the 10 weeks, all groups strengthened their abilities to keep time and concentrate together, to coordinate musical exercises, and to take turns effectively. Activities were flexibly challenging and engaged the interest of groups and individuals. As the weeks progressed, there were markedly fewer instances of participants leaving the circle, and in the profound group repetitive behaviors appeared less frequently, with fewer instances of fingers being placed in ears. Those who initially struggled to make eye contact or speak to others began to respond musically and vocally, to watch what others were doing, and to volunteer comments and suggestions. In particular, three individuals who had been assumed by the observers in the first week to lack speech began to talk and sing during the second or third week. Participants increasingly talked and joked with music staff and other participants during the break and at the beginning and end of the music sessions, getting to know people from centers other than their own.

Some attendance was irregular. Although staff and participants liked the venue, transport difficulties in getting there meant that some participants were late, early, or unable to attend some of the workshops. Also, because participants were recruited through resource centers with existing provision, other activities sometimes clashed with the workshops, forcing participants to choose between them. Observation indicated that the groups varied in their engagement and progress. Those from the younger and less disabled group showed the greatest musical accomplishment and interest in learning new material or shaping it in their own way. Those in the groups with profound disabilities developed strongly in their abilities to remain engaged and attentive, to act as a group, and to contribute to activities, but were reluctant to move on from the material and format of the earliest weeks. Attendance among the third group, who were somewhat older than the others, was the most irregular. It was established later that staff and center users had thought it best to vary who attended from week to week to "give everyone a turn." A few participants who stopped coming after a week or two may have felt that the activities in those weeks were not what they had been expecting, or that they were covering ground they felt familiar with through previous musical activity. This was an issue for a workshop program designed for a consistent group attending for 10 weeks; while those participants who came along each week enjoyed themselves at that session, the group as a whole showed less clear musical development than the others.

Participants may have been apprehensive at attending an unfamiliar setting to carry out unfamiliar tasks with unfamiliar people. One interviewee put it in these terms:

\section{First time I thought some of the people I'd never met before, they all looked quite indifferent... [felt] quite nervous, and trying to get to know them (Pavel)}

In these words, Pavel articulates the difficulties in new social settings that were identified in the section "Disadvantages and Barriers Faced by Participants in Their Lives." However, in the interviews that took place during the workshop program, participants described the sessions as "good fun" or "a great laugh," and said they felt happy or "great" during and after the sessions. They spoke enthusiastically about the reward of overcoming challenges to play or sing something they did not think they were able to, or gaining the confidence to sing to a room full of people. Key workers too perceived a lasting buzz of excitement when they returned from the music workshops. Staff noted a change in mood back at the centers during the program. One participant said "I actually look forward to a Monday morning"; another's mother noted that "she's full of smiles when she comes in, so she's had a good day."

Taken together, the observations and interviews around the workshop program indicate important musical and social behaviors emerging among the group as they participated in accessible and enjoyable group music activities over 10 weeks. Their musical abilities and decisions became more sophisticated, confident, and coordinated, with heightened capacity to attend to what was taking place. They overcame initial apprehensions, substantial in some cases, about an unfamiliar and sometimes loud social setting, to the point where they were able to contribute effectively to reciprocal activities or conversations. Enjoyment emerged as a particularly strong motivation for sustaining participation. Therefore, it is important to show how enjoyment of group music-making can best be enhanced. 


\section{Impacts of Musical Participation on Participants}

All those interviewed after the program felt that the workshops had made important differences for those taking part:

He participates very well. So from that aspect it brings Justin out of his shell and allows him to participate with other members of the group more easily... Dean, for example, is very quiet, normally shows almost no emotion. But if you watch him here you'll see him participating, banging the drum, he's very active. He's very focused. If you look at him, he'll stop... And for Donald a lot of it is turn taking and focus, concentration. It's about emotional management for him. Being happy, effectively. And this is what he's gained, effectively (staff)

This extract highlights important psychological developments for the participants. The phrase "it brings Justin out of his shell" suggests two features: Justin is introverted and possibly could benefit from opportunities to socialize; secondly, music can facilitate this type of important social engagement. The speaker explicitly draws a causal relationship between the musical activities and important psychological and social developments for three individuals.

Participants acquired an enthusiasm to engage in music and were able to build identity around a renewed sense of themselves as musical and accomplished:

\section{I: What kind of things do you think you've got out of playing?}

June: A lot. I didn't think I could play a guitar.

Some described the reward of overcoming challenges to play or sing something they did not think they were able to, or gaining the confidence to sing to a room full of people. Others were particularly taken with the opportunity to play instruments they did not usually have access to.

\section{I: Thinking about the drums when you played them, how easy did you find it? \\ Jeff: No, it's not difficult. It's easy... Aye. My hands and feet. \\ I: Did you just work it out yourself or did somebody show you how to use it?}

\section{Jeff: No, no, I noticed myself.}

In this conversation, participant Jeff demonstrates his confidence not only by asserting that playing the drums is "easy" for him, but by identifying that this is because he is able to understand what to do from what he has "noticed" himself. One father described his daughter's photo of "her new hobby" on Facebook, "proud of the fact that she played drums." Staff were surprised at participants' achievements and how these changed their relationships to music, and were keen to support them in further musical activities: ...you wouldn't necessarily witness when the guys are going back to the bus [to return to the centres], there's like African songs and things like that. (service staff)

A few participants' families were reported to have bought instruments for the participants to pursue interests and abilities they were not previously aware of.

By the end of the program, all groups had manifestly increased their cohesiveness and social interaction. Participant Donald, for example, was able to list a string of new friendships made through taking part in the workshop

\section{I: Do you know anybody from there [another centre]?}

Donald: No. Jackie, June, Sheila. No, I don't know any of them. And Pavel as well, Pavel, he's at [centre name]. Jeff, Colin. Don't know any of them. But I know them now.

Participant Pavel, who reported having been nervous at the start, stated that the workshops had been experienced as having a significant personal impact:

Pavel: Going to the workshop ... made me quite emotional at first, but quite happy... Yeah, it's changed me for the best... they were like a family to me

Here, he embraces the difference he felt this had made to him by seeing this as "for the best," and sees the closeness he perceives with his family as extending to his new acquaintances. Interviews suggested that participants, and those around them, thought this adaptation to a new social environment would stand them in good stead when faced with interacting in other unfamiliar situations.

Isaac doesn't tend to verbalise that much. And I've certainly heard him verbalise at that group (service staff)

The empathic approach and gradually changing nonverbal music tasks allowed participants with more profound difficulties to feel secure and intrigued, engaging in a new physical and social environment to an extent that surprised both staff and parents:

seeing her sitting there for that length of time engaged in that activity without any other supports in place to enable her, facilitate her, to do that is actually amazing. It's because she wants to be there (parent)

Staying involved in activities that lasted for an hour was particularly striking for some staff and families, who reported that this level of sustained focus and commitment to a social situation was unprecedented. One participant who had started out with almost no responses to the group or eye contact became much more physical, concentrated, and involved than staff expected.

Staff and families also observed that many participants' increased confidence in themselves and their abilities increased. Some participants who were interviewed expressed this on their own behalf, for instance: 
Sheila: It's definitely made a difference, because I've enjoyed it.

\section{I: How do you think you've changed over the workshops?}

Sheila: I think I've got a bit more confidence than what I had.

Finally, staff and families noted that participants about whom they had been concerned in relation to low mood, particularly on a Monday, appeared happier, more relaxed, or more enthusiastic following the workshop program. One member of staff, for example, commented that a center user whose mental health she had been concerned about responded dramatically to the opportunities of the workshop program, to the point that over the course of the program she saw him return from a prevailing low mood and avoidance of interaction to his previous happier character when at the center:

you can see that interaction building, and I could see on the last day he was talking, and you just think "yeah, he's coming back to us" (staff)

Interviews suggested a number of features of the workshops that were crucial to the impacts identified here. First, it was vital that participants felt themselves to be having fun, without which they would not have sustained engagement. Music staff were keenly aware of this. They sought to remain open to what participants wanted to do, and identified as part of their approach that amusing participants or engaging in banter between themselves to raise a laugh was important to their approach. Second, the workshops prioritized group activities over one-to-one interaction such as individual guidance on instruments, and included activities that directed participants to engage with each other, for instance looking at the person next to them. In this way, participants were steered into social interaction without having to speak initially. This started from early in the program to help participants feel comfortable with each other. The team sustained an emphasis on interaction during the breaks. These are important social skills that developed through the group music activities and may generalize to other situations.

Third, it was seen as vital that participants felt secure, welcomed, and involved from the outset and throughout. One member of staff spelled out the importance for one participant he worked with of being able to engage at his own speed, and to whatever extent he felt able, without feeling that he was under scrutiny or required to meet others' expectations:

Dean's very reserved, as many people on the spectrum are, because they have difficulties with social interactions and social communication.... within this group you're really in a situation where you can do as you like and you're not the focus of attention, you'repart of a team. So that's where Dean benefits. He gets to interact and participate without any judgement or that sort of thing. (staff)

Key activities in the early stages were songs that introduced each person in the group by turn or that asked them to say something about themselves, and music staff were attentive throughout the sessions to encourage each person to join in to the best of their ability. Fourth, participants were facilitated in exercising choice about what they wanted to play or do, for instance being invited to select musical lines for a song, suggest lyrics, or choose a song they liked for the group to sing. Drumming sections included an activity where each person in the circle in turn had to improvise a rhythm on their drum while the group paused in their playing.

Fifth, some music activities required participants to work with each other on nonverbal tasks; for instance, coordinating in small groups to play melodies with BoomWhackers, or passing a drumbeat around the circle with the challenge of getting faster each time. This brought them to approach tasks together rather than individually, and being part of a group gave them license to experiment creatively with what they were doing:

\section{when I've witnessed him in here, he's very rigid. But the Limelight group seems to have the capacity and the ability, if you like, to bring a wee bit more out them, have a wee bit more expression and a bit more open to other ideas and other people dictating (staff)}

Finally, participants had widely varying abilities and interests; music staff sought to enable each participant to encounter challenge while engaging and progressing at their own rate. Thus, some participants had progressed to conducting the group when using BoomWhackers or accompanying songs on open-tuned guitars, while others had progressed to singing at the same time as other people or not interrupting passages of silence; all were still involved in the same activities, but at a level that engaged them appropriately.

In summary, the interviews with participants and those around them identified a number of key aspects of the experience of these inclusive group music activities which they saw as leading to important changes in participants' abilities, engagement, confidence, sociability, and mood, and allowing them to develop creative expression for themselves.

\section{DISCUSSION}

This paper highlights the potential of music to bring about positive changes in the lives of individuals with learning difficulties. The research has identified that adults with a range of such difficulties face a number of key social inequalities that music participation could address. These include limited access to enjoying or learning music; boredom, isolation, and limited networks; lack of experience of new social contexts; and an associated lack of confidence, low mood or self-esteem. Such experiences may arise through difficulties with noise levels, concentration, or social conventions, but also from other people's assumptions about what these young adults can do, or want to do. The population targeted by this intervention was able to enjoy and sustain engagement with a program of dedicated group music workshops delivered by staff trained in an empathic and inclusive approach. The qualitative results suggest that the unique social, musical, creative, and accessible features of the 
workshop environment facilitate a commitment in the participants to engage with and enjoy the workshops over an extended period of time. Impacts described for participants included an ongoing enthusiasm to engage in music; wider recognition of musicality; increased self-confidence; being happier, more relaxed, and/or enthusiastic after the workshops; better ability to interact with unfamiliar situations and people; and participation in social activities for an unprecedented length of time. Key factors in achieving those impacts are that participants: have fun and interact socially; feel secure, welcomed, and involved at all times; exercise choice; work with others in nonverbal tasks; and encounter challenge while engaging and progressing at their own rate.

Some of these factors have been identified as important to the meaningful engagement of adults more generally in community music-making (Schiavio et al., 2018). However, analysis of our research data suggests several key indicators of impact on the disadvantage faced by the target population in our study:

- Interest or activity in making and enjoying music sustained beyond the workshop program

- Increased and sustained confidence in different social situations

- Reports that participants are more happy, relaxed, or communicative

- Knowing and interacting with a greater number of people, or doing so more often, in workshops or daily life

- Ability to sustain focus and participate sociably for longer in unfamiliar situations

It is important that any investigation of community music interventions for disadvantaged individuals integrates consideration of musical, social, and psychological features. The qualitative findings reported here emphasize that such programs foster not only musical development for the participants, but also psychological and social development. One reported feature of the intervention was that participants enjoyed the classes, and appeared to find the types of musical engagement delivered by Limelight both fulfilling and life-affirming. This result is important as it is a challenge to organize and deliver activities of any sort that individuals with impairments find both enjoyable and rewarding. It is consistent with findings elsewhere that adults' enjoyment of being in a community ensemble is a prerequisite for their collaborative creative practice in group music-making, as is the establishment of socio-musical relationships between them (Kenny, 2014). The observation that the enhanced enjoyment of music continued outside the intervention is also crucial, as a sign that impacts were not restricted to the workshop times. It provides evidence that the current inclusive intervention, flexibly delivered and tailored to the need of individuals, can meet a primary goal of music educators in developing musical tastes and enjoyment in listening and performing.

This finding resonates with other similarly themed research. Research investigating children with autism suggests the ability of song to overcome the structural deficit for speech that arises from alternate mechanisms of speech and music processing in ASD (Sharda et al., 2015). Such children enjoy improvisational music therapy, with a number of important psychological improvements reported (Kim et al., 2009). Improvisational music therapy was more effective at facilitating their joint attention behaviors and nonverbal social communication skills than play alone, resulting in significantly more and lengthier events of eye contact and turn-taking (Kim et al., 2008). The findings reported here underline that engaging in group music can continue to be beneficial to the social and psychological development of individuals with learning impairments as they progress through young adulthood. Individuals with ASD, for instance, have been found able to regulate physiological outcomes associated with mood and emotions using music as a non-pharmacological tool (Hillier et al., 2015).

The approach to understanding the impacts of musical participation with this population reflects recent initiatives to measure outcomes for individuals with learning impairments. Observations of intentional communication in natural settings have been identified as a valuable assessment strategy for research and clinical practice on the basis that they can be quantified to provide a measure of impacts comparable at follow-up (Pasco et al., 2008), but the results here indicate their potential for qualitative investigation in the first instance. Results from the present study were used to design a subsequent phase of the research which is currently underway, to assess the wider impact of the ongoing workshop program, taking into consideration the distinct needs and difficulties of each participant. As well as self-report from participants, staff, and families, key workers have suggested routine assessments that may be accessed, such as mood charts and records of activities undertaken to monitor progress toward outcomes set in Keys to Life, Scotland's learning disability strategy ${ }^{1}$. Video data are being gathered in the next phase of this study to explore quantifiable observational outcomes. The involvement of participants with both learning difficulties and physical impairments in workshops suggests that individuals with other impairments could benefit in similar ways. Comparison of varied populations living in different areas is also part of the next program of work.

There is an imperative to address the opportunities available to individuals with learning impairments in order that they are not disabled relative to the non-impaired population, and do not face worse social outcomes (Goodley, 2011). Access to recreational musical participation and development can enrich adult lives at all ages; this can be sustained through opportunities for informal group music-making but discouraged if musical environments lack encouragement or stimulation (Pitts, 2009; Taylor, 2011). Individuals with ASD in particular may exercise relatively little self-determination, making fewer choices in their daily lives and experiencing fewer relationships than other groups (Mehling and Tassé, 2015). Evidence from research in music therapy highlights a number of key psychological mechanisms with therapeutic potential in the creative nonverbal interaction through music (MacDonald and Wilson, 2014). The developments in individual choice and social relationships observed in the present study suggest strongly that an inclusive music intervention tailored to individual tastes, abilities, and creative objectives offers a powerful, practical, and engaging way to address key disadvantages for this population.

${ }^{1}$ https://keystolife.info/ 


\section{DATA AVAILABILITY}

The datasets for this study will not be made publicly available because the terms of consent for research participation did not specify that data would be accessible other than by the research team.

\section{ETHICS STATEMENT}

This research received ethical approval from the Edinburgh College of Art Research Ethics Sub-Committee, University of Edinburgh, which includes consideration of any risk of disadvantaging individuals through the research. Informed consent for research participation was gathered in advance from participants, or from an identified person with responsibility if they lacked capacity for this. To inform potential interviewees, accessible printed or recorded information sheets were prepared detailing the voluntary, confidential and anonymous nature of participation and how data would be used.

\section{REFERENCES}

Bates, P., and Davis, F. A. (2004). Social capital, social inclusion and services for people with learning disabilities. Disabil. Soc. 19, 195-207. doi: $10.1080 / 0968759042000204202$

Becker, H. S. (1986). Doing things together: Selected papers. (Evanston: Northwestern University Press).

Cacioppo, J. T., and Cacioppo, S. (2014). Social relationships and health: the toxic effects of perceived social isolation. Soc. Personal. Psychol. Compass 8, 58-72. doi: $10.1111 / \mathrm{spc} 3.12087$

de Jong Gierveld, J., van Tilburg, T., and Dykstra, P. A. (2006). "Loneliness and social isolation" in The Cambridge handbook of personal relationships. eds. A. L. Vangelisti and D. Perlman (Cambridge: Cambridge University Press), 485-500.

DeNora, T. (2000). Music in everyday life. (Cambridge: Cambridge University Press).

DeNora, T. (2013). Music asylums: Wellbeing through music in everyday life. (Farnham: Ashgate).

Denzin, N. K., and Lincoln, Y. S. (2017). The SAGE handbook of qualitative research. (London: Sage).

Frith, S. (1996). Performing rites: Evaluating popular music. (Oxford: Oxford University Press).

Goodley, D. (2011). Disability studies: An interdisciplinary introduction. (London: Sage).

Green, L. (2006). Popular music education in and for itself, and for 'other' music: current research in the classroom. Int. J. Music. Educ. 24, 101-118. doi: $10.1177 / 0255761406065471$

Hawthorne, G. (2008). Perceived social isolation in a community sample: its prevalence and correlates with aspects of peoples' lives. Soc. Psychiatry Psychiatr. Epidemiol. 43, 140-150. doi: 10.1007/s00127-007-0279-8

Hillier, A., Kopec, J., Poto, N., Tivarus, M., and Beversdorf, D. Q. (2015). Increased physiological responsiveness to preferred music among young adults with autism spectrum disorders. Psychol. Music. 44, 493-513. doi: $10.1177 / 0305735615576264$

Kenny, A. (2014). Collaborative creativity' within a jazz ensemble as a musical and social practice. Think. Skills Creat. 13, 1-8. doi: 10.1016/j.tsc.2014.02.002 Kenny, A. (2016). Communities of musical practice. (London: Routledge).

Kim, J., Wigram, T., and Gold, C. (2008). The effects of improvisational music therapy on joint attention behaviors in autistic children: a randomized controlled study. J. Autism Dev. Disord. 38, 1758-1766. doi: 10.1007/s10803-008-0566-6

Kim, J., Wigram, T., and Gold, C. (2009). Emotional, motivational and interpersonal responsiveness of children with autism in improvisational music therapy. Autism 13, 389-409. doi: 10.1177/1362361309105660

\section{AUTHOR CONTRIBUTIONS}

All authors listed have made a substantial, direct and intellectual contribution to the work, and approved it for publication.

\section{FUNDING}

This research was supported by a grant from the Scottish Government/European Union Social Innovation Fund, ref. no. SIF-R1-S1-LUPS-006. The funders had no influence on the gathering or analysis of data, nor on the writing of the manuscript.

\section{SUPPLEMENTARY MATERIAL}

The Supplementary Material for this article can be found online at: https://www.frontiersin.org/articles/10.3389/fpsyg.2019.01300/ full\#supplementary-material

MacDonald, R. A., Kreutz, G., and Mitchell, L. (2012). Music, health, and wellbeing. (Oxford: Oxford University Press).

MacDonald, R. A., and Miell, D. (2002). "Music for individuals with special needs" in Musical Identities. eds. R. A. R. MacDonald, D. J. Hargreaves, and D. Miell (Oxford: Oxford University Press), 163-178.

MacDonald, R. A. R., Miell, D., and Hargreaves, D. J. (2017). The Oxford handbook of musical identities. (Oxford: Oxford University Press).

MacDonald, R. A., and Wilson, G. B. (2014). Musical improvisation and health: a review. Psych. Well-Being. 4:20. doi: 10.1186/s13612-014-0020-9

Mehling, M. H., and Tassé, M. J. (2015). Impact of choice on social outcomes of adults with ASD. J. Autism Dev. Disord. 45, 1588-1602. doi: 10.1007/ s10803-014-2312-6

Milner, P., and Kelly, B. (2009). Community participation and inclusion: people with disabilities defining their place. Disabil. Soc. 24, 47-62. doi: 10.1080/09687590802535410

Pasco, G., Gordon, R. K., Howlin, P., and Charman, T. (2008). The Classroom Observation Schedule to Measure Intentional Communication (COSMIC): an observational measure of the intentional communication of children with autism in an unstructured classroom setting. J. Autism Dev. Disord. 38, 1807-1818. doi: 10.1007/s10803-008-0569-3

Pitts, S. (2005). Valuing musical participation. (Aldershot: Ashgate).

Pitts, S. (2009). Roots and routes in adult musical participation: investigating the impact of home and school on lifelong musical interest and involvement. Br. J. Music Educ. 26, 241-256. doi: 10.1017/S0265051709990088

Roy, W. G., and Dowd, T. J. (2010). What is sociological about music? Annu. Rev. Sociol. 36, 183-203. doi: 10.1146/annurev.soc.012809.102618

Schiavio, A., Van Der Schyff, D., Gande, A., and Kruse-Weber, S. (2018). Negotiating individuality and collectivity in community music. A qualitative case study. Psychol. Music. doi: 10.1177/0305735618775806 [Epub ahead of print].

Scottish Government (2016a). Regional employment patterns in Scotland: Statistics from the Annual population survey, 2015. (Edinburgh: Scottish Government).

Scottish Government (2016b). Scotland's people: Results from the 2015 Scottish household survey. (Edinburgh: Scottish Government).

Scottish Government (2016c). Scottish surveys core questions 2014. (Edinburgh: Scottish Government).

Sharda, M., Midha, R., Malik, S., Mukerji, S., and Singh, N. C. (2015). Frontotemporal connectivity is preserved during sung but not spoken word listening, across the autism spectrum. Autism Res. 8, 174-186. doi: 10.1002/ aur. 1437

Shochet, I. M., Saggers, B. R., Carrington, S. B., Orr, J. A., Wurfl, A. M., Duncan, B. M., et al. (2016). The Cooperative Research Centre for living 
with autism (Autism CRC) conceptual model to promote mental health for adolescents with ASD. Clin. Child. Fam. Psychol. Rev. 19, 94-116. doi: 10.1007/s10567-016-0203-4

Simpson, K., and Keen, D. (2011). Music interventions for children with autism: narrative review of the literature. J. Autism Dev. Disord. 41, 1507-1514. doi: 10.1007/s10803-010-1172-y

Taylor, A. (2011). Continuity, change and mature musical identity construction: using "Rivers of Musical Experience" to trace the musical lives of six matureage keyboard players. Br. J. Music Educ. 28, 195-212. doi: 10.1017/ S0265051711000076
Conflict of Interest Statement: The authors declare that the research was conducted in the absence of any commercial or financial relationships that could be construed as a potential conflict of interest.

Copyright (ङ 2019 Wilson and MacDonald. This is an open-access article distributed under the terms of the Creative Commons Attribution License (CC BY). The use, distribution or reproduction in other forums is permitted, provided the original author(s) and the copyright owner(s) are credited and that the original publication in this journal is cited, in accordance with accepted academic practice. No use, distribution or reproduction is permitted which does not comply with these terms. 


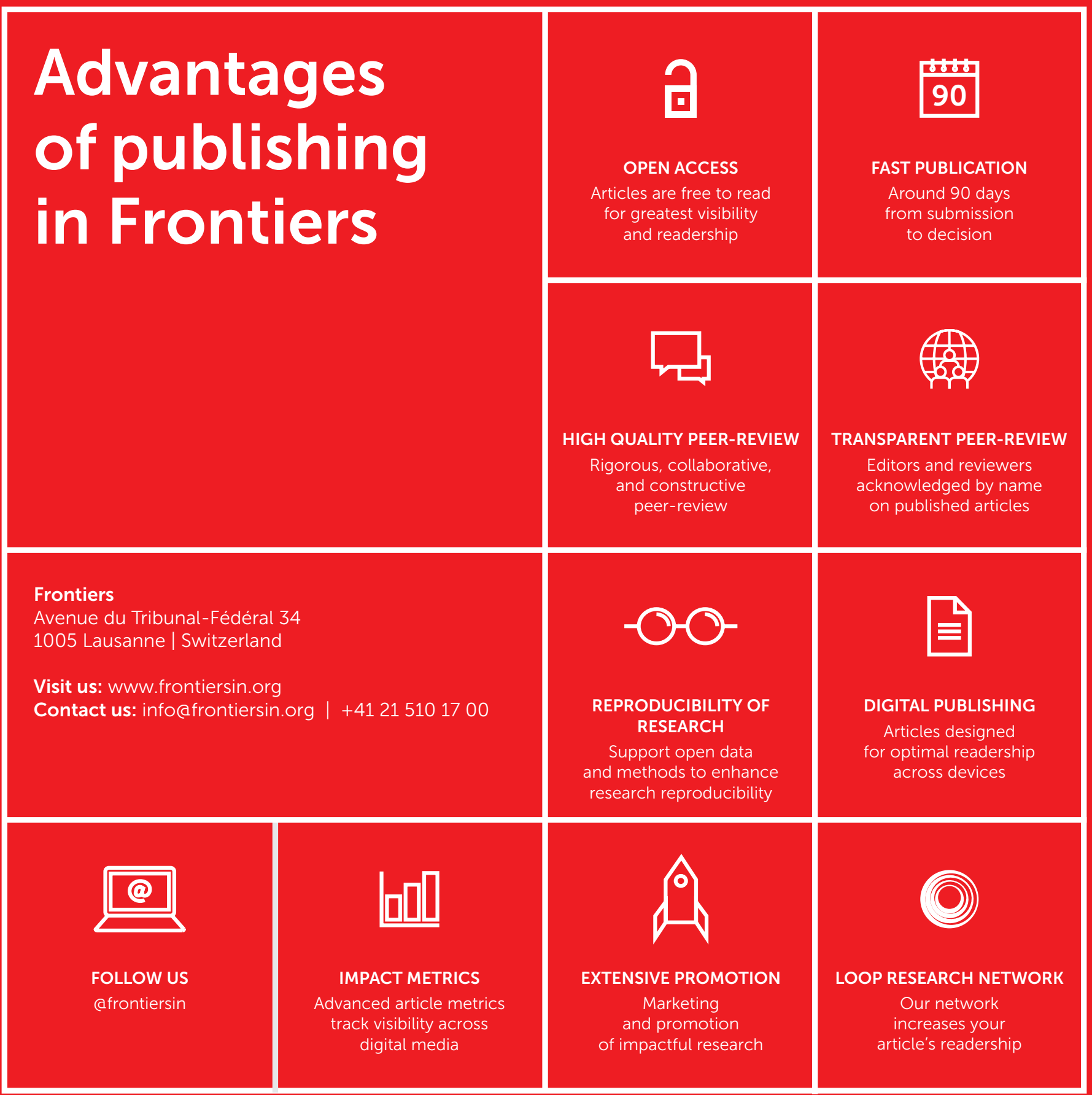

